\title{
European Association of Nuclear Medicine Congress
}

\section{0-24 August 1994, Düsseldorf, Germany}

SATURDAY, August 20, 1994

Pre-Congress Meetings - Opening

\begin{tabular}{|l|l|l|l|l|l|l|l|l|}
\hline & Room 1 & Room 2 & Room 3 & Room 6 & Room 7 & Room 8 & Room 9 & Room 10 \\
\hline 09.00 & & & & $\begin{array}{l}\text { Clinical } \\
\text { PET }\end{array}$ & $\begin{array}{l}\text { Radiation } \\
\text { Protection }\end{array}$ & $\begin{array}{l}\text { Radionuclide } \\
\text { Therapy }\end{array}$ & $\begin{array}{l}\text { Cardiac } \\
\text { SPECT }\end{array}$ & \begin{tabular}{l} 
ISORBE \\
\hline
\end{tabular}
\end{tabular}

10.45 COFFEE BREAK

\begin{tabular}{|l|l|l|l|l|l|l|l|}
\hline 11.15 & & & $\begin{array}{l}\text { Clinical } \\
\text { PET }\end{array}$ & $\begin{array}{l}\text { Radiation } \\
\text { Protection }\end{array}$ & $\begin{array}{l}\text { Radionuclide } \\
\text { Therapy }\end{array}$ & $\begin{array}{l}\text { Cardiac } \\
\text { SPECT }\end{array}$ & \begin{tabular}{l} 
ISORBE \\
\hline
\end{tabular} \\
\hline
\end{tabular}

12.30 LUNCH BREAK

\begin{tabular}{|l|l|l|l|l|l|l|l|}
\hline 13.30 & & & $\begin{array}{l}\text { Clinical } \\
\text { PET }\end{array}$ & $\begin{array}{l}\text { Radiation } \\
\text { Protection }\end{array}$ & $\begin{array}{l}\text { Radionuclide } \\
\text { Therapy }\end{array}$ & $\begin{array}{l}\text { Cardiac } \\
\text { SPECT }\end{array}$ & \begin{tabular}{l} 
ISORBE \\
\hline
\end{tabular} \\
\hline
\end{tabular}

16.00 COFFEE BREAK

\begin{tabular}{|l|l|l|l|l|l|l|l|l|}
\hline 16.30 & & & & & & & $\begin{array}{l}\text { ISORBE } \\
\text { ends 17.00 }\end{array}$ \\
\hline 18.00 & $\begin{array}{l}\text { Official } \\
\text { Opening }\end{array}$ & & & & & & \\
\hline
\end{tabular}

\begin{tabular}{|l|l|}
\hline $\begin{array}{l}19.30- \\
21.00\end{array}$ & WELCOME PARTY - Foyers \\
\hline
\end{tabular}

SUNDAY, August 21, 1994

Scientific - Educational - Technologists - Lunch Sessions

\begin{tabular}{|c|c|c|c|c|c|c|c|c|c|c|}
\hline & Room 1 & Room 2 & Room 3 & Room 6 & Room 7 & Room 8 & Room 9 & Room 10 & Room 26 & Room 27 \\
\hline 09.00 & & $\begin{array}{l}\text { Invited } \\
\text { Lecture I: } \\
\text { PET/ } \\
\text { SPEC T }\end{array}$ & $\begin{array}{l}\text { Invited } \\
\text { Lecture II: } \\
\text { Nuclear } \\
\text { Medicine } \\
\text { in Eastern } \\
\text { Europe }\end{array}$ & & & & & & & \\
\hline 09.45 & & $\begin{array}{l}\text { Invited } \\
\text { Lecture III: } \\
\text { MYOCAR- } \\
\text { DIAL } \\
\text { SCAR }\end{array}$ & $\begin{array}{l}\text { Invited } \\
\text { Lecture IV: } \\
\text { BRAIN/ } \\
\text { PSYCHIA- } \\
\text { TRY }\end{array}$ & & & & & & & \\
\hline
\end{tabular}

10.30 COFFEE BREAK 
SUNDAY, August 21, 1994 (continuation)

\begin{tabular}{|c|c|c|c|c|c|c|c|c|}
\hline 11.00 & $\begin{array}{l}\text { CARDIO- } \\
\text { VASCU- } \\
\text { LAR } \\
\text { Viability I } \\
001 \text { - } 006\end{array}$ & $\begin{array}{l}\text { ONCO- } \\
\text { LOGY } \\
\text { Lympho- } \\
\text { ma } \\
007 \text { - } 012\end{array}$ & $\begin{array}{l}\text { NEURO- } \\
\text { LOGY } \\
\text { Recep- } \\
\text { tors I } \\
013 \text { - } 018\end{array}$ & $\begin{array}{l}\text { TECHNO- } \\
\text { LOGISTS } \\
\text { Opening } \\
\text { Lecture: } \\
\text { Tech's } \\
\text { Role } \\
\text { Session }\end{array}$ & $\begin{array}{l}\text { TUMOR } \\
\text { MAR- } \\
\text { KERS/ } \\
\text { ASSAYS } \\
019 \text { - } 024\end{array}$ & $\begin{array}{l}\text { LUNG I } \\
025-030\end{array}$ & $\begin{array}{l}\text { ENDO- } \\
\text { CRINOL- } \\
\text { OGY } \\
\text { Para- } \\
\text { thyroid } \\
031 \text { - } 036\end{array}$ & $\begin{array}{l}\text { PEDIA- } \\
\text { TRICS } \\
\text { Neurol- } \\
\text { ogy/ } \\
\text { Lung } \\
037 \text { - } 042\end{array}$ \\
\hline
\end{tabular}

\begin{tabular}{|l|l|l|l|}
\hline 12.30 & LUNCH \\
BREAK & $\begin{array}{l}\text { LUNCH } \\
\text { SESSION: }\end{array}$ & LUNCH \\
Amersham & SESSION: & Immuno- \\
& medics \\
\hline
\end{tabular}

\begin{tabular}{|c|c|c|c|c|c|c|c|c|c|}
\hline 14.00 & $\begin{array}{l}\text { CARDIO- } \\
\text { VASCU- } \\
\text { LAR } \\
\text { Viability II } \\
\mathbf{0 4 3 - 0 4 8}\end{array}$ & $\begin{array}{l}\text { ONCO- } \\
\text { LOGY } \\
\text { In Vitro } \\
\text { Studies I } \\
049-054\end{array}$ & $\begin{array}{l}\text { NEURO- } \\
\text { LOGY } \\
\text { Recep- } \\
\text { tors II } \\
\mathbf{0 5 5 ~ - ~} \mathbf{0 6 0}\end{array}$ & $\begin{array}{l}\text { CON- } \\
\text { TINUING } \\
\text { EDUCA- } \\
\text { TION } \\
\text { Lecture } \\
\text { Brain }\end{array}$ & $\begin{array}{l}\text { TECHNO- } \\
\text { LOGISTS } \\
\text { Lecture: } \\
\text { PET } \\
\text { Session }\end{array}$ & $\begin{array}{l}\text { GASTRO- } \\
\text { ENTEROL- } \\
\text { OGY } \\
\text { Liver/ } \\
\text { Biliary } \\
\mathbf{0 6 1 - 0 6 6}\end{array}$ & LUNG II & $\begin{array}{l}\text { ENDO- } \\
\text { CRINOL- } \\
\text { OGY } \\
\text { Thyroid I } \\
\mathbf{0 7 3 - 0 7 8}\end{array}$ & $\begin{array}{l}\text { NEPH- } \\
\text { ROLOGY } \\
\text { Kidney/ } \\
\text { Tracers } \\
\text { 079-084 }\end{array}$ \\
\hline
\end{tabular}

15.30 COFFEE BREAK

\begin{tabular}{|c|c|c|c|c|c|c|c|c|}
\hline 16.00 & $\begin{array}{l}\text { CARDIO- } \\
\text { VASCU- } \\
\text { LAR } \\
\text { Cardio- } \\
\text { myo- } \\
\text { pathy I } \\
\mathbf{0 8 5} \text { - } 090\end{array}$ & $\begin{array}{l}\text { ONCO- } \\
\text { LOGY } \\
\text { Breast } \\
\text { Cancer I } \\
091 \text { - } 098\end{array}$ & $\begin{array}{l}\text { NEURO- } \\
\text { LOGY } \\
\text { Brain I } \\
099-104\end{array}$ & $\begin{array}{l}\text { TECHNO- } \\
\text { LOGISTS } \\
\text { Lecture } \\
\text { Oncology } \\
\text { Session }\end{array}$ & $\begin{array}{l}\text { GASTRO- } \\
\text { ENTEROL- } \\
\text { OGY } \\
\text { Motility/ } \\
\text { Pancreas } \\
\mathbf{1 0 5 - 1 1 0}\end{array}$ & $\begin{array}{l}\text { PHYSICS } \\
\text { PET I } \\
\text { 111 - } 116\end{array}$ & $\begin{array}{l}\text { ENDO- } \\
\text { CRINOL- } \\
\text { OGY } \\
\text { Thyroid II } \\
117-\mathbf{1 2 2}\end{array}$ & $\begin{array}{l}\text { PEDIA- } \\
\text { TRICS } \\
\text { Liver/ } \\
\text { Neuro- } \\
\text { blastoma } \\
\\
\mathbf{1 2 3} \text { - } \mathbf{1 2 8}\end{array}$ \\
\hline
\end{tabular}

MONDAY, August 22, 1994

Scientific - Educational - Technologists - Lunch Sessions

\begin{tabular}{|c|c|c|c|c|c|c|c|c|c|c|}
\hline & Room 1 & Room 2 & Room 3 & Room 6 & Room 7 & Room 8 & Room 9 & Room 10 & Room 26 & Room 27 \\
\hline 08.00 & & & & $\begin{array}{l}\text { CON- } \\
\text { TINUING } \\
\text { EDUCA- } \\
\text { TION }\end{array}$ & $\begin{array}{l}\text { CON- } \\
\text { TINUING } \\
\text { EDUCA- } \\
\text { TION }\end{array}$ & $\begin{array}{l}\text { CON- } \\
\text { TINUING } \\
\text { EDUCA- } \\
\text { TION }\end{array}$ & & & & \\
\hline 09.00 & & $\begin{array}{l}\text { Invited } \\
\text { Lecture V: } \\
\text { TUMOR }\end{array}$ & $\begin{array}{l}\text { Invited } \\
\text { Lecture VI: } \\
\text { RADIO- } \\
\text { CHEM- } \\
\text { ISTRY I }\end{array}$ & & & & & & & \\
\hline 09.45 & & $\begin{array}{l}\text { Invited } \\
\text { Lecture VII: } \\
\text { THYROID }\end{array}$ & $\begin{array}{l}\text { Invited } \\
\text { LectureVIII: } \\
\text { RADIO- } \\
\text { CHEM- } \\
\text { ISTRY II }\end{array}$ & & & & & & & \\
\hline
\end{tabular}

10.30 COFFEE BREAK

\begin{tabular}{|c|c|c|c|c|c|c|c|c|c|}
\hline 11.00 & $\begin{array}{l}\text { CARDIO- } \\
\text { VASCU- } \\
\text { LAR } \\
\text { Myocar- } \\
\text { dial Per- } \\
\text { fusion I } \\
\mathbf{1 2 9} \text { - } 134 \\
\end{array}$ & $\begin{array}{l}\text { ONCO- } \\
\text { LOGY } \\
\text { Gastro- } \\
\text { intestinal I } \\
135 \text { - } 140\end{array}$ & $\begin{array}{l}\text { NEURO- } \\
\text { LOGY } \\
\text { Dementia/ } \\
\text { Cognitive } \\
\text { Functions } \\
141 \text { - } 146\end{array}$ & $\begin{array}{l}\text { CON- } \\
\text { TINUING } \\
\text { EDUCA- } \\
\text { TION } \\
\text { Lecture } \\
\text { Thyroid }\end{array}$ & $\begin{array}{l}\text { TECHNO- } \\
\text { LOGISTS } \\
\text { Lecture: } \\
\text { Kidney } \\
\\
\text { Session } \\
\end{array}$ & $\begin{array}{l}\text { BONE } \\
\text { and } \\
\text { JOINTS I } \\
147 \text { - } 152\end{array}$ & $\begin{array}{l}\text { PHYSICS } \\
\text { Modelling } \\
153 \text { - } 158\end{array}$ & $\begin{array}{l}\text { ENDO- } \\
\text { CRINOL- } \\
\text { OGY } \\
\text { Miscel- } \\
\text { laneous I } \\
159 \text { - } 164\end{array}$ & $\begin{array}{l}\text { NEPH- } \\
\text { ROLOGY } \\
\text { Kidney/ } \\
\text { Function I } \\
\\
165 \text { - } 170\end{array}$ \\
\hline 12.30 & $\begin{array}{l}\text { LUNCH } \\
\text { BREAK }\end{array}$ & $\begin{array}{l}\text { LUNCH } \\
\text { SESSION: } \\
\text { Du Pont }\end{array}$ & $\begin{array}{l}\text { LUNCH } \\
\text { SESSION: } \\
\text { Polaroid }\end{array}$ & & & & & & \\
\hline
\end{tabular}


MONDAY, August 22, 1994 (continuation)

\begin{tabular}{|c|c|c|c|c|c|c|c|c|}
\hline 14.00 & $\begin{array}{l}\text { CARDIO- } \\
\text { VASCU- } \\
\text { LAR } \\
\text { Myocar- } \\
\text { dial Per- } \\
\text { fusion II } \\
171 \text { - } 176\end{array}$ & $\begin{array}{l}\text { ONCO- } \\
\text { LOGY } \\
\text { Urogeni- } \\
\text { tary } \\
177 \text { - } 182\end{array}$ & $\begin{array}{l}\text { NEURO- } \\
\text { LOGY } \\
\text { Epilepsy } \\
\mathbf{1 8 3 - 1 8 8}\end{array}$ & $\begin{array}{l}\text { TECHNO- } \\
\text { LOGISTS } \\
\text { Lecture: } \\
\text { Pediatry } \\
\text { Session }\end{array}$ & $\begin{array}{l}\text { BONE } \\
\text { and } \\
\text { JOINTS II } \\
189 \text { - } 194\end{array}$ & $\begin{array}{l}\text { PHYSICS } \\
\text { Data } \\
\text { Analysis } \\
195-200\end{array}$ & $\begin{array}{l}\text { CHEMIS- } \\
\text { TRY } \\
\text { General I } \\
201-206\end{array}$ & $\begin{array}{l}\text { PEDIA- } \\
\text { TRICS } \\
\text { Kidney/ } \\
\text { Tracers } \\
\mathbf{2 0 7 - 2 1 2}\end{array}$ \\
\hline
\end{tabular}

15.30 COFFEE BREAK

\begin{tabular}{|c|c|c|c|c|c|c|c|c|}
\hline 16.00 & $\begin{array}{l}\text { CARDIO- } \\
\text { VASCU- } \\
\text { LAR } \\
\text { Myocardial } \\
\text { Infarction } \\
\mathbf{2 1 3}-\mathbf{2 1 8}\end{array}$ & $\begin{array}{l}\text { ONCO- } \\
\text { LOGY } \\
\text { Endocrine } \\
\mathbf{2 1 9 - 2 2 4}\end{array}$ & $\begin{array}{l}\text { NEURO- } \\
\text { LOGY } \\
\text { Cerebro- } \\
\text { vascular } \\
\text { Disease } \\
\mathbf{2 2 5} \text { - 231 }\end{array}$ & COST & 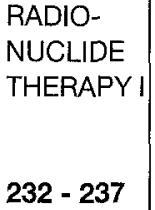 & $\begin{array}{l}\text { HEMA- } \\
\text { TOLOGY I } \\
238 \text { - } 242\end{array}$ & $\begin{array}{l}\text { CHEMIS- } \\
\text { TRY } \\
\text { lodine/ } \\
\text { SPECT } \\
\mathbf{2 4 3 - 2 4 8}\end{array}$ & $\begin{array}{l}\text { PEDIA- } \\
\text { TRIC } \\
\text { INTEREST } \\
\text { GROUP }\end{array}$ \\
\hline
\end{tabular}

ends 17.45

TUESDAY, August 23, 1994

Scientific - Controversy - Poster - Lunch Sessions

\begin{tabular}{|c|c|c|c|c|c|c|c|c|c|c|}
\hline & Room 1 & Room 2 & Room 3 & Room 6 & Room 7 & Room 8 & Room 9 & Room 10 & Room 26 & Room 27 \\
\hline 08.00 & & & & $\begin{array}{l}\text { CON- } \\
\text { TINUING } \\
\text { EDUCA- } \\
\text { TION }\end{array}$ & $\begin{array}{l}\text { CON- } \\
\text { TINUING } \\
\text { EDUCA- } \\
\text { TION }\end{array}$ & $\begin{array}{l}\text { CON- } \\
\text { TINUING } \\
\text { EDUCA- } \\
\text { TION }\end{array}$ & & & & \\
\hline 09.00 & $\begin{array}{l}\text { CARDIO- } \\
\text { VASCU- } \\
\text { LAR } \\
\text { Autono- } \\
\text { mic Car } \\
\text { diac Inner- } \\
\text { vation } \\
\mathbf{2 4 9}-\mathbf{2 5 4}\end{array}$ & $\begin{array}{l}\text { ONCOL- } \\
\text { OGY } \\
\text { Brain I } \\
\\
\mathbf{2 5 5 - 2 6 0}\end{array}$ & $\begin{array}{l}\text { INFEC- } \\
\text { TION } \\
\text { New } \\
\text { Techni- } \\
\text { ques } \\
\mathbf{2 6 1 - 2 6 6}\end{array}$ & & $\begin{array}{l}\text { CON- } \\
\text { TINUING } \\
\text { EDUCA- } \\
\text { TION } \\
\text { Lecture } \\
\text { Heart }\end{array}$ & $\begin{array}{l}\text { TECHNO- } \\
\text { LOGISTS } \\
\text { Lecture: } \\
\text { Quality } \\
\text { Assurance } \\
\\
\text { Session }\end{array}$ & 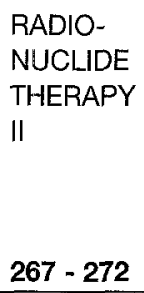 & $\begin{array}{l}\text { PHYSICS } \\
\text { SPECT I } \\
\mathbf{2 7 3 - 2 7 8}\end{array}$ & $279-284$ & $\begin{array}{l}\text { HYPER- } \\
\text { TENSION }\end{array}$ \\
\hline
\end{tabular}

10.30 COFFEE BREAK

\begin{tabular}{|l|l|l|}
\hline 11.00 & Contro- & Contro- \\
& versy & versy \\
Session & Session \\
CARDIOL- & CARDIOL- \\
& OGY & OGY \\
\hline
\end{tabular}

\begin{tabular}{|l|l|l|}
\hline 12.30 & $\begin{array}{l}\text { LUNCH } \\
\text { BREAK }\end{array}$ & $\begin{array}{l}\text { LUNCH } \\
\text { SESSION: } \\
\text { Tumor Im- } \\
\text { munology }\end{array}$ \\
\hline
\end{tabular}

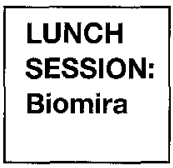

\begin{tabular}{|l|l|c|l|l|}
\hline 13.30 & \multicolumn{1}{|c|}{$\begin{array}{c}\text { POS TER SES S I O N } \\
332-648\end{array}$} & $\begin{array}{l}\text { TECHNOL- } \\
\text { OGISTS } \\
\text { Session }\end{array}$ & P OSTER SES S I O N \\
\hline 15.00 & $\begin{array}{l}\text { Members } \\
\text { Assembly } \\
\text { and Task } \\
\text { Group } \\
\text { Reports } \\
(18.00)\end{array}$ & & \\
&
\end{tabular}


WEDNESDAY, August 24, 1994

Scientific Sessions - Closing

\begin{tabular}{|c|c|c|c|c|c|c|c|c|c|c|}
\hline & Room 1 & Room 2 & Room 3 & Room 6 & Room 7 & Room 8 & Room 9 & Room 10 & Room 26 & Room 27 \\
\hline 09.00 & $\begin{array}{l}\text { CARDIO- } \\
\text { VASCU- } \\
\text { LAR } \\
\text { Ventricu- } \\
\text { lar Func- } \\
\text { tion II } \\
\mathbf{2 9 0 - 2 9 5}\end{array}$ & $\begin{array}{l}\text { ONCOL- } \\
\text { OGY } \\
\text { Miscel- } \\
\text { laneous } \\
\text { II } \\
\mathbf{2 9 6 - 3 0 1}\end{array}$ & $\begin{array}{l}\text { INFEC- } \\
\text { TION } \\
\text { Clinical II } \\
\mathbf{3 0 2} \text { - } \mathbf{3 0 7}\end{array}$ & & $\begin{array}{l}\text { MRI / MRS } \\
308-313\end{array}$ & & $\begin{array}{l}\text { RADIO- } \\
\text { BIOLOGY } \\
\mathbf{3 1 4} \text { - } \mathbf{3 1 9}\end{array}$ & $\begin{array}{l}\text { PHYSICS } \\
\text { Quality } \\
\text { Assurance } \\
\mathbf{3 2 0} \text { - } \mathbf{3 2 5}\end{array}$ & $\begin{array}{l}\text { CHEMIS- } \\
\text { TRY } \\
\text { Technetium } \\
\\
\mathbf{3 2 6}-\mathbf{3 3 1}\end{array}$ & \\
\hline
\end{tabular}

10.30 COFFEE BREAK

\begin{tabular}{|l|l|}
\hline 11.00 & $\begin{array}{l}\text { HIGH- } \\
\text { LIGHTS } \\
\text { LECTURE }\end{array}$ \\
\hline 12.00 & $\begin{array}{l}\text { CLOSING } \\
\text { CERE- } \\
\text { MONY }\end{array}$ \\
\hline
\end{tabular}


Sunday, 21 August 1994

Sunday, 21 August 1994

1

B. L. Gerber, J-L. Vanoverschelde, A. Bol, C. Michel, A. Robert, W. Wijns, J. A. Melin

University of Louvain Medical School, Brussels, Belgium.

COMPARISON OF T1-201 SPECT AND PET TO PREDICT VIABLE MYOCARDIUM IN PATIENTS WITH ISCHEMIC LEFT VENTRICULAR DYSFUNCTION.

Quantitative exercise-redistribution-reinjection Tl-201 SPECT has been shown to accurately predict myocardial viability. Whether quantitative PET imaging improves prediction of functional recovery has not yet been shown in the same patients (pts). Therefore, we measured relative anterior Tl-201 uptake at exercise, redistribution and reinjection by SPECT as well as absolute and relative anterior myocardial blood flow (MBF) with $\mathrm{N}-13$ ammonia and absolute and relative FDG uptake (RFU) during glucose clamp by PET in 24 coronary pts with anterior wall dysfunction before coronary revascularization. $12 \mathrm{pts}$ had viable myocardium, defined as improved regional anterior wall motion score by $>1$ full grade and reduced end-systolic volume at 2D-echo 2 months after revascularization, while the remaining 12 pts had nonviable myocardium. Visual anterior Tl-201 redistribution was present in $7 / 12$ pts (sensitivity $58 \%$ ) with and absent in $8 / 12$ pts (specificity $66 \%$ ) without viable myocardium. Viable myocardium at baseline had higher relative anterior $\mathrm{Tl}-20 \mathrm{l}$ uptake at redistribution $(51 \pm 11$ vs $37 \pm 12 \%, p<.02)$ and at reinjection $(53 \pm 12$ vs $40 \pm 13 \%, p<.03)$ than nonviable myocardium. With a computed cutoff value of $47 \%$, relative thallium uptake at reinjection correctly identified $9 / 12$ pts with and $9 / 12$ pts without viable myocardium ( $75 \%$ sensitivity and specificity). With PET, viable myocardium had higher relative $(70 \pm 15$ vs $51 \pm 9 \%, p<.002)$ and absolute MBF (97 \pm 31 vs $58 \pm 16 \mathrm{ml} / \mathrm{min} / 100 \mathrm{~g}, \mathrm{p}<.001)$, higher relative ( $77 \pm 13$ vs $44 \pm 12 \%, \mathrm{p}<.001)$ and absolute FDG uptake ( $46 \pm 20$ vs $22 \pm 9$ $\mu \mathrm{mol} / \mathrm{min} / 100 \mathrm{~g}, \quad \mathrm{p}<.002$ ) than nonviable myocardium. According to a computed cutoff value of $60 \%$ for RFU, 11/12 pts with (sensitivity $92 \%$ ) and $11 / 12$ pts without (specificity $92 \%$ ) viable myocardium were correctly identified. All pts misclassified by Tl-201 were correctly identified by PET. Thus, FDG PET imaging during glucose clamp has better predictive accuracy for identification of viable myocardium than quantitative Tl-201 exercise-redistribution-reinjection imaging.

2

J. vom Dahl, C. Altehoefer, F. H. Sheehan, I. Beilin, P. Buechin, R. Uebis, B. J. Messmer, U. Buell, P. Hanrath

Depts. of Internal Medicine I, Nuclear Medicine, Cardiothoracic Surgery, RWTH Aachen, D, Univ. of Washington, Seattle, USA

QUANTITATIVE COMPARISON OF REGIONAL MYOCARDIAL FUNCTIONAL RECOVERY FOLLOWING CORONARY ANGIOPLASTY OR BYPASS SURGERY IN RELATION TO PREOPERATTVE MYOCARDIAL VIABILITY

200 patients (pts) underwent assessment of myocardial viability by combined nuclear imaging using resting SPECT with Tc-99m MIBI for evaluation of myocardial perfusion and metabolic imaging by PET and F-18 FDG. During a $17 \pm 7$ month follow-up 108/200 pts $(53 \%)$ underwent coronary revascularization either by PTCA (46 pts) or CABG (62 pts) while 90 pts were treated medically and 2 pts underwent cardiac transplantation. $72 / 108$ pts had follow-up angiography $6 \pm 1$ months after revascularization ( 35 pts with $3 \pm 1$ CABG, 37 pts with single-vessel PTCA). Results of regional wall motion (RWM) comparison (RWM1=baseline, RWM2=follow-up) using the centerline method are expressed in SD of a normal population with negative values indicating hypokinesis. RWM comparison in regions with reduced perfusion (regional MIBI uptake below -2SD of a normal population) is given for three groups categorized by preoperative viability imaging: A: FDG $>70 \%$ of normal perfused region (viable with "mismatch"), B: FDG $51-70 \%$ ("intermediate") and C: FDG $\leq 50 \%$ ("scar")

\begin{tabular}{|l|c|c|c|c|c|c|}
\hline & \multicolumn{3}{|c|}{ PTCA } & \multicolumn{3}{c|}{ CABG } \\
\cline { 2 - 7 } & RWM1 & RWM2 & $\Delta$ RWM & RWM1 & RWM2 & $\Delta$ RWM \\
\cline { 2 - 7 } $\mathbf{A}$ & $-2.7 \pm 1.0$ & $-1.6 \pm 1.1$ & $1.1 \pm 1.0$ & $-2.2 \pm 1.0$ & $-0.6 \pm 0.8$ & $1.6 \pm 1.0^{*}$ \\
B & $-1.4 \pm 0.9 \dagger$ & $-0.9 \pm 1.9 \dagger$ & $0.5 \pm 1.1 \dagger$ & $-1.1 \pm 0.8 \dagger$ & $-1.4 \pm 0.7 \dagger$ & $-0.3 \pm 0.6 \dagger$ \\
$\mathbf{C}$ & $-2.2 \pm 0.7$ & $-2.2 \pm 0.5$ & $0 \pm 0.3 \dagger$ & $-2.4 \pm 0.7$ & $-2.8 \pm 0.6 \dagger$ & $-0.4 \pm 0.5 \dagger$ \\
\hline
\end{tabular}

Following PTCA, EF improved from $49 \pm 11$ to $52 \pm 10 \%$ and after CABG from $43 \pm 11$ to $50 \pm 12 \%$ ( $<<0.05$ ). Thus, functional outcome of RWM was similar following either PTCA or CABG for the tissue subgroups. However, recovery of RWM in "mismatch" regions and of global function was slightly better after CABG which might indicate more severe preoperative ischemia or the effect of complete revascularization in patients with multivessel disease.
3

J.J. Bax, J.H. Cornel*, F.C. Visser, P.M. Fioretti*, A. van Lingen, G.J.J. Teule, C.A. Visser.

Free University Hospital Amsterdam and * Academic Hospital Rotterdam, The Netherlands.

F18-FLUORODEOXYGLUCOSE AND SPECT TO PREDICT RECOVERY OF WALL MOTION ABNORMALITIES.

Recently, we have demonstrated the feasibility of imaging myocardial uptake of F18-fluorodeoxyglucose (FDG) with SPECT. The aim of the present study was to determine whether FDG SPECT could predict reversibility of regional wall motion (RWM) abnormalities in patients undergoing revascularization. We studied 15 patients prior to revascularization ( 9 CABG and 6 PTCA); all had RWM abnormalities as assessed with 2D echo. Each patient underwent resting thallium-201 SPECT (to evaluate regional perfusion), and a FDG SPECT during a hyperinsulinemic glucose clamp. After revascularization $2 \mathrm{D}$ echo was repeated to study change in RWMA (15 segment analysis). The segmental wall motion was analyzed using a $0-3$ score index $(0=$ normo, $1=$ hypo, $2=$ akinesia and $3=$ dyskinesia). For comparison of SPECT imaging with 2D echo, the myocardium was divided in 5 corresponding segments (apex, anterior, lateral, inferior and septal). The SPECT images were analyzed using PET criteria for viable tissue (hypoperfusion with relatively enhanced FDG uptake) and necrotic tissue (both decreased perfusion and FDG uptake). We identified 13 viable and 18 necrotic segments. In 10 viable segments RWM recovered, and in 16 necrotic segments no change in RWM abnormalities was seen. RWM score decreased in viable segments after revascularization from $2.8 \pm 1.7$ to $1.2 \pm 1.2(p<0.005)$. In the necrotic segments the RWM score remained unchanged: $3.3 \pm 2.1$ vs $3.3 \pm 1.8 \quad(p=N S)$. The sensitivity of FDG SPECT imaging to detect improvement in RWM abnormalities was $83 \%$ and the specificity was $84 \%$. These data suggest that FDG SPECT can identify viable myocardium.

4

J. vom Dahl, C. Altehoefer, U. Buell, P. Hanrath

Depts. of Internal Medicine I and Nuclear Medicine, RWTH Aachen, Germany

THE CORRELATION BETWEEN MYOCARDIAL VIABILITY IDENTIFIED BY F-18 FDG PET AND THE SEVERITY OF PERFUSION DEFECTS AS ASSESSED BY TC-99m MIBI SPECT VARIES WITH THE DEFECT LOCALIZATION

Maintained glucose metabolism assessed by FDG and PET indicates preserved viability of hypoperfused and dysfunctional myocardium. To evaluate the regional relationship between the severity of MIBI perfusion defects and FDG uptake, resting MIBI SPECT studies of 174 patients $(85 \%$ male, $58 \pm 9$ years, $84 \%$ with previous myocardial infarction) were compared with data acquired by PET and FDG. MIBI and FDG uptake was evaluated by a computerized semiquantitative analysis for 13 left ventricular regions. The region of maximal MIBI uptake was set as $100 \%$ for both data sets and regional uptake of both tracers was normalized to this reference region. FDG uptake $>70 \%$ was defined as lower threshold of preserved viability (v) and FDG uptake $<50 \%$ was categorized as definite evidence for scar (s) tisssue. Results are given in the territories of the 3 coronary arteries and are divided in moderate (31-50\% of individual maximum) and severe $(\leq 30 \%)$ defects.

\begin{tabular}{|c|c|c|c|c|c|c|c|c|c|}
\hline $\begin{array}{c}\text { MIBI } \\
\text { defect }\end{array}$ & \multicolumn{3}{|c|}{ LAD } & \multicolumn{3}{c|}{ LCx } & \multicolumn{3}{|c|}{ RCA } \\
\hline moderate & 191 & 13 & 5 & $\mathrm{n}$ & $\mathrm{v}$ & $\mathrm{s}$ & $\mathrm{n}$ & $\mathrm{v}$ & $\mathrm{s}$ \\
severe & 69 & 4 & 87 & 39 & $23^{*}$ & 39 & 102 & $39^{* * *}$ & 27 \\
\hline
\end{tabular}

There were significantly more hypoperfused but viable regions in the $L C x$ and RCA territories as compared to the LAD territory where the MIBI defect severity reflected more accurately myocardial viability as identified by FDG PET. Regression analysis in regions with reduced MIBI-uptake (<-2SD of a normal population) revealed a correlation between MIBI and FDG uptake with $r=0.60$ in the LAD, $r=0.43$ in the $\mathrm{LCx}$, and $\mathrm{r}=0.35$ in the RCA territory.

These regional differences are probably due to soft tissue attenuation artifacts and have to be considered in the clinical evaluation of patients with coronary artery disease if the severity of MIBI defects is used as an indirect parameter of myocardial viability. 
5

B.L. Gerber, J.-L. Vanoverschelde, A. Bol, C. Michel, W. Wijns, J.A. Melin

University of Louvain Medical School, Brussels, Belgium

PREDICTION OF VIABLE MYOCARDIUM BY 0-15 WATER AND N-13 AMMONIA QUANTITATIVE PET. WHICH ARE THE PARAMETERS TO DERIVE ?

Myocardial blood flow (MBF) estimates with PET and O-15 water or N-13 ammonia have been shown to distinguish viable from nonviable myocardium. To evaluate whether other model parameters, such as the recovery coefficient in the water model and $\mathrm{K} 1 / \mathrm{K} 2$ from the ammonia model, may provide additional information, 14 coronary patients (pts) with anterior wall dysfunction underwent PET with $\mathrm{O}-15$ water and $\mathrm{N}-13$ ammonia before revascularization. In addition to $\mathrm{MBF}, \alpha$, the recovery coefficient from the 1-compartment water model, and K2, the backward transport rate constant from the 3-compartment ammonia model, were computed. Because overestimation of MBF has been noted in infarcted segments with 0-15 water and the standard 3-parameter model (WA 1) where recovery is a fitted parameter, MBF was also determined from a water model with a priori knowledge of finite resolution effects (WA 2). Five pts had viable myocardium, defined as improved regional anterior wall motion score by $>1$ full grade and reduced end-systolic volume at 2D-echo 2 months after revascularization, while the remaining $9 \mathrm{pts}$ had nonviable myocardium. Baseline PET parameters in the 2 groups are shown in table:

\begin{tabular}{|l|c|c|c|}
\hline & Viable & Nonviable & p value \\
\hline K1 & $1.10 \mathrm{ml} / \mathrm{min} / \mathrm{g}$ & $0.53 \mathrm{ml} / \mathrm{min} / \mathrm{g}$ & $<.002$ \\
\hline $\mathrm{K} 2$ & $0.34 \mathrm{ml} / \mathrm{min} / \mathrm{g}$ & $0.47 \mathrm{ml} / \mathrm{min} / \mathrm{g}$ & N.S. \\
\hline $\mathrm{K} 1 / \mathrm{K2}$ & 3.3 & 1.3 & $<.001$ \\
\hline $\mathrm{MBF}$ (WA 1) & $0.93 \mathrm{ml} / \mathrm{min} / \mathrm{g}$ & $0.91 \mathrm{ml} / \mathrm{min} / \mathrm{g}$ & N.S \\
\hline$\alpha$ (WA 1) & 0.77 & 0.55 & $<.02$ \\
\hline MBF (WA 2) & $1.06 \mathrm{ml} / \mathrm{min} / \mathrm{g}$ & $0.72 \mathrm{ml} / \mathrm{min} / \mathrm{g}$ & $<.03$ \\
\hline
\end{tabular}

Thus, viable myocardium is associated with higher MBF by ammonia or WA 2 , higher $\mathrm{K} 1 / \mathrm{K} 2$ and a larger recovery coefficient than nonviable myocardium.

6

M. Reinhardt, U. Ganschow*, E. Vester ${ }^{*}$, A. Wirrwar, B. Strauer*, H. Vosberg, H.-W. Müller-Gärtner

Departments. of Nuclear Medicine and "Cardiology, Heinrich-Heine-University Düsseldorf, FRG

MISMATCH BETWEEN GLUCOSE METABOLISM AND PERFUSION IN ARRYTHMOGENIC FOCI OF PATIENTS WITH VENTRICULAR TACHYCARDIAS

The aim of this study was to investigate myocardial glucose metabolism and perfusion in the arrhythmogenic foci of patients with monofocal ventricular tachycardias (VT).

We studied 10 patients with VT. The arrhythmogenic foci were localized by endocardial catheter mapping of the left ventricular wall (ECM). Myocardial glucose metabolism was evaluated using the PET-camera PC-4096 and F-18-FDG after oral glucose load. Myocardial perfusion was determined with Tl- or MIBI-SPECT. Perfusion and glucose metabolism were analyzed in 19 myocardial regions using a fivepoint rating scale ranging from missing to maximal accumulation. A mismatch between myocardial glucose metabolism and perfusion was diagnosed when the FDG score was at least two points higher than the perfusion score.

In 9 of 10 patients at least one area of mismatch between perfusion and glucose metabolism was found. The ECM localization of the VT foci was in 6 of these patients within zones of mismatch; in 2 patients the ECM localization of the VT focus was in the margin of such a zone; one patient had no colocalization of the arrhythmogenic focus and his zones of mismatch.

The data suggest that most arrhythmogenic foci are localized in regions of hibernating myocardium. The noninvasive localization of zones of mismatch between perfusion and glucose metabolism may be of importance in planning revascularization or electrical ablation of foci in vT patients.
A. Mastorakou, C. Glannakenas, J. Tselfes, D. Apostolopoulos, T. Gorilas, N. Mastronikolis, M. Matsouka*, P.J. Vassilakos

Depts. of Nuclear Medicine and "Hematology, Regional University Hospital, University of Patras, Medical School, Patras, Greece.

SCINTIGRAPHIC IMAGING OF MALIGNANT LYMPHOMAS WITH 111 -InOCTREOTIDE : COMPARISON TO OTHER IMAGING MODALITIES.

The object of this continuing study was to assess the sensitivity of 111 . In labeled Octreotide $(\mathrm{OC})$ in the detection of malignant lymphomas and/or the extent of known disease. The results were compared to CT and/or MRI, U/S and extent of known disease.
$67-\mathrm{Ga}$ Citrate scintigraphy.

Thirty seven patients, mean age $60 y$, (range 23-78) underwent planar scintigraphy, ( 9 also underwent SPECT), 6 and $24 \mathrm{hrs}$ after the injection of 2.8 $3,0 \mathrm{mCi}$ of $111-\mathrm{In}-\mathrm{OC}, 34$ patients had histologically confirmed Iymphomas 113 Hodgkin's disease and $21 \mathrm{NHL}$, while the remaining 3 patients were under investigation for suspected lymphoma confirmed histologically as Hodgkin's lymphoma in 2 and infectious mononucleosis in 1). All patients had previous investigation with other modalities ( $C T, U / S$ and/or MRI ). In 30 patients whole body 67-Ga-Citrate planar scans were performed at 24 and $48 \mathrm{hrs}$ p.i. Superimposition imaging techniques were applied between SPECT transaxial slices and the corresponding CT sections.

The OC-scan was true positive in 34 patients $(91.9 \%)$ in 1 case it proved true negative (inf mononucleosis), while it was false negative in 2 patients (5.4\%). Concerning the extent of the disease, 109 different sites of 111-In-OC accumulation were detected. In 7 patients $O C$ led to re-staging of the disease, while in 12 patients OC confirmed the presence of the disease in suspected sites. In comparison to other modalities OC proved superior in detecting more lesions in 17 of the 34 positive cases $(50 \%)$, similar in 12 patients (35.3\%) and inferior in $5(14.7 \%)$ cases. The 67-Ga-Citrate scans failed to detect the disease in 6 of 30 patients and proved inferior in defining the extent of the disease. Lesions in the upper abdominal region were more readily detected by SPECT in 9 cases. Superimposition techniques permitted the comparative imaging of the lesions. All the above mentioned findings were confirmed either by needle biopsy and/or by surgery

In conclusion it appears that $O C$ is superior to the conventional modalities in Iymphoma imaging often leading to the restaging of the patients. Also, with the implementation of superimposition techniques and SPECT the detection of the disease and its extent is much enhanced. This becomes even more evident in sites located within the abdominal region.

\section{8}

V. Ivanđević, C. Nauck, B. Wörmann, J. Meller, D. sandrock, A. Kögler, W. Hiddemann, D.L. Munz, D. Emrich.

Div. of Nuclear Medicine and Div. of Haematology and Oncology, University of Göttingen, Germany

SOMATOSTATIN RECEPTOR SCINTIGRAPHY (SRS) WITH 111-IN-PENTETREOTIDE IN HODGKIN- (HL) AND NONHODGKIN LYMPHOMAS (NHL).

The aim of our study was to assess the value of SRS in 21 patients $(9$ females and 11 males, age 24-86 years) suffering from malignant lymphomas. Five patients had HL (1 remission), 8 low-grade (1 remission), and 8 high-grade NHL. A total of 105 lesions was verified during routine staging. Planar whole-body images were taken 4 and 24 hours after i.v. infusion of $110 \mathrm{MBq}$ of $111-\mathrm{In}-$ Pentetreotide.

The patient-based analysis yielded an overall sensitivity of $89 \%$. 4 of 5 patients with HL were true positive, 1 was true negative. 13 of 16 patients with NHL were true positive, 1 false positive, and 2 false negative. The lesion-based analysis revealed different results; in the lowgrade NHL group there were 17 true positive, 2 false positive, and 29 false negative lesions. The high-grade NHL contained 12 true positive and 30 false negative foci, whereas in the HL group there were 10 true positive and 7 false negative lesions. The respective sensitivities were $37 \%$, $29 \%$, and $59 \%$. All the foci seen on SRS had been known before scintigraphy.

In conclusion, SRS does not seem to improve the diagnostic management of malignant lymphomas. This may be due to low receptor densities and/or to the presence of receptor subtypes for which 111-In-Pentetreotide has low affinities. 
9

L. Sarda, O. Mundler, JM. Zini, M. Duet, B. Berolatti, S. Benelhadj, G. Tobelem, Y. Grall.

Depts. of Nuclear Medecine and Hematology, Lariboisiere hosp., Paris France.

\section{In-OCTREOSCAN SOMATOSTATIN RECEPTOR SCINTIGRAPHY IN MALIGNANT LYMPHOMAS.}

To evaluate the interest of Somatostatin Receptors Scintigraphy (SRS), 13 patients with histologically proven nonhodgkin lymphomas were investigated before treatment. Static acquisitions over the whole body were performed 4 and 24 hrs after $148 \mathrm{MBq}$ IV administration of 111 In-Octreoscan. SRS was compared with clinical and conventional imaging data: chest $X$-ray $(n=13)$, abdominal and thoracic computed tomography $(n=13)$, abdominal ultra-sonography $(n=2)$. Only 25 of the 41 confirmed tumoral sites $(61 \%)$ were visualized with SRS. Detection accuracy was higher for extra-abdominal $(77 \%)$ than for abdominal sites $(33 \%)$. Tumoral uptake of 111 In-Octreoscan, visually estimated, was highly variable. In 1 patient, none of the tumoral sites (mediastin, liver, coeliac area) was visualized. In patients with no or low tracer uptake $(n=7)$, only $52 \%$ of the confirmed localizations were detected ( $83 \%$ for the other 5 patients). 6 previously unknown localizations were visualized in 5 patients with multiple other confirmed tumoral sites, suggesting a potential additional diagnostic value. Histological confirmation was not yet performed. Conclusion: because tumoral tracer uptake in malignant lymphomas is highly variable, SRS alone is not enough reliable for an exhaustive evaluation of tumoral extension. This probably reflects different patterns in Somatostatin receptors distribution, as demonstrated by in vitro studies. SRS seems to be more accurate for the detection of extraabdominal tumoral sites and may be complementary with conventional imaging.

10

J.A.Schönberger, J.C.Stollfuß, F.Kocher, J.Tobuschat, M.Bangerter, N.Frickhofen, H.Heimpel, S.N.Reske

Department Nuclear Medicine and Department Hematology, University of Ulm, Germany

WHOLE BODY 18-FDG-PET FOR STAGING OF MALIGNANT LYMPHOMAS

Malignant lymphomas are characterized by highly increased glucose utilisation, wich can be imaged non-invasively with F-18fluorodeoxyglucose (FDG) and positron emission tomography (PET). We thus evaluated the feasibility of whole body PET with FDG for lymphoma staging. Seventeen untreated patients (pts) - seven pts with malignant NonHodgkin's lymphoma (NHL) and ten pts with Hodgkin's disease (HD) were included in the study. Whole body positron emission tomography using FDG was performed with an ECAT 931-08-12 scanner (Siemens-CTI, Knoxville, TN). After transmission scanning for attenuation correction, 300 $\pm 100 \mathrm{MBq}$ FDG were administered i.v. and emission scans were started $45 \mathrm{~min}$ later. All patients were in fasting state for at least $6 \mathrm{hrs}$ prior to the study. Iterative reconstruction was performed and images were reorientated in three views. Lymph node groups visualized by FDG-PET were compared to CT-findings and overall clinical staging. All CT lesions could be demonstrated by PET with high contrast. In 8 pts in stage II ( 3 NHL, 5 HD), $5 / 6$ pts in stage III ( 3 NHL, $3 \mathrm{HD}$ ) and in $1 / 2$ pts in stage IV $(1$ NHL, $1 \mathrm{HD}$ ) PET-staging was concordant to CT-findings and clinical staging. In $1 / 1$ cases in stage I, $4 / 8$ cases in stage II, $1 / 3$ cases in stage III and in $1 / 2$ in stage IV addional lesions, previously unknown, were found by PET leading to an upstaging of a total of 4 patients. Additional lesions were mainly found in abdominal localisations. Furthermore extranodal affections of liver, spleen, bone marrow, lung and breast - which were histologically verified - could be also demonstrated by FDG-PET. Lymphoma invasion of a main bronchus was not correctly diagnosed by PET leading to a downstaging in one case (stage IV). We conclude that PET with FDG provides for reliable and fast staging in patients with untreated malignant lymphoma and Hodgkin's disease. Our results encourage further evaluation of this promising technique for diagnostic workup of patients with suspected lymphoma especially in early tumour stages.
11

A. Dimitrakopoulou-Strauss, L.G. Strauss, H. Goldschmidt, W.J. Lorenz, W. Maier-Borst, G. van Kaick

Department of Oncologic Diagnosis and Therapy, German Cancer Research Center, Heidelberg, FRG

EVALUATION OF TUMOR METABOLISM AND MULTI DRUG RESISTANCE IN PATIENTS WITH TREATED MALIGNANT LYMPHOMAS

One of the main problems in patients with lymphomas after first line treatment is the diagnosis of residual viable tumor tissue for further treatment planning. We used PET in patients with recurrent Hogkin's (HL) and Non-Hodgkin's
(NHL) disease to quantify the tumor perfusion and metabolism prior and after (NHL) disease to quantify the tumor perfusion and metabolism prior and after second line chemotherapy. The primary aim of the study was the detection and quantification of residual tumor metabolism during restaging in order to optimize the further chemotherapeutic treatment protocol. Furthermore, followup studies with PET were used to evaluate the effect of the treatment protocol. SPECT with TC-99m-Sestamibi was used for the evaluation of mult drug resjstance (MDR). The PET results were compared to morphologic (CT) and clinical data. The preliminary evaluation includes 32 ( $\mathrm{HL}, 15$ patients) and 33 ( $\mathrm{NHL}, 17$ patients) lesions. FDG (130-350 MBq) is injected for tumor metabolism studies, while $0-15$ labeled water $(2088-2811 \mathrm{MBq})$ is administered to assess the tumor perfusion. SPECT studies were performed $30 \mathrm{~min}$ after intravenous injection of $370-555 \mathrm{MBq}$ Tc-99m-Sestamibi for the evaluation of MDR.

The quantitative evaluation of the iteratively reconstructed PET data was performed using a ROI-technique. SUV were calculated from the ROI data. The perfusion values ranged from 1.63 to 3.31 SUV in all lesions, while significantly lower values were calculated for the normal soft tissue ( 0.64 SUV, mean value). The metabolism was increased in the malignant lesions and with a large range between 1.42-and 13.35 SUV. The FDG uptake in normal soft tissue and scar tissue was $0.66-0.90$ SUV. No significant correlation was noted for tumor perfusion and metabolism. Furthermore, no significant differences were found for $\mathrm{HL}$ and $\mathrm{NHL}$.

We were able to compare the FDG uptake prior and after one chemotherapeutic cylce (dexamethasone, BCNU, etoposide, alexane, melphalan for HL; alexane, mitoxantrone for $\mathrm{NHL}$ ). While the tumor metabolism was significantly lower after therapy in 5 of 9 lesions, no change was noted in 3 tumors. A decrease in tumor metabolism and no change in morphology (CT) was noted in three lesions responding to therapy.

The accumulation of Tc-99m-Sestamibi and the FDG metabolism prior and after therapy was compared in 10 patients. Again, the change in FDG metabolism correlated with the restaging data. The uptake of TC-99m-Sestamibi was predictive for MDR. Progression of disease was observed in four patients without Sen stamibi accumulation, while response to chemotherapy or stable disease was associated with Sestamibi uptake ( $n=4$ )

Our results show, that PET studies with FDG are a promising tool for both diaOur results show, that PET studies with FDG are a promising tool for both diagnostic evaluation during restaging and therapy planning in $\mathrm{HL}$ and $\mathrm{NHL}$ patithe size and location of the lesion permit a qualitative evaluation.

12

B.M. Dohmen, R. Bares, J. Ammon, R. Osieka, U. Buell

Departments of Nuclear Medicine, Radiotherapy and Intemal Medicine IV, Technical University of Aachen, Germany

\section{FDG-PET FOR ASSESSEMENT OF BONE MARROW METABOLISM IN CANCER PATIENTS}

Energy metabolism of hematopoetic bone marrow (BM) is mainly based on glucose utilization. Aim of the present study was to evaluate FDG-PET for characterization of BM metabolism of cancer patients $(n=26)$. Static PET was performed $46 \pm 18$ minutes after i.v. injection of $224 \pm 68 \mathrm{MBq}$ FDG. Differential uptake ratios (DUR) for bone marrow of the thoracic or lumbar spine were determined using the ROI technique $(21 \pm 12 \mathrm{ROI} / \mathrm{scan}$; coronal and sagittal slices). BM diagnosis (normal, normal after chemotherapy [0-90 d], malignant infiltration, stimulation before planned autologues BM transplantation, irradiated BM) was established by histology, CT/MRI and/or clinical followup.

Compared to normal (DUR: $1.93 \pm 0.43 ; n=13$ ), increased FDGuptake was observed in patients with malignant $\mathrm{BM}$ infiltration (DUR: $3.41 \pm 0.43 ; n=4$ ) and after external BM-stimulation (DUR: $3.86 ; n=1$, however, not during physiological BM-regeneration (DUR: $1.94 \pm 0.55 ; n=6$ ). Radiotherapy caused a marked decrease of FDG-uptake (DUR: $1.19 ; n=2$ ).

We conclude that FDG-PET is suitable for characterizing hematopoetic bone marrow metabolism. It might be useful to improve noninvasive treatment control in patients with neoplastic bone marrow involvement. 
13

K. Tatsch, M. Kerner, J. Schwarz, W.H. Oertel, C.-M. Kirsch, K. Hahn

Department of Nuclear Medicine and Department of Neurology, Klinikum Grosshadern, University of Munich, FRG

ROC-ANALYSES OF ${ }^{123}$ I-IBZM SPECT DATA TO OPTIMIZE DISCRIMINATION BETWEEN PARKINSONIAN SYNDROMES

This study employs ROC (receiver operator characteristic) methodology a) to evaluate the diagnostic performance of IBZM-SPECT for discriminating idiopathic (IPS) from other parkinsonian syndromes (PS) and b) to investigate how pretreatment and variation of the diagnostic system (S/FC vs. S/OC ratios) may alter the discrimination capacity.

126 pts with IPS (de novo: 29 , pretreated: 25 ) and PS (de novo: 31 , pretreated: 41 ) were investigated with SPECT $2 \mathrm{~h}$ after injection of 185 MBq I-123 IBZM. Transverse slices corrected for attenuation were used to calculate striatal to frontal (S/FC) and occipital cortex (S/OC) ratios. Diagnosis of IPS/PS was established clinically by pos./neg. response to apomorphine and/or longterm dopaminergic therapy. According to this classification SPECT data were categorized for various confidence thresholds. The relevant results are listed below: de novo patients pretreated patients

$\begin{array}{ccccc}\text { thresholds } & \text { S/FC } & \text { S/OC } & \text { S/FC } & \text { S/OC } \\ \text { for the ratios: } & \text { SN/SP } & \text { SN/SP } & \text { SN/SP } & \text { SN/SP } \\ 1.42 & 97 / 42 & 66 / 45 & 92 / 24 & 76 / 34 \\ 1.44 & 97 / 52 & \mathbf{6 2 / 6 5} & 92 / 32 & 72 / 44 \\ 1.46 & 93 / 61 & \mathbf{5 9 / 7 4} & 92 / 37 & \mathbf{6 8 / 5 4} \\ 1.48 & \mathbf{9 3 / 6 8} & 55 / 81 & \mathbf{7 6 / 5 1} & \mathbf{5 6 / 5 6} \\ 1.50 & \mathbf{8 3 / 7 7} & 41 / 81 & \mathbf{6 4 / 6 3} & 40 / 63 \\ 1.52 & 55 / 87 & 35 / 90 & \mathbf{6 0 / 6 8} & 28 / 68\end{array}$

The diagnostic performance of IBZM-SPECT for discriminating idiopathic from other parkinsonian syndromes is acceptable (sensitivity: $83 \%$, specificity: $77 \%$ ). Optimal thresholds varied in the different entities. Discrimination capacity was evidently superior for using S/FCratios compared to S/OC-ratios, thus supporting the $\mathrm{FC}$ as reference De novo patients may be classified more accurately than those pretreated with dopaminergic drugs prior to the SPECT study.

\section{4}

B. Mazière1, C. Lochl, C. Halldin 2 , M. Bourguignonl, A. Syrotal, M. Mazièrel, L. Farde 2 1- Service Hospitalier Frédéric Joliot, DRIPP/CEA, F-91406 Orsay, 2- Department of Clinical Neurosciences, Karolinska Institute, S-17176 Stockholm.

SPECT IMAGING OF DOPAMINE D-2 RECEPTORS IN BABOON AND HUMAN USING I-123 NCQ-298.

Previous studies have shown that NCQ-298, a salicylamide ((S)-3iodo- $\mathrm{N}$-[(1-ethyl-2-pyrrolinidyl)methyl]-5,6-dimethoxysalicylamide labelled with $\mathrm{I}-125$ and $\mathrm{I}-123$, possesses the prerequisite pharmacological properties to be used in vivo for the visualisation of dopamine D2 receptors (Hall et al, 1991, Psychopharmacology, $103 ; 6-18$ ).

In SPECT studies in baboons, $1 \mathrm{~h}$ after injection of I-123 NCQ-298 the radioactive concentration in the striatum reached a plateau that remained constant for $4 \mathrm{~h}$. The striatum to cerebellum radioactivity concentration ratio values: $\mathrm{S} / \mathrm{C}$ (uncorrected for partial volume effects) were 10 and $2 l$ at $1 \mathrm{~h}$ and $4 \mathrm{~h}$, respectively.

In humans, brain SPECT studies were performed with a double head DST Sophy y-camera equipped with ultra high resolution parallel hole collimators, after i.v. administration of $150 \mathrm{MBq}$ of $1-123 \mathrm{NCQ}-298$. Sequential acquisitions of 64 images in $128 * 128$ (pixel $=3.4 \mathrm{~mm}$ ) were obtained over $360^{\circ}$ with the smallest circular orbit. Tomographic images were reconstructed by filtered back projection with resolution recovery Butterworth apodisation. The observation of the radioactivity distribution in the brain showed an accumulation of the radioactivity in the basal ganglia as soon as $0.5 \mathrm{~h}$ after injection with a maximum at $3-4 \mathrm{~h}$; on the contrary the radioactivity in the others structures cleared continuouly from an early maximum $(10 \mathrm{~min}) .6 \mathrm{~h}$ post injection, the $\mathrm{S} / \mathrm{C}$ ratio value reached 40 .

These results suggest that I-123 NCQ-298 has the potential of being used for clinical SPECT investigations of cerebral D-2 receptors in the human brain.
15

M. Jacobt, Th. Müller*' D. Biert, W. Kuhn*', +Clinic and Policlinic for Nuclear Medicine, University Essen, *Department of Neurology Hospital, Germany

LISURIDE SPECT - A VALUABLE TOOL TO VISUALIZE DOPAMINERGIC DEGENERATION IN PARKINSON'S DISEASE (PD)

PD is characterized by the degeneration of dopaminergic neurons in the basal ganglia. In vivo visualization and measurement of the copaminergic system in brain structures of Parkinson patients is enabled by the SPECT technique with the I-123 labeiled postsynaptic dopamine D2 receptor agonist Lisuride.

Aim of our study was to correlate SPECTfindings with clinical data of 17 untreated patients with idiopathic PD lage $48-75$, mean 59.6, SD 7.7), which showed response to dopaminergic treatment after the SPECT study. SPECT scans were performed 2 hrs after injection of approximately $185 \mathrm{MBg}$ I-123 Lisuride. Transverse slices without at tenuation correction were used to calculate ratios between the right and the left side of the basal ganglia ( $R / L$ ) and between basal ganglia and frontal cortex (B/FC)

Significant correlation between $R / L$ and the ratio of the scores of the UPDRS-scale for the right and left side is found $(p=0.0181$, $r=0.5650$ ). Additionaliy we observed a negative correlation between the age of the patients and $B / F C(p=0.037, r=-0.4448)$.

Our results suggest, that the Lisuride-SPECT visualizes dopaminergic degeneration in $\mathrm{PD}$ on the base of the quantitative correlation between $R / L$ uptake ratio and severeness of disease.

\section{6}

W. Pirker, S. Asenbaum, S. Kasper*, H. Walter*, P. Angelberger ${ }^{\text {, }}$ G. Koch, A. Pozzera, I. Podreka, T. Brücke

Neurological and Psychiatric (*) University Clinic, Clinic for Nuclear Medicine Vienna, Forschungszentrum Seibersdorf $\left({ }^{\circ}\right)$, Austria

B-CIT SPECT DEMONSTRATES BLOCKADE OF 5HT-UPTAKE STTES BY CITALOPRAM IN BRAIN STEM OF DEPRESSIVE PATIENTS

Serotonin (5HT) uptake inhibitors are substances which are widely used for the treatment of depression and other psychiatric syndromes such as obsessive compulsive disorders.It also has been hypothesized that changes of 5HT uptake sites (US) in the brain are involved in the pathogenesis of depression. Up till now it has not been possible to measure 5HT-US in the brain in humans in vivo. The cocaine analog 2$B$-carbomethoxy-3-B-(4-iodophenyl)-tropane (B-CIT) is a ligand for both DA- and 5HT-US which in its [I-123] labeled form can be used for SPECT.SPECT-experiments in primates have shown that B-CIT binds to 5HT-US in the brain stem and to DA-US in the striatum. The purpose of the present study was to compare [123I]-B-CIT binding in the brain stem (pons, midbrain and hypothalamus) of normal controls and patients under treatment with citalopram. After informed consent 12 depressive patients under $20 \mathrm{mg}(\mathrm{n}=6)$ or $40 \mathrm{mg}(\mathrm{n}=6)$ citalopram daily and 10 normal controls were studied $2,4,16,20$ and 24 hours after i.v. injection of 111 to $148 \mathrm{MBq}$ using a triple head gamma camera (Siemens Multispect). After reconstruction and attenuation correction ROIs were drawn in axial slices in the brain stem, striatum and cerebellum using a brain atlas.Cpm's per pixel were calculated, decay corrected to the time of injection, and corrected for body weight and injected dose. Specific binding was defined as target minus cerebellum. Results show a highly significant reduction of B-CIT binding in the brain stem in patients under citalopram compared to controls (41 \pm 13 vs $83 \pm 48$ cpm's, 4 hrs p.inj, $p<0.0001$ ) which was dosedependent. No changes were found in the striatum.

To our knowledge this is the first report directly demonstrating the effect of a selective 5HT uptake inhibitor in the brain in humans in vivo. SPECT measurements of SHT uptake sites in patients with depression and other psychiatric disorders might provide better insights into the pathophysiology of these disorders and into mechanisms of drug action. 
17

J.T. Kuikka, J. Tiihonen, K.A. Bergström, J. Karhu, H. Viinamäki, J. Lehtonen, B. Hakola, E. Länsimie

Kuopio University Hospital and University of Kuopio, Kuopio, Finlarid

SPET IMAGING OF SEROTONIN AND DOPAMINE UPTAKES IN THE HUMAN BRAIN

Dysfunction of serotonergic (5-HT) and dopaminergic (DA) activity in central nervous system has been observed in several neuropsychiatric disorders. Using of iodine-123-labelled cocaine congener ( $\beta$-CIT) it has been possible to image in vivo with SPET concommittant summarized 5-HT and DA uptake in human brain.

We studied 10 healthy control subjects (age range: 19-60 years) and 4 violent psychiatric patients (type 2 alcoholics; age range: $19-48$ years). The first SPFT scan was started 1 hour after injection of I-123 $\beta$-CIT (110-160 MBq). The second scan was performed 2 hours and the third scan 21 hours after the injection of tracer. The following semiquantitative parameters were calculated: R1) ratio of medial frontal cortex-to-white matter at 1 hour, R2) basal ganglia-to-medial frontal cortex at 1 hour and R3) basal ganglia-to-medial frontal cortex at 21 hours.

The mean values \pm SD of these ratios were $R 1: 1.67$ \pm 0.10 and $1.34 \pm 0.08, \mathbf{R 2}: 1.23 \pm 0.06$ and $1.62 \pm$ $0.13, R 3: 12.5 \pm 0.11$ and $13.0 \pm 1.0$ in controls and in patients, respectively. The results show that 5-HT transporter was markedly decreased and DA transporter increased in the habitually violent offenderg when compared to healthy controls.

\section{8}

C. Halldin, L. Farde, H. Hall, T. Suhara, C.-G. Swahn, G. Sedvall. Department of Clinical Neuroscience, Psychiatry and Psychology Section, Karolinska Institute, Stockholm, Sweden

\section{EXAMINATION OF EXTRASTRIATAL DOPAMINE RECEPTORS IN THE HUMAN BRAIN USING SELECTIVE RADIOLIGANDS}

The function of extrastriatal brain regions may play an important role in diseases such as schizophrenia. PET and SPECT has hitherto been used for the determination of dopamine receptors in the major basal ganglia. In extrastriatal regions the potential for examination of dopamine receptors is limited by the low receptor densities, which are 10-100 times lower than in the basal ganglia. For previously used radioligands the affinity is too low to visualize these receptors.

Suitable radioligands for extrastriatal dopamine receptors should be selective and have a very high affinity to provide high specific binding at low free radioligand concentration in brain. The radioligands examined were [C-11]FLB 457, which is a selective benzamide with a very high affinity (Kd) of $20 \mathrm{pM}$ for dopamine D2 receptors in vitro, and another potent dopamine D2 receptor ligand [1-125] epidepride which is structurally closely related to FLB 457 but contains an iodine. Both were prepared with a specific radioactivity $>1500 \mathrm{Ci} / \mathrm{mmol}$.

In PET examinations on healthy human subjects [C-11]FLB 457 accumulated not only in the basal ganglia but also in several extrastriatal regions. Uptake in the thalamus, amygdala, substantia nigra, colliculus and neocortex was 2-5 times higher than in the cerebellum. In extrastriatal brain regions radioactivity reached a plateau at about 50 minutes after injection. Assuming that radioactivity in the cerebellum reflects the level of free and non-specific binding, the specific binding in extrastriatal regions was on a maximal level within 60 minutes. Human post-mortem whole hemisphere autoradiography using [I-125]epidepride demonstrated labelled receptors in nucleus caudatus, putamen and pallidum and also in extrastriatal areas such as thalamus and the substantia nigra. Specific [C-11]FLB 457 binding should be appropriate for a quantitative equilibrium analysis of extrastriatal dopamine receptor densities with PET. With high resolution SPECT cameras it should be possible to visualize extrastriatal dopamine D2 receptors using [I-123]epidepride.

\section{9}

P.I. Bates ${ }^{1,2}$, H.L. Sharma ${ }^{1}$, B.A. Murrer ${ }^{3}$, C.A. McAuliffe ${ }^{2}$ lDepartment of Medical Biophysics, University of Manchester, ${ }^{2}$ Department of Chemistry, UMIST, Manchester, U.K. and ${ }^{3}$ Johnson Matthey Technology Centre, Sonning, Reading, U.K. THE TISSUE DISTRIBUTION IN MICE OF C-14 LABELLED JM 216, AN ORALLY ACTIVE PLATINUM ANTITUMOR AGENT

A new class of platinum(IV) antitumor agents, the platinum(IV) ammine/amine dicarboxylates has been developed by Johnson Matthey, The Institute of Cancer Research and BristolMyers Squibb. These compounds are orally active, and are undergoing clinical trials at present.

We are at present developing processes for labelling one such compound, $\left[\mathrm{PtCl}_{2}\left(\mathrm{OCOCH}_{3}\right)_{2} \mathrm{NH}_{3}\left(\mathrm{C}_{6} \mathrm{H}_{11} \mathrm{NH}_{2}\right)\right]$ (JM216) at the platinum metal centre and at the carboxylate carbon. These syntheses are from platinum and $\left[\mathrm{PtCl}_{2}(\mathrm{OH})_{2} \mathrm{NH}_{3}\left(\mathrm{C}_{6} \mathrm{H}_{11} \mathrm{NH}_{2}\right)\right]$ (JM149) respectively.

C-14 labelled JM 216 has been prepared from JM 149 and C-14 labelled acetic anhydride. The labelling efficiency was $92 \%$, and the purity $96 \%$ (Shown by HPLC) after recrystallisation.

C-14 labelled JM 216 has been administered orally (Dose:$\left.200 \mathrm{mg} / \mathrm{kg}, \mathrm{LD}_{10}\right)$ to balb/C mice and detailed tissue distribution data obtained (blood plasma, kidney, liver, spleen, brain, lung, muscle and skin) for time points of 2 hours and 2, 6 and 10 days. Comparison of these data with earlier reported data for platinum has shown distinct differences, especially for the liver and the kidney. This clearly indicates a difference in behaviour of the C-14 label and the platinum centre suggesting detachment of the ligand from the metal centre in vivo

Current investigations are taking place to produce Pt-191 labelled JM 149 for a dual labelling experiment.

20

S. Vallabhajosula, B.R. Moyer, J. Lister-James, W.J.McBride, S. Buttram, H. Lipszyc, H. Lee, D.A. Bastidas, R.T. Dean.

Mount Sinai Medical Center, New York, NY and Diatech, Inc., Londonderry, NH, USA.

EVALUATION OF TC-99m LABELED SOMATOSTATIN RECEPTOR-BINDING PEPTIDES IN RATS WITH PANCREATIC TUMOR: COMPARISON WITH In-111-[DTPA]-OCTREOTIDE

Tumors expressing Somatostatin receptors (SSTR) can be detected with high accuracy using In-111[DTPA]-Octreotide (In-Oct). We have developed SSTR-binding peptides $(>50)$ which can be labeled with Tc-99m to very high specific activity ( 300 $\mathrm{Ci} / \mathrm{mMOl})$. The tumor uptake-kinetics of these TCpeptides were compared with In-oct in rats bearing Longnecker tumors (pancreatic tumor line CA20948). Each of the peptides was prepared to contain a $\mathrm{N}_{3} \mathrm{~S}$ or $\mathrm{N}_{2} \mathrm{~S}_{2}$ chelator, labeled with TC$99 \mathrm{~m}$ by ligand exchange, purified by HPLC and assayed in vitro for SSTR binding affinity. Rats were injected i.v. with $0.2 \mathrm{mCi}$ of Tc-peptide
$(16 \mu \mathrm{g} / \mathrm{Kg})$ or $10-50 \mu \mathrm{Ci}$ of $\operatorname{In}-\mathrm{Oct}(2.0 \mu \mathrm{g} / \mathrm{Kg})$. To $(16 \mu \mathrm{g} / \mathrm{Kg})$ or $10-50 \mu \mathrm{Ci}$ of In-Oct $(2.0 \mu \mathrm{g} / \mathrm{Kg})$. To
assess in vivo tumor specificity of Tc-peptides, selected rats were preinjected s.c. with a receptor blocking dose of oct $(4 \mathrm{mg} / \mathrm{Kg})$. The tumor uptake-kinetics were studied by gamma camera imaging and $\frac{1}{1} \mathrm{I} / \mathrm{g}$ of the tumor was determined at 30,60 and $90 \mathrm{~min}$ following sacrifice. The mean 30,60 and $90 \mathrm{~min}$ following sacrifice. The mean
\%ID/g (n=5) and Tumor/muscle (T/M) ratio for selected peptides are as follows:

Peptide peptio./g of tumor at

$\frac{30}{3.81} \quad \frac{60}{300} \quad 90 \mathrm{~min} \quad \mathrm{~T} / \mathrm{M}$ at

$\begin{array}{lllll}\text { TC-P587 } & 3.81 & 3.90 & 3.43 & 33 \\ \text { TC-P617 } & 3.41 & 4.63 & 6.70\end{array}$

$\begin{array}{lllll}\text { TC-P617 } & 3.41 & 4.63 & 6.70 & 34\end{array}$

$\begin{array}{llllr}\text { Tc-P639 } & \overline{3} & \overline{3} & 0.18 & 4 \\ \text { In-Oct } & 3.10 & 3.00 & 2.91 & 64\end{array}$

Blocking studies with cold oct reduced the $90 \mathrm{~min}$ tumor uptake of TC-P587 and TC-617 by $70 \%$ and Inoct by 80\%. Although P587, P617 and P639 had similar in vitro receptor binding affinity $\left(K_{1}<1\right.$ nM), TC-P639 showed minimal tumor uptake in vivo, suggesting that radiotracer performance must be assessed in vivo and not just in vitro. 
21

E. Seregni, A. Bogni, P. Foa*, M. Sala*, F. Crippa, E. Bombardieri and G.L. Buraggi

Nuclear Medicine Division- National Cancer Institute- Milan

$\left.{ }^{*}\right)$ Marcora Center- Ospedale Maggiore Policlinico-Milano

Evaluation of the new tumor marker Cyfra 21.1 in resectable non-small cell lung cancer (NSCLC): comparison with TPA, CEA, SCC and NSE.

The new tumor marker assay Cyfra 21.1 utilizing two monoclonal antibodies against well-characterized epitopes of a water soluble fragment of cytocheratin 19 has been recently developed. The analytical performances of this assay were previous verified by us in a multicenter trial. In this study we evaluated the diagnostic sensitivity and specificity of Cyfra 21.1 in 75 patients with newly diagnosis of NSCLC, in 91 patients with non-neoplastic respiratory diseases and in the follow-up of 31 patients submitted to curative resection of lung cancer. In the same groups of patients TPA, CEA, SCC and NSE were also determined. The adopted cut. off for Cyfra 21.1, TPA, CEA, SCC and NSE were $1.9 \mathrm{ng} / \mathrm{mL}, 90 \mathrm{U} / \mathrm{L}, 5$ $\mathrm{ng} / \mathrm{mL}, 3.0 \mathrm{ng} / \mathrm{mL}$ and $13 \mathrm{ng} / \mathrm{mL}$, respectively. In squamous cell carcinomas $(\mathrm{N}=40)$ the sensitivity of Cyfra 21.1 was as high as that of TPA $(75 \%)$, while the sensitivity of CEA, SCC and NSE was $35 \%, 20 \%$ and $15 \%$. In adenocarcinomas $(\mathrm{N}=35)$ the best sensitivity was reached by CEA $(62.9 \%)$, followed by Cyfra $21.1(51.4 \%)$, TPA $(48.6 \%)$, NSE and SCC $(8.8 \%)$.In non-neoplastic respiratory lung diseases the specificity of Cyfra 21.1 was worse than those of CEA $(95 \%)$, TPA ( $86 \%)$, NSE $(86 \%)$ and SCC $(94 \%)$. Twenty-one patients during the follow-up had no clinical evidence of disease (NED), while 10 patients had local relapse or distant metastes. Three out of 21 NED patients displayed positive values of Cyfra 21.1 and 7 out 10 with disease during the follow-up had high value of the marker. In this group of patients CEA showed a sensitivity of $90 \%$. Cyfra 21.1 was also analyzed in the post-operative period in order to determine the kinetic behaviour of the marker. In our experience Cyfra 21.1 had a very quick elimination from the bady with a $T / 2$ of about 12 hours. In conclusion, this study supports the use of the senum fragments of cytocheratin subunit 19 as an interesting tumor marker in patients affected by non-small cell lung cancers. Further investigations are needed to verify the clinical role of Cyfra 21.1 evaluation either for prognostic purposes and for follow-up survaillance of radically treated patients.

\section{2}

P.Koutsiouba, L.Oros, I.Armeniakos, M.Pagou, P.Natsis

Nuclear Medicine Department,METAXA'S Memorial Hospital Pireus, Greece.

CQRRELATION OF BONE SCAN AND TTMOR MARKERS IN DIF'ERENT MALTCNANCIES .

The breast, lung and prostate cancer, are considered to give frequently bone metasteses. Tumor markers (T.M) are usefull in the follow-up of these diseases and in early diagnosis of the relapse.

The aim of this study, was to correlate bone scan(B.S) and sensitive(T.M) in each case, to find out the (T.M) that we can use as the most sensitive or what is the combination for best results and finally to determine what is the role of positive (T.M) in negative (B.S).

We examined $158 \mathrm{pts}, 62$ with breast cancer, 30 with lung cancer and 64 with prostate cancer. We measured serum levels of CEA, Ca-125, Ca 15-3, TPA, PSA, b'microglobulin, ferritin, and alkaline phosphatase. 58 pts had positive(B.S). The best sensitivity of (T.M) in breast cancer was $88,2 \%$ forCa $15-3,86,6 \%$ for CEA and 76,4\% for TPA. In lung cancer was $88,8 \%$ for $\mathrm{Ca}-125$ and $87,5 \%$ for TPA and in prostate cancer $70,8 \%$ for PSA and $70,4 \%$ for $b^{\prime}$ microglobulin. Of the 100 pts with negative (B.S) 31 had 1(T.M) elevated, 9 had 2 (T.M)above the normal values and only 7 pts had 3 (T.M) elevated ( 3 with liver metastasis 3 with lung metastasis and 1 with brain metastasis).

Conclusion. In the follow up of pts with malignant diseases, when we search for bone metastases, its usefull to combine(B.S) and(T.M) as Ca 15-3 and TPA in breast cancer, Ca-125 and TPA in lung cancer and PSA and alkaline phosphatase in prostate cancer.

When the(B.S) is negative and(T.M) levels are above normal limits (in three of them at least), we must search for other metastases as hepatique,lung or brain.

\section{3}

J.M. Riedinger, I. Barillot, S. Lizard-Nacol, M. Toubeau, A. Riedinger-Berriolo, C. Touzery, Y. Cottin, F Brunotte. Services de Biologie, d'Immunologie et de Médecine Nucléaire, Centre G.-F. Leclerc, Dijon, France

EPITHELIAL OVARIAN CARCINOMA : PROGNOSTIC VALUE OF SERUM CA 125 DOUBLING TIME PRIOR TO CLINICALLY EVIDENT RELAPSE

The rise kinetic of serum CA 125 level prior to diagnosis of clinically evident relapse showed agressivity of the recurrence. The aim of this work was to evaluate the prediction value of the doubling time (T1/2) calculated according the formula : $\mathrm{T} 1 / 2=(\log 2 * t) / \log [(\mathrm{CA} 125) 2 /(\mathrm{CA} \mathrm{125}) 1]$ in which $\mathrm{t}$ is the interval in days between the final and the initial measurement. CA 125 levels were first estimated with ELSA CA 125 and then with EIA CA 125 (CIS Bio International).

The doubling time of CA 125 was calculated in 42 patients with epithelial ovarian carcinoma : $55 \%$ of half-lives were inferior to 60 days. They were inversely correlated with initial serum half-lives of CA 125 measured during the first cycles of chemotherapy $\left(p<10^{-6}\right)$. The more acute rises of serum antigen level were observed in sera of patients who did not responded quickly to firstline chemotherapy. The relative risk of relapse and death in patients with doubling time of less than 60 days were respectively 4,3 $\left(\mathrm{p}<10^{-4}\right)$ and 5,3 times $\left(10^{-4}\right)$ higher than in patients in patients with doubling time of 60 days or more. The median time of relapse free was 21 months for patients with doubling time of 60 days or more and 11 months for the others patients. The median time of overall survival was 36 and 16 months for each category respectively.

In conclusion, the measurement of the serum $\mathrm{CA} 125$ doubling time prior to clinically evident relapse allowed the prediction of the prognostic of the recurrence and the adaptation of a appropriate therapeutic.
24

B. Donnerstag, R.P. Baum, J.B. Oltrogge, K. Henzel, M. Skierlo, L.
Träger, G. Hör.

Depts. of Biological Chemistry and Nuclear Medicine, University Medical Center, Frankfurt/Main, Germany

NEW ASSAYS FOR DETERMINING THE IMMUNOLOGICAL PROFILE OF OVARIAN CANCER PATIENTS UNDER
IMMUNOTHERAPY

The injection of murine monoclonal antibodies for immunoscintigraphy of ovarian cancer patients induces human anti-mouse antibodies (HAMA) Longer survival rates have been reported in related to the enhancement of the anti-tumor response induced by antirelated to the enhancen
idiotypic antibodies.

We have studied the phenotypes and the cytotoxic activity of We have studied the phenotypes and the cytotoxic activity of peripheral blood lymphocytes (PBL) in patients before and during the development of HAMA comparing the immunological parameters and
the clinical course. For phenotyping of lymphocytes, interfering HAMA were eliminated by Ficoll gradient centrifugation and the following antigens were determined by flow cytometry: CD4, CD8, $\mathrm{CD} 3, \mathrm{CD} 19, \mathrm{CD} 56, \mathrm{CD} 2, \mathrm{CD} 25, \mathrm{CD} 45$ and $\mathrm{HLA}-\mathrm{DR}$, For measuring the cytotoxic activity, NK-cell and non-MHC restricted T-cell activity was determined with a fluorescence-based assay. In addition the IL-2 sera levels of the patients were regularly determined. Until now, PBL sera levels of the patients were regularly determined. Until now, $9 B \mathrm{BL}$
of 40 patients injected 1 to 7 times with 1 to $2 \mathrm{mg}$ intact $\mathrm{MAb}$ Tc-99m

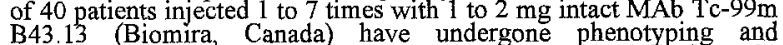
B43.13 (Biomira, Canada) have undergone phenotyping and
functional analysis at intervals of 4 to 8 weeks over a period of 3 to 20 months. In comparison, the PBL of 10 healthy controls, not previously exposed to murine immunoglobulins, served as controls. The results demonstrate that high HAMA titers $\left(10^{3}-5 \times 10^{5} \mathrm{ng} / \mathrm{ml}\right)$ are often associated with remarkable cytotoxic activity and phenotypes of peripheral blood lymphocytes which remain on a persisting level with usually high values. Whereas HAMA-titers below $1000 \mathrm{ng} / \mathrm{ml}$ are often accompanied by PBL with low cytotoxic activity, sometimes often accompanied by PBL with low cytotoxic activity, sometimes immunological instability. Furthermore it was possible to define immunological instability. Furthermore, it was possible to define immunological parameters which correlate with the progression of
tumor growth. Concerning the functionality of non-MHC restricted cytotoxic T-lymphocytes we noticed an increase of more than $100 \%$ indicating a decrease in specificity concerning T-cellular activity. These immunologial parameters obtained by long-term functional analysis, phenotyping of peripheral lymphocytes and serum Interleukin-2 determination are useful in accompanying future immunotherapy of cancer patients. 
Sunday, 21 August 1994

25

S. Dresel $^{1}$, A. Stäbler ${ }^{2}$, N. Holzknecht ${ }^{2}$, K. Tatsch ${ }^{1}$, K. Hahn ${ }^{1}$

Department of Nuclear Medicine', Department of Radiology ${ }^{2}$ Klinikum Grosshadern, University of Munich, FRG

\section{DIAGNOSTIC APPROACH IN ACUTE PULMONARY} EMBOLISM - PERFUSION SCINTIGRAPHY VS. SPIRAL-CT

For diagnosing acute pulmonary embolism, perfusion scintigraphy is a well established and sensitive method. Recently, computed tomography (CT) in angiographic technique was introduced for evaluating the pulmonary artery system without breathing-artefacts. The present study compares lung scan with spiral CT data to establish the diagnostic value of the latter approach.

Twentyfive patients with clinical suspicion of acute pulmonary embolism were examined using both methods. Radionuclide perfusion scans were performed after i.v. injection of $170 \mathrm{MBq}{ }^{99 \mathrm{mTC}} \mathrm{T}-\mathrm{MAA}$ in 8 projections. Spiral-CT data (Somatom plus, Siemens) were obtained after bolus-injection of $100 \mathrm{ml}$ contrast-medium.

In most patients pulmonary embolism was concordantly diagnosed by both methods. However, results were interpreted differently. Particularly if centrally, on both pulmonary arteries localized emboli spiral- perfusion-scan were present, the correct estimation of the CT 2 pos. neg. more affected side was difficult in $\mathrm{CT}$ and \begin{tabular}{l|c|c|c} 
pos. & 18 & 1 & dependent on the experience of the readers
\end{tabular} \begin{tabular}{l|l|l} 
neg. & 4 & 2
\end{tabular} Small, (sub-)segmental embolism could not contrast enhancement of vessels in the periphery. This fact may well explain the false positive and the false negative results, respectively.

Spiral-CT enables to exactly demonstrate thrombosis in severe, centrally localized embolism. In incompletely occluded vessels, the degree and the location of perfusion defects is better estimated by scintigraphy. Due to the results presented and with respect to radiation exposure and the necessary technical equipment for spiral CT, perfusion scintigraphy remains the method of choice in diagnosing acute pulmonary embolism.

\section{6}

A.L. Martel and W.B. Tindale

Department of Medical Physics and Clinical Engineering, Royal Hallamshire Hospital, Sheffield, England

\section{A COMPARATIVE STUDY OF THE EFFECT OF Tc-99m TECHNEGAS AND TC-99M DTPA AEROSOL ON IMAGE APPEARANCE AND LUNG SCAN REPORTING}

The image quality of lung ventilation studies carried out using Tc-99m Technegas was compared to that obtained with Tc-99m DTPA aerosol. The effect of choice of tracer on the final ventilation-perfusion (VP) scan report was also assessed. A total of $192 \mathrm{VP}$ scans from four groupsof patients were used in the assessment. Groups 1 and 3 were ventilated using Tc-99m Technegas, groups 2 and 4 were ventilated using Tc-99m DTPA aerosol. For each scan the age of the patient, the presence or absence of aerosol clumping, the number of ventilation defects (no defects, one or two defects or $>2$ defects) and the final clinical report (obtained using the Biello criteria) was noted. No significant differences were found between groups 1 and 3 or groups 2 and 4 therefore these results were pooled to give two groups of 96 patients, one for Technegas and one for DTPA aerosol. All comparisons of the two ventilation agents were performed using the Pearson chi-squared test.

The mean age of the patients was 56 years and there was no significant difference in the distribution of ages in the two groups $(p>0.05)$. There was a significant difference $(p<0.001)$ in the number of scans with aerosol clumping present, with $19 \%$ of the Technegas scans compared to $41 \%$ of the DTPA scans showing this feature. Multiple ventilation defects were present in $23 \%$ of the Technegas scans and $35 \%$ of the DTPA scans but the differences were not significant $(\mathrm{p}=0.054)$. Despite the differences in image quality, the choice of ventilation agent had no significant effect on the final report $(p>0.05)$

\section{7}

EJR van Beek, A van den Ende, DPM Brandjes, PMM Bossuyt and HR Büller.

Depts of Hemostasis/Thrombosis and Clinical Epidemiology, Academic Medical Centre, Amsterdam, the Netherlands.

\section{THE ROLE OF PLASMA D-DIMER IN THE EXCLUSION OF CLINICALLY SUSPECTED PULMONARY EMBOLISM.}

Physicians often suspect pulmonary embolism (PE), and are then faced with the difficulty of proving or refuting the diagnosis. Lung scanning is the diagnostic test of first choice. Recently, plasma Ddimer, a cheap non-invasive blood test, has been suggested as a useful addition. However, its exact place in a diagnostic strategy, its role and which test to use are presently being debated.

We evaluated 4 commercially available ELISA D-dimer methods, determined the cut-off value to obtain a sensitivity and negative predictive value of $100 \%$, and compared the possible utility for each assay in various diagnostic strategies (in terms of exclusion efficacy and cost-effectiveness).

A consecutive series of 179 patients (78 inpatients, 101 out-patients) underwent lung scintigraphy and/or pulmonary angiography. PE was proven in 73 patients (58 high probability scan, 15 angiogram positive). Comorbid conditions were present in 74 patients.

D-dimer was able to exclude PE in 8-18\% of all patients, and in 11$27 \%$ of outpatients. Two of the 4 assays were significantly better in excluding PE. Similarly, the exclusion rate was significantly better (13-27\%) in patients without comorbid conditions. The cost-effectiveness analysis indicated that $D$-dimer may reduce costs by $10 \%$ and angiography requirements by $28 \%$ without affecting mortality. D-dimer may be a cost-effective addition to lung scintigraphy for the exclusion of PE. Further management studies are advised.

\section{8}

E. Bombardieri, F. Crippa, A. Chiti, E. Seregni, I. Cataldo*, E Soresi ${ }^{\circ}$, R. Boffi ${ }^{\circ}$, G.L. Buraggi

Nuclear Med. Dept., $\left({ }^{*}\right)$ Surgical Oncol. Dept. Natl. Cancer Institute Milano, $\left(^{\circ}\right)$ Dept. Pneumatology, Niguarda Hospital Milano (Italy)

SCINTIGRAPHY OF SMALL CELL LUNG CANCER (SCLC) USING 111 -In DTPA-OCTREOTIDE

In vivo visualization of SS receptors has recently been described using the somatostatin analogue $111 \mathrm{In}$ - Labelled octreotide. Twenty-one patients with histologically proven SCLC were examined : 17 at tumor presentation, 4 had previous therapy.Patients were staged on the basis of chest X-ray, serum neuroenolase (NSE) determination, fiberoptic bronchoscopy, bone scintigraphy, abdominal US, chest and brain CT scan. In few patients planar scintigraphy was performed, 1,5 , and 24 hours after $\mathrm{i} v$ injection of $110 \mathrm{MBq}$ of $111 \mathrm{In}$-Octreoscan. The $111 \mathrm{In}$ Octreotide tumor uptake registered at 5 hours from the injection was good and the imaging was comparable to that of late scan; for this reason the standard examination was manteined at 5 hours. Scanning was done with a Toshiba GCA 901/A gamma camera equipped with a medium energy collimator. SPECT of the thorax and of particular regions of interest was performed. In 20 out of 21 patients lung tumors were visualized at 5 hours scan. The negative case had undergone to a previous chemotherapy. The last scan, when performed, confirmed in all patients the positivity or the negativity of the imaging obtained with the early scan. Octreotide uptake was in generally more extensive than expected from RX and CT studies. The somatostatine analogue visualized all the lesions were demostrated by means of other instrumental examination with the exception of 2 liver metastatic sites, 2 lymph-nodal metastases, 1 adrenal metastases detected by US and /or CT scans; on the contrary 111 In-Octreotide scintigraphy revealed 5 unknown distant lesions ( 3 lymph-nodes, 1 bone, 1 brain) previously not identified with standard examinations. Octreoscan was effective in imaging tumor sites also in patients with low NSE values. 
29

$$
\begin{gathered}
\text { G. Önsel, K. Sönmezoğlu, G. Camsarı*, s. Atay, S. Cetin", } \\
\text { T. Y. Erdil, T. Usiu, A. Uzun", B. Kanmaz, T. Kapıcıŏlu } \\
\text { Department of Nuclear Medicine, Cerrahpaşa Medical Faculty } \\
\text { and Chest Clinic of Yedikule*, Istanbul, Türkiye }
\end{gathered}
$$

THE VALUE OF TC-99m MIBI SCINTIGRAPHY IN PULMONARY TUBERCULOSIS

Ga-67 scanning has been proposed as a sensitive indicator of the presence of active tuberculosis (TB). However, Ga-67 is not readily available and required at least $24 \mathrm{hr}$ between injection and scanning. Recently, TC-99m MIBI (MIBI) has been shown to accumulate in some tumors as well as in some inflammatory lesions. The purpose of this study is to investigate the value of MIBI for the detection and follow-up of active pulmonary tuberculosis (APTB).

The study was carried out in 31 patients $(27 \mathrm{M}, 4 \mathrm{~F}$, age range 17-54). 23 patients had APT (sputa positive for acid-fast bacilli), 4 patients with chronic TB had suspicious of reactivation (equivocal chest $x$-ray and sputa negative for acid-fast bacilil) and 2 patients had miliary TB. All patients were free of chemotherapy at the time of MIBI scintigraphy. In 10 patients with APTB, MIBI scintigraphy were repeated two months after chemotherapy. MBBI scintigraphies were performed in anterior and posterior projections of the chest at 15th 60th and 120 th min. following IV injection of $370 \mathrm{MBq}$ of TC-99m MIBI. The scintigraphies were compared with chest $x$-rays. Of 23 patients with APTB, 22 showed increased uptake of MIBI consistent with the findings of chest $x$-ray and 1 (with small infiltration) had no pathologic uptake of MIBI. Of 6 patients with equivocal disease, 4 showed focal increased MIBI uptake and 2 had negative scintigraphies. Both patients with miliary TB showed remarkably diffuse uptake in both lungs. After chemotherapy, 7 patients had slightly diminished of MIBI uptake concordant with chest $x$-rays. 3 patients with clinically worse had also more increased MIBI uptake.

As a result, TC-99m MIBI scintigraphy is sensitive to detect APTB and could be used in assessing the response to chemotherapy and establishing reactivation of the disease.

\section{0}

W.Brenner, *B.Kroker, K.H.Bohuslavizki, H.Wolf, M.Clausen, J.-U.Eberhardt, *J.Werner, *G.S.Godbersen, E.Henze Clinic of Nuclear Medicine and ${ }^{*}$ Clinic of Oto- Rhino- Laryngology Christian-Albrechts-University, Kiel, Germany

\section{VENTILATION SCINTIGRAPHY OF THE TYMPANIC CAVITY WITH XENON-133}

In this study, an attempt was made to administer $\mathrm{Xe}-133$ by ventilation into the tympanic cavity in order to quantitate uptake and clearance of the tracer as a measure of Eustachian tube function.

$50 \mathrm{MBq}$ of Xenon-133 gas filled in a $50 \mathrm{ml}$ syringe were administered to 15 ENT patients testing 3 different approaches. In 2 patients the gas was injected directly into the tympanic cavity via an in-place Armstrong tube. In another 5 patients Xe-133 was insufflated into the epipharynx using a nasopharyngeal catheter. Subsequently the patients were asked to perform Valsalva manoeuvres three times to imitate physiological ventilation of the middle ear. In a third group of 8 patients Xe-133 was administered via a nose olive while having Valsalva manoeuvres done simultaneously. Immediately after gas application sequential gamma camera imaging for $30 \mathrm{~min}$ and semiquantitative evaluation by ROI-techniques were performed.

As a main result of this study it is to state that all three approaches were successful in trapping Xe-133 within the middle ear space without major problems. Initial accumulation of Xe-133 in the tympanic cavity during the first two minutes yielded a right to left ratio of $51.1 \pm 10.2 \%$ to $48.9 \pm 10.2 \%$ in 10 patients with normal Eustachian tube function. The clearance half life ranged from $11.5 \mathrm{~min}$ to $217.9 \mathrm{~min}$ with a median of $96.2 \mathrm{~min}$. One-sided malfunction of the Eustachian tube proven by impedance mesurements or even the beneficial effects of one-sided application of decongestive drugs could nicely be quantitated both by initial trapping as well as by clearance half life, respectively.

In conclusion, tympanon $\mathrm{Xe}-133$ ventilation scintigraphy is an easyfo-perform and low-radiation-exposure test for evaluating the middle ear ventilation and, thus, Eustachian tube function. Normal ranges are provided by this study, yet the clinical impact needs to be documented by especially designed ENT trials.
31

A Sundin ${ }^{1}$, P Hellmañ ${ }^{2}$, H Ahlstrôm², C Juhlin ${ }^{2}, M$ Bergström ${ }^{1}, G$ Akerström ${ }^{2}$ and $J$ Rastad ${ }^{2}$

${ }^{1}$ Uppsala University PET-centre and ${ }^{2}$ Department of Surgery, Uppsala University, Sweden

PREOPERATIVE LOCALIZATTON OF PARATHYROID TTSSUE BY POSITRON EMISSION TOMOGRAPHY

The purpose of this study was to assess the diagnostic accuracy of preoperative PET in hyper parathyroidism (HPT).

Material: Before operation $(n=10)$ or reoperation $(n=11) 21$ patients with HPT were examined by PET following intravenous (i.v.) injection of $400-$

$800 \mathrm{MEq}$ of $\mathrm{L}$-methyl- ${ }^{11} \mathrm{C}$-methionine. In $6 \mathrm{pa}-$ tients PET was repeated during or immediately after i.v. infusion of $\mathrm{Na}_{2}$-EDTA. A General Electric whole body 4096 PET camera was used. After a 10 min transmission scan a dynamic sequence was performed and an average image (14-45 min) produced and recalculated to provide images of standardized uptake values (SUV). Regions of interest were drawn by a standardized procedure. Contrast enhanced computed tomography (CT) was carried out in 17 patients on a Siemens Somatom Plus CT-scanner using $4 \mathrm{~mm}$ contiguous slices. In 18 patients ultra sonography (US) was performed (Acuson $128,5 \mathrm{MHz}$ ).

Results: Sugery revealed enlarged parathyroid tissue in 20 patients but not in 1 patient. PET was true positive in $76 \%$, false positive in $19 \%$ and true negative in $5 \%$ of the patients. Cr was true positive in $65 \%$ and US in $50 \%$.

The results suggest the PET may provide novel possibilities for preoperative parathyroid imaging in HPT.

\section{2}

J.Y. DEVAJXX, Y. FULLA, Y. CHAPUIS, P. BONNICHON, B. RICHARD

E.A. 215 "Biophysique", Service de Chirurgie, C.H.J. Cochin, Paris, France

TC MIBI SCINTIGRAPHY, A NEW STRATEGIC TOOL FOR THE PREOPERATIVE LOCALIZATION OF PARATHYROID ADEANOMAS.

Recent papexs have demonstrated the usefuliness of TC MIEI scintigraphy (MS) and its potential superiority over thallium-201 for the diagnosis of parathyrold (PT) enlargement. In order to determine where would be the place of this new imaging procedure in the decision tree before surgery, we performed MS in 100 patients (pts) with suspected hyperparathyroldism (HPT). All 100 pts had both echographic and scintigraphic examinations. MS was performed using a double phase method, i.e. imaging time post injection was $15 \mathrm{mn}$ and then $75 \mathrm{mu}$. Up to now, only 58 of these $100 \mathrm{pts}$, had undergone surgical exploxation. Based on the histologic control of these cases, we have establiched three major indications of MS in identifying PT adenomas. MS is superior to all other techniques for the diagnosis and localization of ectopic glands (6 pts in our group). MS is determinant in all cases of recurrent or persistent HPT. When concordance is found between echography and MS on a single site, local instead of general anaesthesia can be proposed. The recent increasing demand for HPT exploration, coupled with the cardiac use of MIBI, allowed a dramatic reduction of the cost pex patient, falling below that of thallium. We conclude that MS is a powerful tool for the strategy of localizing PT aclenomas, especially when ectopic situation is suspected, and should be therefore performed in all patients with clinical and biological hyperparathyrolidism. 
Sunday, 21 August 1994

33

C. Als, M. Listewnik, E. Zingg*, L. Mazzucchelli**, L. Geiger, E.P. Ritter, K. Thiele, M. Sulzer, J. Kinser, H. Roesler

Departments of Nuclear Medicine, Urology*, Pathology**, Inselspital, University of Berne, CH-3010 Berne, Switzerland

Superior visualization of parathyroid adenomas with 3Ddouble-peak-SPET

The established preoperative visualization method of parathyroid (PT) adenomas (AD) is planar subtraction scintigraphy (PSS). To optimize those results, we investigated prospectively 44 patients presenting a clinical diagnosis of hyperparathyroidism (elevated intact parathormone (PHi)) by 3D - SPET scanning technique.

Patients: 44 adults $(2 / 3 \mathrm{f}, 1 / 3 \mathrm{~m})$, aged $23-80$ years (average: 59 years), PHi: 1.2-20 times above the normal limit (average: 6.9 times). The histological diagnosis was confirmed by neck dissection in each case.

Methods: 24 hours after oral administration of $22 \mathrm{MBq} 1231$ and 30-60 minutes after intravenous administration of $280 \mathrm{MBq} 99 \mathrm{mTc}$ Hexamibi (Dupont), a double peak 3D - SPET of the neck was acquired on a PRISM camera (134 and $164 \mathrm{keV}, 10 \%$ window each, LEHR parallel collimators, 40 paces, each \pm 50 kcounts, filtered backprojection, Bandpass filter). The results were compared with those of the planar, weighted, graphical PSS (Macintosh computer, NucLear - programme, own macrofunction). Criteria for diagnosing a pathological PT: additional, Hexamibi-positive area in the PSS and in the 3D-SPET scanning.

Results: For an experienced scientist, the global detection sensitivity for PT-AD was $84 \%$ and $86 \%$ with PSS and 3D-SPET scanning respectively. False negative results were due to: iodine exposure and functional autonomy of the thyroid. False positive results were due to: thymus, lipomatous PT or lymphnodes. The main advantage of 3D-SPET was the preoperative exact localization of PT, especially in case of retrothyroidal PT-AD. Both non - invasive methods allow a functional diagnosis of $A D$ and, more seldom, of hyperplasias of the PT.

\section{4}

C. Schvartz, J.C. Liehn and M.J. Delisle

Department of Nuclear Medicine, Institut Jean Godinot, Reins, France

OOMPARISON OF LATE SESTAMIBI AND SESTAMIBI-PERIECHNETATE SUBIRACTION IMACAS BASED ON 31 SURGICALLY PROVEN PARATHYROID LESIOASS

The purpose of this study is to campare the diagnostic value of SESTAMIBI LATE imaging, as proposed by Taillefer and SESTAMIBI-FERIECHEETATE imaging techniques, in the preoperative localization of parathyroid lesions.

Twenty eight patients were examined. SESTAMIBI images were recorded with a pinhole collimator just after and two hours after injection of $300 \mathrm{MBg}$ of $99 \mathrm{milC}$ SESTAMIBI . The thyroid image was obtained after injection of $300 \mathrm{MBq}$ of $99 \mathrm{~m}$ पc PERTIECHNETATE. The subtraction was performed with an already validated software. Late and subtraction images were interpreted blindly by three observers in each of the four quadrants of the thyroid field. Then 112 quadrants were analysed. Ectopic lesions were not taken into acoount in this study. Thirty one lesions (24 adenomas and 7 hyperplasias) were found at surgery. The sensitivities and specificities obtained are :

\begin{tabular}{lcccccc} 
& \multicolumn{2}{c}{ OBS $\# 1$} & \multicolumn{2}{c}{ CRS $\# 2$} & \multicolumn{2}{c}{ ORS $\# 3$} \\
& SENS & SFEC & SENS & SFEC & SFNS & SFEC \\
LATE & 58 & 98 & 67 & 96 & 64 & 96 \\
SUET & 83 & 96 & 87 & 96 & 87 & 96 \\
& $\mathrm{p}<.02$ & NS & $\mathrm{p}<.05$ & NS & $\mathrm{p}<.05$ & NS
\end{tabular}

We conclude that although specific, late MIBI images lack sensitivity and miss about $23 \%$ of the surgically proven lesions seen on subtraction images.
35

K. Wurm, U. Dörr, D. Bach, H. Bihl

Dept. of Nuclear Medicine, Katharinenhospital Stuttgart, FRG

\section{DETECTION AND LOCALISATION OF PARATHYROID ADENOMAS WITH TC-99m MIBI USING A TRIPLE-HEAD CAMERA.}

The purpose of this study was to evaluate the usefulness of Tc- $99 \mathrm{~m}$ MIBI for the detection and localisation of parathyroid adenomas in patients with primary hyperparathyreoidism. An increase of sensitivity and accuracy of this scintigraphic procedure was expected by the use of a triple-head camera when compared to one-head camera systems.

Between may/93 and january/94, 30 patients suspected for primary hyperparathyreoidism underwent scintigraphy with Tc-99m MIBI. $560 \mathrm{MBq}$ Tc- $-99 \mathrm{~m}$ MIBI were applied i.v. and planar images of the neck were performed $2 \mathrm{~h}$ p.i. followed by SPECT acquisition with a 3 head camera (Prism 3000, Picker). MIBI scans were judged positive for parathyroid adenoma, if increased focal activity was seen - compared to normal thyroid tissue. All 30 patients underwent surgery and histological examination after scintigraphy.

MIBI scans were true positive in 16 of 30 patients, with 12 patients being positive in both planar and SPECT scans, 4 patients positive in SPECT images only. In 6 of the 30 patients, MIBI-scintigraphy was false negative. In the remaining 8 patients MIBI-scintigraphy was unable to detect any pathological finding, so did surgery too. False positive scans did not occur.

In conclusion, Tc-99m MIBI scintigraphy seems to be well qualified for the detection and localisation of parathyroid adenomas, especially if SPECT with modern triple-head cameras is used. Sensitivity, image quality and low costs enable Tc-99m MIBI to replace the often used Tl-201/ Tc-99m subtraction scintigraphy in most diagnostic situations.

\section{6}

I. Poncioni, A. Boubaker, M. Simko, A. Bischof Delaloye, G. Chapuis, B. Delaloye Dept. of Nuclear Medicine, dept. of Surgery, CHUV, LAUSANNE, Switzerland

$\begin{array}{llcc}\text { PREOPERATIVE } & \text { SCINTIGRAPHIC } & \text { LOCALISATION } & \text { OF } \\ \text { ABNORMAL } & \text { PARATHYROIDS } & \text { (PT) } & \text { IN }\end{array}$
HYP ERPARATHYROIDISM

We present the results of a retrospective study of 89 patients (mean age $58.4 y ; 34 \mathrm{M}, 55 \mathrm{~F}$ ) with hyperparathyroidism (67 I, 22 II or III) having undergone a PT scintigraphy $(86$ Tl201 with Tc99m substraction, 3 MIBI) before surgery. 12 patients had been previously submitted to PT surgery. 1 scintigraphy was unreadable. There were 44 patients with adenoma, 31 with hyperplasia. In 10 patients, diagnosis adenomahyperplasia was uncertain; 4 were negative. The scintigraphic localisation of abnormal PT was complete $(+)$ in $64 / 88(72.78)$, partial $(+/-)$ in $9 / 88$ (10.2\%) and incorrect (-) in 15/88 (17.1\%) (table). In 5 patients with adenoma, the concordance was estimated complete when only the

lateralisation was correct.

PT scintigraphy

allows preoperative

localisation complete in cases of hyperplasia. In rare cases, an associated thyroid pathology made the interpretation more difficult, but without significant change of the results. 
37

E.Einton ${ }^{\circ}$ C.Chiron*, J.Motte\#, O. Duiac*, A.Syrota ${ }^{\circ}$ 'SHEJ, CEA, Orsay. *Dept de Neuropédiatrie, Hop St Vincent de Paul, Paris, . \#Dept de Neuropédiatrie, American Memorial Hosp, Reims, France DIAGNOSTIC VALUE OF EARLY SRECT (SINGIE
RHOTON EMTSSION COMPUTED TOMOGRARHY) IN STURGE WEBER SYNDROME (SWS)

Diagnosis of SWS may be difficult during the first year of life because neurological signs can be absent and radiological investigations can be normal. In order to test diagnostic value of SPECT at this age, regional cerebral blood flow was measured using Tomomatic $56 \mathrm{~A}$ and 133-Xenon in 8 children with confirmed SWS, aged 1 to 17 months, and in 16 controls of the same age. Three children with sws never experienced seizures before SPECT ; they showed hyperperfusion in the damaged hemisphere $(+10 \%)$ and particulary in the cortex underlying meningeal angioma $(+20$ 은. Compared to controls, interhemispheric and local asymetries were not significant $(0.05<p<0.1)$ probably because patients were too few. The 5 other children had seizures before SPECT; they showed hypoperfusion in the affected hemisphere (- 20 움) and particulary in the region underlying the angioma $(-25$ 号). Compared to controls, these asymetries were very signifiant $(p<0.0005)$. Hyperperfusion could be related to the accelerated myelination reported on the pathological hemisphere in the first months of life. Later ischaemia which progressively develops, probably due to angioma and worsened by seizures, could change hyper to hypoperfusion. Early SPECT imaging can therefore be an aid for diagnosis of sws in infants before neurological and radiological characteristics appear.

38

A. Hertel, C. Seipp, G. Hör, P. Hernaiz ${ }^{*}$, S. Siegler*, R.P. Baum, D. Schwabe*, G. Jacobi* B. Kornhuber* Department of Nuclear Medicine and Division of Pediatrics, Goethe University Medical Center, Frankfurt, FRG.

IMAGING OF PEDIATRIC BRAIN TUMOURS WITH THALLIUM AND RECEPTOR SCINTIGRAPHY

Diagnosis and therapy of pediatric brain tumours is a difficult and challenging task. To evaluate the role of nuclear medicine procedures in this special group of children regarding primary diagnosis and evaluation of therapy was the purpose of this investigation using routine thallium imaging combined with receptor avid substances like somatostatin analogue octreotide ${ }^{R}$.

Patients and methods: 13 children (age 1-21, mean 8.9 (age 21: recurrency from childhood tumour) (were imaged with Thallium-201 and Somatostatin-receptor ligand $\mathrm{in}-111$ Octreotide ${ }^{\mathrm{P}}$ (Mallinckrodt, FRG). After having obtained informed consent from the parents according to the rules of the ethical comittee, SPECT imaging was performed 30 minutes after injection (for Tl-201) or $3 \mathrm{~h}$ p.i. (Octreotide) followed by $24 \mathrm{~h}$ SPECT in some cases. In case of two tracers applied, a dual isotope SPECT was done. Tumour/to non-tumour uptake was calculated from ROls over the tumour and normal brain tissue in the same slice. TI was done in 11 , octreotide in $9_{r}$ repeat imaging for therapy follow-up in 3 ( $1-3$ controls). Histology was mainly obtained by surgery and ranged from Pineocytoma, astrocytoma II, III to dysgerminoma and medulloblastoma. Differentiation according to WHO grading was $1: 3,11: 2,11: 3,1 \mathrm{~V}: 5$.

Results: Uptake values in therapy controls demonstrated tumour response to chemotherapy, in one case of a pinealoblastoma encouraging further successful low dose therapy. Mean TI uptake values (according to grading): I: $1.99 ; 11: 3.34 ; 111: 4.86 ;$ IV: 3.08 , actreotide uptake: 11: 4.08 ; IV: 6.75 .

Conclusions: The use of different tracers in primary childhood brain tumours is of importance to define the best tracer for further therapy control. Tl and octreotide uptake values do not seem to be able to differentiate low and high malign tumors. The high uptake of somatostatin analogue in vivo in highly malignant tumours contradicts previous in vitro results.
39

K. Buga, L. Pávies, L. Sztriha, J. Kóbor, L. Csernay

Albert Szent-Györgyi Medical University, Departments of Nuclear Medicine and Pediatrics*, Szeged, Hungary

RELATIONSHIP BETWEEN HUMAN BRAIN MATURATION AND REGIONAL CEREBRAL BLOOD FLOW (STUDY IN ASPHYXIAL NEONATES

Maturation of the human brain is not yet complete at birth, but continues in the first few years of life. It has been shown that brain development is associated with regional changes in glucose cerebral metabolic rate and regional cerebral blood flow ( $\mathrm{TCBF}$ ). $\mathrm{rCBF}$ was studied by SPECT, using $99 \mathrm{~m}$-Tc-HMPAO. Eleven neonates were investigated; follow-up studies were performed in 4 cases [from 10 to 24 days of age, med: 20 days $]$. They were all full-term, mature babies, but they all had perinatal asphyxia and/or neurological symptoms such as convulsions, marked hypotonia or rigidity. Examinations were performed in the first, fourth, seventh and twelfth months of life. In first month the cortical rCBF was found to be lower than the rCBF in adults, mainly in the frontal and parietal regions. There was a strikingly higher cerebellar and brain stem uptake. In the first four months the activity of the cortical regions continued to increase, the only exception being the frontal area. After the sixth month the rate of this frontal hypoperfusion showed a futher decrease. By the end of first year, no clinical signs of neurological defects were detected and all these babies were normaly developed. During brain SPECT examinations of neonates, consideration must be paid to these changes in $\mathrm{rCBF}$, which always depend on the state of maturation of the brain.

\section{0}

S. Wynchank, E. Dywer, M.D. Mann.

Medical Research Council, Parow and Dept of Nuclear Medicine, Red Cross Children's Hospital and Institute of Child Health, University of Cape Town, Rondebosch, South Africa.

\section{LARYNGEAL INCOORDINATION IN PAEDIATRIC PATIENTS}

Laryngeal incoordination was sought in 1112 studies of paediatric patients referred for gastro oesophageal scintigraphy. of 69 patients in whom entry into the respiratory passage accompanied deglutition, (mean age 9.9 months, range 37 days 7.5 years, 41 male, 28 female) principal reasons for referral were repeated chest infections, (51), excess vomiting (9) and cough (2). ${ }^{99 \mathrm{~m}} \mathrm{Tc}$ sulphur colloid (4-22 MBq, depending on age) was swallowed in $5 \mathrm{ml}$ of liquid (fruit juice, the usual infant formula or expressed breast milk). Images of oesophageal transit in the erect position were obtained using a framing rate of $2 \mathrm{~Hz}$ for $60 \mathrm{~s}$ and a matrix of $64 \times 64$ pixels, with a right lateral or oblique view. The maximum depth of entry into the respiratory passage (D) was obtained by measurements on individual ciné images using a reflex microscope. The mean number of such entries into the laryngeal region per patient was 4.9 (range 1-12) and mean value of $D$ was $43 \mathrm{~mm}$ (range 16$91 \mathrm{~mm}$ ). A total of 317 entries was tecorded of mean duration $1.2 \mathrm{~s}$ (range $0.5-7.5 \mathrm{~s}$ ). $67 \%$ of such descents were of duration $2 s$ or less and most patients were asymptomatic during laryngeal entry of swallowed liquid. All patients except 12 had concurrent pathological gastro oesophageal reflux (GOR). Only 7 patients had later scintigraphic evidence of pulmonary aspiration. In conclusion an appropriate novel scintigraphic study is useful to diagnose and quantify laryngeal incoordination. This is of particular value in planning therapy for paediatric patients, who have repeated respiratory infections and proven GOR. 
41

I. Szilvási, E. Petrovics, S. Takács

Dept. of Nuclear Medicine, Postgradual Medical School, Budapest, Hungary

VENTILATION SCINTIGRAPHY IN SMALL CHILDREN

In this study results of ventilation scintigraphy in small children with congenital bronchopulmonary abnormalities using an effective aerosol producing equipment are presented. 45 children under three years of age (the youngest was 14 months old) were studied. Diagnosis was made by complex invasive bronchologic examinations. 24 children had bronchiectasia, 14 had deformed bronchial tree ( 9 with lobar and 5 with segmental deformation), 7 had other types of morphologic abnormalities. Multiple views ventilation scintigraphy was performed. 20-40 MBgs of TC99m-DTPA aerosol was inhaled within $2-3$ minutes. Procedure was well tolerated by children even with acute respiratory symptoms. Good-quality images were taken in all of the 67 scintigraphic studies. Grade and localization of the regional ventilation abnormality is essential for adequate therapy (bronchoscopic or surgical intervention or conservative treatment) in this patient group. Ventilation scintigraphy using an effective aerosol producing equipment proved to be useful in the follow-up of patients, because improvement or deterioration of regional ventilation contributes to making therapeutic decision in patients with morphoanatomic abnormalities of the bronchial system and invasive techniques may be avoided. In our opinion this scintigraphic technique should routinely be used in small children with congenital bronchopulmonary abnormalities.

42

S Wynchank, E. Dywer, M.D. Mann.

Medical Research Council, Parow and Dept of Nuclear Medicine, Red Cross Children's Hospital and Institute of Child Health, University of Cape Town, Rondebosch, South Africa.

\section{PULMONARY ASPIRATION IN PAEDIATRIC PATIENTS}

The incidence of pulmonary aspiration (PA), a serious consequence of gastro oesophageal reflux (GOR) and other foregut malfunction, was established in 1112 paediatric gastro oesophageal studies. Patients were referred principally for repeated respiratory infections. They were studied, using ${ }^{99 \mathrm{~m}} \mathrm{Tc}$ labelled sulphur colloid in liquid, in a composite investigation, including an oesophageal transit study, a search for GOR in the supine position (with 10 s per image for $30 \mathrm{~min}$ ) and at least 2 searches for PA at 30 and 120 min after deglutition, with others up to $24 \mathrm{~h}$ later, if there was a very high index of suspicion. Mean age was 18.6 months (range 37 days to 12.7 years) and the sex ratio was 29:18 (M:F). PA was detected in 47 studies. Associated pathological findings in these patients were laryngeal incoordination in 7, pathological GOR in 43 and abnormal oesophageal transit in 39. One patient had PA with normal oesophageal transit and no GOR. 32 PAs were identified in the right lung field, most frequently in the mid zone (15), and 17 PAs were in the left lung field, with 2 patients having two sites of PA. The abnormal oesophageal transit was most commonly holdup at various locations in 27 patients and slow passage of the inferior oesophagus in 11 . Multiple oesophageal malfunctions were seen in 15 patients. In conclusion scintigraphic PA is a relatively rare event, even in patients with repeated respiratory infections. It usually coexists with other foregut malfunctions.
43

A.Giordano, C.Trani, M.L.Calcagni, B.Rossi, A.Lombardo, R.Patrizi, L.Natale, R.Manfredi, G.Galli, A.Maseri. Istituti di Medicina Nucleare, Cardiologia e Radiologia, Università Cattolica del S.Cuore, Rome, Italy

NITRATES 201TL SPET (NSPET) VS. DOBUTAMTNE ECHO (DE) vS. MAGNETTC RESONANCE (MRT) IN DETECTING VLABLE MYOCARDTUM

In order to perforth a reliable multimodality comparison in detecting myocardial viability, we strictly followed a severe methodological approach: 1 - all techiques were tomographic; 2- anatomical correspondence of slices and spatial thickness was optimized, referring all techniques to the standard 16 segments model of the Am. Soc.of Echocardiography; 3- only a-diskinetic segments at preop.echo were considered for analysis; 4- gold standard was the contractile recovery postrevascularization (1 month). 20 pts with previous MI performed the following exams before a suc cessfull revascularization: NSPET (rest injection after i.v. Isosorbide Dinitrate $5 \mathrm{mg}$ with SPET after 4 hours), DE $(5-10 \mathrm{ug} / \mathrm{kg} / \mathrm{min}$ of Dobutamine) and MRT (with determination of Diastolic Thickness, DT.ness, and Systolic Thickening, ST ing. Criteria for viability were: 20171 uptake >50\% of peak activity at SPET; improvenent of WM from adiskinetic to hypo-normal at DE; DT ness $>9.5 \mathrm{~mm}$ and ST ing $>30 \%$ at MRT. Up to now the follow-up time was reached by $15 \mathrm{pts}$. At prop. echo $64 / 240$ segments were a-diskinetic; $25(39 \%)$ became hypo normal at postop. control, therefore were considered viable. $17 / 25$ were viable by NSPET, $14 / 25$ by DE, $7 / 13$ by DT.ness, $10 / 13$ by ST.ing. Sensitivity and Specificity values were: $68 \%$ and $55 \%$ for NSPET; $56 \%$ and $67 \%$ for DE; $54 \%$ and $56 \%$ for DT.ness; $77 \%$ and $61 \%$ for ST. ing. In conclusion; no single method seems; to reach the ac curacy of FDG-PET in predicting myocardial viability in pts with previous myocardial infarction.

44

A. Cuocolo, S. Maurea, A. Soricelli, L. Castelli, A. Nappi, F. Squame, M. Imbriaco, C. Morisco, B. Trimarco, M. Salvatore. University Federico II and National Cancer Institute, Napoli, Italy.

TECHNETIUM-99m MIBI CARDIAC TOMOGRAPHY AFTER NITRATES ADMINISTRATION IN THE IDENTIFICATION OF VIABLE MYOCARDIUM IN CORONARY ARTERY DISEASE.

In patients with coronary artery disease (CAD) and left ventricular (LV) dysfunction resting technetium-99m methoxy isobutyl isonitrile (MIBI) imaging may underestimate the presence of viable myocardium. Rest-redistribution (RR) thallium scintigraphy is able to identify hypoperfused hibernated myocardium. The aim of this study was to assess the capability of MIBI imaging after nitrate administration in differentiating severely ischemic but viable myocardium from fibrotic tissue. Thirty patients ( 28 men, mean age $56 \pm 10$ yrs) with angiographically proven $C A D$ and $L V$ dysfunction (ejection fraction $45 \pm 14 \%$ ) underwent resting and nitrate (nitroglycerine $0.005 \mathrm{mg} / \mathrm{kg}$ per os) MIBI ( $20 \mathrm{mCi}$ iv) tomography in two separate days and RR thallium-201 ( $3 \mathrm{mCi}$ iv) tomography within one week. A total of 660 segments were quantitatively analyzed. On resting MIBI imaging, 292 segments were normal (uptake $\geq 75 \%$ ), 180 segments showed moderate reduction $(51-74 \%)$ and 188 severe reduction ( $\leq 50 \%$ ) of tracer uptake. Of these 188 segments with severe reduction of resting MIBI uptake, $47(25 \%)$ showed increased MIBI uptake ( $210 \%$ vs resting study) after nitroglycerine administration (from $43 \pm 7 \%$ to $60 \pm 8 \%, \mathrm{p}<0.001$ ). All these 47 segments were identified as viable on RR thallium ( 2 normals, 26 reversible defects, and 19 mild-moderate irreversible defects). Of the $141(75 \%)$ segments with severe reduction of resting MIBI uptake and no change after nitroglycerine administration, the majority (100 segments) were identified as nonviable on RR thallium (severe irreversible defects); however, the remaining 41 segments showed evidence of viable myocardium on RR thallium (18 reversible defects and 23 mildmoderate irreversible defects). In conclusion, nitrate administration improves the differentiation between severely ischemic but still viable myocardium from irreversibly fibrotic tissue using MIBI cardiac tomography in patients with chronic ischemic $L V$ dysfunction. 
45

PR. Franken, F. De Geeter, P. Dendale, D. Demoor, A. Bossuyt. Departments of Nuclear Medicine and Cardiology, Free University of Brussels (AZ VUB), Brussels, Belgium.

BETA-METHYL IODOPHENYL PENTADECANOIC ACID UPTAKE IN POSTISCHEMIC DYSFUNCTION MYOCARDIUM: COMPARISON WITH DOBUTAMINE ECHOCARDIOGRAPHY TO PREDICT LONG TERM FUNCTIONAL RECOVERY.

Beta-methyl iodophenyl pentadecanoic acid (BMIPP) allows for myocardial metabolic studies with SPECT. BMIPP uptake reflects the activity of acyl-CoA synthetase, a common enzyme in triglyceride synthesis and beta-oxydation. More reduced BMIPP uptake than flow tracer uptake (mismatching) may be observed in postischemic dysfunctioning myocardium early after acute myocardial infarction (AMi).

To determine if mismatching is an indicator of viability, rest BMIPP $(148 \mathrm{MBq})$ and rest MIBI $(740 \mathrm{MBq})$ myocardial SPECT studies were obtained in 18 patients with left ventricular dysfunction $7 \pm 3$ days after AMI. The relative uptake of the 2 tracers was compared $(9 \mathrm{segs} / \mathrm{pt})$ to the inotropic reserve assessed by $2 \mathrm{D}$ echo and low-dose dobutamine stimulation $(5-10 \mathrm{mcg} / \mathrm{kg} . \mathrm{min})$. Long term functional recovery was assessed in all patients by $2 \mathrm{D}$-echo after a 6 months follow-up.

Of the 54 segs with dysfunction, 34 showed mismatching ( $12 \mathrm{pts}$ ) and 20 demonstrated inotropic reserve (7 pts). Of the 29 segs $(9 \mathrm{pts})$ with improved wall motion at 6 months, 27 segs (9pts) showed mismatching and 16 segs ( $6 \mathrm{pts})$ demonstrated inotropic reserve $(p<0.01)$. The predicitve value $(\mathrm{PV})$ of a positive or a negative test and their percent accuracy $(\mathrm{Acc})$ to predict functional recovery were:

\begin{tabular}{lcccccc} 
& \multicolumn{3}{c}{ SPECT mismatching } & \multicolumn{3}{c}{ ECHO dobutamine } \\
Patient study & 69 & PV - & Acc & PV + & PV- & Acc \\
Seg analysis & 77 & 89 & 78 & 86 & 73 & 78 \\
Se & 81 & 80 & 62 & 69
\end{tabular}

We conclude that mismatched BMIPP and MIBI uptake in dysfunctional segments following AMI, is indicative of residual viable myocardium, whereas matched defects represent scar tissue. SPECT is at least equivalent to ECHO in the prediction of functional recovery.

46

Margot Jonas, W. Brandau, B. Vollet, F.-J. Gildenaus, A. Fahrenkamp ${ }^{+}$, M. Weyand*, C. Puskás, J. Sciuk, H. H. Scheld*, W. Böcker+, O. Schober

Departments of Nuclear Medicine, Cardiac Surgery* and Pathology+, Minster, FRG

\section{I-PHIPA 3-10: A SPECT TRACER FOR ASSESSMENT MYOCARDIAL VIABILITY}

Fatty acid metabolism is altered in ischemic or necrotic myocardium as PET-investigations have shown. To evaluate myocardial metabolism with SPECT radioionated fatty acids have been developed, however without clinical acceptance so far. In contrast to other radiolabeled fatty acids (e.g. 123I-IPPA) 123I-PHIPA 3-10, a phenylene bridged long chain fatty acid, is accumulated in the myocardium with a long biological half life of more than $15 \mathrm{~h}$, indicating a "metabolic trapping".

In order to evaluate the clinical relevance of 123I-PHIPA 3-10 myocardial viability has been assessed in 9 patients with severe coronary heart disease. SPECT investigation using 123I-PHIPA 3-10 were compared with 201T1 (stress / redistribution) and $99 \mathrm{mTc}$-Sestamibi studies. In 8 patients myocardial scares were overestimated by $201 \mathrm{~T}$-redistribution. in 13 regions with persistent $201 \mathrm{Tl}$-defects hibernating/stunned myocardium could be identified as well with 1231-PHIPA as with $99 \mathrm{mTc}$ Tc-Sestamibi. In 4 lesions no perfusion with $99 \mathrm{mTc}$-Sestamibi was observed, but a significant uptake of 123I-PHIPA implicated residual viable myocardium. This could be confirmed by follow-up studies after revascularization where the perfusion with $99 \mathrm{mTc}$-Sestamibi was improved.

During a heart transplantation a double nuclide study using 1311-PHIPA and 99 mTc-Sestamibi was performed. Scintigraphies of the explanted heart were compared to the preoperative scans, the activity of tissue heart were compared to the preoperative scans, the activity of tissue
samples and the corresponding histology. 131I-PHIPA and $99 \mathrm{mTC}$-Sestamibi were accumulated in hibemating or stunned myocardium in a ratio of $2: 1$ according to their percent I.D. In scares however, the accumulation ratio of PHIPA / Sestamibi reversed to $1: 2$.

These results imply that residual viable myocardium can be more accurately differentiated from scares with PHIPA 3-10 than with Sestamibi. Furthermore the different biodistribution of PIIPA and Sestamibi in hibemating myocardium and scares indicates that not only the perfusion, but the metabolic state of the myocardium can be evaluated with PHIPA 3-10. As a metabolic marker of the heart PHIPA 3-10 has the potential to improve the assessment of myocardial viability with SPECT.
47

A. Bockisch, K.J. Henrichs, U. Wenderoth, J. Andreas, J. Meyer, H. Oehlert, K. Hahn. Klinik für Nuklearmedizin, II. Medizinische Klinik HTG-Chirurgie; J. Gutenberg-Universität, D-55101 Mainz, Germany

ACCURRACY OF MYOCARDIAL SCINTIGRAPHY - A COMPARISON OF ${ }^{99} \mathrm{Tc}^{\mathrm{m}}$-MIBI- AND ${ }^{201}$ TICI-FINDINGS WITH THE OUTCOME AFTER BYPASS SURGERY.

In our prospective study, 3 protocols of myocardial scintigraphy were performed in the same patients (pts) suffering from high grade coronary artery stenosis. The results were compared with first pass radionuclid ventriculography (FP-RNV), coronarangiography (CA) and in each case with post surgical outcome. Scintigraphy was performed both prior to and 3 to 6 months after bypass surgery. The scintigraphic investigation included always FP-RNV at rest and under stress, and myocardial SPECT using ${ }^{99} \mathrm{Tc}^{\mathrm{m}}$-MIBI in a 2-days-protocol, ${ }^{201} \mathrm{Tl}$ chloride standard protocol with 3 hours redistribution images, and in addition a rest reinjection (reinj.) 2 days after exercise. Up till now 43 pts have entered the study, which is completed for 20 of them. The results of all modalities are compared.

Result for the myocardial segments rated as scares prior to surgery:

\begin{tabular}{|l|c|c|c|}
\hline findings after surgery $\rightarrow$ & normal & ischemia & scar \\
\hline${ }^{201} \mathrm{TlCl}-$ redistribution & 36 & 14 & 32 \\
\hline${ }^{201} \mathrm{TlCl}-$ reinjection & 10 & 15 & 30 \\
\hline${ }^{90} \mathrm{Tc} \mathrm{C}^{\mathrm{m}}$-MIBI & 18 & 8 & 27 \\
\hline
\end{tabular}

Compared to CA, the 3 myocard. scinti. methods were found to have a very high sensitivity and specificity (98\% of pathological areas) in the detection of coronary heart disease. However, concerning viability, the results differ considerably. Viability was missed in $61 \%$ of the akinetic areas diagnosed as scars by ${ }^{201} \mathrm{Tl}$ standard protocol but only in $45 \%$ by ${ }^{201} \mathrm{TI}$ reinj. and $49 \%$ by MIBI 2-days protocol. In our highly selected patient population, therefore, the use of a reinj. technique (MIBI and $\mathrm{TlCl}$ ) is mandatory.

48

P. Melon, Th. Benoit, V. Legrand, A. Luxen, H. Kulbertus, P. Rigo Divisions of Cardiology and Nuclear Medicine, Cyclotron Research Center Medical Unit, University of Llege, Liege, Belgium

VALUE OF REST TC-99m SESTAMIBI TO IDENTIFY VIABLE MYOCARDIUM IN PATIENTS WITH SEVERE CHRONIC ARTERY DISEASE: COMPARISON WITH POSITRON EMISSION TOMOGRAPHY

To assess whether rest Tc-99m Sestamibi (Mibi) could identify viable myocardium, we studied 15 patients with severe chronic coronary artery disease (three-vessel disease in 9 and wo-vessel disease in 6) and left ventricular dysfunction (ejection fraction: $30 \pm 11 \%$ ), reterred to positron emission tomography (PET) for identification of viable myocardium. $A$ combined study of perfusion (potassium-38 or $\mathrm{N}-13$ ammonia) and glucose combined study of perfusion (potassium-38 or $\mathrm{N}-13$ ammonia) and glucose
utilization (18-FDG) was performed under hyperunsulinemic euglycemic utilization (18-FDG) was performed under hyperunsulinemic euglycemic
glucose clamp. For each study, potassium-38 or $\mathrm{N}-13$ ammonia and 18-FDG activities were determined in 19 myocardial segments. The segment with the highest potassium-38 or N-13 ammonia uptake was set as $100 \%$ for both data sets and segment uptake was normalized to this reference segment. Based on results obtained in control subjects, the thresholds for segment viability were defined as perfusion tracer uptake $<70 \%$ and FDG uptake $>70 \%$ of the reference segment. For each patient, Mibi activity (expressed as percent of peak activity) was determined in the 19 segments by circumferential profile analysis.

Comparison between PET and Mibi data was realized in a total number of 212 segments. According to PET criteria, 43 segments $(20 \%)$ were non viable and 61 segments (29\%) were viable. Mibi uptake was $50 \pm 17 \%$ and $59 \pm 17 \%$ in non viable and viable segments, respectively $(p=0.01)$.

\begin{tabular}{|c|cccc|}
\hline Mibi uplake (\%) & $<40$ & $40-49$ & $50-59$ & $\geq 60$ \\
Viable segments by PET & 11 & 8 & 11 & 31 \\
Non viable segments by PET & 13 & 12 & 5 & 13 \\
\hline
\end{tabular}

The positive and negative predictive values for segment viability using a threshold of $60 \%$ of peak activity of Mibi uptake were $70 \%$ and $50 \%$, respectively.

Conclusion: On the basis of direct comparison with perfusion-FDG PET, Mibi uptake at rest is a poor indicator of myocardial viability in patients with severe coronary artery disease and left ventricular dysfunction. 
H. Wolf, V. Stein, C. Stauch, K.H. Bohuslavizki, M. Schramm, W. Brenner, M. Clausen, E. Henze.

Division of Nuclear Medicine, Christian-Albrechts-University of Kiel, Germany

\section{EFFECT OF INSULIN ON F-18 FDG AND TL-201} UPTAKE IN BREAST CANCER CELLS

F-18-fluorodeoxyglucose (FDG) is widely used to study tumour metabolism by means of positron emission tomography (PET). The purpose of this study was to determine the effect of insilin on the uptake of FDG in cultures of breast cancer cells in comparison to Ti-201. Measurements of both tracers were performed in 160 cell culture tubes with incubation intervalls ranging from 1 to $240 \mathrm{~min}$. Cellular FDG- and Tl-201 accumulation was measured in a gamma counter and normalized to the activity added to the medium and to one million cells expressed as $\%$, with an average of $8.5 \pm 0.7$

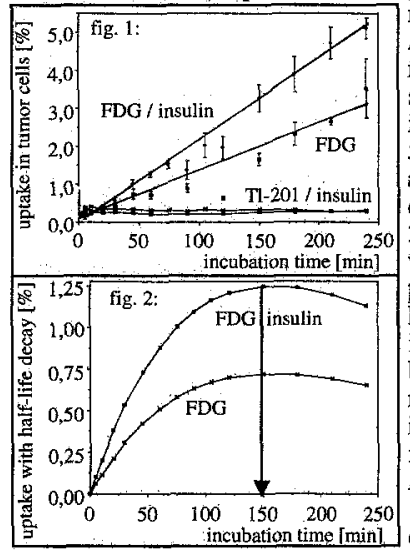
illion cells / culture tube.

As shown in fig. 1, linear accumulation of FDG over time was seen. A further significant increase from $3.52 \pm 0.74 \%$ to $5.10 \pm 0.32 \%$ at $240 \mathrm{~min}$ was attained after adding insulin. In contrast, cellular uptake of $\mathrm{Tl}$ 201 did not differ significantly with and without insulin. Extrapolating these results to FDGPET tumor imaging a markedly improved tumor targeting might be obtained with simply replacing the common FDG injection by a continuous infusion together with insulin. An optimum imaging period at $150 \mathrm{~min}$ after starting infusion considering the decay of F-18 as shown in fig 2 . The potential improvement of PET image quality in tumor visualization with FDG by the approach suggested ought to be tested clinically.

\section{0}

J.A.Schönberger, J.C.Stollfuß, M.Port, K.G.Grillenberger, M.Büchler*, H.G.Beger*, S.N.Reske

Department Nuclear Medicine and Department General Surgery, University of UIm, Germany

ASSOCIATION OF GLUCOSE-TRANSPORTER 1 GENE (GLUT-1) ACTIVATION TO FDG-UPTAKE IN HUMAN PANCREATIC ADENOCARCINOMA

Recent studies indicated increased expression of glucosetransporter-1 gene in ras and sic transformed fibroblasts. Interestingly, increased expression of ras oncogene is found in more than $90 \%$ of human pancreatic adenocarcinomas. We therefore analysed the relation between GLUT-1 gene-expression and glucose-uptake measured with F-18-fluorodeoxyglucose (FDG) and positron emission tomography (PET) in human pancreatic adenocarcinoma (ACP). Seven patients (pts) with ACP were preoperatively examined with FDG-PET ( 3 male, 4 female; mean age 60 years, 52-67). Six pts with chronic pancreatitis (CP), served as controls (6 male; mean age 44 years, 32-57). PET scans were obtained with an ECAT 931-08-12 scanner (Siemens-CTI, Knoxville, TN) $1 \mathrm{hr}$ after administration of $300 \pm 50 \mathrm{MBq}$ of FDG. All patients were fasted for at least 6 hrs prior to this study. FDG-uptake was quantitated by standard uptake values. Tissue specimens were obtained from these $13 \mathrm{pts}$ during surgical pancreas resection for reverse transcriptasepolymerase chain reaction (rt-PCR) and northern blot analysis. FDG-uptake in pts with ACP was significantly higher compared to those with $\mathrm{CP}(2.8 \pm 1.4 \mathrm{vs} 1.2 \pm 0.39 ; \mathrm{MV} \pm \mathrm{SD} ; \mathrm{p}<0.05)$ Northem blot analysis and rt-PCR showed presence of GLUT-1 mRNA in all pancreatic carcinomas; by contrast no GLUT-1 encoding mRNA was detectable in tissue from pts with CP. These results indicate that the difference in glucose utilisation between $\mathrm{CP}$ and $\mathrm{ACP}$ is related to the over-expression of the GLUT-1 gene in pancreatic adenocarcinoma.
51

K. Matzen, J. Mattes, R. Truckenbrodt, R. Senekowitsch, M. Schwaiger

Nuklearmedizinische Klinik und Poliklinik der Technischen Universität München, Munich, Germany

CHANGE IN UPTAKE OF C-14-FDG AND H-3-THYMIDINE AS EARLY RESPONSE OF TUMOR CELL SPHEROIDS TO IRRADIATION

The aim of the study was the evaluation of FDG and thymidine uptake with time in tumor cell spheroids following irradiation in relationship to cell viability and proliferative capacity. Spheroids of a human adenocarcinoma cell line (SW 707) were incubated in media containing C-14-FDG or $\mathrm{H}-3$-thymidine $(37 \mathrm{kBg} / \mathrm{ml})$ for $1 \mathrm{~h}$ at $1,4,8,24$ and $48 \mathrm{~h}$ after irradiation with $6 \mathrm{~Gy}$. Tracer uptake was measured per spheroid. The number of viable celis was determined using the mitochondrial enzyme activity (MTT-test) and the proliferative capacity of dispersed spheroid cells by the clonogenic survival assay. The uptake changed in the following manner (uptake of control = $100 \%$ ).

\begin{tabular}{|c|c|c|c|c|c|c|}
\hline & & $1 \mathrm{~h}$ & $4 \mathrm{~h}$ & $8 \mathbf{h}$ & $24 \mathrm{~h}$ & $48 \mathrm{~h}$ \\
\hline \multirow[t]{2}{*}{ FDG } & $\begin{array}{l}\text { uptake/viable } \\
\text { cell.(z) }\end{array}$ & 52 & 192 & 238 & 61 & 67 \\
\hline & uptake/sph (8) & 40 & 149 & 183 & 44 & 40 \\
\hline \multirow[t]{2}{*}{ Thy } & $\begin{array}{l}\text { uptake/viable } \\
\text { cell }(\%)\end{array}$ & 105 & 89 & 190 & 120 & 206 \\
\hline & untake/sph ( & 85 & 7.0 & 145 & 86 & 121 \\
\hline
\end{tabular}

The proliferative capacity decreased to $4 \%$ of control at $1 \mathrm{~h}$ recovering to $21 \%$ at $48 \mathrm{~h}$. The results indicate, that tracer uptake is changing with time and is higher for the viable cells than for the whole spheroid. The difference is depending on the number of surviving cells. The high uptake at $8 \mathrm{~h}$ is an indication for the recovery of the proliferative capacity.

\section{2}

U. Haberkorn, M. Lindauer, F. Oberdorfer, J. Gebert, K. Weber, I. Morr, Ch. Herfarth, G. van Kaick, H.K. Schackert.

German Cancer Research Center (dkfz), Heidelberg, Germany and Dept. of Surgery University of Heidelberg.

MONITORING OF GENE THERAPY WITH 5-FLUOROCYTOSINE: IN VITRO STUDIES WITH HUMAN CANCER CELLS.

The transfer of genes converting nontoxic agents to cytotoxic ones is a promising approach in cancer therapy. Cytosine deaminase $(C D)$ is a bacterial or fungal enzyme ordinarily not found in mammalian cells, which catalyzes the deamination of cytosine (fluorocytosine) to uracil (fluorouracil). Therefore genetically modified mammalian cells, which express the $\mathrm{CD}$ gene should be able to convert 5fluorocytosine (FC) to 5-fluorouracil (FU) and be selectively sensitive to $\mathrm{FC}$ as compared to unmodified cells. To optimize and individualize the therapeutic protocol it is necessary to assess the enzymatic activity of cytosine deaminase at an early stage of therapy.

Human colon carcinoma and glioblastoma cell lines were transfected with the bacterial $C D$ gene. Cells bearing the gene and cells without the gene (controls) were incubated in the presence of $20 \mu \mathrm{Ci}{ }^{3} \mathrm{H}-\mathrm{FC}$ for 4 hours. After washing and lysis with perchloric acid a high performance liquid chromatography (HPLC) analysis was done of the lysates and the incubation media. Moreover, the uptake of FC was measured after 30 minutes. $1 \mathrm{~h}, 6 \mathrm{~h}, 24 \mathrm{~h}$ and $48 \mathrm{~h}$ incubation in a medium containing $5 \mu \mathrm{Ci} 3 \mathrm{H}-\mathrm{FC}$ and cold $\mathrm{FC}$ with an end concentration of $0.5 \mathrm{mM}$ to quantitate the $\mathrm{FC}$ accumulation during therapy.

${ }^{3} \mathrm{H}-\mathrm{FU}$ was found in the lysates and the media of the CD-bearing cells, whereas in the controls only ${ }^{3} \mathrm{H}-\mathrm{FC}$ was seen, showing the selectivity of therapy. Moreover, the FU found in the media may account for the bystander effect observed in former experiments. However, the uptake studies revealed a moderate accumulation of radioactivity in the tumor cells. This indicates that transport may be a limiting factor for this therapentic approach. Since the synthesis of ${ }_{18} \mathrm{FC}$ has already been established, in vivo data can be obtained with positron emission tomography. 


\section{3}

M. Hosone A. Kondo, Z. Yao, H. Kobayashi, M.N. Hosono, H Sakahara, K. Imada, M. Okuma, T. Uchiyama, J. Konishi. Depts Nucl Med. and Int. Med., Inst. Virus Res., Kyoto University Japan

\section{LOCALIZATION OF INFILTRATION OF HUMAN LEUKEMIA CELLS IN SCID MICE}

Adult $T$ cell leukemia (ATL) is caused by human $T$ cell leukemia virus type I (HTLV-1). This disease can be modeled in severe combined immunodeficiency (SCID) mice inoculated with the HTLV-l-infected leukemic cell line 43T. The purpose of this study was to examine the early and late distributions of these cells in vivo using in-111-oxine labeled $43 \mathrm{~T}$ cells and radiolabeled antiTac (IL-2 receptor $\alpha$ chain) monoclonal antibody (MAb) respectively. Early phase distribution was examined 2 and 6 days after the i.p. injection of $10^{8} \mathrm{ln}$-111-oxine-43T cells into SCID and normal mice. By day 6 significant accumulations of radioactivity were found in the spleen and thymus of SCID mice (33.3 \pm 9.4 and $10.0 \pm 3.6 \% 10 / g$, respectively) in comparison with normal mice $(19.1 \pm 2.5$ and $3.7 \pm 0.9 \% \mathrm{DD} / \mathrm{g})$. The accumulations in other organs of SCID mice were not significantly higher than in those of normal mice. Late phase distribution was monitored in 43T-inoculated-SCID mice starting 28 days after i.p. injection of the cells (not labeled with in-111-oxine) by localization with I125 and In-111 labeled anti-Tac MAb (IgG2a) and an isotypematched control MAb. The biodistributions were examined 1, 2 and 4 days after I.v. injection of MAb. Significant amouts of in111-anti-Tac MAb were found in the spleen and thymus of SCID mice $(22.5 \pm 6.9$ and $22.8 \pm 6.9 \% \mathrm{D} / \mathrm{g}$, respectively at day 2$)$ which were higher than those in the same organs of mice given In-111-control MAb $(12.0 \pm 5.1$ and $7.5 \pm 4.6 \% \mathrm{ID} / \mathrm{g})$. Similar results were obtained with $1-125$ labeled anti-Tac and contro MAbs. These results were coincident with the histologicallyconfirmed infiltration of $43 \mathrm{~T}$ in SCID mice, suggesting that the radiometric techniques in this study could be used to evaluate the proliferation sites of $43 \mathrm{~T}$ cells.

\section{4}

H.Wolf, E.Niemöller*, D.Kutzner, B.Möhrer, C.Stauch, K.H. Bohuslavizki, W.Brenner, M.Schramm, M.Clausen, E.Henze. Division of Nuclear Medicine and * Institut of Pharmacy, Christian-Albrechts-University of Kiel, Germany

I-123-LABELLED ACRIDONES AS POTENTIAL IN. VIVO TUMOUR TRACERS INVESTIGATED IN CELLS OF BREAST CARCINOMA

Acridones show biological effects on various tumours, possibly mediated by tumour cell uptake. The aim of this study was to label selected acridone derivatives and to determine the cellular uptake in comparison to Tl-201 as a known non-specific tumour tracer.

Acridone (AC) and 10-methyl-acridone (MAC) were radioiodinated with I-123 by the Iodogen method. The monoiodinated products were separated by HPLC. The accumulation was determined in breast cancer cell cultures $(n=300)$ with incubation times ranging from 1 to 240 minutes. The uptake was calculated as the percentage (mean $\pm 1 \sigma$ ) of the added activity and standardized to one million tumour cells.

\begin{tabular}{|llll|}
\hline \multicolumn{4}{|c|}{ uptake during different incubation times in breast cancer cells } \\
\hline incubation time & \multicolumn{5}{c|}{ uptake [\%] of } \\
[min] & $\frac{\mathrm{I}-123-\mathrm{AC}}{0.32 \pm 0.05}$ & $\underline{\mathrm{I}-123-\mathrm{MAC}}$ & $\mathrm{Tl}-201$ \\
20 & $0.60 \pm 0.10$ & $0.21 \pm 0.06$ \\
60 & $0.40 \pm 0.07$ & $0.71 \pm 0.05$ & $0.42 \pm 0.07$ \\
120 & $0.31 \pm 0.07$ & $0.72 \pm 0.09$ & $0.43 \pm 0.07$ \\
240 & $0.65 \pm 0.06$ & $0.42 \pm 0.06$ \\
\hline
\end{tabular}

The uptake of 2-iodoacridone (I-123-AC) was similar to TI201. The higher lipophilic, methylated analogon (I-123-MAC) showed an uptake twice as high.

In conclusion, I-123-MAC seems to have potential for tumour imaging. Further research is needed in other types of cancer cells as well as in-vivo studies with lipophilic N-substituted analogons.

\section{5}

L. Schmid, M. Bottlaender, J. Delforge, C. Fuseau, D. Fournier and M. Mazière

CNRS URA 1285, Service Hospitalier Frédéric Joliot, DSV, CEA, 4, Place du Général Leclerc 91401 Orsay (France).

\section{THE INFLUENCE OF ISOFLURANE ON IN VIVO BENZODIAZEPINE RECEPTOR BINDING: A PET MODELING ANALYSIS IN LIVING BABOONS}

Short acting benzodiazepine derivates have found wide clinical use in anesthesia. From previous studies we know that in vivo the cerebral uptake of flumazenil (FMZ), a benzodiazepine receptor (BZR) antagonist, is considerably increased in rats anaestetized with isoflurane. However neither the benzodiazepine receptor density (Bmax) nor the affinity (Kd) is affected by isoflurane in vitro. In an attempt to explain these observations we investigated the interference of isoflurane on the BZR in living baboons. An in vivo modeling analysis for [11C]FMZ kinetics was used which provides an estimation of the apparent $B \max \left(B^{\prime} \max \right)$ and the equilibrium dissociation rate constant KdVR (VR = volume of reaction).

Results show that the apparent Bmax and KdVR of FMZ are significantly increased by about $100 \%$ in animals anaesthetized with isoflurane/nitrous oxide $(1 \% / 70 \%)$ in oxygen compared to animals treated with nitrous oxide alone. We suggest that B'max enhancement is due to isoflurane nonspecific interaction with the lipid bilayer structure, altering the membrane fluidity which might result in a facilitated receptor accessiblity for FMZ. This increased volume of reaction may also explain the increase of KdVR in our study.

These results suggest that the anaesthetic effect of inhalational anaesthetics and BZR agonists in combination results not only from an additive but from a synergistic interaction by increasing the available binding sites for benzodiazepine derivates.

\section{6}

J.Bathmann, S. Braune, G. Deuschl, C.H. Lücking, E. Moser Depts. of Nuclear Medicine and Neurology, University of Freiburg, Freiburg, Germany

EVALUATION OF MYOCARDIAL SYMPATHETIC INNERVATION IN PARKINSON'S DISEASE (PD) AND AUTONOMIC FAILURE (AF) BY J-123 METAIODOBENZYLGUANIDINE (MIBG)-SCINTIGRAPHY

PD can be associated with AF. Standard physiological and pharmacological tests do not allow to distinguish pre- and postganglionic neurons. So far the pattern of affection of the autonomic system in $\mathrm{PD}+\mathrm{AF}$ has remained unclear.

5 patients with $\mathrm{PD}$ and $\mathrm{AF}$ were investigated with standard autonomic testing, magnetic resonance imaging (MRI) and J-123MIBG-Scintigraphy. No patient was on any medication interfering with MIBG-Uptake during the study. Planar whole-body and anterior images of the chest were acquired 1 and $4 \mathrm{~h}$ following injection of $260 \mathrm{MBq} J-123 \mathrm{MIBG}$. SPECT images of the heart were recorded after 4,5 h. 2 days later a myocardial perfusion SPECT at rest, $1 \mathrm{~h}$ after injection of $370 \mathrm{MBq} 99 \mathrm{~m}$-Tc-MIBI was obtained. MIBG imaging was also performed in 8 normal subjects, that served as controls.

In all five patients no myocardial MIBG-Uptake was seen, neither planar nor by SPECT. Heart/Mediastinum ratios in patients and controls were $1,04 \pm 0.07$ and $1,98 \pm 0.24$ respectively ( $\mathrm{p}<$ 0,0001 ). Myocardial perfusion imaging revealed normal findings in all patients.

These results show that in pts with $P D$ and AF postganglionic sympathetic efferents are severely affected. MIBG-imaging may be helpful for early diagnosis of AF in PD-pts by discriminating postganglionic damage from central nervous diseases with preganglionic affection of sympathetic neurons. 
A. Cifelli', J.M. Lavalaye', A.P. Aldenkamp ${ }^{2}$, J. Overweg' ${ }^{2}$, E.A. van Royen'.

'Dept of Nuclear Medicine, AMC, Amsterdam and ${ }^{2}$ Dept of Neurology and Dept of Neuropsychology, Meer B Bosch, Heemstede, The Netheriands.

GAMMA-VINYL-GABA AS ANTIEPILEPTIC THERAPY MAY INFLUENCE CENTRAL BENZODIAZEPINE RECEPTOR IMAGING WITH ${ }_{123}$ I-IOMAZENIL SPECT

Gamma-Vinyl-GABA (GVG/Vigabatrin) is used as an add-on drug in patients with partial epilepsy. It protects GABA from elimination by irreversibly inhibiting GABA transaminase. GVG might also have a benzodiazepine effect on GABA receptors (GABA ), due to the functional coupling of GABA and $B Z$ receptors, and so effecting ${ }^{123}$ I-lomazenil SPECT scans. We speculated on a possible inhibitory effect of GVG on the binding of 1231-lomazenil to the $B Z$ receptors of the human brain.

Three patients suffering from partial epilepsy were examined on and off Vigabatrin medication. Immediately after injection of 152 $\mathrm{MBq}{ }^{123}$-lomazenil i.v. [Mallinckrodt Diagnostica] 12 slices of $5 \mathrm{~min}$ each (128*128 matrix) located at $4 \mathrm{~cm}$ above the orbitomeatal line were acquired for $60 \mathrm{~min}$, followed by a multislice study $120 \mathrm{~min}$ after injection on a SME 810 multi-detector system. Uptake velocity and final binding of ${ }^{123}$-lomazenil was measured by drawing cortical ROIs after correction for dose, bodyweight and decay.

Within 15 minutes a steady state was reached. Between 15 and $60 \mathrm{~min}$, measured at 5 min intervals, uptake did not change $(\mathbb{P}>$ 0.2 , slope of regression curvel for all curves individually. The uptake, after the steady state was reached, was significantly lower $(44$ SMU) for 2 of the 3 patients (each $P<0.001$ ) when on Vigabatrin medication. After 150 min counts in 6 slices gave an average decrease of 115 SMU off GVG to 105 SMU on GVG.

Conclusion. High doses of GVG seem to have an apparent effect on ${ }^{123}$-lomazenil binding to cerebral $\mathrm{BZ}$ receptors in vivo. We conclude that patients with partial epilepsy referred for ${ }^{123}$-lomazenil SPECT in the detection of epileptic foci should temporarily withdraw from GVG medication.

\section{8}

M. Reinhardt, F. Götz, G. Stadtmüller, T. Krause, M. Berger, E. Moser

Depts. of Nuclear Medicine, Clinic of Radiology, Clinic of Psychiatry, University of Freiburg, Freiburg, Germany

I-123 IOMAZENIL SPECT IN MAJOR DEPRESSTVE DISORDERS (MDD) - COMPARISON TO TC-99m HM-PAO SPECT AND MRI There are recent indications that GABAergic transmission may play an important role in MDD. However, little is known about the in-vivo activity of GABA receptors in depressed patients whereas many studies report about diminished cerebral blood flow and metabolism especially in the frontal lobe. Additionally, structural changes and reduced volume of the frontal lobe in patients with severe depression are described in $\mathrm{CT}$ or MRI studies. Thirteen patients ( $50-80$ years) with a mean score on the Hamilton Rating Scale for Depression (HRSD) of 26 and without evidence of relevant physical illness, history of drug abuse or other preexisting psychiatric disorders were investigated. The DSM-III-R criteria for MDD were fulfilled. Iomazenil-SPECT was performed 90 min. and HM-PAO SPECT $20 \mathrm{~min}$. after i.v. injection. Uptake to 8 anatomically defined ROI's of the frontal lobe (4 for each side in consecutive slices, $0.7 \mathrm{~cm}$ thick) was normalized to the cerebellum. An uptake ratio $\leq 0,9$ was determined to be pathological. Neuroradiological examination was done on a 2,0-T MRI-system using $T_{1}$ and $T_{2}$-weighting and disclosed slight frontal atrophy in 6 patients. They all showed a diminished blood flow in one or more ROI's. Seven patients had a normal MRI and HMPAO scan. GABA receptor activity was diminished in 12 of 13 patients in the frontal lobe. Patients with normal HMPAO and abnormal Iomazenil SPECT were characterised by a lower HRSD-score than those with abnormal pattern in both investigations ( $22 \mathrm{vs.} 29, \mathrm{n} . \mathrm{s}$.). In course of depression, reduced GABA receptor activity precedes disturbance of regional blood flow and anatomical changes of brain structure (MRI). Exept of 2 cases of chronic and severe depression, the extend of lesions in the Iomazenil SPECT exceed those of HM-PAO SPECT. This observation confirms the GABAergic hypothesis of depression and may be of relevance for therapeutic studies especially in the earlier stage of MDD.

\section{9}

Weckesser M.1, Ziemons K.1, Langen K.J. ${ }^{1}$, Holschbach M. ${ }^{2}$, Stöcklin G. ${ }^{2}$, Wagner H.N. ${ }^{3}$, Müller-Gärtner H.W.1

1) Institute of Medicine and 2) Nuclear Chemistry, Research Center Julich, 3) The Johns Hopkins University School of Medicine, Baltimore

QUANTIFYING MUSCARINIC CHOLINERGIC RECEPTOR CHARACTERISTICS IN HUMAN BRAIN IN VIVO WITH SINGLE PHOTON EMISSION TOMOGRAPHY AND [123] IODODEXETIMIDE

Muscarinic cholinergic receptors $(\mathrm{mAChR})$ are distributed throughout the cerebral cortex and are supposed to modulate neocortical activity. mAChR are possibly involved in epilepsy and may play a role in dementing processes. We address the issue of quantifying specific kinetic characteristics of these receptors in the living human brain.

Dynamic and static single photon emission tomography (SPET) was quantitatively performed in 3 normal volunteers after intravenous quantitatively performed in 3 normal volunteers after intravenous
injection of $204-252 \mathrm{MBq}[123 \mathrm{~T}$-4-iododexetimide (I-Dex), using a 4 head SPET camera. The input function was determined and corrected for metabolites using arterialized venous blood samples. Kinetic variables were extracted from the time activity curves of various brain structures using a 4-compartment model.

The results (mean $\pm \mathrm{SD}$ ) are summarised in Table 1:

\begin{tabular}{|l|l|l|l|}
\hline Region: & $\mathrm{K} 1(\mathrm{ml} / \mathrm{g} / \mathrm{min})$ & $\mathrm{k} 3\left(\mathrm{~min}^{-1}\right)$ & $\mathrm{k} 4\left(\mathrm{~min}^{-1}\right)$ \\
\hline Frontal lobe & $0.295 \pm 0.005$ & $0.092 \pm 0.01$ & $0.003 \pm 0.0003$ \\
\hline Caudate nucleus & $0.319 \pm 0.007$ & $0.099 \pm 0.008$ & $0.003 \pm 0.0003$ \\
\hline Thalamus & $0.289 \pm 0.005$ & $0.076 \pm 0.01$ & $0.003 \pm 0.0004$ \\
\hline Cerebellum & $0.352 \pm 0.006$ & $0.042 \pm 0.01$ & $0.006 \pm 0.002$ \\
\hline
\end{tabular}

These data support the view that after non-specific delivery to brain tissue (K1) Y-Dex binds specifically $(\mathrm{k} 3$ ) to areas known to be rich in mAChR (cortex, caudate nucleus and to a lesser extent thalamus) but binds with a much lower affinity to structures with a low content of this receptor. The results validate the use of I-Dex for quantifying $\mathrm{mAChR}$ in the human brain in vivo.

\section{0}

E.A.Dubois, G.A. Somsen, J.J.J. Borm, A.G.F.Janssen, E.Busemann-Sokole, K.de Bruin, M. Pfaffendorf, E.A. van Royen, P.A.van Zwieten

Depts. of Nuclear Medicine, Cardiology, Pharmacotherapy, Academic Medical Centre, University of Amsterdam, Amsterdam, The Netherlands Cygne B.V. Technical Unversity Eindhoven, The Netherlands PRELIMINARY EXPERIENCE IN USING [123I]IODODEXETIMDE IN HEALTHY VOLUNTEERS FOR THE IMAGING OF MUSCARINIC RECEPTORS IN THE HUMAN HEART AND BRAIN

Muscarinic receptors may play a significant role in neurological (Parkinson's disease, Alzheimer's disease, epilepsy) as well as in cardiac disorders (myocardial infarction, cardiac failure). In the present study we evaluated the biodistribution of [123I]-Iododexetimide (IDEX) in three healthy volunteers as a possible radioligand for the imaging of muscarinic receptors in both the human brain and the human heart. IDEX (specific activity $>5000 \mathrm{Ci} / \mathrm{mmol}$ ) was synthesized in house and showed high specificity in an animal model. Three male healthy volunteers with an average age of 32 years received approximately $185 \mathrm{MBq}$ i.v.. Dynamic images of the thorax were made during the first hour after injection ( $1 \mathrm{~min} / \mathrm{frame}, 64 \times 64$ matrix). Static images were acquired until 9 hours after injection ( $5 \mathrm{~min} / \mathrm{frame}, 64 \times 64$ matrix). Whole body studies were made at 5,9 and 24 hours after injection.

Liver activity increased throughout the study: $5 \mathrm{~h}$ post injection approximately $5 \%$ of the injected dose was measured in the liver. Lung rapidly increased after injection and diminished 1 hour after administration. The myocardium proved already well defined by 15 minutes after injection. Heart to lung ratios reached maximum values $1.5 \mathrm{~h}$ after injection. At $24 \mathrm{~h}$ post injection, $2 \cdot 3 \%$ of the injected dose was still present in the liver and brain. Heart and lung activity had almost completely disappeared at that time. Cardiac SPECT images with a 3headed camera ( 60 angles, 20 sec/frame, $64 \times 64$ matrix) were obtained in p.i. and were of high quality. Myocardial uptake of DEX varied from 5.3 to $5.7 \mathrm{~Bq} / \mathrm{ml} / \mathrm{MBq}$ injected (BORSOM program v 1.0). Maximum activity in the human brain was reached at $9 \mathrm{~h}$ p.i., which is in accordance with literature data Brain SPECT images (Strichman SME 810, Multislice, $1 \mathrm{~cm} / \mathrm{slice}, 5 \mathrm{~min} / \mathrm{slice}, 128 \times 128 \mathrm{matrix}$ ), were made at $9 \mathrm{~h}$ p.i.. From these in vivo studies in humans, we conclude that IDEX is a promising radioligand for the imaging of muscarinic receptors in the human brain and heart. 
61

A.Kurtaran, M.Raderer, S.R.Li, W.Scheithauer, Ch.Mtlier, J.Pidlich, P.Hübsch, P.Angelberger and I. Virgolini

Depta. of Nuclear Medicine, Oncology, Radiology and Gastroenterology, University of Vienna, Austria.

${ }^{123}$ I-Tyr-14-INSULIN AND ${ }^{9}$ TC-NEOGALACTOALBUMIN (NGA) SCANNING IN PATIENTS WITH HEPATOMAS: COMPARATIVE IN VITRO AND IN VIVO-STUDY

Hepatocellular carcinoma (BCC) ist the most frequent hepatic malignancy and its early detection by conven-tional meang is still very difficult. In recent years, (99mTC-NGA) has successfully been used as hepatocyte receptoras have investi in have in vitro and in vivo receptor binding of ${ }^{2}$-Tyr-14-insulin (200 MBg/ $0.5 \mathrm{I}$.$) and of Te-NGA (200 MBg/50 nimol)$ for the exploration of liver morphology in patients with HCC confirmed by histopathology and CT. Scintigraphic studies were performed in 20 patients (10 females, 10 males; mean age $56 \pm 14$ yearg) with HCC, and in 20 control subjects. In vitro studies demonetrated a $10^{3}$ to $10^{5}$-fold higher number of receptors for ${ }^{123} \mathrm{I}-\mathrm{TYr}-14$-insulin on HCC as compared with normal liver, whereas HCC do not express receptors specific for ${ }^{99}$ Tc-NGA. Due to the signifi-cant higher number of insulin receptors expressed, HCC became visible as "hot spots" after injection of $123 \mathrm{I}-\mathrm{yr}-14-1$ igul in. In the after injection of patients, cold spots" over cr-verified legions at 5 minutes after injection. Good matching of "cold" and "hot" spots was observed. We conclude that our new double-tracer methodology using ${ }^{99}$ Tc-NGA and ${ }^{123}$ Iearly diagnosis of patients with HCC.

62

Madácsy L., Velơsy B., Lonovics J., Csernay L.*

First Department of Medicine and Department of Nuclear Medicine*, Albert Szent-Györgyi Medical University, Szeged, Hungary

EVALUATION OF THE RESULTS OF PROSTIGMINE MORPHINE TEST WITH QUANTITATIVE HEPATOBILIARY SCINTIGRAPHY - A NEW METHOD IN THE DIAGNOSIS OF THE SPHINCTER OF ODDI DYSKINESIA

Introduction: The diagnosis of sphincter of Oddi dyskinesia is usually based upon the positive prostigmine-morphine provocation (Nardi) test which has been recently improved by simultaneous measurements of the serum aspartate aminotransferase (ASAT) levels. Due to the lack of the objective parameters, this test has long been criticized. In order to make Nardi test more objective we have tried to visualize the sphincter spasm Nardi test more objective we have tried to visuali

by quantitative hepatobiliary scintigraphy (QHBS). biliary pain were included in this study. Organic billiary and extrabiliary disorders were excluded. Sphincter spasm was evoked by $0,5 \mathrm{~m}$ prostigmine and $10 \mathrm{mg}$ morphine administration and visualized by QHBS using $140 \mathrm{MBg} 99 \mathrm{mTc}$-EHIDA. Digital images were obtained at one frame $/ \mathrm{min}$. for $90 \mathrm{~min}$. Time-activity curves were generated from regions of the liver parenchyma (LP), hepatic hilum (HH), common. bile duct of the liver parenchyma (LP), hepatic hilum (HH), common bile duct
(CBD) and dtodenum. The time to peak activity (Tmax), the half-time of (CBD) and duodenum. The time to peak activity (Tmax), the half-time of
excretion (T1/2) and the duodenum appearance time (DAT) were excretion (T1/2) and the duodenum appearance time
calculated. Serum levels of ASAT were also determined.

Results: From the 22 patients 12 responded with typical biliary pain and ASAT elevation - in 3 cases less than twofold - to prostigminemorphine provocation. The time-activity curves showed a marked biliary obstruction, and a significantly increased DAT. In 10 patients - 4 indicated abdominal pain, but neither of them had enzyme changes - the time activity curves proved free transpapillary flow of the tracer and the time activity curves proved free transpapillary flow of the tracer and the
DAT was normal. In patients with positive Nardi test the Tmax DAT was normal. In patients with positive Nardi test the Tmax parameters of the $\mathrm{HH}$ and $\mathrm{CBD}(32.0+4.1 ; 62.8+4.7)$ were significantly increased when compared to the Nardi negative group $(19.6+1.0$ were also significantly increased in Nardi positive group $(154.7+27.5$; $159.6+30.2 ; 246.5+29.3 ; 72.9+6.2)$ compared to the Nardi negative group $(36.3+5.0 ; 42.6+5.7 ; 46 . T+5.2 ; 29.5+6.2$ respectively).

Conclusion: QHBS proved to be a valuable method in visualization of the sphincter of Oddi spasm during the prostigmine-morphine the sphincter of Oddi spasm during the prostigmine-morphine
provocation. Application of QHBS may be recommended to improve the sensitivity and specificity of the conventional Nardi test.
63

B.BRUNOT ${ }^{1}$, A CONSTANTINESCO ${ }^{1}$, KBOUDJEMA ${ }^{2}$, Ph.GERMAIN 1 Ph.VINEE ${ }^{1}$, L.MERTZ ${ }^{1}$, M.P.CHENARD ${ }^{3}$, J.P.BELLOCQ ${ }^{3}$, J.D.TEMPE ${ }^{3}$ Services de Médecine Nucléairel, de Chimurgie et Transplantation ${ }^{2}$, d'Anatomie Pathologique ${ }^{3}$ et de Réanimation Médicale. Avenue Molière, 67098 STRASBOURG, France.

\section{VALUE OF QUANTITATIVE HEPATOBLIARY SCINTIGRAPEY IN THE FOLLOW-UP OF ORTHOTOPIC AUXILLIARY LIVER GRAFT (OALG).}

Since October 1992, 6 patients ( 3 children and 3 adults) with hepatitis fulminans were treated with OALG in our hospital. One child received a second OALG because of primary non functioning first transplant. In these patients; 34 hepatobiliary scintigraphies $(150$ to $300 \mathrm{MBq}$ of $99 \mathrm{mTc}-\mathrm{Br}-\mathrm{IDA}$ ) were performed including two phases : an angioscintigraphic phase of $602 \mathrm{sec}$-images and a functional phase of 40 1min-images. In 3 patients ( 2 children and 1 adult) scintigraphic examinations were performed systematically every ten days and the results were compared to clinical, biological and histological data. In the other 3 patients, scintigraphies were indicated if any vascular or functional complication was suspected either in the native or in the transplant liver. Quantitative evaluation of both phases permitted to assess separately the perfusion (arterio-portal index) and the function (uptake and excretion parameters) in each liver. For the native liver, scintigraphic data allowed to follow the process of hepatocyte regeneration (size increase and functional recovery) within a delay of about 40 to 50 days. For the liver graft, owing to scintigraphic results, we were able to evaluate the capacity of substitution function and to give valuable informations about the most usual dysfunctions (cholestasis / rejection and anastomosis patency). In 2 children functional recovery of the native liver was completed 2 months after OALG and a transplantectomy could be decided. In an adult patient, the regeneration and the function recovery were slower and only a progressive decrease of immunosuppressant treatment was adopted. In the 3 remaining patients, transplantation was too recent and no definitive results were available.

\section{4}

S. Lourens, M. Voigt, B.K. Adams, V.V. Naidoo, M. Mann, D. Lüischer

Depts. of Nuclear Medicine, Universities of Berne, Switzerland and Cape Town, South Africa; Liver Research Centre, University of Cape Town

INVESTIGATION OF HEPATIC SPACE-OCCUPYING LESIONS WITH TECHNETIUM 99M RED BLOOD CELL SCINTIGRAPHY: FALSE POSITIVE RESULTS

The distinction between hepatic cavernous haemangioma (HCH) and hepatic malignancy is frequently a dilemma in the investigation of patients with hepatic mass lesions (HML). In the presence of hepatic malignancy, a diagnosis false positive for $\mathrm{HCH}$ could be catastrophic. The high specificity of Technetium 99m labelied RBC scintigraphy (RBC scintigraphy) for $\mathrm{HCH}$ reported in the literature, is a reflection of the high prevalence/prior probability of this lesion in the patient populations studied. We performed a prospective study in a population of 45 patients with HML. The population was balanced for $\mathrm{HCH}$ and non-HCH pathology. With planar and SPECT imaging the specificity was 0.632 , positive predictive value 0.720 and false positive ratio 0.368 . Diagnoses false positive for HCH were found in 7 patients with hepatic malignancy (hepatocellular carcinoma, $n=5$; liver metastases, $\mathrm{n}=2$ ).

These results seriously question the adequacy of RBC scintigraphy to differentiate the mostly innocuous HCH from hepatic malignancy, particularly in the presence of a high clinical suspicion of the latter. Support for a scintigraphic diagnosis of $\mathrm{FCH}$ by other imaging modalities should be mandatory. 
65

Ll. Mairal; M. Rota ${ }^{1}$; M. Castell; P. Romerol; R. Puchal and J. Martin-comin.

Serveis de Medicina Nuclear i Unitat de Trasplantament Hepaticl. CSUB Hospital Princeps d'Espanya. Barcelona. spain.

QUANTIFICATION OF ARTERIOVENOUS SHUNTING IN LIVER TRANSPIANTATION USING $99 \mathrm{~m}_{\text {TC-ALBUMIN-MACROAGGREGATES }}$ SCINTIGRAPHY.

Hypoxia is a frequent finding in end stage liver cirrhoses The mechanism of production relies on precapilar dilation and pulmonary arteriovenous shunts. The aim of this work is
to evaluate and guantify those shunts using $99 \mathrm{~m}$ TcAlbuminmacroaggregates scintigraphy.

Twelve liver graft recipients have been studied before and 2 months post-transplantation. Each patienta received $5 \mathrm{mci}$ 185 in) of $99 \mathrm{~m}$ and

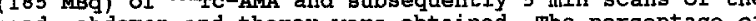
head, abdomen and thorax were obtained. The percentage of shunted activity (SA) was quantified as previously described: systemic activity $=$ (head + abdomen) $/ 0.39$. sA systemic Activity/(System+Lung). Normal values < 6.08 (1) Results: Before grafting, 9 patients showed a SA $<6$, of then remained within normal values at 2 months posttransplantation while 6 of them increased to abnormal values (range $7.9-2 B \quad 8$ ), none of them showed bypoxia. In the 3 remaining patients $S A$ was $>6 \%$ before grafting and normalized at 2 months, only one of these 3 patients presented hypoxia.

conclusion: Those preliminary results suggest the method's hision: Those preliminary results suggest the method's high sensitivity in quantifying pulmonary shunt, even in the absence of clinical hypoxia. The changes observed posttransplantation may be due to the haemodynamic changes that progress to clarify the reason of those changes.

(1) J.M. Mourani et al. AJM 90:693-700, 1991.

66

Th. Krause ${ }^{1}$, V. Siegerstetter ${ }^{3}$, K. Haag ${ }^{3}$, K.-H. Hauenstein ${ }^{2}$, M. Rössle ${ }^{3}$.

Depts. of Nuclear Medicine ${ }^{1}$, Radiology ${ }^{2}$, Gastroenterology $^{3}$, Universitätsklinik Freiburg, Germany.

EVALUATION OF THROMBOGENICITY OF METALLIC STENTS IN PATIENTS WITH TIPS USING TECHNETIUM-99m LABELLED PLATELETS.

Thrombosis and intima proliferation of metallic stents are, at least in part, caused by platelet aggregation. Therefore, Tc-99m HMPAO labelled platelet scintigraphy of stent-shunt may clarify the mechanism and risk of shunt stenosis/occlusion. At the time of dilatation of the stent for TIPS (transjugular intrahepatic portosystemic stent shunt), $120-290 \mathrm{MBq}$ of Tc-99m labelled autologue platelets were injected i.v. in 22 patients. Scintigraphic imaging was done at $20,60,90,180 \mathrm{~min}, 6$ and $24 \mathrm{hrs}$. All patients had patent TIPS at the end of the procedure as assessed by duplex-sonography.

Visual evaluation revealed no or minimal platelet accumulation in 11 patients, moderate accumulation in 5 , and marked accumulation in 6 patients. The stent/liver ratio initially amounted to 1.69 (range 1.0-3.1) and was 1.42 (range 1.0-2.0) at $24 \mathrm{hrs}$. At the same time points the stent/heart ratio was 1.07 (range $0.6-1.8$ ) with a maximum value of 1.13 (range 0.6 2.2) at $90 \mathrm{~min}$. Accumulation in patient with Wallstent was more distinct without than with heparin (stent/heart ratio: 1.6 times, stent/liver ratio: 1,4 at $60 \mathrm{~min}$ ). Stent/liver ratio $>2.3$ at 90 and 180 min was only found in all 4 patients in whom early thrombosis was proven duplex-sonographically at day 2 .

Tc- $99 \mathrm{~m}$ labelled platelet scintigraphy allows detection and quantification of early platelet aggregation at the stent. This technique may be of importance in the evaluation of the risk of stent occlusion and in selection of anticoagulants in patients with TIPS.

\section{7}

J.M. James ${ }^{1}$, J.J. Lloyd ${ }^{2}$, K.J. Herman', B.C. Leahy ${ }^{3}$, R.A. Shields ${ }^{2}$, H.J. Testa

Depts. 'Nuclear Medicine \& ${ }^{2}$ Medical Physics, Manchester Royal Infirmary, Manchester M13 9WL, "3Dept. Medicine, Trafford General Hosp., Davyhulme, Manchester M31 3SL

PERTECHNEGAS LUNG CLEARANCE STUDIES: A NEW METHOD FOR STUDY OF PULMONARY FUNCTION.

Addition of $3 \%$ oxygen to the argon atmosphere within the Technegas generator results in production of a modified form of Technegas, "Pertechnegas", which is Pertechnegas for lung clearance studies has advantages over other radioaerosols in terms of delivery efficiency, radiation safety and patient acceptability. The aim of this study was to measure and compare The aim of this study was to measure and compare Pertechnegas clearance in normal volunteers and in in particular pulmonary fibrosis. Pertechnegas was administered using a single inhalation followed by a 5 second breathold. Dynamic acquisition commenced at inhalation using 10 second frames (64 $x$ 64 matrix) for 30 minutes. Subsequent analysis following definition of regions of interest around the lungs produced a time-activity curve to which a biexponential fit was applied. A half time for the fast component of clearance was then evaluated for the upper and lower zones and for the whole of both lungs.

Results from 11 volunteers and 13 patients studied to date reveal a more rapid mean clearance half-time (fast component) for patients with pulmonary fibrosis ( $n=6$, mean $\left.t_{\frac{1}{2}}=3.6 \mathrm{mins}, p=0.03\right)$ and for smokers $(n=6$, mean $\left.t_{\frac{1}{2}}=3.6 \mathrm{mins}, \mathrm{p}=0.06\right)$ compared to normal non-smoking

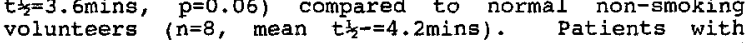
pulmonary fibrosis exhibited more rapid clearance of radioaerosol from the lower zones of the lungs when compared to the upper zones whereas in smokers and normals the converse applied. No significant differences were found between normals and the other patient groups. It is envisaged that sequential measurement of Pertechnegas clearance may be of value to measurement of Pertechnegas clearance may be of value

\section{8}

F.Rogowski,L. Malinowska, I.Skiba, W.Eauer, D. Jurgilewicz, M. Malinowska, A.Sienizę tkowski, E. Jaroszewicz, A.Citko, T.Budlewski,E.0czeretko,B.Kiersnowska-Rogowska. Departments of Nuclear Medicine, Anaesthesiology and Haenatology, Miedical Acaderay, Biažystok, Poland.

PERFUSION ABNORMALITY ON LUNG SCANS AND SERLM CONCENTRATIONS OF ADHESION MOLECULES IN THE PATIENTS WITH ARDS.

The aim of the study was to investigate the behaviour of serum concentrations of intercellular adhesion nolecule-1 (ICAM-I) and P-selectin ( $\mathrm{P}-\mathrm{SeI}$ ) in conjuction with perfusion ganra scanning and lung injury score ( $L$ IS- Murray et al. /1993/) in patients with ARDS.

The study was performed in 8 patients with ARDS caused by: riultitrauma-4, sepsis-2, acute pancreatitis-1, burn-1. As control served 9 volunteers. Venous blood sanples were taken in 12 hours intervals during the first three days and once daily during next 10-14 days or to death of patient $(n=3)$. The concentrations of ICAir-1 and P-Sel were determined by ELISA kits (BBP,UK). The perfusion lung scintigraphy was perforned following intravenous injection of Tc-99m microspheres (CIS,France) using gamma canera.

The results showed continuous increase of ICAM-1 concentrations from slightly higher to about 5-fold of contral in survivors and maintained high values fron beginning of observation to the death of patients. The initial high levels of P-Sel in all patients decreased in the second week of disease in nonsurvivors and renlained high for 3-4 weeks in survivors. In all images many focal perfusion defects in periferal regions and decreased relative radioactivity in the low and middle zane of each lung were found. These abnormalites were more evident during the worsening of the patients state (increase of LIS value).

The concentration of adhesion molecules may be a measure of excessive or inapropriate endothelial or leukocyte activation in the ARDS. Alternations in perfusion nay reflect leukocyte sequestration and releasing of vasoconstri ctors in lung vasculature.Obtained results may be useful for monitoring and prognosis in this disease. 
69

P.G.H.M. Raijmakers, A.B.J. Groeneveld, G.J.J. Teule, L.G. Thijs.

Depts of Internal Medicine and Nuclear Medicine. Free University Hospital; Amsterdam, The Netherlands. PULMONARY MICROVASCULAR PERMEABILITY FOLLOWS THE
CLINICAL COURSE OF PATIENTS WITH THE ADULT RESPIRATORY DISTRESS SYNDROME (ARDS).

ARDS is a major cause of morbidity and mortality in critically ill patients. Pulmonary vascular permeability measurements using radiolabeled proteins may be useful in identification of increased permeability during ARDS. We studied the transvascular passage of Gallium-67, considered to bind to circulating transferrin, as a measure of pulmonary microvascular permeability in 13 patients (female/male: $6 / 7$, age: $47 \pm 18 \mathrm{Yr}$, mean \pm SD) within 72 hours after the development of ARDS. In 9 patients with an improvement of the gas-exchange, defined as a reduction of the positive end-expiratory pressure (PEEP) level to 0 or extubation, the permeability measurement was repeated. Furthermore, 8 patients with congestive heart failure (CHF) and pulmonary edema were studied (female/male:0/8, age: $67 \pm 10$ yr). Kinetics of i.v. injected Ga-67 citrate (4 Mbq) and in vitro labeled red blood cello (Tc-99m, $11 \mathrm{Mbg}$ ) were recorded in blood and over both lungs during $60 \mathrm{~min}$, using probes. The pulmonary leak index (PII) was calculated from the rate of increase in time, of the lung/blood ratio of the Ga$67 / \mathrm{Tc}-99 \mathrm{~m}$ count ratio. The mean PLI ISD of CHF patients was $10.4 \pm 3.910^{-3} \cdot \mathrm{min}^{-1}$ and $11.9 \pm 2.9$ in the left and right lung, respectively. The PLI of ARDs patients was $34.5 \pm 8.510^{-3} \cdot \mathrm{min}^{-1}$ and $34.2 \pm 7.7$ in the left and right lung, respectively (p<0.0005 va. CHF group). In the 9 patients with an improvement of gas-exhange the chest phe 9 patiente while the arterial fraction ratio $\left(\mathrm{P}_{2} \mathrm{O}_{2} / \mathrm{F}_{1} \mathrm{O}_{2}\right)$ increased ( $\mathrm{p}<0.01$ ) from $146 \pm 58$ at a PREP level of $11 \pm 5 \mathrm{~cm} \mathrm{H}_{2} \mathrm{O}$ to $217 \pm 51$ at a PEEP level of 0 . During improvement of gas-exchange the PLI decreased Bignificantly $(p<0.01)$ to $15.6 \pm 4.910^{-3} \cdot \mathrm{min}$ specifically increased in patients with ARDS. The PII may be an index of the clinical severity of ARDs.

70

J. Kotzerke, A. Strumpf, H.-J. Schäfers*, H. Hundeshagen

Dept. of Nuclear Medicine and ${ }^{*}$ Dept. of Thoracic and Cardiovascular Surgery, Hannover Medical School

\section{ALVEOLAR PERMEABILITY AFTER HEART-TX}

An increased lung clearance of Tc-99m-DTPA has been found in smokers and pts. wtih lung fibrosis and pneumocystis pneumonia. A decreased clearance has been documented for diabetic patients with vascular complications or proteinosis. Normally Tc-99m-DTPA is used for estimating an impaired epithelial integrity. Today a new imaging tracer Pertechnegas $(\mathrm{Ptg})$ is available which is easy to produce and to deliver. After diffusion through the alveolar membrane it is rapidly washed out of lungs; the half time of the clearance $\left(T_{1 / 2}\right)$ is $=11 \mathrm{~min}$ in normal pts.

After transplantation immunosuppression prevents a rejection of the organ. To evaluate the influence of long-term immunosuppressive therapy on lung permeability pts. with heart-transplants were examined. 20 non-smoking males (age: $51 \pm 10$ ) and without any signs of rejection inhaled $\approx 20$ MBq Ptg. A dynamic study with 60 frames was acquired over a period of 15 mins and a monoexponential fitting procedure was done on the lung clearance curve.

The clearance rate $\left(T_{1 / 2}\right)$ was $13.6+2.3$ mins (range: 10.3 19.1). There was a significant difference compared to a normal reference group. $\left(n=12, T_{1 / 2}=11.1 \pm 1.1\right.$ mins, range: 9 -13). The reason for that is unclear.

Perhaps immunosuppression causes a thickening of the alveolar membrane. It may also be possible that the clearance is not solely dependant on diffusion but is linked to some active cellular functions which are reduced by immunosuppression, as well. The initial disease which had made transplantation necessary and the following rejection treatment must also be taken into consideration.
71

W.Yu.Ussov, A.M.Peters, J.M.B.Hughes, D.M.Glass, M.Carpani, C.D.Pusey, G.Gaskin, A.Spencer. Hammersmith Hospital, London, UK.

QUANTITATIVE STUDY OF PULMONARY GRANULOCYTE KINETICS IN RELATION TO THE INTEGRITY OF THE ALVEOLOCAPILLARY BARRIER

We used a double In-111/Tc-99m granulocyte labelling technique for the study of granulocyte intravascular and extravascular kinetics in the lung in extrapulmonary inflammatory lesions. The pulmonary vascular granulocyte pool (PGP) and parenchymal migration were compared with inhaled DTPA aerosol clearance as an index of injury to the alveolocapillary barrier. Neutrophil activation was measured in vitro by a shape change assay [as (activated cells)/(total cells)]. PGP was measured by a first-pass integration technique using the $\mathrm{Tc}-99 \mathrm{~m}$ signal, and expressed as a fraction of the total blood granulocyte pool (TBGP). Extravascular granulocyte migration was measured as the 24 hour pulmonary In-111 counts corrected for the chest wall bone marrow background.

Compared with controls $(0.09$, SD $0.013, \mathrm{n}=5)$, PGP:TBGP index was increased in extrapulmonary inflammatory conditions e.g. in inflammatory bowel disease $(0.28$, SD $0.07, n=7, p<0.05)$, systemic vasculitis $(0.33, S D$ $0.03, \mathrm{n}=6, \mathrm{p}<0.001)$ and bone marrow transplant recipients $(0.30, \mathrm{SD} 0.09$, $\mathrm{n}=7, \mathrm{p}<0.001)$. PGP:TBGP correlated with granulocyte activation $(\mathrm{r}=0.76$, $p<0.01)$. Granulocyte migration was increased in vasculitis $(1.35$, sd $1.11 \mathrm{cpm} / \mathrm{pix} / \mathrm{MBq}, \mathrm{p}<0.05, \mathrm{n}=5)$ and bone marrow transplants $(0.96, \mathrm{sd}$ $0.50 \mathrm{cpm} / \mathrm{pix} / \mathrm{MBq}, \mathrm{p}<0.02, \mathrm{n}=5$ ) versus zero in controls, but did not depend on granulocyte activation status. Nevertheless, the migration signal correlated both with $T 1 / 2$ of the lung DTPA clearance curve $(r=0.54$, $p<0.05)$, and with fast clearance exponent $(r=0.77, p<0.01)$.

The fact, that inflammatory activation of granulocytes correlates with delayed transit through the pulmonary vascular bed, but not with extravascular migration argues in favour of endothelial factors in the regulation of granulocyte migration. On the other hand, an increase in PGP:TBGP is not in itself associated with alveolocapillary damage, which seems to require extravascular neutrophil migration.

72

JR Buscombe I Khakhali, A Serafini, F Datz, RF Miller, PJ Ell, WJG Oyen, FHM Corstens,

UCL Medical School, Harbor-UCLA Medical Center, University of Miamir University of Utah Medical Center, University of Nijmegen.

QUANTITATIVE ANALYSIS OF LUNG/HEART RATIO OF In-111 HIG IN PATIENTS WITH AIDS: RESULTS OF A MULTICENTRE TRIAL

Patients with AIDS (the acquired immunodeficiency syndrome) have a high mortality and morbidity from lung infections. Early diagnosis is essential for effective treatment. In-111 labeled pooled human lgG (In111HIG) has been proposed as an effective method for early diagnosis of lung infection in these patients.

The aim of this study was to use a quntitative approach to verity that AIDS patients with lung infection have increased lung uptake of In-111HIG. This lung/heart $(\mathrm{L} / \mathrm{H})$ ratio was calculated from anterior and posterior images of the chest performed, in 106 individuals, 12 hours after the administration of $37 \mathrm{MBq}$ of $\mathrm{ln}-111 \mathrm{HIG}$. The mean age of the patients studied was 32 (range 17-64)., 24 were healthy volunteers without AIDS, 32 had Pneumocystsis carinii pneumonia, 18 had lung infection from ther pathogens and 32 had no evidence of active lung infection. The resulting mean $\mathrm{LH}$ ratio (t/-s.d.) for each group was as follows

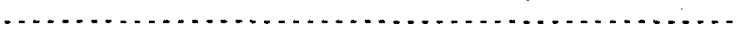
Patient group

\section{I $\underline{\mathrm{UH} \text { ratio }} \mathrm{P}$ (compared with} healihy volunteers)*

Healthy volunteers $\quad 240.46(0.05)$.

AIDS patients, no lung infection $32 \quad 0.47(0.06)$ not significant

$\begin{array}{llll}\text { Pneumocystis carinipneumonia } 32 & 0.54 & (0.07)<0.001\end{array}$

Lung infection, other pathogens $18 \quad 0.55 \quad(0.11)<0.001$

"unpaired $t$ test

In Conciusion this study provides objective evidence that there is more lung activity of In-111 HIG in AIDS patients with lung infection than in those without. In-111 HIG imaging at 24 hours can be recommended in these patients. 
73

Chr.Reiners, J.Biko, W.Bauer, K.Wuttke, C.Streffer, G.Voigt, H. Paretzke, N.Kruglova, E.Demidchik, L.Asthakova, V.Kazakov

Clinic for Nuclear Medicine and Institute for Radiation Biology, University of Essen, Institute for Radiation Protection GSF Munich, Center for Thyroid Tumors Minsk, Belarus

RADIOIODINE TREATMENT OF ADVANCED THYROID CANCER IN CHILDREN FROM BELARUS LIVING IN HIGHLY CONTAMINATED AREAS

The incidence of thyroid cancer in children from Belarus has increased after the Chernobyl reactor accident from 2-4 cases in 1986 to 66 cases in 1992. In April 1993, we started the joint Belarussian-German project "Scientists Help Chernobyl Children" on optimization of childhood thyroid cancer, which is sponsored by German electricity companies. From 1.4 .93 to $28.2 .94,36$ children with advanced thyroid cancer from Belarus have been treated with $1-131$ in Essen. The 22 girls and 14 boys aged $7-18$ years (mean age $11.1 \pm 2.9$ years) histology had shown 34 papillary and 2 follicular cancers. 31 cases had to be classified as pT4, 33 as pN1 and 25 as pM1 cancers respectively. Distant metastases were localized in the lung in 24 patients and in 1 patient in bone. Pulmonary metastases mainly were of disseminated, miliary type.

Up to now 60 courses of high dose $1-131$ treatment have been given to the children. Despite the fact that fractionated 1-131 treatment has not been finished, in all but one cases at least a partial response could be observed. However, in childhood thyroid cancer with disseminated lung metastases |-131 induced pulmonary fibrosis may limit the maximum cumulative therapeutic activity of $1-131$.

With respect to the question of radiation carcinogenesis, biological dosimetry by micronucleus assays in peripheral blood lymphocytes revealed a higher frequency of abnormalities in cancer children as compared to a control group of children without cancer from uncontaminated areas of CIS countries.

\section{4}

* Füzy M., Környei J

National Institute of Oncology,

Isotope Research Institute of Hung Academy of Sciences, Budapest, Hungary

$131_{\text {I-MIBE }}$ therapy of widespread medullary thyroid

\section{carcinoma}

The treatment of primary MTC is surgery. The value of external radiotherapy and chematherapy is debated. Fiveyear survival is $70-80 \%$. Therefore ${ }^{131}$ I-MIBG therapy as a metabolic, internal radiotherapy seems promising in the treatment et widespread MTC. Dverall 34,5\% of MTC con-

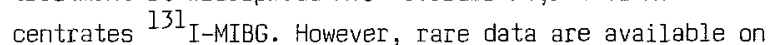
treatment of widespread MTC with high dose of $131_{\text {I-MIBG. }}$.

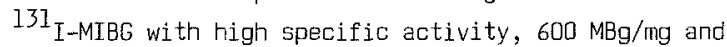
chemical purity $>95 \%$ was produced by the use of a specific unorganic polymer catalyzer. The new Hungarian

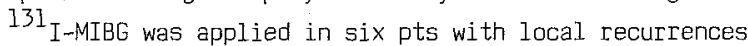
and in one with liver and lung metastases. Single dose, $3700 \mathrm{MBg}$ was injected in slow drop-infusion and the treatment was repeated in two pts. In four pts ${ }^{131}$ I-MTBg uptake was demonstrated and the first three days 50-60\% of radioactivity was excreted in the urine. In one patient $\mathrm{CP}$, in two pts $\mathrm{PR}$, and in one patient minor regression was achieved lasting more than one years. Patient with distant metastases showed palliation for more then one year. Bne patient is not evaluable. Calcium and CEA levels diminished during remission. Side effect: palpitation.
75

D. Huysmans, A. Hermus, J. Barentsz, F. Corstens, P. Kloppenborg.

Depts. of Nuclear Medicine, Endocrinology and Radiology, University Hospital Nijmegen, The Netherlands.

SUCCESSFUL TREATMENT OF LARGE, COMPRESSIVE MULTINODULAR GOITERS WITH RADIOIODINE AND L-THYROXINE.

We evaluated the anatomical and functional results of treatment with 1311 and $L$-thyroxine $\left(L-T_{4}\right)$ in patients with large, compressive multinodular goiters.

18 patients, 16 females and 2 males, aged $65 \pm 13$ yr (meantsd; range $46-86$ yr) were studied. 7 patients had previously undergone thyroid surgery. 131! was administered as a single gift, aiming at $3.7 \mathrm{MBq}$ retained per gram of thyroid tissue at 24 hours. After 131 , therapy $(2.6 \pm 1.0 \mathrm{GBq}$; range 1.48-5.55 GBq), euthyroid patients $(n=14)$ were treated with $L-T_{4}$ in order to keep serum TSH levels $<1.5 \mathrm{mU} / /$; for hyperthyroid patients $(n=4) L-T_{4}$ was combined with methimazole. Before and 1 year after 131 therapy, anatomical and functional parameters were evaluated in 17 of 18 patients (one patient had to be operated 10 months after 131| therapy; thyroid volume reduction at that time was only $10 \%$ ). Thyroid volume (TV) was measured with magnetic resonance imaging (MRI), using planimetry of serial slices. The smallest cross-sectional areaof the tracheal lumen (SCAT) was also determined with MRI.

Exacerbation of obstructive symptoms after 131 i was not observed. Before treatment TV was $274 \pm 161 \mathrm{ml}$ (range 109-825 ml; $n=17$ ). After one year, a volume reduction of $116 \pm 98 \mathrm{ml}$ (range $24-444 \mathrm{ml}$ ) was reached $(40 \pm 15 \%$; range $19-68 \%$ ). Neck circumference diminished by $3+2 \mathrm{~cm}$ (range $1-8 \mathrm{~cm}$ ). SCAT was $0.75 \pm 0.37 \mathrm{~cm}^{2}$ (range $0.29-1.70 \mathrm{~cm}^{2}$ ) before therapy and $0.99 \pm 0.49 \mathrm{~cm}^{2}$ (range $\left.0.30-2.19 \mathrm{~cm}^{2}\right)$ after therapy $(n=16)$. Mean increase of SCAT was $32 \pm 35 \%$. In 12 of 16 patients SCAT increased more than $10 \%$ (range $13 \%$ to $124 \%$ ). In the remaining 4 patients it virtually did not change $(<5 \%)$. Significant improvement (i.e. $>10 \%)$ of forced inspiratory volume in 1 second (FIV-1) was observed in 7 of 16 patients (range $11-48 \%$ ). FIV-1 improved in 4 of the 6 patients in whom this parameter was clearly subnormal before therapy (the other 2 patients had vocal cord paralysis due to prior surgery). Symptoms of superior vena caval obstruction disappeared in one patient and elevated central venous pressure normalized in another patient.

In conclusion, treatment with 131 I and L-T4 of patients with a large, compressive multinodular goiter resulted in a mean reduction of thyroid volume of $40 \%$ in one year. This was accompanied by improvement of compressive signs (tracheal compression, reduction of inspiratory pulmonary function, venous obstruction). ${ }^{131}$ I therapy is an effective alternative in elderly patients who refuse surgery or in whom surgery is contraindicated

76

M.E. Dottorini, G.Lomuscio, L.Mazzucchelli, A.Vignati, L. Colombo.

Division of Nuclear Medicine, General Hospital, Busto Arsizio, Italy.

CAN 131-I WHOLE-BODY SCAN (WBS) REALLY BE REPLACED BY THYROGLOBULIN (TG) MEASUREMENT IN THE MANAGEMENT OF DIFFERENTIATED THYROID CARCINOMA (DTC)? A COMPARATIVE STUDY ON 931 CONSECUTIVE EVALUATIONS OF 785 PATIENTS.

The aim of this study was to evaluate the impact of TG measurement and WBS in the management of patients with DTC. 785 patients (181 male and 604 female) with histological diagnosis of DTC $(623$ papillary and 162 follicular carcinoma) were consecutively submitted to WBS and simoultaneous assay of TG, antiTG and TSH, in the period going from 1st November 1992 to 31 st December 1993. 255 evaluations were done after surgical intervention and before 131-I ablation of thyroid remnants (Group A). In the other 676 cases the exams were performed after 131-I ablation of thyroid remnants (Group B). The results of the examinations in the two groups are reported in Tables 1 and 2

\begin{tabular}{|c|c|c|c|c|c|c|c|}
\hline \multicolumn{4}{|c|}{$\begin{array}{l}\text { Table 1: Post-surgical } \\
\text { Evaluations (Group A). }\end{array}$} & & \multicolumn{3}{|c|}{ 2: Evaluations after } \\
\hline & $\begin{array}{c}\text { TG } \\
<5 \mathrm{n} \sigma / \mathrm{m}\end{array}$ & $\begin{array}{c}\mathrm{TG} \\
5 \mathrm{ng} / \mathrm{m} 1\end{array}$ & Total & & $\begin{array}{c}\mathrm{TG} \\
\langle 5 n \sigma / \mathrm{ml}\end{array}$ & $\begin{array}{c}\mathrm{TG} \\
5 \mathrm{ng} / \mathrm{m} 1\end{array}$ & Total \\
\hline WBS - & 1 & 0 & 1 & WBS & 469 & 141 & 610 \\
\hline WBS + & 122 & 132 & 254 & WBS + & 12 & 54 & 0 \\
\hline Total & 123 & 132 & 255 & Total & 481 & 195 & 676 \\
\hline
\end{tabular}

WBS and TG were discordant in $29.6 \%$ of the cases. The cases with WBS positive and TG negative in Group B were due to extracervical uptakes in six cases. Had WBS not been performed in these last patients, the 131-I treatment might have been inappropriately delayed. The significance for prognosis and survival of an high TO with negative WBS in DTC is still uncertain. The analysis of this large series of patients suggests the need for caution in leaving aside WBS in DTC patients. 
77

M.Possa L.Ruffini R.Sara M.Milella F.Banfi F.Muratori* G.Di Sacco*

Nuclear Medicine Department Division of Endocrinology* Niguarda Hospital, Milan, Italy

111-In-PENTETREOTIDE scintigraphy in recurrence of medullary thyroid carcinoma.

In this study we compared scintigraphy with somatostatin analog 111 In-Pentetreotide (SMS), as a new modality to localize recurrence of medullary thyroid carcinoma (MTC), with other imaging methods (high resolution ultrasound of the neck, TC and NMR). We studied 10 patients ( $6 \mathrm{M} ; 4 \mathrm{~F}$; mean age 42 yrs ranging from 21 to 68 ) treated with total thyroidectomy for MTC with the suspicion of disease recurrence (increasing level of calcitonin (CT) and CEA). 6 patients had familial form of the disease and 4 the sporadic one. Planar (anterior, posterior and lateral views) images of the neck and mediastinum, and in 2 cases of the liver and spine, were obtained 4 and 24 hours after the injection of $110 \mathrm{MBq} 111 \mathrm{In}$-Pentetreotide (OctreoScan(r), Mallinckrodt-Byk Gulden). 7 patients had positive scan. SMS detected mediastimal extention in one patient with a known macroscopic local recurrence. The other 6 patients had no clinical sign of MTC presence: in 3 of them there was concordance with other techniques (TC and neck echo). In 2 patients only after a positive scintigraphic scan echo detected pathologic lymphnodes, suggesting MTC recurrence in spite of a previous negative echo. This result was confirmed by hystology. In one case, with low increase of CT levels and doubtful scan, further studies are carrying on. In 2 patients, known liver and spine recurrences were not found, accordingly with other Authors. SMS and hystology were negative in 3 patients with moderate increase of CT levels and lymphnodal localization assessed by echo. In conclusion, SMS is a sensitive and non invasive method to detecting MTC recurrences even if other imaging are negative. Scintigraphic sensitivity appears to correlate with $C T$ levels.

78

W. Becker, R. Weckermann, A. Bergmann, F. Wolf

Department of Nuclear Medicine of the University of ErlangenNuremberg and Research Department of Henning Berlin, Berlin FRG

\section{DIRECT MEASUREMENT OF SERUM-TPO DURING |-131-} THERAPY OF GRAVES' AND PLUMMERS'DISEASE

Increased serum thyroid peroxidase (TPO) levels in circulation could be measured after thyroid surgery(U. FELDT-RASMUSSEN, Colloque INSERM 1990; 207;173). I-131 leads also to thyroid cell necrosis. We prospectively examined the detectability and the time course of TPO levels before and after I-131therapy of Graves'disease, Plummers'disease and thyroid carcinoma.

Sequential serum samples $(n=53)$ from patients with recurrent thyrotoxicosis in Graves'patients ( $n=10$ ). Plummers'disease $(n=8)$ and thyroid carcinoma $(n=5)$ were examined immediatelly before and monthly after l-131-therapy(up to 8 month). A new commercial available luminometric assay (HENNING BERLIN) with a lower limit of detection of $0.024 \mathrm{ug} / \mathrm{ml}$ was used. $7 / 10$ patients with Graves'disease had significantly increased TPO-levels after I-131-therapy( $96.6 \%$ of all samples), $3.6 \%$ already before therapy. $1 / 8$ patients with autonomy and 3 of 5 patients with metastatic thyroid carcinoma had increased TPO levels. The increase of serum TPO level significantly correlated with the therapeutic effect $(p<0.05)$. Patients with Graves'disease had highest levels 4 to 6 months after 1-131-therapy and immediately before hyperthyroidism. In patients with thyroid carcinoma the increase of TPO levels correlated significantly with a decrease of thyroglobulin levels.

TPO antibodies(IMMU-test) interfered with the TPO detectability, the recovery rate decreased.

In conclusion, TPO levels significantly increase after 1-131-therapy and correlate with the success of I-131 therapy in Graves patients. Interference with TPO antibodies could be observed.
79

R.Müller-Suur and H.U.Gutsche.

Karolinska Institute, Danderyds Hospital, Stockholm Sweden and Institute of Clinical Nephrology, Heide, Germany.

\section{NO EVIDENCE FOR TUBULAR REABSORPTION OF} DMSA

Two sets of micropuncture experiments were performed in rat kidneys in order to study the intrarenal handling of $99 \mathrm{mTc}$-DMSA and pertechnetate. In a first series the concentration profile of DMSA along the nephron was measured according to our earlier micropuncture protocol for MAG-3 (JNM 30:1986, 1991): Fluid from Bowmann's space of surface glomeruli as well as fluid from proximal and distal tubules was collected. Bowman's space urine contained only $15 \pm 2 \%(n=8)$ of the Tc activity of arterial plasma, indicating very low filtration of DMSA likely due to high plasma protein binding $(93 \%)$. The concentration ratio of tubular fluid to plasma was $0.31 \pm 0.4(n=17)$ in the proximal tubulus and $1.13 \pm 0.34(n=5)$ in the distal tubulus suggesting the absence of secretion and reabsorption. In a second series surface loops of proximal tubules were micropunctured and perfused for 10 to 20 minutes with DMSA or pertechnetate dissolved in $0.9 \%$ saline $(10 \mathrm{nl} / \mathrm{min})$. Starting with perfusion onset final urine was collected for at least 100 minutes and the recovery of either substance was determined. $98 \pm 2 \%$ of the DMSA activity was recovered in the ipsilateral final urine. Only $0.5 \pm 0.1 \%$ was found in the urine of the contralateral kidney. Under these experimental conditions, only 57 $\%$ of the pertechnetate was recovered in the ipsilateral urine, indicating considerable reabsorption along the nephron. Conclusion: As reported earlier for MAG-3, very low fractions of DMSA enter the tubule by glomerular filtration. The increasing concentration profile of DMSA as well as the almost complete recovery of microinjected DMSA are clear evidence against tubular reabsorption of DMSA from the glomerular filtrate in kidneys of normal rats.
80

R.A.M. Kengen, S. Meijer, A.K. van Zanten, H. Beekhuis, J.G.W. Kosterink, D.A. Piers

Depts. of Nuclear Medicine, Internal Medicine, Pharmacy, University Hospital, Groningen, The Netherlands

QUALITY REQUIREMENTS OF I-131-HIPPURAN USED FOR EFFECTIVE RENAL PLASMA FLOW (ERPF) MEASUREMENTS.

It is accepted that the radiochemical purity of 1-131-Hippuran (Hipp) used for ERPF measurements with the continuous infusion method with urine collection (with continuous infusion of Hipp over a period of hours) should be minimally $98 \%$ (ie the free 1 -131-iodide (iodide) is maximally $2 \%$ ). This is because iodide and Hipp cannot be counted separatly and in comparison with Hipp, lodide is excreted in urine to a lesser extent. Therefore in the formula ERPF = UXV/P ( $U \times V=$ activity excreted in urine in time; $P=$ plasma activity), iodide will contribute mainly to the denominator and will therefore lower the calculated ERPF.

lodide percentages below $2 \%$ could still have significant influence on calculated ERPF, therefore we investigated the influence on ERPF of labeled iodide impurities below $2 \%$ by adding known amounts $1-123$-iodide to the infusion solution in clearance studies in 9 patients. 1-123-iodide was counted separately from 1-131Hippuran in urine and plasma samples, and the influence of different percentages free iodide on ERPF was determined. It was found, as expected, that the percentage iodide in plasma increased during the infusion time and that the percentage lodide was higher in patients with higher ERPF (in which case 1-131-Hippuran in plasma was lower). Examples of the influence of iodide: the influence of $2 \%$ iodide was substantial: in a patient with an ERPF of about 500 $\mathrm{ml} / \mathrm{min}, 2 \%$ iodide resulted in a drop of ERPF of about $7 \%$ after $1.5 \mathrm{~h}$ and of $13 \%$ after $5.5 \mathrm{~h}$. In this same patient $0.5 \%$ iodide resulted in a drop of ERPF of maximal $5 \%$.

We conclude that one should aim at a radiochemical purity of Hippuran of at least $99.5 \%$ since percentages iodide of 1 a $2 \%$ still have significant influence on ERPF, especially when ERPF is high. 
Sunday, 21 August 1994

81

\author{
L. Kabasakal, S. Atay, B. Kanmaz, K. Sönmezoğlu, M. Demir, \\ H. B. Sayman, K. Özker, T. Y. Erdil, I. Uslu \\ Department of Nuclear Medicine, Cerrahpaşa Medical Faculty \\ İstanbul, TÜRKIYYE
}

\section{EVALUATION OF TC-99m-EC IN RENAL DISORDERS}

$\mathrm{Tc}-99 \mathrm{~m}-\mathrm{Ec}$ is a new renal agent introduced as an alternative substitute for $\mathrm{OIH}$ in evaluation of renal function. Comparative studies in humans have demonstrated that renal clearance of $\mathrm{Tc}-99 \mathrm{~m}-\mathrm{EC}$ is superior to that of Tc-99m-MAG3 with similar imaging quality.

The present study was designed to evaluate the clinical usefulness of Tc-99m-EC in renal disorders using $\mathrm{OIH}$ as an intrasubject standard. 20 patients with various degrees of renal impairement were received $100 \mathrm{MBq}$ Tc-99m-EC and $7.4 \mathrm{MBq} 131-\mathrm{I}-$ OIH. After the injection 11 blood samples were obtained during 60 min. and $0.2 \mathrm{ml}$ plasma samples were counted by a well type counter. Pharmacokinetic data were calculated using double compartment analysis (Sapirstein). $60 \mathrm{~min}$. urine was collected and urine samples were also counted. Protein binding values were determined by ultrafiltration. Imaging were performed for $30 \mathrm{~min}$. duration (Siemens Basicam). The mean plasma clearence of Tc-99m-EC $(264.3 \pm 130)$ was found lower than that of $\mathrm{OIH}(357 \pm 174)(\mathrm{EC} / \mathrm{OIH}$ : 0.76) giving an excellent correlation with OIH $(r=0.64)$. 60 min. excretion of Tc-99mEC was almost identical to that of OIH ( $50 \%$ vs $51 \%$ ). The protein binding of $\mathrm{Tc}-99 \mathrm{~m}-\mathrm{EC}$ was almost half of $\mathrm{OIH}$ protein binding value (33\% vs $61 \%)$ and red blood cell binding of EC was almost negligible with respect to $\mathrm{OIH} \mathrm{(3 \%} \mathrm{vs} 27 \%$ ). The volume distribution of Tc- $99 \mathrm{~m}$ EC was found slightly higher than that of OIH. The elimination of Tc$99 \mathrm{~m}-\mathrm{EC}$ was found longer than that of OIH. Image quality of Tc-99m$\mathrm{EC}$ was of course better than that of hippuran due to $\mathrm{Tc}-99 \mathrm{~m}$ labeling .

In conclusion Tc-99m-EC has excelent imaging charecteristics and its plasma clearence allows to estimate the accurate ERPF. With its prompt availability and simplicity of preparation Tc- $99 \mathrm{~m}-\mathrm{EC}$ can be used in routine renal imaging and functional studies.

\section{2}

C. Sawas-Dimopoulou, P. Papathanassiou*, L. Margaritis* Institute of Radioisotopes and Radiodiagnostic Products, NCSR "Demokritos", 15310 Aghia Paraskevi Attikis, P.O.Box 60228, Greece, *Dept. of Cell Biology and Biochemistry, University of Athens Greece

DISTURBANCE OF 99mTc-DTPA PHARMACOKINETICS AFTER ANTIFUNGAL SYSTEMIC THERAPY - A USEFUL TEST IN EARLY EVALUATION OF ACUTE NEPHROTOXICITY

The incidence of deep-seated fungal infections has dramatically increased these last decades. As they are difficult to eradicate, acute and chronic renal toxicity are among the most common side effects of a number of conventional antifungal treatments. Early evaluation of renal functional insufficiency in the above patients is of clinical significance. The ability of $99 \mathrm{~m}$ Tc-DTPA to detect antifungal therapy nephrotoxicity has been investigated in the present experimental work. Toxic doses $\left(L_{100}, L D_{50}\right)$ of amphotericin $B(A M B)$ have been determined in mice (Swiss SWR/De). Groups of mice intravenously treated by various $A M B$ doses inferior to $L D_{50}$ are weighed daily and urine biochemistry shows normal except protein level which is increased to $30 \mathrm{mg} / 100 \mathrm{ml}$. $99 \mathrm{mTC}$-DTPA biodistribution studies are performed in the above groups of mice $2 \mathrm{hr}$ after the injection of AMB. One hour after the injection of the radiopharmaceutical the mice are sacrificed by an ether overdose. Blood samples, and the various organs are weighed. The urine excreted and the above organs are counted in a well-type counter with reference to a standard dose. After statistical analyses the results show that in a short time interval after $A M B$ injection the blood clearance and urinary excretion are significantly delayed as a consequence of the acute toxicity of $A M B$. Two hours after $3.30 \mathrm{mg} \mathrm{AMB} / \mathrm{kg}$ b.wt, the 99mTc-DTPA concentration in blood $1 \mathrm{hr}$ after inj. is equal to $18.12 \pm 4.63 \%$ of injected dose compared to $1.86 \pm 0.23 \%$ in controls, whereas excretion in urine is equal to $33.20 \pm 6.18 \%$ of injected dose compared to $90.81 \pm 3.54 \%$ in controls. The extent of disturbances in $99 \mathrm{mTc}$-DTPA pharmacokinetics is related to AMB dose. In conclusion, the above results suggest that $99 \mathrm{~m}$ TC-DTPA can be efficiently used in order to evaluate the degree of renal impairment induced by high doses of antifungal agents and to help in the follow-up of the above patients.
83

B. DUGAL

Nuclear Medicine

Østfold Central Hospital

1603 Fredrikstad, Norway

\section{EFFECT OF DOXAZOSIN ON RENOGRAPHY IN MAN.}

Prazosin related alfa 1 adrenoceptors have been known for a long time. Quinazoline derivative, doxazosin messylate (Cardura, trademark firm Pfiser) is also a competative postsynaptic alfa 1 adrenoceptor antagonist, but with a longer half life time. Hypertensive patients were sent to our department for renography because their hypertension was supposed to be due to renal artery stenosis. Four of these patients were pretreated with antihypertensiv doxazosin. In all these cases pronounced changes in the renogrammes were found with doxazosin. In three cases the renogrammes showed reduced bilateral blood flow, prolonged peaktime and delayed excretion of the tracer (Tc99mDTPA) from the kidneys due to bilateral renal artery stenosis. In the fourth case similar results were observed in the right kidney. The renogram of the left kidney was found to be normal. Renal angiography confirmed these results. 10 days after the removal of doxazosin new renogrammes were registered under the same conditions as with doxazosin, showing normal results. We believe that doxazosin also has some effect on the renin-angiotensin system and/or on the renalparenchyme. Renography should be avoided if the patients are taking doxazosin medication. On the other hand double renography without and with doxazosin as shown here may unmask renal artery stenosis.

\section{4}

M. Reinhardt, V. Muller-Mattheis*, A. Wirrwar, H. Vosberg, R. Ackermann $*$ und H.-W. Müller-Gärtner

Departments of Nuclear Medicine and * Jyrology, HeinrichHeine-Universität Düsseldorf, FRG

FDG-PET DETECTS LYMPHNODE METASTASES OF UROGENITAL MALIGNOMAS MORE SENSITIVE THAN X-RAY-CT

The aim of this study was to assess the sensitivity of positron emission tomography with 18-F-FDG (FDG-PET) in the detection of pelvic lymphnode metastases of urogenital malignomas compared to $x$-ray computed tomography (CT). 30 patients were studied; 20 patients presented with prostate cancer, 7 patients with blaclder cancer and 3 patients with penile cancer. FDG-PET was performed in fasting state after bolus injection of $370 \mathrm{MBq} 18-\mathrm{F}-\mathrm{FDG}$ with a PC 4096 PET-Scanner, Patients were scanned dynamically in one scanner position until $80 \mathrm{~min}$ post injection. Additional static frames were aquired to cover the entire pelvic region. All patients recieved a pelvic or inguinal lymphnode dissection.

Postsurgical histological examination revealed lymphnode metastases in $12 / 30$ patients; two of the 12 patients affected had micrometastases $(<1 \mathrm{~mm})$ only. PET detected focal lesions of high FDG-uptake in 10 gatients, whereas CT detected suspicious lymph nodes in only 5 patients. Both FDG $-P E T$ and CT failed to detect metastatic desease in the two patients with micrometastases. FDG-PET detected lymphnode metastases in significantly more patients (ca. $80 \%$ ) with metastatic desease than CT (ca. $45 \%)$ did.

The data suggests that FDG-PET detects pelvic lymphnode metastases of urogenital malignomas with a higher sensitivity than $\mathrm{x}$-ray computed tomography. 
85

I.Ruefini R. Sara F.De Maria*F.Spinelli 0.Parodi*

Nuclear Mecicine Department CNR Institute*

Niguarda Hospital, Milan, Italy

\begin{abstract}
RELATION BETWEEN REGIONAL DISTRIBUTION OF 201.II AND MYOCARDLAL BLOOD FLOW W PATHENTS WTHH ISCHEMIC HEART DISEASE OR DLATED CARDIOMYOPATHY UNDERGONG HEART TRAXSPLANTATHON

Aim of the study was to evaluate the relationship between myocardial blood flow (MPF) and delayed Thallium-201 myocardial uptale and their inplication for the assessment of myocardial viability in dysfunctioning myocardium. We studied 5 with idiopathic dilated cardiomyopathy (IDC) and 5 ischemic heart disease (IHD) by means of Thallium-201 and $99 \mathrm{~m}$ Te-labelled human albumin microspheres, Which were injected during transplant surgery into the left atrium while arterial blood was samled. 11.201 was injected intravenously 4 bours before surgery. Myocardial slices from the excised heart were divided into endocardial-epicardial segments to measure radioactivity (by a well counter) and myocardial fibrosis, expressed as collagen to total tissue protein ratio. MAF was measured by Heymann method. Thallium activity was expressed as percent of the activity measured in the tegion with highest counts. Mean left vestricular MBF and Thallium uptake were similar in $\mathrm{DC}$ and $\mathrm{HWD}$, being more inhomogencous in IHD. In DC, an uncoupling between MBF and Thallum uptake was found. In IFD, at logistic regression analysis, Thallium uptake yielded the best prediction $(91 \%)$ of biochemically determined viability (fibrosis $<20 \%$ ), with a tracer eut-off value of $60 \%$; by this Thallium viability eriterion, $77 \%$ of segments werc judged as viable. Thallium uptake showed two patteras: one linearly related to flow $(r=72, p<0001)$, the other characterized by high Thalium activity and low MBF (flow/viability mismatch); at equivalent low flow rates $(<0.6 \mathrm{ml} / \mathrm{min} / \mathrm{g})$, this mismatch points had less Ebrosis than arcas with MBF/n match $(13+/ 17 \%$ ys $60+1-25 \%$, $\mathrm{p}<0001$ ). In conclusion, in ehronically dysfenetioning hearts of $\mathrm{IHD}$ patients, extensive areas of viable myocardium as detected by Iestredistribution Thallium-201 uptake and confirmed by the absence of relevant fibrosis, can be observed, despite severe MBF impainnent Most of these areas are characterized by a disproportionately elevated Thalliura-201 activity, when compared to the relative MAF values, realizing a pattern of flow/viability mismatch. This "in vitro" signal holds great potential for auclear imaing and may provide accourate ingormation for appropriate treatment of chronic cardiac dysfunction.
\end{abstract}

86

Ignasi Carrió, Montserrat Estorch, Lluis Berná, José López-Pousa, Gustavo Torres.

Hospital de Sant Pau, Barcelona.

\section{ASSESSMENT OF DOXORUBICIN CARDIOTOXICITY BY SEQUENTIAL 111IN-ANTIMYOSIN AND 123I-MIBG STUDIES}

Detection of impairment of adrenergic neuron function with ${ }^{223} \mathrm{~L}$ MIBG and detection of myocyte cell damage with ${ }^{11}$ In-antimyosin during doxorubicin administration may provide early identification of patients at risk of significant cardiotoxicity. We studied 38 cancer patients treated with doxorubicin to compare ${ }^{123} \mathrm{I}-\mathrm{MIBG}$ and ${ }^{111} \mathrm{In}$ antimyosin uptake in the assessment of cardiotoxicity. MIBG scans, antimyosin scans and ejection fraction (EF) measurements were performed before chemotherapy, at intermediate cumulative doses and at maximal cumulative doses of doxorubicin. MIBG uptake was quantified by a mediastinum to heart ratio and antimyosin uptake was quantified by a heart to lung ratio.

All patients had absent antimyosin uptake (mean ratio $1.40 \pm 0.06$ ) with normal MIBG uptake (ratio $1.85 \pm 0.3$ ) before chemotherapy; EF was $61 \pm 8 \%$. At $240-300 \mathrm{mg} / \mathrm{m}^{2}$ of doxorubicin, an increase in antimyosin uptake was observed with a ratio of $1.85 \pm 0.2(\mathrm{p}<0.01)$, whereas a similar degree of MIBG uptake was observed (mean ratio of $1.80 \pm 0.2 \mathrm{p}=\mathrm{NS}$ ); EF was $58 \pm 5 \%$, $\mathrm{p}=\mathrm{NS}$. At $420-600 \mathrm{mg} / \mathrm{m}^{2}$ increased antimyosin uptake was observed with a ratio of $2.02 \pm 0.3$ $(p<0.01)$, whereas a decrease in MIBG uptake was observed (mean ratio of $1.76 \pm 0.2, p<0.05)$; EF was $52 \pm 8 \%(p<0.05)$.

We conclude that assessment of myocyte cell damage by antimyosin studies is more sensitive than the assessment of cardiac adrenergic neuron function and than $\mathrm{EF}$ measurements to detect cardiotoxicity at intermediate doses. At maximal doses, antimyosin and MIBG scans may be abnormal with decreased or maintained EF.

\section{7}

J.Lekakis, V.Prassopoulos, P.Athanassiadis, M.Emmanuel, J.Germanidis, Ch.Palaistidis, P. Kostamis, S.Moulopoutos. Dept of $\mathrm{Cl}$ in. Therapeutics and Dept of Nuclear Medicine Alexandra University Hospita T, Athens, Greece.

IS I123-METAIODOBENZYLGUANIDINE USEFUL FOR EARLY DETECTION OF DOXORUBICIN CARDIOTOXICITY? COMPARISON WITH LEFT VENTRICULAR EJECTION FRACTION.

Previous preliminary report has shown a neurotoxic effect of doxorubicin on the myocardium assessed by I123-metaiodobenzylguanidine (MIBG) scintigraphy. To further evaluate this doxorubicin-induced cardiac neurotoxicity we analyzed data from 36 patients (pts, 26 women, 13 men, mean age $53 \pm 16$ with various malignant diseases; 13 pts were receiving doxorubicin with a mean cumulative dose $353+168 \mathrm{mg} / \mathrm{m}^{2}$ while 23 pts were not on doxorubicin. Cardiac I 123-MIBG uptake was assessed as heart to mediastinum ratio ( $\mathrm{H} / \mathrm{M})$ obtained 4 hours after intravenous injection of $5 \mathrm{mC} i$ I $123-M I B G$. Ejection fraction $(E F)$ by radionuclide ventriculography was $57 \% \pm 10 \%$ in pts on doxorubicin and $60 \% \pm 6 \%$ in pts without doxorubicin (ns). In control pts $\mathrm{MIBG} \mathrm{H} / \mathrm{M}$ was $2,13 \pm 0,2$ while in doxorubicin pts $1,76 \pm 0,2(p<0,001)$. Taking as a cutoff point (mean-2SD) the $H / M, 1,73$, none of the pts without doxorubicin had $\mathrm{H} / \mathrm{M}$ below 1,73 while $4 / 13$ pts on doxorubicin had $H / M<1,73(p<0,01)$. In 10 pts MIBG scintigraphy was repeated after receiving $236 \pm 46 \mathrm{mg} / \mathrm{m}^{2}$ of doxorubicin. Initial $H / M$ was $2,24 \pm 0,2$ and fell to $1,97 \pm 0,29$ ( $p<0,05)$; H/M became abnormal $(<1,73)$ in $3 / 10 \mathrm{pts;} \mathrm{EF}$ changed from $60 \% \pm 7 \%$ to $55 \% \pm 8 \%$ (ns) and became abnorma $(\langle 50 \%)$ in $2 / 10$ pts. An inverse correlation was observed between $H / M$ and doxorubicin dose $(r=-0,51, p<0,001)$, while a weaker correlation was found between $E F$ and $H / M \quad(r=0,38$, $p(0,01)$. A H/M less than 1,73 was observed in none of pts off doxorubicin, $3 / 13$ pts at low-intermediate doses (100$-300 \mathrm{mg} / \mathrm{m}^{2}$ ) and $5 / 10$ pts at higher doses. A $10 \mathrm{w}(<50 \%) \mathrm{EF}$ was observed in $2 / 13$ pts at low intermediate doses and $2 / 10$ pts at higher doses. In conclusion 1123-MIBG uptake decreases in a doxorubicin dose-dependent way indicating a cardiac neurotoxic effect; this phenomenon appears ear$7 y$ in some pts before EF deterioration.

\section{8}

A. Kaldonado, J. Herreros*, M.J. Garoía, I. GarcíaBolao*, L. Rubio, A. Villas, J. Richter.

Depts. of Nuclear Medicine, Cardiology and Cardiovascular surgery*. University Hospital of Navarra school of Medicine. P.O. Box 192, 31080 Pamplona, spaIN.

FUNCTIONAL ASSESSMENT OF DYNAMIC CARDIOMYOPLASTY WITH ISOTOPICAL VENTRICULOGRAPHY AWD ECHO-DOPPLER

teft ventricular function (LV) was evaluated in 13 patients with nyocardiopathy (class III-IV NYHA) and formal contraindication to cardiac transplantation submitted to a dynamic cardionyoplasty (DC) using the latissimug dorsi muscle (LDM). Electrical stimulation of the IDM was performed by means of a train of impulses cardiomyoestimulator (CMS). Two gated equilibrium radionuclide ventriculography (RV) (CMS off and on) were obtained. Data were formatted into 32 frames per cycle $R-R$. LV ejection fraction (EF), peak filling rate (PFR) and the time from end-systolic to PFR (TTPFR) were determined. IV ejection fraction, peak $E$ wave velocity (E) and deceleration time (DT) were evaluated through doppler echocardiography (DE). The mean follow-up during the post-operatory was $9 \pm 5.7$ months. No intraoperative deaths were recoxded and global mortality was $7.6 \%$. An functional improvement through I-II NYHA class was observed in ail patients.

\begin{tabular}{|c|c|c|c|c|c|c|c|c|}
\hline & \multicolumn{2}{|c|}{$E F-O F F$} & \multicolumn{2}{|c|}{ EF-ON } & \multirow{2}{*}{$\begin{array}{c}\text { PFR } \\
\text { (EDV/Sec) }\end{array}$} & \multirow{2}{*}{$\begin{array}{l}\text { TIPFR } \\
\text { (mSec) }\end{array}$} & \multirow{2}{*}{$\begin{array}{c}E \\
(\mathrm{~cm} / \mathrm{sec}\rangle\end{array}$} & \multirow{2}{*}{$\begin{array}{c}\text { DF } \\
\text { (mSec) }\end{array}$} \\
\hline & RV & $\mathrm{DE}$ & RV & $\mathrm{DE}$ & & & & \\
\hline PREOPERATIVE & $20.9 \%$ & $23.6 \%$ & .. & -- & 4.57 & 226.2 & $78+11$ & $127 \pm 37$ \\
\hline 3 MONTHS & $28.7 \%$ & 33.7 & $31 \%$ & $38.8 \%$ & 4.40 & 224.8 & $80 \pm 20$ & $129 \pm 31$ \\
\hline 6 MONTHS & $32.8 \%$ & $31.9 \%$ & $32.4 \%$ & $60.7 \%$ & 3.40 & 176.5 & $80+17$ & $106+9$ \\
\hline
\end{tabular}

Dinamic cardiomyoplasty induces an improvement in systolic function but mild or no change in diastolic function. Differences between cMs on/off are less important in the mid-term than in short-term follow-up. Mechanical and electric aspects of DC must be considered in the evaluation of results from both diagnostic procedures. 
89

G. Notohamiprodio, U. Schmidt, O. Lindner, H. Meyer, U. Gleichmann, R. Körfer

Herzzentrum NRW - Ruhr-University Bochum, Bad Oeynhausen, FRG

\section{FATTY ACID UPTAKE IN TRANSPLANTED HUMAN HEART}

Cardiac transplantation represents an effective and therapeutic alternative for individuals with end-stage heart disease. It is a well-established principle that the success of cardiac transplantation is linked to the control of allograft rejection. In the early phase endomyocardial biopsy is the standard method of quantifying rejection dynamics. In the late follow-up of outpatients, however, there is a gap in noninvasive rejection diagnosis. Therefore, it was of potential interest to find another noninvasive method which allows early recognition of graft rejection. Furthermore, little is known about the myocardial metabolism in denerved transplanted human heart. It is expected that faty acids are still the major energy source of the denerved myocardium. The knowledge of fatty acid transport characteristics in transplanted human heart is theretore of physiological significance because it might represent a site of metabolic control according to the energy requirements of the heart, and in the case of rejections the myocardial fatty acid transport may be altered. 327 patients after orthotopic heart transplantation aging from 1 to 78 years were investigated. The survival time at examination was 2 to 68 months. patients were grouped into: Group 1: patients without or with less than 5 rejections but without coronary anglographic evidence of accelerated than 5 rejections but whout coronary anglographic evidence of accelerated
graft arteriosclerosis $(n=181) ;$ Group 2: patients with chronic repeitive graft arteriosclerosis $(n=181$ ); Group $2:$ patients with chronic repefitive
rejections (more than 5 rejections) but without coronary angiographic evidence of accelerated graft arteriosclerosis ( $n=109$ ); Group 3: patients with documented accelerated graft arteriosclerosis $(n=25)$; Group 4: patients with acute rejection $(n=12)$. For assessment of fatty acid influx rate, a dual tracer lechnique with 20171 as pertusion tracer and $15-(p-123)$-iodophenyl)pentadecanoic acid (IPPA) as fatty acid tracer was used. The measurements were carried out during exercise and at rest. Only in patients of group 4 the investigation was pentormed at rest. Patients after heart transplatation revealed similar myocardial fatty acid transport characteristics as control persons. Chronic repetitive rejections showed diminished maximal velocity vmax. Patients with documented accelerated graft arteriosclerosis showed similar myocardial fatty acid transport characteristics as those chronic repetitive rejections. Patients with acute rejection had pronounced reduction of myocardial fatty acid influxrate. In all patients the myocardial perfusion reserve was reduced compared to normals, particulary those with accelerated graft arterioscierosis. The data indicate that dual tracer technique is a valuable tool for monitoring patients after heart transplantation.

90

Ignasi Carrió, Ricard Serra, David Duncker, Montserrat Estorch, Lluis Berná, Gustavo Torres.

Hospital de Sant Pau, Barcelona.

\section{MYOCARDIAL 111IN-ANTIMYYOSIN UPTAKE IN PATIENTS WITH ECHOCARDIOGRAPHIC FEATURES OF HYPERTROPHIC CARDIOMYOPATHY.}

Pathologic myocardial hypertrophy is associated with myocyte cell damage. We performed antimyosin antibody studies to assess myocyte cell damage in 12 patients who were found to have features of hypertrophic cardiomyopathy at echocardiography. Maximal wall thickness was $\geq 16 \mathrm{~mm}$ in all patients. Septal wall thickness ranged from 13 to $27 \mathrm{~mm}$. Posterior free wall thickness ranged from 12 to $19 \mathrm{~mm}$. Mean left ventricular mass index was $199 \mathrm{gr} / \mathrm{m}^{2}$ (range 144 to 258). None of the patients had valvular stenosis or systemic hypertension. Antimyosin uptake was quantified by a heart to lung ratio (HLR). Ten athletes with physiologic hypertrophy at echocardiography were used as control group.

Antimyosin uptake was observed in 10 patients $(83 \%)$. The pattern of uptake was diffuse in 7 and confined to portions of the ventricle in 3 (1 anterolateral, 1 septal and 1 apical). Mean HLR in the control group was $1.47 \pm 0.09$ ( $p=$ NS versus normal subjects). Mean HLR in the group of patients with features of hypertrophic cardiomyopathy was $1.87 \pm 0.19$, range 1.50 to 2.20 ( $p<0.001$ ). A correlation was found between intensity of antimyosin uptake and ventricular mass index $(r=0.625, \mathrm{p}<0.01)$.

Antimyosin studies may be useful in confirming the diagnosis of pathologic hypertrophy in patients with features of hypertrophic cardiomyopathy at echocardiography.
91

Zeynep BURAK Murat ARCON, Aysenur MEMIS", Serdar ERDEM, Zehra BALKAN, Yusuf DUMAN, Esin E. USTUN*, YId:Z ERHAN**, Hayal OZKLIC

Departments of Nuclear Medicine, Radiology*, Pathology**, Ege University, TURKEY

TECHNETIUM-99m MIBI BREAST IMAGING IN THE EVALUATION OF PALPABLE BREAST MASSES: A COMPERAIIVE STUDY WITH MAMMOGRAPHY

Mammography and US are the basic screening modalities for palpable breast masses. However; those modalities have certain limitations particularly in young women with dense breasts, fibroadenomas and focal fibrous disease of the breast Because of lack mammographical specificity, many biopsies have been done for pathologically proven benign breast masses, In this respect, the aim of this study was to evaluate the feasibility of $99 \mathrm{~m}-\mathrm{TC}$ MBBI as a tumor localsing agent in patients with paipable breast masses in comparison with mammography.

41 patients with palpable breast masses were studied by $99 \mathrm{~m}-\mathrm{Tc}$ MIBI breast maging. All images were evaluated by two nuclear medicine specialistis, and localised increased uptake of MIBI was accepted as positive. Finat histopathological diagnosis was achieved through excisional biopsy.

Mammography could reveal all of the malignant breast masses but differential diagnosis of fibroadenomas could not be achieved. 25 of 27 breast carcinomas were detected using $99 \mathrm{~m}-\mathrm{Tc}$ MIBI scintigraphy, 2 patients with invasive lobular carcinoma showed abcent MIBI accumulation. The smallest breast malignancy detected by $\mathrm{MIBI}$ study had a measured dimention of $7.5 \times 2 \mathrm{~cm}$. Eight of 14 axillary metastases showed positive uptake $(57 \%)$. On the other hand; 12 of 14 patiens with pathologically proven benign breast lesions did not demonstrate any MBI accumulation. Focal $99 \mathrm{~m}-\mathrm{TC} M \mathrm{MB}$ uptake could be observed in two fibroadenomas. The sensitivity and the specificity of mammography in differential diagnosis of breast lesions were $100 \%$ and $78 \%$ and of MIBI analysis were $93 \%$ and $86 \%$, respectively.

Our results indicate that, $99 \mathrm{~m}-T_{\mathrm{C}} \mathrm{M} ! \mathrm{B} /$ scintigraphy may provicle additional information in differentiation of malignant pathologies from benign lesions, in patients with palpable breast anomalies, Especially, in women with high risk for the development of breast cancer and with suspect mammograms, $99 \mathrm{~m}-\mathrm{TC}_{\mathrm{C}} \mathrm{M} / \mathrm{B}$ i study may improve the specificity.

\section{2}

O. Schilllaci, F. Socpinaro, *M. Scarpini, *P.L. Mingazzini, R. Danieli, M. Banci, R. Tavolaro, *M. Zerilli, *M.R.Limiti, A. Centi colella

Dot. Exp.Med. and *I Surgery, Univ."La Sapienza", Rane, Italy

IS 99MTC MIBI UPIAKE IN BREAST CANCER RETATED TO NEQANGIOGENESIS?

Angiogenesis is the main determinant of breast cancer invasiveness and 99mro MIBI uptake by invasive breast cancer has been alreacy reported by us. Aim of this study is to establish if a correspondence exists between tumor-induced necangiogenes is and tumor uptake of 99 till MIBI. 19 patients, 10 with node positive (NH) and 9 with node negative (N-) breast cancer have been studied with 99mTlC MTBI. Node positivity has been established by histological exam of nodes after sungery. Angiogenesis has boen assessed by endothelial staining with anti factor VIII antibodies and microvessels counted by means of a Quantimet 500 device for histological images analysis. A cut-off of 135 vessels/ming corresponding to $90 \%$ probability of spreading in the Weinder's logistic regnession was established. Two cbservers blindly classified 99mIC MIBI scans as positive or negative on the basis of the presence or the absence of tumor image. Tumor/heart (T/H) activity ratio was also measured. Immunohistochemistry showed $71.6 \pm 12.1$ vessels/may in the $\mathrm{N}-$ pts and $146.6 \pm 20.6$ vess $/ \mathrm{mmg}$ in the $\mathrm{N}+$ pts $(\mathrm{P}<$ $0.01)$. All the N-pts had a MIBT- scan whereas all the $\mathrm{N}+$ pts showed a MIBI+ scan. T/M latio was $0.266 \pm 0.035$ in $\mathrm{N}-$ and $0.367 \pm 0.23$ in $\mathrm{N}+$ pts $(\mathrm{P}<0.01) .9 / 10 \mathrm{NH}$ pts had more than 135 vess/mm and 1 showed 99 vess/mng which corresponds to $70 \%$ probabily of node metastasis in the Weinder's regression. In conclusion our study shows that 99mIC MIBI uptake is an irdirect measunement of angiogenesis but by a clinical point of view its sensitivity in detecting invasive cancers is higher than that of irmunohistochenically measured angiogenesis. 
93

\section{G. Demonceau, P. De Maesschalck.}

Dpts of Nuclear Medicine and Gynaecology, St Elisabeth Hospital, Zottegem, Belgium

VALUE OF TC-MIBI FOR THE DETECTION OF BREAST CANCER: A PROSPECTIVE STUDY.

Planar scintigraphy of the breast with T1-201 was recently found to be useful in differentiating between malignant and benign lesions in patients presenting with a palpable mass. Systematic screening for breast cancer is recommended from the perimenopause onward, but mammography may be difficult to interpret. We therefore performed a prospective study on the subject, using Tc-MIBI and planar as well as tomographic scintigrams.

In 120 females, a mammogram of each breast (with in doubtful cases, an ultrasonography) and scintigrams were performed within a 3 -day period. The scintigrams were obtained 1 hour after injection of $740 \mathrm{Mbq}$ of Tc-99m-MIBI, by means of 1 anterior static acquisition of 3 minutes and followed by a circular tomography of 25 seconds per step and with 32 steps over $180^{\circ}$ anteriorly. Final diagnosis was obtained by means of a biopsy or by a clinical, radiological and scintigraphic follow-up of at least 6 months.

Four cancers were detected in this population, the 4 of them by mammography, 3 on scintigraphy (in both the planar and tomographic views). The patient with false negative scintigraphy presented with an in situ intraductal adenocarcinoma of less than $1 \mathrm{~mm}$ in diameter, whose mammographic suspicion was related not to the tumor itself but to the presence of micro calcifications in an adjoining focus of fibrocystic disease. Ten cases of false positives were noted after mammography with ultrasonography versus 4 cases in static scintigraphic images and 11 in scintigraphic tomograms. Finally, tracer uptake was sometimes found in axillary lymph nodes, particularly in tomograms and, mostly, on the side of the tracer injection (sub clinically paravenous?), there being no obvious reason to relate this finding to malignant invasion.

Circular tomoscintigraphy using Tc-99m-MIBI does not seem, in this series, to be better than a simple anterior view, mainly because of reconstruction artefacts when using small tracer activities. Also, results of screening a larger series would obviously be of value. So far though, our data would seem to imply some clinical usefulness for this technique.

\section{4}

McEwan $A^{1}$, MacLean $G^{1}$, Golberg $L^{2}$, Akram I ${ }^{1}$, Boniface $G^{2}$, McQuarrie $S^{1}$, Golberg $K^{1}$, Sykes $T^{2}$, Amyotte $G^{1}$, Noujaim A. ${ }^{2}$

1, Cross Cancer Institute and 2, Biomira, Inc., Edmonton, Alberta Canada.

EVAIUATING RADIOIMMUNOSCINTIGRAPHY IN PATIENTS WITH BREAST CANCER.

Radioimmunoscintigraphy (RIS) has been postulated as having a role in the management of patients with breast cancer both at presentation and at recurrence. We have performed a Phase II trial of a monoclonal antibody (MAb) to assess its potential role in this clinical setting.

MAb 170H.82 (Tru-Scint $\left(\mathrm{AD}^{\mathrm{TM}}\right.$ Biomira Inc) is derived against synthetic TF antigen and in-vivo reacts with most adenocarcinomata and has been directly labelled with Tc- $99 \mathrm{~m}$. Forty patients with primary or metastatic breast cancer have been included in this evaluation. Doses of $1 \mathrm{mg}$. (21 pts), $2 \mathrm{mg}$. (10) and $4 \mathrm{mg}$. (9), labelled with between 1100 and $1600 \mathrm{MBq}$ of Tc-99m, were administered by slow intra-venous administration. Images were obtained immediately post injection and at 4-6 and 18-24 hrs post injection with a GE 400 AT or Prism 2000 gamma camera. SPECT was performed at 24 hours. Pharmacokinetic analysis was performed on 4 patients and included serial blood sampling and total urine collection to 72 hours post injection.

Eighty-five breast, nodal and bone sites were identified as known lesions. Eighteen of 20 breast lesions were identified, 36 of 40 lymph nodes and 23 of 28 bone metastases. In total, five false positives were identified, 3 of which were in the breast, 1 in the intra-mammary chain, and one in the axilla. Sensitivity for locoregional soft tissue disease was $90 \%$ and specificity $93 \%$. These data suggest high clinical accuracy for this MAb in this group of patients and indicate a possible routine clinical management role for RIS in breast cancer management.
95

M. Granowska, M. Carroll, C. Nimmon, D. Ellison, s. J. Mather, L. Biassoni. V.U. Chengazi, A. Granowski, K.E. Britton

st. Bartholomew's Hospital and Medical College, Imperial Cancer Research Fund, ICRF, Nuclear Medicine Group, London, EC1A 7BE

RADIOIMMUNOSCINTIGRAPHY, RIS, OF BREAST CANCER, BC, USING TC $-99 \mathrm{~m} S \mathrm{M}$

Tailoring the extent of surgery in the individual woman with BC in relation to a prior knowledge of the presence or absence of axillary and other lymph node involvement is an unrealised goal, unmet by previous BC RIS. This study embodies the use of a breast cancer specific ICRF monoclonal antibody SM3: the TC-99m label; and the application of kinetic analysis with probability maping which had previously prospectively detected lesionsping to $5 \mathrm{~mm}$ in ovarian cancer.

9 patients have received $600 \mathrm{MBg}$ Tc-99m SM3 IV up to 10 days prior to subsequent surgery. Anterior and lateral gamma camera images of the breasts and axilla were made at 10 mins, 6 and $22 \mathrm{~h}$, with marker images with Co-57 for the subsequent image repositioning protocol. Application of the change detection algorithm gave sites of significant change between the early nonspecific distribution and the later images with sites of specific SM3 uptake. In this way, image positive and image negative axillary nodes as well as the primary $B C$ have been collated with subseguent surgical and histological findings and are correct in $8 / 9$. Apparent ly involved findings and are correct in 8/9. Apparently involved internal mammary nodes have been imaged, but with no surgery to confirm. These initial results are encouraging and are the basis for a formal clinical
trial.
96

C. Giannakenas, A. Apostolopoulos, J. Tselfes, A. Mastorakou, E. Likaki," "E. Tzorakoeleftherakis", P. Mirilas*, T. Gorilas, P.J. Vassilakos.

Depts. of Nuclear Medicine, "Surgery, " Diagnostic Radiology, Regional University Hospital, University of Patras, Rion-Patras, Greece.

\section{IN-VIVO SCINTIGRAPHIC IMAGING OF BREAST CANCER UTILIZING THE RADIOLABELLED SOMATOSTATIN ANALOGUE OCTREOTIDE.}

In this continuing study the in vivo scintigraphic imaging of somatostatin receptors in mammary tumors is studied utilizing 111/n-DTPA-Octreotide in orde to evaluate this technique for both preoperative detection of the extent of the disease and postoperative follow-up for possible recurrence.

A total of 24 female patients (mean $55.07 y$, range $27-67 \mathrm{y}$ ) with diagnosed or suspected breast tumors were studled. All patients had findings in diagnosed or suspected breast tumors were studied. All patients had findings in administration of $2.2-3 \mathrm{mCj} 111 \mathrm{In}$-DTPA-Octreotide, pianar imaging land SPECT selectively) was carried out at $6 \pm 1$ and at $22 \pm 2 \mathrm{hrs} p$.i. The scintigraphic data was compared to the histological data (procured by FNA / node biopsy or tissue removed by surgery). The prestudy grading was as follows: grade IIA $(n=3)$, grade $\| B(n=4)$, grade $\| A(n=5)$, grade $\| B(n=6)$ and grade $N(n=6)$. The scintigraphic imaging was then evaluated in view of these findings for a possible correlation between the grade and the Imaging and also to assess whether imaging could detect local or distal recurrences of the disease.

In 20 of the 22 patients with cancer imaging was positive isensitivity $90.9 \%$. In two patients $18,3 \%$ ) the scan was falsely positive (histology revealed fibroadenosis). In 2 other patients (grades $(I B$, IIIA) the scan was false negative $18,3 \%$. Pleural effusion was imaged in one patient where aspiration and subsequent cytology revealed malignancy. Bone metastases were imaged in 5 patients (confirmed by $99 \mathrm{mTC}$-MDP scintigraphy). The normal biodistribution of the radiopharmaceutical in the liver, the spleen and the kidneys hindered the the radiopharmaceutical in the liver, the spleen and the kidneys hindered the
imaging of possible nepatic metastases. in two of the patients however formerly unknown metastatic lesions were imaged in the abdominal region. In 8 of the 22 true positive scans $(36.4 \%$ ) previously unknown involvement of maxillary and/or thoracic lymph nodes was demonstrated which had either not been detected or not been confirmed previously by other modalities.

111-In-Octreotide proves to be an important radio-pharmaceutical in the diagnostic imaging of breast cancer. Its ability to image involved Iymphnodes and both local and distal recurrence has been helpful in the grading of the patients
and especially those with grades IIIA-lilB with the relevant clinical significance. Short-comings of this procedure is the fact that a benign fibroadenoma was imaged thus lowering the specificity, and the high cost of the radio pharmaceutical which prohibits its everyday use. The introduction of a larger number of patients in this continuing study and the use of SPECT is expected to provide more objective conclusions. 


\section{7}

N. Avril, F. Jänicke*, J. Dose*, S. Ziegler, S. Bense, M. Hertz, H.R. Langhammer, H. Graeff, M. Schwaiger

Nuklearmedizinische Klinik u. Poliklinik, Frauenklinik*, Klinikum rechts der Isar, Technische Universität München, Germany

\section{IMAGING OF BREAST TUMORS WITH FDG-PET IN COMPARISON} WITH HISTOLOGY

PET-FDG imaging has been shown to accurately identify large breast tumors, but little PET data exist in the initial diagnostic workup of small breast lesions detected by mammography. Twenty-one patients scheduled for exploratory breast surgery were studied with FDG-PET (Siemens ECAT 951R). Dynamic FDG-PET scans of the breast were obtained for 60 minutes followed by PET imaging of the axillas for $15 \mathrm{~min}$. Time activity curves were evaluated and standardized uptake values for FDG calculated based on attenuation corrected images acquired between $45-60$ min after tracer injection.

Twenty-two malignant lesions in 17 patients could be identified with FDGPET (SUV 3.6 \pm 1.8 ). An increased FDG uptake (SUV 3.4 and 3.8) was found in two patients with a carcinoma in situ. Benign lesions such as mammary dysplasia $(n=5)$ and fibroadenomas $(n=2)$ showed a significant lower FDG accumulation (SUV 1.3 \pm 0.2 ) than malignant tumors $(p<0,01)$. Tumor FDG uptake correlated with in-vitro human nuclear cell proliferationassociated antigenbinding (Ki-67) in tumor tissue of 11 patients. Axillary lymph node dissection in 15 patients showed in 7 cases axillary lymph node involvement. True positive FDG uptake was seen in 4 patients. 3 patients had false negative findings with lymph nodes size smaller than $1 \mathrm{~cm}$ in diameter.

in conclusion, FDG-PET imaging identified all malignant lesions in this selected patients. Fibroadenomas and areas with mammary dysplasia had significant lower glucose metabolism. The observed high specificity has to be confirmed in a larger patient population with benign tumors. Detection of lymph node involvement appears to be limited by partial volume effect. FDGPET may be clinically useful in the early diagnostic workup of patients with abnormal mammography.

\section{8}

K. Scheidhauer, A. Scharl*, T. Oepen*, E. Voth, P. Theissen, K. Schomäcker, A. Bolte*, H. Schicha Departments of Nuclear Medicine and Gynecology* University of Cologne, Germany.

\section{[18F]-FDG-PET IN BREAST TUMORS}

Aim of the study was to evaluate Positron emission tomography (PET) using [18F]-Fluorodeoxyglucose (FDG) for visualization and detection of breast tumors. Although unspecific, higher rates in glucose metabolism compared to normal tissue should enable selective tumorimaging. Therefore, in 23 patients, 12 with unclear breast findings and 11 breast cancer patients suspicious for metastatic disease, FDG-PET was compared to histology and other imaging methods.

PET was performed after iv-injection of $370 \mathrm{MBq}$ [18F]-FDG in fasting state on a CTI ECAT Exact scanner with an axial FOV of $16.2 \mathrm{~cm}$, transmission and emission images were taken 15-45 min. p.i., with additional whole body scans in some metastatic patients $(n=4)$. Focally increased uptake was visually judged suspicious for malignancy. PET yielded focal FDG-uptake with high contrast in 7 of 8 primary carcinomas including a patient with only monomorphic microcalcifications. In another patient, only PET correctly visualized multifocal disease ( 3 foci, $\varnothing 0.4$ to $1 \mathrm{~cm}$ ). One carcinoma in a diabetic patient was not seen (false negative). A clinically suspicious cicatrical tissue after tumorectomy (lymphoma) and chemotherapy was false positive (low contrast). All metastases (lymph nodes, lungs, brain, bones, soft tissues) scen by other methods (X-ray, CT, MR, bone scan) showed FDG-uptake. In 3 patients, only PET initiated further diagnostic procedures.

These results indicate, that FDG-PET may provide a fast diagnostic study $(30-60 \mathrm{~min}$.), which allows a onetime accurate tumorstaging of several organ systems for primary tumor and metastases. Whether early use of FDGPET in cases of unclear breast disease and for staging in breast cancer benefits patients, needs to be demonstrated.

\section{9}

T. Kuwert ${ }^{1}$ H. Hefter ${ }^{2}$ H. Kreuzpaintner ${ }^{3}$, W. Stremmel ${ }^{3}$, R. Seitz ${ }^{2}$, A. Schlaug 2 , H. Herzog ${ }^{1}$, K.-J- Langen ${ }^{1}$, H.W. Müller-Gärtner ${ }^{1}$

1)Institute of Medicine, KFA Jülich; Depts. of 2) Neurology and 3) Gastroenterology, University of Düsseldorf, FRG.

EVALUATION OF THERAPY IN WILSON'S DISEASE BY PET MEASUREMENT OF STRIATAL GLUCOSE CONSUMPTION

Wilson's disease (WD) is a hereditary disorder of copper metabolism leading to striatal degeneration and liver cirrhosis. It may be treated either by decoppering drugs such as D-penicillamine (DPA) or by liver transplantation (LT). The aim of this study was to evaluate the effect of these therapeutic modalities on striatal glucose consumption (rCMRGlc).

Using FDG and the PET-camera PC-4096 the striatal glucose consumption was measured in 25 normal volunteers and $14 \mathrm{WD}$ patients. In 3 of the patients LT had been performed 1 to 6 years before their inclusion in the study. 11 of the patients were treated by DPA. Whereas in 4 of these decoppering therapy had just been started before the PET study, the duration of DPA therapy was longer than 5 years in the remaining 7 patients. The normal limits of striatal rCMRGic were calculated as $95 \%$ confidence limits of the normal data using Student's t-values.

While striatal rCMRGlc was markedly reduced below normal in the 4 patients with short duration of DPA therapy, it was in the lower normal range in the patients with long-standing medication. In the latter group striatal glucose consumption correlated negatively with the duration of medication and the urinary excretion of copper $(p<0.01)$. The patients in whom LT had been performed also had normal values of striatal rCMRGIc.

In conclusion, DPA medication leads to a normalization of striatal glucose consumption in WD patients; the observed negative correlation between striatal glucose consumption on the one hand and the duration of medication and the urinary excretion of copper on the other suggests that the dose of DPA given in the patients studied was inappropriately low. There was no decrease of striatal glucose consumption after LT; this is in agreement with the recent theory of a disturbance of the biliary excretion of copper as the cause responsible for the excessive tissue copper accumulation in WD.

\section{0}

E. Donnemiller, G. Grubwieser*, D. Kendler, R. Moncayo, A. sztankay, L. Fridrich, G. Riccabona Depts. of Nuclear Medicine and Neurology*, University of Innsbruck, Austria Tc-99m-HMPAO- AND I-123-IBZM-SPECT IN PATIENTS WITH POSTTRAUMATIC EXTRAPYRAMIDAL, MOVEMENT DISORDERS

Posttraumatic Parkinson-syndrome (PPS) can occur after severe head injuries with inner inferior cerebral trauma. L-DOPA-therapy can be helpful in some cases, while others show no response. Considering the pathogenesis of this syndrome either a destruction of dopamine-receptors (DR) in the striatum or a disruption of neural transport systems could be responsible for PPS. To analyze mechanisms responsible for PPS we studied 5 pts. with. PPS doing 99mTeHMPAO-perfusion-SPECT and 123 I-IBZM-D2-receptor-SPECT 1 week after the perfusion study and after apomorphine tests. SPECT-images were obtained with a single-head-rotating-camera 10 min. p.i. of $555 \mathrm{MBq} 99 \mathrm{mTc}-\mathrm{HMPAO}$ and $2 \mathrm{hrs}$. p.i. of $185 \mathrm{MBq} 123 \mathrm{I}$-IBZM. 3 pts, were also studied after L-DOPA-therapy for 6 weeks and after LDOPA had been withdrawn for 7 days. All pts. showed perfusion defects ( 4 bilateral) initia1$1 y$, in 4 cases also in the striatum ( 2 bilaterai). IBZM-SPECT showed uptake defects in basal ganglia only in 4 pts. ( 1 bilateral) of which 1 had the defect in the contralateral side of the perfusion defect. Control scans in 3 pts. showed normal IBZM-uptake in all pts., which had all improved under L-DOPA-therapy. Our findings suggest that HMPAO- and IBZM-SPECT together with apomorphine tests can predict the response to L-DOPA-therapy in pts. with grossly abnormal perfusion scans but with at least partially functioning DR. 
101

H.M. Abdel-Dayem, J. Masdeu, R. O'Connell, S. Aleksic, N. Habbab, D. Iuck, J. Barrera, R. Lim, J. Kempf, R. Bag, F. Pescatore

Department of Nuclear Medicine, St. Vincent's Hospital and Medical Center, New York, USA

BRAIN PERFUSION ABNORMALITIES FOLLOWING MINOR/MODERATE CLOSED HEAD INJURY: COMPARISON BETWEEN EARLY AND LATE IMAGING IN TWO GROUPS OF PATIENTS

Tc-99m HMPAO SPECT Brain Perfusion (BP) imaging is more gensitive than $X$-ray CT or MRI in detecting abnormalities following closed head injury (HI). The purpose of this study is a retrospective comparison between the findings of study is a retrospective comparison between the findings of SPECT BP imaging and $X$-ray $C T$ or MRI in two groups of patients who suffered from minor to moderate closed HI. All $\mathrm{mCi}$ (1110MBg) and were imaged either early within one week 21 patients (Grp I), or late 3 weeks to one year; 21 are in the following table:

Group 1 (early imaging)

No. of patients

Age (mean, range)yrs

Male/Female

Abnormal SPECT

no. of lesions

Normal SPECT

Abnormal CT/MRI

Normal CT/MRI

21
$21(15-79)$
$13 / 8$
18
30
3
3
18

Group 2

(late imaging)

We conclude that early SPECT BP imaging (within one week) following closed HI is more sensitive than delayed imaging following closed HI more sensitive than delayed imaging (more than three weeks). This is due to recovery and repair of lesions detected in early imaging. We encourage BP imaging following minor to moderate head injury in the behavioral complaints of these patients in the absence of CT or MRI abnormalities. An ideal project would be to follow up the same group of patients with early and delayed BP imaging and to correlate with neuropsychological testing.

102

H.M.L. Jansen, A.T.M. Willemsen, L.G.F. sinnige, A.M.J. Paans, J.M. Hew, J. Pruim, W. Vaalburg, J. Korf, J.M. Minderhoud Dept. of Neurology and PET-Center, Univ. Hospital Groningen, the Netherlands

DETECTION OF NEURONAL DAMAGE IN RELAPSING REMITTING MULTIPLE SCLEROSIS WITH COBALT-55 POSITRON EMISSION TOMOGRAPHY

We suggest cobalt-55 (Co) as a calcium (Ca)-marker to visualize $\mathrm{Ca}$ transport across the neuronal membrane with PET. Elevation of intracellular $\mathrm{Ca}$ is closely linked with the process of neuronal cell-decay. Co-uptake is correlated with Ca-accumulation through divalent cation-permeable kainate (KA)-activated receptor-operated channels in the neuronal membrane

Multiple sclerosis (MS) is a progressive disease characterized by exacerbations and remissions. In order to reveal in vivo signs of neuronal damage in $M S$ as a possible explanation of the chronic progression, we performed Co-PET and MRI on a group of 7 relapsing-remitting MS-patients (no immunosuppressive therapy) and a group of 7 healthy sexmatched volunteers. The patientcroup showed an inhomogeneous co-distribution throughout the brain, suggesting focal accumulation in multiple spots. There was a significantly increased number of co-spots (as compared with the healthy brain) in the MS-brain, which showed partial anatomical correlation with the MS-lesions as detected with MRI. There was no correlation between co-uptake and contrast-enhancement on MRI. In conclusion, our preliminary Co-PET data suggest neuronal damage in MS-patients which could explain the slowly progressive course in the later stage of the disease.

103

U. Cremerius, I. Lossau, B. Lippitz, J. Weis, J.M. Schröder, J. Gilsbach, U. Büll

Depts. of Nuclear Medicine, Neurosurgery and Neuropathology, RWTH Aachen, Germany

TC-HMPAO-SPECT VERSUS FDG-PET AND HISTOPATHOLOGICAL GRADING IN INTRACRANIAL MENINGIOMA CAN GRADING BE PREDICTED BY PET OR SPECT ?

The aim of our study was to evaluate the relevance of TC-HMPAO-SPECT (reflecting vascularisation) and FDG-PET (representing glucose consumption) for a non-invasive grading of intracranial meningioma as compared to histopathological grading.

30 patients with intracranial meningioma received SPECT $15-60 \mathrm{~min}$. p.i. (750 MBq 99mTC-HMPAO) and PET 30-60 min. p.i. (150-300 MBq 18-FDG) 26 meningiomas were classified as grade 1 (WHO), 4 as grade 2 or 3 . SPECT was evaluated semi-quantitatively using the tumor/cerebellum-ratio (TCR-HMPAO) and the tumor /grey matter-ratio (TGR-HMPAO). Glucose consumption (MRGlu) of meningioma was assessed from static PET-scans according to sOKOLOFF.

\begin{tabular}{|l|l|l|l}
\hline grading $n$ & TCR-HMPAO & TGR-HMPAO & MRGlu (*)
\end{tabular}

\begin{tabular}{|cc|l|l|l|}
\hline 1 & 26 & $0.90 \pm .21 \mathrm{~ns}$ & $1.16 \pm .25 \mathrm{~ns}$ & $0.25 \pm .11 \mathrm{~s}$ \\
\hline
\end{tabular}

\begin{tabular}{ll|l|l|l|}
\hline $2-3$ & 4 & $1.06 \pm .24 \mathrm{~ns}$ & $1.40 \pm .22 \mathrm{~ns}$ & $0.39 \pm .15 \mathrm{~s}$ \\
\hline
\end{tabular}

*in $\mu \mathrm{mol} / \mathrm{g} / \mathrm{min}$ (n) $\mathrm{s}=$ (not) significant for $\mathrm{p}<.05$ Grade 2- and 3-meningiomas revealed higher MRGlu and HMPAO-uptake than typical grade 1meningiomas. The difference was significant for MRGlu only. TGR-HMPAO and MRGlu were positively correlated $(r=0.41 ; p<.05)$, however. FDG-PET is superior to TC-HMPAO-SPECT in predicting histopathological grading of meningioma.

104

E. Klemm, F. Grünwald, *P. Danos, **S. Kasper, ${ }^{*}$ C. Krappel, *G. Laux, C. Menzel, L. Pavics, A. Schomburg, A. L. Hotze, *H.-J. Möller, H.-J. Biersack.

Departments of Nuclear Medicine and *Psychiatry, University of Bonn, D-53127 Bonn, Germany, and **Department of Psychiatry, University of Vienna, A-1090 Vienna, Austria

RCBF IN HEROIN AND POLYDRUG ADDICTS EVALUTED BY ${ }^{99 m}$ Tc-HMPAO-SPECT: TEMPORAL LOBE INVOLVEMENT

In drug addicts, namely in cocaine abusers, PET studies have revealed metabolic abnormalities. However, the effects of heroin dependence on cerebral perfusion have not yet been investigated systematically. We evaluated 37 in-patients by means of $99 \mathrm{~m}$ TcHMPAO-SPECT (40 investigations). HIV testing was negative in all individuals. Five patients were dependent only on heroin. Twentythree additionally took cannabis, 22 benzodiazepines or barbiturates, 11 cocaine, and 9 amphetamine. In addition to visual evaluation, 31 ROIs were drawn for semiquantative analysis. Both temporal and frontal regions showed the most striking blood flow abnormalities with right-sided preponderance. Thirty patients $(75 \%)$ had a hypoperfusion. Among them, the right temporal lobe presented with reduced blood flow in $32 \%$, the left temporal region in $18 \%$. A decreased perfusion of the right frontal lobe was observed in $11 \%$. The right parietal and the right occipital areas were involved in $9 \%$. Furthermore, combined addiction of cocaine and heroin significantly correlated with hypoperfusion of the right frontal lobe $(\mathrm{p}<0.01)$. Abuse of amphetamines along with heroin was related to left lateral temporal hypoperfusion $(\mathrm{p}<0.005)$, that of barbiturates along with heroin with left mesial temporal hypoperfusion $(p<0.005)$. However, there were no major perfusion differences. when comparing polydrug addicts and individuals dependent only on heroin. Thus, our most striking finding, the hypoperfusion of the right temporal lobe in heroin (heroin alone or combined with other drugs) addicts, seems to be related to the heroin abuse itself.- In conclusion, the results presented here indicate abnormal cerebral perfusion patterns in heroin addiets predominantly of the right hemisphere. The preponderant right temporal lobe involvement might be related to the marked opiate receptor density of that region. 
105

K.Tatsch, W.Münzing, R.Linke, W.Voderholzer, C.-M.Kirsch, K.Hahn

Department of Nuclear Medicine, Klinikum Grosshadern and Medizinische Klinik Innenstadt, University of Munich, FRG

\section{FOURIER ANALYSIS OF CONDENSED IMAGES FOR}

THE EVALUATION OF GASTRIC CONTRACTIONS

We applied Fourier analysis to condensed images of gastric emptying with the aim to evaluate the amplitude (amp) and frequency (f) of gastric contractions. Patients with various gastric disorders were studied to assess the diagnostic value of the new approach.

In 9 controls and 40 patients conventional gastric emptying studies (TAC, emptying rate, $\mathrm{T}_{1 / 2}$ ) were performed using semiliquid and solid (10/49 pts) test meals. In addition for defined time intervals condensed images from a gastric ROI were established (space- and time matrix consisting of 160 frames with 3 sec/frame) which were used to characterize gastric contraction parameters by Fourier analysis.

Condensed images of gastric emptying precisely depicted the regional distribution of the test meal within the stomach and allowed to qualitatively evaluate the number and strength of peristaltic contractions. The quant. results for the semiliquid studies are listed below.

\begin{tabular}{|c|c|c|c|c|c|c|}
\hline & $N$ & $f\left(\min ^{-1}\right)$ & $\mathrm{p}^{*}$ & ampl. (\%) & $\mathrm{p}^{*}$ & \multirow{2}{*}{$\begin{array}{l}\text { The amp and } f \text { of } \\
\text { gastric contrac- } \\
\text { tions was almost }\end{array}$} \\
\hline & 9 & $3.1 \pm 0.3$ & & $29 \pm 12$ & & \\
\hline diabetes & 16 & 3.4 & $\mathrm{~ns}$ & & $<.05$ & \\
\hline ofti & 8 & $3.1 \pm 0.2$ & ns & $13 \pm 9$ & $<.01$ & \\
\hline tosis & 7 & $3.0=$ & ns & $23 \pm 12$ & ns & $\min ^{-1}$ \\
\hline 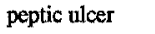 & 5 & 3. & ns & 17 & ns & \\
\hline surgery & 4 & 3. & ns & $9 \pm 3$ & $<, 001$ & (a) \\
\hline & & & & & & 2 \\
\hline
\end{tabular}
significant correlations were observed between gastric emptying $\left(\mathrm{T}_{1 / 2}\right.$ emptying rate) and corresponding contraction parameters.

In conclusion, Fourier analysis of condensed images has shown to be a feasible approach for evaluating gastric contractions in an easy, quick and reliable manner. Amplitude rather than frequency seems the relevant criterium to discriminate between various disorders. Quantitative results have shown to be independent of the composition (semiliquid vs. solid) of the test meal. Data on gastric contractions evidenthy expand the information provided by conventional emptying studies.

106

E. BEADES, J-Y. DEVAUX, R. GUIMBAUD, K. KERROU, I. MORELEC, P. SOGNI, S. CHAUSSADE, B, RICHARD E.A. 215 "Biophysique" CHU Cochin, Paris, France, Gastro-entérologie Hôpital Cochin, Paris, France, C.H. Lyon-Sud, Pierre Bénite, France.

SCINTIGRAPHIC PATHOGNOMONIC ASPECT IN SEVERE ACUTE ATTACK OF ULCERATIVE COLITIS.

Severe acute attack is a Iife-threatening complication of ulcerative colitis (UC), generally suspected on the basis of available clinical and analytical data. Diagnosis is currently established on barium enema and colonoscopy, but with high risk of complications such as colonic toxic dilations or perforations.

In 7 pts out of 36 with known or suspected UC, we have diagnosed severe attacks by $99 \mathrm{mTC}$ HMPAO labelled granulocyte scan, demonstrating a "double track" aspect. In all 7 cases, severe acute attack was confirmed by surgery and histologic findings. In the other 29 pts without severe attack, we never saw this "double track" aspect. Scintigraphic "double track" appears to be pathognomonic of this disease. We explain this phenomenon by the morphologic modification of the inflammatory process which becomes transmural in the colonic wall in case of severe attack of UC.

We conclude that $99 \mathrm{mc}$ FMPAO labelled granulocyte scan is a reliable procedure for the diagnosis of severe acute attack of vC. This method is useful as a diagnosis tool, being easy to perform, fast and without risk of complication.
107

R. Benti, *D. Lisciandrano, A. Bruno, *A.R. Baldassarri, E. Reschini, P. Gerundini

Depts. of Nuclear Medicine and * Gastroenterology;

Ospedale Maggiore and University of Milan, Milan, Italy

SCINTIGRAPHIC ASSESSMENT OF RETROGRADE SPREAD AND ABSORPTION OF A 5-AMINO-SALICYLIC ACID ENEMA IN ULCERATIVE COLITIS AND ILEO-RECTAL ANASTOMOSIS

5-Aminosalicylic acid (5-ASA) enema is frequently given for topical treatment of the rectum in ulcerative colitis (UC) and ileo-rectal anastomosis (IRA).In IRA a significant Retrograde Ileal Spread (RIS) of a 5-ASA enema can unproperly increase its serum levels, being seven fold higher 5-ASA transport efficiency in ileal than rectal mucosa. Ileo-rectal ripartition and absorption of a 5-ASA enema were investigated by nuclear imaging and measurements of 5-ASA plasma levels in seven IRA patients (age 22-68 ys) with UC in remission. Two grams of 5-ASA in $50 \mathrm{ml}$ enema were mixed with $30-40 \mathrm{MBq}$ of $99 \mathrm{~m} T \mathrm{c}$ colloids and administered. Dynamic images (1/min) of the abdomen in anterior view were obtained for $1 \mathrm{~h}$, static (1 min) images from 2nd to 6th hour. Semiquantitative analysis was performed obtaining an lleal Percentual Activity (IPA) from ROIs drawn over the ileum and rectum. Two patterns of rectal wall contraction were recognized in 1st hour images: A) parietal rectal motility with RIS (2$11 / \mathrm{h}$, mean 3.8$)$; B) parietal rectal motility without RIS $(9-21 / \mathrm{h}$, mean 12.1).5-ASA plasma levels were measured with HPLC before, 30,60 min and hourly for $6 \mathrm{~h}$ after administration. 5-ASA absorption was expressed by the integral Area Under time/plasma level Curve (AUC) in $\mu \mathrm{g} / \mathrm{ml} / \mathrm{h}$. Mean IPA were $14.5+16 \%$ and $8.7+6.1 \%$, mean AUC values were $0.5 \pm 0.2$ and $4.3 \pm 3.8$ in the 1 st hour and 2 nd-6th hours periods respectively. IPA and AUC values during the 1 st hour were positively correlated (Spearman rank test, $p=0.03$ ) but not in 2 nd-6th hours period. First hour AUC were lower in IRA than in a control group previously studied.Two IRA patients showed higher AUC than normal, whereas three had lower AUC values.Patients with more than nine RIS/hour had the higher AUC values. The technique detects RIS presence and degree of a 5-ASA enema by quantitative parameters. revealing the rectal motility as a major cause of RIS related high 5-ASA absorption in topically rectal treated UC patients wih IRA.

\section{8}

A Notghi, AB Mostafa, R Hutchinson, N Smith, D Kumar, LK Harding.

Dept Physics \& Nucl Med, Dudley Road Hosp \& Dept of Surgery, Queen Elizabeth Hosp, Birmingham,

Scintigraphic Defaecography: functional measurements in clinical practice

Radionuclide defaecography is now used for assessment of rectal emptyino. Both functional measurements [ ${ }^{2}$ evacuation $(E \&)$, evacuation time (ET), evacuation rate (ER), 咅 initial rise (IRise)] and anatomical measurements [anorectal angel (ARA), pelvic floor descent (PFD)] are used. We have compared the results of defaecography in three groups of patients: 83 with chronic constipation (Cconst), 30 with irritable bowel syndrome (IBS), and 30 with incontinence (Incont). Those who had had surgery were excluded. Patients with Incont were older than those with TBS or cconst ( mean age $57,44 \& 42$ respectively, $p<0.05$ ). There was no difference in the anatomical measurements between the three groups, but the functional measurements in particular between cconst and other two groups were significant.

\begin{tabular}{|c|c|c|c|c|}
\hline & CConst & Incont & IBS & Unpaired $t$-test \\
\hline$\overline{E 8}$ & 60.1 & 76.2 & 65.9 & $\begin{array}{l}\text { CConst/Incont } p<0.001 \\
\text { Incont/IBS } p<0.01\end{array}$ \\
\hline ET (sec) & 72 & 36 & 32 & $\begin{array}{l}\text { CConst/IBS } p<0.01 c \\
\text { CConst/Incont } p<0.01\end{array}$ \\
\hline $\begin{array}{l}\text { ER } \\
(\mathrm{a} / \mathrm{sec}) \\
\end{array}$ & 1.7 & 3.5 & 2.9 & $\begin{array}{l}\text { CConst/IBS } p<0.05 \\
\text { CConst/Incont } p<0.001\end{array}$ \\
\hline TRise (9) & 16.0 & 8.6 & 10.2 & NS \\
\hline ARA (deg) & 131 & 136 & 131 & NS \\
\hline EED $(\mathrm{cm})$ & 3.4 & 2.8 & 3.7 & NS \\
\hline
\end{tabular}

Functional measurements correlate clinical problems more accurately than methods using only anatomical measurements. 
109

R. Bares ${ }^{1}$, P. Klever ${ }^{2}$, St. Hauptmann ${ }^{3}$, J. Faß ${ }^{2}$, U. Cremerius ${ }^{1}$, Ch. Mittermayer $^{3}$, V. Schumpelick ${ }^{2}$, U. Buell ${ }^{1}$

Departments of Nuclear Medicine 1 , Surgery ${ }^{2}$, and Pathology ${ }^{3}$, Technical University of Aachen, GERMANY

FDG-PET FOR CLASSIFICATION OF PANCREATIC DISEASE: CORRELATION WITH PROLIFERATION AND HISTOLOGICAL FINDINGS IN CANCER AND CHRONIC PANCREATITIS

Increased FDG-uptake is a typical PET-finding in malignant tumors and has been observed in inflammatory disease, too. Recent reports upon differentiation between chronic pancreatitis and cancer seem to contradict these results. Purpose of the present study was to compare FDG-uptake measured in 30 patients with malignant $(n=21)$ or benign $(n=9)$ pancreatic tumors with histology of surgical specimens and proliferation evaluated by $\mathrm{Ki}-67$ immunohistochemistry. Static PETscans were performed 40-70 min, after iv injection of $150-300 \mathrm{MBq}$ FDG and quantitated by calculating differential uptake ratios (DUR) of the tumor area shown by CT. Significant differences were found between high, intermediate, or low grade carcinomas (G1: $4.3 \pm 1.1$; G2: $6.2 \pm 0.7 ; G 3: 10.3 \pm 2,1$ [DUR]/p<05) while the correlation between FDG-uptake and proliferation was poor $(r=0.65)$. Tumor size did not influence the magnitude of FDG-accumulation. Chronic pancreatitis present in 8 patients was characterized by low FDG-

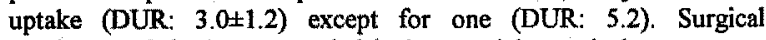
specimens of the latter revealed leukocyte-rich tryptical necroses as sign of active inflammation. In a patient with rapidly proliferating retroperitoneal fibrosis and vast lymphocytic infiltrations a high DUR of 7.9 was calculated and misinterpreted as sign of malignancy.

We conclude that FDG-uptake of pancreatic cancer is strongly influenced by tumor differentiation, less by proliferation suggesting the presence of further cellular determinants of metabolism (e.g. glucose transporter proteins). Leukocytic or lymphocytic infiltrations are the main reason for increased FDG-accumulation in benign conditions.

110

M. Papós, T. Takács, J. Láng, Gy. Farkas, L. Csernay, J. Lonovics

Department of Nuclear Medicine, First Department of Medicine, First Department of Surgery

Albert Szent-Györgyi Medical University, Szeged, Hungary

\section{THE VALUE OF 99mTc-HM-PAO-LEUKOCYTE}

\section{SCINTIGRAPHY IN PANCREATITIS}

Leukocyte scintigraphy (LS) was performed in 22 patients with acute pancreatitis (at least a threefold elevation of the serum amylase level as well as typical ultrasound and/or $\mathrm{CT}$ findings) [group 1] and in 11 patients with pancreatic pseudocyst [group 2]. LS was performed during the first week of hospitalization (mean: 4.3 days). Mixed, autologous leukocytes, labeled with $99 \mathrm{mTc}-\mathrm{HM}-$ PAO in vitro were used. The uptake in the view of the pancreas was classified as none, moderate or intense. Clinical features, laboratory parameters (the baseline amylase level and the amylase level 10 days following $L S$ ) and the outcome were registered during hospitalization.

In group 1,11 of the $22 \mathrm{LS}$ proved positive. 4 of these cases had infected pancreatic necrosis, confirmed by surgery; other patients were successfully treated with antibiotics. The grade of leukocyte accumulation correlated with the duration of hospitalization ( $r: 0.45$, $\mathrm{p}<0.05)$ and with the late amylase level $(r: 0.57, \mathrm{p}<0.01)$. Baseline laboratory findings showed no correlation with these parameters.

In group 2, 4 patients had positive LS; 3 of them were operated on, and pancreatic abscess was detected in all cases. In 3 of the 7 cases with normal LS, surgery revealed a noninfected pancreatic pseudocyst.

Conclusion: Leukocyte infiltration into the pancreas can be demonstrated by LS. Positive LS indicated a severe course of acute pancreatitis, with a special need for antibiotic and/or surgical treatment. LS was also useful for differentiation between infected and noninfected psuedocysts in chronic pancreatitis.
111

I. Andersson, J. Bosaeus, B. Långström

Uppsala university PET-centre, Uppsala, Sweden.

A NEW, RAPID AND ACCURATE METHOD FOR INTRASUBJECT REGISTRATION OF PET IMAGES.

Movements during or between PEF examinations is a common problem causing difficulties in the interpretation of data.

A method to detect and correct such movements is presented. The method maximizes a "similarity function" between two data sets. It uses derivative information from the image sets to determine which parts that contain orientational information and then uses the image correlation between these parts.

It has been validated by applying a known set of translations and rotations to datasets collected from the same subject in the same position. The data sets have been collected either using the same tracer in both, tracers with a similax uptake pattern or tracers with a markedly different uptake pattern.

The results show that for image sets with the same or similarly behaving tracers it is possible to correct for motions with a precision of $0.2 \mathrm{~mm}$ and $0.2^{\circ}$. This precision is achieved for data sets with down to $0.5 \mathrm{milli}$ il counts. For tracers with such marked differences in uptake pattern as water and NMSP it is still possible to reorient the image sets with a precision of $1.0 \mathrm{~mm}$ and $1.0^{\circ}$. The method is fast, and completed in $1-2$ minutes of CPU time on a VAX-station $4000 / 60$.

our results show that the method is adequate to use routinely to register image sets before using subtraction techniques, to register frames within a dynamic sequence to yield accurate TACT-curves and to register examinations performed with different tracers to facilitate the analysis of multi tracer protocols.

112

A. BOUVIER - A. MALLON

CEA / LETI-CENG 38041 GRENOBLE

Positon Axial Tomography (P.A.T.) : a new concept for plans reconstruction for whole body imaging (W.B.I.) which takes benefits from the time of nightt (T.O.F.) technology.

Classical Positon Emission Tomography (P.E.T.) enables to reconstruct either transverse slices of the object in a $2 \mathrm{D}$ mode, or directly a whole volume in a 3D mode. In both cases, the reconstruction process need to get projections of emission measurements all around the object (or patient)

These projections are obtained over $180^{\circ}$ either with many detectors in a ring arrangement around the patient, or with detectors facing only a part of the circumference and rotating around the patient.

The new method called P.A.T. enables to reconstruct directly longitudinal slices of the object without every projection around it. This is realized by using only a sub set of projections with respect to the sub normal direction (typically from $20^{\circ}$ to $40^{\circ}$ ), including the T.O.F.set information. Events so located are then backprojected using a special mode, in order to reconstruct directly the emission information within the object volume. Comparing to the normal projection, the use of oblic projections allows a sensitivity gain with a loss of spatial resolution reduced with the time of flight measurement. An analytic study and a numerical simulation have been performed which enable to quantify for a final spatial resolution of $7 \mathrm{~mm}$ (intrinsec spatial resolution of $6 \mathrm{~mm}$ ) a sensibility gain superior with a ratio 20 (comparing to an aequisition without time of flight) with time of flight measurement of $250 \mathrm{ps}$.

We conclude that T.A.P., thanks to to the T.O.F. information an the plans reconstruction process enables to reconstruct correctly data volumes for WBI, in agreement with the aimed spatial resolution. This technic could dramaticaly reduce the price of the detection system of a PET for WBI applications. 
113

\section{E.Rota Kops, H. Herzog, H.W. Müller-Gärtner}

Institute of Medicine, Research Centre Jülich, and Nuclear Medical Department, Heinrich-Heine-University Düsseldorf; Jülich, Germany

\section{INFLUENCE OF THE TRANSMISSION SOURCE ACTIVITY ON THE RECONSTRUCTED PET IMAGES}

Blank and transmission measurements are necessary for attenuation correction of PET data. The aim of the present study was to investigate the influence of the transmission source activity, e.i. of the number of transmission coincidences, on the quantitative accuracy of reconstructed PET images.

The study was performed with the PET-Scanner PC4096-15WB. A first phase was based on phantom measurements in form of brain simulation, the results of which have been already shown elsewhere. The second phase consisted of patient measurements: five brain studies and four heart measurements. Different transmission scans collected 0.5 to about 12 million coincidences. In case of brain studies, Regions of Interest (ROI) were drawn on whole cortex, thalamus, and white matter; for the heart studies one ROI was drawn on the myocard.

The results of the phantom measurements were confirmed by the patient studies. With transmission coincidences of $\geq 2.5$ million per slice for the brain (left figure) and of $\geq 6$ to 8 million for the heart measurement (right figure) constant values of activity concentration with a variance up to $+1-6 \%$ were found. The corresponding standard deviations yielded values up to about $20 \%$ variance for brain studies, but of only $1 \%$ for heart measurements. For lower values than 2.5 and 7 million of coincidences, respectively, the results confirmed again the phantom measurements: the reconstructed images showed lower concentration values with higher standard deviations, but also image artifacts, due to an erroneous attenuation correction.

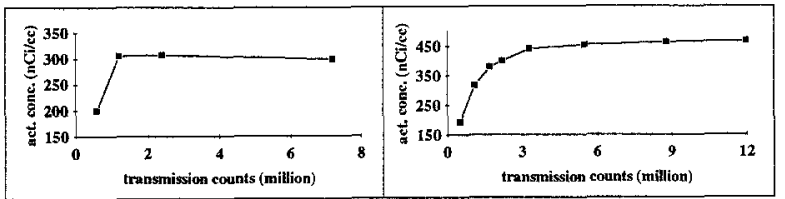

This study, together with the first phase of phantom measurements, confirmes that the transmission source activity influences strongly the quantitative reliability of PET images, but also that the transmission scan length can be shortened to a minimum as result of an optimally planned measurement schedule.

\section{4}

W.Picker, S. Nekolla, S. Ziegler, C. Duvernoy*, J. Sitomer* and M. Schwaiger. Dept. of Nuclear Medicine, Technical University of Munich, Germany and *University of Michigan, Ann Arbor, MI, USA

\section{NEW APPROACH FOR SEMI-QUANTITATIVE DEFINITION} OF CARDIAC TISSUE VIABIIITY BY PET

PET F18-FDG and 13-N Ammonia (NH3) studies are routinely evaluated visualy. The purpose of this study was to develop an automated. semiquantitative analysis by comparing relative cardiac NH3 and FDG uptake. Cardiac PET images of 21 patients were evaluated using a radial 3D maxima activity search based on interactive definition of the ventricular long axis. Relative tracer distributions (NH3, FDG) were displayed in polarmap format. FDG uptake was normalized to the region of maximal NH3 uptake. Ratio (RAT), difference (Dif) and sum (SUM) of regional pixel values on $\mathrm{NH} 3$ and normalized EDG polar maps were calculated. Regional values were compared with visual tissue characterisation (normal, mismatch, scar) by an expert reader.NH3 uptake was significantly reduced in mismatch and scar, but FDG uptake was only significantly reduced in scar tissue. DIF-maps provided best separation between mismatch and scar (t-test <0.01), while sUM allowed best separation of normal tissue(t-test $<0.01)$ from scar. This automated analysis allows data interpretation in good agreement with visual evaluation. Prospective testing of these criteria with functional outcome after revascularization is needed to define the prognostic value of this automated analysis technique.
115

B. Knoop, K. Jordan

Dep. Nuklearmesstechnik u. Strahlenschutz, Medical School Hannover, Hannover, Germany

\section{PROBLEMS WITH ATTENUATION CORRECTION IN PET}

The ability of PET to determine the spatial distribution of activity concentration in vivo is mostly due to the possibility to do an exact attenuation correction. The accuracy of this correction influences strongly the accuracy of quantitation, because typically the correction factors are in the range of $500-2000 \%$. As the correction is based on a transmission scan, in theory complex object geometries with nonhomogeneous attenuation coefficients can be treated.

Practically, however, there are problems mainly due to the limited statistics of the transmission scan. This is especially true for modern scanners with high transverse and axial resolution and therefore fine spatial sampling. So a single correction factor typically is based only on 40 counts for brain- and 0.5-30 counts for abdominal imaging. Without any processing the associated noise completely destroys the emission image. By smoothing the transmission data this effect can be reduced, but the transmission spatial resolution is degraded from $6 \mathrm{~mm}$ to $12 \mathrm{~mm}$. So at the borders between changing attenuation coefficients systematic errors are introduced. So contrast for a cold, airfilled sphere is reduced from $70 \%$ to $65 \%$ (40 $\mathrm{mm}$ in dia.) or from $30 \%$ to $17 \%$ (15 mm dia.), respectively.

Using realistic phantom setups with different attenuation coefficients simulating tissue, lung, and bone the problems of attenuation correction, dependent on the degree of smoothing (2- or 3-dimensional, repeated application), are demonstrated. The data are compared to the results of an analytical correction assuming constant $\mu$, which introduces unacceptable errors (e.g. changes image activity concentration from cold to hot) for these complex geometries.

In addition the degradations caused eventually by external absorbers (patient table, head holder) are discussed.

The problems shown are common to all linear correction methods and demonstrate the need for more sophisticated non-linear correction methods.
116

C.Rossetti, A. Gugiatti, A. Grimaldi, M. Del Vecchio, F. Sassi, E. Borgonovi, F fazio.

Università Bocconi, $\mathrm{H}$ S Raffaele, Università degli Studi, CNR, Milan.

ECONOMIC ASPECTS OF CLINICAL USE OF POSITRON EMISSION TOMOGRAPHY (PET)

Aim of this study is to analyse the economic aspects of $\mathrm{PET}$ as an introduction for a subsequent cost/effectiveness evaluation in selected pathologies. To define the cost of a PET center with complementary equipment (incluaing a cyclotron on site), the PET center operating at $H \quad S$ Raffaele Institute (Milan) has been evaluated. Considering depreciation, maintenance, disposables, training, personnel, other general expenses and financial burdens, the running cost of a PET center - based on obsolescence of 5 years for the tomograph and 10 years for the rest of equipment - was in 1991 1.048.000 ECU. The financial burdens due to investment with an annual interest rate of $7 \%$ were in the same year 132.500 ECU. With this rate of interest, the cost of investment (depreciation and interests) was about 46 응 of the total cost. Considering running costs as fixed, except for the disposables, the average cost per study was between 1.500 ECU ( 800 annual studies) and 1.980 ECU (500 annual studies). These results force a careful evaluation relatively to the acquisition of $\mathrm{PET}$ technology from health care structures specially considering the potential demand of studies. 
117

E. Voth, A.R. Börner, P. Theissen, H. Schicha

Dept. of Nuclear Medicine, University of Cologne, Cologne, Germany

\section{8-FDG PET IN PATIENTS WITH GRAVES DISEASE}

In 30 patients with Graves disease admitted for therapy with 131-1, 18-FDG positron emission tomography (PET) was performed. $99 \mathrm{~m}-\mathrm{Tc}$ scintigraphy, sonography, $131-1$ uptake and $\mathrm{T}_{3}, \mathrm{IT}_{4}$ and TSH were available.

18-FDG PET was performed in all 30 patients before 131-I therapy. Following determination of blood glucose level, $370 \mathrm{MBq}$ of 18-FDG were administered intravenously to the fasting patient. Using a CTI ECAT ECACT $921(16.2 \mathrm{~cm}$ axial field of view, 47 planes), transmission ( $30 \mathrm{~min}$ ) and emission scans ( $6 \times 5 \mathrm{~min}$ ) were performed. Reconstructed data were interpolated in 3-D technique. For quantitative analysis, four coronal $5 \mathrm{~mm}$ slices were added and thyroid FDG-uptake ( $\% / \mathrm{ml}$ thyroid tissue) was determined using ROl-technique.

There was marked thyroid 18-FDG uptake in all patients, increasing with activity of hyperthyroidism and decreasing with age Patients with high 18-FDG uptake showed a short biological half-life of 131-I under therapy conditions. Using multiple regression analysis, significant relations of 18-FDG uptake were determined to biological half-life of $131-1$ under therapy conditions (partial correlation coefficient $r=-0.44, p=0.01$ ), to amount of antithyroidal medication $(r=0.35, p=0.04)$ and to patient's age $(r=-0.33, p=0.04)$ Combination of these three relations resulted in a total correlation coefficient of $R=0.72$ ( $p=0.001)$.

The correlation of thyroid 18-FDG uptake to the biological half-life of 131-I and to the amount of antithyroidal drugs might be explained by activation of glucose dependent transmembrane transporter proteins (P-Glycoprotein), the product of the multidrug resistance genes (MDR1 and MDR 2). Induction of P-glycoprotein and other energy dependent membrane transporters may be the cause of both increased FDG-uptake and short biological half-life of 131-!.

118

C. ALS, M. Listewnik, H. Roesler

Department of Nuclear Medicine, Inselspital, University of Berne, $\mathrm{CH}-3010$ Berne, Switzerland

Toxic adenomas are rarefying in Berne

From a total of $12^{\prime} 320$ patients referred for thyroid scintigraphy to our nuclear medicine department during the years 1976, 1982 and $1991,1^{\prime} 125(9.1 \%)$ were clinically or latent hyperthyroid. The frequency of immunogenic versus non-immunogenic hyperthyroidism ( $\mathrm{IH}$ versus $\mathrm{NIH}$ ) and of toxic adenomas (TA) versus multifocal functional autonomy (MFA) were evaluated in these patients.

\begin{tabular}{|c|c|c|c|c|}
\hline & & 1976 & 1982 & 1991 \\
\hline IH & & $\begin{array}{l}43 \% \\
(106)\end{array}$ & $\begin{array}{l}37 \% \\
(146)\end{array}$ & $\begin{array}{l}44 \% \\
\text { (183) }\end{array}$ \\
\hline \multirow[t]{2}{*}{$\overline{\mathrm{NIH}}$} & $T A$ & $\begin{array}{l}41 \% \\
\text { (101) }\end{array}$ & $\begin{array}{c}30 \% \\
(117)\end{array}$ & $\begin{array}{r}17 \% \\
(71)\end{array}$ \\
\hline & MFA & $\begin{array}{c}16 \% \\
(39)\end{array}$ & $\begin{array}{c}33 \% \\
(129)\end{array}$ & $\begin{array}{c}38 \% \\
(159)\end{array}$ \\
\hline Tota & & 246 & 392 & 413 \\
\hline
\end{tabular}

Results: 50 years after the beginning of a progressive iodine supplementation via kitchen salt in Switzerland, NH is still the most frequent form of hyperthyroidism in Berne. Over the 18 year period evaluated, the $\mathrm{IH}$ proportion remained constant. Within a constant share of $60 \%$ of $\mathrm{NIH}$, the TA/MFA ratio dropped from 2.4 (1976) over 0.9 (1982) to 0.4 (1991). A decreasing proportion of TA and a rising MFA frequency could be markers of the progressively diminishing iodine deficiency. Indeed, the urinary excretion of lodine, from $30 \mu \mathrm{g} / \mathrm{g}$ creatinine in 1920, had risen $(1981-1990)$ to a level above $100 \mu \mathrm{g} / \mathrm{g}$ creatinine (WHO standard), to drop again, in 1992 , to a $87 \pm 36 \mu \mathrm{g} / \mathrm{g}$ creatinine level. A rising proportion of TA seems to be a marker of an increasing or newly installed iodine deficiency, as has recently been observed in several parts of the European continent. Thus, the epidemiology of hyperthyroidism is in continuous change in a prealpine country.
119

T. Y. Erdil, C.. Onsel, I. Uslu, B. Kanmaz, K. Sonmezoğlu, E. Althok, L. Kabasakal, H. B. Sayman, C. Nişli, T. Kapıcıŏ̆lu Department of Nuclear Medicine, Cerrahpaşa Medical Faculty İstanbul, TÜRKIYYE

\section{A COMPARISON OF Tl-201 AND TC-99m SESTAMIBI SCINTIGRAPHIES}

IN THE VISUALIZATION OF SUPRESSED THYROID TISSUE

Because of the unwanted effects of TSH stimulation test, recently Tl-201 has been proposed for the visualization of supressed thyroid tissue in patients with autonomously functioning thyroid nodules (AFTN). It is suggested that TSH control is not a major determinant of Tl-201 uptake. More recently, Tc-99m MIBI has also been recommended to be used for the same purpose. However, the mechanism of thyroid uptake of Tc-99m MIBI is contraversial. In this study, we tried to find out the ideal agent in the visualization of supressed thyroid tissue in patients with AFTN.

Fourteen patients (12F,2M) with toxic solitary AFTN visualized on Tc-99m pertechnetate scan were included in this study. $15 \mathrm{~min}$. images were obtained after IV injection of $80-100 \mathrm{MBq}$ of Tl-201 and $350-400 \mathrm{MBq}$ of $\mathrm{Tc}-99 \mathrm{~m}$ MIBI with 3 days interval. The scintigrams were analysed both visually and semiquantitatively. For the semiquantitative analysis, ROIs were set over AFTN and contralateral normal thyroid tissue and the average counts in every ROIs were determined. After background correction, by dividing nodular counts to normal tissue counts, nodule / extranodular tissue ratios were calculated.The mean tissue ratios for pertechnetate, Tc-99m MIBI and $\mathrm{Tl}-201$ were found to be $10.04 \pm 4.18,4.45 \pm 2.03,1.75 \pm 0.19$ respectively.

Our results showed that Tl-201 uptake of supressed thyroid tissue was more prominent and statistically significant $(p<0.05)$ than that of Tc-99m MTBI in toxic AFTN. This might be due to the higher metabolism and / or TSH dependent uptake of Tc-99m MIBI. In conclusion, T1-201 is superior to Tc-99m MIBI in the visualization of supressed thyroid tissue in patients with AFTN even its poor physical characteristics.

\section{0}

R. Moncayo, A. Dessl*, W. Judmaier*, I. Baldissera§, D. Kendler, Ch. Watfah, A. Sztankay, C. DeCristoforo M. Hackl, L. Fridrich, G. Riccabona

Departments of Nuclear Medicine, *MRI, and §Ophthalmology, University of Innsbruck, Innsbruck, AUSTRIA

\section{OCTREOSCAN AND MAGNETIC RESONANCE IMAGING OF ENDOCRINE ORBITOPATHY}

The previously available imaging procedures - CT and MRI - have been used routinely in cases of endocrine orbitopathy (EO) to characterise morphological aspects of the retrobulbar space. These methods, however, do not provide information related to functional aspects. The capability of the somatostatin analogon Octreotide to localize activated lymphocytes in inflamatory lesions provides investigators with a new tool for this task. The aim of this study was to compare the clinical findings with both MRI and Octreoscan in cases of EO

A series of 11 patients with Graves' disease were included in the study. The ophthalmological score was determined according to Mourits' scale. Both eye muscle thickening and eye muscle oedema were taken as parameters of the MRI investigation. For the Octreoscan studies $111 \mathrm{MBq} 111$-In-Octreotide were injected i.v., planar and SPECT images were taken 2 hours p.i. A study was called positive if tracer accumulation was documented in at least two corresponding slices after SPECT reconstruction of the orbital region

A higher rate of positive findings was seen with the Octreoscan $(88 \%)$. On the other hand, muscle thickening was apparent in $66 \%$ and oedema only in $55 \%$ of the cases. The opthalmological score correlated well with the Octreoscan results.

In this preliminary evaluation, retroorbital changes seem to be more apparent in the Octreoscan as compared to MRI imaging. The underlying pathogenetical mechanism reason for this could be the presence of activated lymphocytes. 
Sunday, 21 August 1994

121

B. Boddenberg, K. Scheidhauer, M. Dietlein, P. Theissen, H. Schicha

Department of Nuclear Medicine, University of Cologne, Germany.

\section{TC-99M-HIG IMAGING IN ENDOCRINE OPHTHALMOPATHY}

Endocrine ophthalmopathy (e.O.) occurs in the course of Grave's disease with signs of inflammation such as periorbital edema and cellular infiltration of retroorbital tissue. Diagnosis is based on clinical and special ophthalmological examinations beside imaging of morphologic changes (CT, US, MR). Activity of the autoimmune process is mostly judged by clinical signs. Aim of this study was to evaluate scintigraphy with human immunoglobulin (HIG) for detection and determination of inflammatory activity of e. $\mathrm{O}$.

8 patients with e.O. and severe clinical symptoms (stage 3 and 4 , Werner classification) were examined. All patients underwent MR $(n=7)$ or CT $(n=1)$ imaging at the same time: $7 / 8$ patients showed typical findings of e.O. 6 patients were without specific therapy at the time of scintigraphy (stopped at least 4 weeks before examination $(n=3)$, or not yet begun $(n=3)) .2 / 8$ patients received low dose steroid therapy. 4 and 24 hours after i.v. application of $555 \mathrm{MBq} \mathrm{Tc}-99 \mathrm{~m}-\mathrm{HIG}$, planar scintigraphy of the head and tomography (triple head gamma camera, LEHR collimators) were performed: None of the 8 patients showed activity accumulation in the region of the orbits, neither in planar nor in SPECT technique.

Conclusion: Tc-99m-HIG scintigraphy does not seem to be a reliable marker in the diagnostis of e.O., neither in primary nor in diagnostic follow up.

\section{2}

P. Theissen, D. Moka, B. Müller-Hübenthal, U. Sechtem, K. Scheidhauer, E. Voth, H. Schicha;

Klinik und Poliklinik für Nuklearmedizin, Klinik III für Innere Medizin, Universität zu Köln, Köln, Germany

INFLUENCE OF HYPOTHYROIDISIM ON MYOCARDIAL ENERGY METABOLISM ? - A PHOSPHOROUS NUCLEAR MAGNETIC RESONANCE SPECTROSCOPY STUDY

Not only the skeletal muscle but also the myocardium is one of the target organs of thyroid hormones. In 15 hypothyroid (TSH $\geq 30 \mu \mathrm{IE} / \mathrm{mI}$ ) patients with thyroid carcinoma after thyreoidectomy and radio iodine therapie, additionally phosphorous31 nuclear magnetic resonance spectroscopy (P-MRS) was performed before iodine131-whole-body-scintigraphy. It was assessed, whether alterations of the myocardial phosphate metabolism occur with hypothyroidism similar as in skeletal muscle. For comparison it was proved, whether left ventricular function of these patients is decreased at rest. In patients and 15 healthy volunteers P-MRS (volume selection method ISIS at the anterior, lateral, and septal left ventricular wall; EKG-triggering, repetition time $=3 \mathrm{~s}$; average of 512 endsystolic measurements) and magnetic resonance imaging (dynamic mid-ventricular transverse and sagittal oblique gradientecho (GE) scans) were applied. The ratio of the peak-areas of phosphocreatine ( $\mathrm{PC} r$ ) and B-ATP has been designed as parameter for the status of the myocardial phosphate metabolism. Values were corrected for partial saturation and contamination with blood. Left ventricular function has been evaluated quantitatively measuring the ejection fraction (EF) by the area length method on the GE-scans. In comparison to the normal subjects with a PCr/B-ATP-ratio of $1.79 \pm 0.21$, this ratio and, therefore, first of all the $\mathrm{PCr}$ of the patients with $1.23 \pm 0.32$ was significantly decreased $(p=0.005)$. Besides the decrease of $\mathrm{PCr}$, only 5 of the 15 patients showed a diminished left ventricle function at rest with $E F$ values below 50 $\%$. Left ventricular dilatation or myocardial thinning was not observed.

This results emphasize that hypothyroidism affects the phosphate metabolism of the myocardium. The drop of $\mathrm{PCr}$ was unexpectedly marked. By this means, at least at rest no high grade reduction of left ventricular function was caused. Presently, the consistency of the existing results is proved by enlargment of the patient group and control examinations at euthyroidism.

\section{3}

D. Francois, J. de Ville de Goyet and S. Pauwels. Depts. of Nuclear Medicine and Surgery, University of Louvain Medical School, Brussels, Belgium.

VISUAL AND QUANTITATTVE ANALYSIS OF HEPATOBLLIARY SCINTIGRAPHY IN THE DETECTION OF BILIARY STENOSIS IN LIVER-TRANSPLANTED CHLDREN.

Development of cholestasis after orthotopic liver transplantation (OLT) in children may be related to various causes, i.e. graft rejection, hepatitis, or segmental biliary stenosis (BS). This latter complication may be suspected by ultrasound (US) when bile ducts are dilated. Since functional biliary alterations may precede morphological alterations, we have now evaluated the usefuiness of hepatobiliary scintigraphy in the early detection of BS when US is still normal. We have reviewed 131 consecutive scans performed in 85 post-OLT children with cholestasis and normal US, including 14 patients (18 studies) with proven BS and 71 patients (113 studies) without stenosis (NBS). Bile ducts activity was visually graded G1 (no or faint bile ducts visualization during less than $60 \mathrm{~min}$ ) or $\mathrm{G} 2$ (bile ducts visualization exceeding $60 \mathrm{~min}$ with or without tracer pooling). Hepatic and bile ducts time/activity curves were generated in order to obtain quantitative parameters of biliary activity and clearance.

The diagnosis of BS was confirmed in 4 of the $108 \mathrm{Gl}$ studies and in 14 of the 23 G2 studies, indicating for the visual method a sensitivity of $78 \%$, a specificity of $96 \%$, a negative predictive value of $96 \%$ and a positive predictive value of $61 \%$.

Although the mean quantitative parameters were different in patients with and without biliary stenosis and correlated with visual grading, all values observed in BS were in the range of those observed in NBS. Conclusion: 1. visual grading of the biliary activity is effective in the early detection of biliary stenosis in post-OLT children, 2. quantitative data analysis does not improve the accuracy of the method.

\section{4}

M.R.Castellani, C.Colombo ${ }^{\circ}$, A.Chiti, M.G.Apostolo ${ }^{\circ}$, L.Maffioli, M.T.Marzano ${ }^{\circ}$, E.Seregni, G.L.Buraggi.

Nuclear Medicine Division, National Cancer Institute - Milano and $\left({ }^{\circ}\right)$ University of Milano. Italy.

\section{Assessment of hepatobiliary function in patients with Cystic Fibrosis-Associated liver disease.}

Chronic liver disease is a main complication of Cystic Fibrosis, and it has been recognized as a risk factor for early death. Aim of the present study is to evaluate the hepatobiliary function by the aid of trimethyl-Br-IDA-99m-Tc (TMBIDA) scan, in 48 children with Cystic Fibrosis and clinical or US signs of known or suspected associated liver disease. Four groups of pts were evaluable: 20 with liver cirrhosis (LC), 13 with not cirrhotic-liver involvement, 15 with no liver involvement ( 8 with biliary sludge and 7 with meconium ileus). In $29 \mathrm{pts}$, the scan was repeated 11-36 months after therapy with Ursodeoxicholic acid (UDCA), with a total of 87 scintigraphy. Morphological abnormalities, hepatic extraction fraction (HEF) and time of visualization of intestine (TI) were evaluated after i.v. inj. of 111-185 MBq of TMBIDA. Parenchymal abnormalities and bile ducts dilatation were observed in $27 / 48$ pts $(56 \%)$ with a slight difference when comparing LC vs the other groups ( $70 \%$ vs $46 \%$ ). HEF and TI were slightly prolonged in pts with LC (30.2 and $28.3 \mathrm{~min}$, respectively) and in pts with elevation of liver function tests (28.8 and $28 \mathrm{~min}$ ), in comparison with all the other pts, irrespectively to the clinical group. Both morphological and functional parameters improved, according with the clinical status, after UDCA therapy in all but 2 LC pts. In conclusion our data demonstrated that scintigraphy well correlates with clinical status and it is a good parameter of treatment responsivity. Moreover it allows to identify liver abnormalities in $2 / 3$ of pts without signs of liver disease. 
125

P. Lass, A. Szarszewski, G. Romanowicz, K. Mizan J.M. Slominiski

University Medical School, Gdańsk, Poland

BILIARY SCINTIGRAPHY IN THE INVESTIGATION OF DUODENOGASTRIC REFLUX IN CHILDREN

Gastric reflux of bile is an important pathogenetic factor of upper digestive tract diseases, including bilary gastritis. The diagnosis is usually based upon endoscopic examination, but the incidence of falsepositive results is high. The papers on gastris bile reflux in adults were published last years, but we are not aware of similar papers in children.

Cholescintigraphy was performed in 32 children with suspected biliary gastritis, aged 4 - 14 years, mean $9.7+/-3.8$ years, 26 girls, 8 boys. In 8 patients the control scanning was performed after the full course of therapy. The dominant symptom was the abdominal pain lasting 3 weeks to 1 year. In all of them endoscopy confirmed gastritis II - IV ${ }^{\circ}$ Sydney scale, together with presence of bile in the stomach. Cholescintigraphy was performed after the administration of mean $5 \mathrm{mC}$ (185 MBa) of Tc- 99m- HEPIDA in upright position with scans performed every $5 \mathrm{~min}$. Patient sat between the examinations.

The biliary reflux was seen in 14 children (44 $z$ ), the results were vague in $6(18 \%)$, the test was negative in $12(38 x)$. Reflux index was $3.4+/-1.1 \%$. Contro 1 scintigraphy was performed in 8 children after the treatment with cisapride and revealed the withdrawal of the reflux in 7 , with persistence in 1 . This was in accordance with clinical symptoms.

Those results are in accordance with the results obtained in similar studies in adults. The authors believe that cholescintigraphy is superior to endoscopy in diagnosis and establishing of therapy of biliary gastritis in children.

126

Charron, M., Fisher, N.A., Kokochis, S. Neigut,

Children's Hospital of Pittsburgh, University of Pittsburgh Medical Center, Pittsburgh, PA 15213

DETECTION OF PROTEIN LOSING ENTEROPATHY WITH TC $99 \mathrm{~m}$ ALBUMIN

Purpose: Hypoproteinemia is a pathological process with many possible etiologies. Occasionally, the source of protein loss is difficult to pinpoint. We have evaluated the applicability of $99 \mathrm{~m}$ Tc-albumin scintigraphy for the detection of gastrointestinal protein loss.

Materials and Methods: Eleven children, five females and six males (ages eight months to 19 years) were and six males (ages eight months to 19 years) were scanned dynamically for one hour post injection. static images were obtained at 2,4 , and 6 hours. All patients had documented total serum proteins and albumins significantly below normal. Most patients had fecal 1-anti-trypsin performed for correlation.

Results: Three liver disease patients with a low likelihood of GI protein loss were normal (controls) as demonstrated by no ${ }^{99}$ Tc-HSA activity within any portion of the bowel. Eight patients demonstrated 99m TC-HSA activity within the bowel and the site of leakage could be determined to assist surgical leakage could be determined to assist surgical planning. In these patients, bowel cort

Conclusion: We believe this is the largest series to date demonstrating that the use of 99 arC-Albumin in detection of GI protein loss offers the following advantages: 1) High sensitivity; 2) Ability to localize accurately the site of leakage, and therefore be useful in surgically correctable disease; 3 ) Reasonable cost.
127

J Farahati. SP Müller, B Kremens, W Sonnenschein, HH Coenen, Chr Reiners.

Nuclear Medicine and Pediatric Oncology, University Hospital Essen, FR Germany.

SCINTIGRAPHY OF NEUROBLASTOMA WITH I-123-MIBG.

For 10 years MIBG has been used successfully in the diagnosis and staging of children with neuroblastoma. Because I-131-MIBG allows delayed imaging, it is reported to have a higher sensitivity than I-123MIBG. To study the sensitivity of I-123-MIBG we evaluated all MIBG scans performed between 1984 and 1992 in 91 children suspicious for neuroblastoma using I-123-MIBG exclusively.

50 males and 41 females were studied. The age of the children varied between 4 weeks and 18 years (average age: 33 months). The results of MIBG scans were compared to those of other imaging techniques (ultrasound, X-ray, MRI, CT, bone scan), of catechol amine levels in urine and plasma, of Neuron Specific Enolase (NSE) levels in plasma, and of bone marrow histology/cytology. Neuroblastoma was confirmed histologically in $81 / 91$ children and excluded in 10 children. The primary tumor was localized in the abdomen in 60 , in the chest in 18 , in the neck in 2 , and in one case in the pelvis. 48 of the children were in stage IV, 19 stage III, 9 stage I or II, and 5 were in stage IVS. 55 out of 81 children had metastases: 12 bone marrow, 4 bone 25 both bone marrow and bone involve ment. 26 children had soft tissue metas tases ( 8 liver, 4 intracerebral, 2 scrotal and 12 lymph node)

Comparing the MIBG results with those of all other methods combined, primary tumors were true positive (TP) in 76 cases, in 10 cases true negative (TN), and in 5 cases false negative (FN). Bone marrow involvement or bone metastases could be diagnosed in 40/41 cases; $50 / 50$ studies were TN. Soft tissue metastases were TP in 22 cases, and $\mathrm{FN}$ in 4 cases ( 2 liver and 2 lymph node metastases). No false positive I-123-MIBG scan was observed in the entire study.

With a sensitivity of $94 \%$ for primary tumor, $98 \%$ for bone/bone marrow involvement, and $85 \%$ for soft tissue metastases without false positives I-123-MIBG results compares favorably with published data for I-131-MIBG. Despite the shorter imaging sequence I-123-MIBG seems to be an equally reliable tool in diagnosis and staging of children with neuroblastoma.

128

L. Diez, M. Mitjavila, C.Lancha, MD. Rueda, ME. Rioja, A. Cr espo.

Nuclear Medic in e D epartme nt. Hospital Ramón y Cajal. Madrid. España.

SHORT AND LONG TERM RESULTS OF ${ }^{131}$ I-MIBG THERAPY IN ADVANCED NEUROBLASTOMA (NBL).

13 children with advanced NBL received ${ }^{131}$ I-MIBG as therapy, They were either refractary to conventional therapy or showed disease relapse after initial successful treatment in one patient the MIBG was given as a consolidation therapy. 11 children were on stage IV and 2 on stage III. Chosen criteria were: positive ${ }^{123} \mathrm{~T}-\mathrm{MIBG}$ in all tumoral lesions and no bone marrow involvement. Many of the patients had received previous intensive therapy, so depression of pre-treatment blood cell count has been in cases a limiting factor for treatment. A total of $59 \mathrm{MrBC}$ courses were administered by infusion. In most children the dose was divided into two portions each infused over a period of 4 hours with a $24 \mathrm{hr}$, interval between them. Courses were repeated up to 4 times and the raximum activity given to one patient has been $22.200 \mathrm{MBq}$. Main toxic efect was thrombocytopenia. Antitumoral effects were evaluated: there was 1 complete remission; 4 minor or subjective response and 8 no response (no changes or disease progression). Children suffering from bone pain became free of complaints during the first three days after treatment. These data suggest that greater effort should be placed to identify the conditions in which NBE may have a real benefit from MIBG therapy in comparision with more standardized treatment schedules. 
Monday, 22 August 1994

129

E. Voth, F. Baer*, P. Theissen, C.A. Schneider*, U. Sechtem*, H. Schicha

Depts. of Nuclear Medicine and Internal Medicine III (Cardiology)*, University of Cologne, Cologne, Germany

DOBUTAMINE STRESS TESTING: ASSESSMENT OF PERFUSION AND WALL MOTION ABNORMALITIES BY MIBISPECT AND MAGNETIC RESONANCE IMAGING

In order to evaluate the significance of dobutamine stress testing, gradient echo magnetic resonance imaging (MRI) and $99 \mathrm{~m}-\mathrm{TC}$ methoxy-isobutyl-isonitrile (MIBI) SPECT were performed in 32 patients at rest and during dobutamine stress. All patients had at least one coronary artery stenosis of $\geq 50 \%$. Dobutamine stress test $(5,10,15,20 \mu \mathrm{g} / \mathrm{kg} / \mathrm{min}$.) was performed during MRI image acquisition with simultaneous MIBI-injection at the maximum dobutamine dose.

Segmental MIBI-uptake and wall motion were evaluated quantitatively on corresponding short axis and transverse tomograms. MIBISPECT and MRI were considered pathologic if dobutamine induced perfusion or wall motion abnormalities occurred in at least two neighbouring segments. For comparison with coronary angiography segmental MIBI-SPECT and MRI findings were compared to the respective coronary artery perfusion territories.

Dobutamine induced perfusion and wall motion abnormalities were detected in $28 / 32(87 \%)$ and $27 / 32(84 \%)$ patients, respectively. Concerning localisation of individual coronary artery stenosis the following values were obtained:

sensitivity:

dobutamine MIBI-SPECT $70 \%$

dobutamine MR

LAD

$\operatorname{RCX}$

RCA

specificity:

dobutamine MIBI-SPECT $100 \% \quad 100 \% \quad 69 \%$

$100 \%$

Dobutamine MIBI-SPECT and dobutamine MRI are qualified techniques for the diagnosis and localisation of coronary artery stenoses. Both techniques agree in the registration of a dobutamine induced ischemic response in coronary artery disease.

130

E. Inglese , G. Bisi, M. Brambilla, A. Migliorato, G.L. Tarolo. on behalf of the SIRT Lnvestigators

Dept. of Nuclear Medicine, Ospedale Maggiore Novara, Multicentre Study Central Office, Novara, Italy.

A MULTICENTRE STUDY (SIRT*) ON THALLIUM-201. REINJECTION AFTER PLANAR STRESS - REDISTRIBUTION" IMAGING: SEGMENTAL ANALYSIS.

To estimate the disagreement between stress (ST)-redistribution (RD) and reinjection (RI) imaging, 12 experienced Nucl. Med. Depts. enrolled 402 patients with at least one ST perfusion defect. To eliminate external sources of variability the same type of gamma camera, acquisition protocol and computer software was used for three views planar imaging of a conventional 3-4 hour ST/RD study. A new set of images was acquired $30 \mathrm{~min}$ after Tl-201 RI performed in the same day (Group A, $\mathrm{n}=230$ ) or in a different day (Group B, $n=172$ ) from to the ST/RD study. Four hour delayed imaging after RI was repeated in Group B only. The left ventricle was divided into 13 segments and blindly evaluated, in a core center, by 3 independent observers, according to a 5-point scale (0) normal, $4=$ no uptake). Reversibility was defined as a shift towards normal of $\geq 1$ grade on subsequent images. Of the 5195 evaluable segments, $2058(40 \%)$ had ST perfusion defects, of which $566(27 \%)$ were severe irreversible defects (SID: score 3-4). After RD, 1313 (64\%) defects remained totally irreversible (TID) and 415 of them (32\%) were SID. After RI the number of TID decreased to $1033(50 \%), 341(33 \%)$ of which were SID. When comparing RI with RD imaging $403(31 \%)$ out of the 1313 TID and 105 (25\%) out of the 415 SID at RD improved after Tl-201 RI. The change in segmental uptake was of only one degree on the scoring scale in $91 \%$ of segments evaluated after RI. In Group B the agreement in the segmental scoring of Tl-201 perfusion defects, at early and delayed imaging after RI, was excellent $(\mathrm{Kw}=0.947)$.

In conclusion the improvment in Tl-201 activity after RI of apparently irreversible defects after conventional ST/RD study is reproducible also when a strictly controlled methodology of image acquisition and analysis is adopted. In a large and unselected population of ischemic pts, the segmental prevalence of this phenomenon is less than previously reported. No significant differences were found when comparing early and delayed imaging after RI

* SIRT: Italian Multicentre Study on Thallium Reinjection.

131

H.M.L. Jansen, E.S. Tan, P.K. Blanksma, J. Pruim, A.M.J. Paans, W. Vaalburg, K.I. Lie, J. Korf.

Dept. of Cardiology and PET Center, Univ. Hosp. Groningen, Netherlands.

MYOCARDIUM AT RISK IMAGED WITH COBALT-55 POSITRON EMISSION TOKOGRAPHY.

After reperfusion of severely ischemic myocardium, prolonged myocardial dyafunction may occur in the salvaged area ("stunning"). This is thought to be caused by calcium (Ca) overload. Co-55 can be used to image Calcium. Co-55 has already been used to visualize ischemic brain damage. To investigate the possibility of Co-55 as a Ca tracer and therefore image myocardium at risk, $2 \mathrm{mCi}$ Co-55 was administered $i . v$. to 2 healthy volunteers and 2 patients with an evolving acute myocardial infarction before reperfusion with balloon dilatation. Co-55 PET was performed ap proximately 24 hours post injection. In healthy volunteers proximately 24 hours post injection. In hea.

The patients clearly show highly selective uptake of co-55 in the ischemic border region. This indicates Ca overload due to reperfusion in these regions, demarcating the area of stunned myocardium.

In conclusion, Co-55 PET visualizing stunned myocardial tissue after reperfusion, may become a promising new method for early detection of ischemic and salvaged myocardium and for monitoring the effect of therapeutic interventions (Ca-antagonist) in these situations. Besides being a relatively inexpensive PET radiopharmaceutical, imaging with co-55 can be delayed 24 hours after the acute ischemic event, making it a convenient tool for aiding patient ment, determining early prognosis and therapy evaluation.

132

C. De Sadeleer, M. Rosseel, P. Dendale, A. Bossuyt, P.R. Franken.

Departments of Nuclear Medicine and Cardiology,

Free University of Brussels (AZ VUB), Brussels, Belgium.

CORONARY COLLATERALS : INFLUENCE OF CORONARY STEAL EFFECT ON THE DETECTION OF CORONARY ARTERY DISEASE BY Tc-99m SESTAMIBI DIPYRIDAMOLE STRESS IMAGING.

The protective role of collateral blood flow on myocardium sustended by stenosed coronary arteries has long been recognized. Its role on the diagnostic performance of dipyridamole stress test has not been established. 26 patients with well-developed intercoronary collaterals and 12 control pts without collaterals were studied at rest and after dipyridamole infusion $(0.84 \mathrm{mg} / \mathrm{kg})$. The regional myocardial activity of SESTAMIBI was measured with SPECT using a 5-point scoring system and 13 segments. The severity of coronary stenoses (Gensini score) was similar in both groups: $12.7 \pm 7.1$ vs $11.3 \pm 6.9(p=N S)$.

Clinical and ECG evidence of acute myocardial ischemia were noted during the test in 15 pts with collaterals and in 2 pts without collaterals $(p=0.004)$. Dipyridamole-induced perfusion changes were observed in 24 pts: 9/20 pts (45\%) with ECG Q-wave vs $15 / 18$ pts ( $83 \%$ ) without ECG $Q$-wave $(p=0.02)$. There was a significant association between perfusion changes and the presence of collaterals in pts without ECG Q-wave: 13/13 pts with collaterals vs $2 / 5$ pts without collaterals showed dipyridamoleinduced changes $(p=0.01)$. Of the 76 diseased vessels ( $>70 \%$ luminal narrowing), 64 vessels supplied territories containing at least $75 \%$ of myocardium with residual SESTAMIBI activity at rest. Dipyridamoleinduced perfusion changes were observed in $40 / 64$ territories $(63 \%)$. A significant association was found between perfusion changes and the presence of collaterals: $18 / 21$ vessels with collaterals $(86 \%)$ vs $22 / 43$ vessels without collaterals $(51 \%)$ showed perfusion changes $(p=0.006)$ The mean difference between stress and rest perfusion scores was higher for collateralized vessels than for non-collateralized vessels ( $3.6 \pm 3.4$ vs $2.0 \pm 3.1 ; \mathrm{p}=0.065$ )

These findings suggest (1) that collateral vessels supplying stenotic coronary arteries are associated with ischemia observed in some patients in response to coronary arteriolar vasodilatation (coronary steal effect);

(2) that collateral vessels play an important role in the diagnostic performance of dipyridamole stress test in patients without ECG Q-wave. Dipyridamole-induced perfusion changes were observed in all pts with angiographic collaterals but in only $40 \%$ of pts with similar CAD severity but without collaterals. 
133

P. Flamen, C. De Sadeleer, M. Rosseel, A. Bossuyt, PR. Franken. Departments of Nuclear Medicine and Cardiology, Free University of Brussels (AZ VUB), Brussels, Belgium.

COMPARISON BETWEEN TC99m TETROFOSMIN AND Tc99m SESTAMIBI FOR DIPYRIDAMOLE STRESS TEST USING A ONE-DAY IMAGING PROTOCOL

Tetrofosmin (Tetro) is a new $\mathrm{Tc} 99 \mathrm{~m}$ labeled myocardial perfusion agent. Biodistribution studies indicate more attractive heart-to-adjacent organ biokinetics than for Tc99m Sestamibi (Mibi) after injection during exercise. To determine whether Tetro could also be more suitable than Mibi for Dipyridamole stress test (Dipy) in a one-day imaging protocol, we compared the distribution of these 2 tracers in patients with proven or suspected coronary artery disease.

Six to $9 \mathrm{mCi}$ of Tc $99 \mathrm{~m}$ Tetro were injected at rest and $23-28 \mathrm{mCi}$ were injected 4 hours later, during Dipy infusion $(0.84 \mathrm{mg} / \mathrm{kg})$. Planar and myocardial SPECT images (MultiSPECT3, High Res collimator, 128 matrix) were obtained 60 minutes after the rest injection, and 30 min and 60 min after Dipy injection. The Mibi study was obtained using exactly the same protocol, either 48 hours before or after the Tetro study. So far, 12 patients have completed the study.

High quality SPECT images with good myocardial delineation and adequate contrast between the heart and background were obtained with the 2 tracers, as well after rest as after Dipy injection. Mean \pm sd myocardial count rate were $5.4 \pm 1.9$ and $5.8 \pm 1.8$ counts $/ \mathrm{pixel} / \mathrm{min} / \mathrm{mCi}$ for Tetro and for Mibi, respectively ( $\mathrm{p}=\mathrm{NS}$ ). Tetro and Mibi heart/ lung ratios averaged $3.1 \pm 0.7$ and $3.2 \pm 0.5$ after rest injection $(p=N S)$ and $3.5 \pm 0.6$ and $3.6 \pm 0.5$ after Dipy injection $(\mathrm{p}=\mathrm{NS})$. The heart/ liver ratio was similar for both tracers 60 minutes after rest injection $(1.4 \pm 0.4$ versus $1.2 \pm 0.6 ; \mathrm{p}=\mathrm{NS}$ ). The Tetro heart/ liver ratio was $0.78 \pm 0.09$ at 30 min after Dipy injection and increased to $1.6 \pm 0.2$ at 60 minutes. At this time, the Tetro heart/ liver ratio was higher than the Mibi heart/ liver ratio $(1.2 \pm 0.5 ; p<0.01)$.

We conclude that Tetrofosmin offers modest biokinetic advantages in comparison with Mibi for Dipyridamole stress test performed with a one-day imaging protocol.

\section{4}

D. Vivegnis, P. Rigo, Th. Benoit, A. Lahiri, R. Itti, S. Braat.

Nuclear Medicine Division, C.H.U. LIEGE, BELGIUM.

\section{IMPROVED MYOCARDIAL IMAGING USING SPECT AND TECHNETIUM-99m TETROFOSMIN.}

Tetrofosmin is a new technetium labelled lipophilic cation evaluated for use as a myocardial perfusion tracer.

Same day stress/rest planar and tomographic images obtained as part of the phase III clinical trial were compared to planar thallium and to angiography in 72 patients with definite or suspected coronary artery disease. Studies were processed on a common computer and read separately by four investigators unaware of patients identification. 42 patients had previous and 30 no previous myocardial infarction. 38 patients had multiple vessel disease (lesions $\geq 50 \%$ ) and a total of 111 arteries had stenosis. Spect tetrofosmin was normal in 8 of the 9 patients without significant coronary lesion, thallium planar and tetrofosmin planar images were normal in 7 of these 9 patients. 12 of 13 patients with previous myocardial infarction and single (10) or no (3) residual vessel lesion had tetrofosmin Spect abnormalities versus 9 with planar thallium and 8 with planar tetrofosmin. 26 of 29 patients with multiple vessel disease and previous myocardial infarction had fixed defects (11 patients) or partially reversible defects (15 patients) as compared to 9 and 13 respectively with planar tetrofosmin and to 7 and 16 respectively with planar thallium. Finally Spect tetrofosmin demonstrated stress abnormalities in 17 of 21 patients without myocardial infarction as compared with 14 using planar tetrofosmin and 13 using planar thallium. Overall, Spect tetrofosmin recognized disease in 69 arterial territories versus 59 for planar tetrofosmin and 58 for thallium.

We conclude that the use of Spect plays a major role in the improved sensitivity and specificity demonstrable using $\mathrm{Tc}-99 \mathrm{~m}$ tetrofosmin.
135

R.P. Baum, B.J. Krause, E. Staib-Sebler, M. Lorenz, G. Hör

Dept. of Nuclear Medicine and Dept. of General Surgery, Johann Wolfgang Goethe University Medical Center, Frankfurt/Main, Germany

FIRST CLINICAL EXPERIENCE WITH A TOTALLY HUMAN MONOCLONAL ANTIBODY (TC-99M-88BV59) FOR DETECTION OF COLORECTAL CANCER

Immunoscintigraphy using TC-99m labelled monoclonal antibodies (Mab) today represents a widely used method in the detection of tumors. However, induction of HAMA after multiple administrations or even after a single injection limits the usefulness of this approach in the follow-up of cancer patients.

This study presents the first immunoscintigraphic results after the injection of the totally human Mab Tc-99m-88BV59 (OncoSpect ${ }^{\top M}$, AZKO-Organon Teknika bv, Turnhout, Belgium) in patients with a history of colorectal cancer.

Mab 88BV59 (IgG Kappa 3 subclass, $170 \mathrm{KD}$ ) is a panadenocarcinoma antibody which detects a cytokeratin-like antigen and has been shown to react with $>90 \%$ of primary colorectal cancers as well as with recurrences and metastases.

ISC (8 anterior/posterior planar images as well as SPECT of the abdomen and pelvis) was performed 18 to $20 \mathrm{hrs}$ after injection of $1.1 \mathrm{GBq}(30 \mathrm{mCi})$ Tc-99m-88BV59 $(9.46 \mathrm{mg})$. The scintigraphic results were compared to CT scan and sonography; all patients subsequently underwent surgery thus allowing to evaluate the immunoscintigraphic findings histologically.

There were no adverse effects and no human anti-human (HAHA) responses. Labelling efficacy reached from 97 to $99.9 \%$. The detection rate was $2 / 2$ for primary tumors, $6 / 7$ for liver metastases, $2 / 2$ for lymph node involvement, and $2 / 2$ for recurrences. All lesions detected scintigraphically were confirmed by histology (no false positive findings).

In conclusion, this first totally human Mab proved useful in the detection of colorectal primary tumors, recurrences and lymph node metastases providing the advantage of no induction of HAMA and thus allowing repeated studies in the follow-up of patients suffering from colorectal cancer.

\section{6}

Th. Behr, W. Becker, M. Klein*, H.-J. Bair, Ch. Stühler*, J. Scheele*, F. Wolf

Departments of Nuclear Medicine and Surgery* of the Friedrich-Alexander-University of Erlangen-Nuremberg, Erlangen, FRG

INTRAINDIVIDUAL COMPARISON OF COMPLETE VERSUS FRAGMENTED ${ }^{99} \mathrm{~m}$ TC-LABELED ANTI-CEA MAbs FOR IMMUNOSCINTIGRAPHY IN COLORECTAL CANCER PATIENTS

The aim of this study was to compare diagnostic accuracy and kinetics of two $99 \mathrm{~m}$ Tc-labeled monoclonal anti-CEA antibodies (MAbs) (complete versus fragmented) in colorectal cancer patients intraindividually.

22 patients were investigated with both MAbs within one week: $740-1295 \mathrm{MBq}$ of the complete $\mathrm{IgG}_{1}$ MAb BW431/26 (1.5 $\mathrm{mg}$ of protein; Behringwerke FRG) and of the $F\left(a^{\prime}\right) 2 / F^{\prime} b^{\text {. }}$ fragment mixture F023C5 (0.35 mg; Sorin Biomedica, Saluggia, Italy) were injected intravenously. Planar and whole body scans were performed 1, 4, 24 and in special cases $48 \mathrm{~h}$ p.i., SPECT after 4 and 24 hours.

The following localizations could be visualized (F023C5 pos./BW431-pos./all lesions): Primary tumors 2/1/2, locoregional recurrences $10 / 8 / 10$, liver metastases $12 / 10 / 14$, kidney met. $0 / 1 / 1$, lung met. $0 / 1 / 1$, lymph node met. $6 / 5 / 6$, bone met. $2 / 2 / 2$, brain met. $2 / 2 / 3$, peritoneal carcinoses 4/3/4. SPECT was superior to planar scans in $35 \%$, especially in locoregional recurrence, liver, kidney and lung met. With MAb fragments, tumor detection was possible in $17 \%$ after $1 \mathrm{~h}$, in $94 \%$ after $4 \mathrm{~h}$, whereas with complete MAb, in $48 \% 24 \mathrm{~h}$ or even $48 \mathrm{~h}$ scans were necessary. The tumor/background ratio of liver metastases was significantly lower with fragments, compared to complete $\mathrm{MAb}(1.24 \pm 0.10$ vs. $1.67 \pm 0.32 ; \mathrm{p}<0.01)$.

In conclusion, with fragments (due to faster whole body clearance), tumor detection is possible with higher sensitivity earlier p.i. in comparison to whole $\mathrm{IgG}_{1}$. Therefore, for Tclabeling with its short physical half-life, fragments seem to be more suitable. The lower tumor / background ratio of liver metastases results probably from the lower antigen affinity known for fragments, despite their higher sensitivity. 
Monday, 22 August 1994

137

A. Akamune, Y. Kimura, S. Nakamura, T. Tsuda, T. Fujii, M. Kawamura, S. Tanada and K. Hamamoto.

Dept. of Radiology, Ehime University Shool of Medicine, Shigenobu-cho, Onsen-gun, Ehime, Japan

\section{SUPPRESSION OF LIVER UPTAKE OF ${ }^{11}$ In-LABELED MONO-} CLONAL ANTIBODY IN RADIOIMMUNOSCINTIGRAPHY

Significant non-specific accumulation of ${ }^{111}$ In-labeled diethylenetriaminepentaacetic acid-monoclonal antibody ( ${ }^{111}$ In-DTPA-MoAb) into normal tissues such as liver and kidney makes it difficult to detect the malignancies in the abdomen. We have so far shown that DTPA could reduce ${ }^{111}$ In accumulation to liver in animal model.

This in vitro and in vivo sudies were performed to evaluate the effect of asialoglycoprotein receptor (ASGPR) on the surface of hepatocyte on the accumulation of ${ }^{111}$ In-DTPA-MoAb to liver.

In vitro studies : The uptake of ${ }^{111}$ In-DTPA-MoAb by isolated rat hepatocytes was suppressed by adding MoAb itself, human IgG, galactosyl human serum albumin (GSA) and GSA-DTPA which were proportional to their concentrations. ${ }^{11}$ In-DTPA-MoAb uptake was much more suppressed by GSA-DTPA than by GSA alone. The hepatocyte uptake of ${ }^{125}$ I-GSA-DTPA was higher than that of ${ }^{225}$ I-GSA. $\mathrm{MoAb}$ which is glycoprotein, was considered to be uptaken in part by hepatocytes through ASGPR.

In vivo studies : Biodistribution and imaging studies with ${ }^{111}$ InDTPA-MoAb depicted that the administration of cold GSA-DTPA reduced the undesirable uptake of ${ }^{11}$ In-DTPA-MoAb by the liver and kidney both in normal and tumor-bearing mice.

These results show the usefulness of administration of cold GSADTPA in ${ }^{111}$ In-DTPA-MoAb scintigraphy.

\section{8}

$\underline{\text { R. Bares }}^{1}$, B.M. Dohmen ${ }^{1}$, J. Fass ${ }^{2}$, H.J. Kaiser ${ }^{1}$, C. Altehoefer ${ }^{1}$, P. Klever $^{2}$, V. Schumpelick ${ }^{2}$, U. Buell ${ }^{1}$

Departments of Nuclear Medicine ${ }^{1}$ and Surgery ${ }^{2}$, Technical University of Aachen, GERMANY

FDG-PET OR CEA-IMMUNOSCINTIGRAPHY FOR DETECTION OF COLORECTAL CANCER? RESULTS OF A COMPARATIVE STUDY

FDG-PET and immunoscintigraphy (IS) have both been shown to be suitable for detection of tumor lesions independent from morphological changes caused by malignant disease. Purpose of the present study was to compare the efficiency of both procedures in a group of 26 patients with a history of colorectal cancer. Static PETscans of suspected tumor areas (3-4 bed positions $=15-20 \mathrm{~cm}$ axial field of view) were obtained $45 \mathrm{~min}$. after iv injection of $150-300$ MBq FDG. Planar and SPECT-IS was performed 4 and 24 hours after iv injection of $2 \mathrm{mg}$ anti-CEA antibodies (BW 431/26) labelled with $1100 \mathrm{MBq}$ Tc-99m. Results of PET and IS were validated by surgery or follow-up ( $>6$ months). Liver metastases were confirmed in 9 patients, IS revealed "hot spots" in 6 and unspecific "cold lesions" in 3 of them. PET-scans of the liver were available for 11 patients (7 truet, 4 true-, among them 2 hemangiomas with doubtful findings of IS). All lymphnode metastases were identified by PET, 3 out of 5 by IS. Local recurrencies $(n=6)$ were correctly localized by both procedures. In 2 patients with a history of radiotherapy because of rectal cancer, PET yielded false positive findings within the portal while IS was normal.

The results indicate that PET is superior in diagnosing liver or lymphnode metastases, which might be attributed to better spatial resolution of PET-scanners and higher tumor/non-tumor ratios of FDG. IS proved to be more specific in case of previous irradiation (no false positives) and is the method of choice in case of unknown tumor location (screening by use of whole body scanning).
139

Stollfuss JC, Schönberger JA, Fries H, Beger HG, Reske SN

Department Nuclear Medicine and Department General Surgery, University of UIm, Germany

IMPROVED DIAGNOSIS OF PANCREATIC CARCINOMA WITH FDG-PET COMPARED TO CT IN NON-INVASIVE IMAGING MODALITIES

Despite the fact that $\mathrm{CT}$ is the mainstay for diagnosis and staging of pancreatic carcinoma, differential diagnosis to chronic pancreatitis may be difficult. We and others have demonstrated the utility of FDG-PET for detecting pancreatic adenocarcinoma. In this study the discriminative power of PET with FDG for differentiating pancreatic carcinoma from chronic pancreatis was compared to CT. 76 consecutive patients with a known pancreatic mass lesion had been included in the study. Histological diagnosis was obtained by either surgery or needle biopsy in all patients and used as gold standard. PET was performed using an Siemens ECAT scanner. After tranmission scanning for attenuation correction, $290 \pm 70$ $\mathrm{MBq}$ 18-F-FDG were administered and scans were started 50 min later, covering a field of view of at least $21 \mathrm{~cm}$. After iterative reconstruction, slices were reorientated in three views. Qualitatative and quatitative evaluation was performed using both standadized uptake values and tumor/non-tumor ratios. Both SUV-values $(3.2 \pm 2.1$ vs. $1.1 \pm 0.6)$ and tumor/non-tumor ratios $(7.6 \pm 4.7$ vs. $3.1 \pm 2.3)$ were significantly higher in patients with pancreatic cancer versus patients with chronic pancreatitis $(p<0.01) .43$ of 45 (96\%, sensitivity) patients with pancreatic cancer had a positive PET-scan in qualitative image analysis. 33 of 45 (72\% sensitivity) patients with proven panreatic cancer had positive (suspicious tumor mass, or indirect signs of malignancy) findings using computed tomography. False positive results in patients with chronic pancreatitis were found in 4 of $31(87 \%$, specificity) cases by PET and in 6 of 31 ( $81 \%$, specificity) cases by CT. PET with FDG had a specificity for differentiating malignant from benign pancreatic mass leasions comparable to CT. Sensitivity of FDG-PET, for detecting pancreatic adenocarcinoma however, was significantly higher than $\mathrm{CT}\left(\mathrm{p}<0.01, \mathrm{Chi}^{2}\right)$.

140

N. Leners, F. Jamar, C. Beckers and S. Pauwels

Dept. of Nuclear Medicine, University of Louvain Medical School, Brussels, Belgium.

ORGAN AND TUMOR UPTAKE OF ${ }^{n}$ IN-PENIETREOTDE IN PATIENTS WTTH GASTROENTEROPANCREATIC NEUROENDOCRINE TUMORS (GEPnt).

The biodistribution of ${ }^{111}$ In-pentetreotide $\left({ }^{111} \mathrm{In}-\mathrm{P}\right)$ was measured $i n$ vivo in 44 patients with suspicion of GEPnt. Patient preparation included administration of laxatives and withdrawal of octreotide therapy. Patients were imaged 4 and $24 \mathrm{~h}$ after injection of $\sim 200 \mathrm{MBq}$ "In-P. Organ and tumor activity was determined using the geometric mean method and expressed as \% injected dose (\%ID) or by quantitative SPECT and expressed as \% $\% / 100 \mathrm{ml}$.

The diagnosis of GEPnt was proven in 36 patients and ${ }^{111} \mathrm{In}-\mathrm{P}$ was positive in 31 cases and negative in 13. Total liver (L) and spleen (S) activity was similar at $4 \mathrm{~h}(\mathrm{~L}: 3.7 \pm 1.2, \mathrm{~S}: 2.8 \pm 1.6 \% \mathrm{ID})$ and $24 \mathrm{~h}$

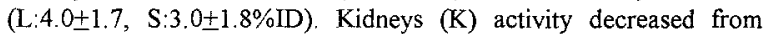
$5.9 \pm 2.5 \% \mathrm{ID}$ at $4 \mathrm{~h}$ to $5 . \mathrm{I} \pm 1.8$ at $24 \mathrm{~h}(\mathrm{p}<.001)$ and a mean decrease of $62 \%$ was measured over background areas. At $24 \mathrm{~h}$, the activity expressed as $\% \mathrm{ID} / 100 \mathrm{ml}$ was 4 -fold lower in $\mathrm{L}(0.2 \pm 0.1)$ compared to $S(0.8 \pm 0.5)$ or $K(0.8 \pm 0.4)$. No differences in $L, S$ or $K$ uptake were observed in patients with positive or negative scans. At $24 \mathrm{~h}$, the thyroid was visualized in $92 \%$ of patients $(0.02 \pm 0.02 \% \mathrm{ID})$, the pituitary in $74 \%$, gallbladder in $14 \%$ and breast in $18 \%$ of females. Bowel activity was seen in $19 \%$ of patients at $4 \mathrm{~h}$ and $91 \%$ at $24 \mathrm{~h}$. The tumor activity was similar at $4 \mathrm{~h}(0.01$ to $20 \% \mathrm{ID}$; median $0.28 ; n=50)$ and $24 \mathrm{~h}(0.02$ to $18 \% \mathrm{ID}$; median 0.31$)$. At $24 \mathrm{~h}$, the median tumor uptake was $0.81 \% \mathrm{ID} / 100 \mathrm{ml}$ with a median tumor/liver ratio of 3.8 (range: $0.8-29.3, \mathrm{n}=59$ )

Conclusions: 1) with the exception of kidneys, the tumor and organ uptake of "In-P remains stable between 4 and $24 \mathrm{~h}, 2$ ) in GEPnt patients, ${ }^{111}$ In-P provides a high target-to-nontarget ratio. 
141

K.A. Jobst, A.D. Smith, N.J. Hindley, N. Soper, M.M.Esiri, A.J.Molyneux, B.J.Shepstone, E.King, B.McDonald.

Oxford Project To Investigate Memory and Ageing (OPTIMA),

Oxford University, Radeliffe Infirmary, Oxford, OX2 6HE

TC-99M HMPAO SPET \& X-RAY CT CAN PREDICT CONFIRMED ALZHEIMER'S DISEASE UP TO 5 YEARS BEFORE DEATH AND PROVIDE CLUES ABOUT THE NATURE OF THE DISEASE.

In a prospective longitudinal study of 197 cases of dementia (mean age 73.2 range 39-95) and 114 controls (mean age 70 range 34-94) annual medial temporal lobe (MTL) oriented X-ray CT and Tc-99M HMPAO SPET scans are used to evaluate the diagnostic and prognostic potential of structural and functional neuroimaging in the differential diagnosis of dementia. Some subjects have had seven annual evaluations. So far of 90 who have died, 87 have come to post mortem (PM)(97\%). 52 had more than 1 annual scan $(1 \times 6,5 \times 4,19 \times 3$ 27x2). Histology is known for 77: 52 had AD ( $27>1$ annual scan), 17 had other dementias $(12>1$ annual scan) and 8 controls had no CNS pathology $(7$ $>1$ annual scan). The combination of MTL atrophy and parietotemporal (PT) hypoperfusion on SPET is thought to predict AD. We assessd scans for significant MTL atrophy ( $<0.79$ multiples of median for age), PT hypoperfusion, and the combination of both, at intervals before PM and up to 28/2/94 in the living controls (see table). Both criteria have been positive at least 56 months before death in confirmed $\mathrm{AD}$, are much less common in other dementias and virtually absent in controls.

months AD other dems pm controls living controls CT Spet both CT Spet both CT Spet both CT Spet both

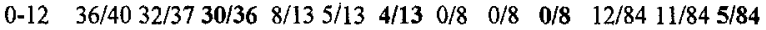

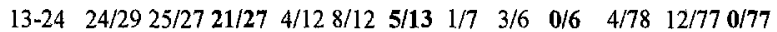

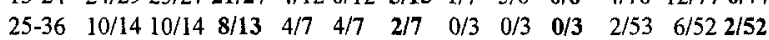
$\begin{array}{lllllllllllll}37-48 & 5 / 8 & 6 / 8 & 4 / 8 & 1 / 4 & 1 / 4 & 1 / 3 & 0 / 8 & 0 / 6 & 0 / 3 & 1 / 27 & 1 / 27 & 0 / 27\end{array}$

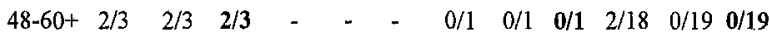
The rate of MTL atrophy in $\mathrm{AD}(1.89 \mathrm{~mm} / \mathrm{yr}, 95 \% \mathrm{CI} 1.25-2.54)$ is an order of magnitude greater than controls $(0.21 \mathrm{~mm} / \mathrm{yr}, 95 \% \mathrm{CI} 0.02-0.39)$ and correlates directly with cognitive decline. Such dramatic MTL atrophy suggests that AD is a disease rather than the inevitable consequence of ageing. These findings have important aetiologic, diagnostic and therapeutic implications.

\section{2}

EA van Royen, WA van Gool, GJM Walstra, $S$ Teunisse, $F$ van der Zandt, HC Weinstein.

Dept. of Nuclear Medicine and Neurology, Academic Medical Centre, Amsterdam, Netherlands.

\section{ROUTINE CEREBRAL BLOOD FLOW SPECT IS NOT USEFUL IN THE EVALUATION OF ELDERLY MILDLY DEMENTED} PATIENTS.

Based on the observation of bilateral temporoparietal hypoperfusion in Alzheimer's disease (AD), cerebral blood flow SPECT has been advocated as a powerful diagnostic tool in the evaluation of demented patients. We assessed if routine SPECT in elderly, mildly demented outpatients increases the a priori diagnostic sensitivity and specificity of a careful clinical and neuropsychologic examination alone.

A total of 110 consecutive patients, referred by general physicians for memory disorders, were studied. SPECT images were acquired on a multidetector brain SPECT system (SME810) after the injection of $550 \mathrm{Mbq}{ }^{99 \mathrm{~m}} \mathrm{TC}$ HMPAO. A diagnosis of probable AD (McKhann criteria) was made in 68 patients (mean age $79.3 \mathrm{yr}$ ) based on clinical examination, neuropsychologic tests (CAMDEX-N). ancillary investigations and a 6 month follow-up. Temporoparietal (TP) perfusion, quantified as the ratio of the activity in the cerebellum was significantly lower in AD patients than in non-demented age matched controls. ROC analysis revealed a cut-off level of 0.79 for the TP ratio to be most favourable. Using that value the speciffcity was $89 \%$ and the sensitivity only $43 \%$ for detecting probable AD. SPECT imaging proved to have contributed to the final diagnosis in only $8 \%$ of the demented patients investigated.

We conclude that in this large series of patients, routine cerebral blood flow SPECT does not contribute substantially to diagnostic accuracy after clinical examination using current diagnostic criteria. Clinical guidelines should be developed for the use of cerebral blood flow SPECT in this type of patients.
143

M. Huguet, A. Catafau, F. Lomeña, P.Santacruz*, R. Blesa*, R.M. Manero**, J. Peña** and J. Setoain.

Depts. of Nuclear Medicine and Neurology*, Hospital Clinic, University of Barcelona. Neuropsychology Unit**, Hospital del Mar, Barcelona. Spain.

Te-99m HMPAO BRAIN SPECT IN PROGRESSIVE APHASIA (PA)

The validity of clinical testing is not possible in all patients with PA, due to frequent difficulties in applying neuropsychometric tests. Structural neuroimaging can be normal or show typically left perisylvian atrophy in some patients. The aim of our study was to evaluate the usefulness of HMPAO-SPECT as a diagnostic tool in PA, in comparison with MRI and clinical follow-up.

HMPAO brain SPECT was performed on 11 patients $(10 \mathrm{~F}$, mean age $68 \pm 8$ yrs) with a mean of 4 yrs of language dysfunction (range 2-15 yrs). All patients underwent a MRI study. Criteria of inclusion were: 1) a history of unexplained progressive language disturbance and 2) the absence of other cognitive impairment for at least 2 yrs after the onset of aphasia. During a 8-33 months follow-up, 5 patients were re-examined by SPECT ( 4 once and 1 twice). The presence of atrophy (MRI) and hypoperfusion (SPECT) were graded as absent (a), mild (ml), moderate (md) or severe (s).

All the SPECT and MRI abnormalities were located in the left hemisphere. Perfusion defects were found in the posterior temporal region (PT) in 11/11 patients $(5 \mathrm{ml}, 3 \mathrm{md}, 3 \mathrm{~s})$, in the anterior temporal region (AT) in $10 / 11(9 \mathrm{ml}, 1 \mathrm{md})$ and in the frontal lobe $(\mathrm{F})$ in $3 / 11$ (3ml). MRI showed atrophy in the PT in $8 / 11$ patients $(6 \mathrm{ml}, 1 \mathrm{md}, 1 \mathrm{~s})$, in the AT in $6 / 11(4 \mathrm{ml}, 2 \mathrm{md})$ and in the $F$ in $2 / 11(1 \mathrm{ml}, 1 \mathrm{md})$. In agreement with clinical deterioration, follow-up SPECTs showed impairment in 5/5 patients. No relationship between perfusion defects and atrophy was found (Fisher's exact test N.S.)

Patients with PA show temporal perfusion defects, mainly in the posterior region, including Wernicke's area. HMPAO-SPECT shows a higher number of severe abnormalities than MRI, and may demonstrate functional impairment in PA patients with normal MRI.

\section{4}

L.ö. Kapucu, S. Candansayar, B. Erbaş, B.Turgut, Z . Arıkan.

Gazi Univ., and Hacettepe Univ, Depts. of Nuclear Med.and Psychiatry. Ankara/TuRkEY.

THE EFFECT OF LOW-DOSE DIAZBPAM ON TCBF IN VOLUNTEERS AT REST AND AFTER COGNITIVE ACTIVATION STUDIED WITH SPLITDOSE $99 \mathrm{mTC}$ THMPAO METHOD.

To investigate the effect of i.v. diazepam on rCBF at rest and following activation, 99mTC-HMPAO/SPECT study was performed in 12 healthy right-handed volunteers (mean age: $23 \pm 1.3$ yrs). Baseline anxiety levels were rated by

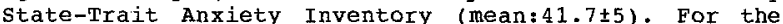
baseline SPECT study, $300 \mathrm{MBg} 99 \mathrm{mTC}-\mathrm{HMPAO}$ was injected. After i.v. administration of $0.1 \mathrm{mg} / \mathrm{kg}$ diazepam, 2 nd dose of $600 \mathrm{MBg} 99 \mathrm{mTC}-\mathrm{HMPAO}$ was given. Brief Cognitive Rating Scale was performed in 6 subjects just before the baseline injection (mean scale:57.33 \pm 0.75 ) and after i.v. diazepam before second $99 \mathrm{mTC}$-HMPAO injection (mean scale: $57.16 \pm 1.07$ ). Their results were compared to those of subjects given no activation test. The mean counts/pixel was calculated for 9 ROIs on 8 sequential trasaxial slices and for cerebellum. Left/right region, region/occipital cortex, region/cerebellum and region/hemisphere ratios were determined for each region. After diazepam, ratios of right and left prefrontal, After diazepam, ratios of right and left prefrontal, significantly. The mean left/right hemisphere ratio was decreased significantly after diazepam with activation (1.025 vs $1.002, p=0.001)$, but not in patients without activation ( 1.018 vs 1.019$)$. The mean frontal/cerebellax cortex ratio of subjects without activation was significantly higher than those of other group, either at baseline $(1.011$ vs 0.971$)$ or after diazepam $(1.013$ vs $0.944)$.

According to our results, the decrease of $\mathrm{CBF}$ in left hemisphere is more remarkable during activation, whereas CBF decreases significantly at rest in both frontal and right parietotemporal regions in comparison to other regions.99m TC-HMPAO/split-dose injection method used in this study seems to be a promising tool in the evaluation of cerebral challenge studies. 
Monday, 22 August 1994

145

R.Mielke, K.Herholz, U.Pietrzyk, A.Jacobs, K.Wienhard and W.D.Heiss

Max-Planck-Institut für Neurologische Forschung and

Klinik für Neurologie der Universität Köln, Germany

\section{METABOLIC CORRELATES OF IMPAIRED FRONTAL HMPAO UPTAKE - A PET-SPECT-STUDY}

Impairment of frontal HMPAO uptake has been described not only in many neuropsychiatric diseases such as Alzheimer's disease (AD), Pick's disease (PD), frontal lobe dementia, major psychoses and thalamic infarction but also in normal aging. We performed SPECT of Tc-99m-hexamethylpropylene amine oxime (HMPAO) and PET of F-18-fluoro-2-deoxyD-glucose (FDG) under identical resting conditions within $3 \mathrm{~h}$ in 53 cases (23 AD, 14 patients with vascular dementia (VD), $2 \mathrm{PD}, 1$ patient with minor depression and 13 normal controls). Reduced frontal HMPAO uptake relative to whole brain uptake was seen in 20 cases ( 7 AD, 6 VD, 2 PD and 5 normals). 14 of these subjects had also impairment of frontal glucose metabolism ( $4 \mathrm{AD}, 4 \mathrm{VD}, 2 \mathrm{PD}$ and 4 normals) while there was no metabolic correlate of low frontal HMPAO uptake in 3 patients with $A D, 2$ patients with VD and 1 normal control. Low frontal HMPAO uptake is an unspecific finding that is frequently seen also in aged normal individuals and is not always accompanied by corresponding reduction of frontal glucose metabolism.

\section{6}

O. MIGNECO ${ }^{*}$, J. DARCOURT*, M. BENOIT**, Ph. ROBERT** M. GRAY*, J. BENOLIEL*, G. DARCOURT**, F. BUSSIERE*. * Department of Biophysic and Nuclear Medicine, University of Nice, France. ** Department of Psychiatry, University of Nice, France.

\section{CEREBELLAR ACTIVATION DURING COGNITIVE
STIMULATIONS USING 99mTC-HMPAO SPECT.}

Cognitive activation studies concentrate on cortical activation and very little attention is paid to the cerebellum. We studied the cerebellar activation in 2 groups of subjects during 2 visuospatial tests.

Group 1 (GI): 7 patients (mean age=75.7; MMSE=17.5) fulfilling the criteria of the NINCDS-ADRDA work group for probable Alzheimer's disease (AD) and 5 elderly controls (mean age $=71 ;$ MMSE $>24$ ). Group 2 (G2): 11 non demented patients with objective memory disorders (mean age=67.1; MMSE $=27.1$ ) and 4 normal controls (mean age $=58.2 ;$ MMSE $=28.7$. Each subject underwent two SPECT studies: one during cognitive stimulation (STIM) and one during baseline conditions (BAS). For G1, STIM consisted in a visuospatial task and for G2 in a visual memorization task. During BAS, all subjects were looking at a blank sheet. For each study, $740 \mathrm{MBq}$ of $99 \mathrm{mTc}$-HMPAO were injected via an IV line during the tasks. One hour later, imaging was performed with a conventional rotating gamma camera. 128 projections of 25 seconds each were gathered over 360 degrees on $64 \times 64$ pixels matrices. The SPECT data were analyzed on reconstructed lateral views of each hemisphere using the Talairach atlas localization system with automatic ROI definition. The activation effect was measured for each ROI by the index: (STIM-BAS/BAS) $\times 100$ In normal patients, a significant cerebellar activation was observed on the right side ( $11 \%$ for $\mathrm{G} 1 ; 4.8 \%$ for $\mathrm{G} 2$ ). In $\mathrm{AD}$ patients in $\mathrm{G1}$, this activation was significantly lower $(3.2 \% ; p<0.05)$ as well as in patients with objective memory disorders in G2 $(-0.55 \% ; \mathrm{p}<0.01)$. Beside the cerebellum, in normals, right associative visual cortex was activated in $\mathrm{G} 1$ and right inferior temporal region in $\mathrm{G} 2$.

These results point out the involvment of cerebellum in cognitive tasks. They also question the use of this region as an internal reference due to its sensitivity to both conditions of injection and pathology.
147

Logemann J., Kocher F,, Grab B.M., Reske S.N.

Dept. Nuclear Medicine, University of Ulm, Ulm, Germany

THE SPECIFICITY OF BONE MARROW SCINTIGRAPHY

Immunoscintigraphy of bone marrow with Tc- $99 \mathrm{~m}$-labelled anti-NCA-95-antibodies has been used for efficient detection of skeletal metastasis in a variety of malignant tumors. Although sensitivity for detecting skeletal metastasis is regarded as high, some authors reported a very low specificity of only $11 \%$ of this procedure for detecting metastatic skeletal deposits.Therefore specificity of immunoscintigraphy was tested in 109 consecutive patients $(\mathrm{m}: \mathrm{f}=62: 47$ ), mean age 47 years $(9-91)$ between $9 / 92$ and $12 / 93$. Clinical indication for the investigation was localisation of potential inflammatory lesions in patients without indication of malignant disease After i.v. injection of 420 (380-460) MBq 99m-Tc-NCA whole body scintigraphy was peformed about 4 hours p.i.. Scans were visually assessed for presence of photopenic lesions. In 88 patients out of 109 we found a completely homogenous hematopoietic bone marrow. Additionally we found peripheral focal increased activity as a sign for inflammation in 47 patients. In 13/21 patients bone marrow defects resulted from bone marrow biopsy ( 3 pts), proven spondylodiscitis ( 3 pts.), state after surgery ( $3 \mathrm{pts}$.), acute compression fracture of a single vertebral body ( 2 pts.), total hip prothesis ( $1 \mathrm{pt}$.) and known hemangioma of vertebral body (1 pt). Among the residual 8 patients there were two patients with fever of unknown origin and spondylodiscitis was supposed but not yet proven. 6 patients showed solitary unexplained defects. These were solitary lesions in 2 patients located in lumbal vertebral body $\mathrm{V}$, in one patient in lumbal vertebral body III/IV, in 2 patients in the iliosacral joint and in one patient in pubic bone. In summary we found in only 6 of 109 patients $(5.5 \%)$ unexplained photopenic defects in bone marrow. We conclude that immunoscintigraphy of hematopoietic bone marrow shows a homogenous bone marrow scan pattern in 95 $\%$ of a medium aged patient population. These data suggest a rather high specificity of bone marrow scintigraphy used for evaluation of metastatic spread to skeleton.

148

D.Brecht-Krauss, M.Schulte*, E.Hartwig*, F.Kocher, J.Stollfuss, S.Glatz, R. Weller, S.N.Reske

Dept. Radiology III and * Surgery III

University of $\mathrm{Ulm}$, Germany

FDG-PET OF OSTEOSARCOMAS BEFORE AND AFTER NEOADJUVANT CHEMOTHERAPY: IS A NONINVASIVE ESTIMATION OF TUMOR REGRESSION POSSIBLE?

In a current prospective study we investigated the uptake of (fluorine-18) 2-deoxy-2-fluoro-D-glucose Yr, mean a 21 yr) with remale, male, range $14-45$ yr, mean age 21 yr) with high malignant osteosarcomas immediately before definitive surgical tramed again detect possible changes in glucose mettabolitment to tumor after neoadjuvant chemotherapy. Chemotherapy the performed according to COSS $86 \mathrm{c}$ protocoll biopsy 5 tumors were intially classified according to Enneking in stage IIB osteosarcoma. In 1 case. We observed a stage III lesion with pulmonary metase. We Differentiation of the tumors was osteoblastic in 3 cases, chondroblastic in 2 cases and parosteal in 1 case. The tumor response to thentiated was determined histologically within the resected specimen and classified in grade of regression according to salzer-Kuntschik. PET was performed with a Siemens ECAT-931-08-12 scanner. After acquisition of a transmission scan 200-300 MBg 18-FDG were administered intravenously. Patients were fasted for at least $8 \mathrm{hr}$ before the study. Emmission scan was started 45 minutes later. Dose/uptake ratios (DUR) and tumor/background ratios (TBR) were determined in tumor and control ROI's in the contralateral extremity. Tumorbiopsies and PET examinations were done within 3 days before and after neoadjuvant chemotherapy. ranged between 2.26 and 3.33 (mean 2.37) and TBR between 5.8 and 12.0 (mean 8.5 ). After chemotherapy DUR ranged between 0.95 and 1.07 (mean 0.98) and TBR between 2.13 and 7.79 (mean 3.39 ). 2 patients with excellent response to the therapy (regression grade 1) displayed a conspicuous decrease of TBR (12.0 to 2.13 , (regression to $2.87,68 \%$ ). 1 case of partial response (regression grade 4) showed a moderate decrease of TBR a remarkably increase of FDG-uptake in osteosarcomate Preliminary follow-up studies suke in osteosarcomas. tumoral EDG-uptake for monitaring potential of chemotherapy. 
149

C. Schiepers, P Broos, J Nuyts, L Mortelmans, A Verbruggen, M De Roo

Depts. of Nuclear Medicine, Traumatology, and Emergency Medicine, University Hospital Gasthuisberg, Leuven, Belgium

POSITRON EMISSION TOMOGRAPHY USING $18 \mathrm{~F}^{-}$- FLUORIDE FOR THE EVALUATION OF FEMORALHEAD OSTEONECROSIS.

Fluoride scintigraphy has been used in the past to evaluate skeletal flow. PET permits quantitative assessment of regional flow and tracer influx rate, and permits tomographic imaging of the skeleton. We investigated the bone blood flow (BBF) and influx rate $(K)$ in 5 patients with a remote femoral neck fracture that was treated with a high risk for developing ostenecrosis. The contralateral hip was normal. A dose of $300 \mathrm{MBq}{ }^{18} \mathrm{~F}^{-}$was administered iv and dynamic imaging performed over $60 \mathrm{~min}$. Plasma fluoride clearance was measured from arterial blood samples. With kinetic modeling diffusion rates were estimated between plasma, extra-cellular fluid and bone compartments. Net Fluoride influx $K$ was used as measure of remodeling rate. For every patient the fiow in the affected head was higher than the contralateral control. Necrosis was seen as absent fluoride uptake, accompanied by a hyperactive rim. The range of $B B F$ and $K$ values in $\mathrm{ml} / \mathrm{min} / \mathrm{ml}$ were:

$\begin{array}{lll}\text { Normal Head } & 0.03-0.06 & 0.02-0.04 \\ \text { Affected Head } & 0.05-0.12 & 0.04-0.06 \\ \text { ratio A/N } & 1.4-2.7 & 1.8-4.2\end{array}$

The bladder is hot because of Fluoride in the urine, causing streak artefacts in the images. An iterative reconstruction technique (Maximum Likelihood Estimation) was also evaluated. The image quality improved significantly with a well defined hot bladder without streaks. Surprisingly, the estimated diffusion rates were similar. Interpretation about areas with necrosis was not influenced.

Conclusions: Regional skeletal bone blood flow and influx rate can be determined in vivo with PET and Fluoride. BBF in necrotic areas was decreased. In principle, flow values can predict normal healing cq necrosis. Improved reconstruction techniques (MLE) yielded superior images but did not change parameter estimates significantly

150

M. Rajtár, T. Séra, z. Czipott*, L. Csernay A. Szent-Györgyi Medical University, Departments of Nuclear Medicine and orthopaedics* Szeged, Hungary

Planar and SPECT imaging in patients with disabled/painful knees

$P l a n a r$ and SPECT radionuclide imaging were performed in 158 patients ( 82 male, 76 female) with disabled/painful knees 3 hours after the injection of $750 \mathrm{MBq} 99 \mathrm{mTC}-\mathrm{MDP}$. The final diagnoses established by arthroscopy in all pts and also by surgery in 110 pts were: meniscal tear: 51 pts, meniscal degeneration: 33 pts, pateliofemoral osteoarthritis: 52 pts, anterior cruciate ligament tear: 23 pts, osteoarthritis: 43 pts, osteomalatia patellae: 9 pts. Planar bone scans showed no abnormality in 13 pts $(8.2 \%)$. Equivocal localization of the tracer accumulation was seen in 33 pts $(20.9 \%)$. The extent of the process was underestimated in 67 pts $(42.4 \%)$. Planar imaging provided correct diagnoses in only 45 pts $(28.5 \%)$. The SPECT images were misinterpreted in 18 pts $(11.4 \%)$, with old meniscal tears associated with cartilage damage. In 140 patients $(88.6 \%)$, the SPECT images provided a correct specific diagnosis. comparison of the abnormalities seen at surgery with those on arthroscopy and the SPECT images revealed good agreement in 60 pts $(54.5 \%)$. The SPECT images were inferior to the arthroscopy results in 16 pts with old meniscal tears, and were superior in 34 pts (30.9\%) with patellofemoral osteoarthritis $(29$ pts) or with abnormalities of the posterior compartment (5 pts). We consider that SPECT imaging should be performed not occasionally, but regularly in patients with disabled/painful knees.
151

E. Bock, M. Moritz, J. Marienhagen, E. Weise, B. Lengert, P. Held, H. Niederdellmann, Ch. Eilles

Depts. of Nuclear Medicine, Oral and Maxillofacial Surgery and Radiology, University of Regensburg, Germany

INTERNAL DERANGEMENT OF THE TEMPOROMANDIBULAR JOINT: IMAGING WITH A HIGH-RESOLUTION SPECT-SYSTEM

Internal derangement is found among the most frequent disorders of the temporomandibular joint (TMJ), characterized by a disruption of the internal aspects of the TMJ with abnormal relationship and structural alterations of the components. A classification is possible according to MRI findings. However, clinical observations not always coincide with MRI results. Aim of this prospective study was to detect osseous changes of the TMJ with high-resolution SPECT and to correlate scintigraphic results with different stages of the disease. 37 patients with suspicion of internal derangement were investigated with 555-740 MBq Tc-99m-DPD using planar and SPECT technique. SPECT was performed with a 3-headed camera equipped with an UHR collimator (Multispect 3, Siemens), matrix size 128x128. Visual and semiquantitative evaluation was carried out by two independent observers. Uptake values greater than those in the ipsilateral petrous bone were considered as pathological. MRI images were obtained in $69 \mathrm{TMJ} / 37$ patients: Stage I $(\mathrm{n}=17)$ displacement of the disc with reduction, II ( $\mathrm{n}=4)$ nonreducing disc, III $(\mathrm{n}=20)$ significant deformity of the disc, moderate osseous alterations and/or adhesions, IV $(n=4)$ progressive osteoarthrosis. All TMJ could be discriminated with high resolution by SPECT. Pathologic uptake was seen in 31/69 TMJ, all of them having positive MRI findings: $\mathrm{n}=11$ belonged to stage I, $n=2$ to stage II, $n=14$ to stage III, $n=4$ to stage IV.

The results of this study indicate a good correlation between SPECT and MRI findings in the later stages of internal derangement. False positive results have not been observed. The diagnostic benefit of SPECT seems to lie in the detection of minimal osseous changes in the early stages of the disease.

152

W.J.G. Oyen, J.A.M. Lempnens, R.A.M.J. Claessens, J.R. van Horn, T.J.J.H. Slooff, F.H.M. Corstens.

Depts of Nuclear Medicine, Radiology and Orthopedics; University Hospital Nijmegen, Nijmegen, The Netherlands.

\section{DIAGNOSING TOTAL HIP PROSTHESIS LOOSENING WITH} COMBINED NUCLEAR AND RÖNTGENOLOGIC ARTHROGRAPHY.

Röntgenologic arthrography (Rö.A.) and bone scintigraphy are diagnostic procedures used for evaluation of possible total hip prosthesis (THP) loosening. In this study, both examinations are combined and nuclear contrast imaging is added.

107 patients were i.v. injected with $600 \mathrm{MBq}$ Tc-99m-MDP. Thereafter, standard Rö.A. was performed. The radiographic contrast medium Omnipaque $300^{\circ}$ was mixed with insoluble In-111 colloid ( $5 \mathrm{MBq}$ per $20 \mathrm{ml}$ ). After completion of the Rö.A., nuclear arthrography (Nu.A.) was performed: multiple view, dual isotope images (In-111: $247 \mathrm{keV}$ peak only) were recorded. Images were interpreted by superposition of the In-111 image and the corresponding Tc-99m-MDP image, the latter serving as a landmark for the position of the THP and osseous structures. 33\% of the acetabular and $29 \%$ of the femoral components were uncemented.

\begin{tabular}{|l|c|c|c|c|c|}
\hline Component & \multirow{2}{*}{$\begin{array}{l}\text { Loosening } \\
\text { at surgery }\end{array}$} & \multicolumn{2}{|c|}{ Nu.A. } & \multicolumn{2}{c|}{ Rö.A. } \\
\cline { 2 - 6 } & + & - & + & - \\
\hline \multirow{2}{*}{ Acetabular } & + & 74 & 2 & 68 & 8 \\
\cline { 2 - 6 } & - & 16 & 15 & 21 & 10 \\
\hline \multirow{2}{*}{ Femoral } & + & 56 & 10 & 47 & 19 \\
\cline { 2 - 6 } & - & 9 & 32 & 13 & 28 \\
\hline
\end{tabular}

Filling of bursae - causing overprojection of In-111 over the joint space and occasional intraarticular In-111 resorption may interfere with image interpretation. In conclusion, Nu.A. is a sensitive technique for detection of THP loosening, offering significant added value over Rö.A. alone, especially for evaluation of the femoral component. Rö.A. remains necessary not only for adequate deposition of the contrast agents but also for detailed evaluation of osseous structures. 
Monday, 22 August 1994

153

G.Westera, A. Buck, C. Burger, K. Leenders*, P.A. Schubiger*, G.K. v.Schulthess.

Division of Nuclear Medicine, University Hospital, Zurich, * PaulScherrer-Institute, Villigen, Switzerland.

\section{IOMAZENIL: A DIRECT C-11-PET - I-123-SPECT COMPARISON}

[I-123]Iomazenil is well known as a benzodiazepine receptor tracer for SPECT in epilepsy.

We have prepared [C-11]iomazenil by methylation of demethyliomazenil with [C-11] MeI, which makes a direct PET-SPECT comparison with [123I]iomazenil possible.

Five healthy volunteers were studied. After i.v. administration ( $4 \mathrm{~min}$ infusion) dynamic PET (during $90 \mathrm{~min}$ ) and SPECT (during $270 \mathrm{~min}$ ) scintigrams were made. The plasma input functions were corrected for metabolites by chloroform/water extraction of arterial (PET) or venous (SPECT) blood samples.

The data were evaluated by fitting the tissue time activity curves to a three compartment model yielding K1 (the rate constant for blood-tobrain transport) and the distribution volume DV.

$\begin{array}{llllllll} & & \text { occ } & \text { front } & \text { cer } & \text { thal } & \text { str } & \text { pons } \\ \text { K1* } & \text { PET } & 0.42 & 0.39 & 0.36 & 0.42 & 0.32 & 0.33 \\ \text { (SD) } & & (0.14) & (15) & (0.11) & (0.19) & (0.11) & (0.11) \\ \text { K1* }^{*} & \text { SPECT } & 0.50 & 0.41 & 0.32 & 0.41 & 0.39 & 0.34 \\ \text { (SD) } & & (0.24) & (0.19) & (0.11) & (0.19) & (0.19) & (0.14) \\ \text { DV } & \text { PET } & 28 & 21 & 12 & 8 & 5 & 3 \\ \text { (SD) } & & (13) & (9) & (4) & (3) & (4) & (3) \\ \text { DV } & \text { SPECT } & 30 & 22 & 13 & 10 & 10 & 4 \\ \text { (SD) } & & (7) & (7) & (6) & (4) & (4) & (2)\end{array}$

* $\mathrm{K} 1$ in min $^{-1}$

The rate constants $\mathrm{k} 2$ - $\mathrm{k} 3$ also showed very good agreement between PET and SPECT.

Direct comparison of PET and SPECT with the same compound allows optimisation and validation of SPECT measurements. Quantification with SPECT is feasible from dynamic data.

\section{4}

M.H.Bourguignon, J. Delforge, C. Loc'h, M.A. Mazière, M.Bottlaender, A. Syrota and B. Mazière,. Service Hospitalier Frédéric Joliot, CEA, Orsay, France.

\section{SPECT IMAGES OF RECEPTOR LIGAND DISTRIBUTION MAY NOT DEMONSTRATE RECEPTOR DENSITY}

As the potential of image investigation of in vivo human biochemistry has been well validated, nuclear medicine must now turn its attention to the development of original radiopharmaceuticals with unique biochemical properties. For receptor studies in SPECT, numerous radioligands libelled with $I-123$ have been described. They are mostly antagonists and possess high affinity for binding sites. One should be careful in the interpretation of "receptor innages" since these ligands are initially distributed with flow and then may demonstrate an uptake which nay not be necessarily correlated with receptor density. For example, PET dala obtained with C-11 MQNB indicate that its cardiac uptake represents only flow at all times after injection. For C-11 Flumazenil, early cerebral cortex uptake images are mostly flow images while late uptake images are more correlated with receptor density. A precise understanding of receptor ligand uptake can be obtained with PET through dynamic acquisitions and compartmental modeling to quantify receptor binding parameters (density Bmax and affinity $\mathrm{Kd}$ ). SPECT technology does not allow so far rapid dynamic imaging of receptor ligand distribution and compartmental modeling cannot be obtained. Consequently, in SPECT, the interpretation of the distribution of a receptor ligand is not straight forward and simplifications are needed. A transfer of knowledge from PET to SPECT may be an adequate mean for establishing simple and direct evaluation of SPECT images. For example, from the modeling the striatat uplake with $\mathrm{Br}-76$ Lisuride on $\mathrm{D} 2$ receptors, one can show that, all parameters being kept constant, Bmax is well correlated with the striatum to cerebellum ratio of radioactive concentrations $S / C$. Although this conclusion cannot be directly extrapolated in patients, the ratio $\mathrm{S} / \mathrm{C}$ has been proved to be useful in clinical practice. In conclusion, the future impact of receptor ligands in SPECT depends on the selection of those radiopharmaceuticals with adequate pharnacological characteristics allowing their radionctive distribution to parallel specilic binding.
155

SP Müller ${ }^{1,2}$, MF Kijewski1 , SC Moore' ${ }^{1}$, Chr Reiners².

Harvard Medical School Boston', USA, Clinic for Nuclear Medicine Universily Essen²,FR Germany.

THE RELATIONSHIP BETWEEN PRECISION IN NONLINEAR QUANTITATION TASKS AND THE DATA VARIANCE.

The parameter variance in quantitation tasks is usually assumed proportional to the variance of the image data. While this assumption is valid for certain simple tasks, e.g., calculation of the mean activity concentration within a ROI, it may not always be valid for more realistic tasks, e.g., estimation of activity within a lesion whose size must also be estimated from the data. For these nonlinear quantitation tasks, the Cramer-Rao bound (CRB) also predicts a linear relationship between parameter variance and data variance. At low signal-to-noise ratio (SNR), however, the CRB may not be achievable. We compared quantitation performance in nonlinear quantitation tasks based on CRB calculations and Monte Cario simulation.

We modeled a spherical object embedded in a uniform background (BG), imaged by a system with a Gaussian PSF (FWHM 4 pixels). We calculated the CRB for the simultaneous estimation of the activity concentrations of the sphere and $B G$ and the sphere diameter. We determined these 3 parameters by maximum-likelihood estimation (MLE) from simulated data (adaptive Gauss-Newton algorithm), calculating the parameter variances over 1000 replications per condition. In regimes of discrepancies between CRB and MLE, the estimation was repeated with the Nelder-Mead and the simulated annealing algorithms. We varied the sphere diameter (3-32 pixel), the axial length of the data set (1-16 slices), the pixel noise (white Gaussian noise with variance 10-3-105), and the object contrast (activity concentration in the sphere 1-200, in the BG 100).

At high SNR MLE achieves the CRB; i.e., it is efficient in the statistical sense. The parameter variance is proportional to the pixel variance. At low SNR, when the coefficient of variation (CV) for the nonlinear parameter (size) is larger than $10 \%$, the variance of the ML-estimates for the linear parameters exceeds the $\mathrm{CRB}$ and is no longer proportional to the pixel variance.

At high SNR, the parameter variance for nonlinear quantitation tasks is proportional to the variance of the data; here the CRB predicts the best possible performance. At low SNR, however, the usual assumption of proportionality between parameter and data variance may break down. This implies, e.g., that averaging estimates over several low count images leads to higher quantitation errors than estimating from the sum of the images.

156

N R Williams, ${ }^{* P}$ B Iles, W H Thomson, and L K Harding

Departments of Physics and Nuclear Medicine, and *Medicine, City Hospital NHS Trust, Dudley Road, Birmingham B18 7QH, UK.

A COMPUTER MODEL TO ASSESS RADIOACTIVE GAS DISTRIBUTION WITHIN THE LUNG

A computer model has been developed to simulate the transport of short-lived radioactive gases within the lung.

The erect lung is divided into upper, middle and lower zones. Associated with each zone is an alveolar compartment and a regional deadspace compartment; the deadspace compartments are connected via a common deadspace volume. All compartment volumes are derived from Weibel's Lung Model A (1963). The alveolar compartments are trumpet shaped, corresponding to approximately the final $6 \mathrm{~mm}$ of distal airways and $90 \%$ of the total lung volume. The alveolar compartment is divided into 21 subcompartments (radial-slices). The regional specific ventilation, breathing rate and pattern, inspired gas concentration profile and radioactive half-life are taken into consideration. Gas movement in the deadspace compartments is considered to be by convection only and in the alveolar compartments by convection and diffusion.

Using the model, the feasibility of utilising radioactive gas washout data to determine regional specific ventilation or gas turnover in patient studies has been assessed. For example, regional specific ventilation can be deduced from the ${ }^{81} \mathrm{Kr}^{m}$ washout time-activity curves, with a typical error of $13 \%$ (normal young adult). Including regions of impaired function within the model gives errors of $40 \%$ In conclusion, the computer lung model is a useful tool to simulate the distribution of short-lived radioactive gases within the lung and has demonstrated that existing quantitative techniques are inaccurate when applied to patient data where pulmonary airways abnormality is present. 
157

I. van den Hoff, W. Burchert, H.-G. Wolpers*, G.-J. Meyer, H. Hundeshagen

Depts. of Nuclear Medicine, Cardiology*, Medical School

Hannover, Germany

\section{A DETAILED MODEL OF $\left[11^{11}\right.$ C]-ACETATE KINETIC IN AEROBIC TISSUE: MMPLICATIONS FOR ASSESSING MYOCARDIAL VIABILITY WITH DYNAMIC PET}

$\left[1-{ }^{11} \mathrm{C}\right]$-acetate has been recognized as a suitable tracer for assessing myocardial oxidative metabolism with dynamic PET. The close correlation between tissue clearance and oxygen consumption has been demonstrated using mono- and bi-exponential fitting as well as mean transit time approaches. A direct biochemical interpretation of the various parameters could not be given up to now.

We have developed a detailed kinetic model which incorporates the known information of the metabolic fate of acetate in myocardium in order to investigate which factors influence the actual behaviour of the tissue response. Our simulations show that the reproduction of measured impuls responses requires at least a 4-tissue-compartment model. Within our model the relation between the parameters of the usual mono- and bi-exponential fits and the rate constants of the model have been established. Model simplifications are discussed. Further, we address the problem of adequate corrections for spillover and recovery which are necessary in order to assess myocardial blood flow in addition to oxidative metabolism. We show that the formal treatment of spillover as effective fractional blood volume leads under realistic conditions implicitely to a rather good de facto recovery correction. Under these circumstances it is therefore erroneous to perform an additional recovery correction as has frequently been done in the literature.

We conclude that the simultaneous quantitative assessment of myocardial blood flow and metabolism with $\left[1-{ }^{11} \mathrm{C}\right]$-acetate is feasible with effective one- and two-tissue compartment models. Exponential fitting should generally be avoided because of the bias introduced by the effects of finite input duration and recirculation.

\section{8}

H.Herzog, E. Rota-Kops, T.Kuwert, S.Mosconi, H.W. Müller-Gärtner Institute of Medicine, Research Center Jülich and Department of Nuclear Medicine, Heinrich-Heine-University Düsseldorf, Jülich, Germany

THE IMPACT OF IMAGE RESOLUTION ON THE ACCURACY OF RATE CONSTANTS IN KINETIC MODELING

It is well known that the image resolution of reconstructed PET images influences the quantitation of the observed activity concentration. The lower the image resolution the more unterestimated is the activity uptake in small structures due to the partial volume effect. The aim of this work was to investigate the influence of reconstructed image resolution on the kinetic analysis of dynamic PET data, i.e. on the rate constants resulting from a nonlinear regression fitting analysis of the time-activity curves.

Dynamic sequences of brain images recorded for $60 \mathrm{~min}$ after intravenous injection of F-18-deoxyglucose (FDG) in 4 patients were reconstructed with a Hanning filter and 4 different cutoff-frequencies leading to an image resolution of $5.5,7,9$, and $11 \mathrm{~mm}$ (full width at half maximum, FWHM). Cortical regions of interests (ROI) were defined at an isocontour level of $50 \%$ at the maximum of the FDG-images summed from 30 to $60 \mathrm{~min}$ after injection. The ROIs became larger with decreased image resolution. Using these ROIs cortical timeactivity curves were obtained. The plasma time-activity curve was derived from arterialized venous blood. Applying the well-established three-compartment model for FDG the rate constants were determined by a nonlinear regression fitting procedure, which was based on the Marquardt-Levenberg algorithm.

For the best image resolution of $5.5 \mathrm{~mm}$ FWHM the resulting values of $\mathrm{K}_{1}, \mathrm{k}_{2}, \mathrm{k}_{3}$ and $\mathrm{k}_{4}$ were $0.121 \pm 0.011,0.223 \pm 0.076,0.116 \pm 0.026$ and $0.005 \pm 0.005$, respectively. All rate constants became higher when the image resolution was decreased. With the lowest image resolution of $11 \mathrm{~mm} \mathrm{FWHM} \mathrm{K}, \mathrm{k}_{2}, \mathrm{k}_{3}$ and $\mathrm{k}_{4}$ increased to $105 \pm 5 \%, 134 \pm 33 \%$, $113 \pm 15 \%$ and $175 \pm 86 \%$, respectively, compared to the results for 5.5 mm FWHM.

This investigation demonstrates that not only static PET data, but also dynamic ones are dependent on the image resolution. As known from animal research (Schmidt et al., JCBM, 1991), the high increase of $k_{4}$ might be caused by effects of tissue heterogeniety which become higher for lower resolution.
159

M.Possa R.Sara L.Ruffini M.Milella R.Cozzi*P.Orlandi* F.Spinelli

Nuclear Medicin Department Division of Endocrinology*

Niguarda Hospital, Milan Italy

111-In-Pentetreotide scintigraphy in pituitary adenomas. The somatostatin (SS) analog 111-In-Pentetreotide has recently allowed to disclose in vivo the presence of SS receptors on pituitary adenomas. We studied 47 patients with pituitary adenoma. Planar images of the brain were acquired after the injection of $110-200 \mathrm{MBq}$ 111-In-pentetreotide. We scored the images by an "uptake index" (UI - ratio between the adenoma radioactivity and the mean intracranial activity). In 4 patients without pituitary disease the mean score was 2 (range 1.8-2.2), GH secreting adenomas $(n=18)$ : the mean U.I. was $3.8+/-0.4$. A direct correlation $(I=0.54 ; p>0.05)$ was found between UI scores and the percentages of GH decrease after acute SS injection (100 gamma sc), calculated as mean of the first 6 hrs GH levels vs baseline. In 3 patients with NMR evidence of empty sella, the scan did not visualize any intrasellar accumulation of pentetreotide. Sllent $\mathbf{G H}$ secreting adenomas $(\mathrm{n}=3)$ : U.I. was very high $(4.8,9.6,38)$; SMS treatment markedly depressed the accumulation of the ligand in the patient with the highest score.Non functioning (NF) adenomas $(n-22)$ : mean UI was $4.1+/-0.9$. Scan was positive in $14(63 \%)$ patients. This \% of positivity is close to the frequency of SS receptors observed in these tumors in vitro. In 6 patients with positive scan, long term SMS treatment did not change the accumulation of ligand neither the size of adenoma.PRL secreting adenomas $(n=4)$ : UI was low (mean score 2.5).Concluslons: Pentetreotide scintigraphy shows the presence of SS receptors in a large portion of pituitary tumors. In acromegaly the observed correlation with GH changes after SMS indicates their functionality. In NF adenomas, the poor results so far obtained with SMS treatment on numor size even in patients with positive SS receptors, point out the lack of functional role of the receptors evidenced by scintigraphy in controlling cell proliferation.

160

H. Sinzinger, H. Kritz, Margarida Rodrigues, M. Wenger, Ingrid Blazek, Susanne Granegger Bettina Mosing, N. Leitinger, Andrea Kugi Dept. of Nuclear Medicine, University of Vienna, Austria

123I-INSULIN SCINTIGRAPHY FOR IN-VIVO IMAGING OF INSULIN RECEPTORS IN METABOLIC SYNDROME (MS) AND NIDDM

Insulin resistance is one of the earliest events in the development of syndrome $\mathrm{X}$ and NIDDM. Radioligand studies (muscle, liver) revealed a significantly different binding ( $\mathrm{Kd}, \mathrm{Bmax})$. We examined 24 patients with MS and NIDDM. In-vitro red blood cell 123Iinsulin binding and in-vivo imaging after administration of $1 \mathrm{mCi} 123 \mathrm{I}$-insulin (4 IU) were performed. Time activity curves over the liver, the kidney and a background region were monitored; liver uptake was quantified in $\frac{a}{6}$. Data from fasting and non-fasting patients were compared (intraindividually) with euglycaemic hyperinsulinemic clamping (administration of 0.1 IU cold insulin/kg/min for 90 minutes followed by $123 \mathrm{I}$-insulin bolus and continuouing glucose clamping for further 60 minutes.

123I-insulin scintigraphy is able to discover differences at the receptor level. The euglycaemic hyperinsulinemic clamping improved significantly the sensitivity of the method. with a new kit preparation (work in progress) this technique could prove very useful for diagnostic purpose and to monitor the spontaneous course of the disease as well as (therapeutic) dietary and/or drug interventions in the future. 
161

R.M.Aigner and G.F.Fueger

Karl-Franzens-University of Graz, Department of Radiology, Division of Nuclear Medicine, A-8036 Graz, Auenbruggerplatz 9, Austria

DIAGNOSTIC CRITERIA OF PARATHYROID ADENOMAS BASED ON TRACER KINETICS WITH $99 \mathrm{~m}$-TC-MIBI

In recent studies $99 \mathrm{~m}$-TC-Methoxy-Isobutyl-Isonitrile (MIBI) was said to be a most useful tracer for detecting parathyroid adenomas. Diagnostic difficulties may arise because of thy - roid adenomas. Our prospective study of 30 patients analyses initial perfusion and washout of MIBI and their possible value in differentiating thyroid from parathyroid adenomas.

We used $7 \mathrm{mCi}$ of $99 \mathrm{~m}-\mathrm{TC}-\mathrm{MIBI}$ iv. and imaged the neck from anterior (first: 16 frames of $1 \mathrm{~s}$, secondly: 16 frames of 1 min, thirdly: static images up to $3 \mathrm{hrs}$. Evaluation was based upon visual interpretation and computer analysis; the functional state of the thyroid was characterized by Iodine-123 uptake and clearance. The initial perfusion was greater in hyperfunctional thyroid adenoma and parathyroid carcinoma than in parathyroid adenomas; it was inconspicuous in normal-and hypofunctional thyroid nodules. The wash-out of MIBI from thyroid tissue was found proportional to the functional state of the thyroid ranging between 35 and $115 \mathrm{~min}$ effective half-time (mean 64 min). The wash-out of MIBI from parathyroid adenomas yielded the same mean value of $64 \mathrm{~min}$. We are led to believe that the perfusion- and wash-out analysis of MIBI in the neck aids in differentiating the functional states of the thyroid but does not obviate the need for dual isotope techniques.

\section{2}

A. Bockisch, S. Walgenbach, R. Görges, G. Meyer, C. Gregel, K. Hahn. Klinik für Nuklearmedizin, Chirurgische Klinik, Johannes Gutenberg-Universität, 55101 Mainz, Germany

EVALUATION OF SENSITIVITY AND SPECIFICITY OF PARATHYROID IMAGING WITH ${ }^{99} \mathrm{Tc}^{\mathrm{m}}$-MIBI - A KINETIC STUDY IN PARATHYROIDAL AND THYROID ADENOMAS.

In our prospective study, we investigated during the last 3 years 50 patients (pts) with suspicion of parathyroidal adenoma on a routine basis with ${ }^{99} \mathrm{Tc}^{\mathrm{m}}$-MIBI, 31 of them - mostly with symptomatic hyperparathyroidism - underwent surgery. 20 patients with established thyroidal nodules were added, 15 pts with hot and 5 with cold nodules. All pts were scanned $10 \mathrm{~min} ., 1,2$, and $4 \mathrm{~h}$ after intravenous injection of $350 \mathrm{MBq}{ }^{99} \mathrm{Tc}^{\mathrm{m}}$-MIBI and digital scintigrams were acquired. The tracer kinetics was analyzed for the site of suspected parathyroidal adenoma (showing up as hot spot or known after surgery), the thyroid, and soft tissue of the neck. The activity of suspected lesions was compared with thyroidal activity and the ratio was found to increase with time for parathyroidal adenomas. 14 of the $31 \mathrm{pts}$, for whom histology is available, were diagnosed true positive. 3 of them were ectopic, intrathoracic in 1 case, autografts in the forearm in 2 cases. 2 findings were true negative, the remainder were false negative resulting in a sensitivity of app. $50 \%$. The scintigraphic finding for the 19 pts, who were not operated and who mostly were under dialysis, was negative and the final diagnosis most likely excluded an adenoma. None of the pts with hot or cold thyroidal nodules showed any lesion, that could be misinterpreted as a parathyroidal adenoma. The sensitivity of the method was found to be moderate in our unselected patient population. However, the specificity is very high (no false positives in our study), as proven by histological comparison and in the prospective study with selected thyroidal disease.
163

\author{
Bailliez A., Nocaudie M., Huglo D., Ziegels P., \\ Proye C., Marchandise X. \\ CHU de LILLE - 59037 LILLE - FRANCE
}

PARATHYROID LESIONS DETECTED USING ${ }^{99 \mathrm{n} \text { TC-MIBI }}$ Aim. This study was designed to assess ${ }^{99 \mathrm{~m}} \mathrm{Tc}-\mathrm{MIBI}$ scan with respect to any parathyroid lesions, as they are found by surgeon.

Patients. Among 65 investigated patients by MIBI scan, 23 patients with primary hyperparathyroidism were cured by surgery and had fully documented data. 91 glands were syste. matically exhibited by surgeon : 34 were abnormal ( 2 ectopic) : 21 adenomas [180-8820 mg], 13 hyperplasias [ $47-433 \mathrm{mg}$ ]. Chief cells were involved in $\mathbf{2 4}$ glands, oxyphil cells in 10.

Methods. $550 \mathrm{MBq}$ (98m-MIBI were injected, images were performed $20 \mathrm{mn}$ and $2 \mathrm{~h}$ later; ${ }^{123} \mathrm{I}$ was then injected and thyroid scan was obtained $2 \mathrm{~h}$ later. Parathyroid sean was + if abnormal focus corresponded to surgical finding, - if not. A ROC analysis was performed to study the mass threshold. Evolution of Contrast Ratio lesion/thyroid from $20 \mathrm{mn}$ to $2 \mathrm{~h}$ (ECR) was studied in $15+$ lesions with normal thyroid.

Results. When multiple lesions, 1 at least was detected in 6 patients (over 9), but all in only 1.12 adenomas $>300 \mathrm{mg}$ (over 13), $3<300 \mathrm{mg}$ (over 8 ) and 2 hyperplasias (over 13) were seen. Locations were right and there was no FP. ROC analysis demonstrated a $280 \mathrm{mg}$ visibility threshold. Mean ECR was $\mathbf{1 . 4}$ [1.02 - 2.12], no correlated with mass, greater in ectopic lesions, but in others, ECR was significantly greater in oxyphil lesions.

\section{4}

H. Sinzinger, N. Leitinger, H. Kritz, Andrea Kugi, Susanne Granegger, Ingrid Blazek

Dept. of Nuclear Medicine, university of Vienna, Austria

111 IN-LDL-LIVER IMAGING FOR DIAGNOSIS OF HYPERCHOLESTEROLEMIA AND TREATMENT MONTTORING

Familial hypercholesterolemia is a consequence of a liver receptor defect. 111 In-IDI has been shown to have preferable binding characteristics both in-vitro and in-vivo as compared to $99 \mathrm{mTC}$ and $123 \mathrm{I}$. LDL was isolated by sequential ultracentrifugation and labeled using cyclic DTPA-anhydride. 111 In-LDL-liver imaging $(500 \mu \mathrm{Ci} ; 32$ minutes, 64 frames, matrix $128 \times 128$ ) was performed after an overnight fasting. Time-activity curves over the iiver, total iiver uptake $\left(\frac{0}{b}\right)$ and plasmatic decay were monitored and compared to in-vitro binding data ( 125 I-LDL). There was a stong correlation between in-vitro binding results and in-vitro trapping by the liver. Therapeutic interventions laiet, $n=8$, drugs [clofibrate analogues, $n=14$, HMG-COA-reductase inhibitors, $n=8$ ] and LDL-apheresis, $n=5$ ) resulted in a significant improvement in in-vitro and in-vivo LDL-binding, which significantly correlated to LDL-cholesterol lowering. 
165

B. Erbas, N. Elahi, M.K.Ahmedi, N. Atakan, A. Karaduman, Z. Koray, G. Erbengi, S. Akkaya

Hacettepe University, Ankara-Turkey.

ACUTE AND LONG-TERM EFFECT OF CYCLOSPORIN A ON RENAL FUNCTIONS AND CIRCULATING ENDOTHELIN LEVELS IN PATIENTS WITH NORMAL KIDNEY.

Nephrotoxicity is a major problem in patients treated with cyclosporin A (CyA). It is used after transplantation and for the treatment of various autoimmune disorders. To examine the short- and long-term renal effects on normal kidneys and circulating endothelin levels, we investigated renal perfusion and glomerular function after oral CyA and one month later.

Study was performed on 18 patients (mean age: $28.7 \pm 14.3$, range: $7-58$ years) with diagnosis of psoriasis or alopecia (duration: 10.2+7.9) whose renal functions were normal. Renal function of pts were assessed using 99mTc-DTPA scintigraphy. Following i.v. administration of $370 \mathrm{MBq}$ $99 \mathrm{mTc}$-DTPA, 64 serial images/per sec and 80 serial images/per $15 \mathrm{sec}$. were recorded. GFR was calculated according to the Gates' technique. Perfusion index (PI) was determined using Hilson's method. All patients received $5 \mathrm{mg} / \mathrm{kg} /$ day of CyA. Renal scintigraphy was performed at baseline, at $2 \mathrm{nd} \mathrm{hr}$ following $\mathrm{Cy} \mathrm{A}$ administration and after one month. Simultaneously blood levels of CyA (2 and $4 \mathrm{hrs}$ after CyA administration), circulating endothelin, plasma and urine beta-2 microglobulin(B-2) were determined using RIA.

The mean GFR was $81.4 \pm 16 \mathrm{~mL} / \mathrm{min}$ (baseline). Following CyA, total GFR was decreased in 12 pts acutely, whereas it was increased in 4 and remained unchanged in 2 pts. CyA levels were $400.3 \pm 330 \mathrm{ng} / \mathrm{ml}$, $399.9 \pm 222 \mathrm{ng} / \mathrm{ml}$ and $269.77 \pm 320 \mathrm{ng} / \mathrm{dl}$, respectively at $2 \mathrm{nd}, 4$ th $\mathrm{hrs}$ and one mo. later. The mean endothelin level $(10.6 \pm 7.8 \mathrm{pg} / \mathrm{ml})$ was increased acutely $(26.9 \pm 22 \mathrm{pg} / \mathrm{ml})$. B-2 levels were within normal limits. The mean percent change of GFR $(0.11 \pm 22$, range: $-38,+57,7)$ and mean percent change of $\mathrm{PI}(-4.97 \pm 36$, range: $-87.9,87.2)$ were not correlated acutely with CyA, endothelin or B-2 levels. However, \% change of endothelin at 2nd hour was significantly correlated with CyA level $(p=0.02 r=0.78)$. One mo. later, the mean GFR was changed to $74.4 \pm 21.2 \mathrm{ml} / \mathrm{min}$, insignificantly. The mean \% change of GFR $(-6.6 \pm 25.2)$ was not correlated with CyA and B-2 levels.

Our results indicated that CyA might cause an insignificant reduction of GFR in patients with normal kidneys being independently of CyA levels immediately after oral administration of drug and at long-term. Acute increase of endothelin correlated with CyA levels might be responsible for acute functional changes.

\section{6}

MaT. Bajén, J. Martin-Comin; R. Puchal, J. Mora; Y. Ricart; J.M. Griño; A.M. Castelao and A. Gonzalez

Serveis de Medicina Nuclear i Nefrologial. CsuB Hospital Princeps d'Espanya $i$ Dept. de Bioenginyeria $\mathrm{UCB}^{2}$. Barcelona. spain.

99mTC-MAG3 RENOGRAM DECONVOLUTION ANALYSIS AS A DIAGNOSTIC AID IN KIDNEY GRAFT MONITORTNG.

AID IN KIDNEY GRAFT MONITORING. The aim of the work was to study the application of MAG3 deconvolution analysis to transplant kidneys and its
usefulness in the differencial diagnosis of postrenal transplant complications.

We have studied 100 renograms from 86 transplanted patients. Mean Transit Time (MTT), T20(time at 208 of the initial height of renal retention function $\mathrm{H}_{0}$ ) and Initial Uptake (IU $=B^{\prime} .100$ ) of whole kidney have been calculated by deconvolution analysis of $99_{\mathrm{mC}-M A G 3}$ renograms.

Based on the clinical, biochemical, ultrasonography and biopsy findings, patients have been categorized into 5 biopsy findings, patients have been categorized into 5 groups: 37 functioning grafts (FG), 32 acute tubular necrosis (ATN), 21 acute rejection (AR), 6 urinary
obstructions (OBS) and 4 cyclosporine nephrotoxicity (NTX). The regults are shown in the next table (mean \pm s.d.):

\begin{tabular}{|l|c|c|c|}
\hline & TTM (s) & T20 (s) & IU \\
\hline FG & $219 \pm 63$ & $279 \pm 97$ & $2.6 \pm 0.96$ \\
\hline ATN & $322 \pm 201$ & $651 \pm 533$ & $0.6 \pm 0.39$ \\
\hline AR & $296 \pm 174$ & $581 \pm 523$ & $0.5 \pm 0.49$ \\
\hline OBS & $437 \pm 43$ & $985 \pm 186$ & $1.5 \pm 0.99$ \\
\hline NTX & $275 \pm 108$ & $493 \pm 413$ & $3.0 \pm 0.99$ \\
\hline The NTT and T20 in OBS is significantIy longer than in FG
\end{tabular}
$(\mathrm{p}<0.001)$.

The IU in ATN, AR and oBs is significantly decreased in reference to FG ( $p<0.001$ in $A I N$ and $A R ; p<0.05$ in $O B S$ ) The IU in NTX is imilar to FG and significantly different from ATN and AR. A significant correlation was found petween serum creatinine and IU values $(I=-0.74$,

to clinical evolution. In conclusion, MTT, T2O and IU are useful in evaluating kidney allograft function and may be of help in the
differential diagnosis of post-transplant renal differential
disfunction.
167

A.Biggi, A. Viglietti, M.C. Farinelli, C. Bonada, G.F. Camuzzini

Department of Nuclear Medicine, Santa Croce Hospital, Cuneo, Italy

ESTIMATION OF GLOMERULAR FILTRATION RATE (GFR) USING $99 \mathrm{mTC}$ DTPA AND $51 \mathrm{Cr}$ EDTA

The pourpose of the study were: a) to compare the clearances rate of $99 \mathrm{mTCDTPA}$ and 51CrEDTA using Sapirstein equation (Method $A$ ); b) to compare for both $99 \mathrm{mTCDTPA}$ and 51CrEDTA, the results obtained with Method $A$ with those

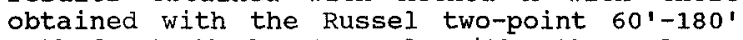
method (Method B) and with the slopeintercept method according to the one-pool model (180'-240' points) (Method $C$ ).

A dose of $100 \mathrm{uCi}$ of 51CREDTA (AMERSHAM) followed by $1 \mathrm{mci}$ of 99mTcDTPA (SORIN) was injected in 44 adults pts referred for routine determination of GFR. Measurement of the injected activity for each radionuclide was obtained by weighing technique. 8 blood samples were collected from $10^{\prime}$ to $240 \mathrm{~min}$ after injection. GFR was determined for both tracer according to Method A, B and C. Linear regression and paired $t$ test were used for comparison. The results $\mathrm{ml} / \mathrm{min}, \mathrm{M}(\mathrm{SD})$ were EDTA (A) DTPA (A) $\quad 69.0(36.8) 72.3 \quad(38.3)$ EDTA (A) VsDTPA (A) $r=0.97 ; p=0.013$ EDTA (B) EDTA(C) $69.2(34.4) \quad 73.5(42.5)$ EDTA:AvsB $r=0.98 ; p=0.96$ AvsC $r=0.99 ; p=0.0001$ DTPA (B) DTPA(C) $70.8(36.9) \quad 78.3(43.9)$

DTPA: AvsB $r=0.99 ; p=0.03 ;$ AvsC $r=0.99 ; p=0.0001$

The mean values for DTPA are higher than EDTA by $4.6 \%$. The difference is of little clinical significance. The Russel method is accurate in clinical practice for both DTPA and EDTA. systematically higher results are obtained with the slope-intercept method.

168

\author{
M.Poropat, D.Batinić, M.Bašić, D.Dodig, Lj.Nižić \\ Dep. of Nucl. Med. and Radiation Protection and \\ Dep. of Nephrology of Pediatric Clinic
}

University Hospital Center, Rebro, Zagreb, Croatia

\section{Tc-99m-DTPA RENAL SCINTIGRAPHY AND DETECTION OF INTRARENAL REFLUX}

The intrarenal reflux plays the key role in the etiology of reflux nephropathy and its detection is of utmost importance in evaluating possible damage in kidney with reflux.

In 176 kidneys (113 children) with different degree of vesicoureteric reflux (VUR), dynamic renal scintigraphy with Tc$99 \mathrm{~m}$-DTPA in zoom mode was performed. From each study 6 functional images of mean time were generated, kidney contour superimposed on each, and time activity curves (TAC) over possible areas of increased mean time were generated. In these study we analysed only areas of increased mean time over the outer contour of the kidney which corresponds to the renal parenchyma.

In later functional images of the mean time we found 53 focal retentions over the part of the kidney which corresponds to the renal cortex (33 in upper, 5 in middle and 15 in lower part of the kidney). TAC-s generated over these areas exibited a sharp increase of activity on the descending part of the curves. We propose that the return of activity from the collecting system to the kidney cortex represents intrarenal reflux.

In our opinion, analysis of functional images of the mean time could be a method for more accurate detection of intrarenal reflux and indicating the children with high risk to acquirfe renal scarring. 
169

A.Viqnati, L.Mazzucchelli, G.Lomuscio, M.E.Dottorini, I. Colombo.

Division of Nuclear Medicin - General Hospital

Busto Arsizio, Italy.

CYLINDRICAL MULTI-SLICE DIAGRAMS FROM RENAI SPECT.

Coronal, sagittal and transaxial sices (CST-slc) from SPECT TC-99m-DMSA allow a good detection of renal scarring but are not easy to read also because they are oblique as regards the longitudinal axis (LA) of the kidneys. The aims of this study were: 1) to obtain slices parallel (PA-slc) and perpendicular (PE-slc) to the LA of each kidney and a cylindrical multi-slice diagram (MSD) of each kidney; 2) to evaluate the effectiveness of both MSD and PA+PE-slc for the detection of renal scarring, in comparison with planar images (PI) and CST-Slc. 26 patients (age range 1-71 yrs) were imaged 2 hours after administration of $80-200 \mathrm{MBq}$ of Tc-99m-DMSA. Planar and SPECT studies were performed. SPECT data were processed both in the usual fashion (to obtain CST-slc) and with a new algorithm. During reconstruction, the SPECT transaxial slices of each kidney were rotated to annihilate the angle between the LA and the sagittal plane. So PA-sIC and PE-slc of each kidney were obtained. From each PE-slc a count distribution angular profile was obtained and represented as a horizontal row of pixels of a matrix (cylindrical MSD). The PI, the CST-SIC, the PA+PE-slc and the MSD of each of the 52 kidneys were analyzed separately. Upper. central or lower part of each kidney was considered as a positive zone if it contained scars. The positive zones were $52,70,81$ and 101 with the four techniques, respectively. 33 positive zones were always observed. Some more positive zones (not always the same) were reported with each technique: 19 on PI; 37 on CST-slc: 48 on PA+PE-slc and 68 on MSD. In conclusion SPECT $P A+P E-s i c$ and especially MSD were easier to read and allowed to find more renal scars than PI or CST-slc.

170

S.Mohammadtaghi, D.Bradley, W.Ussov, M.J.Myers and A.M.Peters. Hammersmith Hospital, London, UK.

\section{DYNAMIC DMSA IMAGING FOR QUANTIFICATION OF TUBULAR} FUNCTION

Whether DMSA is taken up by the tubular cell directly from peritubular capillaries or by reabsorption following filtration of the unbound fraction in plasma, the rate of uptake should reflect tubular function. Since MAG3 and hippurate are taken up even in ATN, they are unlikely to be sensitive markers of tubular injury. The aim of this study was to examine the feasibility of dynamic DMSA imaging, with measurement of renal extraction efficiency (EE) of DMSA, for the assessment of tubular injury. Renal blood now (RBF) and DMSA EE were measured using quantitative techniques previously developed for DTPA. These were respectively based on the first-pass integration technique and the Patlak plot applied up to $15 \mathrm{~min}$ after injection. Since DMSA behaves almost as a pure intravascular marker, RBF can be obtained in units of $\mathrm{ml} / \mathrm{min}$. 24 patients were studied; 14 with native kidneys and 10 with recent transplants. As expected in this heterogeneous group, RBF was highly variable, with maximal values approaching $1000 \mathrm{ml} / \mathrm{min}$ in some transplants. The Patlak plot was consistently bi-phasic, giving a higher EE from $40 \mathrm{sec}$ to $3 \mathrm{~min}$ than over 3-15 min. EE in both phases was consistently higher in native than in transplanted kidneys. In the first phase, it ranged from 1.5 to 7.3 and in the second, from 0.65 to 5.8. Five transplant recipients with biopsy documented ATN had low values of EE, 1.05 (sd 0.5) for the first and $0.28(0.06)$ for the second phase, whereas in 5 with vascular and/or parenchymal rejection, it was significantly higher, 2.35 (1.3; $p<0.05)$, and $1.45(1.0 ; p<0.05)$, respectively. The range of the second phase EE for the ATN group (0.21-0.38) did not overlap with that for the rejection group (0.4-2.8). We conclude that dynamic DMSA imaging yields useful functional data, including RBF, and may be able to distinguish between rejection and causes of tubular damage, such as ATN or cyclosporin toxicity, The timing of the initial higher EE is consistent with DMSA filtration; if $80 \%$ was protein-bound in plasma and filtration fraction was $20 \%$, then EE over this phase would be $4 \%$. The subsequent EE reflects tubular uptake.

\section{1}

R.Bachmann, U.Sechtem ${ }^{*}$, E.Voth, S.Kaspers* ${ }^{*}$ R.Füssi ${ }^{*}$, H.W.Höpp*, H. Schicha.

Klinik für Nuklearmedizin and Klinik III für Innere Medizin*, University of Cologne, Germany.

MIBI-SPECT AFTER SUCCESSFUL CORONARY INTERVENTION: DOES INTRAVASCULAR ULTRASOUND PROVIDE AN EXPLANATION FOR PERSISTENT MYOCARDIAL ISCHEMIA?

Despite angiographically successful interventions, residual ischemia in the perfusion territory of the treated coronary artery is not uncommonly observed in postinterventional perfusion scintigrams. Intracoronary ultrasound (IVUS) studies have demonstrated that the extent of residual intracoronary plaque and obstruction may be underestimated by angiography. Therefore, 24 patients with angiographically successful (residual diameter stenoses $<50 \%$ ) coronary interventions (techniques: 14x PTCA, 6x stenting, 2x directional atherectomy, $2 x$ rotablation; vessels: $15 x$ LAD, $7 x$ RCA, $2 x$ $R C X$ ) were examined by IVUS immediately after the intervention. 24 and $48 \mathrm{~h}$ after the intervention all patients underwent myocardial scintigraphy with $99 \mathrm{~m}$-Tc-methoxyisobutyl-isonitrile after dipyridamole stress and at rest using a triple head SPECT camera.

The perfusion territory of each treated vessel was estimated from the coronary angiogram and assigned to a segmental left ventricular model. Areas of myocardial ischemia were assigned to the same segmental model. The residual area stenosis was determined from IVUS images as (area within lamina elastica interna - luminal area) / area within lamina elastica interna.

13/24 patients had myocardial ischemia during dipyridamole MIBISPECT. Median area stenosis was $71 \%$ in patients with ischemia and $53 \%$ in patients without ischemia ( $p=.008$; Mann-Whitney test). The best concordance between residual area stenosis and residual ischemia was found for IVUS area stenoses $\geq 60 \%$.

Patients with stress induced myocardial ischemia immediately after successful coronary interventions show high grade residual stenoses when examined by IVUS which could at least partially explain the discrepancy between angiographic and scintigraphic findings.

\section{2}

Th. Krause ${ }^{1}$, A.M. Zeiher ${ }^{2}$, V.Schächinger ${ }^{2}$, E. Moser ${ }^{1}$

Depts. of Nuclear Medicine' ${ }^{1}$, Cardiology², Universitätsklinik Freiburg, Germany.

MYOCARDIAL PERFUSION DEFECTS IN EXERCISE THALLIUM201 SPECT CORRELATE WITH ENDOTHELIAL VASODILATER DYSFUNCTION IN PATIENTS WITH NON-OBSTRUCTIVE CORONARY ARTERY DISEASE.

Coronary angiography is regarded as the gold standard in the detection of coronary artery disease (CAD). Therefore, myocardial perfusion scintigraphy is frequently regarded as false positive. Since the endothelium plays a pivotal role for coronary blood flow regulation, we tested the hypothesis that impairment of endothelium-dependent dilation of the microvasculature even in the absence of obstructive CAD could contribute to the failure of coronary blood flow to increase. In 16 patients (p) with normal perfusion and $14 \mathrm{p}$ with exercise induced ischemia evaluated by TI-201 SPECT, coronary blood flow (CBF) was measured after infusion of the endothelium-dependent dilator (EDD) acetylcholine (ACh, $10^{-8}$ to $10^{-6} \mathrm{M}$ ), and of the smooth muscle relaxant papaverine (Pap, $7 \mathrm{mg}$ ) into the $L A D$ via a Doppler catheter. Flow-limiting epicardial CAD was excluded in all p. After ACh, CBF increases were significantly greater in all normal $p$ compared to $\mathrm{p}$ with exercise induced $\mathrm{Tl}-201$ defects (ACh $10^{-8} \mathrm{M}: \quad 43.3 \pm 55.4 \%$ vs $14.4 \pm 12.6 \%$ NS, $10^{-7} \mathrm{M}$ : $119.8 \pm 59.5 \%$ vs $52 \pm 39.8 \%$ p $=0.001,10^{-6} \mathrm{M}: 173.8 \pm 79.9 \%$ vs $72.8 \pm 28.5 \% p=0.002$ ). No difference was found after Pap: $360.6 \pm 141.4$ vs $342 \pm 76.6 \%$ NS. The ACh/Pap-ratio was significantly lower $(\mathrm{p}<0.0001)$ in $\mathrm{p}$ with myocardial perfusion defects. Thus, impaired endothelium-dependent dilation of the coronary resistance vasculature contributes to exercise induced myocardial ischemia detected by TF-201 SPECT in $p$ with nonobstructive CAD. 


\section{3}

J.Lekakis, M.Mavrikakis, M.Emmanue1, V.Prassopoulos, S. Papazoglou, N.Agrios, T.Athanasoul is, D.Moulopoulou, P.Kostamis, S.Moulopoulos.

Dept of Clin. Therapeutics \& Dept of Nuclear Medicine, Alexandra University Hospital, Athens, Greece.

COLD PRESSOR THALLIUM IMAGING MAY DETECT CORONARY RAY;NAUD'S PHENOMENON IN PATIENTS WITH SYSTEMIS . SCLEROSIS

Raunaud's phenomenon is frequent in patjents (pts) with systemic sclerosis (SS). To examine whether cold-induced Raynaud's phenomenon in SS may involve the coronary arteries, we examined 20 pts with known SS. Age was $52 \pm 12$ years (range 38-66 years), duration of SS was $7,2 \pm 5,5$ years (range 6 months -17 years), 18 pts were women. 19 pts had definite Raynaud's phenomenon with duration 10 $\neq 7,6$ years (range $3-24$ years); 4 pts had esophageal involvement. A1l pts underwent dipyridamole-thallium SPECT imaging $(0,56 \mathrm{mg} / \mathrm{kg}$ for $4 \mathrm{~min})$ to exclude the possibility of myocardial ischemia. Within one week thallium SPECT imaging was repeated after immersion of thoth hands to ice water (temperature $0^{\circ}$ to $2^{\circ} \mathrm{C}$ ) for 3 min (Cold pressor, $C P)$. During $C P$ systolic blood pressure rose to $172+27 \mathrm{~mm} H \mathrm{~g}$, diastolic blood pressure rose to $99 \pm 14 \mathrm{mmHg}$ and heart rate to $88 \pm 15 / \mathrm{min}$. No pt developed ischemic thallium defects during dipyridamole thallium imaging. During $C P 6$ pts developed definite ischemic thallium defects ( 2 anteriorly, 2 inferiorly, 1 septal, 1 lateral). All pts with cold-induced defects had Raynaud's phenomen. Compared to pts without cold induced defects, pts with defects had similar rise in blood pressure and heart rate but a significantly longer duration of Raynaund's phenomenon. In conclusion, pts with longstanding SS and Raynaud's phenomenon may present abnormal myocardial perfusion during cold pressor stimulation, indicating a coronary Raynaud's phenomenon in those pts.

174

G.Rubini, A.Bortone*, F.Lauriero, D.Rubini, A.Chiddo*, P. Rizzon*, A. D'Addabbo.

Depts. of Nuclear Medicine, *Cardiovascular Diseases - University of Bari- Italy

MICROVASCULAR ANGINA: $99 \mathrm{mTC}-M I B I$ SPET WITH INTRACORONARY ACETYLCHOLINE TO DETECT REGIONAL PEREUSION DEFECTS DUE TO ENDOTHEIIAI DYSEUNCTION

Endothelium-dependent vasodilatation impairment seems to be responsible for perfusion defects in pts with angina and normal coronary arteries. The aim of this study was to verify whether Acetylcholine (Ach), in conjunction with perfusional scintigraphy (99mTC-MIBI SPET), was able to identify distrectual paradoxis vasoconstriction and quantify the perfu-sion defects. In 13 normotensive pts $(3$ male, 10 fe-male; mean age 52 yrs) with typical chest pain, negative stress test, absence of ischaemic signs after i.v. ergonovine and angiographically normal coronary arteries, Ach $\left(10^{-4} \mathrm{M}\right)$ was administered in the LMCA for $1.5 \mathrm{ml} / \mathrm{min}$ and the coronary sinus flow achieved by thermodilution technique. At the end of perfusion, $500 \mathrm{MBq}$ of $99 \mathrm{mTc}$-MIBI was injected and SPET acquisition was performed 2 hours after. The day after, 99mTC-MIBI SPET was repeated in basal conditions. In $7 / 13$ pts $99 \mathrm{mTc}$ MIBI SPET showed reversible myocardial perfusion defects in the left coronary related area, affecting only part of the distribution area. The coronary sinus flow was reduced in all 7 pts, but not significantly. Ach induced typical angina and ST segment depression $(>1.5 \mathrm{~mm})$ in $3 / 7$ pts. $99 \mathrm{mTC}$-MIBI SPET in conjunction with intracoronary infusion of Ach is a useful method to detect pts and quantify regional myocardial perfusion defects due to endothelium-dependent vasodilatation impairment.
175

P. Theissen, M. Reichenbach, E. Voth, K, Scheidhauer, H. Schich

Klinik unđ Poliklinik für Nuklearmedizin, Universität zu Köln, Köln, Germany

QUANTITATIVE REDISTRIBUTION ANALYSIS OF THALLIUMSPECT MYOCARDIAL SCINTIGRAMS AFTER DIPYRIDAMOL STRESS TEST

Thallium-201 myocardial scintigraphy with dipyridamol-(Dipy)-stress is considered to be equivalent to the scintigraphy after ergometer-(ergo)-stress. In order to ensure the finding "ischaemia", quantitative wash-out evaluation seems to be advantageous. Though, the value of the redistribution analysis for detection of ischemia after Dipy-stress has been judged differently.

In order to prove the significance of the redistribution analysis thallium-SPECT myocardial scintigraphy (MySc) after Dipy-stress wash-out was calculated in 48 patients with coronary artery disease (CAD) verified by coronary angiography (CA). In 42 patients MySc was performed only after Dipy-stress and in 6 patients with a 14 day interval after both, Dipy- and ergo-stress. $0.75 \mathrm{mg} / \mathrm{kg}$ body mass dipyridamol and $74 \mathrm{MBq}$ thallium-201 chloride were administered. The time interval between stress and redistribution scintigram was 3 hours. Polar tomograms resulting from stress and redistribution scintigrams have been divided into 17 Regions of Interest (ROD). For the total 144 ROIs after Dipy-stress and additionally for 18 ROIs after ergo-stress wash-out rates were calculated. The wash-out rates belonging to the 3 vascular regions of coronary blood supply were referred to the average value of the 2 ROIs with the highest wash-out values. Thereby, a reduction of the washout by more than $15 \%$ compared to the maximum has been considered as pathological. Coronary stenoses of $60 \%$ or more deemed to be relevant.

In 113/144 (79\%) ROIs corresponding findings between CA data and washout calculation occured. In 31 ROIs discrepancies were found. Most of the different findings occurred in multi vessel CAD ( 13 cases with either 2 or 3 stenosed coronary arteries). In 8 cases, ROIs with discrepancies between CA and washout data were affected by a myocardial infarct. Comparing Dipy- and ergo-stress, 12 of the 18 ROIs agreed. In 4 ROIs with discrepancies Dipy-stress and in the other 2 ROIs with discrepancies ergo-stress were positive for ischaemia. Herewith, the Dipy and ergo scintigraphy were conform with the CA data in 3 times, respectively.

Wash-out calculation of the MySc after Dipy-stress shows similar results as the redistribution analysis of the $\mathrm{MySc}$ with ergo-stress. The results indicate that also for MySc after Dipy-stress wash-out calculation makes the results more reliable and should, therefore, be added to the routine MySc evaluation.

\section{6}

G. Glatting, R. Weller, M.M. Henrich, S.N. Reske Abteilung Nuklearmedizin, Universität Ulm, Ulm, Germany

INVESTIGATION OF THE UNIDIRECTIONAL EXTRACTION FRACTION OF THE HUMAN MYOCARDIUM WITH RUBIDIUM81/82-PET

The dependence of the extraction fraction $E$ on flow $F$ in the human heart is essential for the noninvasive determination of myocardial blood flow. We investigated this flow dependence in the human myocardium by comparing the Rb-clearance ( $F^{*}$ ) with the flow $F$ determined by the Argon-method. For 5 patients (age $60.6 \pm 7.4 a, 3 / 2=\mathrm{m} / \mathrm{f}$ ) extraction was measured under rest and under

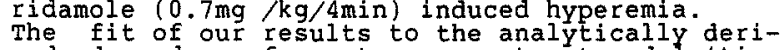
ved dependence for a two compartment model (tissue and vascular compartment, $P$ permeability, $S$ surface) yields

$E=P S /(P S+F), \quad P S=(0.90 \pm 0.09) \mathrm{ml} / \mathrm{g} / \mathrm{min}$.

This result deviates for large and low flows from the standard formula determined by Mullani and Goldstein (for dogs)

$E=0.55 \exp (-\mathrm{bF}), \quad b=0.22 \mathrm{~g} * \mathrm{~min} / \mathrm{ml}$.

We showed that their extraction fraction $E$ is not equivalent to the usual unidirectional extraction fraction and that the data of these investigators are also adequately described by equation (1) with $\mathrm{PS}=0.87 \mathrm{ml} / \mathrm{g} / \mathrm{min}$ - a common value for mammals - if the usual unidirectional extraction fraction is used. The differences between both equations may cause the lower myocardial values for flow $F$ obtained with equation (2) compared to flow measured by microspheres.

Therefore we claim that the functional dependence of equation (1) should be used for the extraction fraction, as it is the theoretically consistent description for a two compartment system. 
Monday, 22 August 1994

177

JC Roos, A Perkins, A van Hof, Q Davies, C Molthoff, W den Hollander, A Wilhelm, R Verheijen, $M$ Frier, J Vermorken, $\dot{A}$ van Lingen, $T$ Baker, $E$ symonds, ? Kenemans. Free Univ tosp Amsterdam, The Netherlands and Celltech Ltd, Slough and Queen's Medical Centre, Nottingham, UK. In-111-hCTMOl NONOCLONAL ANTIBODY (MAb) IN PATIENTS WITH OVARIAN CANCER: IMMUNOSCINTIGRAPHY AND BIODISTRIBUTION

hCTMO1 is a CDR-grafted humanised IgG4 MAb binding polymorphic epithelial mucine (PEM) which is present on the membrane of secretory epithelia and overexpressed in malignancies derived from these tissues.

13 Patients (pts) suspected of primary or recurrent ovarian cancer were injected intravenously with 0.1 $\mathrm{mg} / \mathrm{kg}$ In-111-hCTMO1 macrocycle conjugate (100 $\mathrm{MBq}$ ). Radiochemical purity was $\geq 96.48$ (TLC) and immunoreactivity (coated antigen bead assay) was $\geq 66 \%$ binding. Al1 pts were assayed for circulating CA $15-3$ prior to injection. Immune complexes were detected (HPLC) in all blood samples from $15 \mathrm{~min}$ post injection (pi). The mean biological Th of IgG in blood in cancer pts was $61 \pm 20$ $h$, and longer in pts with high levels of circulating antigen (CA $15-3$ assay). The mean biological $T \frac{1}{2} \beta$ of the complexes was $54 \pm 13 \mathrm{~h}$.

Immunoscintigraphy up to $120 \mathrm{~h} \mathrm{pi}$ showed malignancy in 6 out of 8 pts with surgically proven cancer. Bone marrow, liver and spleen were seen in all pts. Of the 5 pts with benign or borderline tumour, 1 was false positive.

Mean (SD) tissue uptake ( $\mathrm{BD} / \mathrm{kg}$ ) at surgery (6 days $\mathrm{pi}$ ) was for tumour (T) 4.9 45.3 (range 1.3-17.4), normal tissue (NT: mean of skin, fat, muscle and peritoneum) $0.7 \pm 0.6(0.2-2.2)$, blood (B) $2.3 \pm 2.3(0.5-6.4)$ and liver $22.1 \pm 11.6(5.4-35.6)$. Mean $T / N T$ was $7.7 \pm 5.9(2.4-19.8)$ and $T / B 2.7 \pm 1.7(0.8-6.3)$. In one pt with a high $\mathrm{CA} 15-3$ the highest tumour and lowegt liver uptake was found.

In conclusion, hCTMO1 selectively targets ovarian cancer with positive immunoscintigraphy. The possibility of an inverse relationship between circulating CA 15-3 level and liver uptake requires further investigation. The MAb dose is now to be increased to $1 \mathrm{mg} / \mathrm{kg}$ to elucidate the effect of a higher MAb dose on complex formation and liver and tumour uptake.

178

K.F. Hübner, T.W. McDonald, G.T. Smith, K. Hunter, H.R. Gould, E. Buonocore

Biomedical Imaging Center, Depts. of Radiology and $\mathrm{Ob} / \mathrm{Gyn}$, The University of Tennessee Medical Center at Knoxville, Tennessee, USA

ASSESSMENT OF PRIMARY AND RECURRENT OVARIAN CANCER BY F-18 LABELED DEOXYGLUCOSE (FDG) AND POSITRON EMISSION TOMOGRAPHY (PET)

Laparoscopy, CT of abdomen and pelvis, and the CA-125 marker are alternatives to "second-look" laparotomy in ovarian cancer. The purpose of this study was to evaluate FDG PET for detecting, staging, and monitoring ovarian cancer. Ninety-five patients with primary or suspected recurrent ovarian cancer had a total of 119 PET scans. Dynamic emission scans were obtained after i.v. bolus of 185-370 MBq of FDG Whole-body PET images were also obtained for 33 patients. Images were analyzed by visual interpretation, time-activity curve (TAC) profiles and standardized uptake vaIues (SUV) PET results were correlated with $C T$ and histological findings and survival ( $\geq 12$ months post PET). A SUV of $3.08 \pm 1.68$ was the cutoff separating benign from malignant tissue.

Overall, PET had a sensitivity and specificity of $83 \%$ and $80 \%$, compared to $82 \%$, respectively $53 \%$ of CT. In patients with suspected recurrent ovarian cancer, PET had a sensitivity, specificity and accuracy of $58.3 \%, 95 \%$ and $81 \%$. More importantly, the combination of $\mathrm{PET}$ and $\mathrm{CT}$ had a negative predictive value close to $100 \%$ (CT alone: 62\% and PET alone: 76\%). Whole-body PET provided complementary information in six of 33 patients. FDG PET is sufficiently specific, especially in light of a negative CT scan, that it may obviate "second-look" operations.

(Supported by US NIH Grant CA59615-0I)
179

Kocher F., Stollfuß J.C., Schönberger J.A., Logemann J., Bachor R.*, Hautmann R.*, Reske S.N

Department Nuclear Medicine and Department Urology*, University of Ulm, Ulm, Gêrmany

\section{FEASIBILITY OF 18- FDG-PET IN LYMPH NODE}

\section{STAGING OF UROGENITAL CARCINOMAS}

Staging criteria for lymph node involvement in kidney- and bladder cancer presumes actual biopsy of nodal tissue. Since glucose utilisation is markedly increased in a variety of malignant tumors and their lymph node deposits, the feasibility of non-invasive assessment of lymph node involvement with FDG-PET was studied in 11 patients with bladder cancer and 10 patients with carcinoma of the kidney. After performing transmission scans for attenuation correction PET-Scans were acquired with ECAT-931-08-12 PET-Scanner ( Siemens-CTI, Knoxville TN ) 50 minutes after intravenous injection of $240+1-60 \mathrm{MBq}$ 18-FDG. The axial field of view was $30 \mathrm{~cm}$. Iterative reconstruction was performed in eight iterations and images were reconstructed in three views. To avoid artefacts due to 18-FDG contamined urine $20 \mathrm{mg}$ furosemide was given i.v. and the urinary bladder was purged continuously with an irrigation catheter. Results were compared to histological findings of intraoperativly picked lymph nodes. PET-scans were classified correctly positive, when a focally increased FDG-uptake was found in all correct anatomical sites of verified infiltrated lymph nodes. Pet scans were classified as correctly negative when activity distribution in small pelvis and lower abdomen showed no focally increased uptake in major lymphatic pathways and histology of lymph nodes was negative. In patients with bladder carcinoma lymph node staging was correctly negative in 7 patients, correctly positive in 3 patients and false positive in one patient. In patients with carcinoma of the kidney, lymph node staging was correctly negative in 7 patients and correctly positive in three patients. Despite renal excretion even the primary tumor was detectable in 4 out of 10 patients with kidney carcinoma.These preliminary results suggest that 18-FDG-PET provides a highly promising tool for demonstrating or excluding metastatic spread to regional lymph nodes in bladder- and kidney carcinoma.

\section{0}

Karl-Mikael Kälkner, Claes Ginman, Jan-Erik Westiin, Henry Letocha, and Sten Nilsson Department of Oncology, Akademiska sjukhuset, University Hospital, Uppsala, Sweden

Somatostatin Receptor scintigraphy in Metastatic Hormone-Refractory prostatic Carcinoma

The present study was initiated with the aim of discovering whether octreoscanili could detect somatostatin receptors in hormonerefractory prostatic carcinoma. Seventeen patients with skeletal metastases of hormonerefractory prostatic carcinoma were investigated scintigraphically with the 111 indium-labelled somatostatin analogue, [DTPA-D-Phe1]-octreotide (Octreoscan111). The patients also underwent a $99 \mathrm{mrc}-\mathrm{HDP}$ bone scintigraphy. In $16 / 17$ (94\%) patients, at least one of the metastases disclosed an uptake of the radiolabelled somatostatin analogue. of all the lesions detected at bone scintigraphy, $44 / 112$ (39\%). were positive for somatostatin analogue uptake. These results reflect a heterogeneity in the metastatic lesions of prostatic carcinoma and show that somatostatin receptors are present in the hormone-refractory type of this malignancy. The data are in accordance with previous reports on the neuroendocrine nature of this disease and form a basis for future studies with somatostatin analogues in the treatment of hormone-refractory prostatic carcinoma. 
181

$\underline{\text { R. Bares }}^{1}$, P. Effert ${ }^{2}$, St. Handt ${ }^{3}$, C. Altehoefer ${ }^{1}$, U. Cremerius ${ }^{1}$, D. Wittstock $1^{1}$, A. Boecking 3 , Ch. Mittermayer ${ }^{3}$, G. Jakse ${ }^{2}$, U. Buell ${ }^{1}$ Departments of Nuclear Medicine ${ }^{1}$, Urology ${ }^{2}$, and Pathology ${ }^{3}$, Technical University of Aachen, GERMANY

\section{PROGNOSTIC CLASSIFICATION OF PROSTATIC CANCER: IS GLUCOSE METABOLISM MEASURED BY FDG-PET A USEFUL PARAMETER?}

Although various well-established prognostic parameters of prostatic cancer are known today, assessment of individual prognosis is still difficult. Aim of the present study was to evaluate FDG-PET for classification of untreated prostatic cancer. A total of 51 patients with suspected carcinoma was examined by dynamic PET after iv injection of 150-300 MBq FDG. Prostatic FDG-uptake (differential uptake ratio [DUR]) as well as the metabolic rate of glucose (MRG $[\mu \mathrm{mol} / 100 \mathrm{~g} / \mathrm{min}] ; \mathrm{LC}=1$ ) were calculated and compared with tumor stage (TNM), histopathological grading (G), DNA-ploidy, and proliferation. In 45 patients with cancer both DUR and MRG were not influenced by tumor extension $\left(\mathrm{T}_{1 / 2}: 3.1 \pm 0.3 / 8.2 \pm 1.1 ; \mathrm{T}_{3 / 4}\right.$ : $3.7 \pm 0.8 / 9.7 \pm 2.5)$, grading $\left(\mathrm{G}_{1}: 4.2 \pm 0.9 / 8.5 \pm 3.1 ; \mathrm{G}_{2}: 4.1 \pm 1.2\right.$ $8.9 \pm 1.8 ; G_{3}: 3.8 \pm 1.1 / 10.2 \pm 3.4$ ), or DNA-ploidy (aneuploid: $3.9 \pm 1.3 /$ 9.3 \pm 3.8 ; euploid: $4.1 \pm 1.2 / 8.7 \pm 4.4$ ) and did not significantly differ from values found in benign hyperplasia $(2.0 \pm 0.7 / 4.1 \pm 1.7 ; n=6)$ except for patients with generalized disease $\left(\mathrm{N}_{+} / \mathrm{M}_{+}: 4.8 \pm 1.4 /\right.$ $10.9 \pm 4.1 ; \quad p<.05$ ). Proliferation evaluated by quantitative Ki-67 immunohistochemistry correlated slightly with DUR $(r=0.61)$ and more closely with MRG ( $\mathrm{r}=0.72)$.

We conclude that FDG-PET can differentiate 2 subgroups of prostatic cancer. Glucose metabolism of one group equals that of benign hyperplasia while the other group, which mainly comprises patients with metastatic disease, is clearly hypermetabolic. Thus, identification of patients with high risk of progressive disease might be possible by PET and needs to be further investigated by long-term follow up studies.

\section{2}

V.U. Chengazi, M. Feneley, M. Granowska, S.J. Mather, D. Elis ison, M.J. Carroll, A.R. Granowski, R.S. Kirby, K.E. Britton.

ICRF Nuclear Medicine Group, St. Bartholomew's Hospital, West Smithfield, London EC1A 7BE, U.K.

RADIOIMMUNOSCINTIGRAPHY OF SOFT TISSUE RECURRENCE OF PROSTATIC CANCER WITH TC-99m LABELLED MONOCLONAI ANTIBODY

A Tc-99m labelled antibody has been used in 14 patients for imaging recurrent prostatic malignancy. $600 \mathrm{MBq}$ of Tc-99m labelled CYT-351 (Cytogen corporation, USA) was administered, with planar imaging at 10 minutes, 6 hours and 24 hours and pelvic SPET at 6 and 24 hours.

All patients had bone scans (BS) and immune scans (IS). 2 patients had negative BS and IS. 4 patients had positive $\mathrm{BS}$ and positive IS. In these patients, the IS picked up local soft tissue recurrence but not the BS lesions, while in one IS showed all BS lesions. In 6 patients with a negative $B S$ and a rising PSA, IS demonstrated local recurrence. In 2 patients with extensive BS lesions, IS did not demonstrate any disease. Both had longstanding metastases and were responding to treatment.

This procedure offers all the advantages of using Tc-99m and is comfortable for the patient, the entire study being completed in 24 hours with no special preparation.

These early results seem to indicate that TC-99m labelled CYT-351 may have a role in identifying soft tissue recurrence, especially in patients with a negative workup and a rising PSA.
183

H.Feistel, M.Merkl, W.Siegfried, C.Möller, S.Dertinger, J.H.Ficker E. Hahn, F. Wolf.

Medicine I, Friedrich- AlexanderUniversity of Erlangen- Nuremberg, Erlangen, FRG

BRAIN PERFUSION DURING SLEEP APNEA A STUDY WITH TC-99M-HMPAO IN SLEEP LABORATORY

The sleep apnea syndrome (SAS) is comprised by daytime hypersomnolence, snoring, obesity and frequent cessation of breathing during sleep. Complications are alterations of blood pressure, brain pressure and cardiac output, lethargy, loss of cognitive functions and memory, and brain infarction as well. Aim of the study was (1) to find feasability of imaging rCBF during noctumal phases of sleep apnea and (2) to document therapeutic effects of transnasal continuous positive airway pressure (CPAP) breathing on rCBF. 13 patients ( 12 male, 1 female, mean age $50+1.9 .8$ y, mean body weight $107+1-13.6 \mathrm{~kg}$ ) were suspected to have SAS as indicated by a device for ambulant SAS monitoring (MESAM box) and were enrolled to the study. Polysomnography (PS) was started in the late evening hours. Patients were injected during a PS- documented apnea phase with 700 MBq Tc99m-HMPAO by rapid inflush of saline solution into i.v. indwelling cannula. Injection took place between 2.00 and 4.00 hrs a.m., SPECT data acquisition at $7.30 \mathrm{a} . \mathrm{m}$. Two nights later the investigation was repeated under therapeutic transnasal CPAP breathing. Two injections coincided with REM phase. Injection times were doumented to allow for precise analysis of PS. All SPECT studies were evaluated by means of a semiquantitative algorithm and regional perfusion indices were established. Under CPAP breathing a drastic improvement of the former pathologic sleeping behaviour could be documented the arterial $\mathrm{pO}_{2}$ saturation increased $(p<0.02)$, the apnea index decreased $(p<0.01)$ significantly. More patients attained deeper states of sleep (from I and II to III and IV). in 5 patients a suprafronta hyperperfusion, in two pat. an additional increased basal ganglia perfusion could be found, five pat revealed temporo- parietal decreased perfusion pattern. All findings improved under CPAP, in two patients normalizing completely. SPECTs of the REM phase showed increased occipital perfusion in spite of closed eyes, revealing hyperactivity of visual cortex. We conclude that HMPAO- SPECT is capable to image nocturnal regional cerebral blood flow. Frontal hyperperfusion is discussed to have two reasons: dilatation of brain vessels due to increased $\mathrm{pCO}_{2}$ tension with superposition of frontal cognitive activation during pathologic light sleep behaviour.

\section{4}

C. Menzel, F. Grünwald, A. Hufnagel, L. Solymosi, L. Pavics, E. KJernm, A. Schomburg, C.E. Elger and H.J. Biersack. Depts. of Nuclear Medicine, Epileptology and Radiology, University of Bonn, Germany

The Relevance of InTerictal RCBF SPECT Using TC-99M HMPAO IN The Presurgical Evaluation of Epilepsy

A total number of 126 patients suffering from epilepsy have been evaluated retrospectively for the predictive value of diagnostic imaging using MRI and interictal HMPAO-SPECT. In 106 patients these results could be compared to the EEG findings, the site of surgical intervention and the post-op outcome. 10 - 30 minutes after i.v.-injection of $740 \mathrm{MBq}(20$ $\mathrm{mCi}$ ) $99 \mathrm{mTc}$-HMPAO a brain-SPECT was performed. MRI scanning included sagittal T1transversal proton- and T2- and coronal T1-weighted IR-sequences and in most cases T2 weighted TSE-sequences using a temporal angulation. Areas of hypoperfusion were seen in 113 patients, whereas only 13 patients showed an inconspicious cerebral perfusion pattern. MRI revealed lesions in 74 patients and normal results in 52 patients. In 7 cases both techniques described defects, that did not match concerning their localization. The distribution of the remaining results is given below.

\begin{tabular}{lcc} 
Table 1: & SPECT normal & SPECT abnormal \\
MRI normal & 9 & 43 \\
MRI abnormal & 4 & 63 \\
\hline
\end{tabular}

$87 / 106$ patients underwent surgery, 78 of which suffered from mediacally refractory partial seizures. Table 2 compares presurgical imaging using MRI and SPECT as well as the EEC results with postsurgical outcome in these 78 patients. Postsurgical outcome was measured as a $100 \%,>75 \%$ or $<75 \%$ decrease in seizure frequency.

\begin{tabular}{llccc} 
Table 2: & & $100 \%$ & $>75 \%$ & $<75 \%$ \\
Complete match & $(\mathrm{N}=40)$ & $\mathbf{8 2 . 5 \%}$ & $10 \%$ & $7.5 \%$ \\
HMPAO/EEG match & $(\mathrm{N}=16)$ & $75 \%$ & $12.5 \%$ & $12.5 \%$ \\
EEG only & $(\mathrm{N}=13)$ & $69 \%$ & $31 \%$ & - \\
MRI/HMPAO match & $(\mathrm{N}=3)$ & - & $33 \%$ & $67 \%$ \\
MRI/EEG match & $(\mathrm{N}=6)$ & $100 \%$ & - & - \\
\hline
\end{tabular}

MRI corresponded to the SPECT findings in $60 \%$ of cases but was normal in approx. $40 \%$ of cases in our collective (SPECT $8 \%$ ). Interictal SPECT corresponded to the EEG findings in $72 \%$ of all cases (MRI $59 \%$ ).

In conclusion both, MRI and interictal SPECT, must be seen as being supportive to the substantial EEG, if no differentiation is made between superficial and invasive EEG and on basis of post-op decrease of seizure frequency only. However, morphological and functional informations of imsportance for $\mathrm{OP}^{-}$-and invasive EEG-planning are given 
185

C. Menzel, F. Grünwald, J. Bauer, L. Pavics, A. Hufnagel, R. Sakowski, C.E. Elger and H.J. Biersack. Departments of Nuclear Medicine and Epileptology, University of Bonn; Du Pont Pharma, Bad Homburg, Germany.

\section{ICTAL AND INTERICTAL RCBF SPECT USING TC-99M-BICISATE}

Tc-99m-Bicisate is now available as a new tracer for cerebral blood flow (rCBF). We initiated a study aimed at evaluating its usefulness in the detection of epileptogenic foci. We report on the first 11 cases who underwent an ictal and interictal rCBF SPECT so far.

All patients received an EEG and a MRI of the brain including transverse protonTSE-sequences using a temporal angulation.

Prior to injection the radiochemical purity of the tracer was determined to be at least $90 \%$ in all cases using thin layer chromatography. Approximately $590-770$ $\mathrm{MBq} 99 \mathrm{mTc}$-Bicisate were i.v.-injected 2 - 20 seconds (mean $5 \mathrm{sec}$.) after the onset of a seizure. SPECT-studies were started 1 to 3 hours after injection, using an annular crystal SPECT-system (Ceraspect, DSI). Interictal studies were performed 30 minutes to 1 hour after i.v.-injection of $740 \mathrm{MBq} 99 \mathrm{mTc}$-Bicisate using the same SPECT-system.

MRI was normal in $7 / 11$ patients. During interictal phase $10 / 11$ patients showed areas of hypoperfusion according to the EEG findings. Ictal SPECT-studies revealed significant changes in perfusion involveing especially these areas in all patients. Circumscript areas of ictal byperperfusion were detected in 5 patients. These preliminary results indicate that Bicisate is valuable for the assessment of epileptogenic foci, if the tracer injection is carried out rapidly after the onset of a seizure, which underlines the value of a tracer with a high in-vitro stability over several hours. In addition ictal rCBF SPECT using high resolution SPECT systems is able to detect circumscript ictal hyperperfusion suggested to represent the focus itself in a considerable number of patients.

This study is partly covered by a trial supported by Du Pont Pharma. and T2-weighted sequences, as well as coronal T1-weighted IR- and T2-weighted

187

I. Podreka, Ch. Baumgartner, G. Koch, D. Wimberger, G. Lindinger, A. Olbrich, S. Aull, U. Pietrzyk*, S. Lurger, W. Serles, M. Pruckmayer, S. Stanculovic, Th. Brücke

Neurological University Clinic, Clinic of Nuclear Medicine and MRI Institute of Vienna, Austria, *Max Planck Institute Cologne, Germany HMPAO - SPECT AND PROLONGED VIDEO - EEG FINDINGS COMPARED TO SEIZURE SYMPTOMATOLOGY

This study was performed in order to correlate ictal CBF changes to seizure symptomatology and to estimate the value of combined video EEG and HMPAO - SPECT findings for the presurgical lateralisation of epileptogenic foci. In 15 patients (18 - 65 yrs) prolonged video EEG recording (mean 5 days) and HMPAO - SPECT (Siemens Multispect 3 ) were obtained during a seizure (HMPAO inj. 40-60 sec after seizure onset). MRI and a second SPECT study were performed in the seizure free intervall. Nine patients had temporal lobe epilepsy (TLE) while 6 patients suffered from extra - temporal seizures (ETE). For anatomical reference SPECT studies were realigned and superimposed to MRI images. rCBF changes between the two SPECT studies were estimated visually and localized on MRI images. Loss of consciousness, postictal amnesia and automatisms were associated predominantly with activation of the lower mesial and lateral portion of the temporal lobe. Additional activation of the lateral frontal lobe was also seen in 3 patients with automatisms. Two patients with paranoid symptoms revealed increased HMPAO uptake in the mesio- and laterofrontal regions as well as in the anterior basal ganglia. One patient with TLE showed an activation in one temporal lobe and in the contralateral frontal and parietal region, suggesting no direct contralateral temporal seizure propagation, as already postulated by electrophysiological investigations in man. Optical hallucinations were paralleled by CBF increase in the temporal lobe, basal ganglia, sup. and inf. occipital lobe. During seizure activity elevated CBF extended from the extratemporal focus to the ipsilateral occipital and temporal lobe or even in both mesio - temporal regions in the ETE - group. One case with negative motor seizures had a circumscribed ICBF increase in the prefrontal cortex. Lateralisation of the epileptogenic focus was achieved by SPECT in $90 \%$, EEG $60 \%$, and MRI (hippocampal atrophy) in $70 \%$ of the cases. In summary, high resolution SPECT combined with prolonged EEG recording considerably increases the accuracy of focus lateralisation and the knowledge on seizure propagation related to seizure symptomatology.

\section{8}

W. Reiche ${ }^{1}$, J.Ree ${ }^{2}$, R.Weber ${ }^{2}$ K.P.Westphal ${ }^{2}$, H.H.Kornhuber ${ }^{2}$, S.N.Reske ${ }^{3}$

Institute for Neuroradiology, University Hospital Homburg; ${ }^{2}$ Neurological Clinic in the Reha-Hospital Ulm; ${ }^{3}$ Department of Nuclear Medicine, University Ulm

COMPARATIVE EVALUATION OF 18FDG-POSITRONEMISSION-TOMOGRAPHY (PET) AND NEUROPSYCHOLOGICAL EXAMINATIONS IN PATIENTS WITH MEDICALLY REFRACTORY EPILEPSY

Save and effective surgical treatment for medically refractory epilepsy depends upon acurate identification of the epileptogenic brain area. Confirmatory tests of focal functional deficits are applied to increase confidence in EEG-localization. The goal of this study was to compare the findings of 18 FDG-PET and neuropsychological tests with the EEG-localization in the presurgical evaluation in patients with medically refractory focal epilepsy.

We examined 15 patients ( 12 females, 3 males, medium age 26.3 \pm 14.4 years $)$ with long-standing $(17.7 \pm 10.2$ years $)$ medically refractory focal temporal lobe epilepsy by EEG-videometry, 18 FDGPET and additional 7 patients with neuropsychological tests. Interictual PET-investigation was acquired $30 \mathrm{~min}$. after intravenous injection of $185-250 \mathrm{MBq}{ }^{18 F D G}$. We applied for neuropsychological testing the testbattery of the helmstaedter working-group (BonnGermany).

In all cases the PET-findings, a hypometabolic temporal area, correlated well with EEG-localization. In $4 / 7$ patients we revealed a temporal memory deficit with a clear side classification, which correlated significantly with the EEG- and PET-lateralization of the epileptogenic area. In $2 / 7$ cases there were bitemporal neuropsychological deficits. One neuropsychological test was rejected because of both-handing.

These results demonstrate, that (1) noninvasive information about epileptogenic brain areas can be gained from ${ }^{18} \mathrm{FDG}$ and PET. (2) If the findings of EEG, PET and neuropsychological tests agree referring to the lateralization, this may spare patients a stressful invasive diagnostic by EEG with subdural records.
M.A. Antar, S.R. Benbadis, N.K. So, W.J. McIntyre

State University of New York, Stony Brook, NY, Cleveland Clinic Foundation, Cleveland, $\mathrm{OH}$.

EVALUATION OF PRE-OPERATIVE F-18-FDG PET SCANNING IN BITEMPORAL EPILEPSY; CORRELATION WITH OTHER MODALITIES.

Effective surgical treatment for medically intractable bitemporal epilepsy complex partial seizures depends upon accurate identification of the epileptogenic brain zone. In bitemporal epilepsy (BTE), this remains difficult and requires extensive evaluation by several modalities and invasive depth electrodes. The aim of this study is to evaluate FDG PET findings in the lateralization of BTE in 23 patients (12 women and $11 \mathrm{men}$ ) and correlate them with other modalities. The dose for PET was 5-10 $\mathrm{mCi}$ of F-18 FDG (i/v). All patients had interictal F-18 FDG PET scan, MRI, prolonged EEG/video monitoring and invasive depth electrode study. MRI was abnormal in 5 patients, of these only 3 showed lateralization (13\%). Scalp prolonged EEG showed definite lateralization in 2 patients (9\%). PET scan revealed lateralization (hypometabolism in one temporal lobe) in 13 patients. In these patients there was agreement in lateralization between PET and Ictal depth electrodes in 11 patients and discordance in 2 patients. In the remaining 10 patients, there was bilateral temporal hypometabolism (non-lateralization) or equivocal PET scan. In this group, ictal depth electrodes findings agree with PET findings (nonlateralization) in seven out of 10 patients. However, there was lateralization by ictal depth electrodes in 3 patients; two patients were shown to have a right temporal focus and underwent a right temporal lobectomy and the third patient showed a left temporal focus. In patients with temporal epilepsy, MRI and scalp EEG prolonged monitoring revealed lateralization in a small percentage FDG PET and depth EEG findings in $18 / 23$ patients $(78 \%)$. of patients. In contrast, there was overall concordance between 
189

EMC Manning, M Critchley*, C Charlwood, J Robinson, MA Al-Janabi*, A Shenkin, WD Fraser. University Dept of Clinical Chemistry and "Dept of Nuclear Medicine, Royal Liverpool University Hospital, Prescot Street, Liverpool, L7 8XP. RADIONUCLIDE BONE SCAN ABNORMALITIES IN OSTEOPOROTIC PATIENTS GIVEN A NON-STANDARD CYCLICAL ETIDRONATE REGIMEN

Etidronate $400 \mathrm{mg} /$ day given cyclically 2 weeks out of every 12 weeks for $2-3$ years is a recommended treatment for osteoporosis (Regimen 1). In an attempt to improve efficacy, the same dose of etidronate was given but for 8 weeks of each 12 week cycle (Regimen 2).

An open, randomised trial was undertaken to compare Regimens 1 and 2 given for 2 years. 32 patients were randomised to Regimen $I$ and 36 patients to Regimen 2. During the etidronatefree period, calcium and vitamin D intake was optimised in both groups.

During their second year of treatment, two Regimen 2 patients developed bone pain and lower limb weakness. Radionuclide bone scans suggested metabolic bone disease and iliac crest biopsies confirmed osteomalacia. The trial was terminated and radionuclide bone scans were performed on consenting participants.

Bone scans suggested metabolic bone disease in only 1 of 17 patients on Regimen 1 (bone biopsy awaited) compared with 22 of 27 patients on Regimen 2 . 16 bone biopsy results are available in Regimen 2 patients to date: 12 show evidence of osteomalacia.

The high incidence of osteomalacia makes Regimen 2 an unsuitable treatment regimen.

Bone scintigraphy is not routinely performed in osteoporotic patients but it played a vital role in this study in the identification of a serious adverse event.

\section{0}

J. Földes, P. Lakatos, J. Zsadányi

I Department of Medicine, Semmelwe is

University, Budapest, Hungary

IS DECREASED IGF-I PRODUCTION AN ADOITIONAL RISK FACTOR FOR OSTEOPOROSIS IN POSTMENOPAUSAL WOMEN WITH SUBCLINICAL HYPERTHYROIDISM?

The aim of the present study was to elucidate whether changes in serum IGF-I and IGFBP-3 levels act as additional and potential risk factors for the development of osteoporosis in postmenopausal women with endogenous subclinical hyperthyroidism. Premenopausal and postmenopausal females with a solitary autonomously functioning thyroid nodule $(n=44)$ were categorized into subclinical hyperthyroid and toxic nodular goiter groups and the results were compared with those of age-matched euthyroid females $(n=20)$. Lumbar spine and femoral neck bone mineral densities (BMD) were measured by DEXA. Serum IGF-I was assayed by Nichols and IGFBP -3 by Diagnostic Systems Lab. kits. Results indicate that in a fraction of postmenopausal subclinical hyperthyroid patients serum IGF-I and IGFBP-3 levels are normal or elevated, while in other subjects their values are lower than normal ( $p<0.01$, respectively). In the group with decreased IGF-I and IGFBP-3 levels the mean BMD of the femoral neck but not of the lumbar spine was significantly lower than the bone density in the group with normal or elevated IGF-I and IGFBP-3 values $(p<0.01)$. Present study refers to the possibility that diminished IGF-I production may have an additional role in the development of osteoporasis in postmenopausal women with subclinical hyperthyroidism.
191

J. Andreas ${ }^{1}$, T. Forst ${ }^{2}$, R. Lobmann ${ }^{2}, H$. Schäfer $^{2}$, R. Piepenburg ${ }^{1}$, K. Hahn', J. Beyer².

Klinik für Nuklearmedizin ${ }^{(1)}$ and III. Medizinische Klinik ${ }^{(2)}$; Johannes Gutenberg-Universität Mainz, Germany.

\section{IS THERE ANY RELATIONSHIP BETWEEN BONE MINERAL CONTENT AND THE STATE OF DIABETIC POLYNEUROPATHY?}

Aim of the study: Diabetic polyneuropathy leads to an alteration in bone perfusion by the opening of arterio-veneous shunts. $A$ relationship between sympatholytic increased bone perfusion and diabetic osteopathy is discussed. Aim of the investigation is to show a possible correlation of bone density, examined by double-photon-absorptiometry (DPA) and the state of diabetic polyneuropathy.

Performance: The study includes 60 diabetics and 30 control persons. Diabetic polyneuropathy was found in 30 diabetics. Exclusion criteria were neurological diseases of non-diabetic origin, osteological diseases as well as medications with an effect on the nervous system or on the bone turnover. Bone density was measured in the left femoral neck and in a defined region of the left distal lower leg.

Findings: In the diabetic group we found a significantly reduced bone mineral content $\mathrm{g} / \mathrm{gcm}$ hydroxilapatidequivalent in the area of the femoral neck $(0,87 \pm 0,17$ vs. $0,93 \pm 0,14 ; p<0,05)$ and in the area of the distal lower leg $(0,92 \pm 0,13$ vs. $1,01 \pm 0,13$; $p<0,01)$. However, no significant difference between diabetics with or without polyneuropathy could be found.

Conclusions: There is no correlation between the existence and state of diabetic polyneuropathy and the bone mineral content appointed by DPA.

192

H.Elser, M.Eisenhut, M. Seibel*, M.Hofmann, P.Georgi

Departments of Nuclear and *Internal Medicine (I), University of Heidelberg, Heidelberg, Germany

BONE KINETICS OF I-131 LABELED DIPHOSPHONATE FOR THE DIAGNOSIS OF METABOLIC BONE DISEASE: PRELIMINARY CLINICAL RESULTS WITH I-131 BDP3.

The kinetics of $\alpha$-amino-(3-[ ${ }^{131}$ I]iodo-4-hydroxybenzylidene)diphos-<smiles>NC([OH2+])([18OH])c1ccc(O)c(Br)c1</smilesphonate ( ${ }^{131}$ I-BDP3) was investigated in patients with various metabolic bone disease. A series of three whole-body retention measurements, $10 \mathrm{~min}$., 1 and 7 days p.i. of $40 \mathrm{MBq}$ 131I-BDP3 in 14 patients with suspected high ${ }^{131}$ I-BDP3 bone turnover (8 osteoporosis (OP), 2 osteomalacia (OM), 1 Paget's disease (PD), 1

primary hyperparathyreoidsm (PHP), 1 high turn over of unknown origin (UTO), 2 normal controls (C)) revealed pronounced differences in the kinetics of the title compound. Depending on the state of disease the effective half lives of ${ }^{131}$ I-BDP3 resulted to: (OP) 100-116 hours, (OM) 123-139, (PD) 154, (PHP) 24, (UTO) 45, and (C) 140. The percent retained activity at time zero, also known as whole-body retention, varied within the following ranges: (OP) 38-59\%, (OM) 55-62, (PD) 59, (PHP) 74, (UTO) 48, and (C) 42.

While the whole-body retention values reflect the elevated binding capacity of the bone due the liberation of hydroxyl apatite binding sites the effective half lives indicate the accelerated kinetics of bound ${ }^{131}$ I-BDP3 as a consequence of the enhanced turnover rate. The faster liberation of radioactivity in patients with $\mathrm{OP}$ and $\mathrm{PHP}$ as well the delayed turnover in $\mathrm{PD}$ as compared to the control demonstrates the sensitivity of the method. Measurements of ${ }^{131}{ }^{1}$ I-BDP3 kinetics in bone may therefore be useful in the diagnosis and therapy monitoring of metabolic bone disease. 
193

J. Kuyvenhoven, G. Forger, J. De Rycke, D. Cleppe,

R. Crombez.

Depts. of Nuclear Medicine, Physical Medicine and

Rehabilitation, St Lucas-St Jozef Hospital, Brugge and Orthopaedics, Elisabeth Hospital, Sijsele, Belgium.

DIAGNOSTIC VALUE OF DYNAMIC TC-HSA SCAN FOR THE DETECTION OF REFLEX SYMPATHETIC DYSTROPHY (RSD).

Dynamic TC-HSA (DHSA) scan is used for staging RSD. Its diagnostic value was never established nor compared with bone scan. Based on clinical criteria, 116 patients were assigned to low, medium or high RSD probability prior to Tc-MDP bone scan and DHSA scan. Clinical criteria were used as reference for RSD during follow-up. Receiver Operator Characteristic, employed to assess test criteria, showed DHSA scan to be more specific (79\%) than bone scan (19\%) for $100 \%$ sensitivity of both. Bone scan of the affected part of the body was test negative if poolphase displayed equal or slightly diffusely increased activity and if delayed phase displayed equal activity when compared to the contralateral side. Other bone scan patterns of the affected part of the body with juxtaarticulary and diffusely increased or diffusely decreased activity when compared to the contralateral side were test positive. DHSA scan was test positive if the timeactivity curve of the affected part of the body displayed a curve grade I-II or grade I with a countrate ratio to the contralateral curve of more than 1.16 at 12 min; other curve patterns were test negative. Bone scan sensitivity was over $97 \%$ and specificity was below $54 \%$ in all groups; DHSA scan sensitivity was $81 \%$ and specificity was over $86 \%$. Diagnostic accuracy of DHSA scan was higher than of bone 5 can ( $86 \%$ versus $63 \%$ ) in the law probability group and was similar in the medium probability group ( $87 \%$ and $85 \%$ respectivi7y). Performing DHSA scan only in case of a positive bone scan yields a specificity and a diagnostic accuracy higher than $89 \%$ in all groups.

Considering the cost of therapy for RSD, a specific test is required. DHSA scan, aTone or combined with bone scan, may be suitable in this regard.

\section{4}

YW Bahk, YH Park, SH Kim, SK Chung

Depts of Radiology and Nuclear Medicine, Catholic University

Medical College, Seoul, 137 KOREA

\section{PINHOLE BONE SCAN SIGN OF CHONDROMALACIA} PATELLAE

Chondromalacia patellae $(\mathrm{CP})$, pathologically characterized by a series of degenerative changes in the retropatellar cartilage, is one of the most common causes of anterior knee pain. Its primary diagnostic approach is radiography augmented with arthrography. But radiography is gradually becoming replaced by arthroscopy, CT and MRI, which are either invasive, expensive or not readily available. Technetium-99m MDP/HDP bone scan is a well established adjunct in the study of bone and joint diseases, and recently pinhole scintigraphy (PS) has been shown to provide important and often pathognomonic information.

In the present communication we wish to describe a hitherto undescribed scan sign of $\mathrm{CP}$ as revealed by PS. The scan changes were prospectively analyzed in 10 patients with $\mathrm{CP}$, and findings were compared with those of osteoarthritis, rheumatoid arthritis and Reiter's syndrome. In all four disease groups studied the planar bone scans showed simple "hot patella" sign that has no distinguishing feature. But, interestingly enough PS portrayed in all ten CP cases a characteristic spotty tracer uptake that was localized in the central retropatellar facet. In contrast, in 12 (75\%) of 16 cases of osteoarthritis, rheumatoid arthritis and Reiter's syndrome PS showed increased patellar uptake to be not localized but diffused in the retropatellar facet. The uptake was spotty in the remaining 4 cases, but none of these were in the central facet. Small spotty tracer uptake localized in the central retropatellar facet is pathognomonic of $\mathrm{CP}$.
195

P.M. KOULIBALY*, P. CHARBONNIER**, J. DARCOURT* O. MIGNECO*, M. BARLAUD**, L. BLANC-FERRAUD**. * Department of Biophysic and Nuclear Medicine, University of Nice, France.

** Laboratoire I3S University of Nice-Sophia Antipolis, France.

\section{ITERATIVE SPECT RECONSTRUCTION WITH GIBBS PRIOR REGULARIZATION COMPARISON OF MAP-EM-OSL AND ARTUR.}

The EM (Expectation-Maximization) algorithm is one of the most employed in iterative SPECT reconstruction because it takes into account the Poisson statistic assumption.

Due to the ill-posed nature of tomographic reconstruction, iterative methods cease to converge at a high number of iterations. Therefore, $a$ priori information on the solution must be introduced (so called regularization) to assure the convergence. Various a priori can be used. Gibbs distributions are popular in image processing because they can model many different distributions. We choose one of them which assumes that all neighbouring pixels differences should be smoothed below a defined threshold and preserves above. This provides a regularization which preserved the discontinuities in the image.

When regularized and in order to derivate the total energy term, the EM becomes a MAP-EM OSL (Maximum a posteriori-Expectation maximization one step late). ARTUR is an adaptative deterministic relaxation algorithm which relax the Poisson statistic. It models the photon counts by a Gaussian likelihood.

We compared MAP-EM OSL and ARTUR, both using such a regularization on numerical and physical phantoms. Our data were acquired for different count rates and the numerical simulations were corrupted with Poisson noise. For the same image quality in terms of Signal to Noise ratio (SNR), at a number of counts in current use in clinical diagnoses, 60 iterations of $10 \mathrm{sec}$ were necessary with ARTUR and 250 iterations of $20 \mathrm{sec}$ for the MAP-EM OSL. At a lower count rate the same result need 120 iterations for ARTUR and 250 iterations for the MAP-EM OSL.

These results show that if the performances of both algorithms are equal, even at low count rates, computation time make a consequent difference in favour of ARTUR.

\section{6}

C. Vanhove, C. Monte, H. Colaert, G. Demonceau

St-Elisabeth Hospital, Zottegem, Belgium; Trionix Res. Lab., Twinsburg, USA.

\section{STANDARDISED ATLAS FOR BRAIN STUDIES}

The purpose of this study was to simplify the detection of brain lesions applying a standard stereotaxic coordinate system.

The automated comparison of brain studies with a normal anatomical and functional database requires transformation of a $3 \mathrm{D}$ image into a standardised coordinate system. Conversion to the stereotaxic Talairach atlas (1988) was obtained after identification of the mid-sagittal plane, the bicommissural line (ACPC) and the brain surface contour. Following activity normalisation, a "mean-activity-map" and a "variance-activity-map" of the brain was computed. Mid-sagittal plane localisation was done by a registration procedure where the left hemisphere is compared with the right hemisphere and vice versa. Three landmarks - a frontal, an occipital and a sub-thalamic point were detected in the mid-sagittal plane to extract the ACPC-plane. A $3 D$ segmentation procedure was applied to determine the brain contour. Activity normalisation was achieved by computing the modus of the ratio obtained by dividing the reference image data with the patient image. Afterwards, the anatomical ROIs, based on the Talairach co-ordinate system, could be created. Moreover, a map of the significant differences from the functional normal database ( 28 patients ) was generated.

In a series of 24 patients (10 CVA's, 8 TIA's and 6 normals) the transformation had to be corrected manually in 5 cases ( 1 half-brain CVA, 1 thalamic CVA, 1 image with low signal-noise ratio and 2 probable movements during acquisition). There was a significant difference between the pathological and the normal patients using the mean count ratio between the suspected region and the cerebellum $(p=0.01)$ or the contra-lateral region $(p=0.008)$ as a reference.

These results suggest that this program can objectively and reproducibly detect and quantify brain lesions in $80 \%$ of the cases. 


\section{7}

L.Tellmann. A.Wirrwar, H.Herzog, H.W.Müller-Gärtner

Institute of Medicine, Research Centre Jülich and Department of Nuclear Medicine, Heinrich-Heine-University Düsseldorf, Jülich, Germany

A WIENER RESTORATION FILTER FOR ITERATIVELY RECONSTRUCTED OI5-BUTANOL-RCBF-IMAGES

Restoration filters which usually employ the specific system modular transfer function (MTF) improve the contrast of small structures and reduce high frequency noise simultaneously. This study applies an individual Wiener restotation filter (WRF) to iteratively reconstructed O15-butanol-images in dynamic studies exhibiting low count-statistics and insufficient resolution which is in part caused by the long positron range of 015 (positron energy $1.7 \mathrm{MeV}$ ).

To circumvent the difficulties of generating the MTF for the ultra short-life isotope $\mathrm{O} 15$, we used $\mathrm{Tc} 94 \mathrm{~m}$ ( $\mathrm{T} 1 / 2=52 \mathrm{~min}$ ) with a positron energy of $2.5 \mathrm{MeV}$ and compared it to F18 $(0.6 \mathrm{MeV})$. Count dependent signal $(S(f)$ and noise $(N(f))$ spectra were determined and fitted using a $2 \mathrm{D}$-Hoffman brain phantom tilled with Tc94m. Based on these data, we designed a WRF described by: opt $(f)=M T F(f) /\left(M T F\left(f^{\wedge} 2+\left(N(f)^{\wedge} 2 / S(f)^{\wedge} 2\right)\right)\right.$.

Because of rotational symmetry of the frequency spectrum, the filter function was calculated one-dimensionally and applied to the low count-images of the Hoffman phantom and of ol5-brain-activity in patients in the frequency space two-dimensionally (fig. left).

The comparison of the MTFs shows the dependency on the positron energy of the isotope used, the transfer characteristics at high frequencies decreased for the greater positron energy (fig. right). Visual inspection of the images reveals an improvement in noise reduction and restoration of small structures. Ouantitative analysis of the resolution yields an improvement of $11 \%$ from $8.7 \mathrm{~mm}$ to $7.8 \mathrm{~mm}$. The maximum value of a whole-brain region increased by $50 \%$ at the same average of the regions.
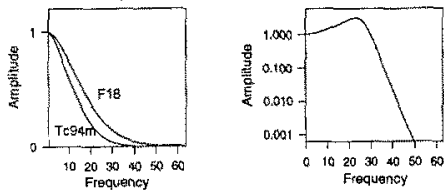

In conclusion, we designed a Wiener restoration filter based on an isotope specific MTF for O15 rCBF studies, which improves resolution and quantitative tracer recovery in brain activation studies.

\section{8}

P.F. Kao, Z. Szabo, R.F. Dannals, H.D. Burns* , R.E. Gibson*, H.T. Ravert, T.G.Hamill*, W.B.Mathews, J.L.Musachio, H.N. Wagner. Johns Hopkins Medical Institutions, Baltimore, MD, and Department of Pharmacology, ${ }^{*}$ Merck Research Laboratories, West Point,PA,USA. IMPULSE RESPONSE FUNCTION OF THE ANGIOTENSION II/AT1 RECEPTOR IMAGNG AGENT: CARBON-11 L-159,884

Carbon-11 L-159,884, is a radiolabeled antagonist of the angiotensin XI/AT1 (AT1) receptors. The purpose of this work was to investigate the binding properties of this new PET agent using the renal impulse response function.

Carbon -11 L- 159,884 was injected to three dogs under pentobarbital anesthesia before $(4,30 \pm 0.46 \mathrm{mCi}, 3624 \pm$ $1657 \mathrm{mCi} / \mu$ mole $)$ and after $(4.46 \pm 0.67 \mathrm{mCi}, 2699 \pm 1450 \mathrm{mCi} / \mu \mathrm{mole})$ pretreatment with $1 \mathrm{mg} / \mathrm{kg}$ of MK-996, another potent antagonist of the ATI receptors. A dynamic PET study was performed over 105 minutes. The input function was obtained from metabolite corrected plasma samples. The impulse response function (IRF) obtained by model-free deconvolution demonstrated three distinct components corresponding to vascular activity, non-specific binding, and specific binding. An average value of the third component (IRF60-105) was calculated 60-105 min. p.i.. Using the compartmental model the following parameters were calculated by the Marquardt algorithm: $k 1$ and $\mathrm{k} 2$ (tissue uptake and release) $\mathrm{k} 3$ (specific binding), as well as $\mathrm{k} 3 / \mathrm{k} 4$ (binding potential). ATl blockade reduced the specific binding of the tracer by $\sim 80 \%$ measured both by the binding potential $(\mathrm{k} 3 / \mathrm{k} 4)$ and the model-free IRF (Table). Thus, [C-11]L-159,884 has favorable kinetic properties for PET studies of these receptors.

\begin{tabular}{|l|c|c|c|}
\hline Parameter & Control & Blockade & \% difference \\
\hline $\mathrm{k} 1$ & $0.1382 \pm 0.1071$ & $0.0832 \pm 0.0265$ & $25.22 \pm 30.22$ \\
\hline $\mathrm{K} 2$ & $0.1238 \pm 0.0475$ & $0.1581 \pm 0.0621$ & $-27.85 \pm 27.13$ \\
\hline $\mathrm{K} 3$ & $0.0500 \pm 0.0224$ & $0.0244 \pm 0.0079$ & $49.48 \pm 6.08$ \\
\hline $\mathrm{K} 3 / \mathrm{k} 4$ & $13.330 \pm 10.3523$ & $2.0979 \pm 1.0111$ & $81.75 \pm 7.32$ \\
\hline $\mathrm{KRF} 60-105$ & $0.0383 \pm 0.0407$ & $0.0056 \pm 0.0025$ & $76.28 \pm 14.14$ \\
\hline
\end{tabular}

\section{9}

$\frac{\text { M. Samal }}{1}{ }^{1}$, W. Backfxieder ${ }^{2}$, K. Kletter ${ }^{2}$, M. Kärný3 ${ }^{3}$,

"Charles University Erague, czech Republic, 2vienna General Hospital AKH, Austria, Acadeny of sciences of the Czech Republic, ${ }^{4} \mathrm{~L}$. Boltzmann Institute of Nuclear Medicine, Vienna, Austria

REPRODUCIBIIITY OF DNAMIC STRUCNURES EXTRACTED FROM DYNAMIC RENRL STUDTES BY FACTOR ANAIIYSIS

Most elinical demonstrations with factor analysis of image sequences reported until today can be characterised as feasibility studies rather than rigorous clinical as frials. Considering the comolexity of aiagnostic problems trials. Considering the complexity of diagnostic problems attempted to be solved by factor analysis, it is surprising that basic quantitative dependences of extraded factors on the temporal and spatial sampling of input data are still not known. The aim of this study is to partially fill the gap. Six high-frequency Mags renal
studies $300-400$ images $64 \times 54 \times 2$ recorded in $3-$ second intervals during $15-20$ minutes in total) have been collected and analyzed using a workstation and an automatic procedure of factor analysis. Oblique rotation of factors was performed in the spatial domain and a Bayesian procedure used to automatically control the rotation. Using this method, the date heve been analysed repeatediy while this method, the datea have been analysed repeatediy white varying input conditions systematicaliy in wide limits. The hypothesis was that the extxacted factors (dymamio structures, compartments) should be lrvariant since the input data reflect the structure and function of real parameters investigated were the time interval and region of interest to be used in the analysis, the effect of smoothing, the reduction in temporal and/ox spatial sampling, and the number of factors. The results confirmed the hypothesis over a wide range of input parameters. when comparing the factor curves with the corresponding curves obtained from standard RoIs the main difference Found was that the factor curves appmoached aero at the same time when the factor structures lost their contre same time when the factor structures lost their contrast againt background. The conclusion is that the reproducibility of factors confin both thetr usefuhness ith clinical studies and their physiological meaning. Additional facts produced by the experiment help to underatand the interpretation of factors, predict their appearance, and formulate more accurate advice for users.

\section{0}

E. Oczeretko, W.Skarbek*, F.Rogowski, A.Pietrowcew*, T.Budlewski.

Department of Nuclear Medicine, Medical Academy and *Institute of Informatics, Technical University, Bialystok, Poland.

FRACTAL COMPRESSION AND SMOOTHING OF SCINTIGRAPHIC IMAGES.

Fractal compression is a new method for image data encoding which competes with traditional transform coding. The Banach fixed point theory had given effective tools for the design of fast image reconstruction algorithm. We used "local patchwork" approach, based on $4 \times 4$ pixel blocks in order to estabijsh the best fit local transformation of image blocks.

We applied fractal method for compression of scintigraphic images. We analysed $37 \mathrm{liver}$ and 22 bone scintigrams (byte mode aquisition, $128 \times 128$ matrix size) before compression - group I and after reconstruction of these compressed images - group II. The program was written in C. The reduction of image data size, we have obtained, was between 7 and 12 times as a function of local search radius, chosen of affine transformations group and entropy efficiency. For optionally selected regions of interest (R0I-s $32 \times 32$ and $64 \times 64$ ) we found that the total number of counts for regions in scintigrams from group II was slightly higher than in regions from group 1 . The increase number of counts ranged from $0.017^{\%}$ to $0.26^{*} \frac{1}{4}$ for RoI-s $32 \times 32$ and from $0.06 \%$ to $0.7 \%$ for R0I-S $64 \times 64$ (the regions of interest $64 \times 64$ included areas outside of the organ).

The images from group II were smoothed and appeared better (subjective measure!) than original images. The fractal smoothing may be used as a separate option.

In conclusion, the fractal compression of scintigrams is useful because of disk space saving and the reduction of transmission time by about $90 \%$.Decompresion of images does not affect significantly their quality but their smoothing improves the visual perception. 


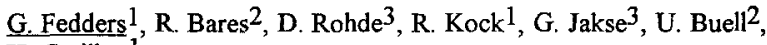
H. Greiling 1

Departments of Clinical Chemistry 1 , Nuclear $M^{2} e_{i c i n e}{ }^{2}$, and Urology $^{3}$, Technical University of Aachen, GERMANY

METABOLIC PATHWAYS OF 2-FLUORO-2-DEOXY AND 2DEOXY-D-GLUCOSE IN HUMAN PROSTATE CANCER CELLS

During the past decade fluorine-18 labelled 2-fluoro-2-deoxy-Dglucose (FDG) has been frequently used for in-vivo assessment of glucose metabolism by positron-emission-tomography (PET) Assuming that metabolization of phophorylated FDG is slow and therefore neglegible within the first 2 hours after injection, the distribution of F-18 measured by PET is usually regarded to reflect the local amount of phosphorylated FDG. The aim of our study was to check this assumption using human prostate cancer cells as model (LnCap-well differentiated/PC3-less differentiated). After serial incubation of culture plates with C-14 labelled FDG or 2-deoxy-Dglucose (DG) the time course of cellular FDG/DG accumulation was measured, and intracellular metabolites were identified by HPLC Thirty minutes after incubation significant quantities of FDG-6-P and FDG-1-P, and after 2 hours also UDP-FDG, UDP-FDGal, and FDGluconate-6-P were detected. In LnCap cells FDG-6-P increased constantly for 4 hours while in PC3 cells an early maximum was reached after 60 minutes. DG-turnover was considerably faster compared to FDG, the main products were DG-6-P and DGluconate-6-P.

The results indicate that metabolic pathways of cancer cells are highly variable which may cause underestimation of glucose metabolism if quantification is exclusively based upon static PET-scans. The observed differences between LnCap and PC3 cells can be explained by limitations of substrate transport ( $\mathrm{PC} 3$ ) emphasizing the relevance of transport mechanisms (e.g. expression of glucose transporter proteins) in addition to enzyme activities. Thus, for accurate estimation of tissue metabolism dynamic PET scanning is required

\section{2}

S. Vallabhajosula, B.R. Moyer, H. Lee,

H. Lipszyc, D.A. Bastidas, J. Shani, J. Machac, $\mathrm{J}$. Lister-James, R.T. Dean

Mount Sinai Medical Center, New York, NY and Diatech Inc., Londonderry, NH, USA.

ACUTE FOCAL INFLAMMATION (INF) MODEL IN RATS: COMPARISON OF SEVEN DIFFERENT RADIOTRACERS

INF can be induced in rats within $24 \mathrm{hrs}$ following i.m. injection of $\mathrm{E}$. Coli. This animal model has been used extensively to develop and screen radiotracers for imaging INF. The model was never evaluated with different tracers under similar conditions. We therefore compared the kinetics of accumulation and clearance of seven different radiotracers (In-111-IgG, I-125-HSA, Ga-67, In111-WBC, Cr-5i-EDTA, TC-99m-glucohepto-nate, F$18-F D G$ at $0.12,0.5,1,2$, and 4 hrs in a total of 37 groups and 5 rats/group.

$8 \mathrm{I} . \mathrm{D} \cdot \mathrm{g}$. INF

0.12 i

$\frac{0.12}{0.09} \quad \frac{1}{0} 14 \quad \frac{4 \mathrm{hr}}{0.29}$

$\begin{array}{llll}\text { IgG } & 0.09 & 0.14 & 0.29\end{array}$

$\begin{array}{llll}\text { WBC } & 0.10 & 0.14 & 0.21 \\ \text { HSA } & 0.30 & 0.47 & 0.75\end{array}$

$\begin{array}{llll}\mathrm{Ga}-67 & 0.42 & 0.68 & 0.67\end{array}$

$\begin{array}{llll}\text { EDTA } & 0.59 & 0.08 & 0.01 \\ \text { Gluco } & 0.67 & 0.09 & 0.04\end{array}$

FDG $\quad 0.38 \quad 0.50 \quad 0.42$

\begin{tabular}{lll}
\multicolumn{3}{c}{ INF/CM } \\
$\frac{0.12}{3.1}$ & $\frac{1}{5.6}$ & $\frac{4 \mathrm{hr}}{9.0}$ \\
2.2 & 2.3 & 3.5 \\
3.5 & 6.6 & 9.1 \\
2.2 & 2.4 & 3.0 \\
2.3 & 2.1 & 2.1 \\
2.8 & 2.3 & 2.4 \\
2.6 & 3.9 & 2.5
\end{tabular}

The $\%$ I.D. $/ \mathrm{g}$ in the INF tissue for IgG, WBC, HSA and Ga-67 showed a gradual increase with time while EDTA and Gluco showed a rapid decline. In control muscle (CM) there was no gradual increase with IgG, WBC and HSA. The INF/CM ratio also showed a gradual increase for IgG, WBC and HSA while there was not much change for other tracers. FDG showed the highest INF/blood ratio compared to all other tracers. This model may be compared to all other tracers. This model may be The kinetics of uptake and clearance at the site of INF are more important than INF/CM ratio. Due to increased capillary permeability and edema at the site of INF, cautious interpretation of the data is criticai to distinguish specific from non-specific radiotracers.
203

V.S.Skuridin, V.I.Chernov, G.G.Glukhov, N.G.Krivonogov, Yu.B.Lishmanov, A.I.Komov

Insitute of Nuclear Physics and Institute of Cardiology, Tomsk, Siberia, Russia

LOW-ENERGY CYCLOTRON PRODUCTION AND ORGAN DOSIMETRY OF SHORT-LIVING THALLIUM-199

Thallium still remains unbeatable isotope for myocardial perfusion scintigraphy, particularly for the diagnosis of myocardial viability, and gains new oncological applications. Newertheless, its long physical and biological half-life times as well as high radiation dose make impossible the usage of this isotope for frequent follow-up studies and control of myocardial perfusion under surgical or medical treatment. Thus we have developed a technique for low-energy cyclotron production of Thallium- 199 chloride $(\mathrm{T} 1 / 2=7.4 \mathrm{hr})$, compared its technological parameters with Thallium-201 and tested biokinetics and dosimetry of the short-living radionuclide.

Thallium- 199 has been produced by $\mathrm{Au}(\mathrm{He}, 2 \mathrm{n}) \mathrm{Tl}-199$ reaction using low-energy cyclotron U-120 with the yield of $19.6 \mathrm{MBq} / \mathrm{mkA} / \mathrm{hr}$. Cost of the isotope dose production per study was 4.4 times cheaper than an equivalent one for the Thallium-201. Biokinetics did not differ significantly between T1-199 and Tl-201 either in rabbits or in clinical testing. Organ dosimetry of Tl-199 had been calculated by MIRD technique and was as follows (in rads):

$\begin{array}{lcc} & \mathrm{Tl}-199,185 \mathrm{MBq} & \mathrm{Tl}-201,74 \mathrm{MBq} \\ \text { Whole body } & 0.55 & 4.7 \\ \text { Testes } & 0.34 & 2.5 \\ \text { Ovaries } & 0.76 & 11.0 \\ \text { Bone Marrow } & 0.73 & 8.9 \\ \text { Myocardium } & 1.73 & 38\end{array}$

We conclude that Thallium-199, which gives equivalent clinical information to that of $\mathrm{Tl}-201$, has more reliable technoeconomic characteristics and provides lower radiation doses, and so makes possible repeat follow-up myocardial pertusion studies with physiological tracer.

204

S. Czekalski, T.Miazgowski

Dept. of Endocrinology and Metabolic Diseases, Pomeranian Academy of Medicine, Szczecin, Poland.

RADIOIMMUNOLOGICAL DETERMINATION OF SERUM OSTEOCALCIN IN THE DIAGNOSIS OF OSTEOPOROSIS.

The aim of the study was an evaluation of serum osteocalcin (GLA) determinations in the diagnosis of osteoporosis (OP). The study was performed on the random sample of 653 individuals of Szczecin population (341 women, 312 men), age 40-80 yrs. Gl.A was measured by RIA using Incstar Corporations kits (normal range: $4.2 \pm 1.8 \mathrm{ng} / \mathrm{ml}$. Two X-ray pictures of both thoracic and lumbar spine were taken in the lateral position in all examined subjects and evaluated for $O P$. The diagnosis of $O P$ was based on the following radiological criteria: 1) rarefaction of bone structure appearance of strations on the vertebra, 2) intracorpora intussepsception of upper of both limiting membranes of vertebrae, 3) wedge-shape, compression or biconcave deformities of vertebrae. OP was diagnosed in 193 women $(56.6 \%)$ and 105 men $(33.7 \%)$ and the prevalence was parallel to the age of subjects in both sexes. Serum GLA was significantly higher in women than in men $(3.7 \pm 1.9 \mathrm{ng} / \mathrm{ml}$ vs $3.3 \pm 1.6 \mathrm{ng} / \mathrm{mI}, \mathrm{p}<0.005)$. Women with OP had slightly higher GLA than women without OP $(3.8 \pm 1.8 \mathrm{ng} / \mathrm{ml}$ vs $3.5 \pm 2 \mathrm{ng} / \mathrm{ml}, \mathrm{n} . \mathrm{s}$.) but in the subgroup of women in perimenopausal period (45-55 yrs) GLA was significantly higher in women with OP than without 'OP $(p<0.05)$. The latter was similar in men: GLA was elevated in men with OP when compared without $O P(3.7 \pm 1.8 \mathrm{ng} / \mathrm{ml}$ vs $3.1 \pm 1.5 \mathrm{ng} / \mathrm{ml}, \mathrm{p}<0.005)$. In conclusion, serum GLA determinations may be useful in screening evaluation of bone remodelling in osteoporotic individuals, especially in men and perimenopausal women with diagnosis of OP. 
205

M. Martin, P. Blockx

Department of Nuclear Medicine, Antwerp University Hospital, Wilrijkstraat. 10, 2650 Edegem, Belgium

THREE DIFFERENT RADIOTMUNOASSAYS (RIA) FOR THE DETERMINATION OF INSULIN-LIKE GROWTH FACTOR-I (IGF-I) .

Three RIA's for the determination of IGF-I were compared analytically and clinically: IGE-I Nichols Institute (NICH), SM-C-RIA-CT Medgenix Diagnostics (MX) and IGF-I Mediagnost GmBH (MD). NICH (double antibody liquid RIA) was agerformed without any (double antibody liquid RIA) was performed without any extraction in order to obtain
only free IGF-I. The procedure includes a predilution, 6 pipetting steps, an overnight incubation and a centrifugation/aspiration step. MX and MD determine as well free as bound IGF-I, MX by an acid-ethanol extraction and MD by blocking IGF-I binding sites by IGF-II. MX (coated tube RIA) needs 4 pipetting and 1 centr, step for the acid-ethanol extraction, an overnight incub, and one wash cycle. MD (double AB liq. RIA) uses an acidic dilution, 3 pipetting steps and a 2 days+1hr incub.. The centr./asp. step has to be periormed 2x to obtain better precision.

Data reduction was performed with linear smothed spline.

Data reduction was performed with linear smoothed splin.

Curve fitting performance for $\mathrm{NICH}$ and $\mathrm{MX}$ revealed no problems (iterations 1, variance ratio o). For MD curve the patient samples (duplicates) had a within-assay the patient samples (duplicates) had a within-assay
precision (CV) <10\%, for MX and MD resp. $91.7 \%(33 / 36)$ and $90.6 \%(29 / 32)$ of the results had $C^{\circ} \mathrm{s}<10 \%$.

Clinical correlation between $M X$ and $M D$ showed $a r=1.00$ with a slope of 0.79 . Correlation factors between $\mathrm{MX} / \mathrm{NICH}$ and $\mathrm{MD} / \mathrm{NICH}$ were both 0.93 with resp. slopes of 0.15 and 0.19 . All results were classified according to the method specific reference ranges, matched for sex and age. For $26 / 36$ patients the same clinical information was obtained differences in clinical classification were caused only due to borderline differences.

borderline differences. Although the kits evaluated use totally different methodologies, the obtained clinical information is very simi lar. NICH is analytically very good, but the procedure for this direct method (free IGF-I) is rather labourious. $M X$ and $M D$ (freetbound IGF-I) have similar precision profiles. Nevertheless, $\mathrm{MX}$ was preferred due to a better calibration and a more user-friendly procedure.

206

D. Sorger, W. Krüger, L. Otto, R. Kluge, U. Junhold, and W. H. Knapp

Department of Nuclear Medicine, University of Leipzig, Germany

BONE SCINTIGRAPHY AND THE DIAGNOSTIC VALUE OF IMMUNORADIOMETRIC MEASUREMENT OF ALKALINE PHOSPHATASE BONE ISOENZYME IN SUSPECTED METASTATIC DISEASE

The introduction of new methods for biochemical parameters of bone related processes necessitates reevaluation of the diagnostic regimen in bone metastases. We present a study initiated by the question whether the new radioimmunological test for bone specific alkaline phosphatase (BAP) yields additional or more adequate information than routine bone scintigraphy in patients with breast or prostatic cancer.

For 130 post-operative patients ( 95 breast and 35 prostatic cancer) the resuits of two different methods for the determination of BAP were compared: photometric determination after wheat germ precipitation (Boehringet Mannheim) and two site immunoradionetric assay ("Ostase", Hybritech). Bone scans were recorded simultancously $99 \mathrm{~m}_{\mathrm{Tc}}$-HMDP, whole body scanning anterior and posterior views, Siemens-Bodyscan). Patients with bone lesions were $X$ rayed and followed-up for one year.

Results: 62 patients were free of any scintigraphic lesions, 29 had increased focal activity accumulation caused by degenerative processes and 39 patients had lesions caused by solitary or multiple metastases. The information content of the biochemical tests was determined using ROC diagrams. With a $90 \%$ specificity both tests had a sensitivity of $60 \%$. The results of both determinations showed a significant correlation ( $r=0.85, \mathrm{p}<0,01)$. The optimal threshold for differentiating patients with and without metastatic disease was found to be $25 \mathrm{ng} / \mathrm{ml}$ for the immunoradiometric assay.

We conclude that both methods of BAP determination provide similar results and that neither method offers the potential to substitute bone scintigraphy in primary diagnosis of metastatic disease, due to limited accuracy. The role in therapy remains to be defined.
207

T. Martins, L. Oliveira, A. I. Santos, G. Cantinho, J. Colarinha

Instituto de Medicina Nuclear, Faculdade de Medicina de Lisboa, Universidade Clássica de Lişboa, Lisboa, Portugal

ATTEMPT TO ESTABLISH ULTRASONOGRAPHIC PATTERNS OF RENAL SCARS BASED UPON THE TC-99m DMSA RENAL SCAN

The aim of our stady was to establish ultrasonographic (US) criteria that would allow the diagnosis of renal scars (RS), using the Tc-99m DMSA scan as the gold standard.

We selected a group of 33 children with DMSA scans positive for RS (decreased uptake with contour abnormality), who had also undergone US. They had previously diagnosed urinary tract infection or a prenatal diagnosis of dilatation of the pelvis and calices. The group consisted of 23 females and 10 males, whose ages ranged from 3 months to 16 years (<age $>=5,5 \pm 4,1$ years). The renal scan was performed 2 hours after the injection of a weight related dose of Tc $-99 \mathrm{~m}$ DMSA, with a $256 \times 256$ matrix and an acquisition time of 180 seconds, under 3 projections (posterior; left and right posterior obliques) Results obtained with the 3 projections and with the posterior projection alone were compared.

US was performed on the same day, using a 3,5 MHz transducer. Four US criteria were tested: irregular cortical margin, cortical thinning, focal calyceal dilatation and hyperechoic cortical foci.

62 RS in 46 renal units were detected by the 3 projection DMSA scan. 23 of them corresponded to normal US studies; $14(23 \%)$ had 1 positive criterium (focal calyceal dilatation was the most frequent); $14(23 \%)$ had $2 ; 8(13 \%)$ had 3 and $3(4 \%)$ had 4 . Cortical thinning (28\%), irregular cortical contour $(27 \%)$ and focal calyceal dilatation ( $26 \%$ ) were the criteria most frequently found.

The posterior projection of the DMSA scan alone revealed 48 RS $(77 \%)$.

The average number of positive US criteria per RS was 1,3 . If we consider US studies with 2 or more positive criteria (above the average) as being suspect of RS, we find that only $40 \%$ of the US studies would be classified as abnormal. Although calyceal dilatation was the most common finding when only 1 positive criterium was present, it was not the most frequent when the whole group was considered.

We conclude that US, besides having an already known low sensitivity in detecting RS, does not even have a reliable pattern of findings that allows the establishment of levels of suspicion. Renal DMSA scan in only 1 projection (posterior) was still far better than US (77\% of RS detected).

\section{8}

M.R.Castellani, M.Marra ${ }^{\circ}$, L.Maffioli, M.Gasparini, G.L.Buraggi.

Nuclear Medicine Div. National Cancer Institute - Milan ${ }^{\circ}$ University of Milan. Italy.

\section{Tc-99m-MAG3 SCINTIGRAPIIIC DIAGNOSIS OF CONGENITAL RENAL DAMAGE DUE TO VESICOURETERAL REFLUX (VUR).}

Tc-99m-MAG3 scan allows a good renal imaging in neonates and provides a lower dose than Tc-99m-DMSA. Aim of the study is to verify the clinical usefulness of MAG3 scan in evaluating the renal damage in babies with congenital VUR before the onset of upper tract infections (UTI)

34 neonates $(26 \mathrm{M} / 8 \mathrm{~F})$ with prenatal echographic diagnosis of renal pelvic dilation were perspectively studied with cystography, MAG3 scan, urography and urino cultures, during the first month of life. 7 pts with VUR associated with other urinary malformations or obstructive uropathy were excluded. 27 pts with primary monolateral (13) or bilateral (14) VUR have been evaluated for a total of 41 refluxing renal units (RRU). Antibiotic prophylaxis was sistematically performed from VUR diagnosis.

Urino cultures were negative in all patients.

Renal damage was quantified by relative uptake (RU) at $2 \mathrm{~min}$. from i.v. injection of $20 \mathrm{MBq}$ of Tc-99m-MAG3. Kidneys with uptake of at least $43 \%$ were considered normal, those with uptake between 43 and $25 \%$ slightly damaged, and those with uptake of less than $25 \%$ severely damaged. Main results were correlated with VUR grade:

\begin{tabular}{|l|c|c|c|}
\hline VUR grade & $\mathrm{RU} \geq 43 \%$ & $42 \%<\mathrm{RU} \geq 25 \%$ & $\mathrm{RU}<25 \%$ \\
\hline I-III & $13(100 \%)$ & - & - \\
\hline IV & $8(53 \%)$ & $5(34 \%)$ & $2(13 \%)$ \\
\hline V & $2(15 \%)$ & $5(38 \%)$ & $6(46 \%)$ \\
\hline
\end{tabular}

So, MAG3 scan allowed the identification of primitive renal damage in $18 / 41(44 \%)$ RRU before the onset of UTI within the first month of life. 
209

A Garcia-Burillo, I Roca, S Aguadé, F Porta, F Castelló", Vilaplana E*, J. Castell, D. Ortega, FM Domènech-Torné Servei de Medicina Nuclear. Servei de Lactants.

Hospital Universitàri Vall d'Hebron*. BARCELONA

EVOLUTION OF ACUTE PYELONEPHRITIS IN INFANTS UNDER 2 YEARS: FOLLOW-UP DMSA STUDIES

DMSA scintigraphy is useful for the diagnosis of acute pyelonephritis (APN). Infants less than 24 months old are a special interest group due to a higher probability of kidney damage during an urinary tract infection, and to a relatively high incidence of APN. This study tries to increase our knowledge about the evolution of the acute pyelonephritis in these young children.

DMSA scans performed in 29 children during the APN and 12 months after have been analyzed (persistence, improvement or normalization of the hypoactive area(s), variation in differential function, grade of vesico-ureteric reflux).

\begin{tabular}{l|ccc|cc|c|}
\cline { 2 - 7 } $\begin{array}{l}\text { DMSA scan } \\
12 \text { months } \\
\text { follow-up }\end{array}$ & $\begin{array}{c}\text { Focal Multifocal } \\
\text { hypoactive } \\
\text { areas }\end{array}$ & $\begin{array}{c}\text { Abnormal } \\
\text { diffuse } \\
\text { uptake }\end{array}$ & \multicolumn{2}{|c|}{$\begin{array}{c}\text { VUR } \\
=\langle\|\end{array}=$} & TOTAL \\
\hline persistance & 5 & 2 & 7 & 8 & 6 & 14 \\
improvement & 1 & 2 & 4 & 6 & 1 & 7 \\
normalization & 4 & 1 & 3 & 6 & 2 & $\mathbf{8}$ \\
\hline
\end{tabular}

Results show disappearance of hipoactive areas in 8 cases, improvement in 7 and persistence of the abnormality in 14 children. When the presence or not of VUR is analyzed, a higher percentage of bad evolution is found $166 \%$ persistance of the hypoactive area in case of VUR $>=$ III. $40 \%$ in case of VUR $=<$ II). When the VUR is $>=$ III, the probability of persistance of the hypoactive area seems to be higher. DMSA scan is a useful technique to value the evolution of APN in young children, and can contribute to better understand the natural history of this lesions.

\section{0}

P.Flamen, A.Willikens, A. Vanderfaeillie, B. Desprechins, A. Piepsz.

Departments of Nuclear Medicine, Paediatrics and Paediatric Radiology, Free University of Brussels (AZ VUB), Brussels, Belgium.

HOW USEFUL IS Tc99m-DIMERCAPTOSUCCINIC ACID (DMSA) RENAL SCINTIGRAPHY IN CASE OF FIRST URINARY TRACT INFECTION IN CHILDREN OLDER THAN 5 YEARS

It has been suggested that children over 5 years of age with a first documented urinary tract infection (UTI) are unlikely to develop renal lesions and therefore do not require scintigraphic investigations in case of normal renal ultrasound. The aim of the present work was to evaluate, in this category of patients, the frequency of the scintigraphic abnormalities.

Ninety-one children older than 5 years were found in a database of 261 patients who underwent, between January 1991 and December 1992 , DMSA scintigraphy for suspected UTI. The clinical files of those children were closely examined by two experienced paediatricians. Patients with incomplete file data, a history of previous UTI, known obstructive or reflux uropathy were excluded. Twenty-three patients remained who underwent both renal ultrasound and DMSA scintigraphy during the acute phase of a first culture-documented febrile UTI.

Among these 23 patients, cortical defects were found on DMSA scintigraphy in 14 children $(61 \%)$. Follow-up scintigraphy, performed 2 to 12 months after the first one, revealed, in 7 children complete healing or considerable improvement of the defects, confirming that the UTI affected a previously normal kidney. In 4 patients, a small deformed kidney, obviously existing, but unsuspected before the present UTI, was detected on the first scintigraphy and the lesions remained unchanged on follow-up scan. One child developed new lesions due to a second episode of pyelonephritis. Two children with focal renal lesions on DMSA scan had no follow-up scan. Renal ultrasound examination was entirely normal in the 9 patients with normal DMSA images but also in 6 out of the 14 patients showing scintigraphic abnormalities.

In conclusion, although the incidence of a first UTI is low in children older than 5 years, the frequency of scintigraphic abnormalities is comparable to what is found in younger age groups; part of them are clearly related to the current infection. In this age group, a strategy based exclusively on ultrasound examination would miss a significant amount of abnormal kidneys.
211

C. De Sadeleer, F. Keuppens, A. Piepsz.

Departments of Nuclear Medicine and Urology,

Free University Brussels (AZ VUB), Brussels, Belgium.

PREDICTION OF THE RESPONSE TO FUROSEMIDE IN CHILDREN WITH NORMAL RENAL UPTAKE.

Experimental partial ureteral obstruction can lead to reduced GFR in the weeks following surgery, the intensity of this reduction being dependent on the severity of the stenosis. After this transitory period, the function remains stable, suggesting that patients with congenital hydronephrosis (HN) are not at high risk of further degradation of the function. Similarly, it is logical to think that a preserved function, in a patient with congenital HN, is not suggestive for marked obstruction and one might therefore expect a good renal emptying after furosemide. The aim of the present work was to evaluate if a normal GFR on the site of the affected kidney can predict a good response to furosemide.

During the last 4 years, we performed $348 \mathrm{Tc}-99 \mathrm{~m}$ MAG3 pediatric studies because of $\mathrm{HN}$ or vesico-ureteral reflux (VUR). The separate GFR (SGFR) was estimated by combining the left to right MAG3 early renal uptake and the overall GFR obtained with Cr-51 EDTA. The response to furosemide was evaluated quantitatively by measuring the residual renal activity (RA) in percentage of the pre-furosemide value. Among the 348 studies, 202 were not followed by a furosemide test. either because the drainage was already satisfactory at the end of the 20 minute renographic study, or because the main purpose of the examination was the detection of VUR by means of indirect cystography. Among the 146 studies (291 kidneys) completed by a furosemide challenge, 139 corresponded to unilateral $\mathrm{HN}$ and 7 to bilateral HN. SGFR was considered as normal in 92 kidneys and abnormal in 61 . In the group with normal SGFR (age between 1 month and 12 yrs), 90/92 kidneys showed good renal emptying after furosemide (highest RA 40\%). In the group with abnormal SGFR (age between 1 month and $20 \mathrm{yrs}$ ), the RA after furosemide was less than $40 \%$ in 47 kidneys, between 40 and $60 \%$ in 7 kidneys and, in the rermaining 7 , more than $60 \%$, corresponding to poor emptying.

In conclusion, a normal SGFR is highly predictive for a good response to furosemide.

\section{2}

N. Deriaz, A Boubaker, A. Bischof Delaloye, D. Berger, N. Genton, B. Delaloye

Dpt of Nuclear Medicine and Pedriatric Surgery, Centre Hospitalier Universitaire Vaudois, Lausanne, Switzerland

PROGNOSTIC VALUE OF RENOGRAPHY IN INFANTS WITH PRENATALLY DETECTED URINARY TRACT DILATATION.

The aim of this study was to determine if the renal function established by renography with I-123 hippuran within the first three months of life was of any prognostic value in children with urinary tract dilatation detected prenatally.

We have studied 51 children (39 boys, 12 girls) with dilatation of 72 renal units. A total of 180 studies were performed, starting 4-110 (mean 36) d after birth and followed up to $5 y .5$ studies (3\%) were excluded because of incomplete injection. 16 presented unilateral (10) or biTateral (6) lower (LJ0), 23 unilateral (20) or bilateral (3) upper (UJO) urinary junction obstruction. 10 boys had valves of the posterior urethra and 2 bilateral vesicoureteral reflux (VUR). The table shows renal units ( $n$ ) with normal (N) or decreased (D) function according to the type of malformation as well as the outcome after treatment.

\begin{tabular}{lllccc} 
& & \multicolumn{2}{c}{ diagnosis } & \multicolumn{2}{c}{ follow-up } \\
VUR & $n$ & N & D & N & D \\
LJ0 & 04 & 04 & 00 & 04 & 00 \\
UJ0 & 22 & 16 & 06 & 19 & 03 \\
Valves & 26 & 12 & 14 & 16 & 10 \\
& 20 & 10 & 10 & 09 & 11
\end{tabular}

Function was most impaired in UJ0 and valves, response to treatment was favorable in $4 / 14(29 \%)$ UJo, but not in valves. Highly impaired ( $<30 \%$ of normal) function did never return to normal, whereas function of previousty normal kidneys only exceptionnally decreased later on. 


\section{3}

P. Flamen, P. Dendale, L. Kaufman, A. Bossuyt, P.R. Franken. Departments of Nuclear Medicine, Cardiology and Biostatistics, Free University of Brussels (AZ VUB), Brussels, Belgium.

\section{NON-INVASIVE DETECTION OF SEVERE CORONARY ARTERY} DISEASE AFTER UNCOMPLICATED MYOCARDIAL INFARCTION

The aim of the study was to examine the ability of the simultaneous assessment of left ventricular function and myocardial perfusion using one single injection of Tc99m Sestamibi, to identify high risk patients with left main, proximal LAD and three-vessel coronary artery disease (CAD) after an uncomplicated acute myocardial infarction (AMI).

Combined first-pass radionuclide angiocardiography (RNA) and myocardial perfusion SPECT at rest and during submaximal exercise were performed in 52 patients, less than 6 weeks after an uncomplicated AMI, using Tc99m Sestamibi. Patients were classified in two subgroups according to the presence of left main, proximal LAD or three-vessel CAD. Stepwise logistic regression analysis was used to determine the independent predictors of severe CAD.

All patients underwent the exercise testing without any medical complication. On multivariant analysis the wall motion score during exercise was the only independent predictor for the presence of severe CAD ( $\mathrm{p}<0.001, \mathrm{r}=0.6$ ). Analyzing patients with anterior AMI separately, LV EF at submaximal exercise was the most accurate predictive parameter. If a cut-off value of $40 \%$ is chosen, the LV EF at exercise had a sensitivity of $85 \%$ and a specificity of $78 \%$ for the detection of severe CAD. In patients with inferior AMI, LV EF, wall motion nor myocardial perfusion scores were useful to differentiate the two subgroups. In these patients the presence of an additional perfusion defect in one of the anterior wall segments yielded a sensitivity of $70 \%$ and a specificity of $75 \%$ for the presence of severe CAD.

In conclusion, simultaneous evaluation of LV function and myocardial perfusion at submaximal exercise, using one single injection of Tc99m-Sestamibi, is a safe and accurate technique to select patients with severe CAD after an uncomplicated AMI. In patients with anterior AMI the best parameter for selection is the global LV EF at exercise. In patients with inferior AMI an additional perfusion defect in the noninfarcted anterior wall yields the highest predictive accuracy.

214

E. Voth, F.M. Baer ${ }^{1}$, H.J. Deutsch ${ }^{1}$, C.A. Schneider ${ }^{1}$, R. Wagner ${ }^{2}$ K. Wienhard ${ }^{2}$, U. Sechtem 1 , H. Schicha

Depts. of Nuclear Medicine and 1 Internal Medicine III, University of Cologne, 2MPI for Neurological Research, Cologne, Germany

18-FDG-PET AND LOW-DOSE DOBUTAMINE TRANSESOPHAGEAL ECHOCARDIOGRAPHY IN THE ASSESSMENT OF INFARCT REGION RELATED MYOCARDIAL VIABILITY

To assess residual viable myocardium, 40 patients with chronic myocardial infarction (infarct age $\geq 4$ months) and regional akinesia or dyskinesia by ventriculography underwent 18-FDG positron emission tomography (PET) after oral glucose load and transesophageal echocardiography (TEE) at rest and during lowdose dobutamine infusion $(5,10 \mu \mathrm{g} / \mathrm{kg} / \mathrm{min})$. A standardised 26 segment model consisting of 3 short axis tomograms and a transverse midventricular long axis tomogram was used for analysis of FDG-uptake and wall motion in corresponding myocardial regions. Segments with an FDG-uptake $\geq 50 \%$ of the maximum FDG-uptake and with a dobutamine induced wall motion were graded viable by PET and TEE, respectively.

PET diagnosed residual viability within the infarct region in 25/40 $(63 \%)$, TEE in $21 / 40(53 \%)$ patients. Diagnostic agreements between the 2 techniques was $90 \% .235$ of totally analysed 1040 segments were akinetic or dyskinetic by TEE at rest. Within these 235 segments myocardial viability was graded concordantly by PET and TEE in $89 \%$ of segments. Compared to FDG-PET defined viability, positive predictive accuracy of dobutamine-TEE was $81 \%$ and negative predictive accuracy $97 \%$. FDG-uptake in segments with a dobutamine induced contraction reserve $(68 \pm 11 \%)$ was significantly higher $(p=0.001)$ compared to segments remaining akinetic or dyskinetic during dobutamine infusion (45 $\pm 9 \%$ )

Compared to the "gold standard" FDG-PET, dobutamine-TEE yields a good correspondence. Like reinjection or resting scintigraphy with 201-TI, dobutamine-TEE may offer an alternative to FDG-PET for the assessment of infarct region related myocardial viability.
215

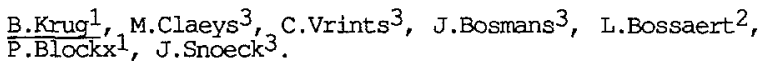

Departments of Nuclear Medicine ${ }^{1}$, Intensive Care ${ }^{2}$ and Cardiology ${ }^{3}$. Antwerp University Hospital, BEIGIUM.

ADENOSINE TECHNETIUM-99M SESTAMIBI SPECT FOR THE EARLY ASSESSMMENT OF JEOPARDIZED MYOCARDIUM AFTER ACUTE MYOCARDIAL INFARCTION

The purpose of this study was to evaluate the accuracy of adenosine TC-99m sestamibi SPECT in the detection of jeopardized myocardium early after acute myocardial infarction.

Coronary arteriography and myocardial scintigraphy were performed early after myocardial infarction in 41 consecutive patients with an uncomplicated myocardial infarction. Tc-99m sestamibi SPECT was performed both at rest and after IV adenosine infusion $(140 \mu \mathrm{g} / \mathrm{kg} / \mathrm{min}$ during $6 \mathrm{~min}$, in $40 \mathrm{ml}$ saline). The SPECT-jmages were analyzed semiquantitatively using a 17 segment model. A reversible perfusion defect in the infarct region occured in 23 patients (56 \%) and was almost solely observed in the presence of jeopardized myocardium [Myocardium was considered jeopardized if the successfully reperfused infarct region was supplied by a residual significant coronary artery stenosis $(>50$ \%). Jeopardized myocardium was present in $24\left(\begin{array}{ll}58 & \%\end{array}\right)$ patients].

Adenosine TC-99m sestamibi SPECT had a sensitivity of $87 \%$, a specificity of $88 \%$ and a accuracy of $88 \%$ for the detection of jeopardized myocardium. Adenosine TC-99m sestamibi SPECT correctly identified 100\% $(20 / 20)$ of patients with single vessel disease, and $75 \%(12 / 16)$ of patients with multivessel disease.

These results suggest that adenosine $\mathrm{Tc}-99 \mathrm{~m}$ sestamibi SPECT is a fairly accurate non-invasive method for detecting jeopardized myocardium and may be a valuable non-invasive test for the early selection of high risk patients surviving acute myocardial infarction.

\section{6}

L. Mortelmans*, A. Maes*, J. Nuyts*, C. Schiepers*, M. De Roo*, H. De Geest ${ }^{* *}$, A. Verbruggent, F. Van de Werf*

Depts. of $\left({ }^{*}\right)$ Nuclear Medicine, $\left({ }^{*}\right)$ Cardiology and $\left(^{+}\right)$ Radiopharmacy, U.Z.Gasthuisberg, K.U. Leuven, Belgium.

STUDY OF THE NO REFLOW PHENOMENON BY PET WITHIN 24 hours AFTER SUCCESSFUL THROMBOLYSIS.

To determine whether a patent coronary artery correlates with restoration of myocardial tissue perfusion after successful thrombolysis, blood flow (BF) measurements using $13 \mathrm{~N}-\mathrm{NH} 3$ were performed in 11 patients (pts) within 24 hrs after onset of infarction.

All patients received thrombolytic therapy within 4 hrs after onset of symptoms. Coronary angiography was performed at $90 \mathrm{~min}$ and at 5 days. Only pts with a TIMI-3 grade patency at 90 minutes were selected. Patients underwent a control flow (N-13 NH3) and metabolic (F-18 FDG) study 5 days and 3 months later. In 8 out of 11 pts, only a moderate decrease of BF was found initially $(71 \pm 8 \mathrm{ml} / \mathrm{min} / 100 \mathrm{~g})$ of whom 5 showed preservation of BF $(73 \pm 9 \mathrm{ml} / \mathrm{min} / 100 \mathrm{~g})$ and metabolism during follow-up. In 1 patient PTCA was performed because of a mismatch pattern at 5 days resulting in improvement of $\mathrm{BF}$ and metabolism. in 2 pts, a decrease of $B F(35 \pm 9 \mathrm{ml} / \mathrm{min} / 100 \mathrm{~g})$ and metabolism was found at 5 days of whom 1 showed reocclusion. On the contrary, in 3 out of 11 pts, a severe reduction of BF $\left(31_{ \pm} 7 \mathrm{ml} / \mathrm{min} / 100 \mathrm{~g}\right)$ was present in the acute stage although the infarct related coronary artery was patent. Control studies in 2 of those 3 pts showed persistently reduced $\mathrm{BF}(32 \pm 6 \mathrm{ml} / \mathrm{min} / 100 \mathrm{~g})$ and metabolism compatible with necrosis. Having a mismatch pattern at 5 days, and a critical stenosis, the third patient underwent a PTCA resulting in a restoration of $\mathrm{BF}(69 \mathrm{ml} / \mathrm{min} / 100 \mathrm{~g})$ and metabolism measured after 3 months.

We conclude that persistent flow after thrombolytic therapy, as shown by acute coronary angiography, is not always associated with optimal tissue perfusion. In patients showing 'no reflow' at $24 \mathrm{hrs,}$ flow/metabolic PET studies are helpful to identify viable tissue. 
217

G. Demonceau, C. Van Hove, J. De Vuyst, D. Bladt, S. Dierickx

St Elisabeth Hospital, Zottegem, Belgium

DETECTION OF MYOCARDIAL INFARCTION BY GATED BLOODPOOL TOMOGRAPHY

Tomoscintigraphy of myocardial parenchyma is highly sensitive but poorly specific as to the detection of infarction. We have compared the usefulness of gated tomography of the heart cavities (4D-angio) to that of TcMIBI tomography (tomo-MIBI) for that condition.

Both investigations were performed at 2-day intervals in 80 patients (acute infarction (50\%), heart failure, angina, dyspnea and valvulopathy). 4Dangio was performed after in vivo labelling of red blood cells with about 1300 Mbq of Tc-pertechnetate. Acquisition was obtained using a 3-head camera (TRIAD) in about 15 minutes. Image treatment was done by automatic segmentation in 4 cavity dimensions. Contraction amplitude was obtained by the "slope method", whereas zones with abnormal phase were identified by comparison with a normal population and the lesions being shown in a Bullseve projection. Only those zones showing concordant pathology in phase and in amplitude were considered positive. Tomography of the parenchyma was obtained $60 \mathrm{~min}$. after injection of $740 \mathrm{MBq}$ of Tc-MIBI and comparison with a normal population (CEQUAL program) was projected as Bullseye. For each test, the lesions found were compared both visually and quantitatively (extent, depth, arterial territory). The existence of a history of infarction was based on clinical data, chemical results and ECG.

Results:

\begin{tabular}{|c|c|c|c|c|c|}
\hline & & \multicolumn{2}{|c|}{ Tomo-MIBI } & \multicolumn{2}{|c|}{ 4D-ANGIO } \\
\hline & & + & E & + & - \\
\hline $\begin{array}{l}\text { Infarcti } \\
\text { on }\end{array}$ & + & 37 & 3 & 26 & $\overline{14}$ \\
\hline & - & 28 & 12 & 6 & 34 \\
\hline
\end{tabular}

4D-angio appears as less sensitive but much more specific than tomo-MIBI, and its accuracy is higher ( $75 \%$ vs $61 \%$ ). False negatives are mainly small inferior infarction, whereas the false positives in Tomo-MIBI were mainly due to attenuation problems.

In the conditions of our study, $4 \mathrm{D}$-angio appears as highly superior to tomo-MIBI for the detection of myocardial infarction.

218

D. Le Guludec, MC. Peker, M. Kebbou, A. Petiet, M. Faraggi, JL. Moretti, L. Hamidi, A. Meulemans.

Laboratoire de Biophysique, Fac. Xavier-Bichat, Paris, France.

TWO-STEP TARGETING OF EXPERIMENTAL INFARCTION WITH STREPTAVDINE-ANTIBODY AND RADIOLABELED BIOTIN.

Because the schedule of early investigation in acute myocardial infarction (MI) is critical, a realistic strategy for the assessment of the infarcted area with In-111 antimyosin-antibody (AM) would imply an immediate injection of cold AM in intensive care unit. Then tracer administration and imaging could be delayed after emergency therapy. Moreover, such a two-step targeting could improve the signal to noise ratio, since liver uptake is critical. The streptavidine (Sav)-biotine (B) system is interesting because of the high affinity binding of B to Sav, and the small size of the labelled ligand. The myocardial uptake (autoradiography), the biodistribution, and the image quality of two different AM were evaluated: In-111DTPA-Fab (Myoscint, Centocor), and two-step Fab' ${ }_{2}$-Sav followed by in-111DTPA-B. A MI was induced in 25 male rats by left descending coronary artery tying. $24 \mathrm{~h}$ after surgery, 15 rats were injected with Myoscint (G1, $10 \pm 1 \mu \mathrm{g})$, and 10 rats with the conjugated Fab' $2(\mathrm{G} 2,11 \pm 2 \mu \mathrm{g}$ ), followed by in-111-B injection $24 \mathrm{~h}$ later. Anterior 5 minutes-images were acquired at $6,12,24,48 \mathrm{~h}$ on a gamma camera with a pin-hole collimator. Biodistribution and autoradiography were performed at the same times.

Results. A better delineation of the infarcted area was obtained on images of $G 2$ since the liver was not visualized. The specificity of myocardial uptake in the infarcted area was confirmed by quantitative autoradiographic data. In $\mathrm{G} 2$, the counts in normal myocardial area were not significantly different than noise. The best blood to myocardial ratio was obtained at $48 \mathrm{~h}$ of the tracer injection for $\mathrm{G} 1$ and at $12 \mathrm{~h}$ for $\mathrm{G} 2(0.52 \pm 0.05$ vs $0.49 \pm 0.07$, ns). Aithough absolute myocardial uptake was 8.2 times lower in $G 2$ vs $G 1$, noise was 55 times lower resulting in an increase of 6.9 times in the signal / noise (heart / fiver) ratio for the conjugated $A M$ $(0.19 \pm 0.02$ in $\mathrm{G} 1$ vs $1.31 \pm 0.2$ in $G 2, p<0.0001)$.

Conclusion: two-step targeting of infarction with AM and Sav-B system allows delayed injection of the tracer and imaging, which is crucial in this clinical situation, and improves image quality.
219

GS Limouris, V Giannakopoulos, N Toubanakis, $A$ Stavraka

Nuclear Medicine Section, Areteion Univ Hosp, Athens

\section{In-111-PENTETREOTIDE, Tc-99m (V)-DMSA AND I-123-mIBG IN THE DETECTION OF MALIGNANCIES}

The aim of the study was to reveal primary and/or secondary foci that are clinically occult in suspected, positive for somatostatin - receptors tumours, and to evaluate their tumouricidal behavior.

Among 38 patients (a) 4 (all females aged 38 to 51 years) suffered from medullary thyroid carcinoma, (b) 6 (2 females and 4 males aged 41 to 57 years) had small-cell lung carcinoma, (c) 11 ( 6 females and 5 males, aged 8 to 46 years) had pheochromocytoma, and (d) 17 (all females aged 32 to 49 years) had breast cancer. $185 \mathrm{MBq}(5 \mathrm{mCi}) \mathrm{Tc}-99 \mathrm{~m}$ (V)-DMSA [Nucl Research Center «Demokritos", Athens] and $185 \mathrm{MBq}$ (5mCi) In-111-Pentetreotide [OctreoScan 111, Mallinckrodt Medical B.V., Petten] were both injected to each patient with medullary thyroid cancer while $148 \mathrm{MBg}(4 \mathrm{mCi}) \mathrm{I}-123-\mathrm{mIBG}$ [Mallinckrodt Medical B.V.,Petten] and $185 \mathrm{MBq}(5 \mathrm{mCi})$ In-111-Pentetreotide to each patient with pheochromocytoma, breast and small cell lung cancer (3-4 days time interval between the two examinations). Tc- $99 \mathrm{~m}$ (V)-DMSA scintigraphy was carried out 2.5 and 5 hrs, In-111-Pentetreotide static images 5 and 18 hrs and I-123-mIBG scans 24 and 48 hrs (delayed scans for comparison) after i.v. application.

\begin{tabular}{lcccc}
\hline Radiopharmaceuticals & $\begin{array}{c}\text { Medullary } \\
\text { Carcinoma }\end{array}$ & $\begin{array}{c}\text { Small Cell Lung } \\
\text { Carcinoma }\end{array}$ & Breast Cancer & $\begin{array}{c}\text { Pheochromo- } \\
\text { cytoma }\end{array}$ \\
\hline Tc-99m(V)-DMSA & $4 / 4$ & $2 / 6$ & $12 / 17$ & $3 / 11$ \\
\hline In-111-Pentetreotide & $3 / 4$ & $6 / 6$ & $17 / 17$ & $9 / 11$ \\
\hline I-123-mIBG & $2 / 4$ & $2 / 6$ & $6 / 17$ & $10 / 11$ \\
\hline
\end{tabular}

According to the results we can conclude: (a) all three radiopharmaceuticals showed higher sensitivity than do conventional imaging techniques, (b) medullary carcinoma showed in all cases a positive $\mathrm{Tc}$-99m (V)-DMSA scan, small cell lung carcinoma and breast cancer a positive in all cases In-111-Pentetrcotide scan, while an interesting accumulation in APUDomas and breast cancer was observed; (c) it is worthwhilc to be noticed that the general sensitivity and spccificity for all three radiopharmaceuticals is high; (d) the cxaminations are easy to performe and safe.

\section{0}

J. Pinkert, L. Oehme,W.-G. Franke, B. Gerbert*

Departments of Nuclear Medicine and Internal Medicine* Technical University of Dresden

\section{PITUITARY UPTAKE INDEX FOR SEMIQUANTITATIVE DETERMINATION OF SOMATOSTATIN RECEPTORS IN ADENOMAS.}

To predict the success of octreotide treatment from endocrine active pituitary adenomas a method for semiquantitative determination of somatostatin receptors with In-111-pentetreotide was developed. 15 patients were examined suffering from pituitary adenomas confirmed by clinical and lab examinations, MRI and histology. The reference group contains 13 patients suffering from medullary thyroid carcinoma which received additionally a In-111-pentetreotideSPECT-Scan of the head and neck area.

SPECT studies $(60 \mathrm{~s} /$ frame, 64 frames at a $64 \times 64$ matrix) were accomplished 4 and $24 \mathrm{~h}$ after i.v. injection of $220 \mathrm{MBq}$ In-111pentetreotide using a dual head camera equipped with medium energy collimators $(\mathrm{FWHM}=15 \mathrm{~mm})$. After reconstruction with a Butterworth filter $(8 / 0.34)$ an attenuation correction using the CHANG-algorithm was performed. Coronal scans were obtained after reconstruction and the slice containing the count maximum of the pituitary gland was selected. A "pituitary uptake index" was built from the ratio of the count maximum in the pituitary gland and neighbouring skull bone at 4 and $24 \mathrm{~h}$ p.i.

The results show an increase of the uptake index from 4 to $24 \mathrm{~h}$ in the adenomas and also in the reference group. This increase was 2-3 times higher as an average for the adenomas and shows the stronger binding and density of receptors in adenomas. Of course a clear seperation from the reference group was only possible for adenomas greater than $15 \mathrm{~mm}$ in diameter.

These results suggest that a estimation of the "pituitary uptake index" could be a useful parameter for receptor density and might be a possibility to predict clinical response on octreotide treatment. 
221

R. Lebtahi, L. Sarda, G. Cadiot, M. Faraggi, E de Kerviler, D. Daou, MC. Peker, M. Mignon, D. Le Guludec.

Depts of Nuclear Medicine and Gastro-Enterology, Hôpital Bichat, Paris, France.

COMPARISON OF 99mTC-HMDP AND 1111n-OCTREOSCAN SCINTIGRAPHY FOR THE DETECTION OF BONE METASTASES IN NEUROENDOCRINE TUMORS.

Therapeutical management of patients (pts) with endocrine gastroenteropancreatic tumors (GEP) is dependent on staging. Scintigraphy with Somatostatin analogs is a sensitive method for the detection of GEP tumors and their metastases. The aim of this study was to evaluate the contribution of 111In-Octreoscan Somatostatin Receptor Scintigraphy (SRS) in the detection of bone metastases compared to conventional bone scintigraphy with Tc-99m-HMDP. 23 patients (pts) with proven GEP tumors were investigated. HMDP hot spots were classified in metastatic (69 sites, $10 \mathrm{pts}$ ) and non metastatic lesions ( 11 sites, 3 pts) according to RX, CT, MRI, and histological data.

Scans were normal with both tracers in 10 pts. For the 11 HMDP hot spots corresponding to degenerative bone disease $(n=6)$ or fractures $(n=5)$, SMS was always negative.

HMDP demonstrated 69 bone metastases (10 pts). SRS was positive in 9 / 10 pts. The last pt had a non-secreting GEP. 23 localizations were missed with SMS. In $1 \mathrm{pt}, 4$ right ribs metastases were hidden by the superimposition of an high uptake in liver metastases. Conversely, SRS revealed 44 others previously unknown bone metastases, associated to a diffuse axial squeletal bone involvement in 4 pts. This extensive involvement was confirmed by the osteo-medullar biopsy (1 pt) or by HMDP performed 6 months later ( $2 \mathrm{pts}$ ).

These preliminary results suggest that SRS seems specific and provides additional informations in bone metastases detection of GEP tumors. Further studies are needed to confirm the earlier detection of bone metastases by SRS compared with HMDP.

\section{2}

W.Becker, Th. Behr, M. Pavel, J. Hensen, F. Wolf

Department of Nuclear Medicine and Internal Medicine I of the University of Erlangen-Nuremberg, FRG

\section{IN-111-OCTREOTIDE SCINTIGRAPHY AND CONCURRENT SOMATOSTATIN THERAPY}

The influence of a somatostatintherapy on the scintigraphic result with In-111-octreotide was prospectively examined in 6 patients with different neuroendocrine tumours( carcinoids $n=3$; metastatic pheochromocytoma $n=1$; metastatic paraglioma $n=1$; not specified neuroendocrine tumor $n=1$ ).

The somatostatin therapy was administered with increasing dose over more than two weeks before In-111 octreotide scintigraphy( $3 \times 150 \mathrm{ug}$; $3 \times 300 \mathrm{ug}$; continuous infusion of $1.5 \mathrm{mg}$ or $3 \mathrm{mg}$ via a pump). 100-185 MBq in-111-octreotide were injected for scintigraphy. Gammacamera images were aquired as anterior and posterior whole body scans $4 \mathrm{~h}$ and $24 \mathrm{~h}$ p.i. The calculation of the tumor uptake ratio in \% of whole body activity and the target/background ratio was performed with the ROl-technique $24 \mathrm{~h}$ p.i. The relative tumor uptake in primary fumours and metastases increased with increasing somatostatin dose (basal: $2 \%+/-0.5 \% ; 1.5 \mathrm{mg}: 3 \%+/-1.1 \% ; 3 \mathrm{mg}: 4.9 \%+/-2.3 \%$ ). The target/background ratio increased under low dose of somatostatin and decreased with increasing dose. A continuous infusion of $3 \mathrm{mg}$ somatostatin completely blocked the receptors. In conclusion, a low dose somatostatin therapy $(3 \times 150 \mathrm{mg})$ increases the detectability of tumours and metastasis and has not to be stopped before scintigraphy. Competetive mechanisms or higher somatostatin receptor expression has to be discussed. A higher dose leads to a blockage of the receptor binding of In-111 octreotide.

Irene Virgolini, M.Raderer, A.Kurtaran, M.Schwarz, S.R.Li, Madosavljevic, P.Angelberger, S.Banyai, W.Scheithauer, W.Pehamberger, A.Luger and P.Valent.

Departments of Nuclear Medicine, Internal Medicine I, III and IV, and Dermatology, University of Vienna, Austria.

VASOACTIVE INTESTINAL PEPTIDE (VIP) RECBPTOR IMAGING: EXPERIEATCE IN 150 EATIENTS WITE VARIOUS CANCERS

Large numbers of high affinity receptors for VIP are expressed on various tumors including adenocarcinomas, endocrine-related tumors, melanomas and lymphomas. We have evaluated the efficacy of scanning with ${ }^{123}$ I-labeled VIP for tumor localization in 150 patienta with colorectal, pancreatic, gastric or pulmonary adenocarcinomas, carcinoids, insulinomas, prolactinomas, ACTH-producing carcinoids, insulinomas, prolactinomas, ACTH-producing hypophyseal adenomas, metastatic melanomas and in lymphomas. Radioiodinated VIP (purified by HPLC) was administered as a single i.v.-bolus injection (150-200 MBgi 300 pmol). ${ }^{123}$ I-VIP was initially bound by the lungs, whereas no tracer accumulation was observed in the bowel. Elemination occured via the kidneys. ${ }^{123}$ I-VIP provided excellent visualization of adenocarcinomas, carcinoids, insulinomas, lymphomas and of metastases spread from melanomas. Binding of ${ }^{123}$ I-VIP by primary tumors as well as by liver-, lung- and lymph node-metastases was visible shortly after injection of ${ }^{223}$ I-VIP and was still demonstrable at 24 hours. Tumors of $0.5-1 \mathrm{~cm}$ in size were vibualized. Primary adenocarcinomas, carcinoids, insulinomas and intestinal lyour Lhereas whereas only $20 \%$ of hypophyseal adenomas were visualized. In 60 patients comparative studies were performed with In-DIfa-phe-1-octreotide or 1-Tyr-3-octreotide demongtrating that ${ }^{123}$ I-VIP maintains a higher sensitivity for adenocarcinomas and some endocrine-related tumors. We conclude that ${ }^{123}$ I-VIP receptor scanning provides a novel and potent technique for the visualization of ${ }^{123} \mathrm{I}-\mathrm{VIP}$ expressing tumors.

224

L. Fridrich, C. Messa, C. Landoni, R. Moncayo, D. Kendler, G. Lucignani, G. Riccabona and F. Fazio

Dept. of Nuclear Medicine University of Innsbruck (Austria), INBCNR, Universityof Milan, H.S. Raffaele, Milan (Italy)

\section{PET/[F-18]FDG AND I-131 SCINTIGRAPHY IN PATIENTS WITH THYROI CARCINOMA(ThC)}

I-131 whole body scintigraphy (I-131 Sc) is used to predict the efficacy of I-13I therapy in patients with metastatic ThC. However, I-131 Sc may fail to show metastases in subjects despite concurrent high thyroglobuline plasma levels. To assess the use of [F-18]FDG scintigraphy (PET ) for ThC metastasis detection, we compared I131 and [F-18]FDG scintigraphy in 12 patients with known ThC suspicious for metastatic disease prior to consecutive I-13I therapy Patients underwent both whole-body I-131Sc with $740 \mathrm{MBq}$ I-131, and [F-18]FDG scintigraphy with $3700 \mathrm{MBq}$ [F-18]FDG. PET scans were performed to obtain bidimensional images of either the superior or inferior part of the body and tomographic images of the region where the presence of metastases was suggested by standard methods, including MDP scans or X-ray. The number of lesions observed by the I-131 Sc and PET in the same field of view was reported by three different observers.

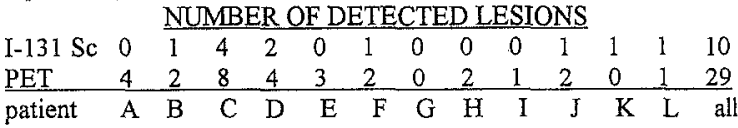

PET appears to be far more sensitive than diagnostic I-131 Sc for detection of metastatic lesions in patients with $\mathrm{ThC}$, as confirmed by the (post)therapeutic scintigraphy (20 positive lesions) too. However, a negative 1-131 scintigraphy as performed is insufficient to exclude the presence of metastases that may respond to I-131 therapy . Positive PET/[F-18]FDG findings without I-131 uptake after I-13I therapy should lead to another therapeutic strategy 


\section{5}

A.Brunetti, A. Soricelli, E. Tedeschi, E.M. Covelli, L. Castelli, L. Aloj, B. Alfano, M. Salvatore

Nuclear Medicine, University Federico II Naples - National Research Council C.N.R. - Cancer Institute 'Fond. Pascale' Naples, Italy

CORRELATION BETWEEN BRAIN PERFUSION DEFECTS ASSESSED WITH 99mTcHMPAO SINGLE PHOTON EMISSION TOMOGRAPHY (SPET) STUDIES AND MAGNETIC RESONANCE ANGIOGRAPHY (MRA)

The clinical role of SPET in the evaluation of brain perfusion (BP) is well established, while the significance of abnormal visualization of intracranial vessels with MRA has to be assessed. The aim of the study was to correlate the appearance of intracranial arteries at MRA with SPET images in 24 patients with $\mathrm{BP}$ abnormalities indicative of cerebrovascular disease (CVD).

SPET studies were performed after i.v. injection of $740 \mathrm{MBg} 99 \mathrm{mTc}$ HMPAO using a high-resolution system (CERASPECT, DSI). The presence of either focal or diffuse cortical perfusion defects or of marked left-right asymmetry were considered abnormal findings at SPET. A conventional MR brain scan (1.5 Tesla, Magnetom, Siemens) was followed by the acquisition of 3D-time-of-flight MRA sequences for the study of intracranial arteries (TR=36-43 $\mathrm{msec}$, $\mathrm{TE}=6-8 \mathrm{msec}$, Flip angle $=15^{\circ}$ ). MRA studies were considered abnormal if major intracranial arteries showed narrowing or reducedabsent signal, or if their peripheral branches had decreased visualization.

SPET and MRA abnormalities were concordant in 12 cases. MRA was rated normal in 5 patients with focal BP defects and/or marked asymmetry, and in 5 patients with globally decreased cortical perfusion. In the remaining 2 patients with frontal and fronto-parietal BP defects, reduced visualization of peripheral cerebral vessels were observed in the contralateral hemisphere.

MRA documented the "anatomic" correlation to the BP abnormalities in $50 \%$ of the cases; on the other hand, several factors may modify blood delivery to the parenchyma independently from the status of feeding arteries, and this could justify the findings in the other patients. MRA seems able to provide useful complementary anatomic information in the diagnostic work-up of patients with CVD, with little extra scan time after a conventional MR brain scan.

\section{6}

O. Sabri, D. Hellwig, H.J. Kaiser, C. Doherty, R. Schneider, M. Mull, U. Buell, A. Thron, E.B. Ringelstein

Depts. of Nuclear Medicine, Diagnostic Radiology-Neuroradiology, Neurology, Technical University of Aachen, Aachen, Germany REDUCTION OF GLUCOSE CONSUMPTION AND ICBF IN CEREBRAL MICROANGIOPATHY (CMA) IS GOVERNED BY ATROPHY AND NOT BY SEVERITY OF. WHITE MATTER CHANGES IN MRI

CMA, indicated in MRI by lacunar infarctions (LI), deep white matter lesions (DWML) and atrophy (ATR), is accompanied by vascular dementia which was reported to be caused by $\mathrm{LI}$ and DWML. 51 pts. with CMA were scrutinized for changes in rCBF and rMRGlu in both WM and cortex. The results were correlated with MRI-findings. A special head holder system provided exact repositioning during examination of $5 \mathrm{CBF}$ (15 min P.1.740 MBQ 99mTc-HMPAR -SPCT cortex were quantitated with ROls defined in MRI and superimposed to corresponding PET/ SPECT slices (overlay). Glucose utilization (rMRGlu) was derived from the autoradiographic method, regional cerebral perfusion ( $\mathrm{rCBF}$ ) from normalization to cerebellum. $\mathrm{Ll}$ and OWML were divided in two groups (= microangiopathy-score): 1) < 4 $L I$, no DWML, 2) > 4 LI, middle to severe DWML. For ATR a two stage grading was used: $A$ ) no to only slight inner and/or outer ATR B) middle to severe outer and inner ATR.

\begin{tabular}{|c|c|c|c|c|c}
\hline group & $n$ & \multicolumn{2}{|c|}{ rCBF } & rMRGlu $[\mu \mathrm{mol} / 100 \mathrm{~g} / \mathrm{min}]$ \\
\hline LI/DWML & & WM & cortex & WM & cortex \\
\hline 1 & 14 & $0.71 \pm 0.09$ & $0.86 \pm 0.06$ & $11.6 \pm 3.6$ & $36.1 \pm 7.3$ \\
\hline 2 & 37 & $0.69 \pm 0.08$ & $0.83 \pm 0.07$ & $12.7 \pm 4.9$ & $38.5 \pm 11.2$ \\
\hline
\end{tabular}
No significantly changed values for rCBF and TMRGlu were found between patients of 1) and 2)

\begin{tabular}{|c|c|c|c|c|c|}
\hline group & $\mathrm{n}$ & \multicolumn{2}{|c|}{ rCBF } & \multicolumn{2}{c|}{ MRR Glu $[\mu \mathrm{mol} / 100 \mathrm{~g} / \mathrm{min}]$} \\
\hline ATR & & WM & cortex & WM & cortex \\
\hline$A$ & 33 & $0.71 \pm 0.07$ & $0.85 \pm 0.06$ & $13.4 \pm 4.2$ & $40.7 \pm 9.3$ \\
\hline$B$ & 18 & $0.66 \pm 0.08^{*}$ & $0.81 \pm 0.05^{*}$ & $10.5 \pm 4.7 \pm$ & $32.8 \pm 10.1^{*}$ \\
\hline
\end{tabular}

$p<0.05 / * p<0.025$ significance of $A$ ) $v$ s. $B$ )

$P$ ts. of $B$ ) showed significant lower $r C B F-$ and $r M R G l u$-values both in cortex and WM than A). Thus, since reduced rCBF- and rMRGlu-va lues in CMA are not correlated to $\mathrm{LI}$ and DWML but to outer and inner ATR, the concept of vascuar dementia may be incorrect. Subsequent inner and outer atrophy may be responsible for functional (and clinical) presentation of such pts.

\section{7}

C. De Sadeleer. K. de Metz, G. Somers, A. Bossuyt. Departments of Nuclear Medicine and Internal Medicine Free University of Brussels (AZ VUB), Brussels, Belgium

\section{RELATIVE QUANTIFICATION OF TC99m HMPAO SPECT BASED ON SECTORIAL REGIONS OF INTEREST IN NORMAL VOLUNTEERS.}

In order to obtain a data base for activation studies, the normal values and the reproducibility of $\mathrm{Tc}-99 \mathrm{~m}$ HMPAO regional cerebral uptake measurements were assessed in 16 young healthy volunteers. 11 studies were repeated in identical conditions within 72 hours. All studies were processed by two observers unaware of each others results.

Five minutes after the injection of $20 \mathrm{mCi}$ Tc-99m HMPAO, a SPECT acquisition, 64 steps of 30 sec over $360^{\circ}$, was started on a $3-$ head Multispect Siemens gamma camera (192 images). After backprojection using a Butterworth filter (cutoff 0.5), Chang's attenuation correction and, reorientation according to the orbito-meatal line, 5 transverse $14 \mathrm{~mm}$ thick slices were defined: the lowest slice containing the cerebellum and temporal lobes, the second through the basal ganglia and thalamus and, 3 consecutive cortical slices. A threshold of $50 \%$ was used in the lowest 2 slices for manual delineation of the regions, according to a cerebral atlas, $(5$ and 6 mirrored regions respectively); a threshold of $65 \%$ was used for the automatic sectorisation in the upper 3 slices ( 6 mirrored regions in each slice) Thus 58 ( $2 \times 29$ ) vascular ROls were defined over transverse slices in each volunteer. All regions were normalized to the maximal cerebellar activity.

For each of the 58 segments, the $95 \%$ confidence interval on the mean uptake values ranged between $2.7 \%$ and $9.37 \%$. The variation coefficient for the left to right ratios varied between $2.1 \%$ for the thalamus and $5.3 \%$ for the latero-frontal upper segment. The interobserver variation coefficient for the different regional uptake values ranged from $1.92 \%$ to $7.29 \%$. The interstudy variation coefficient for the different regional uptake values varied between $2.50 \%$ and $5.91 \%$

Thus, in normal volunteers, using a 3-head multispect gamma camera, very strict reference values were obtained which can be used for interindividual, intergroup and interstudy comparisons.

C.J. Rehmann, M. Limburg, D. Legemate, M.M.C. Tiel-van Buul, E.A. van Royen

Academic Medical Center, University of Amsterdam, the Netherlands

$99 \mathrm{~m}$-TC-HMPAO DIAMOX BRAIN SPECT IN CAROTID ARTERY STENOSIS

In this prospective study, cerebral perfusion reserve (CPR) was evaluated by acetazolamide (Diamox) $99 \mathrm{~m}$-TC-HMPAO brain SPECT in patients clinically suspected for carotid artery stenosis.

Consecutive $99 \mathrm{~m}$-Tc-HMPAO brain SPECT investigations were obtained within one hour before and after a dose of $1000 \mathrm{mg}$ acetazolamide iv. Studies were performed with a SME 810 multidetector neuroSPECT system. After reconstruction mean counts in the parieto-occipital region were calculated on fixed regions of interest (ROI's) in two slices. The calculated activity was corrected for injected dose $99 \mathrm{~m}-\mathrm{Tc}-\mathrm{HMPAO}$ and decay. The corrected counts of the baseline study were subtrated from the post-Diamox study. The increase in perfusion, the CPR, was expressed as a percentage of the baseline results in the same ROI Sixteen studies were performed in 15 patients, 5 women and II men, mean age 66 years, range $42-81$ years. The results of 16 studies (32 carotid arteries) were used for statistical analysis. A carotid stenosis was considered significant above $70 \%$ (duplex sonography or angiography). The results were:

\begin{tabular}{lrll} 
& $n$ & mean CPR & range \\
\hline Normal arteries & 12 & $35.1 \% \pm 13.4 \%$ & $18-53 \%$ \\
Non sign stenosis & 6 & $36.0 \% \pm 16.4 \%$ & $22-58 \%$ \\
Significant stenosis & 10 & $22.0 \% \pm 16.0 \%$ & $8-39 \%$ \\
Occlusive arteries & 4 & $27.3 \% \pm 13.1 \%$ & $12-53 \%$ \\
\hline
\end{tabular}

The patient who was investigated a second time after succesful endarterectomy of a $95 \%$ carotid stenosis showed a significant increase of cerebral perfusion reserve from $12 \%$ to $33 \%$.

Conclusions: In these small series of patients the cerebral perfusion reserve, compared with normal arteries, was significantly lower $(p=0.004)$ in patients with carotid artery stenosis above $70 \%$. No difference was seen between normal and tota! occluded arteries, suggesting adequate collateral blood supply. Lack of difference between normals and non significant stenoses can indicate the clinical non significance of these stenoses. Further studies should be performed to relate the CPR will risk reduction for CVA's in the follow up of these patients. 


\section{9}

E. Vanninen, R. Vanninen, J.T. Kuikka, K. Partanen, M. Puranen, H. Tulla Depts. of Clinical Physiology and Nuclear Medicine, Clinical Radiology, and surgery, Kuopio University Hospital, Kuopio, Finland

ASYMPTOMATIC CEREBRAL EMBOLISM DURING CAROTID ENDARTERECTOMY DECREASES IPSILATERAI REGIONAI PEREUSION RESERVE

The aim of the present prospective study was to evaluate the haemodynamic significance of asymptomatic cerebral embolism in patients undergoing carotid endartectomy (CEA). In a series of 22 patients (two patients had both sides operated sequentially), MRI revealed small single or multiple emboli after five CEAs. Cerebral perfusion reserve (CPR) was measured just before and one month after CEA by performing TC-99m-HMPAO SPET both before and after intravenous injection acetazolamide (dose $1 \mathrm{~g}$ ). CPR was calculated for the whole $10.5 \mathrm{~mm}$ thick transverse slice as well as for the symmetrical ipsi- and contralateral ROIs representing the affected embolic and contralateral areas. Before CEA, regional ipsi- and contralateral CPRs were symmetrical (5 vs 11 , ns). After CEA, ipsilateral CPR decreased to $-3 \%$. The contralateral CPR remained unchanged ( $8 \%$ ), and it was significantly higher than on the ipsilateral side after CEA $(p<0.05)$. The CPR for the whole slice showed a slight decrease (13\% vs $3 \%$ ns). These preliminary findings suggest that asymptomatic cerebral embolism during CEA decreases ipsilateral regional vascular reactivity.

230 L. Pávics*, F. Grünwald, F. Ries, K. Reichmann, C. Menzel, H. Depts. of Nuclear Medicine, Neurology, Neuroradiology, and ENT, University of Bonn, Supported by the A.v. Humboldt Foundation, Germany

REGIONAL CEREBRAL BLOOD FLOW (rCBF) STUDIES WITH 99mTCHMPAO SPECT DURING BALLOON TEST OCCLUSION WITH ACETAZOLAMIDE

In the surgery of tumours, metastases and aneurysms affecting the carotid vessels, in some cases resection of the internal carotid artery (ICA) is needed. To predict the tolerance of permanent ICA resection, a ballioon test occlusion is recommended. The procedure is commonly combined with $99 \mathrm{mTC}$-HMPAO SPECT and recently also sensitized with acetazolamide. The aim of our study was to determine the quantitative criteria of pathological rCBF patterns in this test. In 17 patients with oropharyngeal tumours, a balloon test occlusion with acetazolamide ( $1 \mathrm{~g}$ ) (stress study) combined with high resolution SPECT (CERASPECT) and 99mTc-HMPAO were performed besides continuous clinical and transcranial Doppler sonographical (TCD) monitoring. In 8 cases the rCBF SPECT was repeated under resting condition. The patients in groups with normal and pathological reaction were categorized according to th clinical signs and symptoms, the TCD findings and the postoperative outcome. The rCBF SPECT data with an automatic ROI method were analysed. The asymmetry of $99 \mathrm{mTC}-\mathrm{HMPAO}$ uptake in percent and its changes compared to the rest study were determined at the global cortex, the medial cerebral artery (MCA) territory, and the watershed zones. The rCBF SPECT asymmetry values of the cortex and of the watershed zone showed a statistically significant correlation with the respectively, Spearman rank correlation), but not with the the test occlusion the side difference on SPECT was $5+2 \%$ in the the test occlusion the side difference on cortex, $8 \pm 5 \%$ at the MCA region, and $9 \pm 6 \%$ in the watershed zone. condition $(n=5)$ were $2 \pm 2,2 \pm 2$, and $2 \pm 3$, respectively. In the differentiation between normal and pathological reactions, difrerentiation between normal and pathological reactions, gave a sensitivity of $5 / 6$, and a specificity of $11 / 11$. We concluded gave a sensitivity of $5 / 6$, and a Specificity of $11 / 11$. We concluded: in the interpretation of ICA occlusion tests with acetazolamide. 2. in the interpretation of lCA occlusion tests with acetazolamide. 2 . valuable. 3 . In the suspicion of anamnestic rCBF disturbances rCBF SPECT under rest condition is also needed.
231

JL.Moretti, P Weinmann, G Baillet, G Sebbane, C Meyrignac,

$\mathrm{N}$ Caillat-Vigneron.

Neurosciences Department Creteil, René Muret and Nuclear Medicine Division. Bobigny - FRANCE

Tc-99m HMPAO BRAIN SPECT FOR EFFICIENCY IMAGING OF AZETAZOLAMIDE IN PATIENTS TREATED FOR HYDRO-CEPHALUS.

Normal pressure hydrocephalus (NPH) can be cured by surgical derivation but morbidity of such treatment is high indeed. Iterative lumbar puncture can induce clinical and cerebral blood flow improvement. Chronic treatment with acetazolamide $(0,5 \mathrm{~g}$ per day) can also improve clinical status after a few weeks $(1,2)$. We undertook to measure brain perfusion by $99 \mathrm{~m}$ Tc HMPAO SPECT $(740 \mathrm{MBq})$ before and after 4 to 6 week-treatment with acetazolamide in 5 patients (age 42 to 86 years). All patients demonstrated ventricular enlargment on CT, and were suffering from gait and walking disturbances and anterograde amnesia. Their clinical status was assessed by a Mini Mental Test (3MT) and a Hachinski Test. SPECT demonstrated a decreased regional blood flow in hippocampal areas, in frontal and white matter parietal cortex. Acetazolamide treatment was effective in $4 / 5$ patients, with $3 \mathrm{MT}$ improvement, even normalization in $2 / 5$. The nonresponding patient ( 42 years) had a non communicating congenital hydrocephalus. Accordingly, brain perfusion assessed quantitatively with a standard dose was dramatically improved (range 20 to $75 \%$ ) in all patients with clinical improvement (4/5). The patient not clinically improved did not demonstrate any brain perfusion increase after acetazolamide. We concluded that $99 \mathrm{mT}$ C HMPAO brain SPECT may be a non invasive and objective imaging procedure to assess acetazolamide efficiency in patients suffering from NPH, matching well with clinical status. A on going expertise is done on a cohort of 9 patients with the same protocol to assess regional increase of flow in psychiatric improved patients after DIAMOX.

- (1) Aimard G, Vighetto A, Gabet JY et al : Acetazolamide : une alternative à la dérivation dans l'hydrocéphalie à pression normale. Résultats préliminaires. Rev. Neurol. Paris 1990, 146:6-7 ; 437-439.

- (2) Larson A, Bergh AC, Bilting M, Arlig A, Jacobson L, Stephenson $H$, Wikkelsö $\mathrm{C}$ : regional cerebral blood flow in NPH : diagnostic and pronostic aspects. Eur J Nucl Med 1994, 21:118-123.

\section{2}

M.I.Goris, S.J.Knox, K.Trisler, M.Becker, K.R.Nielsen and S.Fowler. Division of Nuclear Medicine and Dept. of Radiation oncology, stanford U. School of Medicine. Stanford, California, USA.

TREATMENT OF B CELL LYMPOMA WITH 9OY-B1 MONOCLONAL ANTIBODIES .

The safety of and tolerance for radiolabeled monoclonal antibodies was evaluated in a study in which $\mathrm{B}$ cell lymphoma was treated with increased doses of anti-CD20 labeled with yttrium-90. Therapy was preceded by bone marrow or peripheral stem cell harvesting with purging. Doses were planned to increase in four steps $(10,20,30,40)$ to $40 \mathrm{millicurie,} \mathrm{and} \mathrm{bone}$ marrow toxicity to be handled with autologous bone marrow transplantation. The infusion of the radiolabeled agent was preceded by an infusion of $1 \mathrm{mg} / \mathrm{kg}$ of unlabeled antibody. No bone marrow transplantation was necessary for the 9 patients who received up to 30 millicurie (three last ones), but 4 patients received GCSF support: Leukopenia ( $1.9 \mathrm{~K})$ and thrombocytopenia $(<25 \mathrm{~K})$ occurred in 5 and 2 patients respectively, with nadirs lasting up to 6 weeks. The whole body dosimetry was calculated to be $1.40 \pm 0.57 \mathrm{cGy} / \mathrm{mCl}$, for the liver and the spleen the estimated doses were $9.89 \pm 8.91$ and $9.75 \pm 6 \mathrm{cGy} / \mathrm{mCi}$ respectively. Estimated tumor doses were variable, and reached $60 \mathrm{cGY} / \mathrm{mci}$. In eight patients who could be evaluated, stable disease was found in 3, partial response in 3 and complete response in 1. The response of individual lesions was not predictable by the individual lesions' imaging characteristics with 111-indium labeled antibodies, but by the best visualization on a patient basis. 
Monday, 22 August 1994

233

K.E. Britton, H.A. Band, A.M. Creighton, J. Long, C. Bartram and $M$. Granowska

Imperial Cancer Research Fund, Nuclear Medicine Group, St. Marks Hospital, City Road, London

RADIOIMMUNOTHERAPY, RIT, WITH 32P LABELLED ANTIBODIES, A PRET IMINARY REPORT

Phosphorous - 32 is an energetic beta emitter (1.71 MeV) with a two week half life and a recognised clinical application in polycythaemia, bone metastases and malignant effusions. It is a suitable agent for long lived RIT. It has been attached to monoclonal antibodies SM 3 \& PRIA 3 using the Kemptide peptide prior labelled with carrier free 32P. In the current procedure a phosphate-receptor peptide is linked procedure a phosphate-receptor peptice is linked directly to the antibody and phosphorylated enzymatically with 32P-ATP. The product is then separated on a Sepharose column using a semi-automatic FPLC system under sterile conditions with appropriate shielding. Radiochemical yields in clinical preparations have been 40-50\% but have not yet been optimised. New purification procedures should soon alow the use of a much cheaper source of $32 \mathrm{P}$.

In one pilot phase 1 study, 4 polycythaemic patients received single doses of from $1.9-5.5 \mathrm{mCi}$ i.v. (at 1.00 - $1.65 \mathrm{mCi} / \mathrm{mg}$ ) of 32P-labelled SM3. The labelled conjugate cleared from the circulation at a very similar rate to the corresponding macrocyclic $111-$ nn-label rate to antibody. There was good stability of the 32P linkage to antibody. There was no slgnificant effect on haemoglobin, white cells or blood chemistry but in two patients with high platelets receiving about $5 \mathrm{mCi}$ of 32 P-SM3, a significant reduction in platelets was observed to normal levels. In a second study involving hepatic metastases from colorectal primaries, the first two patients were given 2.4 and $5.00 \mathrm{mCi}$ of $32 \mathrm{p}-1 \mathrm{abelled}$ PR1A3 intraarterially (at $0.74-1.00 \mathrm{mCi} / \mathrm{mg}$ ) after angiotensin II infusion without untoward effect. Good stability was again achieved and in the first case, further $5 \mathrm{mCi}$ was given two months later. A fall in serum CEA was noted but with an increase in tumour size. Dose escalation is proceeding.

\section{4}

D.Riva*, A.Arista ${ }^{\circ}$, C.Sturiale ${ }^{\circ}$, G.Franceschi*, A.Spinelli*, G.Moscatelli*, F.Campori*, N.Riva*, M.Casi*, R.Gentile* *Nucl. Med.Dept. Ist it. Oncol . Romagnolo; ${ }^{\circ}$ Neurosurg.

Dept. "M.Bufalini" Hospital Cesena (Italy)

INRRAESIONAL RADIOIMMUNOIHERAPY OF MALTENANT GLIOMAS EMPLOYED IN RECURRENT LESIONS OR AS ADUUVANT SETTING.

Anti-Tenascin MAbs, BC-2 and/or BC-4 (SORIN-BICMEDICA, Italy) labelled with I-131 (dose range 10-55, mean $42 \mathrm{mCi}$ ), were utilized for intralesional Radioimunotherapy (RTI') in 45 patients with malignant gliama. 31 of these had a recurrent lesion following surgery radio and chemotherapy. In 26 cases they underwent further surgery : quite radical removal of neoplasm was achieved in 15.Conversely 12 patients received RTT after resection and external irradiation of primary tumour; 8 of these presented only a resicual minimal disease. Two cases were submitted to treatment as 1st therapy. Usually RIT courses were repeated up to 5 . The therapy was alwrys well tolerated. In same cases (21/45) HAMA production at low titers was recorded. Sequential scintigraphies, carried out up to 50 days after injection, demonstrated an intense and persisting uptake of MAbs in the lesion. The radiation to neoplastic tissue resulted, on average, 23000 ofy per cycle; the curnulative dose, in some patients who received multiple RIT, exceeded 100.000 oGy. The median survival time was significantly prolonged(19 months). In 35 evaluable cases 9 stabilizations of disease for 12 months (median), 7 partial remissions(median 9 months), 6 complete remissions (median 18 months) were achieved. The overall response rate (RR) was $37.1 \%$. In 17 patients with macroscopic disease the $\mathrm{RR}$ was 23.58. In 18 cases with minimal disease after removal of primary or recurrent disease, the RR was 50\%. Moreover, in this last subset, when RIT was given after first intervention and radiotheraoy, we obtained a $\mathrm{RR}$ of $66.6 \%$. Conversely in the patients treated after renoval of recurrence it was 41.6\%. So intralesional RIT could be successfully applied after surgical and radiotherapy treatment of primary tumour, as adjuvant setting . (Work supported by National Research Council (Italy): ACRO, subproject 8)

\section{5}

R. P. Baum, A. Niesen, A. Nancy, J. Oltrogge, B. Donnerstag, A. Hertel, A.A. Noujaim, G. Hör

University Medical Center, Frankfurt/Main, Germany

Biomira Research Inc., Edmonton, Canada

IMMUNOTHERAPY BY HAMA INDUCTION IMPROVES SURVIVAL OF OVARIAN CANCER

In patients (pts) with advanced stages of ovarian carcinoma (OVCA) receiving radiolabeled monoclonal antibodies (MAb) against $\mathrm{CA}$ 125 for diagnostic purposes, a better survival rate than expected was observed by our group and others. A more detailed analysis of the effects caused by the injection of the MAb revealed that the better prognosis was linked to the rise of human anti-mouse antibodies in these pts. The present study was undertaken to evaluate these effects in a larger patient population.

The intact Tc-99m labeled anti-CA 125 MAb B43.13 (Biomira, Edmonton, Canada) was injected repeatedly (161 injections) into $63 \mathrm{pts}$ suffering from recurrent OVCA. Serum HAMA titers (anti-isotypic and anti-idiotypic antibodies) were measured at monthly intervals. 53\% of pts developed HAMA after the first injection and all (except one) became HAMA positive after 3 injections. The HAMA response was very variable in the individual patient (titers from 1,000 to $>500,000 \mathrm{ng} / \mathrm{ml}$, normal cut-off $220 \mathrm{ng} / \mathrm{ml})$. The repeated injections ( $1 \mathrm{mg}$ dose) were well tolerated (only two allergic reactions which needed medical intervention). No toxic long term effects were observed.

Compared to control ( $\mathrm{n}=6170$ patients), the 1-yr survival rate (SR) for ovarian cancer FIGO III stages was $95 \%$ vs. $77 \%$ (control), 2-yr SR was $90 \%$ vs. $50 \%, 3$-yr SR was $73 \%$ vs. $38 \%$ and 4 -yr survival $57 \%$ vs $30 \%$ (Kaplan-Meier analysis; $p<0.002$ ). Most of the pts showed a stable course of the disease under immunotherapy, in few cases also tumor tegressions were observed. These effects might be caused by induction of anti-idiotypic antibodies which trigger a host therapeutic effects directed against the tumor. HAMA also induce T cell activation directed against the tumor cells. A prospective, randomized multicentric study in patients at high risk for recurrence of ovarian carcinoma, including patients with minimal residual disease or NED after second look surgery, is going to start soon.

\section{6}

F.D. Maul, G. Hör, D. Schwabe, R.P. Baum, K.H.Manegold, J. Hesse, A. Hertel, T.Kranert, V. Gerein, B. Kornhuber

Divisions of Nuclear Medicine, Pediatric Oncology, and Radiooncology, Johann Wolfgang Goethe-University, FrankfurtMain, Germany

\section{STANDARD VS: INDIVIDUAL I-131 MiBG ACTIVITY IN NEUROBLASTOMA THERAPY?}

Clinical data of $37 /-131$ MIBG treatments were reevaluated with the question whether the application of a therapeutic standard activity - which is easier to handle - is preferable or an individual activity based on a pretherapeutic dosimetry is mandatory. The goal of pretherapeutic dosimetry was to keep the whole body dose below 1 Gy per treatment. Treatments were devided into 2 groups: Group $1(\mathrm{n}=8)$ receiving tumor doses of more than 50 Gy per treatment (high tumor dose, HTD) and group $2(n=29)$ with tumor doses below 50 Gy (low tumor dose, LTD). The aim was to clarify whether the tumor doses and side effects correlate with the whole body doses. Medians were calculated and U-tests were applied. A p-value $<0.05$ was assumed to be significant. Whole body doses of the HTD group and LTD group were not significantly different $(0.5$ Gy resp. $0.55 \mathrm{~Gy})$. There was a not significantly higher $24 \mathrm{~h}$ uptake of MIBG under therapy by the HTD group (34 vs. 29\%) although, according to the selection of groups, tumor doses of the HTD group were significantly higher than in the LTD group (62.5 Gy vs. 16 Gy). We observed only a trend towards a longer survival of the HTD group ( 9.5 vs. 7.5 months), however, the cured child and the child with the longest survival (> 8 yaers) belonged to the HTD group. Tumor marker responses (VMA, HVA) did not differ between the groups. The immediate toxic bone marrow response based on leucocyte and thrombocyte counts was comparable for both groups. Despite a low tumor uptake a long remission of more than 24 months was achieved in 1 child. In conclusion: Individually measured dosage of MIBG application combines the advantage of keeping acceptable whole body doses along with the change of achieving the highest possible tumor doses. A "lower" tumor dose concept may be reasonable for palliative treatment. 


\section{7}

M. Rodrigues, G. Karanikas, S. Granegger, H. Sinzinger

Department of Nuclear Medicine, University of Vienna, Vienna, Austria

PLATELET UPTAKE OF 131 I-METAIODOBENZYLGUANIDNE MECHANISM, EXTENT AND CLINICAL RELEVANCE

${ }^{131}$ I-metaiodobenzylguanidine (MIBG) is associated with a reduction in the circulating platelets, thrombocytopenia being the major factor limiting the therapeutic application of a ${ }^{131}$ I-MIBG therapy. In order to assess the potencial radiation hazard, we therefore investigated the platelet uptake mechanism of 131 I-MIBG in-vitro and in-vivo. The influence of plateletdensity, temperature and time of incubation and amount of radioactivity were analysed as well as the effect of iv administration of 131 I-MIBG for diagnosing on platelet count, viability and blood cell uptake invivo. Venous blood from healthy volunteers $(n=182)$ and patients with $(n=17)$ and without $(n=9)$ B-blockers was studied.In-vitro studies included varying conditions: platelet-density $\left(1.10^{7}, 1.10^{8}, 1.10^{9}\right.$. platelets $\left./ \mathrm{ml}\right)$, temperature of incubation $\left(4^{\circ} \mathrm{C}, 22^{\circ} \mathrm{C}, 37^{\circ} \mathrm{C}\right)$, time of incubation $(5,10$, $30,60 \mathrm{~min}$ ) and amount of radioactivity $(0.01,0.1,1 \mathrm{uCi})$.

When increasing the number of platelets a progressively higher cellular uptake was found. This uptake was temperature - and time - dependent, while it was not influenced by increasig amounts of radioactivity. No difference in platelet uptake between volunteers and patients without $B$ blockers was found, while platelets derived from patients under Bblockers did not show that selective uptake. After a diagnostic application of $500 \mathrm{uCi}{ }^{131} \mathrm{I}-\mathrm{MIBG}$ in-vivo a selective uptake by platelets occurred too, resulting in a platelet to plasma ratio of up to $1: 32$. Even after a diagnostic dose a nadir of platelet count down up to $36 \%$ (maximum at 7 days) was observed. Patients under B-blockers did not show a change in their platelet count, the platelet plasma ratio did not exceed 1:4.4. These in-vitro and in-vivo findings suggest that ${ }^{131} \mathrm{I}-\mathrm{MIBG}$ is actively taken up by blood platelets by a mechanism being dependent on the concentration of platelets, the temperature and the time, which is inibited by ß-blockers. This selective uptake may explain the major irradiation hazard of ${ }^{131} \mathrm{I}-\mathrm{M} B \mathrm{BG}$.

\section{8}

$\underline{\text { R.P. Baum }}^{1}$, M.J. Blend ${ }^{2}$, G.Q. Mills ${ }^{3}$, G.L. Burraggi ${ }^{4}$, D.M. Goldenberg ${ }^{5}$ Univ. Hospital ${ }^{1}$, Frankfurt, FRG; Humana Hospital ${ }^{2}$, Chicago, IL; Baton Rouge $^{3}$, LA; Istituto Nat. Tumori ${ }^{4}$, Milan, Italy; $\mathrm{CMMI}^{5}$, Newark, NJ

CLINICAL BENEFIT FROM RADIOIMMUNODETECTION (RAID) OF B-CELL LYMPHOMAS UTILIZING A TC-99M LABELED MONOCLONAL ANTTBODY FRAGMENT (LL2): ANALYSIS OF MULTICENTER PHASE I/II TRIALS

These multicentric open-label studies were designed to evaluate the safety, tolerance, pharmacokinetics, and efficacy of the Tc- $99 \mathrm{~m}$ labeled anti-lymphoma antibody MMMU-LL2 Fab' fragment (Immunomedics, Morris Plains, $\mathrm{NJ}$ ) for the defection of malignant lesions of $>0.5 \mathrm{~cm}$ in size in patients with non-Hodgkins's B-cell lymphomas.

In the pilot study, approx. $925 \mathrm{MBq}(25 \mathrm{mCi})$ of the $\mathrm{Tc}-99 \mathrm{~m}$ labeled $\mathrm{Mab}$ $(1 \mathrm{mg}$ ) was injected iv in 16 patients. At $30 \mathrm{~min}, 3-4 \mathrm{~h}$, and $24 \mathrm{~h}$ p.i, whole body images were obtained. Planar imaging was performed at $1 \mathrm{~h}, 3-4$ and 24h p.i.; SPECT 4-5h p.i. HAMA were determined using the ImmuStrip ${ }^{\circledR}$ HAMA ELISA (Immunomedics). The agent was tolerated well with no adverse reactions. The half life for the total body ranged between 44 and $58 \mathrm{~h}$. Dosimetry data revealed the highest dose for spleen and kidney (0.15-0.24 and $0.28-0.33 \mathrm{cGy} / \mathrm{mCi}$, respectively; total body $0.01-0.02 \mathrm{cGy} / \mathrm{mCi}$ ). In the Phase 1 study (10 pts), 3 different doses $(0.5,1$, and $5 \mathrm{mg})$ of LL2 were administered, all revealing similar elimination half-times. $24 \mathrm{pts}$ were enrolled in the Phase II trial ( $30 \mathrm{mCi} \mathrm{Tc}-99 \mathrm{~m}, 0.25-1 \mathrm{mg}$ Fab'). All pts underwent non-invasive imaging (CT, chest $\mathrm{x}$-ray, MRI, bone scan, Ga-67 derwent non-invasive imaging (CT, chest $\mathrm{X}$-ray, MRI, bone scan, $\mathrm{Ca}$, 6 ,
scand the results were compared to RAID scans. The efficacy analysis in the 43 evaluable pts demonstrated a sensitivity of $86 \%$, an accuracy of $86 \%$, and a positive predictive value of $100 \%$. Only $1 \mathrm{pt}$ at the $5 \mathrm{mg}$ dose and another pt at the $1 \mathrm{mg}$ dose developed a positive HAMA response. The Tc-99m-IMMU-LL2 antibody fragment appears to be a safe and accurate imaging agent. With regards to staging, in 11/43 cases, RAID correctly identified all areas of disease previously diagnosed by 2-3 separate, different diagnostic modalities and in another $11 / 43$ cases, identified additional tumor sites not detected by any other previous diagnostic procedure; 10/58 pts were upstaged. Based on managing physician judgements, 29/43 (67\%) of pts had clinical benefit as defined by confirmation of disease extent ( 25 pts), upstaging (6 pts), or change in management. (Supported in part by pts), upstaging ( 6 pts), or change in mant
USPHS grant CA 39841 from the NIH).
239

M.Stoffel, F. Jamar, N.Leners, C.Beckers, A.Ferrant and S.Pauwels. Depts. of Nuclear Medicine and Hematology, University of Louvain Medical School, Brussels, Belgium

STAGNG OF LYMPHOMAS WTTH ${ }^{111}$ INmPENTETREOTWE ( ${ }^{111}$ IN-P).

Discordant ${ }^{11}$ In-P results have been reported in lymphoma patients (pts) according to the cell-type and grade of malignity. Our study was performed in order to evaluate the sensitivity of ${ }^{111} \mathrm{In}-\mathrm{P}$ in $17 \mathrm{pts}$ with histology-proven lymphoma, including 4 pts with Hodgkin's (HL, 4 studies) and 13 non-Hodgkin's lymphomas: 5 low-grade (INHL, 7 studies), 2 intermediate-grade (iNHL, 3 studies) and 6 high-grade (hNHL, 8 studies). Planar (4h, 24h) and SPECT (24h) acquisitions were performed after injection of $\sim 200 \mathrm{MBq}{ }^{111}$ In-P. The scintigraphic findings were compared with conventional imaging modalities (CIM) and histology when available.

${ }^{111}$ In-P was +ve in 7 new cases, 3 recurrences and 5 residual diseases. One of 2 refractory pts was ${ }^{111}$ In-P +ve (iNHL) whereas the other was -ve (INHL). ${ }^{111}$ In-P was -ve in 4 of the 5 complete remissions whereas in $1 \mathrm{pt}$ a stable lymph node remained +ve after radiotherapy.

${ }^{111}$ In-P defected 21 of the 28 lesions recognized by CIM: the sensitivity per lesion was $100 \%, 78 \%, 80 \%$ and $64 \%$, in $\mathrm{HL}, \mathrm{hNHL}$, iNHL and INHL respectively. The -ve sites included 2 lymph nodes, 1 spleen infiltration, 1 liver, 1 bone and 1 meninges lesion. ${ }^{11}$ In-P detected 9 unsuspected lesions: 1 true +ve, 5 unconfirmed and 3 false tve ( 2 infectious sites, 1 external contamination). Nine of the 11 biopsied lesions ( 9 pts) were ${ }^{111} \mathrm{In}-\mathrm{P}+\mathrm{ve}(82 \%)$. In all but 2 pts ( 2 NHL), ${ }^{111}$ In-P and CIM resulted in an identical pt staging.

Conclusions: 1) our results indicate the potential usefulness of ${ }^{111} \mathrm{In}-\mathrm{P}$ for staging of lymphomas, particularly in HL patients, 2) data interpretation should be cautious in patients with potential infectious complications.

240

I. Szilvási, M. Petö, Z. Nagy, I. Jánoskuti

Depts. of Nuclear Medicine and Medicine, Postgradual Medical School, Budapest, Hungary

PROBLEM-ORIENTED USE OF BONE MARROW SCINTIGRAPHY IN PATIENTS WITH MALIGNANT LYMPHOMAS.

Bone marrow (BM) involvement in patients with malignant lymphoma is of therapeutic and prognostic relevance. Bone scintigraphy (BS) is not sensitive enough, and whole-body imaging is not possible by CT or MRI. BM scintigraphy (BMS) has been proposed to detect focal BM replacement by space-occupying lesions. In our study 56 patients with various types of malignant lymphomas were evaluated by BS and BHS. TC-99m-MDP and TC-99m-labeled antigranulocyte antibody were used. In selected cases Blf biopsy, X-ray, CT or HRI examinations of suspected regions were also performed. Diagnostic values of BS and BMS in different patients groups and regions (skull, vertebra, ribs, pelvic region and extremities) were evaluated on a lesion-by lesion basis.Results: BMS proved to be insensitive for detection of $B M$ involvement of the skull and the ribs, but is an accurate method for detecting vertebral lesions in all groups of patients (sensitivity:.88 specificity:.96). In the pelvic region BMS is not sensitive(.34). No lesion of the extremities was found in patients without localized pain. Diagnostic accuracy of BMS is higher than that of BS in patients with malignant lymphoma (.76 vs .52). BMS proved to be reliable for evaluation of BM reserve function in patients with multiple myeloma. In conclusion:problem-oriented use of BMS using antigranulocyte antibody is indicated in patients with malignant lymphomas. 
J. Kropp ${ }^{1}$, M. Heer ${ }^{2}$, R. Gerzer ${ }^{2}$, H.-J. Biersack ${ }^{3}$.

Depts. of Nuclear Medicine Tech. Univ. of Dresden ${ }^{1}$ and Univ. of Bonn ${ }^{3}$, Dept. of Aerospace Medicine DLR-Cologne ${ }^{2}$; Germany

\section{MICROGRAVITY AND SODIUM INTAKE INDUCED HÄMATO- LOGIC AND BLOOD VOLUME CHANGES.}

Weightlessness induces changes on blood cells, body fluids and electrolytes which can be simulated by head-down-tilt studies (HDT). Sodium $(\mathrm{Na})$ intake might be responsible for some changes. We investigated 12 healthy volunteers with $4 \mathrm{MBq}{ }^{51} \mathrm{Cr}$ in-vitro labeled red blood cells (RBC). Governmental permission was obtained. Group I $\left(\mathrm{GI}, \mathrm{n}=6\right.$ ) was evaluated with 10 days $6^{\circ}$ HDT with strict diet control. Group II (GII, $n=6$ ) was investigated with increasing Na intake (three 8 days periods: $2.8->5.6->8.4 \mathrm{mmol} \mathrm{Na} / \mathrm{kg} \mathrm{BW}$ ). Half-life of $\mathrm{RBC}$ (T1/2RBC) was calculated by using ${ }^{51} \mathrm{Cr}$ counts $(\mathrm{CrC})$ of blood samples (BS). To calculate blood volume (BV) the $\mathrm{CrCs}$ were corrected by the T1/2RBC, the total volume of $\mathrm{BS}, \mathrm{T} 1 / 2$ of ${ }^{51} \mathrm{Cr}$ and an activity loss of the RBCs of $2 \%$ /day. Plasma (PV)- and RBC-volume (RBCV) was then calculated by using the hematocrit. In GI Na loss of $80 \mathrm{mmol}$ was observed during the first days of HDT and $\mathrm{Na}$ retention during recovery. PV was reduced by $16 \%$ and returned to normal during recovery whereas RBCV remained constant. T1/2RBC was slightly reduced $(p<0.4)$. In GII Na- and urodilatin (natriuretic peptide)-excretion increased with increasing levels of $\mathrm{Na}$ intake $(\mathrm{p}<$ 0.006 ) whereas urine flow remained unaffected. BV increased linearily with $\mathrm{Na}$ intake resulting in a raise of maximally $0.61 \pm 0.131$ (p $<0.05$ ). This was accompanied by a decrease in vasopressin, and renin-aldosteron levels. The metabolic $\mathrm{Na}$ balance revealed a $\mathrm{Na}$ storage without changes in serum Na concentration and -osmolality. T1/2RBC decreased significantly by 7.5 days $(p<0.05)$. We conclude, that microgravity and $\mathrm{Na}$ intake significantly affects the composition of body fluids and cells which can be accurately measured by the ${ }^{51} \mathrm{Cr}$-method and urodilatin appears to contribute to the day-to-day $\mathrm{Na}$ balance.

\section{2}

C. Aprile, G. Merlini,V. Bellott, G. Marinone, Fond.Cl inica Lavoro, IRCCS; Osp.S. Matteo, IRCCS-Pavia(I) and Hammersmith Hosp.- London(UK)

SCINTIGRAPHY AND KINETIC STUDY OF AMYLOID DEPOSITS WITH 131-I SAP

In this study we report on 7 pts with AL amyloidosis (13 studies) investigated employing SAP, a normal plasma glycoprotein that deposits on amylotd fibrils, labelled with 131-I. Prevtous injection of 25-40 MBq of 131-I SAP, a whole body scan was carried out and repeated after $1,2,3,5$ and 7 days; venous blood samples and urine were collected throughout the study. Whole body and argan retention of radioactivity were calculated from geometrical mean values and compared with the baseline scan. Six studies were performed in 4 pts after experimental therapy with an anthracycilne derivate. Acceleration of initial plasma clearance and increase in whole body retention were noted in pts with extensive amylold deposits. In 4 pts imaging showed involvement of the spleen, in 3 of the liver, in 2 of kidneys and bone marrow; 3 pts had an equivocal uptake respectively in heart, kidneys and adrenals. One pt with low whole body retention and normal plasma clearance, in whom biopsy did not conftrm the presence of amyloidosts, showed no significant focal uptake. A11 cases but one, presenting a large amyloid deposit involving the sacrum, showed no apprectable differences after treatment, although in 2 pts there were minor changes in plasma clearance and whole body retention indicating an increased sequestration of SAP into amyloid deposits. Despite the unfavourable physical carachtertstics of $131-\mathrm{I}$ and the low dose administered, these studies provide detailed information on the quantity and distribution of visceral AL amyloid deposits.
243

T. Lutz, C. Vo, and D.M. Lyster.

TRIUMF, Department of Pharm. Sci., University of British Columbia, Vancouver General Hospital, British Columbia, Canada.

SYNTHESIS OF AN I-123 LABELLED IODO GLUCOSE ANALOGUE AS A POTENTIAL IMAGING AGENT

Although F-18-2-fluoro-2-deoxyglucose (FDG) is the optimum agent used in heart and brain imaging, it is not routinely available. Due to the advantages of using a gamma emitting nuclide, several researchers have studied I-123 iodo glucose analogues, but none have shown any localization in heart or brain tissues.

Unfortunately 2-IDG is not stable enough to be isolated. Therefore we proposed the synthesis of 2-iodo-2-deoxy-1,5-anhydro-D-glucitol (2IDAG) where the C1 hydroxyl has been replaced by a hydrogen so as to stabilize the compound. This was a synthetic challenge starting with 1,5-anhydro-D-mannitol $(1,5$-AM) due to the similar reactivity properties of the $\mathrm{C} 2$ and $\mathrm{C} 4$ hydroxyls. 3,6-dipivaloy 1-1,5-anhydro-Dmannitol $(3,6-\mathrm{PM})$ was prepared by the addition of a $2: 1$ ratio of pivaloyl chloride:1,5-AM in $50 \%$ yield. $3,6-$ PM was then iodinated to give 3,6-dipivaloy1-2-iodo-1,5-anhydro-D-glucitol (3, 6-PIG) which was purified by HPLC. Compounds were characterized by elemental analyses, and NMR. 3,6-PM was also selectively mesylated at the C2 position to give 2-Ms-3,6-PM, which was used as a precursor for radioiodination. Under various reaction conditions, no radiolabelling was observed using I-123. We therefore exchange labelled 3,6-PIG obtaining $97.5 \%$ radiochemical purity. Biodistribution studies in mice showed $1.67 \%$ heart and $0.83 \%$ brain uptake at 30 minutes post injection.

\section{4}

C. Krummeich, M. Holschbach, G. Stöcklin

Institut für Nuklearchemie, Forschungszentrum Jülich $\mathrm{GmbH}$, 52425 Jülich, Germany

\section{A NEW RADIOIODINATED AMINO ACID FOR MEASURING AMINO ACW TRANSPORT WITH SPET}

3-Iodo-L- $\alpha$-methyltyrosine (IMT), has been shown to be a useful tracer for amino acid transport and has found application in tumour studies with Single Photon Emission Tomography (SPET) (Biersack et al., J. Nucl. Med. 1989, 30: 110-112; Langen et al., J. Nucl. Med. 1990, 31: 281-286, and ibid. 1991, 32: 1225-1228). However, the brain uptake of IMT is poor, and only allows the visualization of increased rather than decreased incorporation. We have therefore prepared and evaluated O-methylated radioiodinated tyrosines in order to increase their lipophilicities. Direct electrophilic n.c.a. radioiodination of the anisol-like aromatic moieties using the well known Chloramine$\mathrm{T}$ (CAT) method resulted in unsatisfactory yields. Using trifluoroacetic acid (TFA) containing $10 \mathrm{vol} \%$ water as a reaction solvent radiochemical yields of $40 \%$ of $3-\left[{ }^{123} \mathrm{I}\right]$ Iodo-O-methyl-L-tyrosine (IOMT) and 3-[123]]Iodo-O-methyl-L- $\alpha$-methyltyrosine (IOMMT) could be obtained at $60^{\circ} \mathrm{C}$ in 5 min reaction time. Use of Iodo-gen ${ }^{\mathrm{TM}}$ as an oxidant instead of CAT gave rise to RCY of $60 \%$ at $60^{\circ} \mathrm{C}$ in 45 min reaction time. In contrast to the normally used two phase system Iodo-gen ${ }^{\mathrm{TM}} /$ water our labelling reactions were performed in a homogeneous medium

IMT can be obtained in RCY's of $80 \%$ in 10 min at RT using Iodogen ${ }^{\mathrm{TM}}$ in the normal aqeous two phase system at $\mathrm{pH}=\mathbf{8}$.

Biodistribution studies with NMRI mice revealed a maximal brain uptake of the compounds that parallels their lipophilicities: IMT $<$ IOMT $<$ IOMMT. IOMMT exhibits a maximum brain uptake of $5 \%$ dose $/ g$ at $10 \mathrm{~min} \mathrm{p.i.} \mathrm{This} \mathrm{is} \mathrm{by} \mathrm{a} \mathrm{factor} \mathrm{of} 5$ higher than that of IMT used so far. In vivo deiodination of the O-methylated tyrosines is significantly inhibited and can be neglected. Thus, IOMMT seems to be a useful tracer for studying amino acid transport in brain with SPET. 


\section{5}

W. Brandau, F.-J. Gildehaus, C. Puskás, M. Jonas, B. Vollet, O. Schober

Department of Nuclear Medicine, University of Münster, FRG

\section{[I-123]IODO-PHIPA 3-10: A METABOLIC MARKER FOR MYOCARDIAL SPECT INVESTIGATIONS}

Recently 14-[F-18]Fluoro-6-thia-heptadecanoic acid has been proven to be a metabolically trapped fatty acid (DeGrado et al., JNM 32: 1888 [1991]) suitable for myocardial PET investigations. A phenylene-bridged LCFA (13-(p-[1-123]iodophenyl)-3-(p-phenylene)-tridecanoic acid, ([I-123]-PHIPA 3-10) which is trapped in the myocardium has been reported (Eisenhut et al. ARI 39: 639 [1988]).

In the current study we compared the influence of Etomoxir as a specific inhibitor of palmitoyl-carnitine-transferase I (CPT 1) on the biodistribution of [I-123]PHIPA 3-10 in animals. In humans, basic biokinetic data were determined in volunteers.

Using cuprous chloride as catalyst, labeling yields of $65 \pm 18 \%(n=34)$ were obtained by non-carrier-added [I-123] / Br-exchange at $180^{\circ} \mathrm{C}$. Purification by HPLC gave the final product in a radiochemical purity of $>99 \%$ and a specific activity of $>4 \mathrm{~GB} q / \mu \mathrm{mol}$ within $2 \mathrm{~h}$.

Animal studies were performed in non-fasted Sprague-Dawley rats. While myocardial biokinetic was comparable to the results obtained in fasted animals, liver activity drops from $5 \% \mathrm{I} . \mathrm{D} . \mathrm{g}(5 \mathrm{~min}$ p.i.) to $1.5 \% \mathrm{I} . \mathrm{D} . \mathrm{g}$ ( $30 \mathrm{~min}$ p.i.) and $0.6 \% \mathrm{IDD} . \mathrm{g}(4 \mathrm{~h}$ p.i.), in contrast to $3 \% \mathrm{I.D} . \mathrm{g}$ ( $4 \mathrm{~h}$ p.i.) retained in the liver of fasted rats. Treatment of non-fasted animals with Etomoxir $(20 \mathrm{mg} / \mathrm{kg})$ prior to injection of [I-123]-PHIPA 3-10 resulted in significant biokinetic differences. Myocardial activity accumulates significantly with time from $3.8 \%$ I.D./g (5 $\min$ p.i.) to $6.8 \%$ I.D. $/ \mathrm{g}(4 \mathrm{~h}$ p.i.) compared to $3.6 \%$ I.D. ( $5 \mathrm{~min}$ p.i.) and $3.2 \%$ I.D. $(4 \mathrm{~h}$ p.i.) for non-treated animals, whereas the biokinetics for blood, lung, liver etc. are not influenced.

In 4 volunteers planar and SPECT-studies were performed up to $24 \mathrm{~h}$ post injection of $150 \mathrm{MBq}$ [I-123]-PHIPA 3-10. Myocardial accumulation was $2.1 \pm 1.1 \%$ I.D. with a heart/lung ratio of $1.9 \pm 0.230 \mathrm{~min} \mathrm{p.i.} \mathrm{and} \mathrm{a} \mathrm{biological} \mathrm{half}$ life in the heart of about $27 \mathrm{~h}$. Quality of SPECT-images obtained with [I-123]PHIPA 3-10 was at least comparable to [TI-201]- or [Tc-99m]-MIBI-scans.

The results from the animal experiments indicate that the retention of [I-123]PHIPA 3-10 in myocardial tissue is influenced by CPT 1, and that this tracer could serve as a metabolic marker in myocardial SPECT-investigations.

\section{7}

K.Dutschka, H.H.Coenen, J.Farahati, Chr. Reiners Nuklearchemie und Radiopharmazie, Klinik und Poliklinik für Nuklearmedizin, Universitätsklinikum Essen, D-45122 Essen, Germany

CU(I)-ASSISTED RADIOSYNTHESIS AND HUMAN PLASMA ANALYSIS OF N.C.A. 2- $\beta$-CARBOMETHOXY $-3-\beta-(4-$ [123I] IODOPHENYL) TROPANE ([123I]RTI-55)

The cocaine-analogue RTI-55 ( $\beta$-CIT) exhibits a high affinity for the dopamine reuptake transporters. [123I]RTI-55 holds great diagnostic potential for Parkinson's disease with SPECT. For routine application its labelling by non-isotopic $123 \mathrm{I}$-for-Br exchange and its metabolic stability were examined.

The halogen exchange was performed on the bromine analogue (RTI-51, gift from Dr. Carroll, Research Triangle Institute, $\mathrm{NC}$ ) in a $0.01 \mathrm{mM}$ solution of $\mathrm{CuCl}$ in acetic acid. A radiochemical yield of 30 to $40 \%$ was obtained at $160^{\circ} \mathrm{C}$ within lomin. In comparison, iododestannylation yielded 60 to $80 \%$ with a max. specific activity of $<100$ $\mathrm{GBq} / \mu \mathrm{mol}$. This is due to intrinsic contamination of the tin-precursor with cold RTI-55. In contrast, with non-isotopic halogen exchange a specific activity of $4500 \mathrm{GBq} / \mu \mathrm{mol}$ is achieved which strongly reduces the pharmacological burden to the patient.

Analysis of [123I]RTI-55 in human plasma was performed by solid phase extraction (SepPak $\mathrm{C} 18$ plus) with $\mathrm{CH}_{3} \mathrm{CN}$ and subsequent isocratic HPLC on a $250 \times 4.6 \mathrm{~mm}$ Kromasil 5-C18 column with $\mathrm{CH}_{3} \mathrm{CN} / \mathrm{H}_{2} \mathrm{O} / \mathrm{Et}_{2} \mathrm{NH}=60 / 40 / 0.2$ as eluant. Relative plasma contents of $50 \%$ and $10 \%$ of the tracer were found at about $2 \mathrm{~h}$ and $18 \mathrm{~h} \mathrm{p.i.,} \mathrm{respec-}$ tively. Degradation probably proceedes in the liver as indicated by whole body scintigraphy and leads to one major polar metabolite.

\section{6}

J. Sartor, S. Guhlke, P.O. Zamora*, H.J. Biersack

Klinik for Nuclear Medicine, University of Bonn, D-53127 Bonn, Germany, *RhoMed Inc., Albuquerque, NM (USA)

NEW THIOL REACTIVE IODOVINYL AND IODOAROMATIC PROSTHETIC GROUPS FOR LABELING PEPTIDES AND PROTEINS

Radiolabeling peptides and proteins for diagnosis and therapy necessitates new special demands of peptides and proteins.

Two new reagents, iodoacylated p-Iodoaniline and iodoacylated 1-Iodoallylamine were prepared. For the aniline derivative yields of about $70 \%$ were obtained by a two step one-pot synthesis (iodination of aniline and subsequent iodoacylation of $p$-iodoaniline). In case of the vinyl derivative the radio-synthesis requires a stannylated precursor giving rise to almost quantitative iodination yields in one step.

Both reagents reacted quickly and quantitative with cysteine at $\mathrm{pH} 7.5$. Labeling of proteins was demonstrated using BSA as a model compound. In this case, coupling rates were higher using the aniline derivative and reached $40 \%$ RCY at $\mathrm{pH} 9$, where amine labeling would predominate. Lower yields of $25 \%$ (aniline) and $15 \%$ (vinyl) were obtained at $\mathrm{pH} 7.4$, where thiolate labeling would be expected. This result is consistent with the known low number of thiols in BSA. With the model peptide PA-22-2 (CSRARKQAASIKVAVSADR) labeling by both reagents was equally successful. The conjugation proceeded with yields of $77 \pm 15 \%$ within $30 \mathrm{~min}$ at $\mathrm{pH} 7.4$.

These reagents can be applied to a variety of peptides and proteins.

\section{8}

J. Mertens $^{1}$, J.E. Leysen ${ }^{2}$, D. Terriere', P.M .F. Janssen ${ }^{2}$, W. Gommeren'.

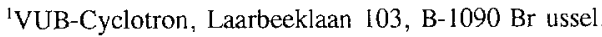
${ }^{2} J a n s s e n$ Research Foundation,2340 Beerse, Belgium. In vitro and in vivo evaluation of Radioiodo-4-amino- $\mathrm{N}-[1-[3-(4-$ fluorophenoxy) propyl]-4-methyl-4-piperidinyl]-5-iodo-2-me thoxybenzamide as a potential $5 \mathrm{HT}_{2}$ receptor tracer for SPET. Within a series of halogenated derivatives of 4-amino-N-[1-[3-(4fluorophenoxy)propyl]-4-methyl-4-piperidinyl]-5-halo-2-methoxybenzamide, a new class of potential $5 \mathrm{HT}_{2}$ antagonists, it was shown that the 5-iodo-2-methoxybenzamide analogue showed a high affinity ( $\mathrm{Ki}$ for inhibition of $\left[{ }^{3} \mathrm{H}\right]$ ketanserin binding to rat frontal cortex membranes $0.22 \mathrm{nM}$ ) and high selectivity for $5 \mathrm{HT}_{2}$ receptors (selectivity with regard to other neurotransmitter receptorsites of at least a factor 50 ). Therefore the pure N.C.A. radioiodinated analogue was synthetised and evaluated in vitro and in vivo in rats. Scatchard analysis of saturation binding to rat frontal cortex membranes of the I-125 labelled compound revealed a linear plot yielding a $\mathrm{Kd}$ of $0.11 \pm 0.01 \mathrm{nM}$ and a Bmax value of $37.79 \pm 0.75 \mathrm{fmol} / \mathrm{mg}$ tissue. The binding occured reversibly on one single type of receptor, i.e. $5 \mathrm{HT}_{2}$. From 1 hour up to 3 hours after i.v. injection into the tail vain of rats the frontal cortex to cerebellum ratio showed a steady state value of about 8-9 and the frontal cortex to blood ratio a value of $5-6$. The striatum/cerebellum and striatum/blood amounted to about half that value. Inhibition and displacement by Ritanserin and Ketanserin showed that binding to $5 \mathrm{HT}_{2}$ receptors was involved. Two hours after injection the $\% \mathrm{ID} / \mathrm{g}$ in frontal cortex was 0.24 and in striatum was 0.12 while in the total brain (frontal cortex and striatum included) this value amounts to 0.087 which means a ratio of at least 3 for frontal cortex and 2 for the striatum versus the rest of the brain. These results show that, if the behavior in human will be comparable, this new compound is really promising for visualisation of $5 \mathrm{HT}_{2}$ receptors with SPET 
Tuesday, 23 August 1994

\section{9}

Montserrat Estorch, Ignasi Carrió, José Lopez-Pousa, Lluis Berná, Gustavo Torres.

Hospital de Sant Pau, Barcelona.

\section{INTENSITY OF MYOCARDIAL 123I-MIBG UPTAKE RELATES TO AGE}

Fourty cancer patients, enrolled in a study to assess the effect of doxorubicin administration on adrenergic neuron function, underwent baseline studies with ${ }^{123} \mathrm{I}-\mathrm{MIBG}$ before chemotherapy. None of the patients had a history of previous cardiac disease or previous chemotherapy or mediastinal radiotherapy. MIBG uptake was quantified by a mediastinum to heart ratio (MHR) 4 hrs after i.v, administration of $5 \mathrm{mCi}$ of ${ }^{123} \mathrm{I}-\mathrm{MIBG}$.

Mean age of patients was 36 years, ranging from 16 to 75 . Six patients were below 20 years, 9 patients were between 20 and 40 years, 12 patients were between 40 and 60 years and 13 patients were over 60 years of age. Myocardial ${ }^{123} \mathrm{I}-\mathrm{MIBG}$ uptake was observed in all patients with a mean MHR of $1.85 \pm 0.29$, tange 1.31 to 2.62. MHR correlated to age $(r=-0.6264, p<0.001)$. A decline in ${ }^{123} \mathrm{I}$-MIBG with increasing age was observed. Mean MHR of patients of $<20$ years was 2.06 , of patients between $20-40$ years was 1.90 , of patients between $40-60$ years was 1.86 , and of patients $>60$ years was 1.55 . The best separation was observed comparing patients who were $>60$ years (mean MHR of $1.55 \pm 0.15$, range 1.31-1.78) with patients who were $<60$ years (mean MHR of $1.95 \pm 0.26$, range $1.45-2.62, p=0.003$ ); mean $\mathrm{EF}$ in both groups was similar: $56 \pm 7 \%$ and $60 \pm 7 \%, \mathrm{p}=\mathrm{NS}$.

We conclude that intensity of myocardial ${ }^{123}$ I-MIBG uptake relates to age. A decrease in MIBG uptake is observed with aging, specially in those patients over 60 years. This has to be taken into account when designing studies to assess cardiac adrenergic neuron function with ${ }^{123}$ I-MIBG.

\section{0}

Ch. Guertner, R.J. Werner ${ }^{\star}$, Th.Wendt**, B.J. Krause, G. Winten $^{\star \star}$, C. Teupe ${ }^{\star \star}$, F.D. Maul, H. Holzmann*, G. Hör

Depts. of Nuclear Medicine, Dermalology*, Cardiology**, University Hospital, Frankfurt am Main, Germany Evaluation of Cardiac Sympathetic Nervous System in
Systemic Sclerosis Using 123-1-Metaiodobenzylguanidine (MIBG) and SPECT

Autonomic dysfunction is known as a feature of systemic sclerosis. In order to evaluate the cardiac sympathetic nervous system in systemic sclerosis 9 patients (age 25-70 years, 8 female, 1 male) were investigated by $1-123-$ MiBG scintigraphy.1123-MIBG is an analogue of norepinephrine and therefore serves as a tracer of the integrity of the sympathetic nervous system. Supplementary myocardial stress and rest scintigraphy and cardiological examination including long-term ECG, stress $\mathrm{ECG}$ and echocardiography were performed. Static anterior images were aquired $15 \mathrm{~min}$ (extraneuronal) and $4 \mathrm{~h}$ (intraneuronal) after i.v. injection of $250 \mathrm{MBq} 123-1-\mathrm{MIBG}$. A $4 \mathrm{~h}$ SPECT aquisition was added. Image quantitation was based on the ratio of intraneuronal myocardial to mediastinal MIBG uptake.

None of the patients showed scintigraphic or electrocardiographic stress induced ischemia. Long-term ECG gave no evidence of pathological arrhythmias. showed a slight impairment of the diastolic compliance. On all SPECT images the MIBG uptake was homogenous without any regional defects. The intraneuronal MIBG uptake ratios ranging from 1,28 to 1,86 (average 1,57) were reduced indicating homogenous norepinephrine depletion. We conclude that sympathetic newous dysfunction can be detected by MIBGscintigraphy prior to noninvasive cardiological examinations. It therefore seems to be an early indicator of cardiac involvement in systemic sclerosis due to microangiopathy.

\section{1}

J.Lekakis, J.Nanas, V.Prassopoulos, Z.Margari, N.Kouvelas, P.Palazis, P. Kostamis, S.Moulopoulos.

Dept of $\mathrm{Cl}$ in Therapeutics \& Dept of Nucl. Medicine,

"Alexandra" University Hospital, Athens, Greece.

CARDIAC I-123 METAIODOBENZVLGUANIDINE UPTAKE MAY PREDICT 6-MONTH MORTALITY IN PATIENTS WITH CONGESTIVE HEART FAILURE.

Myocardial adrenergic innervation is disturbed in patients (pts) with congestive heart failure (CHF). I-123-metaiodobenzylguanidine (MIBG) is useful in assessing adrenergic cardiac innervation in those pts. To examine possible prognostic value of I-123 MIBG scintigraphy in CHF we analyzed data from $31 \mathrm{CHF}$ pts. Age was $52 \pm 13$ years, 30 pts were men, 13 pts were in Class I/II and $18 \mathrm{pts}$ in Class III/IV. 15 pts had dilated cardionyopathy, 12 ischemic cardiomyopathy and 4 pts valvular heart disease. Ejection fraction was $16 \pm 9 \%$, mean right atrial pressure (RA) $10 \pm 5 \mathrm{mmHg}$, mean pulmonary artery pressure (PA) $41 \pm 11$ mintg and mean pulmonary capillary wedge pressure (PCWP) $26 \pm 7 \mathrm{mmHg}$. Scintigraphy was performed 4 hours after intravenous injection of $5 \mathrm{mC}+\mathrm{I}-123 \mathrm{MIBG}$; uptake was assessed as heart to mediastinum ratio $(H / M)$. Mean $H / M$ in

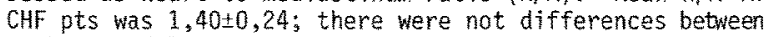
ischemic, dilated and vaivular disease and between Class I/II and III/IV pts. H/M showed a significant correlation with $E F(r=0,53, p<0,01)$ but not with RA, PA and PCWP pressures. All pts were followed up for a mean period $9,1 \pm 4,3$ months (range 2-16 months). 1 pt was transplanted (excluded from further analysis) and 6 pts died.From the remaining alive pts, 12 pts improved in NYHA Class, 2 pts deteriorated and the remaining were stable during follow-up. In 6 pts with a severeiy depressed I-123 MIBG uptake $(H / M<1,2), 2$ pts died within 6 months and 1 pt deteriorated. From the pts with $H / M>1,24$ pts died during follow-up; within 6 months 1 pt died and 1 pt deteriorated $(0,01<p<0,05)$. Thus a $H / M<1,2$ may identify pts at high risk for a subsequent cardiac death at 6 months. In those pts early transplantation is warranted.

\section{2}

Ch. Guertner, Ch. Schacherer*, J. Zickmann, B.J. Krause, F.D. Maul, H. Klepzig Jr.": G. Hör

Depts. of Nuclear Medicine, Cardiology ${ }^{*}$, University Hospital, Frankfurt am Main, Germany

\section{Evaluation The Sympathetic Nervous System With I-123- Metaiodobenzylguanidine in Silent Ischemia}

Myocardial ischemia may be strictly silent or a combination of symptomatic and silent episodes. Angina pectoris is mediated through afferent sympathetic nerve fibres. I-123-MIBG serves as an analogue of sympathetic neurotransmitter norepinephrine. In order to examine the integrity of the sympathetic nervous system 13 patients (age 48 - 69 years, male) with angiographically proven coronary artery disease and ECG documented asymptomatic ST-segment depression were investigated by 1-123-MBBG scintigraphy. Patients with diabetes mellitus were excluded. $15 \mathrm{~min}$ and $4 \mathrm{~h}$ after i.v. injection of $250 \mathrm{MBq}$ 1-123-MIBG anterior static images and a $4 \mathrm{~h}$ SPECT acqusition was obtained. Complementary myocardial stress and rest scintigraphy and long-term ECG was performed. MIBG quantitation was based on the myocardial to mediastinal ratio. The intraneuronal $(4 \mathrm{~h})$ MIBG uptake ranged from 1,35 to 1,84 (average 1,62) indicating reduced myocardial norepinephrine content. On SPECT images all patients showed regional MIBG depletion which corresponded with scintigraphically identified infarcted or ischemic regions. Well perfused myocardial regions matched with regular MIBG uptake. There was no evidence of increased arrhythmias in long-term ECG.

Our findings of regular MIBG uptake next to infarction or ischemia associated regional sympathetic nervous dysfunction endorse that silent ischemia is not due to a global sympathetic nervous dysfunction in a sense of cardiac polyneuropathy. 
253

B.J. Krause, C. Gürtner, F.D. Maul, T.W. Kranert, R.P. Baum and G. Hör

Department of Nuclear Medicine, J. W. Goethe-University Hospital, Frankfurt am Main, GERMANY

Double Isotope Technique Validation in the assessment of Sympathetic Reinnervation after Heart Transplantation using I-123 MIBG and TI-201: Patient and Phantom Studies

Sympathetic reinnervation was evaluated in 15 patients following hear transplantation by a double isotope technique using I-123-MIBG (MIBG) as tracer of the integrity of the sympathetic nervous system and T1-201 for landmarking to allow better delineation of the myocardium due to faint MIBG accumulation in heart transplants.

The goal of this study was to assess the influence of crossover from Tl201 in the I-123 energy window on the myocardial MIBG uptake ratio by patient and phantom studies.

In phantom studies (cylindrical phantom (CP), heart phantom (HP)) mean crossover from $\mathrm{Tl}-201$ in the I-123 window was $10.27 \%$ (CP) and $10.02 \%$ (HP). Depending on attenuation in water (depth of water 0 to $5 \mathrm{~cm}$ ) crossover increased from $10.27 \%$ to $14.03 \%$ (CP) and from $10.02 \%$ to $15.83 \%$ (HP)

8 Patients undergoing myocardial scintigraphy served as reference: after injection of Tl-201 an acquisition. was performed using two energy windows to assess crossover from Tl-201 to the I-123 window. Mean crossover was $15.35 \%$ being as high as the crossover assessed by phantom studies taking into account attenuation and scatter caused by the chest wall.

In order to reduce crossover, I-123-MIBG activity (370MBq) administered in patients following heart transplantation was six times as high as Tl-201 activity (37MBq). In phantom studies the I-123/Tl-201 activity ratio was varied $(1: 4,1: 5,1: 6,1: 7,1: 8)$. Mean crossover from Tl201 to the I-123 window ranged from $1,61 \%(1: 8)$ to $3.14 \%$ (1:4).

In conclusion 8 of 15 patients showed an increased MIBG uptake in the anterior basal region indicating sympathetic reinnervation following heart transplantation with a crossover from $\mathrm{Tl}-201$ in the I-123 window in the order of $2.5 \%$ leading to a negligible overestimation of the myocardial MIBG uptake ratio.

254

N.T.B. Nguyen, T.R.DeGrado, P. Chakraborty, D.Wieland, M.Schwaiger

University of Michigan, Ann Arbor, MI, U.S.A

EVALUATION OF C-11 EPINEPHRINE IN ISOLATED WORKING RAT HEART.

C-11Epinephrine (EPI) is a new PET tracer for the study of sympathetic nerve terminals in the myocardium. To study the kinetics of EPI, isolated working rat hearts were perfused with $\mathrm{K}-\mathrm{H}$ buffer. Control hearts were perfused with EPI in buffer for $20 \mathrm{~min}$ followed by a $40 \mathrm{~min}$ washout phase. To evaluate uptake specificity of the tracer, 50nM Desipramine (DMI), an uptake 1 blocker, was added to the perfusate in a second group of hearts. DMI was added to the perfusate during the washout phase only in a third group to study the effect of DMI on EPI clearance. A forth group of rats were pretreated with reserpine, which blocks vesicular uptake. The results show that $\mathrm{EPI}$ is avidly retained in the myocardium with slow clearance during washout phase. DMI significantly reduced both uptake and retention during washout (91\%) indicating low extraneuronal retention. There was also a significant decrease in both uptake and retention in the reserpine pretreated hearts (95\%), indicating storage of EPI in the neuronal vesicles In contrast to previous results with C-11 Hydroxyephedrine, EPI clearance is not accelerated by DMI during washout phase which is consistent with stable vesicular storage. Thus, EPI traces both neuronal uptake and storage, providing an excellent marker for neuronal turnover of stored catecholamines.

\section{5}

D. Apostolopoulos, J. Tselfes, C.Clannakenas, A. Mastorakou, B. Michalopoulos, A. Masmanides*, N. Papadakis*, P.J. Vassilakos.

Depts. of Nuclear Medicine and *Neurosurgery, Regional University Hospital, University of Patras, Medical School, Patras, Greece.

DUAL ISOTOPE IMAGINC OF BRAIN TUMORS WITH TL-201-CI AND IIn-111 DTPA-PhE-11-OCTREOTIDE : PREOPERATIVE TUMOR CHARACTERIZATION?

Radioisotopic imaging of brain tumors provides valuable information concerning tumor viability. residual tumor or recurrences. Preoperative characterization of these tumors is a standing challenge for Nuclear Medicine. In this respect, the object of this study was to define the potential value of combined imaging with $\mathrm{Tl}-201-\mathrm{Cl}$ and $\mathrm{In}-111$-Octreotide (Oct).

Dual tracer studies were performed in 26 patients, prior to open brain surgery (6 meningiomas, 14 gliomas, 3 metastatic tumors, 2 acoustic neuromas and 1 NHL) $74 \mathrm{MBg}$ of $\mathrm{Tl}-201$ were injected and planar images were acquired 10-30 $\mathrm{min}$ later, followed by the administration of $111 \mathrm{MBg}$ in-111-0ct and imaging 6 his later. SPECT was also performed in 8 of the patients. Tumor/Non Tumor hrs later. SPECT was also performed in 8 of the patients. Tumor/Non Tumor
$(\mathrm{T} / \mathrm{NT})$ ratios of both tracers, as well as TI/Oct ratios were calculated from planar (T/NT) ratios of both trace
images or SPECT slices.

To date the results of the study are as follows:

\begin{tabular}{|l|cc|cc|c|}
\hline Tumor type & $\begin{array}{c}\text { TI-201-Cl } \\
(+1\end{array}$ & $\begin{array}{c}\text { Mean } \\
\text { T/NT }\end{array}$ & $\begin{array}{c}\text { In-111-Oct } \\
(+)\end{array}$ & $\begin{array}{c}\text { Mean } \\
\text { T/NT }\end{array}$ & $\begin{array}{c}\text { Mean } \\
\text { TV/Oct }\end{array}$ \\
\hline Gliomas Grade II & $2 / 3$ & 1.39 & $3 / 3$ & 3.83 & 0.36 \\
Grade III & $6 / 7$ & 2.57 & $7 / 7$ & 2.98 & 1.12 \\
Grade N & $4 / 4$ & 3.36 & $2 / 4$ & 1.42 & 3.01 \\
& & & & & \\
Meninglomas & $6 / 6$ & 4.72 & $6 / 6$ & 7.94 & 0.63 \\
Metastatic & $3 / 3$ & 2.07 & $3 / 3$ & 3.27 & 0.74 \\
Neuromas & $0 / 2$ & 1.00 & $2 / 2$ & 1.96 & 0.52 \\
Lymphomas & $1 / 1$ & 2.23 & $1 / 1$ & 2.96 & 0.75 \\
\hline
\end{tabular}

In-111-Oct imaging is generally superior to Ti-201 in terms of tracer uptake. Apart from meningiomas, which show a fairly high uptake of both tracers, other types of intracranial tumors cannot be differentiated by means of any numerical values. in the case of gliomas, a double tracer study seems worthwhile, because some high grade astrocytomas do not concentrate in-111-0ct, while some of some high grade astrocytomas do not concentrate In-111-Oct, while some of
low grading may be missed by TI-201 alone. Moreover, the Tl/Oct ratio clearly
correlates with histological grading. This index can prove valuable for the correlates with histological grading. This index can prove valuable for the
noninvasive grading of gliomas, its implications thus affecting treatment strategles.

\section{6}

Renata Andrysiak, Leszek Królicki, Witold Mazurowski, Sławomir Budrewicz

Nuclear Medicine and Magnetic Resonance Imaging Department, Medical Acadery in Warsaw, Folard.

SemTC-MIBI and MUI In Diagnosis of CVS Tmours

There is some data indicating that MIBI-scan can be used in dlagncsis of the brain malignancy. Most of the observations were howevel performed before therapeutic treatment.

The aim of this study was to evaluate MIBI-scintigraphy in patients with equivocal MRI pattern after operation and raditherapy of the brain tumor. In all cases a recurrence process was suspected. The examinations were perfonned in 15 patients. Additianally, examinations were made in 9 patients (as a control group) with other, non-malignant diseases: vascular lesions $(n=6)$, toxoplasmosis $(n=1)$, brain traura $(n=1)$, brain abscess $(n=1)$. Somatom SF-G3 was used for MR imaging. Examinations were performec before and after Magnevist injection (T1, T2 and $P D$ seguences). Scintigraphic examinations were performed $1 \mathrm{~h}$ after injectlon of s9mTC-MIBI (15 mCi) using SEECT method. A gama camera ZLC Siemens with microdel ta computer system were used.

In 9 cases a true positive and in 6 cases a true negative results of MIBI scan were obtained in the group of patients with suspiclor: of recurrence process.

In the second group, in all cases, a nomal scintigraphic pattern was observed.

On the basis of present experiences we concluded that MIBI-scan could be a useful method in examination of the neoplastic recurrence of the brain tumours. This trethod might be helpful when MRI pattem is equivocal. 
257

L.Maffioli, M.Gasparini, M.R.Castellani, A.Gramaglia, V.Mongioj, M.scorsetti, A.Chiti, C.Giorgi, G.L.Buraggi. Nuclear Medicine Div. and Radiotherapy Div. of National Cancer Institute - Milan. Neurosurgical Div. of "C.Besta" Neurologic Institute - Milan - Italy

\section{TC-99M SESTAMIBI SPET IN DETECTING} BRAIN TUYOURS REIAAPSES

One of the most important problems for neurologist is the distinction between brain tumour relapse and necrosis after therapy. Recent investigations have shown that TC-99m-SestaMIBI could be used to image several tumours.

Aim of the study was to evaluate clinical usefulness of Tc-99m-SestaMIBI SPET in imaging brain tumours and differentiating between local relapse and necrosis. Based on clinical and radiological (CT or MR scan) suspicion of relapse of brain tumours after surgery. chemo and radiotherapy, 30 patients (M/F $=20 / 10$; age range $=18-70$ years) were enrolled in this study. SPET was performed $I$ hour after i.v. injection of $20 \mathrm{mci}$ of Tc-99m-SestaMIBI. The acquisitions were executed with a Toshiba GCA-901A on a $64 * 64$ matrix, zoom $=1.5,30$ sec/proj, 60 steps over $360^{\circ}$. Results were compared with clinical status and radiological findings during the follow-up.

SPET resulted positive in $24 / 30$ pts: $7 / 8$ glioblastomas, $8 / 10$ astrocytomas, $1 / 2$ oligodendrogliomas, $1 / 1$ gliosarcoma, $1 / 1$ meningioma, $1 / 2$ medulloblastomas, $0 / 1$ pinealoblastoma, $0 / 1$ chordoma, $1 / 1$ melanoma, $3 / 3$ metastatic ca. In 6 cases SPET showed no pathological uptake. Choroidal plexuses were visualized in all patients. After a mean follow-up time of 6 months, these findings appear as follows: $18 \mathrm{TP}$; $3 \mathrm{TN}$; $1 \mathrm{FP} ; 4 \mathrm{FN}$. The remaining 4 pts are still under observation. We are also correlating tumour uptake with histological grading. In conclusion, Tc-99m-SestaMIBI seems to be a promising tracer for brain imaging, to distinguish between tumor relapse and radionecrosis.

258

Q. Schober ${ }^{1}$; S. Assmann ${ }^{1}$, W. Wagner ${ }^{2}$, J. Sciuk ${ }^{1}$, P. Bartenstein ${ }^{1}$, W. Brandau 1 , N. Willich ${ }^{2}$, H. Wassmann ${ }^{3}$.

Departments of Nuclear Medicine ${ }^{1}$, Radiotherapy ${ }^{2}$ and Neurosurgery ${ }^{3}$, University Hospital, Münster; Germany

\section{THEASSESSMENT OF 123 THE FOLLOW UP OF PRIMARY BRATY TOSIN (IMT)}

PET (amino acids, FDG) has been successful in differentiating between recurrence, radiation necrosis and oedema in brain tumours. This was confirmed in a comparative study with 123 I- $\alpha$-IMT-SPECT. It was the aim of this study evaluate the grade of malignancy and to assess the diagnostic and prognostic usefulness of IMT-SPECT in the follow up of patients after surgery and intra operative radiotherapy (IORT).

52 patients with suspected brain tumours ( 13 controls, 3 metastases, 36 gliomas [histopathology grading: low grade II: 6; high grade IIIII,III: 17, III-IV,IV: 13]) (age 2-59 y) were additionally characterized by morphologically oriented methods (MRI/CT) and functional imaging (99mTc-HMPAO; 123I-IMT; three headed-SPECT-system, SIEMENS Gammasonics; LEUHR). The scans were visually compared and evaluated by ROIs and T/NT-ratios. 14 patients were followed (up to $3 \mathrm{x}$ ) by SPECT imaging after IORT.

Results:

1. Tumor extent and uptake: IMT $\geq$ HMPAO in $51 / 52$ patients (exception 1 abcess).

2. IMT uptake and histopathologic tumor grading showed only a weak correlation (low grade: T/NT: II: 1.2, 1.1-1-3; high grade: II-III, III: 1.6, 1.2-3.1, III-IV,IV: 1.7, 1.3-2.1) (median, range).

3. IMT-SPECT: up to 6 months earlier positive than morphologic changes (CT/MRT).

4. Effect of therapy (surgery and IORT) on IMT-uptake: T/NT pre 1.55 $(1.2-3.9)$ vs. T/NT post 1.15 (1.0-3.1) ( $p<0.001$; median, range). Conclusions:

1. The IMT uptake in cerebral gliomas is dominated by amino acid transport and not by perfusion (HMAPO).

2. IMT-SPECT provides important prognostic and additional information in all types of primary brain tumors, esp. after IORT

3. IMT-SPECT is comparable to PET in patients with brain tumors. The uptake indicates early a poor prognosis regardless of histologic diagnosis or previous therapy.
259

J. Pruim, A.M.J. Paans, A. van Waarde, A.T.M. Willemsen, G.M. Visser, M.A.A.M. Heesters, K.G. Go, W. Vaalburg.

National Research PET-Centre, Dept. of Radiotherapy, and Dept. of Neurosurgery, University Hospital, Groningen, The Netherlands.

QUANTIFICATION OF THE PROTEIN SYNTHESIS RATE OF BRAIN TUMOURS WITH L-1-[1" C]-TYROSINE PET: A MODALITY FOR THE ASSESSMENT OF THERAPY.

Functional parameters are needed to assess the response of tumours to therapy. Quantitation of the protein synthesis rate (PSR) is possible with the tracer L-1- $\left[{ }^{11} \mathrm{C}\right]$-tyrosine (TYR). Here we report the first results with TYR in patients with brain tumours, including the response to therapy.

Sixteen patients with a suspected primary or recurrent brain tumour were studied. Patients received $370 \mathrm{MBq}$ TYR i.v. A dynamic protocol was used and arterial blood samples were taken for measurement of the input function and the assessment of metabolites. PSR was calculated using a modified Patlak-analysis. Two of the patients underwent a second TYR-PET shortly after the end of radiotherapy. Histology showed: astrocytoma $(n=5)$, oligodendroglioma $(n=2)$, glioblastoma $(n=4)$, metastasis $(n=1)$, meningioma $(n=1)$, vasculitis $(n=1)$. No histological confirmation was obtained in 2 patients: 1 with a positive signal on TYR-PET, 1 with a negative signal.

All tumours gave a positive signal. The PSR varied between 0.68 and $2.05 \mathrm{nmol} / \mathrm{ml} / \mathrm{min}$ in the tumours and between 0.42 and $1.64 \mathrm{nmol} /$ $\mathrm{ml} / \mathrm{min}$ in the contralateral hemisphere. Tumor to background ratio was 1.64 \pm 0.46 . In 2 patients an -unexplained- low PSR was found (tumour: 0.15 and 0.17 , hemisphere: 0.12 and 0.07 ). The vasculitis patient had a PSR of 0.82 in the lesion, while the meningioma did not show up on the scan. Both patients that were studied after radiotherapy too showed no respons in the PSR, indicating no effect on tumour metabolism. ${ }^{1} \mathrm{H}$-spectroscopy and clinical symptoms pointed towards similar findings.

In conclusion: TYR is a useful diagnostic tool for the visualisation of brain tumours. The possibility of calculating a PSR allows the use of TYR-PET in the evaluation of radiotherapy.

\section{0}

K.-J. Langen ${ }^{1}$, K. Ziemons ${ }^{1}$ J.C.W. Kiwit ${ }^{3}$, H.Herzog ${ }^{1}$ T. Kuwert ${ }^{1}$, M. Weckesser ${ }^{1}$ M. Holschbach ${ }^{2}$ G. Stöcklin2, W.J. Bock ${ }^{3}$, H.-W. MüllerGärtner ${ }^{1}$ 1) Institute of Medicine and 2) Nuclear Chemistry, Research Center Jülich, 1) Department of Nuclear Medicine and ${ }^{3}$ Neurosurgery, University of Düsseldorf, Germany

QUANTIFICATION OF AMINO ACID TRANSPORT USING DYNAMIC 1-123- $\alpha$-METHYLTYROSINE SPECT IN PATIENTS WITH BRAIN TUMORS

3-[I-123]iodo- $\alpha$-methyltyrosine (IMT) has been shown to be a tracer of amino acid transport. This study investigates the determination of rate constants of amino acid transport in brain tumors and normal brain using dynamic IMT SPECT.

Ten patients with cerebral gliomas were investigated by dynamic SPECT using a triple-headed system after i.v. injection of no carrier added (n.c.a.) IMT (8 scans á 2 min., 9 scans á 5 min.). For comparison, dynamic PET studies after injection of n.c.a. [methyl-C-11]-L-methionine (MET) were performed on the same day. The plasma input functions were determined and corrected for metabolites. The SPECT scanner was calibrated against the well counter by brain phantom studies and the SPECT scans corrected for attenuation by individual contour finding. The kinetics of IMT in plasma brain and brain tumors were evaluated by Logan plots for 2-compartment models and the kinetics of MET by Patlak plots to determine the rate constant of unidirectional influx, $\mathrm{Ki}$.

The Logan plots of IMT kinetics were linear for tumors and normal brain in all subjects and allowed the calculation of the influx and backflow rate constant K1 and k2 of IMT transport. K1 values were significantly higher for tumors than for normal brain $(0.120 \pm 0.044$ vs. $0.055 \pm 0.020, \mathrm{n}=10$ $\mathrm{p}<0.01))$ as were the $\mathrm{Ki}$ values for MET $(0.044 \pm 0.018$ vs $0.020 \pm 0.007$ $\mathrm{n}=10, \mathrm{p}<0.01$ ). However, no significant correlation of the K1 values of IMT and the $\mathrm{Ki}$ values of MET could be found in this group of patients.

In conclusion, dynamic IMT SPECT appears to be capable to determine the rate constants of amino acid transport in brain and brain tumors. The lack of correlation with MET influx may indicate that SPECT quantification has still to be improved and needs sophisticated scatter correction and attenuation correction by measured transmission scans. 
261

I. van der Laken, O.C. Boerman, W.J.G. Oyen, M.T.P. van de Ven, J. Makarewicz, R,A.M.J. Claessens, J.W.M. van der Meer, F.H.M. Corstens. Dpts. of Nuclear Medicine and Internal Medicine, University Hospital Nijmegen, Nijmegen, The Netherlands.

\section{RECOMBINANT HUMAN INTERLEUKIN-1: A POTENTIAL AGENT TO IMAGE INFECTIOUS FOCI}

Infection and inflammation are characterized by an influx of activated leukocytes, being predominantly interleukin-1-receptor positive cells. Interleukin-1 (IL-1) has a high affinity for its receptor and might therefore be able to specifically localize in the infectious or inflammatory tissue. We investigated whether recombinant human $\mathrm{IL}-1$ can be used for imaging infectious foci in vivo in mice and rats.

IL-1 $\alpha$ was radiolabeled with I-125 via the glucose-oxidase/lactoperoxidase method, resulting in a specific activity of $60-120 \mu \mathrm{Ci} / \mu \mathrm{g}$. The receptor binding fraction in vitro, determined on EL-4 thymoma cells, was 70-95\%. $24 \mathrm{Hr}$ after induction of a S. Aureus abscess in the left calf muscle, 25 Swiss mice $(20-25 \mathrm{~g})$ were i.v. injected with $0.2 \mathrm{ml} 10 \mu \mathrm{Ci}$ 1-125-IL-1 $\alpha$. Groups of five mice were sacrificed at $2,6,12,24$ and $48 \mathrm{hr}$ p.i. Activity uptake was assessed in the blood, muscle, abscess, thymus, thyroid, lung, spleen, kidney, liver and intestine. Gamma camera images were obtained by injecting rats i.v. with $400 \mu \mathrm{Ci}$ I-131-IL-1 $\alpha$.

I-125-IL-1 $\alpha$ was rapidly cleared from the blood; $12 \mathrm{hr}$ p.i. the activity had decreased to less than $0.5 \% \mathrm{ID} / \mathrm{g}$. After $12 \mathrm{hr}$, the abscess was the organ with the highest activity $(1.02 \pm 0.23 \% \mathrm{~m} / \mathrm{g})$. The abscess/muscle ratios sharply rose from $3.2 \pm 0.5$ at $2 \mathrm{hr}$ p.i. to an ultimate value of $37.0 \pm 13.2$ at $48 \mathrm{hr}$ p.i., whereas the ratios of a control agent, B-Lactoglobulin, only slightly increased from $3.1 \pm 0.6$ to $5.0 \pm 0.8$ at 2 and $48 \mathrm{hr}$ p.i. respectively. The ratios of I-125-IL-1 $\alpha$ were significantly higher from $12 \mathrm{hr}$ onwards (Wilcoxon-Rank test, $\mathrm{p}<0.008$ ). The abscesses were clearly visualized at 12,24 and $48 \mathrm{hr}$ p.i.

Our results demonstrate specific retention of IL-1 in the abscess, presumably by interaction of IL-1 with its receptor on the inflammatory cells. The high target-to-background ratios that were obtained relatively shortly after injection indicate that $I L-1$ may be a valuable agent to image infectious foci.

\section{2}

A. Goodbody, J. Ballinger*, L. Tran, M. SumnerSmith, F. Lau, K. Meghji, A. Pollak.

Resolution Pharmaceuticals Inc., Mississauga and *ontario Cancer Institute, Toronto, Ontario, Canada

A NEW TC-99M LABELLED FEPTIDE INFLAMMATION IMAGING AGENT

Tuftsin, a tetrapeptide derived from the Fc portion of IgG, promotes phagocytosis and chemotaxis of neutrophils and monocyte/ macrophages by a receptor-mediated mechanism. Given the uniqueness of the receptor for these cells, we have attempted to use tuftsin antagonist (TKPPR) as a targeting moiety for inflammation imaging.

The peptide was synthesized with an N3S peptidic chelation site (Pic-SC(Acm)G; Pic = picolinic acid) attached at the N terminus. It was easily labelled with Tc $99 \mathrm{~m}$ by transchelation from glucoheptonate. Typical radiochemical purities were in the range of 91 97\%. Labelled peptide was administered intravenously $(100 \mu \mathrm{Ci} / 0.6 \mu \mathrm{g}$ dose) to rats with infectious ( $E$, coli) inflammation of the right thigh and excellent scintigraphic images of the site were obtained within $0.5 \mathrm{~h}$ of injection. Measurements of radioactivity in tissues excised $0.5,3$ and $17 \mathrm{~h}$ post-injection revealed target to background ratios of 3.6 5.0 and 16.2 respectively $(n=2-4)$ Elimination was primarily renal with $80.6 \%$ of the dose in the bladder at 3h.

In conclusion, the tuftsin antagonist TKPPR has excellent potential as a targeting agent for inflammation imaging.

\section{3}

R.A.M.J. Claessens, E.B. Koenders, W.J.G. Oyen, O.C. Boerman, M.J. Abrams, H.F. Solomon, and F.H.M. Corstens.

University Hospital, Nijmegen, The Netheriands, Johnson Matthey Pharm. Research, West Chester, PA, and R.W. Johnson Pharm. Research Institute, Spring House, PA.

RETENTION OF TECHNETIUM IN AND RELEASE OF PROTEIN METABOLITES FROM INFECTIOUS FOCI AFTER I.V. ADMIN ISTRATION OF TC-99m-HUMAN POLYCLONAL IgG TO RATS.

in-111 and Tc-99m labeled human polyclonal immunoglobulin $G$ (hlgG) are of clinical use for detection of sites of infection. Previously we reported on the role of release of in-111 from In-111-hlgG in the retention of the indium label in foci of infection. This study focuses on the release of Tc-99m from Tc-99m-higG in infectious foci, comparing two different Tc-99m-hlgG preparations with in-111-hlgG.

Tcr 99m-labeled nicotinyl hydrazino higG (Johnson Matthey, JM) and Tc-99m-labeled 2-iminothiolane-modified hlgG (Mallinckrodt Diagnosti$\mathrm{ca}, \mathrm{MD}$ ) were both labeled with $\mathrm{C}-14$ by methylation, using $\mathrm{C}-14$ formaldehyde, and purified by gel filtration. DTPA-conjugated higG was labeled with $\mathrm{In}-111$ by citrate transchelation and used as such. HPLC was performed as quality control. Young Wistar rats with a $S$ Aureus infection of the left calf muscle were i.v. injected with either 6 $\mathrm{MBq} \mathrm{Tc}-99 \mathrm{~m}$ and $40 \mathrm{KBq} \mathrm{C}-14$ labeled to 130 or $50 \mu \mathrm{g} \mathrm{hlgG} \mathrm{(JM} \mathrm{resp.}$ $\mathrm{MD}$ ) or $2 \mathrm{MBq}$ in-111 labeled to $80 \mathrm{\mu g}$ higG. Rats were sacrificed at 2,6 , or $24 \mathrm{hr}$ p.i... Averages $\pm S D(n=5)$ of percentages of dose per gram (\%/D/g) were determined for plasma, urine, abscess, and various other tissues. Plasma and urine samples were analyzed by HPLC.

The radiochemical purity was $>96 \%$. Tc-99m-hlgG (JM) was better retained in abscesses and had a slower plasma clearance than both Tc-99m-higG (MD) and in-111-higg (24 hr p.i. \%ID/g abscess: JM 1.48; MD: $.84 ;$ in: $1.10 ; p<0.01$; plasma: 3.24 resp. 1.69 and 1.28 p<0.01; abscess/muscle: 9.0 resp. 6.5 and 6.3) In abscesses the Tc $99 \mathrm{~m} / \mathrm{C}-14$ ratio increased over time. The renal uptake of Tc-99m-hlgC (JM) at $24 \mathrm{hr}$ p.i. was lower than for both other agents (\%ID/g kidney: $\mathrm{JM}: 1.45 ; \mathrm{MD}: 5.07 ; \mathrm{In}: 4.57 ; \mathrm{p}<0.01)$. In plasma a small quantity of only one Tc-99m-labeled metabolite was found with a higher molecular weight than hlgG.

In conclusion: Tc-99m-labeled nicotinyl hydrazino hlgG (JM) seems an attractive agent for localization of infections. As in-111, Tc-99m is retained in infectious foci, whereas IgG or its metabolites are released.

\section{4}

W. Becker, H. Streckenbach, J. Bair, H.J. Hansen, D.M. Goldenberg, F. Wolt

Department of Nuclear Medicine of the University of ErlangenNuremberg, FRG; Immunomedics, Inc. Morris Plains, $\mathrm{N} J$ and

Center of Molecular Medicine and Immunology, Newark, NJ, USA

KINETICS OF A NEW TC-99m-ANTIGRANULOCYTE FAB'FRAGMENT(NCA-90; IMMU-MN3) FOR THE DIAGNOSIS OF INFECTIOUS DISEASES

The kinetic data and whole body distribution of a new Tc-99mantigranulocyte Fab'-fragment (NCA-90; IMMU-MN3) were prospectively examined in 10 patients with suspected osteomyelitis and/or fever, who were injected with 185-900 MBq(0.1-1.0mg) of the labelled fragment. Blood specimens ( $5 \mathrm{ml}$ ) were drawn $5,15,30,45$ and $60 \mathrm{~min}$, $4 \mathrm{~h}$ and $24 \mathrm{~h}$ postinjection for calculation of the recovery rate of the antibody in whole blood. After isolation of pure granulocytes on a PERCOLL/plasma gradient, the antibody distribution in the gradient was imaged with a gamma camera and calculated by ROl-technique. Whole body scans $1 \mathrm{~h}, 4 \mathrm{~h}$ and $24 \mathrm{~h}$ p.t. in anterior and posterior projections were performed to calculate whole body distribution.

The recovery rate of the fragment in whole blood was $38.8 \%$ (5min), $24.4 \%(1 \mathrm{~h}), 12.2 \%(4 \mathrm{~h})$ and $3.2 \%(24 \mathrm{~h})$. The recovery rate for the cell associated Tc-99m-fragment was $4 \%-6 \%$ (5min$60 \mathrm{~min}$ ). In-vivo infectious lesions could already be detected in post injection. These results prove the low antibody binding to circulating granulocytes and a high in-vivo availability of the unbound antibody.

In summary, this high in-vivo availabiltiy of the unbound Fab'fragment seems to allow a binding of the fragment in p.l. to granulocytes in the foci and imaging of an infection independent from leucocyte migration. 


\section{5}

W. Becker, C. Palestro, J. Winship, D.M. Goldenberg, F. Wolf Department of Nuclear Medicine of the University of ErlangenNuremberg,FRG; Long Island Jewish Medical Center,NY, Center for Molecular Medicine and Immunology, Newark, NJ, and Immunomedics, Inc., Morris Plains, New Jersey, USA

TC-99m-LABELLED ANTIGRANULOCYTE FAB'-FRAGMENTS(IMMU-MN3) FOR IMAGING INFECTION-RESULTS OF A MULTICENTER STUDY

The diagnostic accuracy for imaging infection of a Tc-99m-label led antigranulocyte Fab'-fragment(NCA-90;IMMU-MN3) was prospectively examined in a multicenter study Scintigraphy was performed in 53 patients $(27 \mathrm{~m}$; 26 ; ;age: $26-86$ years) between 1-6 $\mathrm{h}$ and $24 \mathrm{~h}$ p.i. after injection of 0.1-1.00 $\mathrm{mg}$ of antibody fragment labeled with $166-1036 \mathrm{MBq}$ of Tc- $-99 \mathrm{~m}$ In total, 39 infectious lesions were detected with histology, cytology, other imaging procedures, or by follow-up.38 patients were additionally examined with Tc-99m-HMPAO or In-111oxin labelled granulocytes within of one week of the MN3 study.

Sensitivity, specificity and diagnostic accuracy of Tc-99m-MN3 was $92.3 \%, 85.2 \%$ and $89.4 \%$, of autologous leukocyte scintigraphy $86.7 \%, 77.8 \%$ and $83.3 \%$, respectively. The sensitivity was independent of the amount of the labelled antibody injected (0.1-0.5mg: $96.6 \% ;>0.5-0.9 \mathrm{mg}: 100 \% ;>0.9-1.00 \mathrm{mg}: 71.4 \%$ ). False positive lesions were detected in a periprosthetic calcification a frontal hyperostosis and two prosthetic hips which had loosened. Human antimouse antibody (HAMA)could not be detected in any of the 13 patients 1 or 3 months postinjection In summary, Tc-99m-IMMU-MN3 is suitable for imaging infectious lesions and has diagnostic advantages over autologous leukocyte scintigraphy. The sensitivity is independent of the in jected antibody dose.HAMA could not be detected.

\section{7}

Kairemo KJA, Korppi-Tommola T, Salo Jo, Taavitsainen MJ, Rannikko $S$

Depts of Clinical Chemistry, Radiology and Urology, Helsinki University Central Hospital

Dosimetric and kinetic characteristics of Re-186-HEDP administered due to skeletal pain in prostate cancer Patients with verified skeletal metastases of prostate cancer were studied for pain relief by injecting 1300 MBq Re-186-HEDP ( $\beta 1.07 \mathrm{MeV}, \gamma 137 \mathrm{keV}(9 \%), t_{1 / 2} 89.3 \mathrm{~h}$ ). These patients were studied quantitatively using gamma imaging (2-headed Picker Prism 2000 with Odyssey computer) at 4,28 and 100 hours, and prior to therapy a quantitative bone scan with Tc-99m-HDP. They have several multiple (>15) foci (I), superscan (II) or a few (<15) strong uptakes (III).

The lesions were analyzed using a conjugate view method (ROIs from $A P$ and $P A$ views, geometric mean and size of the lesion, std activity source). The lesions were analyzed separately in skull (S), vertebrae (V), ribs (R),pelvis (P) and extremities(E) separately using background subtraction (mirror technique.

The skeletal Tc-99m-HDP-uptake at $4 \mathrm{~h}$ varied from $24.5 \%$ to $44.0 \%$ ID, whereas the Re-186-HEDP-uptake at $4 \mathrm{~h}$ varied from $25.4 \%$ to $58.6 \%$ ID. In lesions, eg.in a patient with 97 lesions, the uptakes of TC-99m (at 4h) were as follows: $S 0.030, \mathrm{~V} 0.073, \mathrm{R} 0.29, \mathrm{P} 0.051$, and $E 0.14 \% I D / \mathrm{cm}^{3}$; and with Re-186 (at $4 \mathrm{~h}$ ) S $0.034, \mathrm{~V}$ $0.068, R 0.35, \mathrm{P} 0.053$, and $\mathrm{E} 0.16 \% \mathrm{ID} / \mathrm{cm}^{3}$. The $\mathrm{Re}-186$ HEDP-half lives in lesions varied from 41 up to 113 hours. The dissappearance rate from the skeleton was quite constant, the Re-186-uptake of ID was usually higher than that of Tc-99m. The highest uptake in a single solitary lesion was observed in III, being $0.36 \%$ ID $/ \mathrm{cm}^{3}$, and the whole lesion was $11.2 \%$ ID at $4 \mathrm{~h}$.

This data indicates that a proper quantitation should be performed in order to estimate the real dose delivered by palliative bone pain treatment because of large individual and interlesional variation.

\section{6}

Reske SN, Fleischmann $W^{*}$, Brecht-Krauß D, Schulte M, Kinzl L.*

Department Radiology III and *Surgery III, University Uln, Germany.

Sensitive detection of musculoskeletal inflammation with FDG-PET

Based on a highly stimulated glycolysis of activated leucocytes and macrophages, we examined performance of FDG-PET for detecting and defining anatominal extent of musculoskeletal inflammatory disease. In total, $25 \mathrm{pa}-$ tients (pts) with suspected chronic recurrent posttraumatic osteitis (19 pts) or spondylodiscitis (6 pts) were examined with FDG-PET in the fasting state 45 min after injection of $350-450 \mathrm{MBq}$ FDG $i . v$. with an ECAT 931-08-12 PET scanner (Siemens/CTI, Knoxville TN). Transverse, frontal and sagittal images were generated with an iterative reconstruction technique. In all pts, infection imaging was done additionally with a Tc-99m labeled monoclonal antigranulocytic antibody (TcNCAA) 4 and $24 \mathrm{hrs}$ after injection of $350-450 \mathrm{MBq}$ antibody i.v. Presence or exclusion of active inflammation was determined by intraoperative and histological findings in 12 pts and by clinical follow-up in 13 pts. Focally increased FDG-uptake correctly identified $18 \mathrm{pts}$ with active inflammation, whereas normal FDG-uptake excluded correctly active disease in $6 \mathrm{pts}$. Intramedullary osteitis, fistulas and paraosseal soft tissue involvement could only be differentiated by FDG-PET whereas TCNCAA only allowed the distinction of presence or absence of active disease. Active spondylodiscitis could not be diagnosed with TcNCAA but was clearly imaged as focally increased FDG-uptake in all 6 pts with PET. Sensitivity and specificity of FDG-PET - $100 \%$ and $96 \%$ respectively were significantly superior to TcNCAA (61\% and $86 \%$, $\mathrm{p}<0.01, \mathrm{~N}=25$ ). These first results indicate a significant potential of FDG-PET for detecting and localizing acitive musculoskeletal inflammatory disease.

\section{8}

GS Limouris ${ }^{a}$, V Giannakopoulos", A Gouliamos ${ }^{\text {b }}$, SK Shukla ${ }^{\mathrm{c}}$, A Stavraka ${ }^{\text {a }}$ a. Nuclear Medicine Section, Areteion Univ Hosp b. Dept of Radiology, Areteion Univ Hosp, c. CNR+Nuclear Medicine Dept, St Eugenio Hosp, Rome

\section{PAMIDRONATE AND RENIUM-186-HEDP APPLICATION IN MIXED OSSEOUS METASTASES}

The specificity and potency of Pamidronate as an inhibitor of osteoclastic bone resorption provides the rationale for its therapeutic use in osteolytic bone metastases. The mode of its action comprises: a) adsorption onto the surface of hydroxyapatite crystals in mineralized bone matrix, thercby reducing the solubility of the mineralized matrix and rendering it more resistant to osteoclastic resoption and b) impairment of the attachment of osteoclast precursors to the mineraljzed matrix, thereby blocking their subsequent transformation into mature, functioning ostcoclasts. On the other hand, Re-186-HEDP exclusively accumulates by chemisorption onto the surface of hydroxyapatite crystals of the osteosclerotic osscous lesion.

The efficacy of Pamidronate (dealing with the osteolytic bone activity) and of Re-186-HEDP (dealing with the osteosclerotic bone activity) combined treatment was evaluated in 7 patients with mixed (osteolytic lesions, surrounded by osteosclerotic halo) bone metastases due to osteosarcoma (1 male aged $24 \mathrm{yrs}$ ), lung ( 3 males aged 37 to 57 years) and colon ( 1 female and 2 males aged 62 to 70 yrs) cancer.

In all 7 cancer patients parallel to $1400+100 \mathrm{MBq}$ Re-186-HEDP i.v therapy [Mallinckrodt Medical B.V., Petten] an anti-osteolytic treatment with Pamidronate [Aredia, Ciba-Geigy Ltd, Basel] was performed via i.v. infusions in a total monthly amount of $120 \mathrm{mg}$ and for a three months duration.

The follow-up period covered thirty weeks. The efficacy of the treatment was assessed by a) a pain and performance questionnaire that the patients were asked to complete daily b) a CT and a bone density scan comparison of a randomly preselected osseous lesion before and 30 wecks after Pamidronate and radio labelled Renium application.

All 7 patients responded to therapy. The osteosarcoma patient became free of pain while the other 6 patients experienced obvious pain improvement. None manifested a flare response to treatment. All patients showed a definite decrease of platelets and absolute number of polymorphonuclear white blood cells up to the fourth week following treatment. Two out of 3 colon cancer patients underwent a whole blood transfusion.

Combined Pamidronate and Re-186-HEDP therapy appears to be very promissing for the palliation of painful mixed bone mctastases. 
269

L. Oliveira, G. Cantinho, A.I. Santos

instituto de Medicina Nuclear, FML - Lisboa - Portugal

STRONTIUM BS IN PAINFUL BONE METASTASIS: SHOULD WE ADJUST DOSAGE TO METASTATIC BURDEN Bone metastases (BM) occur in $80 \%$ of patients (pts) with prostate carcinoma, the 2nd most common cancer in males and in $50 \%$ of patients with breast carcinoma, the most common cancer in lemales. Many other primary tumours frequently metastasize to the skeleton and pain reilef often requires long term narcotic analgesia when surgical, hormonal and chemoterapy treatment have failed. External beam radiotherapy may be effective for isolated BM but is difficult to apply in multifocal disease.

Strontium- 89 localizes in osteoblastic BM. Its beta particles have sufficient energy to be therapeutically useful, However, several reports show very different results in pain palliation, ranging from not different of a placebo response to $82 \%$ true response. This report details the results of a retrospective evaluation of 20 therapeutic administrations of $89 \mathrm{Sr}$ (about $4 \mathrm{mCi}$ iv) to 19 elderly, severely ill pts (1 retreatment) with painful $B M_{1}$ not aleviated by any of the usual therapeutic means. 16 male and 3 fermale pls were entered, aged berween 54 and 82 years old, with a mean age of $68,4 \pm 6,3$ years. The primary tumor was prostate carcinoma in $16 \mathrm{pts}$, breast carcinoma in 2 and bladder carcinoma in 1 . One fernale and 1 male pts were lost to follow-up; 4 aditional pts died in a short period ( $<3$ months after $89 \mathrm{Sr}$ )

The criteria for admission were (1) biopsy-proven tumor, (2) 99mTc-MDP bone scanning with evidence of extensive BM the week preceding therapy, (3) failure of prior conventional therapy and (4) total WBC count $>4000 / \mathrm{mm}^{2}$, total plalelet count (TPC) $>100000 / \mathrm{mm} 3$ and serum creatinine concentration $<1,6 \mathrm{mg} / \mathrm{dl}$. Blood profiles were repeated at intervals of 2 weeks for 3 months. All pts required regular narcotics for relief of pain from BM. Pis were encouraged to keep their anaigesic as needed, increasing or decreasing according to pain; $89 \mathrm{Sr}$ was utilized as adjunctive therapy. Metastatic spreading was graded according to: 1) sites in pelvis and lumbar spine; 2) additional sites in trunk skeleton; 3 generalization. Pts were asked to indicate analgesic intake and level of pain (none, less, same, worse) after $89 \mathrm{Sr}$ administration. Response to therapy was assessed at 2 week intervals to 12 weeks. The pain index was regardad as the primary end point for statistical analysis. Imaging was repeated at 3 months (i) possible). In one pt who experienced complete pain relief we performed a second therlapy.

Only $6(32 \%)$ of the 19 pls experienced substancial $(4=21 \%)$ or complete $(2=11 \%)$ relief of pain. Pain relief occured within 20 days of $89 \mathrm{Sr}$ administration, and time to maximum response was 2 months, during up to 3 months. 4 pls died early during treatment, with no pain changes. The cause of cleath was related with the primary tumor, not $89 \mathrm{Sr}$. In $1 \mathrm{pt}$ who experienced complete relief there was recurrence of pain at 3 months, so a retreatment was perlormed, with no effect; this pt died soon atter. No pt experienced any clinically evident acute side effects or reactions following the injection. $4(21 \%)$ had transient increase in pain (flare response) within 10 days of injection, subsiding the following days. At weeks 2 through 4 , individual declines in the TPC from the baseline level were siatistically significant. By 8 weeks after administration, TPC returned to baseline levels in all but 1 pt. Follow-up bone scan revealed maintenance or progression of disease, without the disappearance of any previously described abnormalities, in 7 treatments. As the extension of metastases into bone determines the relative nuclice dose and appears as an important parameter of survival, we believe the amount of $895 \mathrm{~S}$ should be adjusted to the BM volume.

270

D. Sandrock, T.v. Rothkirch, A. Kögler, D.L. Munz, V. Ivancevic, D. Emrich. Departments of Nuclear Medicine and Orthopedics, Georg August University, Göttingen, Germany.

\section{RADIONUCLIDE-SYNOVIORTHESIS VERSUS CORTICO- STEROID-INJECTION IN PATIENTS WITH RHEUMATOID ARTHRITIS}

Aim of this study was to compare the therapeutic effect of radionuclide-synoviorthesis with that of intraarticular corticosteroid-injection in patients with rheumatoid arthritis.

In 66 patients (aged between 38 and 73 years), joint motility, pain (4 step score), and palpation status (4 step synovitis-score) was assessed before and at intervals up to 6 months after intraarticular radionuclide-injection ( $n=70$ joints; Y-90, Re-186, Er-169, depending upon the joint), corticosteroid-injection ( $n=66$ joints) or a combination of both given simultaneously ( $n=97$ ). The joints were put in a resting position for at least $48 \mathrm{hrs}$ p.i. and the patients requested to avoid physical exercise for 2 weeks.

Overall, 233 joints were treated. At follow-up, the mean pain score was 1.5 steps lower as compared to that before treatment and the palpation score 2 steps lower, the combination of nuclide and corticosteroid being more effective than either component alone. The percentage of full remission (combined score) after $\mathbf{6}$ months was $\mathbf{5 2} \%$ in the group receiving nuclide plus corticosteroid, $34 \%$ in the corticosteroid, and $\mathbf{2 5} \%$ in the nuclide group.

In conclusion, the combination of simultaneous intraarticular radionuclide- and corticosteroid-injection is superior to the injection of either component alone in patients with rheumatoid arthritis.
271

X. MALEZIEUX* ${ }^{*}$ C. GETE*, J-P. DREYER*, J. PETERSCHMITT**

Services de médecine nucléaire* et de rhumatologie** Hôpital PASTEUR - F.68024 COLMAR - FRANCE

BREMSSTRAHLUNG IMAGING IN YTTRIUM 90 AND ERBIUM 169 RADIATION SYNOVECTOMY

Since more than 20 years, Yttrium 90 and Erbium 169 colloids are used in our nuclear medicine department for radiation synovectomy in several disease as rheumatoid arthritis, hemophilic sinovitis, psoriasis arthropathy. More than thousands joins have been traited. $90-Y$ are used for large joins: knees, hips, shoulders; 169-Er for others (elbows, metacarpophalangeal cavities, digits, ankle). $90-Y$ and $169-\mathrm{Er}$ do not emit gamma rays suitable for quality images but bremsstrahlung imaging can be used to control the quality of the diffusion in the synovial cavity.

Because a misadministration of radioactive material can induce a injection into the bloodstream or in the lymphatic system, it is very important to evaluate the quality of injection.

We use $222 \mathrm{MBq}(6 \mathrm{mCi}) 90-Y$ in knees and hips, $148 \mathrm{MBq}$ (4mCi) $90-Y$ in shoulders, $111 \mathrm{MBq}$ (3 mCi) 169-Er in elbows and ankles, $37 \mathrm{MBq}(1 \mathrm{mCi}) 169-\mathrm{Er}$ in small joins. Prior to the delivery, we remove as much synovial fluid as possible and the delivery is followed by a flush of $1 \%$ lidocaine and corticoid.

15 to 30 minutes after the intra-joint injection, an image is realised, using a conventional gamma camera (Siemens Rota) with a low energy, middie resolution colilimator. A $100-250 \mathrm{KeV}$ window $(90-Y)$ or a 50-150 KeV window (169-Er) are used for a 3 minutes acquisition.

Results from 854 treated joints are presented. In approximatively seven percent, the image shows a single point, i.e. extra synovial deposit.

So, prior to initiating clinical evaluations of the efficiency of the radiation synovectomy, it is important to know if the synovectomy agent is realy in the synovial cavity. Our method, easy to use, provides this kind of information.

\section{2}

A.R. Haldemann, J. Kinser, U. Noelpp, A. Fikrle, C. Ozdoba* T. Vonlanthen, G. Schroth*

Departements of Nuclear Medicine and Neuroradiology ${ }^{*}$ Inselspital and University of Berne, $\mathrm{CH}-3010$ Berne, Switzerland

DIAGNOSTIC RADIOEMBOLISATION OF CNS TUMOURS: METHODS, DOSIMETRY AND FIRST CLINICAL EXPERIENCE IN REGARD TO THERAPY

Our experience with over 30 selective transarterial radioembolisations using $90 Y$ resin particles $(\varnothing 45-75 \mu \mathrm{m}$ ) has shown good palliative results in patients with inoperable hepatocellular carcinoma. This method may be successful for many inoperable tumours or symptomatic metastases, but selective tumour embolisation without shunting to the lungs or neighbouring organs must be documented with diagnostic radioembolisation prior to therapy.

We have started examining highly vascularized tumours of the skull base, the meninges and the spinal canal which are already routinely embolised mecanically with microparticles of different sizes in order to reduce the perioperative risk of hemorrhage. In 14 patients (5 meningiomas, 3 dural angiomas, 2 metastases, 2 chemodectomas, 1 hemangioblastoma and 1 dural fibrosarcoma) $100 \mathrm{MBq} 99 \mathrm{mTc}$ labelled macroaggregates of albumin $(\varnothing 25-50 \mu \mathrm{m})$ were injected intraarterially after transfemoral cathererization of the tumour-feeding artery. Approximation of pulmonary shunting was done immediatly after diagnostic radioembolisation in the angiography room using a hand-held detector. Afterwards, the definite activity in the area of embolisation and in the lungs was recorded using a gamma camera, and the pulmonary shunt rates calculated.

In this ongoing study, we have found three different patterns of embolisation: I) with pulmonary shunt (up to $76 \%$ of injected activity; 4 patients), II) without pulmonary shunt but sometimes considerable peritumoural embolisation (5 patients) and III) superselective embolisation without significant pulmonary or peritumoural embolisation (5 patients). In patients who would qualify for therapeutic radioembolisation (Group III), dosimetric calculations (MarinelliQuimby-Hine) resulted in tumour doses of 200-1000 Gy for $370 \mathrm{MBq}$ $90 Y$ resin particles. 
273

D. D. Patton, H. H. Barrett, J. C. Chen, W. P. Klein, I. W. Pang, D. L. Richards, M. M. Rogulski, J. Sain, W. E Smith, T. A. White

Division of Nuclear Meojicine and Optical sciences Center, University of Arizona, Tucson, Arizona, USA

FASTSPECT: A 4-DIMENSIONAL STATIONARY BRAIN IMAGING DEVICE We have developed a SPECT device with no moving parts, which acouires dymamic 3 -dimensional (3-D) images of the brain. The unit consists of 24 individual gamma cameras (T1) crystal with 4 photomultiplier tubes; each views the entire brain through one or moxe $2-5 \mathrm{~mm}$ pinholes, forming a true 3-D image of the brain. FASTSPECT has collected $3-D$ images at frame rates of 1 every $2 \mathrm{sec}$, and is capable of frame rates of $30 / \mathrm{sec}$; it is therefore a 4 -dimensional imaging system. It offers image quality comparable to that of state-of-the whole-brain rapid dynamic imaging. ln

images at $2 \mathrm{sec} /$ frame after IV injection of $740 \mathrm{MBg}(20 \mathrm{mC}$ i) TC-99m HMPAO clearly showed transit of tracer through common and internal carotids, anterior and middle cerebral artery groups, and major veins, all displayed in cine mode in each of 24 slices encompassing the entire brain. (study approved by Human Subjects Committee.) Structures resolved on delayed images include temporal and cerebellar cortex and white matter, ventral and dorsal thalamus, caudate nucleus, lenticular nucleus (components not yet resolvable). Eleven million the Good spatial resolution is essential in the brain, where location of a lesion is of utmost importance.

The FASTSPECT design offers some interesting potentials: 1) more and smaller pinholes improve resolution without sacrificing sensitivity; 2) measuring system response to a known activity at every point in object space enables guantitation of uptake in every voxel; 3) fast 3-D dynamics enables study of perfusion sequences in the brain. clinical studies are underway in epilepsy and stroke.

Resolution on the $1 \mathrm{~mm}$ scale (using semiconductor detectors) will pose some challenging technical problems: lerance, and patient restraints, and gated-acquisition brain imaging.

274

G.A. Somsen, J.J.J. Borm, E.A. Dubois, B. van Vlies, P.A.R. de Milliano, E.A. van Royen

Depts. of Nuclear Medicine and Cardiology, Academic Medical Centre, Amsterdam, The Netherlands

\section{METHODOLOGICAL EVALUATION OF MYOCARDIAL} I-123 MIBG - SPECT QUANTIFICATION

Myocardial sympathetic nerve ending integrity and function is visualized with I-123-MetaIodoBenzylGuanidine (MIBG)

Absolute myocardial MIBG-uptake can not be obtained by conventional techniques. The pulmonary or mediastinal tissue is not a suitable reference because of high variation in uptake. We evaluated various other methods of measuring MIBG uptake in LAO and SPECT studies. Left ventricular cavity (VC) may be suitable, as it can be quantitated using a bloodsample and any systematic reconstruction errors are the same for both myocardia uptake (MYO) and VC. Standardised concentric elliptic ROI's of MYO and VC of all short-axis slices were quantitated using the BORSOM program (v. 1.0). Manual ROI's were drawn in planar $\mathrm{LAO}$ views and in single short-axis slices.

We used data from 5 patients with congestive heart failure (60 angles, 64 matrix, $60^{\prime \prime} /$ frame) 4 hours after injection of $185 \mathrm{MBq}$ MIBG.

Reproducibility of LAO methods was good (intra-observer (IA) coefficient of variation (CV) 0.025 typically, inter-observer (IR) $\mathrm{CV}$ of 0.012 ), but the loss of dynamic range down to 1.25 is intolerable. IA-CV of single short-axis slice method was 0.2 typically. This was due to poor VC counts statistics. After optimisation of the VC size; the multiple short-axis slice method with a standardised VC had a good reproducebility (IA-CV 0.05 , IR-CV 0.06, mean ratio 7.3). MYO/CV ranged from 4 to 25 . Bloodpool activity was varying between 400 to $1000 \mathrm{~Bq} / \mathrm{ml}$ at the time of acquisition.

Conclusion: Semi-automated multiple slice quantification with standardised VC regions combined with a single bloodsample is a promising technique in myocardial MIBG-uptake measurement.
275

K. Knesaurek, M.Ivanovic, J. Machac. The Mount Sinai Medical Center, New York, NY, USA and Brookhaven National Laboratory, Upton, NY, USA

\section{A NEW DUAL-ISOTOPE CONVOLUTION CROSS-TALK CORRECTION TECHNIQUE: A TL-201/TC-99m SPECT PHANTOM STUDY}

Simultaneous dual-isotope SPECT imaging provides a clear advantage in situations where two concurrent metabolic, anatomic or background measurements are desired. It obviates the need for two separate imaging sessions, reduces patient motion problems, and provides exact image registration between two studies.

However, a potential limitation of dual isotope SPECT imaging is energy spectral cross-talk in both energy windows. Therefore a new convolution method of energy spectral cross-talk correction was developed. The sophisticated, nonuniform RH-2 thoraxheart phantom was used to test the correction technique. Three separate acquisitions were performed, each with two $20 \%$ wide energy windows, centered at $140 \mathrm{KeV}$ and $70 \mathrm{KeV}$. The data were collected with phantom filled with 1) $\mathrm{Tc}-99 \mathrm{~m}$ solution only, 2) Tl201 solution only, and 3) mixture of Tc-99m + Tl-201. The main step in our correction technique was development of the filters which, when convolved with primary energy window images gives scattered images as viewed at other energy windows. In this way it was possible to subtract mutual cross-talk from dualisotope images. The results of our method have improved the quality of simultaneous dual-isotope SPECT imaging.

\section{6}

C. Vanhove, D. Bladt, S. Dierickx, J. De Vuyst, G. Demonceau.

St Elisabeth Hospital, Zottegem, Belgium and Trionix Res.Lab., Twinsburg.

STUDY OF SEGMENTAL DYSKINESIAS BY 4D VENTRICULOGRAPHY Segmental contraction of the left ventricle can be evaluated in 4 dimensional (4D) ventriculography by using either the Fourier or the slope method. This information may be represented in 3D (reprojection) or in 2D (Bullseye) in which case, comparison with normal cases is easy. These representations may be expressed either in relation to the maximum value of the movement, or (this only in case of the slope method) in absolute values. We have been trying to find the most suitable method by comparing their results to these of myocardial scintigraphy.

During the same week, a Tc-MIBI tomography as well as a gated tomogram with labelled red blood cells (RBC) was performed in 45 patients having suffered from myocardial infarction before at least 2 weeks, but otherwise without symptoms of non-ischemic cardiomyopathy. Reorientation and representation in $2 \mathrm{D}$ and $3 \mathrm{D}$ were obtained automatically, using information derived from the $4 \mathrm{D}$ segmentation. Comparison with normal controls was obtained after normalisation on the modal value of the distribution pattern of the pixels. Comparison between Tc-MIBI and Tc-RBC obtained from quadrants mimicking the arterial territories was made both visually and from quantitative movement data.

3D-representation, although more spectacular, proved to be less accurate than 2D-representation, particularly concerning the inferior wall (in the case of horizontal rotation) and the septal wall (in the case of vertical rotation). Using the bullseye without comparing to normal values overestimates the existence and the size of septal hypokinesias (particularly inferior ones) Both visually and quantitatively, the slope method proved significantly better than the Fourier method in all territories, but particularly so in the inferosepta and posterobasal aspects. Using the optimal method, only 1 patient out of 45 still presented with discrepancies in the location of lesions between both tomoscintigraphies.

In conclusion, the slope method in a $2 \mathrm{D}$-representation and corrected for normal values, appears as the most appropriate one for the representation of hypo- or akinetic zones in the case of proven myocardial infarction. It also has the added advantage to yield absolute values of movement, more particularly in territories that are both least contractible (fibrous septum) and most contractible (used for normalisation in the relative methods). 
277

P. Wanet, M. Stegen, A. Sand, J. Abramovici

Nucleaire Geneeskunde, De Bijloke Ziekenhuis, Gent, Belgium and Département des Radioisotopes, Centre Hospitalier Etterbeek - Ixelles, Bruxelles, Belgium.

HIGH RESOLUTION PINHOLE SPECT OF THE THYROID : METHOD AND CLINICAL EVALUATION.

High resolution SPECT imaging using a rotating scintillation camera equipped with a pinhole collimator provides a new method of investigation of small organs.

Projections are collected over 180 or 360 degrees. Before the reconstruction, the projections are corrected for camera efficiency and decay of $\mathrm{Tc} 99 \mathrm{~m}$. The 3D-reconstruction algorithm used to reconstruct the normalized data is based on a modified backprojection algorithm. Two methods have been investigated : a 1D Metz filter followed by a 3Dconverging back projection or a $2 \mathrm{D}$ prefiltering followed by a $1 \mathrm{D}$ ramp filter and the same converging backprojection. The reconstruction program gives the exact size of the organ and provides a correct determination of the volume. A series of 20 slices is reconstructed in about $25 \mathrm{sec}$

One of the most interesting applications is the tomography of the thyroid. The acquisition is performed with the camera tilted at an angle of 15 degrees in order to be close as possible to the thyroid. In routine practice. the pinhole is located at $10 \mathrm{~cm}$ of the center of rotation. With a pinhole of $2 \mathrm{~mm}$, we get a resolution of $5 \mathrm{~mm}$ and a sensitivity of $0.2510^{-4}$. The $4 \mathrm{~mm}$ pinhole gives a sensitivity of $1.010^{-4}$, but with a resolution of $7 \mathrm{~mm}$. The resolution obtained with a parallel collimator is always above $12 \mathrm{~mm}$ since the distance between collimator and center of rotation cannot be decreased below 20 to $25 \mathrm{~cm}$, because of the shoulders.

Several tests of our program have been made on a thyroid phantom a different distances, with different filters, with and without tilt of the camera. Studies on patients are carried out in 15 minutes, after an injection of 185 to $260 \mathrm{MBq}$ ( 5 to $7 \mathrm{mCi}$ ) of Tc99m-pertechnetate. Coronal slices and 3-D volumetric images are used for diagnostic purposes. Height, width and volume of the thyroid can also be calculated by the program.

conclusion, these SPECT studies on thyroid have shown that ultra-high resolution can be obtained using pinhole collimators.

\section{8}

L Geworski, W Sonnenschein, SP Müller, J Farahati, Chr Reiners.

Clinic for Nuclear Medicine, University Essen, FR Germany.

PHYSICAL COMPARISON OF LESION CONTRAST FOR SPECT WITH Tc-99m, l-123, AND Tl-201 AND PET WITH F-18.

There is increasing interest in SPECT and PET studies with multiple radionuclides for the comparison of radiopharmaceuticals or for the assessment of complex physiological processes with several tracers. We investigated whether differences in the gamma energies of the labelling isotopes or different imaging systems have to be taken into account to allow meaningful ROl quantitation of activity concentrations and, hence, the comparison of derived pharmacokinetic or physiological quantities.

We characterized the imaging properties of SPECT with Tc-99m, TI201 , and $1-123$ compared to PET imaging with $F-18$. We imaged a $20 \mathrm{~cm}$ diameter cylindrical phantom (Data Spectrum) with 6 cold spheres (13 - $38 \mathrm{~mm}$ diameter) or a cardiac insert (activity concentration ratio 4:1 for myocardium to background or ventricular cavity) with a $5.3 \mathrm{ml}$ anterior and a $3.0 \mathrm{ml}$ inferior wall defect on a Siemens ZLC 3700 camera equipped with a low-energy, all-purpose (LEAP) collimator $\left(180^{\circ}\right.$ and $360^{\circ}$ acquisitions) and on a CTI ECAT 953 PET scanner. We acquired high-count studies and reconstructed all images with calculated attenuation correction. The ratio lesion to background for the spheres was calculated from circular ROI's ( $0.5 \times$ sphere diameter) and the ratio lesion

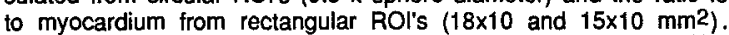

\begin{tabular}{lccccc} 
LEAP $360^{\circ}$ & \multicolumn{3}{c}{ cold spheres } & \multicolumn{3}{c}{ myocardial defects } \\
& $25 \mathrm{~mm}$ & $32 \mathrm{~mm}$ & $38 \mathrm{~mm}$ & $3.0 \mathrm{ml}$ & $5.3 \mathrm{ml}$ \\
\hline TC-99m & $58 \%$ & $45 \%$ & $32 \%$ & $71 \%$ & $55 \%$ \\
l-123 & $66 \%$ & $54 \%$ & $44 \%$ & $69 \%$ & $62 \%$ \\
TI-201 & $66 \%$ & $57 \%$ & $46 \%$ & $76 \%$ & $61 \%$ \\
F-18 (PET) & $31 \%$ & $16 \%$ & $15 \%$ & $30 \%$ & $28 \%$ \\
\hline Th
\end{tabular}
The improvement of lesion contrast for $180^{\circ}$ vs. $360^{\circ}$ SPECT acquis tions was more pronounced for TC-99m than for 1-123 or Tl-201.

The effects of differing physical characteristics of the labelling isotopes and imaging systems must be taken into account for quantitative comparisons. SPECT acquisition with $180^{\circ}$ helps to improve geometrical resolution but only to a lesser degree the contrast degradation due to scatter and collimator penetration.
279

C.A. Guhlmann\#, K. Krauss\#, F. Oberdorfer\#, Th. Siegel\#, P.H. Scheuber*, S. Ziegler\#, H. Ostertag\#, D. Keppler\# \#Deutsches Krebsforschungszentrum, 69120 Heidelberg, FRG *Dept.Clin.Chem.Clin.Biochem., 80336 München, FRG

C-11 LABELLED N-ACETYL-LEUKOTRIENE E 4 TO MEASURE HEPATOBILIARY FUNCTION BY POSITRON EMISSION TOMOGRAPHY

$N$-Acetyl-leukotriene $E_{4}\left(L_{T E} N A c\right)$ has been identified as an endogenous, biologically less active cysteinyl leukotriene metabolite in rodents and man. In order to evaluate the ratio of hepatobiliary to renal elimination of leukotrienes (LTs) noninvasively by PET, we synthesized N-[11C]acetyl-leukotriene $E_{4}$ by chemical $N$-acetylation of $L T E_{4}$. Following the i.v.injection of [11C]LTE 4 NAc in normal rats and monkey, uptake by the liver and subsequent excretion into bile were responsible for its rapid elimination from blood. In the monkey, excretion of the LT into urine played an additional role. Kinetic modeling using a gamma variate function indicated a mean transit time through the liver of $17 \mathrm{~min}$ and $34 \mathrm{~min}$ in rat and monkey, respectively; the corresponding hepatic excretion halftimes amounted to $8.5 \mathrm{~min}$ and $16 \mathrm{~min}$. In a mutant rat strain deficient in the hepatobiliary excretion of cysteinyl LTs, the mean liver transit time was extended to $54 \mathrm{~min}$ and the hepatic excretion half-time was 29 min indicating prolonged processes of organ storage and metabolism. Following transport from the liver back into the circulating blood of $\omega$-oxidized and $\beta$ oxidized metabolites of [11C]LTE 4 NAc, renal excretion compensated for impairment of hepatobiliary elimination. A similar shift from hepatobiliary to renal cysteinyl LT elimination was monitored in rats with cholestasis due to bile duct obstruction. C-11 labelled LTE 4 NAc enables the assessment of hepatobiliary function by PET as well as the quantitative and non-invasive evaluation of the contribution of liver and kidney to LT elimination under normal and various pathophysiological conditions.

\section{0}

\section{H.J. Wester, S. Guhlke, G.Stöcklin}

Institut für Nuklearchemie, Forschungszentrum Jülich $\mathrm{GmbH}$ 52425 Jülich, Germany

COMPARATIVE EVALUATION OF N.C.A. ${ }^{18_{F}}$ FLUORINATION AGENTS FOR PEPTIDES AND PROTEINS

${ }^{18} \mathrm{~F}$-labelled peptides and proteins are becoming increasingly important in tumor research with PET. The labelling methods should be simple and mild. We developed and compared the following 18 F-fluorination agents: $2-\left[{ }^{18} \mathrm{~F}\right]$ fluoropropionyl-4-nitrophenyl ester (1) 2- $\left[{ }^{18}\right.$ F $]$ fluoropropionyl- $N$-hydroxysuccinimidyl ester (2) $2-[18$ F]fluoropropionic acid chloride (3) and 4-azidophenacyl${ }^{18}$ F] fluoride (4). (1) was used to label small peptides such as OCTREOTIDE in the presence of 1-hydroxybenzotriazole (HOBt). Furthermore, regioselective $N(\alpha)$-or $N(\varepsilon)-\left[{ }^{18}\right.$ F $]$ fluoroacylation of model peptides was possible using (1) in the presence of HOBt and $\mathrm{pH}$ control. Due to competing $18 \mathrm{~F}$-acylation of the hydroxyl group of the tyr residues, 18 F-labelling of HSA with (1) yielded a partially instable product. Labelling of HSA with (2) gave rise to stable products and could be carried out using low protein concentrations $(80 \mu \mathrm{g}$ $\mathrm{HSA} / 100 \mu \mathrm{l} ; \quad 42 \pm 6 \% \quad \mathrm{RCY}$ ). However, regioselective [18F]fluoroacylation of peptides with (2) was not possible. (3) can be used for the $2-\left[{ }^{18} \mathrm{~F}\right]$ fluoropropionylation of small peptides which are soluble in organic solvents. Like (2), (4) is a potential agent for ${ }^{18} \mathrm{~F}$ labelling of MAbs. In contrast to the syntheses of (1),(2) and (3) (3 steps; RCY 50-60\%; 75-90min), preparation of (4) can be done in a single step within $30 \min$ (RCY $72 \pm 5 \%$ ). Conjugation with the in situ generated corresponding nitrene of (4) is achieved by UV irradiation within $5 \mathrm{~min}$ in the presence of the protein. However, when compared with (2), the time advantage is compensated by a lower conjugation yield and a higher protein concentration $(15 \% \mathrm{RCY}$ at $500 \mu \mathrm{g} / 100 \mu \mathrm{l})$. 
281

P.Goethais, N. Lameire, D. Kesteloot, M. van Eijkeren, H. Thierens

Depts. of Analytical Chemistry, Nephrology, Nuclear Medicine and Medical Physics, University Gent \& University Hospital, Belgium

CAN THE PET QUANTIFICATION DATA OBTAINED WITH [METHYL- $\left.{ }^{11} \mathrm{C}\right] T$ TR GIVE INFORMATION ABOUT CELL PROLIFERATION?

[Methyl-11 C]TdR has been used for qualitative imaging of tumours. Studying the metabolites of TaR in plasma showed that they became dominant (>75\%) from $5 \mathrm{~min}$ post injection on. To correlate the quantification data with cell proliferation, knowledge of metabolites contribution to the total tissue activity is a prerequisite.

In this study using an animal model (rats), two independent pathways were followed.

(1) The total activity in fast dividing tissue after i.v. injection of [methyl-11 C]TdR resp. [methyl-12C]thymine (first metabolite) was compared. For the rats injected with $T d R$, the tissue activity consists of labeled TdR and its metabolites. For those injected with thymine, tissue activity consists only of labeled metabolites. Depending on the model, the fraction of labeled metabolites yielded 3 to $30 \%$.

(2) The liver was surgically clamped. Catabolism of TdR mainly takes place in the liver. Comparing the specific activity in fast dividing tissue in normal and in rats with "locked" liver function showed no significant difference between both series. The metabolite fraction was less than $10 \%$.

As conclusion, we can say that the major activity in fast dividing tissue consists of [methyl-11C]TaR. Consequently, the pET quantification data can give information about cell proliferation and makes it possible to evaluate therapeutic techniques.

\section{2}

C. Loc'h1, J.E. Ombetta2, C. Fuseau1, I. Lena2, M. Ottaviani1, D. Guilloteau2, B. Mazière1. 1-Service Hospitalier Frédéric Joliot DRIPP/CEA, F-91406 Orsay, 2 Laboratoire de Biophysique Médicale et Pharmaceutique, INSERM U316, F-37200 Tours.

PREPARATION AND EVALUATION OF Br-76-2-BROMOPARGYLINE : A POSITRON EMITTING MAO-B INHIBITOR.

Pargyline is a MAO-B inhibitor which has been labelled with I-123 in order to perform SPECT studies (J Nucl Med, 1993 34;5, 335p). For assessment of MAO distribution in brain by PET, Br-76bromopargyline ( $\mathrm{N}-2$-bromobenzyl- $\mathrm{N}$-methylpropargylamine) was prepared from the iodinated analogue using heteroisotopic exchange. The $\mathrm{Cu}+$ assisted substitution reaction between nca radioactive bromine and the cold iodine of iodopargyline takes place at $165^{\circ} \mathrm{C}$ using gentisic, ascorbic and citric acids as reducing agents. A RPHPLC purification gave Br-76-bromopargyline in a $75 \%$ radiochemical yield with a specific activity of $20 \mathrm{MBq} / \mathrm{nmol}$

The inhibitory activity of 2-bromopargyline was assessed in vitro by measuring its potency to inhibit the MAO activity in rat brain homogenates. The $\mathrm{IC}_{50}$ values toward MAO-A $(26 \mathrm{nM})$ and MAO-B (3.3 $\mathrm{nM}$ ) demonstrated that 2-bromopargyline possess a good selectivity for $\mathrm{MAO}-\mathrm{B}$ and a higher affinity than pargyline itself ( $\mathrm{IC}_{50}$ $\mathrm{MAO}-\mathrm{B}=20 \mathrm{nM}$ )

In rats, biodistribution kinetic studies showed a high uptake $(0.5 \%$ $\mathrm{ID} / \mathrm{g})$ of $\mathrm{Br}$-76-bromopargyline in brain. Using ex vivo autoradiographic studies, a preferential localisation of the radiotracer was observed in the pineal gland and the thalamus, brain structures which are known to possess high concentrations of MAO-B

These preliminary results suggest that $\mathrm{Br}$-76-bromopargyline could be a useful radiotracer to study, by PET, the variation of MAO-B activity in neurodegenerative pathologies where monoaminergic neurotransmissions play an important role
283

K. Någren ${ }^{*}$, C. Halldin, C.-G. Swahn, T. Suhara, L. Farde. *Radiopharmaceutical Chemistry Laboratory, Turku University Cyclotron/PET Center, Turku, Finland and Department of Clinical Neuroscience, Psychiatry and Psychology section, Karolinska Institute, Stockholm, Sweden.

[C-11]METARAMINOL, A FALSE NEUROTRANSMITTER. PREPARATION, METABOLITE STUDIES AND PET INVESTIGATION IN MONKEY.

Recently we described the selective synthesis and preliminary validation of [C-11]norepinephrine (NE) as a tracer for adrenergic neurons. Metaraminol is a NE congener which has a high affinity for the uptake-1 mechanism and, unlike NE, is not metabolised by MAO and COMT. [F-18]6-Fluorometaraminol has previously been prepared from [F-18]acetyl hypofluorite (Mislankar et al., 1988). However, this approach gave a product with too low specific radioactivity corresponding to a dose which is too high for PET studies in humans.

In the present work, no-carrier-added preparation of [C-11]metaraminol was achieved by a selective condensation of [C-11]nitroethane with $m$-hydroxybenzaldehyde followed by reduction with Raney nickel. Chromatographic purification with reversed phase HPLC enabled the separation of the racemic erythroand threo-forms of the product. [C-11]Metaraminol was prepared in $13-20 \%$ decay corrected radiochemical yield within $45-55$ minutes with a specific radioactivity of $300-800 \mathrm{Ci} / \mathrm{mmol}(11-30 \mathrm{GBq} / \mathrm{mmol})$. PET examination of racemic [C-11]erythro-metaraminol (META) in a Cynomolgus monkey showed a high uptake of radioactivity in the heart. The radioactivity in the myocardium was more than 10 times higher than in adjascent lung tissue and more than 5 times higher than in plasma. In a pretreatment experiment using the NE reuptake inhibitor desipramine, radioactivity uptake in the myocardium was decreased by $80 \%$. This observation demonstrates the specificity of META for the NE reuptake in the heart.

The metabolism of META, determined in plasma by HPLC, was rapid. The fraction of total radioactivity representing META in plasma was $14 \%$ at $6 \mathrm{~min}$ and $8 \%$ at $34 \mathrm{~min}$. High specific radioactivity META should be useful as a radioligand for imaging of cardiac sympathetic neurotransmission in the human heart.

\section{4}

C. Loc'h1, P. Hantraye 1, L. Müller2, E. Brouillet1, C. Halldin2, L. Farde2, M. Mazière1, B. Mazièrel. 1- Service Hospitalier Frédéric Joliot, DRIPP/CEA, F-91406 Orsay, 2- Department of Clinical Neurosciences, Karolinska Institute, S-17176 Stockholm

PREPARATION AND PET STUDIES OF Br-76 $\beta$-CBT, A NEW PET TRACER FOR IN VIVO IMAGING OF THE MONOAMINE UPTAKE SITES

For PET studies of the monoamine uptake sites, $\beta$-CIT and its fluoro analog $(\beta-C F T)$ have been labeled with $\mathrm{C}-11$. To study the role of the halogen in the selectivity of the tracers for the dopamine reuptake sites, we have labelled the bromo analog $\beta$-CBT $[2 \beta$-carboxy- $3 \beta-(4-$ bromophenyl)-tropane] with $\mathrm{Br}-76\left(\mathrm{~T}_{1 / 2}=16 \mathrm{~h}\right)$.

$\mathrm{Br}-76 \beta$-CBT was prepared either by electrophilic substitution from the tributyl-stannyl derivative with $\mathrm{Br}-76$ using peracetic acid as oxidant or by nucleophilic substitution using $\beta$-CIT and a $\mathrm{Cu}^{+}$ assisted bromodeiodination exchange. Polar by-products were eliminated by SPE using a $\mathrm{C} 18$ cartridge and $\mathrm{Br}-76 \quad \beta$-CBT was purified by RP-HPLC. Using the two techniques, the radiotracer was obtained in $80 \%$ and $60 \%$ radiochemical yields, respectively, with specific radioactivities $\sim 20 \mathrm{GBq} / \mu \mathrm{mol}$

PET imaging of $\mathrm{Br}-76 \quad \beta-\mathrm{CBT}$ biodistribution in baboons demonstrated a rapid and high uptake in the brain (5\% ID) and $1 \mathrm{~h}$ after injection, the radioactive concentration in the striatum reached a plateau $(0.13 \% \mathrm{DD} / \mathrm{g})$ that remained constant for $4 \mathrm{~h}$. No specific uptake in the cortex was observed. Due to the constant wash-out in the cerebellum, 4 hours after injection, the striatum to cerebellum ratio was 10 . In a baboon with unilateral MPTP induced substantia nigra lesion, the uptake in the diafferented striatum was reduced by $50 \%$, a value similar to that obtained using C11- $\beta$-CFT

These results suggest that $\mathrm{Br}-76 \beta$-CBT has the potential of being developed as a useful PET radiotracer for imaging monoamine uptake sites in pathological conditions. 


\section{5}

G.Picclotto A. Sargiot to, R. Ropolo.P.G.DeFi11ppl Nuclear Medicine pepartment - ospedale Molinette - Torino (Italy)

EXPECTED RENOGRAM : A NEW METHOD TO COMPARE POST-CAPTOPRIL RENOGRAPHY WITH BASELINE

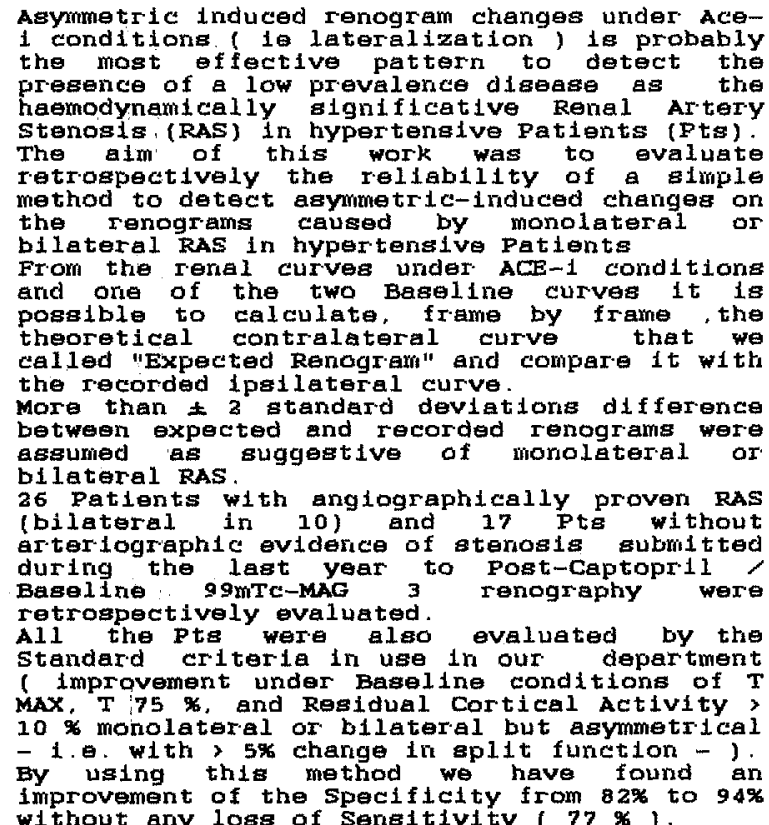

without any loss of Sensitivity ( $77 \%)$.

286

E.A. Dubois, K.L. Kam, G.A. Somsen, A.G.F. Janssen, A. Maas, K. de Bruin, M. Pfaffendorf, E.A. van Royen, P.A. van Zwieten Depts. of Nuclear Medicine, Pharmacotherapy, Cardiology, Academic Medical Centre, University of Amsterdam, Amsterdam, The Netherlands Cygne BV, Technical University Eindhoven, The Netherlands

\section{[123I]-MIBG ACTIVITY AS AN INDICATION FOR SYMPATHETIC} ACTIVITY IN HYPERTENSIVE/DIABETIC RATS

Diabetes mellitus and hypertension often coincide. In order to study this relation, we assessed sympathetic activity, by measuring [123П]-MIBG activty after intravenous injection, in an animal model where these disorders exist either simultaneously or separately. In male Wistar Kyoto rats (WKY) and in spontaneously hypertensive rats (SHR), diabetes was induced at the age of 12 weeks by a single injection of $60 \mathrm{mg} / \mathrm{kg}$ streptozotocin (STZ) i.v. At the age of 20 weeks the rats received $10 \mu \mathrm{Ci} / \mathrm{kg}$ body weight [123I]-MIBG. Thirty minutes after injection of [123I]-MIBG, blood samples were taken to measure noradrenaline levels (NA) and the rats were sacrificed. Organs were weighed and total radioactivity was established. Two weeks later $\beta$-adrenoceptor ( $\beta-A R$ ) density in the heart was measured. NA levels, heart/lung radioactivity ratios and $\mathrm{B}-\mathrm{AR}$ densities were compared as listed in the table:

$\begin{array}{lllr} & \begin{array}{c}\text { MIBG } \\ \text { (heart/lung) }\end{array} & \begin{array}{c}\text { NA } \\ \text { (nmol/L) }\end{array} & \begin{array}{c}\text { B-AR density } \\ \text { (fmol/mg prot) }\end{array} \\ \text { WKY } & 1.28 \pm 0.11 & 2.37 \pm 0.66 & 52 \pm 3.5 \\ \text { STZ WKY } & 0.92 \pm 0.09^{*} & 3.60 \pm 0.93 & 36 \pm 3.9^{*} \\ \text { SHR } & 1.06 \pm 0.11 & 1.98 \pm 0.49 & 53 \pm 4.9 \\ \text { STZ SHR } & 0.73 \pm 0.10 & 0.46 \pm 0.10^{*} & 35 \pm 5.0^{*}\end{array}$

Means \pm SEM, $n=7 * *$ * $<0.05$ vs control or \#: vs diabetic WKY. Concomitantly, [123I]-MIBG activity in the hearts of diabetic animals appears to decrease, probably as a result of an enhanced release of endogenous NA. The impaired cardiac activity of [1231]-MIBG may reflect an increase in sympathetic activity in the diabetic state.

\section{7}

A. Prigent, M. Froissart, M. Azizio, P.-F. Plouin ${ }^{\circ}$ M. Paillard.

Depts of Physiology, Nuclear Medicine and Hypertension", Broussais Hospital, Paris, France.

SPLIT AND GLOBAL RENAL FUNCTION AFTER PERCUTANEOUS TRANSLUMINAL RENAL ANGIOPLASTY IN PATIENIS WTTH UNILATERAL RENAL ARTERY STENOSIS.

Global renal function using inulin clearance

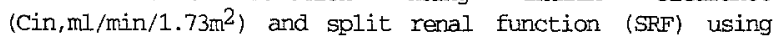
99mTc-DTPA renal scintigraphy were determined in 18 patients ( 8 men) with significant unilateral renal artery stenosis (8 dysplasia:FMD and 10 atheroma:ATH), 2 days before (TO) and 3 months after (T1) successful percutaneous transluminal renal angioplasty (PTRA). After 6 months of follow-up, 16/17 patients had cure or improvement of their hypertension. A moderate restenosis $(<50 \%)$ was observed in $3 / 15$ control angiograms.

\begin{tabular}{lccccccc} 
& \multicolumn{3}{c}{ FMD } & & \multicolumn{3}{c}{ ATH } \\
\cline { 2 - 4 } \cline { 5 - 8 } (meantSD) & T0 & & T1 & & T0 & & T1 \\
Cin & $95 \pm 15$ & $*$ & $107 \pm 8$ & & $81 \pm 20$ & ns & $83 \pm 25$ \\
SRF-SK (8) & $28 \pm 16$ & $* *$ & $43 \pm 8$ & & $40 \pm 19$ & ns & $47 \pm 13$ \\
SBP (mmHg) & $181 \pm 26$ & $* *$ & $135 \pm 10$ & & $178 \pm 27$ & $* *$ & $140 \pm 15$ \\
DBP (mnHg) & $119 \pm 15$ & $* *$ & $92 \pm 11$ & & $102 \pm 15$ & ns & $76 \pm 28$
\end{tabular}

DBP (minHg) $119 \pm 15 * * 92 \pm 11 \quad 102 \pm 15$ ns $76 \pm 28$

SRF-SK: SRF of the stenotic kidney; SBP/DBP: systolic and diastolic blood pressures.* $\mathrm{P}<0,05 ; * * \mathrm{P}<0,01$.

Median [range] of Cin variation was $17 \%[-5 ;+39]$ and $8 \%$ $[-29 ;+30]$ and that of SRF-SK variation was $518 \quad[+10 ;+300]$ and $7 \%[-8 ;+67]$ in FMD and $\mathrm{ATH}$, respectively. There was a significant correlation between the variations in Cin and SRF-SK before (TO) and after (TI) PIRA; neither of these changes was related to corresponding SBP or DBP variations. In conclusion, Cin and SRF imoroved significantly in FMD patients after PTRA, contrasting with that observed in ATH patients. These short-term effects of PTRA on both kidneys especially in FMD (increase in GFR of the stenotic kidney and decrease of GrR of the hyperfiltrating contralateral kidney) might lead to a longterm renal benefit.

\section{8}

M.C. Treluyer, A. Hadj-Aissa, M. Labeeuw, A. Prigent*.

Dpts of Nuclear Medicine, Renal Function Investigation, Nephrology, Hosp. E. Herriot (Pav.P), Lyon, and Hosp. Broussais*, Paris, France.

BILATERAL RESPONSE (BR) IN CAPTOPRIL RENAL SCINTIGRAPHY (CRS) :

Captopril-induced changes of $99 \mathrm{mTc}$-MAG3 renal scintigraphy (CRS) were evaluated in 58 hypertensive patients highly suspected of having renal artery stenosis (RAS) and arteriographically controlled. Eleven showed BR, 3 of which twice (before and after percutaneous transluminal renal angioplasty (PTRA)). In order to distinguish false from true positive $\mathrm{BR}$, changes in these $14 \mathrm{BR}$ were compared with arteriographic data.

Arteriography showed renal artery stenosis (RAS) (bilateral in 2) in 9 patients and no renal artery stenosis in 5. Chronic renal failure (plasma creatinine $\geq 129 \mu \mathrm{mol} / \mathrm{l}$ ) was present in $9 / 11$ and peripheral artery disease in $8 / 11$ patients.

A by kidney and a by patient analysis of time to peak (TP), residual activity (RA) at $24 \mathrm{~min}$, mean parenchymal transit time (MPTT), split renal function (SRF) and of renographic pattern grades (RPG) were done in these $14 \mathrm{BR}$.

In $8 / 9$ patients with RAS and in $1 / 5$ without RAS, the right to left differences in TP and RA changes were $\geq 7 \mathrm{~min}$. and $\geq 7 \%$. These changes were more marked in unilateral RAS than in bilateral RAS. According to RPG the right to left differences in scores changes were $\geq 1$ in patients with RAS and null in patients without RAS. Surprisingly the largest changes were not located to the stenosed kidney in 6/8 patients. MPTT and SRF were not significantly different in patients with or without RAS.

In patients with BR in CRS : i) Asymmetrical BR may help to detect RVH. The capability to differentiate bilateral from unilateral RAS still remains to assess in a larger group. ii) Although asymmetrical $B R$ is often present in patients with RAS the largest captopril-induced changes were not always localized to the stenosed kidney in our short series. 
Tuesday, 23 August - Wednesday, 24 August 1994

289

UJ. Miola, D. Hamilton, D. Mousa, M. AlSulaiman, Z. Rassoul, A. Abdalla, M. Abdur Rehman, A. Al-Khader Riyadh Al Kharj Hospital Programme, Kingdom of Saudi Arabia

\section{THE IMPORTANCE OF PERFUSION INDEX IN THE INTERPRETATION OF CAPTOPRIL RENOGRAPHY IN RENAL TRANBPLANT ARTERY STENOSIB}

Severe renal artery stenosis (RAS) is a relatively uncommon complication following renal transplantation but is a potentially curable entity in which early diagnosis and appropriate treatment can reduce morbidity, mortality and graft loss. Captopril renography has been used for a number of years as a method of detecting RAS, mainly in native kidneys and with only a limited number of studies undertaken in the transplant situation which show a high false positive rate. Interpretation criteria do not presently include a consideration of the changes in renal perfusion between pre- and postcaptopril studies. This communication presents a comparison of 35 captopril renography and arteriography investigations with the additional criterion of perfusion changes incorporated. The results show a high sensitivity ( $89 \%$ ) and indicate that the specificity is improved from $67 \%$ to $81 \%$ and that the predictive value of a positive test is improved from $47 \%$ to $62 \%$ when a consideration of the change in perfusion index (Guy's) between pre- and post-captopril studies in included in the interpretation criteria.

Wednesday, 24 August 1994

290

A. Hertel, F.D. Maul, R. P. Baum, A. Niesen, A. Hartmann, A. Zöller, H. Klepzig*, G. Hör. Departments of Nuclear Medicine and Cardiology*, Goethe University Medical Center, Frankfurt/Main, FRG.

\section{RADIONUCLIDE VENTRICULOGRAPHY COMPARED WITH ANTIMYOSIN IMAGING IN LONG TERM FOLLOW-UP OF HEART TRANSPLANTATION.}

Antimyosin imaging is routinely used for detection of rejection in heart transplant (HTX) patients. Repeat rejections reduce the patients' long term prognosis. Correlation of myocardial cell damage as detected by antimyosin and serial evaluation of heart function with stress radionuclide ventriculography (RNV) was the purpose of this investigation.

Patients: In 20 patients, transplanted between $7 / 86$ and $8 / 92$ In-111 antimyosin (AM) scans were applied (142 scans, mean 7.1/patient, 530 months overall follow-up since HTX, mean 26.5 months, mean every 3.7 months/patient). For detection of rejection a heart-to-lung ratio (48 h p.i.) of $>1.5$ was set. All ratios $<1.5$ were considered negative. Serial RNV was applied in all patients (2-11 studies/patient) at rest and under tread mill stress (25-75 W).

Results: 10 patients had positive AM scans ( maximum values range 1.6-1.98, mean 1.8) and at least 1 rejection episode (1-3 episodes, mean 1.85) and 10 patients were negative (max. values 1.22-1.5, mean 1.41). Regarding RNV, even close to EMB proven rejections, normal rest EF values were obtained. In long term follow-up, in both groups rest global EF decreased, but stayed in the lower normal range $(>50 \%)$. Decisively, stress RNV clearly seperated both groups. $7 / 10$ in the AM negative group maintained a normal stress EF increase, 1 no stress done, in 2 EF decrease. Of the AM-positive group 8/10 demonstrated a global EF decrease under stress with normal rest EF values.

Conclusions: Whereas antimyosin is a reliable screening tool for detection of HTX rejections, radionuclide ventriculography at rest is unreliable for evaluation of acute rejection episodes. Stress RNV is highly significant for evaluating loss of heart function in patients with rejections.
291

S.Dürk, D.Heute, F.G.Haslinger ${ }^{1}$, M.Seyrl ${ }^{1}$, N.Mutz ${ }^{1}$, J.M.Hackl ${ }^{1}$, G.Riccabona and L. Fridrich

Univ.Clinic for Nuclear Medicine, ${ }^{I}$ Univ. Clinic for Anaesthesiology and Intensive Care Medicine; Univ. of Innsbruck, AUSTRIA

RIGHT VENTRICULAR FUNCTION BY CONTINUOUS CARDIOSCINT ASSESSMENT OF RVEF DURING INTENSIVE CARE:EVALUATION OF INVASIVELY MEASURED RVEF

CARDIOSCINT (CSC) is a new miniaturised probe system connected to a $\mathrm{PC}$ that allows continuous Monitoring of ventricular function after $99 \mathrm{mTc}$ labelling of red blood cells at bedside. CSC initially dedicated to LVEF assessment has been validated by gamma camera imaging also for RVEF $(r=0.89, n=70)$ and RVEF - changes $(r=0.85, n=39)$ during interventions. Additional studies were done with a REF - catheter which is a special designed variation of Swan-Ganz catheter for assessment of RVEF by thermodilution. The aim of this study was to compare CSC and REF simultaneously assessed RVEF for evaluation of their clinical reliability. In 10 septic patients of the intensive care unit (age 42-65y) on artificial respiration right heart catheterisation with a REF - Catheter and CSC continuous monitoring of RVEF was performed. 56 REF-RVEF(44+/-10 $\%$ values were correlated to CSC-RVEF (45+/- 9\%). As expected correlation was highly significant $(p<0.001)$ the correlation coefficient $(r=0.64)$, however, was relatively low due to the wide scatter of the REF.-RVEF in contrast to the stable CSC - RVEF values over wide periods. In conclusion: CSC provides continuously stable accurate assessment of RVEF even under conditions of artificial respiration. REF-RVEF values however were characterised by a wide scatter within short haemodynamically stable periods. Thus CSC-RVEF appears as a more reliable tool for evaluation of right ventricular function even if right heart catheterisation seems necessary for pressure monitoring

292

A. Le Helloco, L. Nicol. A. Devillers, M. Bedossa, M. Laurent P. Bourguet. C. Almange.

Departement of Cardiology and Nuclear Medecine, University Hospital Rennes, France.

ASSESSMENT OF LEFT VENTRICULAR VOLUMES FROM TOMOGRAPHIC GATED BLOOD POOL RADIONUCLIDE VENTRIGULOGRAPHY.

The aim of this study was to calculate left ventricular volumes by tomographic radionuclide ventriculography [V.] and to correlate with data obtained from angiographic studies. 20 patients [18 male and 2 female] with mean age 57.4 years \pm 10 were divided in 2 groups: group 1 included 9 patients with normal coronary angiogram [6 pts with normal ejection fraction. 2 pts with aortic valvular disease and 1 pt with dilated cardiomyopathy] : group 2 included 11 patients with significative coronary stenosis and altered ejection fraction : anterior M.I. [4), postero-inferior M.l. [5]. All patients underwent tomographic radionuclide ventriculography and cardiac catheterization with coronary angiography [mean delay : 24 hours]. Tomographic left ventricular volumes were calculated by 2 independent observers from the transaxial sections through the left ventricle at both enddiastole and end-systale.

\section{Radionuclide $\mathrm{V}$. Radialogic $\mathrm{V}$}

EDV $\quad 110 \mathrm{ml}^{2} \mathrm{~m}^{2} \pm 48 \quad 112 \mathrm{ml} / \mathrm{m}^{2} \pm 47$

ESV $\quad 61 \mathrm{ml} / \mathrm{m}^{2} \pm 41 \quad 62 \mathrm{~m} / \mathrm{m}^{2} \pm 42$

EF $\quad 47.3 \% \pm 17.1 \quad 47.4 \%+17.2$

Correlations of tomographic left ventricular volumes and E.F. with angiography were excellent with $r$ values of 0.96 for EDV. 0.94 for ESV and 0.95 for EF.

Gated blood pool tomography is an accurate method far the measurement of left ventricular volumes. 
293

G. Notohamiprodjo, A. Bergmann, N.J. Shah, R. Weise, O. Lindner, H. Körperich, W. Eschner, H. Meyer, U. Gleichmann, R. Körfer Herzzentrum NRW - Ruhr-University Bochum, Bad Oeynhausen, FRG

\section{COMBINED PET AND MR IMAGING FOR THE EVALUATION OF CARDIAC AND MEDIASTINAL TUMORS}

Primary cardiac tumors are less common. It is of potential interest to distinguish between malignant and benign tumors in order to select the best thera. peutic strategy. We report our preliminary experience in combined PET and MR imaging performed in 8 patients with primary cardiac tumors and in 3 patients with mediastinal tumors for evaluation of operative strategy. MR Investigations were performed on a Picker 1.5 T HPQ system using T1. weighted spin-echo, segmented k-space, field-echo and bright blood fieldecho sequences. The investigation was repeated after Gd-DTPA infusion. In 2 patients with large cardiac tumors proton-MR-spectroscopy was attempted. All patients underwent ${ }^{18 F D G ~ P E T ~ b e f o r e ~ a n d ~ a f t e r ~ g l u c o s e ~ l o a d i n g . ~ T r a c e r ~}$ uptake, distribution, and glucose metabolic rates of the tumor and of the surrounding myocardial tissue were evaluated. PET and MR images were also matched by using the software developed in our center. The results were then compared with those from transesophageal echocardiography (TEE), transthoracic echocardiography (TTE), CT and with intraoperative and histologic findings. MR Imaging demonstrated excellent delineation of tumors of the heart and surrounding structures superior to TTE, TEE and CT due to better soft tissue constrast. In all patients but one the preoperative prediction of the resectability of the tumor was achieved correctly. Compared to TEE, MRI failed in most cases to detect tumorous lesions smaller than $0.5 \mathrm{~cm}$ particularly those which involved the papillary muscle and those involving the right ventricle. Slight improvement in the detection of such small lesions was achieved after Gd-infusion. Tumor infiltration and expansion detected in MRI correlates well with enhanced tumor glucose metabolism. These can help in the evaluation of tumor dignity. In 1 Patient with an enlarged hemangioma in the right ventricle with significant obstruction of the outflow tract, the combined PET and MR images were falsely interpreted as well vascularized malign sarcoma of the heart. However, as confirmed by intraoperative findings, the non-resectability of the lesion was correctly diagnosed. It is concluded that combined PET and MR imaging might be a valuable tool for the evaluation of operative strategy of cardiac and mediastinal tumors. Motion artifacts combined with a strong signal from fat resulted in very poor spectra with no diagnostic advantage.

\section{4}

P. Olivier, P.Y. Marie, G. Karcher, N. Danchin, M. Angioi, T. Arsena, N. Quiri, N. David, $Y$. Juillière, $F$. Cherrier and A. Bertrand.

Dept. of Nuclear Medicine, CHU Nancy, France.

COMPARED X-RAY AND RADIONUCLIDE ROUTINE DETER MINATIONS OF LEFT VENTRICULAR EJECTION FRACTION FOR THE LONG TERM PROGNOSTIC ASSESSMENT IN PATIENTS WITH $Q$ WAVE MYOCARDIAL INFARCTION

In order to compare the long term prognostic value of left ventricular ejection fraction (LVEF) routinely determined by single plane $x$ ray angiography and by equilibrium radionuclide angiography (RNA), a 5-year follow-up period was obtained in 103 consecutive pts 199 men, $54 \pm 8$ years) with $Q$ wave $M I$, who had $X$-ray and radionuclide LV-angiography, and who were initially treated medically. X-ray LVEF, determined by cardiac catheterization (area-length method on a $30^{\circ}$ RAO) was higher than that determined by RNA (manual method on a LAO) in the overall population $(p<.05)$. But the difference was marked for pts with anterior MI $(n=51) \quad(46 \pm 13 \%$ vs $39 \pm 14 \%$,

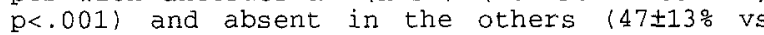
$49 \pm 10 \%$, NS). When we compared pts with cardiac death $(n=13)$ to those without death, cardiac surgery or angioplasty $(n=79)$ during the 5-year of follow-up. MI location was more frequently anterior $(85 \%$ vs $47 \%, \mathrm{p}<.02)$ and both $\mathrm{X}$-ray and RNA LVEF were lower. However, the difference between the 2 groups was slight for X-ray LVEF $(41 \pm 12 \%$ vs $48 \pm 12 \%, p<.05)$ and much more marked for RNA LVEF $132 \pm 12 \%$ vs $46 \pm 12 \%, p<.001)$.

Compared with single plane $\mathrm{X}$-ray angiographic determination, LVEF calculated by RNA appears to be a better long term predictor of cardiac death after $Q$ wave MI. This might be related to improved assessment of $\mathrm{LV}$ function in anterior $\mathrm{MI}$.

\section{5}

M. Rodrigues, H, Kritz, M. Wenger, H. Sinzinges Department of Nuclear Medicine, University of Vienna, Vienna, Austria

EVALUATION OF 111 IN-POLYCLONAL FUMAN TMMUNOGLOBULIN $G$ (HIG) IN HUMAN AND EXPERTMENTAL ATHETOSCLEROSIS.

There is a strong need for non-invasive techniques in imaging early atherosclerotic lesions (AI). The already documented safety and easy preparation of IgG support a study in human atherosclerosis, which has not yet been reported. We evaluated the value of 111 In-HIG for diagnosing $\mathrm{AL}$ in 35 patients (mean age $59 \pm 4.7)$ with ultrasonographically suspected AT in the carotid arteries. A comparative study

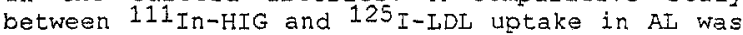
also performed in cholesterol-fed rabbits. Four hours after iv injection of 500uCi 111 InHIG ap images (500000 counts/frame) of the neck were obtained with a gamma camera. The HIGuptake was also continuously monitored with detectors placed over the carotid arteries and a background region and time-activity curves were analysed, After injection of $10 u \mathrm{ul}_{1}$ 111 InHIG and 125 I-LDE, the HIG-entry into aortic segments was evaluated in animels.

Scintigraphy (SC) detected 58 lesions in 27 patients. $13 / 58$ lesions were not evidenced by ultrasonography (US). The overall sensitivity for $S C$ in detecting early AI, according to morphological data, was $77.1 \%$. 111 In - HIG accumlation was higher in reendothelialized areas, foam cells, at the edge of lesions and hypercholesterolemic rabbits. This study indicates that imaging with 111 In-HIG can provide valuable information concerning metabolic aspects of atherosclerosis.

\section{6}

J.F. Chatal, L. Chatenoud, N. Chanteloup, P. Peltier.

INSERM Research Units 211, Nantes and 25, Paris, France.

Can the immunogenicity of radiolabeled murine antibodies be reduced by immunosuppressive treatment at nontoxic doses?

The immunogenicity of murine antibodies is one of the main limiting factors of immunoscintigraphy and radioimmunotherapy. Their replacement with human or humanized antibodies provides a partial solution to this problem. However, in the organ transplant field, where anti-T-lymphocyte murine monoclonal antibodies have been used for more than 10 years, an alternative approach to reduce sensitization frequency consists in associating conventional immunosuppressants with monoclonal antibody treatment protocols. Thus, we tested low-dose, short-term immunosuppressive treatment in 14 patients injected with $1 \mathrm{mg}$ of $F\left(a b^{\prime}\right) 2$ fragments of OC125 antibody for scintigraphic detection of recurrences of ovarian carcinoma. We administered $15 \mathrm{mg} / \mathrm{day}$ of prednisolone and $3 \mathrm{mg} / \mathrm{kg} /$ day (but not more than 150 $\mathrm{mg} /$ day) of azathioprine, for six consecutive days (beginning 2 days before immunoconjugate injection). The appearance of anti-OC125 human antibodies was monitored by a solidphase immunoenzymatic technique (ELISA). Three of these 14 patients $(21 \%)$ developed human anti-mouse antibodies (HAMA), whereas in a control group receiving the same preparation of $F\left(a b^{\prime}\right) 2$ fragments (without immunosuppressive treatment) $7 / 22$ subjects $(32 \%)$ developed HAMA (nonsignificant difference). Thus, this type of immunosuppressive regimen did not significantly reduce immunization against the xenogenic protein. The possibility of using other types of short-term ( $<10$ days) immunosuppressive treatments in association to immunoscintigraphic and radioimmunotherapeutic protocols will be discussed. 
297

L.M. Eig ${ }^{1}$, E.L. Kramer ${ }^{2}$, S.J. Harwood ${ }^{3}$ for the Mrlticenter Trial Group; L. Hughes ${ }^{4}$, C.M. Pinsky ${ }^{4}$. ${ }^{1}$ DVA Medical Center, Ann Arbor,

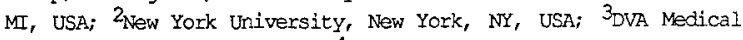

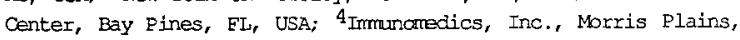
$\mathrm{NN}$, USA.

TC-99M ANTI-CEA FAB' MONOCLONAL ANTIBODY (IMMURAIDCEA) IMMUNOSCINTIGRAPHY IN NON-SMALI CELL LUNG CANCER.

Tc-99m is a nearly optimal radiolabel which to date has been underutilized in immunoscintigraphy. We examined the safety and performance of $\mathrm{TC}-99 \mathrm{~m}$ labeled anti-CEA Fab' monoclonal antibody (ImmuRAID-CEA) in non-small cell lung cancer. In a multicenter trial, fifty four patients were studied (42 male, 12 female; ages 40-93 yr.) who had primary operable (19), primary inoperable (7), occult (9), metastatic (15) or recurrent (4) disease. The injected dose was 20-30 mCi TC-99m labeled to $1 \mathrm{mg}$ protein. Planar and SPECT imaging was performed at $4-8$ and $18-24$ hr in 44 patients and at $15-18 \mathrm{hr}$ only in 10 patients.

There were no clinically significant adverse reactions. There were four minor and transient alterations in biochemical and/or hematologic indices. In 27 patients tested at 4-6 weeks or 3 months, human anti-mouse antibody (HAMA) assays were negative by Immustrip( HAMA fragment kit. Overall sensitivity (Sens) for tumor detection on a per patient basis was 90\%, accuracy (AcC) $85 \%$ and positive predictive value (PRV) $94 \%$. On a region basis sens was $80 \%$, AcC was $84 \%$ and PPV $83 \%$. Sens in the chest on a per lesion basis was 69 \% and 88 용 in the liver. Prominent blood pool activity was noted on the early images but improved target to background ratios with lower count rates were present on the delayed images. A negative scan confirmed equivocal studies as true negative in 5 patients. Foci of tracer uptake at previously unsuspected sites were observed in 24 patients. Of these, cancer was confirmed in 11, not confirmed in 2 , and remains indeterminate in 11.

ImmuRAID-CEA immunoscintigraphy has proven safe and has potential utility in the depiction of tumor extent in non-small cell lung cancer.

\section{8}

J. Bandt, J.F. Verzijlbergen, V.A.M. Duurkens, E.K.J. Pauwels.

Depts. of Nuclear Medicine and Pulmonology, St. Antonius Hospital, Nieuwegein and Academic Hospital, Leiden, The Netherlands.

TL-201 MEDIASTINAL SPECT IS ABLE TO ACCURATELY SELECT PATIENTS WHO NEED MEDIASTINOSCOPY IN THE STAGING OF BRONCHIAL CARCINOMA.

Various techniques are employed to exclude mediastinal lymph node metastates in pts with bronchial carcinoma. T1-201 can discriminate between malignant and benign nodules. The purpose of this study was to prospectively determine the value of mediastinal Tl-201 SPECT in the staging of 68 pts with suspected bronchial carcinoma. 15 min. and 4 hrs after injection of $185 \mathrm{MBQ}$ T1-201 SPECT of the thorax was performed. Clear mediastinal uptake of $\mathrm{Tl}-201$ was denoted positive. The 'gold standard' was mediastinal histology. In 14 pts no mediastinal histology was obtained (mainly small cel ca). In 18 of the remaining 54 pts a benign disease was diagnosed, only one of them being falsepositive (tuberculosis) but not in the mediastinum. In $10 / 36$ of the remaining pts mediastinal tumour spread was confirmed, in all of them SPECT-positive. In none of the 26 mediastinal tumour-free pts $\mathrm{T} 1-201$ uptake was noted. No false-positives and -negatives were found. In 23 pts mediastinoscopy was performed, $8 / 23$ pts showed mediastinal spread. In $3 / 8$ mediastinoscopy was false-negative. In all other pts correct results were obtained.

Mediastinal tumour metastases can be correctly diagnosed with T1-201. Selection of pts for mediastinoscopy, to obtain histology, can be accurately performed.

\section{9}

Steinert $H^{1}$, Huch Böni $R^{2}$, Bōni $R^{3}$, Voellmy $D^{3}$, Berthold $T^{1}$, Burg $G^{3}$ von Schulthess 1

Division of Nuclear Medicine ${ }^{1}$, Departement of Radiology ${ }^{2}$, Departement of Dermatology ${ }^{3}$, University Hospital of Zürich, Switzerland.

\section{DETECTION OF METASTISING MELANOMA BY WHOLE- BODY POSITRON EMISSION TOMOGRAPHY (PET) USING [18 F] FDG}

Staging of malignant melanoma and the early detection of metastases are important factors for the therapeutic procedure and the prognosis. Until now only single body regions could be examined using PET. In a prospective study the potential of a rapid whole-body PET with [18 F] FDG in patients with metastic melanomas was evaluated.

Fifteen patients (age range 28 to 73 years) were studied approximately 40 minutes following the injection of $350 \mathrm{MBq}[18$ F] FDG. Whole-body PET scans were performed using a General Electric Advance PET-Scanner with an acquisition time of 40-50 minutes. The results were compared with clinical findings and findings on other imaging techniques (MRI, CT, US).

Whole-body PET correctly demonstrated 27 metastases in the lung, brain, pancreas, nasal activity, cutis and subcutis and in lymph nodes. The sensitivity of all metastases larger than the spatial resolution of the PET-system (>5 mm) was $100 \%$. Two cutaneous metastases, measuring approximately $3 \mathrm{~mm}$, did not show an increased FDG-uptake, the overall sensitivity was therefore $96 \%$. PET correctly excluded malignancy in 4 cases, where suspicious lesions were found with conventional crossectional imaging modalities but later ruled out by fine needle biopsy.

In summary our data demonstrate the clinical potential of whole-body FDG-PET for the detection of metastising melanoma.

\section{0}

O.E. Nieweg, J. Pruim, J.W. Braams, A. Vermey, J.L.N. Roodenburg, H.J. Hoekstra, J. Oldhoff, W. Vaalburg, H. Schraffordt Koops

University Hospital, Groningen, The Netherlands Cancer Institute, Amsterdam, the Netherlands PET WITH FDG IN PATIENTS WITH UNKNOWN PRIMARY TUMORS

A minority of cancer patients presents with metastatic disease and an unknown primary tumor. Deciding whether or not to treat and how to treat such patients is easier when the primary lesion can be identified. In a pilot study, PET with FDG was used to search for primary tumors after a thorough but unsuccessful conventional diagnostic work-up. Ten patients with various histologic types of metastasis and unknown primary tumors were studied. An i.v. dose of $370 \mathrm{MBq}$ FDG was administered. Whole body scans were made with a Siemens ECAT 951/31 camera.

In one patient the PET study suggested a primary tumor in the lung, in another patient a primary plasmacytoma of the soft tissue with a $3 \mathrm{~mm}$ size was identified on the base of the tongue. The first patient was treated with palliative radiotherapy, the second with surgery aiming for a cure. In one of the eight patients with a negative scan, the subsequent clinical course revealed a papillary thyroid carcinoma. In the remaining seven patients a primary lesion was never found. In all patients the known tumor sites were visualized and in four patients additional metastases were discovered with PET. The treatment plan was adjusted in one of these patients.

In summary, PET with FDG yielded useful information that changed clinical management in three of ten patients with an unknown primary tumor. One patient may be cured, the other two are adequately palliated. 
301

J Kuyama, K Uno, Y Imai, Y Uchida, J Okada, J Itami, K Imazeki, $M$ Saitoh, N Arimizu.

Dept. of Radiology, Chiba University, School of Medicine,

Chiba, JAPAN

\section{EVALUATION OF INDIUM-111 LABELED LYMPHOCYTE SCINTIGRAPHY IN CLINICAL USE.}

To evaluate the usefuiness of In-111 labeled lymphocyte scintigraphy (InLLS) as a staging method in malignant lymphoma(ML), we performed InLLS in comparison with Ga-67 scintigraphy in 39 patients (4Hodgkin's disease[HD] and 35 Non-Hodgkin's lymphoma[NHL]) prior to treatment. 9 patients with inflammatory diseases were also investigated with InLS as a control group. We labeled lymphocytes collected by a cell separator (Haemonetics and Ficoll-paque) with In-111 tropolone. Whole body imaging was performed after 4 and $24 \mathrm{hr}$ after injection. Ga-67 was positive in $\mathbf{3 0}$ patients, but it detected only $\mathbf{5 3}$ out of a total of 78 tumor sites, detected with other modalities in these 30 patients. InUS showed positive imaging in 35 patients, and it detected all 72 tumor sites with no false negative uptake in these 35 . The specificity was similar for both agents: InLLS $83 \%$ and Ga-67 80\%. InLLS, however gave clearer images than Ga-67 for the evaluation of abdominal and hilar uptake. In 4 further patients with NHL, InLLS showed no accumulation in known lymphoma lesions. We could find no abnormalities of $T$ cell function in these 4 patients, as shown by mitogen activation, ratio of $T$ cells to $B$ cells, or ratio of OKT4 to OKT8. We also could not prove any associated abnomalities of lymphocyte uptake in adjacent inflammatory changes in these patients' biopsy specimens. On the contrary, in 9 patients with inflammatory diseases, lymphocyte migration to inflammatory foci correlated with $T$ cell activation by mitogen. We suggest that anergy of lymphocytes in patients with $\mathrm{NHL}$ is due to more complicated factors. In 35 patients with NHL, 4 were negative with InLLS and in 29 patients lymphocyte migration was observed only in the $24 \mathrm{hr}$ images. InLLS showed uptake in tumor lesions on both 4 and $24 \mathrm{hr}$ images in only 2 patients with $\mathrm{NHL}$, but in all 4 patients with $H D$. NHL is distinguished by this slow accumulation from

inflammatory diseases and HD which showed early accumulation.

H.M. Abdel-Dayem, I. DiFabrizio, R. Bag, T. Aras, H.T. Turoglu, J. Rempf, N. Habbab, F. Pescatore, S. Sadik, พ. Kowalsky

Department of Nuclear Medicine, St. Vincent's Hospital and Medical Center, New York, USA

SEQUENTIAI (SEQ) THALLIUM (TI) AND GALLIUM (Ga) SCANS IN PULMONARY COMPLICATIONS OF AIDS PATIENTS

We previously reported the high accuracy of sequentially performed TI +ve, Ga -ve scans in AIDS pts in Dx of
pulmonary Raposi's Sarcoma (PKS). The purpose of this presentation is retrospective analysis of $88 \mathrm{seq}$. TI Ga scans done for 85 AIDS pts. The final Dx was correlated with chest $X$-ray (CXR), bronchoscopy (B), CD4 and findings of $\mathrm{Tl}$ and Ga scans. The results are in the following table.

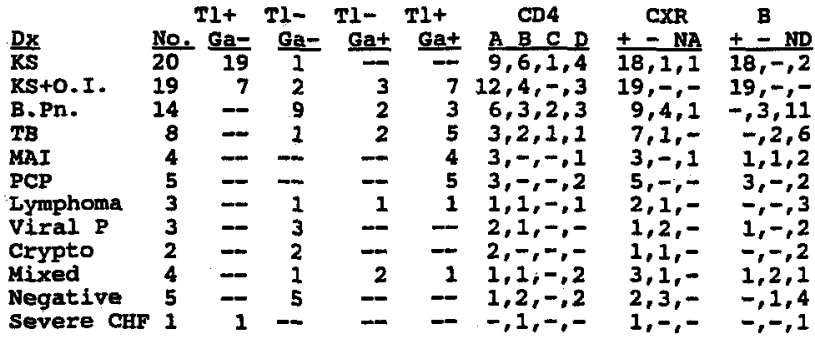

0.I.=Opportunistic Infection. B=Bacterial. Pn=Pneumonia. NA=not available. Negative=gymptoms were not related to chest infection. $C D 4=A=<50, B=50-100, C=100-200, D=n o t$ done within 6 months. ND=no bronchoscopy done within 1 month. Crypto=cryptococcosis.

We conclude that: 1) Tl+ Ga- scans are highly accurate for Dx of PKS. 2) This accuracy decreases in presence of $0 . I$. 3) Tl+ Ga+ lesions are geen in TB, MAI, and PCP but pattern is different. 4) Ga+ scan is not sensitive in bact \& viral pneumonia in these pts. 5) There is no correlation between Gat scang and $\mathrm{CD} 4$ counts.

\section{3}

D.M. Goldenberg, R.M. Sharkey, R. Vagg, M. Juweid, A.I. Markowitz, L.C. Swayne, S. Udem, H.J. Hansen, and P.D. Walzer

Center for Molecular Medicine \& Immunology, Newark, NJ; Immunomedics, Inc., Morris Plains, NJ; and University of Cincinnati, Cincinnati, OH

IMAGING OF PNEUMOCYSTIS CARINII PNEUMONIA WITH A TC99m-LABBLED MONOCLONAL ANTIBODY FRAGMENT

A murine monoclonal antibody (MAb), 7C5, has been developed and shown to be specific for Pneumocystis carinii (PC) surface antigen. The $\mathrm{Fab}$ of this IgGI MAb has been labeled by a direct, 1-step method, with TC-99m, and examined as a noninvasive, simple imaging procedure for diagnosing PC infection in

procedure for diagnosing PC infection in immunocompromised patients, using $1 \mathrm{mg}$ of Mab labeled with 25-30 $\mathrm{mCi}$ Tc-99m and injected $i . v$. Planar images at $24 \mathrm{~h}$ proved to be optimal, and revealed a high rate of accuracy in disclosing PC pneumonia in a series of HIV-patients. Results correlated with sputum and bronchoalveolar cytology, Ga-67 scans, and chest roentgenograms in 15 assessable patients showed 6 true-positives, and 7 true-negatives, indicating a sensitivity and specificity of imaging of over $85 \%$. In an additional 3 patients, the PC MAb disclosed pneumonia whereas a control, similarly prepared, anticancer Mab Fab' did not show specific accretion. These results suggest that this organism-specific MAb These results suggest that this organism-specific MAb may be of use in the rapid and specific diagnosis of PC infection in immunocompromised patients. (Supported in part by USPHS grants CA39841, HL46653, AI31702, AI32899, and contract AI25139 from the NIH, and by the Medical Research Service, Department of Veterans Affairs.)
304 Garcia-Burillo A, Encabo G, Castell J . Ysamat M. González JM, Valenzuela H, Canela T,
Montero Bं, Altisent $\mathrm{C}^{\circ}$.

Servei de Medicina Nuclear, Servei d'Hemotilla", Hospital Universitari Vall d'Hebron. Barcelona.

SERUM $B 2$ MICROGLOBULIN IN HAEMOPHILIAC PATIENTS WITH RELATED HIV INFECTION, PRELIMINARY RESULTS.

$\beta 2$-microglobulin (B2M) is a low molecular weight protein present on the surface of many somatic cells, including $T$ and $B$ lymphocytes. Stimulation of lymphoid cells can increase serum levels of B2M. Serum B2M concentrations therefore seem to reflect the degree of immune-system activation. Several studies have suggested that that quantification of B2M might be useful in the prognosis of human immunodeficiency virus (HIV) infection, showing that high concentrations of it are predictors of acquired immunodeficiency syndrome (AIDS).

The aim of our study was to determine basal serum levels of B2M in order to measure the immune state of patients with haemophilia and reflect the course of HIV related disease.

We carried out a 2 years retrospective study with 152 haemophiliac patients 1137 men, 15 woment. The mean age was 24 years $(5-62$ y). 111 had HIV infection (HIV + ) and 41 were HIV negative (HIV-). A first serum level of $B 2 M$ was measured by a double antibody radioimmunoassay in all patients. They are now being submitted to serial controls of their $B 2 M$ levels trying to predict deterioration of symptoms. All HIV + patients $(111)$ were classified according to Centers for Disease Control (CDC) categories: $89 \mathrm{CDC}$ II or III category and 22 CDC IV category.

The medium B2M concentration in patients with $\mathrm{CDC}$ II or III category was $3.08 \mathrm{mg} / \mathrm{l}$ and in patients with CDC IV category was $4.07 \mathrm{mg} / \mathrm{l}$. Significant differences were found between 2 groups $(p=0.0007)$.

The medium B2M concentration in HIV-patients was $1.81 \mathrm{mg} / \mathrm{l}$ and in HIV+ patients was $3.28 \mathrm{mg} / 1$. Significant differences were found between both groups ( $p<0.000001$ )

Besides, in a subgroup of $50 \mathrm{HIV}+$ patients without zidovudine therapy we studied the relation between serum B2M levels and $\mathrm{CD} 4$ lymphocyte count. $\mathrm{B} 2 \mathrm{M}$ concentration was significantly and negatively correlated with $\mathrm{CD} 4$ count $\{r=0.348, p=0.00083\}$

In view of the importance of an early identification of individual haemophiliac patients with high risk of fast progression to AIDS, our preliminary results seem support the idea that serum levels of B2M adds important prognostic information to CD4 count in determining the risk. We are now carring out a prospective study in these kind of patients in order to predict CD4 cell deplection and disease progression and assess response to treatment. More data are therefore needed about the prognostic significance of serum B2M levels. 
305

T.Banek, D. Dodig, Z. Re1jica-Kostić, P. Predič, G.Kurnik, D. Ivančević

General Hospital, Zagreb, Dept of Nucl. Med. University Hospital Zagreb, Croatia and Hospital Celje Slovenija

THREE-PHASE BONE SCAN, Ga-67 AND 99mTC-NANOCOL SCAN IN DETECTION OF OSTEOMYELITIS CAUSED BY WAR INJURIES

Thirtythree injured solidiers were surgicaly treated because of pierce wounds of extremities. Treatment was either osteosinthesis or external fixation. Two to 4 weeks post treatment clinical signs of osteomyelitis appeared. $X$-ray was negative in all patients. Three-phase bone scan was performed in order to establish diagnosis. Bone scan was positive in all patients. For 11 patients only bone scan was sufficient for decission of further treatment. In 22 patients Ga-67 or 99 mTc-nanocoll or both examinations were performed on surgeon, s request. In 2 patients out of 5 with additionaly Ga-67 scan, Ga-67 scan showed more lesions than it was seen on bone scan. In 3 patients out of 5 with additionaly $99 \mathrm{mTc}$ nanocoll scan, $99 \mathrm{mTc}$ nanocoll scan showed more lesions than it was see on bone scan. In 12 patients with positive bone scan and negative or unclear Ga-67 scan $99 \mathrm{mTc}$ nanocoll scan was performed. In 5 out of 12 patients $99 \mathrm{mTc}$ nanocol1 scan establish diagnosis in others confirmed finding on bone and $\mathrm{Ga}-67$ scan.

Our results showed that in majority of cases 3-phase bone scan was sufficient for diagnosing of osteomyelitis caulsed by war injuries. In selected cases where bone scan was not sufficient for diagnosis and decission of treatment $99 \mathrm{mTc}$ nanocol was more sensitive than Ga-67. In our experience three phase bone scan is method of choice for diagnosing of osteomyelitis in war situation with a lot of causalties.

\section{6}

C. Aktolun, W. Yu.Ussov, A. Arka, D. Glass, R. Gunasekera, A.M. Peters

Department of Diagnostic Radiology, Hammersmith Hospital, London, U.K

EXPLOITING GRANULOCYTE MARGINATION IN THE BONE MARROW FOR THE DIAGNOSIS OF CHRONIC BONE INFECTION BY DOUBLE LABELLING OF THE GRANULOCYTES

Chronic bone infection (CBI) is best imaged with In-111 granulocytes(InG) Since radiolabelled granulocytes(RLG) are taken up by the bone marrow (BM), interfering with the interpretation of the granulocyte scans, a colloid scan is usually necessary to define $\mathrm{BM}$, the distribution of which is significantly altered after surgery. RLG uptake in BM as early as $1 \mathrm{hr}$ after injection is more evident when granulocytes are labelled with $\mathrm{Tc}-99 \mathrm{~m}$ HMPAO, and suggests granulocyte margination, which could be used to define BM. If this assumption is true, then labelling of granulocytes with both Tc-99m and In-111 and using the early Tc- $99 \mathrm{~m}$ signal to define BM and late InLG signal to show the migration to CBI would avoid the need for a colloid scan. The aims of this study were,therefore, 1) to establish the nature of early RLG uptake in BM, 2) to describe a new technique of labelling granulocytes with both In-111 and Tc-99m HMPAO in order to exploit this uptake, 3 ) to verify the functional integrity of these granulocytes, and 4) to predict the feasibility of this technique for the diagnosis of CBI in humans. Fifty seven patients were studied in 2 parts. In the first part, we retrospectively quantified InG uptake in $B M$, spleen (Sp), liver ( $L v$ ) and 3 different types of inflammatory lesions (Le) by comparing the early and late Le/BM ratio with Le/Sp and Le/Lv ratios. In the prospective second part, we labelled granulocytes with both $\mathrm{In}-111$ and Tc-99m HMPAO, studied their kinetics in $\mathrm{Sp}, \mathrm{Lv}$ and $\mathrm{BM}$ in 14 patients with painful prosthetic hip, and compared this technique with colloid BM scan in 9 patients. We showed that $57 \%$ of the BM signal from InG at $3 \mathrm{hr}$ was due to marginated cells. The corresponding values for $\mathrm{Sp}$ and $\mathrm{Lv}$ were $71 \%$ and $47 \%$, respectively. In the second part, cell labelling efficiency was $38.6 \%+12$ and $62.6 \%+14$ for $\mathrm{Tc}-99 \mathrm{~m}$ HMPAO and In-111, respectively. Granulocyte activation, determined as the percentage of cells showing shape change, and recovery of double labelled granulocytes were similar to those previously published for single labelling.

We conclude that granulocytes can be labelled with both In-111 and Tc-99m without cell activation or damage, and double labelling of granulocytes appears to be a promising tool in the diagnosis of CBI, using the early Tc-99m signal to define $\mathrm{BM}$ and the late In-111 signal to demonstrate migration to CBI.
307

M.Milella E.Ruffini R.Sara M.Possa F.Banfi E.Faglia F.Spinelii

Nuclear Medicine Department Division of Internal Medicinet* Niguarda Hospital, Milan, Italy

DETECTION OF FOOT INFECTION BY 99m Tc-HMPAO LEUKOCYTE SCINTIGRAPHY IN DIABETIC PATIENTS.

The aim of this study was to evaluate the sensitivity of Tc-HMPAO labeled leukocytes in identifing bone infection in diabetic foot. In this population radiological investigations may not demonstrate if bone lesions are expression of an acute infection. Ninenteen diabetic patients (15M; $4 \mathrm{~F}$; mean age $62+/-7$ yrs) were studied with $99 \mathrm{~m}-\mathrm{Tc}$ HMPAO leukocytes because of the suspicion of foot infection. Scans were obtained at 4 and $24 \mathrm{hr}$ after the injection of labeled leukocytes (185-370 MBq 99mTc-HMPAO). Seventeen patients had positive scintigrams and two had negative scans for infection. The diagnosis was confirmed by surgical control, histologic findings or by clinical follow-up. In one case leukocyte scan showed infection in spite of a negative bacterial culture by wad. In one case a clinically unknown site was detected. In five patients the study was repeated after clinical and/or surgical therapy : 4 showed an improvement and 1 had a post therapy abscess. In all patients studied antibiotic or surgical therapy was decided also following leukocytes scan indications. In conclusion, 99m-Tc-HMPAO leukocytes scintigraphy had high accuracy in detecting localization and extention of infected foci, in particular it showed if the infective process interests only soft tissue or even bone tissue. The main clinical consequence of this accuracy is the possibility of performing, when surgery is necessary, a conservative approach.

308

B.Sünger, U. Hoppe*, H.-J. Deutsch*, P. Theissen, U. Sechtem*, E. Erdmann*, H. Schicha.

Klinik und Poliklinik für Nuklearmedizin and Klinik IIl für Innere Medizin* University of Cologne

DIAGNOSTIC VALUE OF MAGNETIC RESONANCE IMAGING COMPARED TO TRANSESOPHAGEAL ECHOCARDIOGRAPHY IN 58 ADULTS WITH CONGENITAL HEART DISEASE.

In case of congenital heart disease (CHD) an accurate depiction of anatomy and function of the heart is a main diagnostic issue. As could be shown this is provided with great accuracy by magnetic resonance imaging (MRI). With transesophageal echocardiography (TOE) another imaging technique providing excellent information on cardiac anatomy and function has been developed, that may substitute MRI. To assess the diagnostic value of both methods, a direct comparison was performed by retrospective analysis of 58 corresponding examinations. The collective consisted of patients with ASD (30), VSD (5), single ventricle (4), Tetralogy of Fallot/ puimonary atresia (5), aoritic coarctation (5), complex defects $(9)$.

Pathologic findings were devided into 1. lesions at atrial level, 2. lesions at ventricular level, 3 . valvular defects, 4 . defects of the great arteries

\begin{tabular}{|c|c|c|c|c|c|}
\hline Findings by: & Atrium & Ventricle & Valves & Great Vessels & $\Sigma$ \\
\hline MRI + TOE & 77 & 90 & 55 & 41 & 263 \\
only MRI & 2 & 7 & 9 & 14 & 32 \\
only TOE & 4 & 5 & 7 & 0 & 16 \\
\hline$\Sigma$ & 83 & 102 & 71 & 55 & 311 \\
\hline
\end{tabular}

At atrial, ventricular and valvular level both methods showed nearly equality in depicting the pathologic findings. At the level of the great vessels $25 \%$ of the pathologic findings were seen only by MRI, because of the greater field of view.

In conclusion TOE may substitute MRI in most cases if lesions are found at atrial, ventricular or valvular level. If the great vessels are involved in the disease MRI is superior in depicting pathologic findings. It may be applied additionally if a conclusive diagnosis is not possible by echocardiography alone. 


\section{9}

D.Moka, A. Kaiser, P.Theissen, U.Sechtem*, H.Schicha.

Klinik und Poliklinik für Nuklearmedizin, *Klinik III für Innere Medizin, University of Cologne, Germany.

\section{LEFT VENTRICULAR MYOCARDIAL METABOLISM IN PATIENTS WITH AORTIC VALVE STENOSIS ASSESSMENT BY 31-P-MRS}

To assess myocardial energy metabolism in aortic valve stenosis before valve replacement, 10 patients with severe aortic valve stenosis (effective aortic orifice $\leq 0,8 \mathrm{~cm}^{2}$; pressure gradient across the aortic valve $\geq 70 \mathrm{~mm} \mathrm{Hg}$ ) were examined on a Philips 1,5 Tesla imaging / spectroscopy magnetic resonance system using a surface coil and Gyroscan / ISIS software.

6 patients had a hypertrophic left ventricle but normal ventricular contraction (group A), 4 patients had a dilatation of the left ventricle (LV) (left ventricular enddiastolic diameter (LVEDD) - mean: $59.4 \pm$ $3.6 \mathrm{~mm}$ ) and clinical sign of heart failure (group B). The PCr-/ $\beta$-ATPratio of the myocardium was compared to those of 15 healthy volunteers.

To minimize the influence of blood-ATP, all spectra were acquired at end-systole. The cube-shaped volume of interest was placed after performing gradient-echo magnetic resonance imaging into the apical-septal area of the myocardium. Spectra were evaluated by an iteration program and corrected for $\mathrm{T} 1$-effects and blood contamination.

Mean PCr-/B-ATP-ratio in group B was reduced from 1,79 \pm 0,21 (healthy volunteers) to $1,27 \pm 0,22(p=0,01$ - unpaired $t$-test) Patients (A) without elevated LVEDD had a PCr-/B-ATP-ratio similiar to those of normal controls ( $A: 1,87 \pm 0,22 ; p=0,45$ ).

The reduced $\mathrm{PCr}-/ \beta$-ATP-ratios in patients with severe aortic stenosis and elevated LVEDD is in agreement with the reduced PCr- $/ \beta$ ATP-quotient observed in in vivo 31-P-MRS studies on patients with cardiomyopathy. It is likely caused by structural abnormalities of the LV-hypertrophy after LV-dilatation and decreased total creatine levels and/or altered creatine kinase activity.

To what extend a decrease of the PCr-/B-ATP-ratio is a predictor of a beginning heart failure must be assessed by further investigations.

310

N. J. Shah, A. Spilker, W. Eschner, R. Weise, and $\mathrm{G}$. Notohamiprodjo

Herzzentrum NRW, 32545 Bad Oeynhausen, Germany.

MOVEMENT INDUCED ERRORS IN CARDIAC MR AND PET IMAGE MATCHING USING EXTERNAL MARKERS

Given the lack of suitable common internal markers, the registration of cardiac MR and PET images can be performed using external reference markers. A fundamental assumption of the method is that there is no relative movement between the markers and the anatomy of interest, namely the heart. A number of potential types of movement have been simulated with a view to determining the accuracy of the method.

The lack of ionising radiation makes $M R$ an ideal technique for repeated measurements to determine dominant error sources. Initially however, the non-linearity of the MR gradient system must be determined. Fortunately, the non-linearity can be determined and corrected using software; we have performed this. Three reference markers containing cod liver oil were then placed on a volunteer. The plane containing all three markers was found using a fast field echo sequence. The "3-marker" plane was found after simulating a number of different types of patient movement. The anatomy within the plane could thus be compared anatomy within the plane could thus be compared movement and position of the heart relative to the markers caused by the following: (a) gross patient motion; (b) motion caused by raising and lowering of the arms; (c) sliding around on the patient couch in between measurements; (d) the effect of a full stomach; and (e) imaging on consecutive days.

Additionally, we comment on the problems of matching gated MR images with non-gated PET images. MR and PET images registered using the external marker method will be presented.

\section{1}

K.H. Bohuslavizki, W. Schmidt, W. Brenner, J.-U. Eberhardt, H. Wolf, M. Schramm, M. Clausen and E. Henze

Departments of Nuclear Medicine and Anastheasia, Universities of Kiel and Ulm, Germany

INFLUENCE OF STORAGE TIME AND ADDITIONAL PERFUSION WITH BLOOD ON PORCINE LIVERS INVESTIGATED EX VIVO BY P-31 NMR-SPECTROSKOPY.

NMR fields with both wide bore and high field strengths have become available, allowing the application of magnetic resonance spectroscopy (MRS) in larger mammalian organs. Energy status of these organs as determined by P-31-spectroscopy will depend on the treatment following explantation. Therefore, the aim of this studie was to test different preservation protocols after explantation in a liver model. 30 cadaveric porcine livers were harvested from the slaughterhouse after $11.3 \pm 0.8 \mathrm{~min}$ of warm ischemia. After preparation of the right lobe livers were treated for storage in 5 different protocols: after sudden reperfusion with homologous blood and subsequent perfusion with cold collins solution, livers were stored at $4^{\circ} \mathrm{C}$ for $1-2 \mathrm{~h}$ (No. 1 ) or 4-6 h (No. 2). Protocols No. 3 and No. 4 were identical, except for the omission of blood perfusion. Protocol 5 (No. 5) equaled No. 4, but storage time was extended to $24 \mathrm{~h}$. MRS was performed using a 4.7 $\mathrm{T} / 40 \mathrm{~cm}$ diameter system with $60^{\circ}$ pulses at $81 \mathrm{MHz}$ and subsequent Fourier analysis under identical conditions for all five protocols after reperfusion with warm homologous blood. During perfusion, index of $\beta$-ATP and inorganic phosphate $(\beta-\mathrm{ATP} / \mathrm{Pi})$ was compared at 20 and $80 \mathrm{~min}$, respectively.

Medians ( $\mathrm{n}=6$, each protocol) of $\gamma$-ATP/Pi are given in the table:

\begin{tabular}{|l||l|l|l|l|l||}
\hline & No. 1 & No. 2 & No. 3 & No. 4 & No. 5 \\
\hline $\mathbf{2 0 ~} \mathrm{min}$ & .059 & .091 & .104 & .113 & .054 \\
\hline $\mathbf{8 0 ~} \mathrm{min}$ & .133 & .192 & .331 & .205 & .082 \\
\hline
\end{tabular}

Additional perfusion with warm blood decreases recovery of $\beta$-ATP Increase of storage time from $1-2 \mathrm{~h}$ to $4-6 \mathrm{~h}$ increases recovery of $\beta$ ATP, while storage time of $24 \mathrm{~h}$ worsens $\beta$-ATP recovery. In conclusion, in explanted liver best preservation is received by cold storage for $1-2 \mathrm{~h}$ in collins solution only, possibly due to reduced thrombosis.

312

K.H. Bohuslavizki, W. Schmidt, H. Wolf, W. Brenner, J.-U. Eberhardt, M. Schramm, M. Clausen and E. Henze

Department of Nuclear Medicine, Christian-Albrechts-University, Kiel, Germany

\section{APPLICATION OF P-31 NMR-SPECTROSKOPY TO A NEW EXPERIMENTAL PERFUSION MODEL USING CADAVERIC PORCINE LIVERS FROM SLAUGHTERHOUSE.}

Wide-bore NMR fields with high field strengths have become available, allowing the application of magnetic resonance spectroscopy (MRS) in larger mammalian organs. Energy status of organs may be determined by P-31-spectroscopy may determine enrgy ststus of these organs, which will be dependend on the treatment following explantation. Therefore, the aim of this studie was to test different preservation solutions in a liver model.

35 cadaveric porcine livers were harvested from the slaughterhouse after $10 \mathrm{~min}$ of warm ischemia. After preparation of the right lobe and cooling down by perfusion MRS was performed to examine the energy phosphate pattern as an index of viability using a $4.7 \mathrm{~T} / 40 \mathrm{~cm}$ diameter system with $60^{\circ}$ pulses at $81 \mathrm{MHz}$ and subsequent Fourier analysis. Investigation was carried out as follows: Cooling with Collins (COL) solution ( $\mathrm{n}=17)$ or Bretschneiders (BRET) solution $(\mathrm{n}=18)$, MRS, reperfusion with warm blood for $80 \mathrm{~min}$ to allow $\beta$ - and $\gamma$-ATP-peak to recover completely, MRS, re-cooling with Collins solution or Bretschneiders solution and MRS for $240 \mathrm{~min}$. Vanishing of $\gamma$-ATP-peak was quantified by the index $\gamma$-ATP/MDP-standard Time axis of re-cooling was normalized to index maximum.

\begin{tabular}{|l||l|l|l|l|l|l|l|l||}
\hline \multicolumn{1}{|c||}{} & \multicolumn{7}{|c|}{$\gamma$-ATP/MDP } \\
\hline \hline $\mathrm{t}[\mathrm{min}]$ & 0 & 20 & 40 & 60 & 80 & 100 & 120 & 140 \\
\hline $\mathbf{C O L}$ & .15 & .12 & .07 & .06 & .055 & .05 & .05 & .05 \\
\hline BRET & .15 & .16 & .13 & .11 & .08 & .085 & .09 & 0.1 \\
\hline
\end{tabular}

All results are given as medians in this table. Observe, in Collins solution index of $\gamma$-ATP/MDP decreases faster than in Bretschneiders solution.

In conclusion, in explanted liver Bretschneiders solution may be perferable to Collins solution due to its better preservation function. 


\section{3}

D.Moka, S.Koppelmann*, J.Hahn*, P.Theissen, H.Schicha.

Klinik und Poliklinik für Nuklearmedizin, *Lehrstuhl II für Anorganische Chemie, University of Cologne, Germany.

\section{MRS-QUANTIFICATION OF PHOSPHATE METABOLITES IN HUMAN WHOLE BLOOD}

In-vivo phosphorus spectroscopy (31-P-MRS) has the potential to determine energy/phosphate metabolism noninvasively. In order to evaluate metabolite concentration in human organs with blood contamination (e.g. heart) by 31-P-MRS, a correction factor (CF) for the influence of blood-ATP is necessary. Since 2,3-diphosphoglycerate (DPG) mainly occurs in blood and has a constant relation to bloodATP, quantification of the DPG/ATP-ratio may be a reliable CF for blood contamination e.g. 31-P-MRS of the heart

To assess a CF, fully oxygenated whole blood $(4 \mathrm{ml})$ of 9 healthy volunteers were examined on Bruker 4.7 Tesla spectrometer Quantitative in-vitro 31-P-NMR spectra were obtained spinning (10 rotations per sec.) at $4{ }^{\circ} \mathrm{C}$ for 4 hours ( 4000 scans) using a repetition time of 3 sec and 1-Hupulse decoupling. Chemical shifts were referenced to $85 \% \mathrm{H}_{3} \mathrm{PO}_{4}$ using the chemical shift of internal methylene diphosphonate $(1 \mathrm{mM})$. To keep the whole blood homogeneous, spectra acquisition were carried out using a permanently rotating glas stirrer. Tatrogene cell destruction could be excluded from microscopic analysis and erythrocytes counting of the blood samples, which were performed previous to and after each measurement. Spectra were evaluated by an iterative deconvolution process, the $\mathrm{CF}$ was obtained using the DPG/ $\alpha$-ATP-ratio.

The mean concentration in human whole blood of 2,3diphosphoglycerate is $1,92 \pm 0,32 \mathrm{mM}$, of $\alpha$-ATP is $0,52 \pm 0,08 \mathrm{mM}$, inorganic phosphate is $0,71 \pm 0,12 \mathrm{mM}$. Mean DPG/ATP-ratio of whole blood is $3,84 \pm 0,84$

This study shows, that quantitative determination of high energy phosphate in human whole blood is possible by MRS. To assess a CF for blood contamination in in-vitro, it is sufficient only to evaluate the mean DPG/ATP-ratio, because there is no dependence of the DPG/ATP-ratio on the erythrocyte count or hematocrite in whole blood.

\section{4}

D. Arndt, W.H. Mehnert, W.-G. Franke, P. Woller, G. Laude, A. Rockel, M. Waller

Federal Office of Health, Berlin, Clinic for Nuclear Medicine, Technical University of Dresden, Municipal Hospital Chemnitz, Germany

\section{RADIOIODINE THERAPY DURING AN UNKNOWN REMAINED PREGNANCY AND RADIATION EXPOSURE OF THE FOETUS A CASE REPORT.}

A papillary thyroid cancer staged as $\mathrm{pT}_{2} \mathrm{~N}_{3} \mathrm{M}_{0}$ was treated in a 28 -year old woman by surgery, radiation and radioiodine therapy (10 single applications ranging from 3300 to $3840 \mathrm{MBq}$ ). She was asked to prevent a pregnancy. Nevertheless, in the course of radioiodine therapy an attended gynaecologist found the woman pregnant in the $24^{\text {th }}$ week of gravidity. Consequently, radioiodine has been administered during the $2^{\text {nd }}$ and the $22^{\text {nd }}$ week of pregnancy, each time $3700 \mathrm{MBq}$. The ovarial dose resulting from the radioiodine administrations before pregnancy was estimated to $2200 \mathrm{mGy}(220 \mathrm{rd})$, the foetal doses resulting from the two administrations during pregnancy to $250 \mathrm{mGy}$ ( $25 \mathrm{rd}$ ) in each case, and the foetal thyroid dose to between $90 \mathrm{~Gy}(9000 \mathrm{rd})$ and $900 \mathrm{~Gy}(90000 \mathrm{rd})$ for the $2^{\text {nd }}$ therapy during pregnancy. Because of the risk of malformations this pregnancy was interrupted taking the recommendations of the so-called Danish model as a basis for the decision. The foetus was studied with regard to possible consequences of radiation exposure by pathologico-anatomical cytogenetic and radiobiological methods. In the foetus the most important changes were found within the thyroid gland (atrophy, sclerosis, subcapsular interstitial fibrosis, necrobiosis of follicular epithelial cells). On the other hand, analysing the karyotype using dermal fibroblastic cell cultures, no radiation induced chromosomal aberrations were seen. The results of these studies present in detail give useful information on effects to be expected as a result of radioiodine application during certain periods of pregnancy. They might help to extend the radiobiological knowledge with regard to the foetal period.

\section{5}

G.Sarti*, S.Lazzari*, P.Riva

* Health Physics Department and Nuclear Medicine Department, "Bufalini" Hospital, Cesena Italy

\section{DOSIMETRY MODELS IN RADIOIMMUNOTHERAPY FOR BETA-GAMMA EMITTERS.}

In radioimmunotherapy a dosimetric approach at tissue level is required to analyse the effects of radionuclide distribution.

We have studied the differences in therapeutic effect for the radionuclides commonly used in radioimmunotherapy (I131 Y90 Cu67 Re186 Re188 Sm153) versus various tumours wich differ in size, thikness and shape.

A MonteCarlo code (EGS4 -PRESTA) has bean employed to simulate the emission and to transport the beta and gamma emission in human tissue.

We have calculated the kernel scaled beta dose in water in order to evaluate the dose distribution around a source point.

We have assumed idealized geometries for both the radioactive source volume and the target volume of tumour of our patients and we have calculated the fraction of absorbed dose with a spatial resolution of 10-100 $\mathrm{mm}$ and with 100.000 histoires simulated

\section{6}

J.J.J. Borm, M. J. Bruno, E.A.van Royen

Department of Nuclear Medicine and Department of Gastroenterology and Hepatology, Academic Medical Centre Amsterdam.

\section{PITFALLS IN THE DOSIMETRY OF NEUTRON ACTIVATED} PHARMACEUTICALS

Post-production neutron activation of an intact pharmaceutical preparation is a labelling technique of interest for specific research purposes. However, in-vivo use is limited by the complex dosimetry. We report on our experience with an $170 \mathrm{Er}$ oxide enriched enteric coated pancreatic enzyme preparation used in dual isotopc gastric emptying studies. Other labelling techniques were considered inappropriate because of possible interference with the $\mathrm{pH}$-dependent release of the pancreatic enzymes, which was measured simultaneously.

Neutron activation of biological materials results in many radioactive isotopes besides 171Er. This affects scintillation camera operation and adds to the radiation dose. The activation yields, half lifes, and radio-toxicity vary strongly. Therefore, all these radiocontaminants ( $R C$ ) must be taken into account. Within the limits for adequate imaging, the ratio of committed dose equivalent due to $171 \mathrm{Er}$ and the total committed dose equivalent should be maximised. This ratio varies with the interval between neutron activation and ingestion (IAI). This ratio and the dose are most easily calculated when the ALI (ICRP 61) is used as a common denominator After optimisation, $171 \mathrm{Er}$ accounted for $83 \%$ of the committed dose equivalent of $0.5 \mathrm{mSv}$ per $\mathrm{MBq}$ of $171 \mathrm{Er}$. The isotopic content was verified through instrumental neutron activation analysis. Some pitfalls are :

- $23 \mathrm{Na}$ is omnipresent in biomaterials; $24 \mathrm{Na}$ emits high energy photons that penetrate conventional high-energy collimators and interfere with image quality; - isotopic enrichment (in our case $97.9 \% 170 \mathrm{Er}$ ) does not preclude significant amounts of other lanthanides (i.e. in this case $5400 \mathrm{ppm}$ of other lanthanides);

- many RC may result, each with its own unique physical and biological properties (i.e. beta-emitters such as $32 \mathrm{P}$ and $35 \mathrm{~S}$ );

- RC may be present in a chemical form for which dosimetric data is not available

- for trace contaminants very large batch-to-batch variations must be anticipated (i.e. Lu varying from $<10$ to $700 \mathrm{ppm}$ );

- the neutron beam energy spectrum may include epithermal and fast neutrons resulting in increased activation yields for some elements;

If these pitfalls are taken into consideration, neutron activation of isotopically enriched pharmaceuticals is a suitable labelling technique that does not interfere with specific galenic properties. 


\section{7}

H. Bender, J.G. Emrich*, C.M. Hand*, S.L. Tabor*, P. Black*, Z. Steplewski \#, H.-J. Biersack, L.W. Brady*. Dept. of Nuclear Medicine, University of Bonn

*Depts. of Radiation Oncology and Neurosurgery, Hahnemann University and "The Wistar Institute; Philadelphia, USA.

\section{EFFECT OF LOCALIZED OPENING OF THE BLOOD- BRAIN BARRIER BY LEUKOTRINE C4 ON THE} ACCUMULATION OF MONOCLONAL ANTIBODIES.

The monoclonal antibody (MAb) 425 (produced by the Wistar Institute, Philadelphia) binds to a protein domain of the external part of the epidermal-growth factor receptor as overexpressed in human high grade gliomas. MAb 425 is currently being used in a therapeuticclinical phase II trial with promising results. The low accumulation in intracerebral tumors has partly been attributed to a still intact bloodbrain barrier (BBB) and/or blood-tumor barrier. In a recent autoradiographic study, Leukotriene C4 (LCT4) has been demonstrated to open selectively the blood-tumor barrier of intracerebral tumors, resulting in an enhanced uptake of C14-labeled gamma-aminobutyric$\operatorname{acid}(\mathrm{GABA})$.

The aim of this study was to investigate the effect of LTC4 on the accumulation of MAb 425 in xenotransplanted nude rats. Nude rats were stereotactically transplanted with the human glioma cell line A1235 in the right frontal lobe. Eight days later, rats received an infusion of I-125 labeled MAb $425(300 \mu \mathrm{Ci})$ via the carotid artery. Prior to the MAb, rats were injected with LTC4 $(4 \mu \mathrm{g})$ or saline via the same route. Animals were sacrificed 24 hrs later and radioactivity distribution of tumor, normal brain, and several other organs were determined.

Our biodistribution data showed that LTC4 treated animals showed a 2.7-times higher MAb accumulation in the tumor as compared to the controls. No statistical significant differences were found in the ipsilateral and the contralateral normal brain tissue of LTC4 treated animals as compared to control animals.

These preliminary data demonstrate that LTC4 is able to enhance selectively MAb accumulation in intracerebral tumors. This effect is probably due to opening the blood-tumor barrier. Further studies are necessary to evaluate the therapeuticimplications.

\section{8}

A. Ahonen, P. Karnani, O. Lukkarinen, M. Nurmi, M. Teräs, J. Heikkilä, K. Kairemo

Divs. of Nucl. Med. and Surgery in Univ. Hospitals of Oulu, Turku and Helsinki, Orion Farmos Pharmaceuticals, Finland

PHARMACOKINETICS OF IN-111 PAP-MOAb AFTER THE UNLABELED PAP-MOAb INFUSION

The aim of the present study was to evaluate the effect of unlabeled antibody on the biodistribution and pharmacokinetics of 111-In PAP-MoAb (prostatic acid phosphatase monoclonal antibody).

The human purified PAP was used in the preparation of a spesific MoAb. DTPA-derivates of the antibody $\mathrm{F}\left(\mathrm{ab}^{\prime}\right) 2$-fragments $(1 \mathrm{mg})$ were labeled by $185 \mathrm{MBq} 111-$ In chloride. 111-In was chelated by the DTPA-moiety attached to the antibody fragment. The unlabeled antibody was given slowly intravenously $(30 \mathrm{~min})$ in a randomized fashion to invidual patients at three different doses $(0 \mathrm{mg}, 40 \mathrm{mg}$ and $80 \mathrm{mg} / 100 \mathrm{ml}$ ) diluted with physiological saline to $100 \mathrm{ml}$. The labeled $\mathrm{MoAb}$ was injected $60 \mathrm{~min}$ after initiating the unlabeled MoAb. Until now 15 patients have been imagined.

Biodistribution and pharmacokinetics of 111-In PAP-MoAb in serum was evaluated using the sum of 2 and 3 exponentials. Results indicated that the 3 exponential model was more reliable. The apparent final halflife ( $>20 \mathrm{~h}$ postinjection) was $10 \mathrm{~h}$ for the patients receiving no unlabeled antibody. For the patients given unlabeled antibody the apparent final halflife for 111-In PAP-MoAb was $24 \mathrm{~h}$ in serum. The liver to blood ratio of the patients with no unlabeled antibody was higher than of those patients who received unlabeled antibody. Spot images indicated that radioactivity level in the liver, spleen and bone narrow decreased when unlabeled antibody was available.

The use of unlabeled MoAb before the injection of labeled MoAb scems to enhance the amount of radioactive MoAb in the blood with a simultaneous decrease in the liver accunulation.

\section{9}

R.Hamm, M.Fuchs, A.Schaefer, E.oberhausen

Dept. of Nuclear Medicine, University clinics of Homburg, Homburg/Saar, FRG

RADIATION EXPOSURE OF RADIOIMMUNOSCINTIGRAPHY USING TC-99m LABELED MONOCLONAL ANTIBODY BW $431 / 26$

In recent years radioimmunoscintigraphy (RIS) with Tc-99m labeled monoclonal antibody (MAb) BW 431/26 became of increasing importance in the detection of carcinoembryonic antigen (CEA) positive tumors.To estimate the value of the antibody it is important to consider not only the detection of the tumor but also the radiation exposure of the patients. In a study including 10 patients with CEA positive carcinoma the effective dose has been calculated for selected organs (heart, liver, spleen, kidneys, brain) and for the rest of the body using the concept of the Medical Internal Radiation Committee. For every patient four acquisitions were performed (pool, 1, 5, 24 hours) after the injection of about $950 \mathrm{MBq}$ Tc-99m MAb BW 431/26.

The results revealed the spleen to be the organ with the highest radiation dose $(<=41.7 \mathrm{mSv})$. The organ with the lowest radiation dose was the liver yielding values between $5.25 \mathrm{mSv}$ and $8.7 \mathrm{mSv}$. The commited effective dose of the patients resulting from the organ doses ranged from $2.67 \mathrm{mSv}$ to $4.89 \mathrm{mSv}$. After the application of the MAb BW 431/26 patients showed no significant changes in vital signs and labatory measurements. In conclusion the RIS has no side effects for the patients. The comited dose did not exceed the values of other routinely used examinations in radiology and nuclear medicine.

\section{0}

B.E.Jones, N.E.Herrera, E.Busemann Sokole, V.Volodin. Charing Cross Hospital, London, UK; Danbury Hospital, Ct, USA; Academic Medical Centre, Amsterdam, NL; World Health Organisation, Geneva, $\mathrm{CH}$.

\section{WORLD HEALTH ORGANISATION MULTICENTRE INTERCOMPARISON STUDYUSING A CARDIACGATED BLOOD POOL PHANTOM.}

A multinational gated cardiac blood pool intercomparison study has been performed in Europe under the auspices of the World Health Organisation. This study used mechanical moving plate transmission phantoms (the "Danbury Heart") designed to simulate left and right cardiac ventricular motion with a constant background activity.

One hundred and fifteen Nuclear Medicine Centres in 11 countries took part in the study and provided 144 independent sets of data relating to the equipment and acquisition parameters, details of processing protocols, and measured ejection fraction $(E F)$ for the phantom used.

The distribution of results for the measured EF, as a percentage of the true value for the relevant phantom, was found to be bimodal. Inspection of the data showed that EF results from different makes of computer (20 manufacturers represented) fell into two distinct groups with means of $90.6 \%$ and $98.1 \%(\mathrm{p}<0.001)$. This suggests that there are significant differences in $\mathrm{EF}$ algorithms leading to variations in results even where there is a constant background activity. Further differences are to be expected in the physiological situation with the variations in background subtraction methods.

These results emphasize that a consensus should be reached for standardisation of software analysis protocols for cardiac investigations. 
321

\author{
M. Šămal 1, H.Bergmann ${ }^{2}$ \\ 'Charles University Prague, Czech Republic \\ ${ }^{2}$ Vienna General Hospital AKH and L.Boltzmann Institute \\ of Nuclear Medicine, Vienna, Austria
}

HYBRID PHANTOM FOR QUALITY ASSURANCE OF THE SOFTWARE FOR DYNAMIC RADIONUCLIDE STUDIES

Phantoms represent a standaxd way to control the quality of measurement in nuclear medicine. While physical and mathematical phantoms satisfy the needs of checking the quality of equipment, they may not be fully relevant to check the quality of a clinical software. Recently, the software phantoms (clinically validated patient data software phantoms (clinically validated patient data available in a standard format) have been introduced for the purpose. They meet most of clinical quality control requirements except the need of precise quantification: values of derived diagnostic parameters are estimated in uncertain confidence limits. In order to overcome this drawback we suggest to complete the spectrum of testing tools by a hybrid phantom which combines some features of simulated and software phantoms. The hybrid phantom is made with the help of factor analysis: (1) the standard software phantom is decomposed to oblique factors and noise residuals, (2) factor curves are etther modified or substituted by those generated by a physiological model (3) hybrid dymic dat are reconsti (3) hybrid dynamic data are reconstructed using original factor images and new factor curves, (4) hybrid phantom is completed by an addition of original noise. The hybrid phantom thus represent the sequence of images with real structures and $S / N$ ratio close to original, manifesting the known dynamics generated by physiological model. For the purpose of semiquantitative (comparative) evaluation and/or the separation of spatial and temporal information, factor curves of another patient may also be substituted after adjustment to original factor contributions. In order to test the effect of noise, a series of identical phantoms with different contributions of original noise can be generated The hybrid phantom is (a) considered to be a useful supplement rathex than a substitution of the simulated and software phantoms. Phantoms generated using time-activity curves of different origin are demonstrated together with the source data. Applications ln the quality control of various clinical software are demonstrated and discussed.

\section{2}

Marchandise X., Kotzki P.O., Sabatier J.P., Basse-Cathalinat B. LILLE, MONTPELLIER, CAEN, BORDEAUX - GRIO

(Groupe de Recherche et d'Information sur les Ostéoporoses)

\section{QUALITY ASSURANCE AND BONE ABSORPTIOMETRY}

When medical imaging aims towards in vivo measurement, assurance of quality gains a particular interest : actually, the concepts of accuracy, of precision or of reproducibility of measurement are far from the concepts of contrast, of field homogeneity or of sharpness of the image. We describe here how we set up a national quality assurance in this field.

Assurance quality has been built up from three projects : - absorptiometry workshops : they use both theoretical and practical approaches, in order to make physicians aware of the difficulties of the measurements they perform or of the limits of their clinical interpretation. Through 10 such workshops, more than 180 physicians have been concerned at this time.

- elementary fantoms have been designed in order to appraise each feature of absorptiometers and to characterize their limits. Short and long term reproducibility, accuracy, linearity, homogeneity, soft tissue effects are so studied. Such phantoms are currently circulating through absorptiometric centers (more than 20 centers reached at this time). Hydroxyapatite samples of various quite definite densities are then used in blind studies to check the quality of the measurements.

- relying upon the confidence of measurements in the involved centers, multicentric clinical studies are running.
323

A van Lingen, os Hoekstra, JC Roos, GJJ Teule Free University Hosp, Amsterdam, Netherlands

Performance tests of the C-Trak gamma probe for localisation during surgery.

There is a renewed interest in probe guided surgery. The C-Trak probe (Care Wise) is provided with a cdTe crystal in a $4 \mathrm{~mm}$ tungsten shielding, weighs 600 gram and is fully battery operated. The energy resolution for ${ }^{57} \mathrm{Co}$ proved to be $20 \%$. Spatial resolution (FWHM) is measured $1 \mathrm{~cm}$ from the detector face: $25 \mathrm{~mm}$ (uncollimated), $17 \mathrm{~mm}$ (single collimation) and $7 \mathrm{~mm}$ (double collimation). The counting sensitivity with single collimation is $2.2 \mathrm{cps} / \mathrm{kBq}$ and is proportional with activities in the range of 0.3 to $600 \mathrm{kBq}$. The influence of background is investigated with a theoretical "tumor next to vein"-model consisting of point and line source, both with equal activity concentration. The contrast is expressed as the ratio between point plus line source and line source, at 1,2 and $5 \mathrm{~cm}$ from the detector:

\begin{tabular}{lllll} 
collimation & $\begin{array}{l}\text { depth } \\
(\mathrm{cm})\end{array}$ & \multicolumn{2}{l}{ rel sens (8) } & contrast \\
none & & \multicolumn{1}{l}{ line } & \\
& 1 & 100 & 100 & 1.050 \\
single & 2 & 47.3 & 69.3 & 1.034 \\
& 5 & 12.9 & 31.4 & 1.020 \\
& 1 & 70.7 & 43.1 & 1.081 \\
double & 2 & 36.1 & 31.4 & 1.057 \\
& 5 & 10.3 & 16.1 & 1.032 \\
& 1 & 33.5 & 21.5 & 1.077 \\
& 2 & 16.1 & 15.2 & 1.053 \\
& 5 & 4.1 & 6.8 & 1.030
\end{tabular}

The results show that, without background, the probe is very well suited for localisation. with background activity, localisation depends strongly on activity concentration and volume.

\section{4}

J. Heikkinen, J.T. Kuikka, A. Ahonen, J. Jurvelin, $\mathrm{K}$. Hartikainen and $G$. Kvist

Kuopio University Hospital, Oulu University Hospital, Association of the Finnish Local Authorities, Central Hospital of Vaasa, Finland

\section{A FINNISH MULTI-CENTER OUALITY ASSURANCE PROJECT IN BONE SCINTIGRAPHY AND IN BRAIN SINGLE PHOTON EMISBION TOMOGRAPHY: DOSE CAIIBRATORS}

This study is a part of the national phantom study for bone scintigraphy and brain SPET systems. The purpose of this study was to evaluate differences of dose calibrators. We examined calibrators in 14 nuclear medicine laboratories. Same co-57 source (4 MBq) was measured in supervising by the same physicist. Results were compared to calculated activities. Average percentual error between measured and calculated activity was $3.9 \%$ and the range was $0.9 \%$ $11.4 \%$ Most calibrators showed stable values but in some places the variation of the reading was up to $48 \%$ due to roaming. Reasons for big percentual errors and unstable readings are probably the low activity of the source and short time constant of the calibrator. According to these preliminary results we conclude that some laboratories should pay attention to quality assurance of dose calibrators and a new test with bigger source activity should be performed. 


\section{5}

M.H.Bourguignon ${ }^{1}$, H. Sochor 2 and E. Busemann Sokole ${ }^{3}$. ${ }^{1}$ Service Hospitalier F. Joliot, Orsay - France, ${ }^{2}$ Division of Cardiology, Vienna - Austria and 3 Division of Nuclear Medicine, Amsterdam - The Netherlands.

\section{AN EUROPEAN NUCLEAR CARDIOLOGY DATA BASE.}

A data base of clinical studies is necessary to establish normal values and range and typical values corresponding to diseases. It is also needed to assess variability between operators and centers, and is a mandatory tool for software quality assurance. To fulfil these goals, an European Nuclear Cardiology Data base (ENCD) is being built with the collaboration of the European Association of Nuclear Medicine, the European Society of Cardiology and the European Communities COST B2 program "Quality Assurance of Nuclear Medicine Software". The ENCD includes normal subjects and patients with typical patterns of myocardial ischemia, recent myocardial infarction and cardiomyopathy, selected according to predefined criteria which exclude radionuclide data. Acquisition protocols have been designed for planar gated blood pool studies, planar and SPECT myocardial studies using TI-201 and Tc-99m Cardiolite (Eur J Nucl Med 1993, 20:59-65). Acquisition portfolios have been created and widely distributed for the collection of the necessary clinical and test data, respecting medical privacy. The portfolio keys have been defined in agreement of 41 European nuclear medicine physicians and cardiologists. To participate, one must provide radionuclide acquisition data, converted into Interfile format, with completed portfolios. These will be included in the ENCD after a group of experts have checked that the data meet the required quality. In order to prepare further steps, the acquisition portfolio includes the parameters to be obtained by processing. A processing portfolio, now being created, will be distributed with the ENCD. The numerical values will be processed with variance analysis, to help define ranges. It is planned that the ENCD will be made available on CD-ROM to the nuclear medicine community in 1995. The ENCD can then be applied by any user for all of the above goals

\section{6}

\section{K.O. Mang'era, H. P. Vanbilloen, M.J. De Roo, A.M. Verbruggen}

Laboratory of Radiopharmaceutical Chemistry F.F.W. and Nuclear Medicine, K.U.Leuven, U.Z. Gasthuisberg, B-3000 Leuven, Belgium

\section{EFFICIENCY AND BIOLOGICAL BEHAVIOUR OF COBALT IONS USED TO STABILISE TC-99m HMPAO PREPARATIONS}

$\mathrm{CoCl}_{2}$ has been reported to stabilise Tc-99m d,1-HMPAO (PAO) prepared from a Ceretec kit with $1110 \mathrm{MBq}(30 \mathrm{mCi}) \mathrm{TcO}_{4}^{-}$(RCP at $5 \mathrm{~h}=81-91 \%$; EJNM 1993; 20: 661). In our experience, addition of $200 \mu \mathrm{g} \mathrm{CoCl}{ }_{2} .6 \mathrm{H}_{2} \mathrm{O}$ is even efficient in stabilising multi-dose, high activity ( $150 \mathrm{mCi} \mathrm{Tc}-99 \mathrm{~m}$ ) Ceretec preparations with $\mathrm{RCP} \geq 85 \%$ at $6 \mathrm{~h}$. When used at $5 \mathrm{~h}$, such preparation yielded brain images in a baboon, and human WBC labelling yields comparable to those obtained with a fresh $30-\mathrm{mCi}$ PAO preparation. However, up to now no evidence is available on the clinical usefulness and safety of PAO stabilised in this way. Moreover, the formation of a yellow colour on addition of $\mathrm{CoCl}_{2}$ suggests a Co-HMPAO complexation reaction.

Using $\mathrm{Co}-57$ we have performed biodistribution studies in mice on three preparations, i.e. (a) ${ }^{57} \mathrm{CoCl}_{2}$; (b) ${ }^{57} \mathrm{CoCl}_{2}+0.5 \mathrm{mg} \mathrm{d}, 1-$ HMPAO; (c) ${ }^{57} \mathrm{CoCl}_{2}+0.2 \mathrm{mg} \mathrm{CoCl} 26 \mathrm{H}_{2} \mathrm{O}+$ Ceretec kit $(0.5 \mathrm{mg}$ d,l- $\mathrm{HMPAO}+7 \mu \mathrm{g} \mathrm{SnCl} 2.2 \mathrm{H}_{2} \mathrm{O}$ ), and also compared their behaviour on paper chromatography. The results show that in (b) and (c) a lipophilic Co-HMPAO complex is formed with a favourable biological profile, which significantly differs from that of $\mathrm{CoCl}_{2}$. Its plasma clearance is higher (14\% of I.D. in blood at $2 \mathrm{~min}$ and $3.8 \%$ at $10 \mathrm{~min}$, vs. $16.5 \%$ and $13.5 \%$ respectively for $\mathrm{CoCl}_{2}$ ), and it is excreted by both the renal and hepatobiliary system. The excretion of $\mathrm{CoCl}_{2}$ is slower and almost exclusively by the renal system. Co-57 d,1-HMPAO exhibits negligible brain uptake $(<0.25 \%$ of I.D. at $t=2,10$ or $30 \mathrm{~min}$ vs. $\sim 1 \%$ for Tc- $99 \mathrm{~m}$ d, 1 -HMPAO), despite its lipophilic nature. The results justify a next-phase study with multi-dose $\mathrm{Co}^{2+}$-stabilised PAO preparations, especially with regard to pharmacological safety.
M.Giganti, A.Duatti ${ }^{*}$ L.Uccelli, C.Cittanti, P.Colamussi And A.Piffanelli.

Chair of Nuclear Medicine - University of Ferrara - Italy

*Physics and Inorganic Chemistry Department - University of Bologna Italy

\section{PREPARATION OF Tc-99m NITRIDO RADIO- PHARMACEUTICALS INCORPORATING A GLUCOSE DERIVATIVE}

$\mathrm{Tc}-99 \mathrm{~m}$ radiopharmaceuticals containing the $\mathrm{Tc} \equiv \mathrm{N}$ triple bond have been recently introduced into the field of nuclear medicine after the advent of an improved synthesis of the TcN group at tracer level. These radiopharmaceuticals exhibit high in vitro and in vivo stability and can be easily functionalized to obtain radiopharmaceuticals having diverse biological properties. In particular, the incorporation of a suitable ligand into the structure of these complexes may lead to the preparation of a tracer for monitoring metabolic pathways. Glucose is a fundamental substrate for heart and brain metabolism and the stable incorporation of a glucose derivative into the chemical structure of a Tc-99m complex should be a fundamental step in the effort to obtain a Tc-99m-analogue of FDG. In this paper, we describe the preparation of Tc-nitrido compounds containing two different glucose derivative and their biodistributions in rats. The complexes were prepared by reaction of a prereduced TcN-intermediate with 1 -thio- $\mathrm{B}$-D-glucose and D-glucaminedithiocarbazate, respectively. The yields of formation were always higher than $95 \%$. The compounds were stable in vitro for a period of 4 hours. They have been characterized by TLC chromatography and by comparision with the corresponding Tc- 99 analogues. Both complexes have a square-pyramidal structure with an apical TcN group. In complex 1, two l-thio- $B$-D-glucose ligands were found to be coordinated to the metal center through the negative thiol sulphur atom and one negative hydroxilic oxygen atom, giving a final dianionic compound. In complex 2 , the coordination of two monoanionic dithiocarbamate ligands through the four sulphur atoms led to the formation of a neutral compound. Biodistributions in rats showed that complex 1 is rapidly eliminated throgh the kidneys as a results of its negative charge. On the contary, complex 2 showed both urinary excretion and a significant heart uptake (1.6\% i.d.)

\section{8}

A. Afsan, M. Jehangir, M. Ashraf, E. Chiotell is Nuclear Medicine Oncology, Radiotherapy Inst., PINSTECH, Is lamabad Pakistan, PUNJAP Univ. Lahore Pakistan and N.C.S.R. "Demokritos" Athens, Greece

FORMULATION OF A SINGLE COMPONENT KIT FOR THE PREPARATION OF $99 \mathrm{~m} T \mathrm{~T}$-ECD COMPLEX;BIOLOGICAL EVALUATION IN PRIMATES

Ethyl cysteinate dimer ECD, labelled with technetium-99m has been proposed recently as a promising radiopharmaceutical for brain perfusion imaging. However preparation of $99 \mathrm{~m}_{T C-E C D}$ reported to proceed via a two step kit formulation requiring reconstitution with saline and $\mathrm{pH}$ adjustement prior labelling.

In the present work we describe a single composition instant freeze dried kit for the preparation of $99 \mathrm{~m} T \mathrm{~T}-\mathrm{ECD}$ complex. Various stabilizers such as EDTA, mannitol etc., as well as $\mathrm{pH}$ and $\mathrm{SnCl}_{2}$ concentrations were tested in order to evaluate the optimal ingredients. Optimum formulation contained ECD. HCl $1 \mathrm{mg}, 0.5 \mathrm{mg}$ EDTA, stannous chloride and mannitol as stabilizer. Formulation was performed under controlled temperature and nitrogen atm. at $\mathrm{pH}$ 6.0 . The lyophilized kit was recontrituted with 2 m of pertechnetate eluent, resulting in $97.5 \%$ yield in $99 \mathrm{~m}_{-\mathrm{ECD}}$ complex. A radiochemical method of analys is has been also developed permiting the identification of active complex and impurities. Thus by electrophoress is in Whatman No 1 $(250 \mathrm{~V})$, mobile phase phosphate buffer pH 6.8, 99mTC-ECD migrated towards anode at $2.6 \mathrm{~cm}$, while pertechnetate at $10.5 \mathrm{~cm}$. Further the instant freeze dried kit formulation developed was evaluated in primates, monkeys and human. Data demonstrated that activity in the brain tissue was retained until approx. 90 min $p . i$. , resulting in high qua],ity images of brain. Thus the new formulation can be proposed as a reliable kit for the routine preparation of 99mTC-ECD complex. 
329

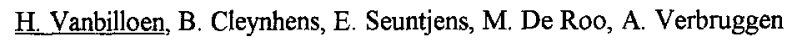

Laboratory of Radiopharmaceutical Chemistry F.F.W. and Nuclear Medicine, K.U.Leuven, U.Z. Gasthuisberg, B-3000 Leuven, Belgium

SYNTHESIS AND BIOLOGICAL EVALUATION OF DERIVATIVES OF 99mTC-ETHYLENEDICYSTEINE WITH ENHANCED LIPOPHILICITY

A striking structural difference between ${ }^{99} \mathrm{~m} T c-L, L-E C$, a new renal function radiopharmaceutical and $\mathrm{OIH}$ is the fact that $\mathrm{OIH}$ has a lipophilic region which is not present in $99 \mathrm{~m}$ Tc-L,L-EC. As OIH is still the renal tracer agent with the most favourable properties, we have now synthesized dimethyl (DM) derivatives (D,D-DMEC, L, L-DMEC) and tetramethyl (TM) derivatives (D,D-TMEC, L, L-TMEC) of EC to enhance the lipophilicity and to create lipophilic region.

HS-CR $\mathrm{R}_{2}-\mathrm{CH}(\mathrm{COOH})-\mathrm{NH}-\mathrm{CH}_{2}-\mathrm{CH}_{2}-\mathrm{NH}-\mathrm{CH}(\mathrm{COOH})-\mathrm{CR}_{3} \mathrm{R}_{4}-\mathrm{SH}$

$\mathrm{EC} \mathrm{R}_{1}=\mathrm{R}_{2}=\mathrm{R}_{3}=\mathrm{R}_{4}=\mathrm{H} \quad$ DMEC R $\mathrm{R}_{2}=\mathrm{R}_{2}=\mathrm{H} \mathrm{R}_{3}=\mathrm{R}_{4}=\mathrm{CH}_{3} \quad \mathrm{TMECR}_{1}=\mathrm{R}_{2}=\mathrm{R}_{3}=\mathrm{R}_{4}=\mathrm{CH}_{3}$ After labelling with $99 \mathrm{~m} \mathrm{Tc}$ the reaction mixtures were analysed by $\mathrm{RP}$ HPLC. DM derivatives eluted as two, probably diastereomeric species (A,B).The TM derivatives occurred as a single radiochemical compound. In mice al derivatives cleared rapidly from the blood. The increased lipophilicity of the new derivatives resulted in a marked rise of the amount cleared by the hepatobiliary system. $30 \mathrm{~min}$ p.i. about $35 \%$ of the i.d. of the $99 \mathrm{~m}$ Tc-TMEC complexes and $16 \%$ to $32 \%$ for three of the DM derivatives (L,L-A; D,D-A; D,D-B) was found in liver and intestines. ${ }^{99} \mathrm{~m} \mathrm{Tc}-\mathrm{L}, \mathrm{L}-\mathrm{DMEC}-\mathrm{B}$, on the other hand, showed a surprisingly low hepatobiliairy handling ( $4.6 \%$ of i.d. $30 \mathrm{~min}$ p.i.), only slightly higher than that of ${ }^{99 \mathrm{~m} T c-L, L-E C ~(2.1 \% ~ o f ~ i . d . ~} 30 \mathrm{~min}$ p.i.). The

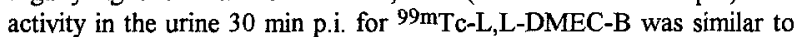
that of $99 \mathrm{~m}$ Tc-L,L-EC ( $90 \%$ of i.d. $30 \mathrm{~min}$ p.i.) and much higher than that of the other DM derivatives $(61 \%$ to $72 \%$ of i.d. $30 \mathrm{~min}$ p.i.) and TM derivatives ( $45 \%$ to $47 \%$ of i.d. $30 \mathrm{~min} \mathrm{p.i.).}$

The results show that besides lipophilicity the configuration of the complex ( $\mathrm{L}$ or $\mathrm{D}$; syn or anti) and the position of the lipophilic substituents seem to play an important role for the biological characteristics

\section{0}

K. Verbeke, G. Schiettekatte, C. Boonen, M. De Roo, A. Verbruggen

Laboratory of Radiopharmaceutical Chemistry, F.F.W., K.U. Leuven and Nuclear Medicine, UZ Gasthuisberg, B-3000 Leuven, Belgium

\section{EVALUATION OF 99mTc -3,4- DIMERCAPTOBUTYRYL CYSTEINYL-HSA AS A POSSIBLE BLOOD POOL AGENT}

The specific binding and technetium chelating strength of proteins can be increased by the covalent attachment of a bifunctional chelating agent (BCA) that forms stable complexes with technetium.

We have now synthesized N-hydroxysuccinimidyl 3,4-di-(S-benzoylmercapto)butyryl S-benzoylcysteinate, a trimercaptomonoamide BCA, and coupled it to human serum albumin (HSA). This was of particular interest because a stable and easy-to-prepare $99 \mathrm{~m} \mathrm{Tc}-\mathrm{HSA}$ derivative would be useful as a blood pool agent in radionuclide ventriculography. After coupling of the BCA to albumin in molar ratios of $8 / 1,16 / 1$ or $25 / 1$, the S-benzoyl groups were removed by incubation with a hydroxylamine solution. Direct labelling could be performed by addition of $10 \mu \mathrm{g} \mathrm{Sn}{ }^{2+}$ followed by $1 \mathrm{ml}$ of a ${ }^{99 \mathrm{~m} T c-g e n e r a t o r ~ e l u a t e . ~}$ The preparations were analysed and purified using FPLC on a Superdex 200 HR 10/30 column eluted with a $0.05 \mathrm{M}$ phosphate buffer pH 7.5 containing $0.15 \mathrm{M} \mathrm{NaCl}$ and $1 \mathrm{mM} \mathrm{NaN}_{3}$.

The biological behaviour and especially the retention in the blood of this new preparation was evaluated in mice and a rabbit. Blood retention was clearly higher than that of unmodified ${ }^{99 \mathrm{~m}} \mathrm{Tc}-\mathrm{HSA}$ and was comparable to that of the reference compound ${ }^{125}$ I-HSA.

Finally, a preliminary study in a human volunteer was performed with the $16 / 1$ preparation. At 2 hours p.i., $83 \%$ of the initial activity was retained in the vascular compartment and only $1.2 \%$ was excreted into the urine vs. respectively $59 \%$ and $17 \%$ for unmodified ${ }^{9} \mathrm{~m} \mathrm{Tc}$-HSA. These results indicate that the preparation of a stable ${ }^{99 m} \mathrm{Tc}-\mathrm{HSA}$ derivative is possible using the bifunctional chelate approach, resulting in a preparation with a clearly higher vascular retention.
331

M. Chianelli ${ }^{1}, 2$, A. Signore ${ }^{2}$, G. Ronga ${ }^{2}$, A. Fritzberg ${ }^{3}, S$. J. Mather ${ }^{1}$

1Dept. of Nucl. Medicine Research, St. Bartholomew's Hospital, London, ${ }^{2}$ Clinica Medica II, University of Rome "La Sapienza" and ${ }^{3}$ NeoRx corp. Seattle, USA.

LABELLING, PURIFICATION AND BIODISTRIBUTION OF 99MTC-INTERLEUKIN-2: A NEW RADIOPHARMACEUTICAL FOR IN VIVO DETECTION OF ACTIVATED LIMPHOCYTES

123I-interleukin-2 (IL2), is currently used for the in vivo detection of lymphocytic infiltration in man. The ready availability and low cost of $99 \mathrm{mTc}$ suggest the use of this isotope for the labelling of IL2. We have previously described a method for labelling biologically active peptides with 99mTc (Eur J Nucl Med. 1992. 19(8):625). The aim of this study was to test different purification methods and to study the biodistribution of 99Tc-IL2 in mice. Human recombinant IL2 was labelled using a bifunctional N3S chelating agent as previously described. Labelled IL 2 was purified by gel filtration (G50M, PDI0 column, Pharmacia), and reversed phase (RP), chromatography using a C18 Classic Sep-Pak column (Waters) $(0.1 \% \mathrm{H} 3 \mathrm{PO} 4,5 \%$ ethanol, $\mathrm{pH}=2$ solvent $\mathrm{A} ; 0.5 \%$ H3PO4 in ethanol, $\mathrm{pH}=3$, solvent B), or a C8 HPLC column (Beckman) (0.1\% trifluoro acetic acid (TFA), $5 \%$ acetonitrile, $\mathrm{pH}=1.7$ solvent $\mathrm{A} ; 0.1 \%$ TFA in acetonitrile, $\mathrm{pH}=2.2$, solvent $\mathrm{B}$ ). The receptor binding capacity of $99 \mathrm{mTc}-1 \mathrm{~L} 2$ was tested by an indirect assay. Biodistribution of $99 \mathrm{mTc}-\mathrm{IL} 2(<1 \mu \mathrm{g})$, purified by either method was studied in Balb/c mice $(\mathrm{n}=8)$. Sep-Pak purified 99mTc-IL2 $(\sim 15 \mu \mathrm{g})$ was also tested in normal volunteers in man $(n=2)$. IL2 LE was $\sim 20 \%$ (SA: $\sim 3 \mathrm{GBq} / \mathrm{mg}$ ). $99 \mathrm{mTc}$-IL2 purified by gel filtration, when injected into mice was taken-up mainly by liver, suggesting aggregation of IL2 during labelling. $99 \mathrm{mTc}-1 \mathrm{~L} 2$ eluted at $\sim 60 \% \mathrm{~B}$ in the RP HPLC. $100 \% \mathrm{~B}$ was used for elution of $99 \mathrm{mTc}-\mathrm{IL} 2$ in a small volume with Sep-Pak. Studies of receptor binding indicated that $99 \mathrm{mT}$ - - IL 2 retained unaffected its receptor binding capacity. Biodistribution of RP purified $99 \mathrm{~m} T \mathrm{Tc}-\mathrm{IL} 2$ showed a kinetics similar to that of iodine labelled IL2. $99 \mathrm{mTc}$-IL2 metabolization, determined by TCA precipitation of sera, showed a higher in vivo stability compared to iodine labelled IL2, probably due to the fast in vivo dehalogenation of tyrosine residues of jodine labelled IL2. Studies in normal volunteers showed that $99 \mathrm{mT} \mathrm{c}-\mathrm{IL} 2$ has a kinetis similar to that of 123I-IL2. Our results suggest the use $99 \mathrm{mTc}-I L 2$ in man for the in vivo detection of lymphocytic infiltration.

\section{Poster presentations}

\section{2}

P. BERTELLI, L. BURRONI, D. VOLTERRANI, A. VELLA and A. VATTIMO.

Nuclear Medicine Unit, University of Siena, Italy.

RADIONUCLIDE IMAGING OF PARATHYROID ADENOMAS: DUAL PHASE SINGLE INJECTION OF MIBI-TC99m VERSUS TL201/TC99M METHOD.

T1201/Tc99m subtraction (Tl-TC) scintigraphy is a well established method in the detection of parathyroid adenomas. Recently Tc99m-Sestamibi (MIBI) has been proven to be rapidly cleared from the thyroid tissue and longer retained in the parathyroid adenomas clearly delineated in a late image. 9 patients ( $8 \mathrm{~F}, 1 \mathrm{M}$; aged $57-79 \mathrm{yrs}$ ) with clinical and biochemical pattern of primary hyperparathyroidism (Ca:10-23 $\mathrm{mg} \%$; $: 2.7-3.8 \mathrm{mg} \%$ Intact PTH: 127$1450 \mathrm{pg} / \mathrm{ml}$ ) were studied with both methods. All of them had surgical confirmation of parathyroid adenoma. AlI patients were injected with $20 \mathrm{mCi}$ of MIBI and imaged at $30 \mathrm{~min}$ and again at 2-3 hrs; one week later the Tl-Tc scintigraphy was performed. MIBI scintigraphy correctly revealed solitary adenoma in the neck in 8 patients and a mediastinal adenoma in one, clearly localized by SPET. Tl-Tc scintigraphy revealed the adenoma in 7 patients, including the mediastinal adenoma. MIBI scintigraphies were of better quality than those obtained with Tl-Tc due to the physical properties of Tc99m and not requiring any subtraction techniques. Unfortunately we found the parathyroid pattern of MIBI in 3 patients with Hurtle cell carcinoma of the thyroid, so limiting the specificity of the method. However the dual-phase technique with MIBI promises to be superior to the dual-isotopes technique in the scintigraphic evaluation of parathyroid adenomas. 


\section{3}

G.Villa, M.Bartolomei, A.Cifelli, G.Calcagno, C.Motta, R.Fiscer, B.Santaniello, P. Eilassoni

Nuclear Medicine Service - DiMI University of Genova - Italy

EVALUATION OF 99MTC-SESTAMIBI UPTAKE IN PARATHYROID ADEMOMAS AND HORYAL THYROID TISSUB.

A single radiotracer method with 99mTcsestamibi (MIBI) has been recently introduced for preoperative localization of parathyroid adenomas. Adenomas show an increased focal uptake which persists on late imaging. Purpose of this study was to compare MIBI uptake of parathyroid adenomas and normal thyroid tissue. 28 pts (mean age 52 yrs) with a clinical and biological diagnosis of hyperparathyroldism were submitted to a MIBI study. Anterior planar imaging of $10 \mathrm{~min}$. were performed 20 and 120 minutes post injection. In 5 Pts aa immediate 20" dynamic acquisition, and delayed static acquistion after 3 and 24 hours were performed. Parathyroid adenoma/normal thyroid tissue activity ratio was $1.21+-0.14$ for early imaging, $145+-0.34$ after 2 hours and $1.44+-0.3$ after 3 hours. In parathyroid and thyroid the peak pf tracer pptake was 5 ' after injection and no difference was observed in activity ratio during dynamic acquistion. We conclude on the usefulness of the evaluation of uptake and wash out index of this tracer from parathyroid enlargements, in order to increase the test sensibility.

334

C. ALS, K. Lauber*, L. Brander*, H. Roesler

Dept of Nuclear Medicine, Central Chemical Laboratory* Inselspital, University of Berne, $\mathrm{CH}-3010$ Berne, Switzerland

\section{In an affiuent society, iodine supply}

via table salt is unstable

lodine supply influences the management and outcome of radioiodine therapy of differentiated thyroid carcinoma. The former iodine deficiency in the Bernese region had been supplemented by a progressive jodination of kitchen salt $(5,10$ and $20 \mathrm{mg} \mathrm{KI} / \mathrm{kg}$ kitchen salt) in the years 1922,1965 and 1980 respectively. The urinary excretion of iodine had risen from a severely deficient 30 $\mu \mathrm{g} / \mathrm{g}$ creatinine level (1920) to a level above $100 \mu \mathrm{g} / \mathrm{g}$ creatinine (WHO standard), during the years 1981-1990. Our study in 1992 in $\mathbf{5 5}$ healthy probands and 234 patients, thyroidectomized because of differentiated thyroid carcinoma, showed still and again an unexpected iodine deficiency: $87 \pm 36 \mu \mathrm{g} / \mathrm{g}$ creatinine. Only $24 \%$ of the healthy volunteers fulfilled the WHO criteria. In a single healthy volunteer evaluated 9 times repeatedly, the average urinary excretion of iodine was $70 \mu \mathrm{g} / \mathrm{g}$ creatinine, the WHO standard was reached only in $11 \%$ of cases. Euthyroid patients on T4 supplementation ( $0.15 \pm 0.04 \mathrm{mg} \mathrm{T} 4 /$ day or $98 \pm 3 \mu \mathrm{g}$ iodine/day) had WHO-insufficient iodine excretion levels only in 19\%: hormonal substitution is thus a provider of a sufficient iodine supply in Berne. Thus, iodine supply via iodinated kitchen salt only is not as stable as had been hoped for. The reasons are multiple: a reduced intake of table salt, increased consumption of imported food, which have not been prepared with iodinated salt, diversified eating habits, increased number of meals taken outside the family table due to a rising female professional occupation, longer transport times to the working place and increasing numbers of single's households. It is to be expected, that a deficient iodine supply will remain a reality in an affluent, goiter endemic society as long as iodinated kitchen salt will not be used generally, for instance in the whole European Community.
335

C. ALS, M. Listewnik, E.P. Ritter, D. Luscher,

H. Ledermann, J. Kinser, H. Roesler

Department of Nuclear Medicine, Inselspital, University

of Berne, $\mathrm{CH}-3010$ Berne, Switzerland

Quantitative evaluation of thyroid autonomy in case of hyperthyroidism

In case of multifocal functional autonomy (MFA) or of toxic adenoma (TA), hyperthyroidism (blocked TRH-test) takes place above a threshold volume of autonomous (A) cells. 1311 therapy is successful, because for an identical specific cellular density, A thyroid (th) areas withhold more radioiodine than functionally suppressed areas. In independence of the functional status, $99 \mathrm{mTC}$ $\mathrm{MIBI}$ (Dupont) is a marker of cellularity. As well in A (AR) as in suppressed (RR) th regions, a dissociation between regional function $(\mathrm{rF})$ and regional cellularity $(\mathrm{rC})$ can be depicted by double tracer scintigraphy. $\mathrm{rF}$ and $\mathrm{rC}$ are then scanned sequentially with $131(123)$ $(7 \mathrm{MBq})$ and $99 \mathrm{mTc}$-Hexamibi $(200 \mathrm{MBq})$. After background subtraction and normalization, the weighted $\mathrm{rC}$ image is subtracted from the weighted $\mathrm{rF}$ image. In the resulting subtraction image [F$C]$, the ratio of count rates: (A area/the most suppressed area) results in the "toxicity" index $\mathrm{T}$.

AR [F-C] $\quad A R=$ counts in $A$ regions

Tw- $\quad R R=$ counts in reference region ("normal" function)

RR[F-C] [F-C] = subtraction scan ("function minus cellularity") Results: Average/1SD of "T" $(\mathrm{n}=77)$, of TT4 (nmol/l), of TT3 $(\mathrm{nmol} / \mathrm{l})$ and of TSH $(\mathrm{mU} / \mathrm{l})$ in 52 hyperthyroid patients (MFA: $n=36$, TA: $n=16)$ before and after 1311 therapy $(150 \mathrm{~Gy} /$ th or 30 Gy/A areas).

\section{Gy/A areas)}

MFA bef. 1311 204 $T^{\top}$

$\pi 4$ TT3 $3 \pm 0.9$ TSH

MFA aft 1311

$25 \pm 3.5 \quad 167 \pm 50.9$

$2.0 \pm 0.4$ $0.2 \pm 0.16$

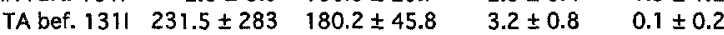

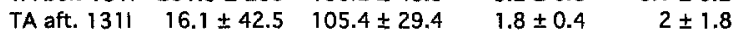

Within a follow-up time of $9-22$ months did none of these " $T$ " residues induce recurrent hyperthyroidism. This new quantitative method is an alternative to the T3-suppression and/or to the TSHstimulation tests, to delineate functionally $A$ th parenchyma.

\section{6}

Chr.Reiners, A.Yavuz, T.Ugur, G.Caspari, W.Sonnenschein, Th.Olbricht

Clinic for Nuclear Medicine and Internal Medicine, University of Essen

SENSITIVE DETERMINATION OF THE INCREASE OF THYROIDAL IODINE CONTENT BY X-RAY FLUORESCENCE

The quantification of stable iodine content of the thyroid (STIC) is of great importance for the evaluation of new therapeutic strategies in the treatment of endemic goiter. We therefore developed a stationary system for $X$-ray fluorescence analysis (XFA) of STIC. This system consisting of a collimated Am-241 source (activity $11.1 \mathrm{GBq}$ ), a HPGe semiconductor detector and a spectrum analyzer has a sensitivity as low as $0.1 \mathrm{mg}$ iodine per $\mathrm{ml}$ of thyroid volume. The coefficient of variation for duplicate measurements amounts to $10 \%$ for a STIC of 0.1 $\mathrm{mg} / \mathrm{ml}$, to $7 \%$ for $0.3 \mathrm{mg} / \mathrm{ml}$ and $5 \%$ for $0.5 \mathrm{mg} / \mathrm{ml}$ respectively.

In normal controls $(n=165)$ STIC averages to $0.32 \pm 0.14$ $\mathrm{mg} / \mathrm{ml}$ (mean $+\mathrm{SD}$ ). In patients with endemic goiter, STIC is significantly lower (diffuse goiter: $0.25 \pm 0.21$; $P<0.01$; nodular goiter: $0.26 \pm 0.15 ; P<0.01$ )

A group of 28 patients with moderate endemic goiters has been treated with $200 \mathrm{ug}$ of iodine per day for a time period of $9.1 \pm 4.5$ weeks. Mean thyroid volume decreased significantly from $26.1 \pm 13.4 \mathrm{ml}$ to $19.8 \pm 9.5 \mathrm{ml}(P<0.001)$. STIC increased with high significance from $0.19 \pm 0.11 \mathrm{mg} / \mathrm{ml}$ to $0.35 \pm 0.14 \mathrm{mg} / \mathrm{ml}(P<0.0001)$

The study shows that a short treatment period with $200 \mathrm{ug}$ of iodine daily may result in a significant decrease of goiter volume, which is correlated with a highly significant increase of STIC. XFA - a genuine nuclear medicine procedure - should be employed on a broader scale in epidemiological or therapeutic studies in thyroidology. 
337

A. Rutkowski

Department of Nuclear biagnostics and Therapy, District Hospitai, Zgierz, Poland

IMPORTANCE OF SCINTIGRAPHIC EXAMINATION IN DETECTION OF COEXISTENCE OF GRAVES ${ }^{\rightarrow}$ DISEASE AND THYROID AUTONOMY

The aim of this work was to investigate the frequency of occurance autonomous foci (AF) in patients (pts) with Graves' disease and to estimate its influence on the treatment efficacy with $131 \mathrm{~J}$. Our material consisted of 71 ciency area. The doses calculated on 90-120 Gy appeared inffective in these cases and following doses were needed. The total applicated activity ranged from 110 to $300 \mu \mathrm{Ci} / \mathrm{g}$ of tissue. The analysis of the thyroid scans in these pts allowed to divide them in two groups: 1-with homogenous distribution of the tracer ( $n=31)$ and $11-$ with heterogenous distribution where the Af were found $(n=40)$. The activity given to the pts from the second group was in the range 160-300 $\mathrm{HCi} / \mathrm{g}$ of tissue and was significantly higher in comparison to growp 1. We suggest existence of Af in many pts suffering from Graves' disease. It refers especialiy to pts from lodine deficiency areas. Diagnosis of the autonomy is particulariy essential before $131 \mathrm{~J}$ therapy pe w11 enable to achieve the sooner euthyreosis in these patients.
A. Karwowska, pts with Graves' disease from iodine defibecause relatively higher doses of the isoto-

339

T. Y. Erdil, C. Onsel, I. Uslu, B. Kanmaz, K. Sönmezoğlu, Enis Altıok, L. Kabasakal, C. Nişli, T. Kapıcıoglu Department of Nuclear Medicine, Cerrahpaşa Medical Faculty İstanbul, TÜRKIYE

\section{TC-99m SESTAMIBI SCINTIGRAPHY IN THE EVALUATION OF AUTONOMOUSLY FUNCTIONING THYROID NODULES}

Tc-99m Sestamibi (MIBI) has been proposed for the visualization of supressed thyroid tissue in patients with autonomously functioning thyroid nodules (AFTN). However, the effect of TSH on thyroid uptake of MIBI is contraversial. The aim of this study is to evaluate the effect of TSH and metabolic activity of AFTN on thyroid uptake of MIBI in patients with toxic and non-toxic solitary AFTN.

Sixteen patients (13F, 3M) with toxic and 12 patients with nontoxic (9F, 3M) solitary AFTN visualized on Tc-99m pertechnetate scan were included in the study. MIBI scans were performed 15 and 90 min. after IV injection of $350-400 \mathrm{MBq}$ of the agent. The scintigrams were analysed both visually and semiquantitatively. For semiquantitative analysis ROIs were drawn over AFTN and contralateral normal lobe and the mean counts in every ROIs were found. After background correction, by dividing nodular counts to extranodular tissue counts nodule / extranodular tissue ratios were calculated. The results are shown on the table.

$\begin{array}{llll} & \text { pertechnetate } & \text { MIBI (15min) } & \text { MIBI (90min) } \\ \text { Toxic } & 10.94 \pm 4.91 & 4.62 \pm 2.14 & 3.91 \pm 1.94 \\ \text { Non-toxic } & 2.97 \pm 0.98 & 1.63 \pm 0.31 & 1.49 \pm 0.19 \\ & p<0.05 & p<0.05 & p<0.05\end{array}$

The results demonstrate that the uptake of MIBI in toxic AFTN is significantly higher than that of non-toxic AFTN. MIBI scintigraphy also shows good visualization of extranodular tissue in non-toxic AFTN compared to toxic AFTN. In conclusion, both metabolic activity of AFTN and TSH might be effective on the thyroid uptake of MIBI

340

338

E. Anterska ${ }^{1}$, W.S. Richter ${ }^{2}$. M. Cordes ${ }^{2}$

1 Robert-Rössle-klinik, 2 strahlenkIinik UKRV

Free University of Berlin, Germany

INITIAL THYROIDAL PERTECHNETATE KINETICS

By use of compartmental analysis a rapid initial thyroidal uptake of pertechnetate in the normal and diseased gland has been described.

In order to estimate the initial thyroidal uptake of pertechnetate, a method based on a dynamic 5-minute Tc-99m pertechnetate thyroid imaging was developed. ROI was set over the thyroid gland and time-activity-curve corrected for background activity was generated. Typically. the time-activity-curves showed three segmentssegment $I$ : vascularisation, segment $I I$ : initial uptake and segment III: postinitial uptake. The incline of segment II was calculated applying the technique of "normalised slope". The results are presented in the form of initial pertechnetate uptake index (IUI).

The study was performed in 74 subjects in whom the diagnosis was established on the basis of clinical evaluation, TSH, thyroid hormones and antithyroglobulin, antithyroid microsomal and anti TSH receptor antibody levels.

The results were as follows: (n) controls

IUI $(x+/-S D)$

euthyroid goiter

$4.06+/-2.31$

euth.thyr Hashimoto

$4.25+/-3.56$

$16.91^{*}+/-7.02$

$29.57^{\star} \star+/-25.44$

multinodular toxic goiter $17 \quad 6.13+/-4.56$

The correlation between the IUI and the Tc-99m uptake at $20 \mathrm{~min}$ was poor $(r=.58, p<.001)$. These results indicate that the IUI may be useful in evaluating autoimmune thyroid status rather than thyroid function.
C. ALS, H. Rösler, L. Brander*, K. Lauber*, D. Lüscher Department of Nuclear Medicine, *Inselspital, University of Berne, $\mathrm{CH}-3010$ Berne, Switzerland

After withdrawal of hormone substitution in thyroidectomized patients, TSH increase and iodine depletion are parallel phenomena.

An endo- and/or exogenously rising TSH level is often considered as a prerequisite for an efficient radioiodine therapy in case of differentiated thyroid carcinoma. Moreover, supply of "cold" iodine should be maximally reduced, in order to increase the uptake of 1311 . Some experimental data were to confirm the hypothesis, that the correlation between TSH increase and iodine depletion is only mimicked.

Cold iodine in plasma (mainly T4) was measured in 55 healthy volunteers $(A, B)$ and in 3 patient groups with differentiated thyroid carcinoma after thyroidectomy: hypothyroid patients with $(C, n=30)$ and without $(D, n=67)$ thyroid remnants and euthyroid patients under T4 substitution ( $E, n=137$ )

Results: The ratio of $131 \mathrm{l} /$ cold iodine was 3 times higher in group $E$ than in group D patients. Euthyroid patients under T4 $(0.15 \pm 0.04 \mathrm{mg}$ T4/day or $98 \pm 3 \mu \mathrm{g}$ iodine/day) (E) had plasma jodine values by $40 \%$ higher than hypothyroid patients (D). An increasing TSH level was associated with a decreasing plasma iodine level: by $40 \%$ after thyroidectomy (C), by $51 \%$ after an additional radioiodine "elimination" of thyroid remnants (D). On the opposite, decreasing TSH was associated with increasing plasma iodine. This - only initally - linear correlation ( $n=0.64 "$ ) is but an illusion. The effective stimulation of an increased 1311 uptake is due to the depletion of competing cold iodine after withdrawal of the substitutive T4: this manoeuvre increases the specific activity of radioiodine. The parallel increase of TSH in itself is not the reason for a better radioiodine uptake in the tumor. 


\section{1}

M.E. Hakman, F.A. Pameijer, J.M.H. de Klerk, H.P.F. Koppeschaar, P.M.J. Zelissen, J.W. van Isselt, J.H.C.M. Fouchier and P.P. van Rijk Depts.of Nuclear Medicine, Radiology and Endocrinology, University Hospital Utrecht, The Netherlands

\section{IODINE-131 THERAPY IN NON-TOXIC GOITER: VOLUME REDUCTION MEASURED BY CT}

Treatment of hyperthyroidism with I-131 is well accepted and usually results in a substantial decrease in thyroid volume. However, few data are available on the effect of I-131 treatment on thyroid volume reduction in non-toxic goiter and the results are seldom evaluated by objective methods. CT is an accurate and reproducible method to determine volume reduction. The aim of this study was to investigate the effect of $1-131$ on goiter volume measured by CT. Twenty-seven patients (mean age: $58.1 \pm$ $10.5 \mathrm{yr}$ ) with complaints of goiter entered the study. All patients had a normal plasma TSH, or a suppressed plasma TSH with a normal plasma T4 (or T3). The administered dose was intended to be $3.7 \mathrm{MBq}$ I-131 per cc thyroid volume, corrected to a $100 \%$ uptake at 24 hours. For the purpose of dose calculation, volume was estimated from a planar Tc-99m pertechnetate scintigraphy. Patients received a dose between 500 and 3700 $\mathrm{MBq}$. Prior to therapy, all patients underwent a CT of the neck, performed with contiguous $5 \mathrm{~mm}$ sections, and without using intravenous contrast material. Volume measurements were made using the summation-of-areas technique. CT was repeated one year after therapy.

The thyroid volume prior to therapy measured by $\mathrm{CT}$ was $203.1 \pm 144.5 \mathrm{cc}$. One year later the volume was $145.8 \pm 115.7 \mathrm{cc}$. Volume reduction was obtained in all patients (mean: $32.8 \pm 17.5 \%$ ). Differences in size, plasma TSH level, radioiodine uptake, and prior thyroid medication did not change the outcome. All but three patients (11.1\%) were satisfied with the result. These three patients showed a reduction of $25.2 \pm 10.0 \%$. Hypothyroidism occurred in four patients $(14.8 \%)$, in whom volume reduction was $43.2 \pm 16.2 \%$. No other side effects occurred.

In conclusion, I-131 therapy for volume reduction in non-toxic goiter is a safe and effective treatment, but the degree of goiter reduction cannot be predicted by pre-treatment thyroid volume, TSH levels, diagnostic radioiodine uptake or thyroid medication.

\section{2}

D. Huysmans, W. Buijs, M. van de Ven, W. van den Broek, A. de Leeuw, A. Hermus, P. Kloppenborg, F. Corstens

Depts. of Nuclear Medicine and Endocrinology, University Hospital Nijmegen, The Netherlands.

DOSIMETRY OF l-131 THERAPY IN LARGE, MULTINODULAR GOITERS; COMPARISON OF EU-AND HYPERTHYROID PATIENTS.

1311 therapy is a widely accepted treatment for large, toxic multinodular goiters. There is, however, a reluclance to use 131 for reducing the volume of large euthyroid goiters for fear of too high absorbed doses in these patients. We compared absorbed doses in thyroid, neighbouring tissues and rest of the body in eu- and hyperthyroid patients with large, nodular goiter $(>100 \mathrm{~g})$

The administered activity of 1311 (AA) was calculated to retain $3.7 \mathrm{MB} / \mathrm{g}$ thyroid tissue at 24 hours. Thyroid weight (TW) was estimated from a rectilinear scan. In 11 eu- and 9 hyperthyroid patients, thermoluminescence dosimeter (TLD) measurements were performed for 5 to 15 days after 131! therapy on 3 locations: on the skin overlying thyroid, submandibular and parotid gland. Extrapolation of monoexponential fits from day 2 onward was used to determine the cumulated absorbed doses on the skin. Furthermore, in 5 eu- and 6 hyperthyroid patients thyroid uptake (lead shielded Nal cristal) and whole-body retention (whole-body counter) were measured daily for 7 to 15 days. Residence times in thyroid and rest of the body (integration of activities over time using extrapolation of monoexponential fits from day 2 onward) were entered into the MIRDOSE2 program to calculate absorbed doses.

TLD measurements. TW, $24 \mathrm{~h} 131$ uplake (RAIU) and AA were $214 \pm 60 \mathrm{~g}$ $33 \pm 9 \%$ and $2.5 \pm 0.8 \mathrm{GBq}$, resp., for eu- and $215 \pm 69 \mathrm{~g}, 50 \pm 15 \%$ and $1.8 \pm 0.9$ $\mathrm{GBq}$ for hyperthyroid patients. Absorbed doses on the skin were $4.1 \pm 1.1 \mathrm{~Gy}$ (thyr), 1.0 $0.4 \mathrm{~Gy}$ (subm) and $0.3 \pm 0.1 \mathrm{~Gy}$ (parot) for eu- and $4.0 \pm 1.5 \mathrm{~Gy}, 1.2 \pm$ 0.5 Gy and $0.4 \pm 0.2$ Gy for hyperthyroid patients (diff. eu-/hyperthyroid n.s.).

Thyroid uptake and whole-body retention measurements: TW, RAIU and AA were $217 \pm 85 \mathrm{~g}, 36 \pm 12 \%$ and $2.4 \pm 1.1 \mathrm{GBq}$ for eu- and $219 \pm 75 \mathrm{~g}, 49 \pm 5 \%$ and $1.8 \pm 0.8 \mathrm{GBq}$ for hyperthyroid patients. Absorbed doses in the thyroid were $82 \pm 19$ Gy for eu- and $76 \pm 19$ Gy for hyperthyroid patients (n.s.). Total body absorbed doses were $0.7 \pm 0.3$ Gy for both groups and absorbed doses in other target organs were also comparable for eu- and hyperthyroid patients.

In conclusion, absorbed doses in thyroid, neighbouring tissues and total body did not differ significantly between eu- and hyperthyroid patients with a large, multinodular goiter treated with 1311. In view of the wide acceptance of ge, multinodular goiter treated with 131]. In view of the wide acceptance of
131 t therapy for large, hyperthyroid goiters, radiation burden is no reason to discourage 131/ therapy for volume reduction of large, euthyroid goiters in elderly people who refuse surgery or in whom surgery is contraindicated.
343

E. Derebek, A. Koyuncu, S. Erdem, M. Ünlü, Y. Yüzer, H. Ozkılıç

Depts. of Nuclear Medicine, Surgery, Ege University, İzmir, Turkey

THE ROLE OF EARLY AND DELAYED TL-201 IMAGING IN THE EVALUATION OF THYROID NODULES : Comparison with Tc $99 \mathrm{~m}$ - pertechnetale perfusion imaging

$\mathrm{TI}-201$ has been used in order to differentiate malign and benign thyroid nodules for a long time. Quite different results were reported. Increased uptake of T1-201 in malign tumor are beliaved related to vascularity of the tumor, basicaly. We aimed to investigate the role of early and delayed TI - 201 in differentiation of malign and benign thyroid nodules, comparing with Tc99m-pertechnetate perfusion images.

28 patients with solid-solitary-hypoactive thyroid nodules were included in the study. 15 minutes and 3 hours after injection of $2 \mathrm{mCi} \mathrm{Tl}-201$, static images of 400-500 Kcounts are obtained, using LEGP collimator. Thereafter, $2 \mathrm{mCi}$ Tc $99 \mathrm{~m}$-pertechnetate were injected intravenously. Soon after the injection first minute perfusion images were obtained. Nodule Activity Index (NAl) was calculated from each static image.

NAI $=\frac{\text { (Nodule Count }- \text { B.G.) }-(\text { Normal Thyroid Tissue - B.G.) }}{(\text { Normal Thyroid Tssue }} \times 100$ (Normal Thyroid Tissue - B.G.)

Results were evaluated semiquantitatively. NAI greater than 100 were accepted as the criteria of malignancy. The patients were operated and histopathologic results were obtained. 8 patients had malign tumors and 20 patients had benign tumors.

\begin{tabular}{llccc} 
& TL (Early) & \multicolumn{1}{c}{ TL(delayed) } & TL(early del) & Tc 99m perf \\
\hline Sensitivity & $\% 100$ & $\% 62$ & $\% 62$ & $\% 87$ \\
Spesificity & $\% 60$ & $\% 85$ & $\% 62$ & $\% 75$ \\
(-) predictive value & $\% 100$ & $\% 100$ & $\% 100$ & $\% 83$ \\
\hline
\end{tabular}

We concluded that TI-201 imaging is useful method in the evaluation of thyroid nodules and Tc99m-pertechnetate perfusion imaging may also be promising method.

\section{4}

L.Dominguez-Gadea, L.Diez, G.Piedrola*,C.Nieto, A. Crespo.

Dept of Nuclear Medicine and *Endocrinoloqy, Hospital Ramon y Cajal. Madrid

SCINTIGRAPHIC DETECTION OF SUB-CLINTCAT CUSHING SYNDROME IN PATIENTS WTTH ASYMPTOMATTC ADRENAL MASSES.

We have studied 51 patienta with asymptomatic unilateral adrenal masses refered to our department for characterize the mass with adrenocortical scintigraphy (AS). We report 5 patients (all females) aged 55 to 70 years in whom the As showed uptake at the side of the mass without visualization of the contralateral gland. Adrenal masses ranged in size from 2.5 to $5 \mathrm{~cm}$. AS were performed with $75-$ Secholegterol on days. 5 and 7 postinjection.

Al1 patients had a 24 h urinary free cortisol and basal plasma ACTH concentrations in the normal range. plasma cortisol showed an intact circadian rhytm in all cases. Low dose dexamethasone suppression test $(1 \mathrm{mg}$ at $23 \mathrm{~h}$ and cortisol determined at $9 \mathrm{~h}$ ) were performed in two patients and all two performed in two patients and all two
demonstrated normal suppression (serum cortisol under $5 \mathrm{mcg} / \mathrm{dl})$. Three out of five patients have undergone unilateral adrenalectomy. In all cases an adrenal adenoma was found. None of them developed adrenal insufficiency after surgery.

In conclusion, As permits the detection of corticoadrenal adenomas in subclinical setting when the disease is not yef demonstrable by biochemical determinations. 
345

G.Rubini, S.Iliceto*, E.Lauriero, D.Rubini, L.Galiuto*, P.Rizzon*, A.D'Addabbo.

Depts. of Nuclear Medicine, *Cardiovascular Diseases University of Bari- Italy

MYOCARDIAI CONTRAST ECHOCARDIOGRAPHY AND 99MTC-MIBI SPET IN THE DETECTION OF VIABLE MYOCARDIUM

The complete assessment of acute myocardial infarction (AMI) requires the evaluation of regional function and myocardial perfusion. Two dimensional echocardiography (2DE) evaluates myocardial dysfunctions, and during infusion of Low Dose Dobutamine (IDD) recognises hypoakinetic Myocardial Segments (MS) with contractile reserve. Myocardial contrast echocardiography (MCE) with intracoronary injection of a sonicated contrast medium gives information about myocardial microvascular integrity. From intracellular uptake of $99 \mathrm{mTc}-\mathrm{MIBI}$ it is possible to obtain the regional perfusion patterns. To evaluate if there is a 99mTC MIBI uptake in postinfarction dysfunctioning $M S$ with contractile reserve and microvascular integrity, we studied 5 pts with AMI (3 anterior, 1 lateral and 1 posterior). All pts underwent $2 \mathrm{DE}$ monitoring (at day $1,3,5,7,15)$, IDD $(5$ $\mathrm{mcg} / \mathrm{kg} / \mathrm{min}$ for $5 \mathrm{~min}$. and $10 \mathrm{mcg} / \mathrm{kg} / \mathrm{min}$ for a further 10 min.) at day 5, MCE at the time of coronary angiography, with selective intracoronary injection of sonicated Ioxaglate, and 99mTC-MIBI SPET. To analyse the data we used a 16 segment model of the left ventricle. 25 of 80 MS were hypo or akinetic at first 2DE. IDD, MCE and 99mTc-MIBI SPET data are reported:

\begin{tabular}{cccc} 
MS & IDD & MCE & 99mTC-MIBI \\
\hline 14 & improved kinetic & microvascular integrity & uptake \\
8 & not improved & absenoe & notake \\
\hline 2 & improved kinetic & microvascular integrity & no uptake \\
1 & not improved & microvascular integrity & uptake \\
There is good agreement (88\%) among IDD, MCE and rest
\end{tabular}
There is good agreement (88:) among IDD, MCE and rest can be considered a marker of postinfarction myocardial viability.

\section{6}

J.T. Kuikka, J. Hartikainen, E. Vanninen, M. Mäntysaari, E. Länsimies

Departments of Clinical Physiology and Medicine, Ruopio University Hospital, Kuopio, Finland

DETECTION OF HIBERNATING MYOCARDIUM BY COMBINING PERFUSION WITH PPPA AND MIBG ASSESSMENT: A ONE YEAR FOLLOW-UP STUDY

We have previously examined the relation of myocardial perfusion, fatty acid uptake and sympathetic denervation in patients with recent myocardial infarction using three tracers Tc-99m MIBI, I-123 MIBG and I-123 PPPA. Here we report the results of a one year follow-up.

The patient series consists of 12 men, aged less than 65 years who had survived their first MI. Ten of the patients were succeeded to re-study after one year follow-up using these 3 tracers. There were no significant changes between these two examinations in the size of the MIBI-defect (9.4 \pm 2.5 vs $10.4 \pm 3.0$ \%, mean \pm SEM), MIBGdefect $(17.0 \pm 2.8$ vg $17.1 \pm 2.5$ \% $)$ and PPPAdefect $(9.4 \pm 3.0$ vs $8.4 \pm 2.5 \%$, respectively). However, the metabolic reserve was significantly $(p<0.05)$ improved $(11.6 \pm 4.18)$ and the size of viable but denervated myocardium was slightly increased $(5.7 \pm 9.5 \% ; p>0.05)$.

The results suggest that there are no significant changes in the infarct size, in the extent of perfusion defect and no re-innervation of the sympathetic nerve endings in the infarcted region during the one year follow-up. The metabolic reserve in the peri-infarcted region was the only parameter that improved during the follow-up.
347

J. Zehelein, R. Zimmermann, B. Bubeck, M. Eisenhut, W. Kübler, P. Georgi

Departments of Nuclear Medicine and Cardiology, Ruprecht-KarlsUniversity, Heidelberg, Germany

SPECT I-123 PHIPA IMAGING IN PATIENTS WITH CHRONIC CORONARY ARTERY DISEASE: A COMPARATIVE STUDY WITH THALLIUM-201

13-(p-[ $\left.{ }^{123} \mid\right]$ iodophenyl)-3-(p-phenylene) tridecanoic acid (PHIPA) is a radiolabelled synthetic long chain fatty acid with prolonged myocardial retention (biological half life up to 70 hours) due to a trapping mechanism caused by the p-phenylene group in the alkyl chain. The present study examined the ability of myocardial scintigraphy with PHIPA in comparison to thallium-201 for the detection and estimation of coronary artery disease.

In 8 patients with chronic coronary artery disease and stable angina pectoris, $180^{\circ}$ SPECT images were acquired $30 \mathrm{~min}$ after injection of $160 \mathrm{MBq}$ PHIPA at stress. Two to three days later, separate rest studies were performed (identical imaging protocol). The regional activity in each of 32 myocardial segments per patient was compared to that of stress/redistribution thallium201 images obtained within 1 week after the PHIPA studies

The PHIPA stress images showed 16.6 \pm 6.1 abnormal segments per patient (thallium-201 stress: $17.0 \pm 5.1$ segments), and the PHIPA rest images showed $16.8 \pm 6.8$ abnormal segments (thallium-201 rest: $13.2 \pm 8.2$ segments). Of 134 abnormal segments with PHIPA during stress, only $9(6.7 \%)$ showed reversibility on rest images as compared to 37 out of $142 \mathrm{seg}$ ments $(26.0 \%)$ on thallium-201 redistribution images $(p<0.001)$.

The virtually identical number of abnormal myocardial segments obtained with stress thallium-201 and rest PHIPA scintigraphy suggests rest PHIPA imaging as a promising tool for the identification of poststenotic myocardium. With respect to the evaluation of myocardial viability, however, an optimal imaging protocol remains to be established.

\section{8}

P.Y.Marie, P. Olivier, G. Karcher, N. Danchin, M. Angioi, T. Arsena, N. Quiri, N. David, Y. Juillière, F. Cherrier and A. Bertrand.

Dept. of Nuclear Medicine, CHU Nancy, France.

UPTAKE OF [123I]-16-IODO-3-METHYL-HEXADECANOIC ACID (MIHA) IN AREAS WITH IRREVERSIBLE DEFECTS ON EXERCISE SPECT-T1201 WITH REST-REINJECTION

Rest-SPECT with MIHA frequently shows an increased level of uptake in areas with irreversible exercise SPECT-T1201 defects. Such a miss-match pattern between a flow (Tl201) and a metabolic (MIHA) tracer might correspond to ischemic but viable myocardium, but the relationship with quantified level of Tl201 uptake remained to be clarified. Exercise SPECT-T1201 with rest reinjection and rest-SPECT with MIHA were performed in 83 pts with myocardial infarction. Exercise defects areas showing an increased level of uptake (Ex-IU), were determined on MIHA and T1201 redistribution and reinjection scans by a visual comparison with exercise Tl201-scans. Compared with T1201 redistribution and reinjection, MIHA scans allowed a higher rate of detection of Ex-IU, especially of a $\geq$ moderate extent $(\geq 15 \%$ of LV) $(40 \%$ vs $19 \%, \mathrm{p}<.001)$. In areas with visually irreversible defects at TI201-reinjection, extent of EX-IU determined on MIHA was related to the quantified extent of areas with a T1201 uptake $\geq 50 \%$ of normal $(p<.001)$, but the correlation was weak $(R=.5)$ ; areas with $\geq 50 \%$ of T1201 uptake were larger than those with Ex-IU on MIHA in $86 \%$ of pts.

MIHA rest-SPECT frequently shows an increased level of uptake in areas with irreversible defects at T1201-reinjection. Such areas are likely to have a $\geq 50 \%$ of T1201 uptake, but are not accurately identified by SPECT-T1201 data. 


\section{9}

P. Olivier, P.Y. Marie, G. Karcher, N. Danchin, M. Angioi, T. Arsena, N. Quiri, N. David, Y. Juillière, F. Cherrier and A. Bertrand.

Dept. of Nuclear Medicine, CHU Nancy, France.

USEFULNESS OF SERIAL REST-SPECT WITH SESTAMIBI TO ASSESS THERAPEUTIC INTERVENTIONS AT THE ACUTE PHASE OF THROMBOLYZED MYOCARDIAL INFARCTION

Serial rest-SPECT with Sestamibi was used to assess the effects of the administration of a beta-blocking agent (bisoprolol) at the acute phase of MI. Eighteen pts with $<8$ hrs acute MI treated with thrombolysis, were randomly assigned to treatment with bisoprolol or placebo. Sestamibi was injected at the time of thrombolysis and before the IV administration of the study agent, and an initial SPECT acquisition was started 1 to 2 hours later. Treatment was continued orally during a 4 -week period in all but 3 pts ( 1 death (bisoprolol) and 2 heart failure (placebo)). A 4 -week evaluation was performed using radionuclide angiography, and rest and execise SPECT with Sestamibi. No difference was observed, between pts with $(n=8)$ and those without $(n=7)$ bisoprolol treatment, on extents of initial rest SPECT defects and on ald data obtained at 4-week (radionuclide LVEF, extents of exercise and rest SPECT defects). However, the decrease of defects size, between initial and 4-week rest-SPECT, was greater in pts treated by bisoprolol than in placebo pts (\% LV-area: $-14 \pm 8 \%$ vs $-6 \pm 4 \%, p<.02$ )

At the acute phase of thrombolyzed MI, betablocking therapy (bisoprolol) enhances extent of salvaged myocardium. This is evidenced on serial Sestamibi rest-SPECT, which appears to be a very sensitive technique to detect beneficial effects of treatments proposed in thrombolyzed MI.

350

J. Mester, T. Forster, M. Csanády, L. Csernay

Dept. of Nuclear Medicine, 2nd Department of Medicine, Albert Szent-Györgyi Medical University, Szeged, Hungary

SIMULTANEOUS DOBUTAMINE STRESS MYOCARDIAL PERFUSION SPECT AND 2D ECHOCARDIOGRAPHY IN PATIENTS WITH OLD MYOCARDIAL INFARCTION (MI)

The results of dobutamine (DOB) stress scintigraphy and 2D echocardiography were compared in 21 patients (mean age 58 years) with old MI. 9 patients had an inferior, 8 an anteroseptal, 2 an apical and 2 an anteroseptal and inferior resting WMA documented by echocardiography. DOB stress was performed by using a stepwise increasing dosage (5-10-20-30-40-40 micrograms $/ \mathrm{kg} / \mathrm{min}$, each step over $3 \mathrm{~min}$ ). During the test, continuous echocardiographic monitoring was performed. If the age-dependent submaximal heart rate was not attained after the second 40 micrograms $/ \mathrm{kg} / \mathrm{min}$ dose, $0.25 \mathrm{mg} / \mathrm{min}$ of atropine was given over $4 \mathrm{~min} .200 \mathrm{MBg}$ of SESTAMIBI was injected immediately after termination of the DOB or atropine infusion. Myocardial perfusion SPECT studies were performed according to a one-day protocol $(750 \mathrm{MBq}$ SESTAMIBI for the resting study). Echocardiography was evaluated from the standard views. SPECT studies were evaluated by a combined consideration of the short-axis, horizontal and vertical long-axis slices and quantitative polar maps. Improved wall motion (WM) at low dose DOB (viability) was signalized in 6 myocardial regions in 5 patients. In these territories scintigraphy documented stress-induced ischemia in 3 cases, an enhanced defect size at rest in 1 patient, and a persistent perfusion defect in 2 cases. Stress-induced WMA was documented in 8 patients. These ischemic segments were concordantly identified with scintigraphy in 6 patients; myocardial perfusion was normal in 2 patients. On the other hand, scintigraphy documented stress-induced myocardial ischemia in 10 regions of 8 patients, without changes in regional WM during stress. 5 of these regions had resting WMA, but 5 did not.

It is concluded that the results of DOB stress myocardial SPECT and 2D echocardiography are different in nearly half of the patients with old MI, and these diagnostic modalities can not replace each other.

\section{1}

D.Moka, P.Theissen, U.Sechtem*, H.Schicha.

Klinik und Poliklinik für Nuklearmedizin, *Klinik III für Innere Medizin, University of Cologne, Germany.

ASSESSMENT OF ENERGY METABOLISM IN PATIENTS WITH NON-TRANSMURAL MYOCARDIAL INFARCTION BY 31-P-MRS

To assess myocardial viability after nontransmural anterior myocardial infarction (MI), 19 patients with critical LAD-stenosis $(>80 \%)$ and anterior wall hypokinesia (A) and 10 healthy volunteers were examined on a Philips 1.5 Tesla imaging/spectroscopy MR system using a surface coil and Gyroscan/ISIS software. All patients had minimum diastolic wall thickness of $6 \mathrm{~mm}$ as assessed by gradient-echo magnetic resonance imaging. Patient spectra were recorded under optimal antiischemic medication. The cube-shaped volume of interest was placed into the apical-septal area of the myocardium. Spectra were corrected for $\mathrm{T}_{1}$-effects and blood contamination.

To cleminate the possibility that alterations of the spectra were mainly caused by LAD-stenosis, 4 additional patients (B) with critical LADstenosis but normal left ventricular (LV) function were examined following the same protocol.

The effect of glyceroltrinitrate (GTN) was evaluated in 4 further patients (C), who had the same clinical features as the 19 patients mentioned above. MRS was performed without antiischemic medication (washout period >one day) and during intravenous application of GTN.

Mean PCr/ATP-ratio (A) was reduced from $1.74 \pm 0.23$ to $1.24 \pm$ 0.18 ( $\mathrm{p}=0.01$ unpaired t-test) as compared to normal controls. Patients with normal LV function (B) had PCr/ATP-ratios similar to those of normal controls ( $p=0.23$ ). After GTN infusion (C) PCr/ATP-ratio rose from $1.12 \pm 0.08$ to $1.32 \pm 0.13(\mathrm{p}=0.04$ paired $\mathrm{t}$-test $)$

The alterations in myocardial metabolism at rest are likely caused by the wall hypokinesia, because patients with critical LAD-stenosis without anterior wall hypokinesia showed a normal PCr/ATP-ratio. A possible reason could be a degeneration of the myocytes after MI. An other possibility is a chronic cellular ischemia produced by a disturbed microperfusion after MI. This theory is supported by an improvement of the energy metabolism in hypokinetic regions by GTN. To differ whether a reduction of the $\mathrm{PCr} / \mathrm{ATP}$-ratio is really a sign of cellular ischemia in coronary heart disease further investigations are needed.

\section{2}

R.Sara L.Ruffini E.Corrada* S.Pirelli M.Milella F.Banfi F. Spinelli

Nuclear Medicine Department *Cardiology Department "De

Gasperis"

COMPARISON BETWEEN DOBUTAMINE BCHOCARDIOGRAPHY AND 201-TL SPECT IN IDENTIFING VIABLE MYOCARDIUM Aim of the study was to evaluate stress echocardiography with low dose Dobutamine ( $5-10$ gamma $/ \mathrm{kg} / \mathrm{min}$ ) ( DSE ) vs 201-T1 delayed SPECT in identifing myocardial viability. We studied 17 patients ( 15 $\mathrm{m}, 2 \mathrm{~F}$, mean age $53+1-9 \mathrm{yrs}$ ) with multivessel coronary artery disease ( $\mathrm{CAD}$ ), previous infarction and reduced ejection ( EF ) at resting echocardiography $(<30 \%)$. We used a sixteen segments ventricular model for DSE and T1 SPECT. DSE was analysed using a score index ranging from 1 (normokinesis) to 4 (dyskinesis). Bach short axis reconstructed from SPECT raw images was divided into six segments and the mean count was calculated for each one. Segmental $\mathrm{Tl}$ uptake was determinated as a percentage of the maximum among all short axis sections. A segment was considered viable when resting dyssynergy ( score $>2$ ) showed an improvement of one grade or more during Dobutamine infusion and when $\mathrm{Tl}$ uptake was $>50 \%$. The findings of DSE and rest $T I$ imaging were compared in a total of 272 segments, 232 of which were dyssynergyc segments: 150 were akinetic and 82 hypokinetic segments. Rest $\Pi 1$ imaging showed viable myocardium in $93 / 150(62 \%)$ akinetic segments. Of them $76(82 \%)$ showed no improvement at DSE. Of 82 hypokinetic segments 75 (91\%) were seen as viable by $\mathrm{Tl}$ imaging. Of them $43(57 \%)$ showed no improvement at DSE. Thus, concordance between TI SPECT and DSE in identifing viable myocardium was clow: DSE showed wall motion improvement in $49 / 168(29 \%)$ viable segments. Concordance was higher for non viable segments: $58 / 64(91 \%)$. In conclusion, in patients with CAD and reduced EF, DSE if compared with TI SPECT as reference test, showed low sensitivity (29\%), high specificity (91\%) and moderate accuracy (46\%) in detecting viable myocardium. 


\section{3}

W.A. Chalela, J.Soares Jr., J.F.Ramires, A. Bottega, M. Izaki, M.C.P.Giorgi, A.P.Moraes, G.G.Cerri, G.Bellotti, J.C.Meneghetti. The Heart Institute, University of Sao Paulo, Brazil.

MYOCARDIAL PERFUSION PRE AND POST-REVASCULARIZATION OF INFARCTED AREAS : COMPARISON BETWEEN THALIIUM-201 AND TC-99m MIBI SPECT IMAGING. To determine the utility of stress(s)-redistribution(RD), rest(R)-reinjection(RI) TI-201 and S-R MIBI for assessing myocardial viability(MV) before a potential revascularization, we prospectively studied 11 pts(all male; $58.2 \pm$ $12.8 \mathrm{yrs}$ ) with coronary artery disease, ventricular dysfunction and previous myocardial infarction(MI). Coronary angiography showed three-vessel disease in 5 pts, two-vessel disease in 5 and single-vessel disease in 1. A total of 16 territories corresponding to the infarcted regions at EKG were analysed visually: 8 anterior MI, 7 inferior MI and 1 infero-lateral MI. All pts were submitted to bypass surgery including the infarcted region and underwent post-operative R-RI TI-201 and S-R MIBI imaging. MV was detected at pre-operative analysis in $11 / 16(68.75 \%)$ by S-RD Tl-201, 15/16(93.75\%) by R-RI TI-201 and in 10/16(62.5\%) by S-R MIBI imaging. Post-operative R-RI TI-201 and R MIBI demonstrated improvement of the perfusional pattern in $14 / 16(87.5 \%)$ infarcted territories. It is important to emphasize that post-operative $R$ MIBI imaging showed better myocardial uptake even in territories considered non-viable by pre-operative S-R MIBI.According to these data scintigraphy with R-RI Tl-201 more accurately identified viable myocardium. The superiority of R-RI TI-201 over S-R MIBI in the detection of MV may be related to different degrees of metabolic changes in hibernating myocardial.

\section{4}

N. Zafrir, B. Vidne, R. Bassevitch, E. Lubin

Nuclear Cardiology Unit \& Cardiothoracic Surgery, Beilinson Medical Center, Petah Tiqva, Israel

EXTENT OF FUNCTION-PERFUSION MISMATCH - A PREDICTOR FOR EFFICACY OF CORONARY ARTERY BYPASS GRAFTING IN PATIENTS WITH SEVERE LEFT VENTRICULLAR DYSFUNCTION

This prospective study was performed to predict the efficacy of coronary artery bypass grafting (CABG) in patients ( $\mathrm{ptg}$ ) with severe left ventricular dysfunction (SLVD) in terms of increasing global ejection fraction (GEF) 3 months after CABG. The study protocol undertaken within a week before CABG included: 1) simultaneous assessment of function (F) and perfusion (P) at rest using TC-MIBI. The left ventricle was divided into 5 regions (apex, septum, anterior, inferior and posterolateral) and the same scoring system (0-normal to 3 -severe) was employed for $F$ and P. The difference (D) between the F-P scores was calculated in each patient; 2) low-dose Dobutamine (Dob) administration $5-10 \mathrm{kr} / \mathrm{kg} / \mathrm{min}$; 3) Isosorbide dinitrate (Iso) given sublingually $5 \mathrm{mgx} 3$ at $5 \mathrm{~min}$ intervals (end point related to side effects). MUGA study was performed before and during Dob and Iso administration to calculate D GEF. Eighteen pts with GEF $\leq 30 \%$ have so far been studied. These were divided into 2 groups (Gr) according to $D$ GEF before and 3 months after CABG: (Gr1 - D GEF <3\%; Gr2 - D GEF $>3 \%$ ). The mean results of the pre-CABG studies were compared in the 2 Grs:

\begin{tabular}{|c|c|c|c|c|}
\hline & D GEF/CABC & D F-P score & D GEF / DOb & D GEF/Iso \\
\hline r. 1 & $-1.7 \pm 2.4$ & $2.0 \pm 1.1$ & $6.6 \pm 3.4$ & $1.0 \pm 1.5$ \\
\hline 2 & $7.1 \pm 3.2$ & 4. $3 \pm 1.3$ & $8.5 \pm 7.2$ & $0.2 \pm 3.5$ \\
\hline value & 0.001 & 0.0024 & 0.56 & 0.66 \\
\hline
\end{tabular}
$\begin{array}{lllll}P \text { values } & 0.001 & 0.0024 & 0.56 & 0.66\end{array}$ It is concluded that the extent of $F-P$ mismatch may be used as a predictor for efficacy of CABG in pts with SLVD.

\section{5}

B.L.F.van Eck-Smit, E.E.van der Wall, A.H.Zwinderman, E.K.J.Pauwels

Depts. of Diagnostic Radiology and Nuclear Medicine, Cardiology, Medical Statistics, University Hospital Leiden, Netherlands

CONCORDANCE BETWEEN THALLIUM-201 STRESS-IMMEDIATE REINJECTION IMAGING AND REST REDISTRIBUTION IMAGING FOR THE IDENTIFICATION OF VIABLE MYOCARDIUM.

Introduction: The accurate assessment of myocardial viability is of major importance in patients(pts) following myocardial infarction.

Purpose: The aim of our study was to determine whether immediate thallium-201(Tl) reinjection(Ri) imaging provides adequate information regarding myocardial viability. In 23 pts with documented anterior wall infarction results of the immediate Ri procedure were compared with 1)TI rest(Re)-redistribution(Rd) images, and 2lleft ventricular wall motion( $\mathrm{Wm}$ ) obtained with radionuclide angiography(RNA)

Methods: For the Ri procedure $T l(75 \mathrm{MBq})$ was injected at maximal $\mathrm{Ex}$ and reinjected ( $37 \mathrm{MBq}$ ) immediately post $\mathrm{Ex}$ imaging followed by imaging 60 min later. $R e$ imaging was performed on a separate day 60 minutes after injection of $\mathrm{T} 1(75 \mathrm{MBq}$ ). In $13 / 23$ pts additional Rd images were acquired $3 \mathrm{~h}$ after Re imaging On each set of 71 images, 8 segments(segm) were visually and quantitatively analyzed. Ex data were compared with Ri images, Re images and $\mathrm{Rd}$ images. Myocardial segments were classified as viable $(\mathrm{V}+$ (normal, ischemic, non-ischemic viable)\}, and non-viable(V-). Left ventricular $W m$ of the infarct region was studied by RNA performed at rest and during Ex. Regional Wm was classified as normal, hypokinetic or a/dyskinetic. All but persistent a/dyskinetic regions were considered viable.

Results: $\quad \operatorname{Ex}-\operatorname{Re}(\mathrm{n}=184 \mathrm{segm}) \quad$ Ex-Rd (n=104 segm) $W \mathrm{Wm}(\mathrm{n}=23 \mathrm{pts})$

\begin{tabular}{cc|cc|cc|c}
$V+$ & $V+$ & $V-$ & $V-$ & $V+$ & $V-$ \\
Ex-Ri & 15 & 3 & 88 & 0 & 5 & 6 \\
\hline$V-$ & 0 & 26 & 2 & 14 & 1 & 11
\end{tabular}

concordance $181 / 184(\mathrm{~K}=.97) \quad 102 / 104(\mathrm{~K}=.97) \quad$ Sens. $96(78-100)$ Conclusion: Immediate $\mathrm{Tl}$ reinjection imaging 1) provides accurate information on myocardial viability comparable to Re and Rd imaging, and 2) adequately identifies viability in infarct regions. These findings show the value of immediate $T$ reinjection imaging for the detection of myocardial viability in pts following myocardial infarction.

356

M. Ciavolelia, N. Schad, R. Wagner*, E. Kreuzer\#

Institute of Radiology, University of Siena, Italy; *staedt. Krankenhaus, Passau, and \#Klinikum Grosshadern, University of Munich, Germany

123I-PHENYLPENTADECANOIC ACID (IPPA) MYOCARDIAI DYNAMIC METABOLISM IN THE ASSESSMENT OF INFARCTED TISSUE VIABILITY

To evaluate the dynamic pattern of IPPA metabolism in viable vs. non viable tissue on left anterior descending artery infarcted territory (according to coronary angiography and left ventriculography), 23 pts (mean age: $59 \pm 9$ years) with documented previous anterior myocardial infarction underwent 2 dynamic studies ( 1 frame/s) by means of $37 \mathrm{MBq}$ of IPPA i.v. bolus injection and a multicrystal gamma camera, before and 3-5 months after surgery. Dynamic technique allowed processing of parametric images showing different patterns of predominant regional IPPA clearing (i.e. beta oxidation) from 4 to 25 min after injection. Cell viability was represented not only by a steep IPPA wash-out, but also by ongoing IPPA accumulation in cytoplasma, indicating a peculiar metabolic situation, potentially improvable through blood flow restoration. In addition, the analysis of initial (100 s) histograms showed an increase in IPPA first pass uptake by myocytes after revascularization, pointing to an improvement in primary celi functions, such as the uptake of substrates necessary to the later recovery of beta oxidation. At post-operative study, regional IPPA uptake and/or clearing improved in 18 pts (78\%); of the remaining pts, 4 showed an increase of IPPA accumulations. Clinical improvement after surgery was detected in 2 Ipts. 
357

M.Cappagli, * D.Bertoli, \#S.Gramenzi, P.Poggi,

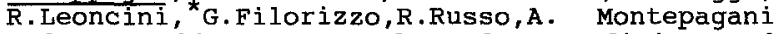
and A. Coli. Depts. of Nuclear Medicine and \#Cardiology, La Spezia and "Dept. of Medicine, Sarzana, Italy.

EFFECTS OF RANDOMIZED TREATMENT WITH NITRATES AND LISINOPRIL IN ACUTE MYOCARDIAL INFARCTION

ON PEAR FILLING RATE AT HOSPITAL DISCHARGE,

The aim of this study was to analyze the effects of randomized (factorial design $2 \times 2$ ) nitroglicerin (N) and lisinopril (L) treatment (started within 24 hours from onset of symptoms) in acute myocardial infarction (MI) on left ventricular (LV) peak filling rate (PFR) evaluated at hospital discharge.

We examined radionuclide angiographic data of 147 pt with a first MI studied in our laboratory as part of a multicenter study elsewhere described (Am J Cardiol 1992;70:62C69C). Age, LV ejection fraction (EF), heart rate (HR) and PFR (end-diastolic counts/sec) of pt submitted to randomized treatments were compared with the control (C) group. Results: Group $n^{\circ}$ Age (y) HR(bpm) EF(\%) PFR

$\begin{array}{llllll}\mathrm{N}+\mathrm{L} & 35 & 62 \pm 11 & 72 \pm 11 & 50 \pm 12 & 2.06 \pm 0.67\end{array}$

$\begin{array}{llllll}\mathrm{L} & 36 & 61 \pm 12 & 63 \pm 10 & 51 \pm 11 & 2.06 \pm 0.48 \\ \mathrm{~N} & 42 & 62 \pm 11 & 65 \pm 10 & 51 \pm 10 & 1.83 \pm 0.48\end{array}$

C $\quad 34 \quad 61 \pm 11 \quad 70 \pm 12 \quad 50 \pm 13 \quad 2.03 \pm 0.48$

No statistically significant differences in $\mathrm{EF}$ and PFR were found between treatment groups and $\mathrm{C}$ group. Nevertheless in $\mathrm{N}$ group PFR was reduced in comparison with $L$ group $(p<0.05)$.

Our data seem to suggest different effects of $\mathrm{N}$ and $\mathrm{L}$ on $\mathrm{LV}$ diastolic function, as evaluated by PFR, probably as a consequence of different actions on preload and afterload.

Analysis on larger sample of patients are required to validate this hypothesis and to study its clinical relevance.

\section{8}

E.S. Tan, J.G. Meeder, H.J.G.M. Crijng, A.C.P. Wiesfeld P.K. Blanksma, J. Pruim, A.T.M. Willemsen, W. Vaalburg, K.I. Lie.

Dept. of Cardiology and PET Center, Univ. Hosp. Groningen, Netherlands.

\section{EVIDENCE OF ISCHPMIA IN PATIENTS WITH VENTRICULAR} FIBRILLATION POSITRON EMISSION TOMOGRAPHY ASSESSMENT OF MYOCARDIAT PERFUSION.

To investigate the potential role of ischemia in ventricular fibrillation (VF) late after myocardial infarction, we compared 6 pts with documented VF late after myocardial infarction (LaVF) and 27 healthy volunteers (HV). All pts and HV underwent quantitative dynamic positron emission tomography (PET) with N-13-ammonia for determination of myocardial perfusion. Patterns of perfusion were assessed in 480 segments. The coefficient per variation (CV) as a measure of ischemia was calculated. of variation (CV) as a measure of ischemia was calculated. the infarct more than 6 month before VF. With conventional studies, such as exercise tests, thallium scan

Perfusion (ml/min/100g)

at rest

$\mathrm{CV}$

after dipyridamole (dip)

ratio (dip/rest)

$\begin{array}{ll}\text { LaVF (6) } & \text { HV (27) } \\ 123 \pm 27 & 96 \pm 5 \\ 24.7 \pm 5.7 & 13.5 \pm 2 \\ 163 \pm 32 & 191 \pm 15 \\ 30.9 \pm 5 & 14.6 \pm 3 \\ 1.36 \pm 0.34 & 1.96 \pm 0.2\end{array}$

Although ischemia was thought to be sufficiently ruled out, the significantly larger CV in LaVF compared to HVs suggests that active ischemia still plays a role in the development of ventricular fibrillation in these patients. This is of importance as it may result in a different selection of therapy. Moreover, it emphasizes the importance of the more accurate assessment of myocardial importance of the
359

M.Cappaqli, *D.Bertoli, \#S.Gramenzi, P.Poggi R.Leoncini, C. De Gaudio, ${ }^{\star}$ G.Filorizzo, R. Russo and A. Coli. Depts. of Nuclear Medicine and \#Cardiology, La Spezia and * Dept. of Medicine, Sarzana, Italy.

EVALUATION OF DIASTOLIC FILLING IN MYOCARDIAL INFARCTION: COMPARISON BETWEEN DISCHARGE AND 6MONTH FOLLOW-UP

In order to evaluate the 6-month behaviour of left ventricular diastolic performance (peak filling rate-PFR-end-diastolic counts/sec) and its relationship with systolic function (ejection fraction-EF) in patients (pt) with acute myocardial infarction (MI), we submitted $107 \mathrm{pt}$ to gated blood pool (GBP) 10 days (D) and 6 months $(6 \mathrm{M})$ after hospital admission for a first MI.

According to GBP data at $D$, pt were divided in 3 groups: A) pt with systolic and diastolic dysfunction $(E F<50 \%$ and PFR<2.5), B) pt with only diastolic disfunction ( $E F \geq 50 \%$ and PFR<2.5), C) pt with preserved systolic and diastolic function ( $E F \geq 50$ and $P F R \geq 2.5$ ). No $p t$ presented only systolic dysfunction. Results:

GROUP $n^{\circ} \quad E F-D \quad E F-6 M \quad$ PFR-D PFR-6M

$\begin{array}{llllll}\text { A } & 47 & 39 \pm 8 & 39 \pm 13 & 1.5 \pm 0.4 & 1.5 \pm 0.5\end{array}$

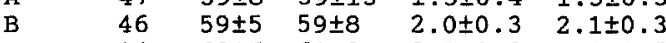

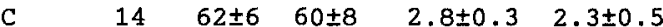

Group $A$ and $B$ did not show any statistically significant difference of diastolic and systolic performances between $D$ and $6 \mathrm{M}$. In group $C$ PFR at $6 \mathrm{M}$ was reduced in comparison with PFR at $D(p<0.01)$.

Post-MI pt with preserved systolic and diastolic phase at $D$ show a trend toward impaired diastolic performance despite no changes of $E F$ at $6 \mathrm{M}$. Reduction of PFR may be a possible signal of incoming ventricular dysfunction.

\section{0}

C.Rossetti, C. Landoni, G. Lucignani, A. Bartorelli, A.Margonato, S.Chierchia, M. Guazzi, F.Fazio. INB-CNR, University of Milan, $H$. Raffaele, centro Cardiologico Fondazione Monzino, Milan Italy.

SPET/TL-201 REST-REDISTRIBUTION AND PET/[F18]EDG PATTERNS IN SEVERE SPET/[TC-99m] MIBI DEFECTS.

To evaluate the suitability of a diagnostic protocol for the assessment of perfusion and viability by the combined use of [Tc-99m]MIBI and Tl-201, twenty-six patients with previous Q-wave MI and chronic stable coronary artery disease underwent the following studies: a) stress and b) rest $\left.360^{\circ} \mathrm{SPET} /[\mathrm{Tc}-99 \mathrm{~m}] \mathrm{MIBI}, \mathrm{c}\right)$ rest-redistribution $180^{\circ} \mathrm{SPET} / \mathrm{T} 1-201$, d) glucose load PET/[F-18]FDG. For each patient 11 myocardial segments were identified on matched tomographic images obtained by the different techniques. The segment with the highest activity on the stress [Tc-99m]MIBI images was selected as the reference region (activity $=100 \%$ ). Segments with $<50 \%$ [TC$99 \mathrm{~m}]$ MIBI activity at rest were considered to be severely hypoperfused $(\mathrm{N}=30)$, and among them the segments with $>50 \%$ [F-18]FDG activity were defined as $[F-18]$ FDG $(+)$. Activity $>50 \%$ of T1-201 redistributed at rest was considered as Ti-201(+).19/30 segments with severe hypoperfusion (63\%) were [F-18]FDG(t), but only $8 / 30(26 \%)$ were $T 1-201(t)$. Among the $8 / 30$ $\mathrm{T} 1-201(t)$ segments, 7 were $[F-18] \mathrm{FDG}(t)$ and 1 was [F-18]FDG(-). In conclusion: although [F18]FDG was present in a higher number of severely hypoperfused segments than Tl-201 rest-redistributed, a first approach to the assessment of both myocardial perfusion and viability can be proposed based on stress-rest [TC-99m]MIBI and TI-201 rest-redistributed. 


\section{1}

G. Cannizzaro,${ }^{\circ} \mathrm{G}$. Calsamiglia, ${ }^{*} \mathrm{M}$. Previtali, ${ }^{*}$ L. Lanzarini, C. Aprile. Service of Nuclear Medicine and ${ }^{\circ}$ Dept. of Cardiology

- Clinica Lavoro Foundation - Montescano - Italy -

*Dept. of Cardiology - Policlinico S. Matteo - Pavia - Italy -.

RECOVERY OF PERFUSION AND FUNCTION AFTER PTCA OF THE INFARCT-RELATED CORONARY ARTERY IN PATIENTS WITH RECENT THROMBOLIZED MI: USEFULNESS OF THALLIUM 201 STRESS-REDISTRIBUTION SCINTIGRAPHY.

In thrombolysed myocardial infarction (MI) akinesia of the infarct area (IA) can be related either to myocardial necrosis or to viable but hypoperfused myocardium that can recover its function after revascularization.

12 pts. (11 males, 1 woman; mean age $56 \pm 6$ years), with recent MI ( 7 anterior, 5 inferior) treated by thrombolysis underwent basal 2D echo (11 segment model; score of the IA from $0=$ normal to $3=$ dyskinesia) and $\mathrm{Tl} 201$ stress-redistribution (ST-RD) ECT (score from $0=$ normal to $3=$ severe reduction or absent $\mathrm{Tl}$ uptake) within 2 weeks from MI. All pts. underwent successful $(<50 \%$ residual stenosis) PTCA of the stenotic or occluded MIrelated coronary artery within 1.5 month from $\mathrm{MI}$ and had a new baseline $2 \mathrm{D}$ echo and T1 201 ECT 2 months after PTCA.

After PTCA 6 pts (GROUP 1) showed functional improvement in $>50 \%$ of basally akinetic sgts., with decrease of wall motion score (WM) of the IA from $5 \pm 2.7$ to $1.8 \pm 1.3$ ( $p<.01$ ), while $6 \mathrm{pts}$. (GROUP 2) hadn't any significant improvement in WM score of the IA (from $6 \pm 1.8$ to $5.3 \pm 1.9 ; \mathrm{ns}$ ).

GROUP 1 GROUP 2 p value

reversible defects before PTCA $16 / 24(67 \%) \quad 6 / 22(27 \%) \quad<.05$ severe irrev.defects before PTCA $3 / 24(12 \%) \quad 9 / 22(41 \%)<.05$ normal sgts after PTCA* ${ }^{*} \quad 9 / 24(37 \%) \quad 3 / 22(14 \%)<.05$ * no normal sgt was found in the IA before PTCA in both groups

In group 1 the ST-Tl score of the IA improved significantly from $8.5 \pm 4.6$ before PTCA to $4 \pm 2.3(p<.05)$ after PTCA, as did the RD score (from 4.6 3.1 to $3.5 \pm 2.1$ ); on the other hand, after PTCA, group 2 pts showed no significant improvement in ST (from $8.2 \pm 3.9$ to $6.3 \pm 3.4$ ) or RD (from $7.1 \pm$ 3.1 to $5.7 \pm 3.6$ ) $\mathrm{Tl}$ score of the IA

In recent thrombolized $\mathrm{MI}$, the recovery of perfusion and function of the akinetic segments in the IA, after PTCA of the MI-related coronary artery, is accurately predicted by the prevalence of reversible ST-RD defects in the IA. No significant improvement can be expected when a high fraction of sever irreversible ST-RD defects is observed. Likely there is no need of reinjection.

\section{2}

G. Calsamiglia,${ }^{*}$ G. Cannizzaro, ${ }^{*}$ C. Aprile, L. Tavazzi

* Service of Nuclear Medicine and Dept. of Cardiology. Clinica Lavoro Foundation -IRCCS - Montescano - Italy -.

THE REINJECTION OF TL 201 IS REALLY USEFULNESS IN THE DETECTION OF RESTOUAL ISCHEMIA AND VIABILITY IN RECENT UNCOMPLICATED THROMBOLIZED MYOCARDIAL INFARCTION ?

The clinical impact of the reinjection (REI) in different groups of patients (pts.) undergoing Tl 201 stress study remains still uncertain. Aim of the study was to assess whether the REI, performed in pts with first myocardial infarction (MI) treated by thrombolysis $(<6 \mathrm{~h})$, gives significant additional information compared with the conventional stress-redistribution (ST-RD) Tl ECT scan.

ST-RD (4h)-REI (injection just after RD, imaging after 30') -Tl ECT was perfomed in 31 patients (pts) $10 \pm 5$ days after a first uncomplicated thrombolized $\mathrm{MI}$ ( median age $56 \pm 7$, echo E.F. $=51 \% \pm 12 \%$ ). The left ventricle was divided into 11 segments (sgts). Tl uptake in each sgt. was scored by consensus of two experienced blinded physicians using a 4 point scoring system ( from $0=$ normal to $3=$ severe reduction of Tl uptake).

\begin{tabular}{|c|c|c|c|}
\hline & normal sgts. & ischemic sgts. $^{\circ}$ & non ischemic sgts. ${ }^{*}$ \\
\hline ST-RD & \multirow{2}{*}{$240 / 341(70.4 \%)$} & $57(16.7 \%)$ & $44(12.9 \%)$ \\
\cline { 1 - 2 } ST-REI & & $67 /(19.6 \%)$ & $34(10.0 \%)$ \\
\hline
\end{tabular}

${ }^{\circ}$ ischemic $=$ sgts. with ST abnormal, RD/REI improved score *non-ischemic $=$ sgts. with ST abnormal. RD/REI unchanged scone

\begin{tabular}{|c|c|c|c|}
\hline & normal & viable & scar \\
\hline ST-RD & $240 / 341(70.4 \%)$ & $68(19.9 \%)$ & $33(9.7 \%)$ \\
\hline ST-REI & & $70(20.5 \%)$ & $31(9.1 \%)$ \\
\hline
\end{tabular}

*viable $=$ sgts with ST aboormal $<3$ and with $\mathrm{ST}=3, \mathrm{RD} / \mathrm{REI}$ improved score patient's analysis:

\begin{tabular}{|c|c|c|c|}
\hline & no ischemia & mild ischemia & marked ischemia \\
\hline ST-RD & 8 & 7 & 16 \\
\hline ST-REI & 5 & 10 & 16 \\
\hline
\end{tabular}

* marked ischemia $=$ score change $\geq 2$ points in at least 2 sgts

Tl ST-RD ECT, performed early after thrombolized uncomplicated MI, identifies residual ischemia and myocardial viability in the infarct related area and/or in other regions; TI ST-REI study seems to give no important additional information, particularly in pts. with marked residual ischemia.
363

J. Kropp ${ }^{1}$, J. Likungu ${ }^{2}$, P.-G. Kirchhoff ${ }^{2}$, H.-J. Biersack ${ }^{3}$.

Depts. of Nuclear Medicine Tech. Univ. of Dresden ${ }^{1}$ and Univ. of Bonn ${ }^{3}$, Cardiovascular Surgery Univ. of Bonn ${ }^{2}$; Germany

ARE THERE DIFFERENCES IN FATTY ACID (FA) UPTAKE AND METABOLISM DUE TO THE KIND OF AORTO-CORONARY BYPASS GRAFT (ACB)? A STUDY WITH [ ${ }^{123}$ 1]-IPPA.

In recent studies we found many segments with persitantly pathologic FA metabolism after revascularization which might be due to the kind of the graft. We investigated 21 patients (pts) before and two months after ACB. 58 grafts revascularized (rev) 41 of 49 $(84 \%)$ territories perfused by a stenosed artery. In the LAD territory revascularization was achieved in $62 \%$ by the internal mammary artery (IMA), in $38 \%$ by vein grafts (SVG) and $34 \%$ of the pts had both, IMA- and SVG-grafts. $200 \mathrm{MBq}$ of IPPA were injected at the end of a submaximal exercise. After exercise SPECT-scintigraphy (ECT-I) was performed which was repeated after 15 min with pts in the same position (ECT-11). Oblique slices were obtained and divided into 5 segments (sgs) which served for quantification. Comparison of sgs of ECT-I before and after therapy served for evaluation of uptake (flow, up) and sgs of ECT-ll for turnover (metabolism, met). In total 126 non-infarcted but ischemic sgs (LAD: 42 rev by IMA; 25 rev by SVG; 23 rev by IMA + SVG) served for the investigation of changes of up and met after therapy.

$\begin{array}{lcccc} & \text { up } \uparrow+\text { met } \uparrow & \text { up } \uparrow+\text { met- } & \text { up- }+ \text { met- } & \text { up } \downarrow+\text { met } \downarrow \\ \text { IMA } & 34 & 48 & 16 & 2 \\ \text { SVG } & 35 & 50 & 9 & 6 \\ \text { IMA + SVG } 51 & 42 & 7 & 0\end{array}$

$\%$ of sgs: $\uparrow=$ improvement; $-=$ abnormal or unchanged, $\downarrow=$ worsened In conclusion, restitution of flow early after reperfusion therapy can be clearly demonstrated by IPPA studies. Many myocardial regions show persitantly abnormal fatty acid metabolism after therapy with no major difference between IMA and SVG grafts. The outcome of the patients in terms of fatty acid uptake and metabolism was best in the case of a combined IMA and SVG therapy.

\section{4}

B.I.F.van Eck-Smit, E.E.van der Wall, A.H.Zwinderman, E.K.J.Pauwels.

Depts, of Diagnostic Radiology and Nuclear Medicine, Cardiology, Medical Statistics, University Hospital Leiden, Netherlands.

CLINICAL VALUE OF IMMEDIATE THALLIUM-201 REINJECTION IMAGING FOR THE DETECTION OF ISCHEMIC HEART DISEASE

Introduction: Immediate thallium-201(T1) reinjection following post exercise(Ex) imaging has been proposed as a novel time-saving approach for the evaluation of patients(pts) with ischemic heart disease.

Purpose: The aim of our study was to establish the clinical value of immediate $\mathrm{Tl}$ reinjection imaging for the detection of the distinct aspects of ischemic heart disease. A total of 138 pts with undiagnosed chest pain and documented coronary anatomy were studied. Fifty-six $(41 \%)$ of the 138 pts had previous myocardial infarction(MI). Of the remaining 82 pts(59\%), 24 pts(17\%) had low likelihood coronary artery disease(CAD).

Methods: Tl (75 MBq) was injected at maximal Ex and reinjected ( $37 \mathrm{MBq}$ ) immediately after completing 3-view planar Ex imaging. Resting images were acquired 60 minutes after reinjection. Each set of Tl images was visually and quantitatively analyzed and compared with arteriographic findings and left ventricular wall motion. Scintigraphic detection of stress induced ischemia was based on T1 Ex data in all 138 pts. detection of reversible ischemia on reversibility of Tl defects in 50 pts with proven CAD and previous MI, and detection of viability on severity of T1 defects in 168 vascular regions of $56 \mathrm{MI}$ pts.

Results:

Stress induced ischemia

$\mathrm{n}=138 \mathrm{pts}^{*}$

sensitivity accuracy

Reversible ischemia

$\mathrm{n}=50 \mathrm{pts}$

Viability

Normalcy rate bas

Numbers between brackets denote the associat $95 \%$ confidence limits

Conclusion: Immediate Tl reinjection imaging shows adequate diagnostic accuracy for the assessment of stress induced ischemia, reverstble ischemia, and myocardial viability in pts with ischemic heart disease. These findings underscore the potential of immediate TI reinjection imaging for use in routine clinical practice. 


\section{5}

J. Stollfuß, G. Glatting, M. Kochs*, R. Weller, M.M. Henrich, V. Hombach*, S.N. Reske

Department of Radiology III and *Internal Medicine II, University UIm, Germany

DETERMINATION OF Rb-81/82 (Rb) BLOOD FLOW/Rb EXTRACTION FRACTION CHARACTERISTICS IN NORMAL HUMAN MYOCARDIUM

Non-invasive determination of myocardial blood flow (MBF) with Rb-PET requires knowledge of the

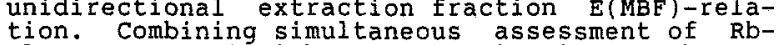
clearance (ExF) with MBF-determination by the argon inert gas-method allowed us for the first time determination of the previously in humans unknown $\mathrm{E}(\mathrm{MBF})-\mathrm{rel}$ ation.

Therefore Rb-clearance (ExF) was determined with a Siemens ECAT-931-08-12 PET scanner. MBF was measured simultaneously with the argon inert gasmethod by coronary sinus sampling of argon desaturation in 5 patients without indication of coronary artery disease. Mean age was $60.6 \pm 7.4$ years ( 2 female and 3 male patients). Measurements were done at rest and during dipyridamol $(0.7 \mathrm{ml} / \mathrm{mg} / 4 \mathrm{~min})$ induced hyperemia. Mean Rb-ExF and argon flow values were $0.33 \pm 0.03 \mathrm{ml} / \mathrm{g} / \mathrm{min}$ and $0.69+0.15 \mathrm{ml} / \mathrm{g} / \mathrm{min}$ at rest and $0.69 \pm 0.15 \mathrm{ml} / \mathrm{g} / \mathrm{min}$ at rest, respectively, and $0.42 \pm 0.06 \mathrm{ml} / \mathrm{g} / \mathrm{min}$ and $1.46 \pm 0.23 \mathrm{ml} / \mathrm{g}$ was derived by dividing Rb-clearance by argon flow values.

A fit to the theoretical dependence of the extraction fraction on flow for a two compartment model yields

$$
E=P S /(P S+F), \quad P S=(0.90 \pm 0.09) \mathrm{ml} / \mathrm{g} / \mathrm{min} \text {. }
$$

We conclude that the simultaneous measurement of $\mathrm{Rb}-\mathrm{clearance}$ and argon flow is a powerful tool for the assessment of the Rb-extraction fraction in dependence on flow in normal human myocardium.

\section{6}

G. Cantinho, A. Pereirinha, A.I. Santos, M. Fiúza, L. Oliveira, H. Chester, E. Dias, M.G. Lopes, F. Godinho

Instituto de Medicina Nuclear and UCIM/Med IV, LA3 CCUL - Hospital de Santa Maria

Lisboa - Portugal

Tc 99m-SESTAMIBI VERSUS ECHOCARDIOGRAPHY DURING DOBUTAMINE STRESS IN THE DETECTION OF MYOCARDIAL ISCHAEMIA

In order to compare stress echocardiography (Echo) with perfusion $99 \mathrm{~m}$ Tc-Sestamibi (MIBI), we have used stress dobutamine to study 110 patients (pts). However, 23 pts were excluded due to inconclusive stress test. The remaining 87 pts, 74 males and 13 females, <age $>=57.4 \pm 10$ years, 56 pts with previous myocardial infarction and 31 with angor. 37 pts $(43 \%)$ had coronariography with significant lesions. Left ventricle ejection fraction by equilibrium angiocardiography was in the anterior infarction = $32.7 \pm 12.7 \%$, in the inferior $=51.2 \pm 8.3 \%$ and in pts witbout infarction $=53.4 \pm 9.5 \%$. Pts were infused with dobutamine until maximal dose of $40 \mathrm{mg} / \mathrm{kg} / \mathrm{min}$ or a new regional wall motion abnormality (RWMA) was detected by Echo or if standard clinical criteria appeared for stopping infusion.

No major side effects were referred. However, in $12 / 23$ pts out of the study, the infusion was stopped due to increased BP in 8 and SVE in the others. During the infusion Echo study was performed and $15 \mathrm{mCi}$ of MIBI was administered at test end, with infusion continuing one more minute. SPECT was performed 1 bour later and rest SPECT at 48 hours. MIBI and Echo were analyzed qualitatively, considering 12 segments of left ventricie. We have considered normal segments and two lesions types, namely necrosis $=$ irreversible MIBI lesions = stress and rest Echo RWMA and ischaemic $=$ reversible MIBI lesions $=$ stress Echo RWMA.

Of a total of $1044(12 \times 87)$ segments analyzed, $813(77.9 \%)$ had the same classification with MIBI and Echo. The most discordant wall was the anterior $(\mathbf{7 2 . 5 \pm 1 6 . 8 \% )}$ and the most concordant studies were in the patients without previous infarction $(86 \pm 14 \%)$. If we consider myocardial ischaemia, we found only $28 \%$ of concordance in a total of 47 pts with ischaemia in at least one of the two methods.

Dobutamine stress echocardiography seems to offer an alternative to $99 \mathrm{~m}$ Tc-Sestamibi, in the identification of $\mathrm{CAD}$ with a $78 \%$ concordance. Our results show, however, a poor match in the detection of ischaemic myocardium, which is the main purpose of the method ( $28 \%$ of concordance). No statistical differences were found related with the infarction localization. However, in pts without previous myocardial infarction we have obtained better results, perhaps because it is easier the valorization of RWMA, when no rest alteration exists, which may account for the principal indication for stress Echo.
367

J.F. Verzijlbergen, A.H. Zwinderman, E.E. van der Wall, C.A.P.I. Ascoop, E.K.J. Pauwels.

Depts. of Nuclear Medicine and Cardiology, st. Antonius Hospital, Nieuwegein and Academic Hospital, Leiden, The Netherlands.

NO INCREMENTAL DIAGNOSTIC VALUE OF PLANAR TCSESTAMIBI MYOCARDIAL PERFUSION IMAGING AND LEFT VENTRICULAR WALL MOTION STUDIES COMPARED TO TL-201 IMAGING.

Logistic regression analysis was used to determine whether the addition of TC-SESTAMIBI myocardial imaging- and left ventricular function parameters or diastolic images could improve the accuracy of $\mathrm{Tl}-201$ scintigraphy to predict the presence and extent of CAD in 60 consecutive pts. Univariate logistic regression analysis demonstrated that all Tc-SESTAMIBI visual and quantitative perfusion parameters, gated wall motion studies, diastolic images and $\mathrm{Tl}-201$ perfusion parameters were significantly related to the probabilities of CAD, multivessel disease and 3-vessel disease.

Using the multiple regression model the 'optimal' combination was stepwise selected. For $\mathrm{Tl}-201$, being the visual score of the stress images and quantitative score of redistribution. For TC-SESTAMIBI, only the visual score of the stress images.

only small and statistically no significant differences with respect to predictive values were seen. Whatever combination of variables of the two protocols were considered, no significant difference with respect to predictive value was found compared to the simple ('optimal') TL-201/TC-SESTAMIBI protocols.

\section{8}

B. Szzumilak, L.M. Brodzki, A. Teresińska, E. Gosiewska - Marcinkowska, S. Konieczna

National Institute of Cardiology, Warsaw, Poland

DIPYRIDAMOLE TC-99M-MIBI MYOCARDIAL SPECT: COMPARISON BETWEEN HIGH AND LOW DOSE.

The aim of the study was to establish if the high dose dip SPECT brings more diagnostic informations than low dose dip SPECT.

32 patients (pts) with coronary artery disease without infarct were examined. Low-dose dip $(0,56 \mathrm{mg} / \mathrm{kg})$ was administered i.v. to $18 \mathrm{pts}$ (group l); high-dose dip $(0,7 \mathrm{mg} / \mathrm{kg})$ was administered i.v. to $14 \mathrm{pts}$ (group 11 .) Administration of dip was connected with minimal exercise. The both groups of pts were matched in age, resting heart rate and systolic blood pressure. All pts underwent two $99 \mathrm{~m}-\mathrm{Tc}-\mathrm{M} \mid \mathrm{BI}$ SPECT studies performed 15 days apart, one after dip administration and another one after submaximal bicycle stress ( $85 \%$ age-predicted heart rate).

Agreement in defects size, their type and localization between dip SPECT and stress SPECT was observed in 17 of 18 pts (group I) and in 13 of 14 pts (group II).

The following side - effects were observed: ST segment depression in group $1-2$ pts, in group || -3 pts; chest pain in group I - 2 pts, in group II -4 pts. Headache, dyspnoe, dizziness and nausea in group I - 3 pts, in group II -7 pts.

1. High dose dip SPECT do not bring more diagnostic informations than low dose dip SPECT.

2. High dose dip caused side effects in the greater number of pts. 
369

G. Romanowicz, P.Lass, K.Gockowski, J.M.Słomiński

Dept. of Nuclear Medicine, Univ. Medical School

Gdańsk, Poland

HEART PERFUSTON DEFECTS IN WOMEN WTTH OISSEMINATED SYSTEMIC LUPUS ASSESSED BY HEART PERFUSION SCINTIGRAPHY Heart involvement is relatively frequent in the course of systemic disseminated lupus (LED).

Aims: assessment of heart perfusion disturbances in $L E D$ by means of heart perfusion scintigraphy with $7699^{\text {h }}$ MIBI. The test was performed in 25 women with established diagnosis of LED ( 4 - 9 criterions of ARA), 12 with positive and 13 with negative exercise test. Both subgroups were comparable in aspects of age (mean 40.1 $+/-4.7$ years) and duration of disease ( 4.9 years). Heart perfusion scintigraphy was performed with the use of TC99-MIBI with the use of gamma-camera DC4 (Picker) The investigations were performed in the LAO45, ANT, LLAT projections in a standard protocol of exercise and rest.

Segmental perfusion defects were seen in 80 of patients, mostly localised in anterior and posterolateral heart wall. Those changes were mostly reversi-

ble at rest. Perfusion defects were seen in $9 / 13$ ( 69

t) and in $11 / 12$ (92 *) patients with positive exercise test.

Conclusions: Subclinical perfusion defects are frequent in LED, also in patients without open clinical manifestations of ischemic heart disease. EKG exercise test may not be sensitive enough in cardiological diagnostics and negative result does not exclude cardiac perfusion defects. Cardiac perfusion scintigraphy may prove superior to other tests in diagnosing patients with LED, because of possibly different pathogenetic mechanisms involved, similar to those of silent myocardial ischemia in diabetes.

\section{0}

K. Blaszyk, A. Baszko, R. Czepczyniski, A. Eukaszewski

A. Ciesliński, M. Gembicki, J. Sowinski

Ith Department of Cardiology and Clinic of Endocrinology, Academy of Medicine, Poznan, Poland

MYOCARDIAL PERFUSION IN PATIENTS WITH ANGINA PECTORIS AND NORMAL CORONARY ARTERIES ASSESSED WITH Tc-99m-MIBI SPECT.

Myocardial ischaemia and anginal pain (ap) in patients (pts) with normal coronary arteries and no other heart abnormality is supposed to be due to limited flow reserve of small vessels. Perfusion defects on planar thallium scans are frequently reported in this group of patients although the limitation of the method obscure their interpretation. Single photon emission computed tomography (SPECT) has improved the nuclear technique to diagnose and localize perfusion defects.

17 pts (age \pm sd: $42.6 \pm 8.0 ; \mathrm{F} / \mathrm{M}:$ 9/8) with ap, positive exercise stress test, normal coronary arteriography and no other heart abnormality were included. Myocardial perfusion was examined with Tc-99m-MIBI SPECT technique (gammacamera DIACAM, SIEMENS). Perfusion was assessed in 9 segments using semiquantitive scale.

Abnormal perfusion was detected in 12 pts (70\%). Of the overall 153 segments, there was fixed defect in 10 , reversible in 8 , and paradoxal deterioration of rest perfusion in 25 segments. The defects were localized mainly in the territory left anterior descending artery (33 segments) and right coronary artery ( 9 segments).

\begin{tabular}{lccc}
$\quad$ & \multicolumn{2}{c}{ DEFECT } \\
WALL & REVERSIBLE & FIXED & REST \\
ANT+APEX & 4 & 9 & 9 \\
SEPTUM & 2 & 0 & 9 \\
INFERIOR & 2 & 1 & 6 \\
LATERAL & 0 & 0 & 1
\end{tabular}

There is a high proportion of perfusion defects on SPECT scan in patients with microvascular angina with no consistent pattern. There is a numerous group of patients with paradoxal perfusion deterioration at rest mainly in the territory of the left anterior descending artery.
371

Li S.R.' Q.Yang, D.Gludovacs, F.Rauscha, Departments of Nuclear Medicine, Cardiology and physiology, University of Vienna, Austria

MODIfIEd LDL DEcraAses THE BINDING OF PROstaglandin $\mathrm{E}_{2}$ AND $\mathrm{I}_{2} / \mathrm{E}_{1}$ ONTO MONOCYTES IN PATIENTS WITH PERIPHERAI VASCULAR DISEASE

Recent data suggest that various prostaglandins (PGs) play an important role in the development of atherosclerotic lesions. Monocytes have been implemented in early atherogenesis because they express receptors that take up modified LDL. In this study we investigated and compared the binding of ${ }^{3} \mathrm{H}-\mathrm{PGE}_{2},{ }^{3} \mathrm{H}-\mathrm{PGE}$, and ${ }^{3} \mathrm{H}-\mathrm{PGI}_{2}$ onto intact blood monocytes isolated from 10 male patients with ischaemic peripheral vascular disease stage II with the results obtained in 8 volunteers. A single class of high affinity binding sites was identified for $\mathrm{PGE}_{2}$ and two classes of binding sites for ${ }^{3} \mathrm{H}-\mathrm{PGE}$ as well as for ${ }^{3} \mathrm{H}-\mathrm{PGI}_{2}$. In patients, the binding capacities were decreased for either ligand indicating an absence of high affinity binding sites. Ex vivo, oxydized or acetylated LDL decreased ( $p<0.1-0,001$ ) binding of PGs onto monocytes ( $\mathrm{PGE}_{2}-60 \%, \mathrm{PGE}_{1}-40 \%, \mathrm{PGI}_{2}-30 \%$ ) as we11, whereas VLDL, LDL, and HDL had no constant effect on PG-binding onto monocytes. These results suggest a direct effect of modified LDI on PG-binding onto monocytes. We conclude that the presence of monocytes in the arterial wall, the complex interactions with $P G s$ and lipoproteins may be important during atherogenesis.

\section{2}

A.Teresińska, L.M.Brodzki, B.Szumilak, E.Gosiewska-Marcinkowska, Z.Juraszyński, Z.Ślipko, A.Dębski

National Institute of Cardiology, Warsaw

QUANTITATIVE Tc-99m-MIBI SPECT PERFUSION ASSESSMENT BEFORE AND AFTER INVASIVE CARDIAC REVASCULARISATION

The aim of the work is to establish if the quantitative (QUANT) results of LV perfusion studies performed before revascularisation (PRErev) allow to predict the perfusion after revascularisation (POSTrev). Thirty five pts were studied PRErev and POSTrev with Tc-99m-MIBI SPECT (according to 2-days protocol) in stress (S) and rest (R). CABG was performed in $23 \mathrm{pts}$, PTCA in $10 \mathrm{pts}$; 2 pts died before planned CABG. The study assessment was performed qualitatively (visual evaluation of LV sections and BULL'S-EYE maps) and quantitatively (normative evaluation of BULL'S-EYE maps). The results of the QUANT evaluation were 4 numbers: the area of the perfusion defects in $S$ and $R$ (As and $A r$ ) and the perfusion level within the defects in $S$ and $R$ as a fraction of the normal perfusion level (Is and Ir). The perfusion defect was defined as $\mathrm{DEFs}=\mathrm{As}$ (1-Is) in $\mathrm{S}$ and as $\mathrm{DEFr}=\mathrm{Ar}(1-\mathrm{Ir})$ in $\mathrm{R}$. Also $\mathrm{S}-\mathrm{R}$ perfusion improvement was calculated as DEFr-s=DEFr-DEFs. Linear correlation was investigated between DEFs, DEFr and DEFr-s PRErev and POSTrev. Results: 1) The QUANT results were rejected in 13 pts because of inaccuracy of the BULL'SEYE technique in cases of completely unperfused apex or because of falsepositive results in cases of very short but normally perfused septum or because of false-negative results in cases of long underperfused septum. 2) In 2 patients who died before revascularisation, DEFs and DEFr achieved the highest values of all the 22 pts evaluated quantitatively PRErev. 3) In 20 pts left, the correlation was statistically significant $(\mathrm{p}<0.05)$ for: DEFr PRErev vs DEFr POSTrev ( $1=0.87)$, DEFs PRErev vs DEFr POSTrev $(=0.82)$, DEFs PRErev vs DEFs POSTrev ( $r=0.78$ ), DEFr PRErev vs DEFs POSTrev $(r=0.78)$. There was no correlation between DEFr-s PRErev and any of POSTrev parameters. Conclusions: 1) The results of QUANT assessment should be neglected in high percentage of pts ( $37 \%$ in present study) because of limitations of BULL'SEYE technique. 2) In pts with reliable results of QUANT assessment, the preliminary comparison shows significant correlation between perfusion defects in PRErev and POSTrev studies performed with Tc-99m-MIBI SPECT. 
373

E.Özalp, N.ö. Küçük, E. Altınyay, K.M. Kır, G. Aras, G. Erbay

Ankara Universty Medical School Dept. of Nuclear Medicine, 06100 ANKARA/TÜRKIYE

\section{EVALUATION HYPOPERFUSION AT LATERAL WALL IN PATIENTS WITH HYPERTENSION AND NORMAL} CORONARIES

The relative hypoperfusion at lateral wall due to septal hypetrophy on the myocardial perfusion scans(MPS) have been previously reported in patients (pts) with hypertension. In our study, we aimed to investigate the frequency and level of lateral hypoactivity on MPS in hypertensive(HT) pts either with septal hypertrophy or not. $\mathrm{Tl}^{201}$ SPECT studies were performed in $33 \mathrm{HT}$ pts and 27 normotensive(NT) pts who have normal coronary angiograms. The images were evaluated by quantitative method(Bull's eye) and lateral to septal(L/S) count ratios. Bull's eye demonstrated fixed perfusion defect in $11 \mathrm{HT}$ pts that 6 of them showed septal hypertrophy on echocardiographic imaging. L/s ratios were found as in following table:

$\begin{array}{lll}\text { HT pts }(33) & \frac{\text { Stress }}{1.02 \pm 0.09} & \frac{\text { Rest }}{1.01} \pm 0.08(p<0.01) \\ \text { NT pts }(27) & 1.11 \pm 0.09 & 1.09 \pm 0.08(p<0.01)\end{array}$

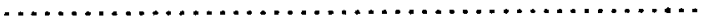

Pto(11) with

Bull's eye defect $0.976 \pm 0.08 \quad 0.960 \pm 0.074$

The ratios of $\mathrm{L} / \mathrm{S}$ in HT pts were found lower than NT pts as statistically significant.

It was concluded that the lateral fixed defects in HT pts which may mimick myocardial infarction on MPS have to be kept in mind during evaluation of MPS. On the other hand, fixed defect at lateral wall may be seen in HT pts without septal hypertrophy and we think that more studies are necessary to explain the causes of the lateral wall defetcts in HT pts.

\section{4}

S. INANIR, M. ÜNLÜ, C. ALATAŞ, B. OKUDAN, H. ALKIM

Gazi University Medical Faculty, Department of Nuclear Medicine. Ankara / TURKEY.

\section{COMPARISON OF SCINTIGRAPHIC PATTERNS AND} CLINICAL STAGING IN DIABETIC FOOT ULCERS

The aim of the study was to search for an objective criterion of healing diabetic foot ulcer, using intraarterial, simultaneous injection technique of Tc-99m MAA (MAA) and $\mathrm{Xe}-133$ (Xe) to identify perfusion and venous capillary blood-flow of lesion side. 12 patients (pts.) with risk toot and or diabetic foot ulcer who had clinical diagnosis of grade (G) 0 to 4 ,were included, after getting their informed consent. All pts. received intra-arterial administration of $1 \mathrm{mCi}$ of MAA (particule size:10$60 \mathrm{um}$ and max. particule amount : $\left.5 \times 10^{4}\right)$ and $\mathrm{Xe}(0.5 \mathrm{mCl})$ simultaneously. No complications or side effects were observed. A two - step dynamic acquisition protocole was applied in dual isotope energy window (1 sec. acq. time for 150 frames and $60 \mathrm{sec}$. for 10 frames). All images recorded in $64 \times 64$ word matrices. Whole-body (WB) images were also obtained within an hour after tracer administration. U/cer zone, surrounding hyperactive area and gangreneous tissue were evaluated as considering MAA uptake of normal $(\mathrm{N})$, hot $(\mathrm{H})$, cold $(\mathrm{C})$, and Xe washout curve patterns of normal $(\mathrm{N})$ fast $(\mathrm{F})$, moderate $(\mathrm{M})$ or poor $(\mathrm{P})$. In , G- 0 (1 pt.) Xe and MAA uptake pattern was normal. In 6 pts. with $G-1$ disease MAA uptak was in $\mathrm{H}$ pattern and 5 of them had $\mathrm{F}$ Xe washout, a case with $\mathrm{N} X e$ washout pattern was found to have two focii in healing process. In a case with $G$ - 2 disease diffusely $H$ MAA uptake and $M X e$ washout was observed due to the serious infection. In a G -3 pt, same uptake patter of MAA and $F$ washout of $X e$ was detected. In 3 cases who had G- 4 disease $H$ / $C$ MAA uptake pattern and F / $P$ Xe washout were noted. In WB images pulmonary uptake of MAA was also searched as an indicator of arterio-venous (A-V) shuntings. Except for a pt. with $G$. 0 disease, in all cases $A-V$ shunts were detected in a pt. With G- Shunt ratios were quantified as $22.8 \pm 14.1$ and $10.3 \pm$ 6.7 in disease side and intact zone, respectively $(p<.025)$. in conclusion, $H$ MAA uptake pattern and $N$ or $F$ washout of $X e$ indicate healing potential of diabetic ulcers. $M$ or $P$ Xe washout curves and mixed uptake pattern of MAA $(H / C)$ indicate serious infection and gangreneous nature of the lesions. Combined use of MAA uptake and $X e$ washout gives an objective criterion to evaluate healing potential of diabetic foot ulcers.
375

WS Richter, D Calder, R Jochens, M Cordes, R Felix Strahlenklinik, Universitätsklinikum Rudolf Virchow, Freie Universität Berlin, Germany

\section{MYOCARDIAL KINETICS OF TC-99m-SESTAMIBI DURING THE FIRST 120 MIN AFTER INJECTION}

Aim of this study was to examine if myocardial activity of Tc$99 \mathrm{~m}$-sestamibi is stable after injection. Sestamibi myocardial scintigraphy was performed in 19 consecutive patients with angiographically proven coronary artery disease (2-day-protocol; $2 \times 300 \mathrm{MBq}$ ). After submaximal bicycle exercise, SPECT was performed $5 \mathrm{~min}$ (early) and 2 hours (late) after injection. The rest study consisted of one scintigraphic acquisition about $100 \mathrm{~min}$ p.i. The left ventricular myocardium was divided into six segments and sestamibi uptake was scored semiquantitatively by 2 experienced observers using a 5-point grading system.

In all studies a sufficient image quality was achieved for interpretation of slices. However, image quality was affected by hepatic activity in early SPECT in some patients. A total of 114 segments was analysed. In 69/114 segments initial defects were detectable. In 72/114 segments no difference in score could be registered between early and late SPECT. In 35/114 segments the score improved within the first $120 \mathrm{~min}$ p.i. (fill-in of initial defects). The early fill-in could be correlated with a significant narrowing of the corresponding coronary artery in most cases. In $7 / 114$ segments a deterioration in score from early to late SPECT was documented (tracer wash-out); in 6 of these 7 segments the score returned to its initial value $(n=2)$ or improved $(n=4)$ in the rest scan. In 1 segment sestamibi was washed-out between early and late SPECT and tracer uptake remained low in the rest study. A clear correlation between tracer wash-out and coronary anatomy could not be detected.

Myocardial Tc-99m-sestamibi activity is not stable after injection: Early tracer washout can be documented as well as a fill-in of initial defects. SPECT should be performed immediately after exercise; later acquisitions may be performed if image quality is poor.

\section{6}

M.Camerani*, R. Bettini, S.Severi*, L.Visonà, E.Lorenzi*, L. Gramegna, F. Furlanello, F. Dalla Palma*

Dep. of Radiology and Nuclear Medicine* - Dep. of Cardiology S. Chiara Hospital - Trento - Italy

123I-MIBG scan: a new approach to silent arrhythmogenic cardiomyopathy. Preliminary report.

The Silent Arrhythmogenic Cardiomyopathy (SAC) includes an inhomogeneous spectrum of life-threatening arrhythmogenic cardiac diseases without valuable functional impairment. In order to establish the efficacy of 123I-MIBG scan as diagnostic marker in SAC 18 patients (18 males - 0 females, aged $18-45 \mathrm{yrs}$ ), with high physical performances, "clinically normal" heart, and hypercinetic ventricular arrhythmias (9/18 with sustained ventricular tachycardia a/o ventricular fibrillation, 2 of them resuscitated) were studied and compared with a control group of 5 subjects.In all of them we performed: a) a complete cardiological study comprehensive of echocardiography $M$ and $2 D$ mode; b) tomographic scan 4 hours after injection of $370 \mathrm{MBq}$ of $123 \mathrm{I}-$ MIBG and 1 hour after injection of $740 \mathrm{MBq}$ of $99 \mathrm{mTC}-\mathrm{MIBI}$ with tomographic camera Orbiter Siemens, HR collimator,64 matrix, 64 views, $180^{\circ}$ rotation. Both data were reconstructed by filtered backprojection (SLH, cut off: 0.5 MIBG, 0.8 MIBI).The Cardiac Magnetic Resonance was also performed in $9 / 18$ and the Coronarography in 7/18. All the investigations were normal in the control group; in the other patients the cardiological study detected a cardiomyopathy in 12/18 (6 Right Ventricular Arrhythmogenic Disease, 4 Biventricular Cardiomyopathy, 1 Left Ventricular Dilated Cardiomyopathy, 1 Coronary Artery Disease [CAD] $) .11 / 18$ patients showed focal defects with MIBG: 6 in the apex, 2 in the anterior wall, 3 in the lateral, 4 in the septal and 7 in the inferior. With MMI we observed defects in 4 patients: 2 defects in the anterior wall, 3 in the lateral and 2 in the inferior.In all cases, except one (CAD), there is a mismatch between MIBG and MIBI defects Versus SAC the MIBG scan has demostred: Sensitivity $83.3 \%$, Specificity $83.3 \%$, Predictive Positive Value 90.9 $\%$, Predictive Negative Value $71.4 \%$, Accuracy $83.3 \%$. These preliminary findings suggest that the MIBG may be a good marker to evaluate defects of the cardiac adrenergic innervation in SAC and that it may play an important role in the difficult detection of these cardiac diseases. 


\section{7}

M.ÜNLÜ, S.INANIR, S. GÜNAYDIN, M.ALKAN, L.GÖKGÖZ

Gazi University, Medical Faculty, Departments of Nuclear Medicine, Cardiology and Cardiovascular Surgery, Ankara - TURKEY.

COMPARISON OF I-123 MIBG SYMPATHETIC CARDIAC INNERVATION IN DIABETIC AND NONDIABETIC CORONARY ARTERY DISEASE PATIENTS. The aim of the study was to compare adrenergic innervation and myocardial perfusion in diabetic and non-diabetic patients who had serious coronary artery stenosis. I-123 MIBG, a guanethidine analague was used for scintigraphic assesment of myocardial symphatetic innervation. A total of 19 coronary artery disease (CAD) patients who had documented multivessel disease in coronary angiography, 8 diabetics (D) and 11 non-diabetics (ND) were included, prior to by-pass surgery. After a $30 \mathrm{~min}$. resting period, all patients received $110 \mathrm{Mbq}$ of I-123 MIBG. SPECT data and planar views were obtained starting at 15 minutes and 4 hours after the injection. In all patients, rest - Tl-201 SPECT study was also performed within two days to asses myocardial perfusion and viability. Heart to mediastinum (H/M) I-123 MIBG activity ratios in early and delayed views were quantified.. Tl-201 and I-123 MIBG SPECT data were compared in respect of defect size, in tomographic planes and polar maps. In D patients globally poor cardiac uptake of I123 MIBG was observed. In all patients segmentally larger denervative areas in disease sites were identified when compared to $\mathrm{Tl}-201$ perfusion defects. A statistically significant difference in $\mathrm{H} / \mathrm{M}$ ratios of I-123 MIBG uptake was detected between D and ND patients in early and delayed views; D: $1.72 \pm .11$, ND: $2.13 \pm .22$, D: $1.51 \pm .24, \mathrm{ND}: 2.30 \pm .15, \mathrm{P}<.005$. However, it has not reached a statistically difference, a slight increase in I-123 MIBG uptake in ND patients and a decrease in D patients was noted in delayed views.

We conclude that, D CAD patients had significantly poor sympathetic myocardial innervation when compared to ND's without any relation with the severity of CAD. Further studies are necessary to determine the clinical importance of viable but denervated myocardium in $D$ patients in respect of prognosis after coronary by-pass surgery.

\section{8}

H. Valette, B. Mazière, C. Loc'h, C. Fuseau, A. Syrota. Physiologie CHU Bicêtre and SHFJ-DRIPP-CEA, Orsay, France.

EFFECT OF METOPROLOL ON MYOCARDIAL UPTAKE OF 76Br-METABROMOBENZYLGUANIDINE (MBBG).

Beta-adrenergic antagonists are increasingly used for the treatment of congestive heart failure. Nevertheless, the effects of these drugs on myocardial presynaptic sympathetic uptake-storage function are not well known. Therefore, the effects of the $\beta 1$-antagonist metoprolol on the presynaptic neuronal uptake of MBBG, a norepinephrine analog, were studied in normal rabbits. This species was chosen because previous study has shown that the rabbit heart, like the human heart, has little capacity for non-neuronal uptake of MIBG, and consequently appears to be a more suitable model than rat to study neuronal uptake. Sixteen rabbits (mean weight: $2.5 \mathrm{~kg}$ ) were studied: $8 \mathrm{control}$ and 8 pre-treated with metoprolol $(0.1 \mathrm{mmol} / \mathrm{kg}$ ip twice a day for one week). Initial myocardial uptake of $\mathrm{MBBG}(6 \mathrm{MBq})$ was measured 30 min after injection of the tracer. Rabbits were killed with an overdose of pentobarbital, their heart quickly removed, dissected and the radioactivity counted. Results (mean value \pm sd in \% kg dose/g; left (LV) and right (RV) ventricles): LV

Control (C)

$\begin{array}{lcc}\text { Metoprolol (M) } & 5.60 \pm 0.74 & 4.42 \pm 0.75 \\ & 3.94 \pm 1.27 & 3.09 \pm 1.00\end{array}$

$\mathrm{p}$ (t-test) $\quad 0.006 \quad 0.01$

Plasma Norepinephrine (NE, pg/ml) remained unchanged and epinephrine (E) remained unchanged: $\mathrm{NE}-\mathrm{C}=245+55$, NE-M= $231 \pm 45 \mathrm{pg} / \mathrm{ml}$ and $\mathrm{E}-\mathrm{C}=18 \pm 4, \mathrm{E}-\mathrm{M}=19 \pm 6 \mathrm{pg} / \mathrm{ml}$, respectively The significant decrease (30\%) in myocardial MBBG uptake cannot be explained an increased release of norepinephrine, the release being reduced by B-blockade. The reduced MBBG uptake cannot be related to a decrease in coronary blood flow which has been show to be invariant after B-blockade in awake animals. These results suggest an interaction between metoprolol and the norepinephrine uptake one carrier.
379

G. Cantinho, E. Silva, T.Martins, A.L. Santos, L.C. Oliveira, F. Godinho Instituto de Medicina Nuclear, FML and Serviço de Cardiologia, HSM Lisbon, Portugal

METAIODOBENZYLGUADINE IN THE EVALUATION OF THE ADRENERGIC NERYOUS SYSTEM IN MITRAL VALVE PROLAPSE SYNDROME PATIENTS

Metaiodobenzylguanidine labeled ${ }^{131}$ I (MIBG), as an analogue of norepinephrine, localizes in the adrenergic neurons, enabling determinations in vivo of disturbances in integrity and function of the sympathetic nervous system (SNS)in the heart. MIBG is taken up in the post ganglionic presynaptic vesicles, remaining intra-vesicular taken up in the post ganglionic presynaptic vesicles, remaining intra-vesicular
representing the true intraneuronal uptake. The non specific extraneuronal uptake is representing the true intraneuronal uptake. The non specific extraneuronal uptake is
of little afinity, with a rapid washout. The aim of our study is to evaluate SNS MIBG uptake in patients with mitral valve prolapse syndrome (MPS) and it's relations with arrhytmogenic activity and plasma cathecolamines levels. We studied 17 patients (pt) with MPS, mean age 49,5 12,8 years old, 11 female and 6 male, all with ventricula disritmias. In 13 the sympathetic adrenal activity was increased (mean orthostatic plasma catecolamine $=867.9 \pm 947.6, \mathrm{~N}<400 \mathrm{pg} / \mathrm{mI}$ ), all without heart failure. After thyroid blocade, all pt were injected iv, with $1 \mathrm{mCl}$ of MIBG. Sets of scintigraphic planar anterior imapes were performed at 4 and 24 hours. Data was collected over planar anterior images were performed at 4 and 24 hours. Data was collected ove $15 \mathrm{mn}$ in a $128 \times 128$ pixel matrix. Uptakes in the heart (H), lung (Lu) and liver ( $L$ ) were measured in regions of interest (ROI, 25 pixels), drawn at the 4 and $24 \mathrm{~h}$ in $\mathrm{H}$

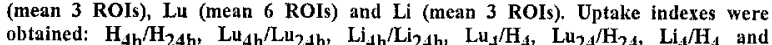
$\mathrm{Li}_{24} / \mathrm{H}_{24}$.

$\mathrm{Lu}_{4} / \mathrm{Lu}_{24}=2.67 \pm 0.6 ; \mathrm{H}_{4} / \mathrm{H}_{24}=2.11 \pm 0.42 ; \mathrm{Li}_{4} / \mathrm{Li}_{24}=2.6 \pm 0.51 ; \mathrm{Lu}_{4} / \mathrm{H}_{4}=0.7 \pm 0.11$ $\mathrm{Lu}_{24} / \mathrm{H}_{24}=0.57 \pm 0.13 ; \mathrm{Li}_{4} / \mathrm{H}_{4}=1.78 \pm 0.38 ; \mathrm{Li}_{24} / \mathrm{H}_{24}=1.49 \pm 0.35$. Two pt had 4 and $24 \mathrm{~h} \mathrm{Lu} / \mathrm{H}$ 1SD superior $(0.84$ and 0.95 ), without increased lung washout (2.22 and 233)Regarding ethical probems in 2.33) Regarding ethical problems in studing normal volunteers, we considered the $\mathrm{Lu}_{24} / \mathrm{H}_{24}$ values obtained by $\mathrm{M}$. Nakago et al. as the normal index $(\mathrm{N}=$
0.57 ). Statistical analysis using paired t Test didn't find any differences between Lu/Lu 0.57). Statistical analysis using paired t Test didn't find any differences between $\mathrm{Lu} / \mathrm{Lu}$
and $\mathrm{H} / \mathrm{H}$ ( 4 and $24 \mathrm{~h}$ ), but there was a significant difference between $\mathrm{Lu}_{4} / \mathrm{Lu}_{24}$ and $\mathrm{Li}_{4} / \mathrm{Li}_{24}$ compared with $\mathrm{H}_{4} / \mathrm{H}_{24}(p<0.001)$. Statistical differences were found between $\mathrm{L} / \mathrm{H}$ and $\mathrm{Li} / \mathrm{H}$ at 4 and $24 \mathrm{~h}(\mathrm{p}<0.05)$.

No inter-patients differences in heart, lung and liver washout was found, suggesting a true neuranal uptake. No correlation was found between plasma cathecolamines concentration and MIBG uptake, stggesting autonomic function. In $88 \%$ of pt, the MIBG uptake normal results suggest normal pre-sinaptic function. In only $2 \mathrm{pt}(12 \%)$ the results did suggest adrenergic nervous system disfunction.
380

J.Nanas, J.Lekakis, Z.Margari, V.Prassopoulos, L. Palazis, N.. Kouvelas, E. Agapitos, P. Kostam1s,S. Moulopoulos.

Dept of Clin. Therapeutics and Dept of Nuclear Medicine Alexandra University Hospital, Athens, Greece.

POSITIVE INDIUM III-MONOCLONAL ANTIMYOSIN IMAGING IN DILATED CARDIOMYOPATHY PREDICTS A FAVORABLE OUTCOME.

The purpose of the study was to identify the relation of positive antimyosin scan to onset of symptoms of congestive heart failure (CHF), myocardial fibrosis (MF) and respunse to medical treatment including digoxin, ACE inhibitors and furosemide. Seventeen patients ( $p$ ts, 13M/4F) $43 \pm 12$ yrs suffering from idiopathic cardiomyopathy diagnosed by endomyocardial biopsy, underwent antimyosin scan, clinical and hemodynamic evaluation and quantitative estimation of the MF. In 9 pts (group A) the antimyosin uptake ratio in the cardiac and right lung regions $(\mathrm{H} / \mathrm{L})$ were $>1.6(2.01 \pm 0.35)$ and in 8 pts (group $\mathrm{B})<1.6$, $(1.43 \pm 0.05, p<0.005)$. The duration of heart failure symptoms in group $A$ was $30 \pm 34$ months (range, $1.5-108$ ) and in group $B, 18 \pm 19$ months (range, $4-62, p=N S$ ). There was no clinical or hemodynamic difference between the 2 groups. The proportion of MF was $7.6 \pm 5.3 \%$ in group $A$ and $3.6 \pm 2 \%$ in group $B(p=N S)$. NYHA functional $c l$ ass was reduced by medical treatment by $1.5 \pm 1.2$ in group $A$ vs $0.4 \pm 0.5$ in group $B \quad(p<0.05)$. There were 2 deaths in group $B$ and none in group $A$. In conclusion, these data suggest that even long standing dilated cardiomyopathy might give a positive antimyosin imaging independent of the level of MF. However, a positive antimyosin scan predicts favorabie response to medical treatment. 


\section{1}

J. Soares Jr., R. Snitcowsky, A. C. S. Tanaka, F. H. Hironaka, M. Izaki, M. C. P. Giorgi, G. G. Cerri, F. Pileggi, J. C. Meneghetti.

The Heart Institute, University of São Paulo, Brazil.

CORRELATION BETWEEN GALLIUM-67 SCINTIGRAPHY AND IAABORATORY TESTS IN RHEUMATIC CARDITIS.

In order to compare the classical laboratory tests for acute phase(AP) of rheumatic fever(RF) and cardiac scintigraphy with Ga-67(Ga) for the detection of active carditis in pts with RF we studied prospectively 142 pts $(80 \mathrm{~F} ; 10.4 \pm 3.6$ yrs). All pts fulfilled the Jones criteria for diagnosing acute RF with carditis. They were submitted to $\mathrm{Ga}$ and acute phase reactants(APR) laboratory tests in the $\mathrm{AP}$ and during the evolution of the disease.

According to the different results observed we divided the pts in 4 groups $(G)$ : GI (Gat $x$ APR+) $=$ 71pts; GII (Gat $\times$ APR-)=30pts; GIII (Ga- $\times$ APR+) $=$ 13 pts; GIV(Ga- x APR-) $=28$ pts. The chi-square test showed association between laboratory and $\mathrm{Ga}$ results $(\mathrm{p}<0.0001)$. The time interval between $A P$ of $R F$ and the procedures were: $10.72 \pm 23.96$ days (GI); $63.73 \pm 53.98$ days (GII); $87.92 \pm 89.51$ days (GIII) and $135.96 \pm 131.63$ days (GIV). Nonparametric analysis by Kruskal-Wallis test showed significant difference between the distribution of the time interval among groups. The best correlation between $\mathrm{Ga}$ and APR occurred at shorter time interval between the procedures and the AP of RF. Discordant cases with Gat $x$ APR- suggest premature negativation of APR with persistent cardiac inflammatory state.Discordant cases with Ga- $x$ APR+ may suggest inflammatory state without carditis. Finally, the results observed at GIV suggest regression of the carditis by both methods.

\section{2}

G. Notohamiprodjo, A.Bentrup, K. Mellwig, O. Lindner, U. Gleichmann

Herzzentrum NRW, Ruhr-University Bochum, Bad Oeynhausen, FRG

\section{IN-ANTIMYOSIN SCINTIGRAPHY FOR THE EVALUATION OF ADDITIONAL MYOCARDIAL INJURY IN PATIENTS WITH SUSPECTED MYOCARDITIS UNDERGOING DIRECT-CURRENT CARDIOVERSION WITH 100-300 J-S}

The development of $111 / \mathrm{n}$-labeled monoclonal antibodies to human myosin allows a widespread clinical application for the investigation of myocardia infarction and myocarditis. Several studies have shown a typical antibody uptake pattern, localized to a segment in case of myocardial infarction and diffuse accumulation in acute myocarditis. We detected the site of myocardial injury secondary to a cardioversion with 100-300 $\mathrm{J}^{-5}$ in 9 patients with atrial or ventricular arrhythmic disorders suspected to suffer from myocarditis. Series of 111 In-AMAB images 24 hours before and 72 hours after DC cardioversion were obtained. In $6 / 9$ patients $A M A B$ images prior to $D C$ cardioversion were positive. In 5/6 patients diffuse accumulation of AMAB in the myocardium was obtained, and the ongoing myocarditis was confirmed. The remaining $1 / 6$ patient had regional accumulation of AMAB. In this patient and in one of the remaining $3 / 9$ patient without significant AMAB accumulation ischemic coronary disease was diagnosed. In the latter patient AMAB accumulation in the apical segment of the myocardium 72 hours after $D C$ cardioversion was registered. In a patient with myocarditis the extension of $A M A B$ accumulation was larger after $D C$ cardioversion. The remaining $2 / 9$ patients showed negative $A M A B$ accumulation prior and after DC cardioversion. In these patients myocarditis and ischemic coronary disease was excluded. The arrhythmia was suggested to be due to valvular disease. We conclude that ${ }^{11}$ In-antimyosin scintigraphy is a valuable tool for diagnosis myocarditis, and can be use to detect additional myocardial injury secondary to DC cardioversion. No significant additional myocardial injury after direct current cardioversion with energy less than $300 \mathrm{Js}$ could be observed. Small additional myocardial injury after $300 \mathrm{~J}^{5} \mathrm{DC}$ cardioversion could be expected in patients with ongoing myocardial injury due to primarily myocardia disease. The distribution of AMAB-accumulation with typical site and localisation can be used to differentiate whether the necrosis is induced by ischemic coronary disease or it is secondary to a myocardial disease.

\section{3}

J.Hashimoto, K. Nakamura, A. Kubo

Dept. of Radiology, School of Medicine, Keio University, Tokyo, Japan

\section{SCINTIGRAPHIC EVALUATION OF THE DIABETIC HEART}

We performed radionuclide myocardial imaging using I-123-MIBG (metalodobenzylguanidine) and 1-123-BMIPP (beta-methyl-paraiodophenyl pentadecanoic acid; fatty acid analog) for the assessment of the severity of the diabetic heart.

Thirty six NIDDM patients(pts) who had no history of CAD (excluded by stress ECG and TI-201 scintigraphy) underwent I-123-MIBG scintigraphy. SPECT images were obtained 3 hours after the intravenous injection of the tracer. Patients were divided into 4 groups according to the MIBG findings. Findings were normal in Groupl (14pts), mild reduced uptakes in Groupll (3pts), decreased uptakes in the inferior wall in Grouplll (10pts) and diffuse marked decreased uptakes in GroupiV (9pts)

Duration of the disease were $6.1 \pm 3.6$ (Groupl), $6.5 \pm 3.5$ (Groupll), $8.8 \pm 3.6$ (Grouplil) and $11.1 \pm 7.3$ (GrouplV) (years; mean \pm s.d.). $\mathrm{HbA} 1 \mathrm{c}$ values were $6.5 \pm 0.6$ (Groupl), $6.4 \pm 0.9$ (Groupll), $7.3 \pm 1.7$ (Grouplll) and $7.3 \pm 1,0$ (GrouplV) (mean \pm s.d.). Diabetic complications (neuropathy, nephropathy and retinopathy) were recognized in 4 pts of Groupl (29\%), 1 patient of Groupll (33\%), 7 patients of Groupll $(70 \%)$ and 6 patients of GrouplV (67\%). I-123-BMIPP scintigraphy was also performed on patients belonging to Grouplll ( $4 \mathrm{pts}$ ) and GrouplV (6pts). Reduced tracer uptakes were not observed in any pts of Grouplli, and those were found in 4 pts of GrouplV $(66 \%)$.

In patients with DM, scintigraphic findings were correlated with duration of the disease, HbA1c values, and presence of diabetic complications. MBBG and BMIPP myocardial scintigraphies might be useful for the evaluation of the diabetic heart.

\section{4}

EE Comans, GW Sloof, G Elzinga, FC Visser. Depts of Physiology, Nuclear Medicine, and Cardiology, Free University and Free University Hospital, Amsterdam, the Netherlands.

QUANTITATION OF MYOCARDIAL FATTY ACID OXIDATION WITH 123 I-HEPTADECANOIC ACID SCINTIGRAPHY DURING CARDIAC CATHETERIZATIOH

The relationship between myocardial oxygen consumption and the externally measured time-activity curves of terminally labelled 123 I heptadecanoic acid (ADA) ag a tracer of myocardial fatty acid (FA) metabolism was measured in six patients with coronary artery disease and one subject with normal coronary arteries. Myocardial time-activity curves were obtained after intracoronary administration of HDA (236 $\pm 41 \mu \mathrm{Ci})$ during 45 minutes. With a continuous sampling technique, arterial (A) and coronary sinus (CS) blood was collected for the determination of $H D A$ and $123 \mathrm{I}$ and to measure myocardial extraction of glucose $(6.1 \pm 15.4$ umol $/ \mathrm{min})$, lactate $(10.6 \pm 12.4 \mu \mathrm{mol} / \mathrm{min})$, FA $(22.7 \pm$ $6.3 \mu \mathrm{mol} / \mathrm{min}$ ), and oxygen (589 $\pm 196 \mu \mathrm{mol} / \mathrm{min}$ ). Coronary sinus blood flow was measured using a continuous infusion technique. Radio-chemical analysis of the plasma samples showed that after approximately 10 minutes the time-activity curves were mainiy determined by ${ }^{123}$ I washout (CS/A HDA ratio 1 , CS/A 123 I ratio 4.5 ). The myocardial time-activity curves were fitted with a mono-exponential function plus a constant from $t=10$ min. The calculated oxygen equivalents of extracted FA, glucose and lactate amounted $90.5 \pm 23.5,11.1 \pm$ 8.6, and $5.8 \pm 5.8 \%$ of the $\mathrm{O}_{2}$ consumption, resp. A positive relation was found between the amplitude of the exponential curve and the myocardial oxidative Iate $(r=0.71, p<0.05)$.

We conclude that scintigraphy enables the quantitation of myocardial oxidative metabolism. 


\section{5}

T. Athanasoulis, N. Sifakis, S. Gerali, Ch. Kalliontzi, P. Kostamis, S. Moulopoulos

Dept of Clinical Therapeutics and Dept. of Nuclear Medidine, ALEXANDRA Hospital, Greece.

CORRECTION OF THE FORWARD RIGHT VENTRICULAR EJECTION FRACTION (F-RVEF) IN PATIENTS WITH SIGNIFICANT TRICUSPID REGURGITATION (TR) BY GATED SPECT BLOOD BOOL IMAGING (GSBP).

Total right ventricular ejection fraction (T-RVEF) is overestimated when TR is present. F-RVEF overcomes this problem but is affected by the increased preload because of $T R$. The aim of this study is to correct the F-RVEF (FC-RVEF) from this influence by using GSBP. In 15 controls and 18 patients (Pts) with TR due to pulmonary hypertension $(P H)(\overline{P A P}=38 \pm 11 \mathrm{mmig})$ GSBP was performed. Left and right ventricular end diastolic counts (RVEDC) as well as left and right ventricle stroke counts (LVSC) and (RVSC) were estimated with the count based method by the horizontal long axis slices. Assuming that the forward stroke volumes of the two ventricles are almost equal and the difference between RVSC and LVSC (DSC) affects only RVEDC we calculated the T-RVEF, F-RVEF and the FC-RVEF from the ratios: RVSC/RDEDC, LUSC/RVEDC and LUSC/(RUEDC-DSC) respectiveTy. The T-RVEF mean value of the controls was $54 \pm 5 \%$ and was found to be significantly different from the Pts" T-RUEF, F-RVEF, FC-RVEF mean values (p 0.001$)$ In the pts ${ }^{\prime}$ group the results were: T-RVEF $42 \pm 5 \%$ versus F-RVEF $28 \pm 6 \%(p<0.001)$. T-RVEF versus FC-RVEF $32 \pm 6 \%$ $(p<0.001)$ and F-RVEF versus FC-RVEF ( $p<0.001)$ (Pair t-test). A negative correlation was found between pts $\overline{P A P}$ and T-RVEF $r=-0,67, F-R V E F \quad r=0,84$, and Fc-RVEF $r=-0,82$ $(0<0.001)$. These results indicate that in the case of significant PH and TR, T-RVEF is higher and F-RVEF is lower than FC-RVEF. We consider that FC-RVEF calculated by this method is less influenced by TR and could be useful in clinical practice.

\section{6}

O. Schillaci, F. Scopinaro, A. Muzio, R. Danieli, F. Messa, R. Tavolaro, M. Ciavolella, IJ* Di. Macio, G. Calcagnini, A. Centi Colella

Dpt. Experim. Medic., University "Ia Sapienza", Rame, Italy

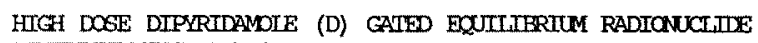
VENIRIOULOGRAPHY (RV)

Ventrioular dysfunction indroed by $D$ infusion (I) is helpful in detecting patients ( $\mathrm{Ts}$ ) with $\mathrm{CAD}$. To evaluate the usefulness of $\mathrm{DI}$ associated with $\mathrm{RV}$, we studied 22 pts (15 males and 7 females, mean age $54.9 \pm 7.34$ years) with effort chest pain and no previous myccardial infarction. Aocording to concmary angiography, 12 pts had $\mathrm{CAD}$ (stenosis $>70 \%$ in at least 1 major coronary artery). $R V$ was perfomed at rest (R), after a first (F) DI $(0.56 \mathrm{mg} / \mathrm{Kg}$ over $4 \mathrm{~min}$ ) and, in absence of angina and/or ST segment depression (18 pts), after a second (S) DI $(0.28 \mathrm{~mm} / \mathrm{Kg}$ over 2 min). Aninophylline (A) was always injected at the end of the stury and another RV performed 2 mirutes later. For each aopuisition global left vericrioular ejection fraction (LVEF) was calculated and regional wall motion (BM) was aralysed and graded on a 4 point scale after dividing IV into 5 segments. The difference between R-IVEF in nomal subjects $(54.0 \pm 8.4 \%$ and $C A D$ pts $(52.3 \pm 8.6)$ was not statistically significant. LVEF decreased after FDI in $9 / 12$ pts with $\mathrm{CAD}$ (sens. 75\%) and in 11/12 after SDI (sens 92\%) whereas it increased in 7/10 momal subjects after FDI (spec 70\%) and in 9/10 (spec 90\%) after SDI. FM decreased of at least one point after FDI in $7 / 12$ pts (58\%) with CAD and in $9 / 12$ (75\%) after SDI. LVEF netumed to baseline values after A administration in $9 / 11$ pts. our results suggest that LV dysfunction caused by DI in CAD pts can be detected by $R V$ and reversed by $A$ : the use of high dose of DI is mandatory: it improves both sens and spec of RV. Moreover, $\mathrm{RV}$ after DI provides an useful altemative to exercise $k V$ : it reduces movement artefacts and can be performed also in pts with limite? exercise tolerano.

\section{7}

Y. COTTIN, C. TOUZERY, A. GLLLES, M. TOUBEAU, D. BERNARD, A BERRIOLO, E. PICHON, JE. WOLF, P. LOUIS, F. BRUNOTTE. Service de Médecine Nucléaire, Service de Cardiologie, CHU de Dijon, 21000, FRANCE.

CARDIOTOXICITY OF DOXORUBICIN - EVOLUTION OF THE DIASTOLIC AND SYSTOLIC PARAMETERS STUDIED BY RADIONUCLIDE ANGIOGRAPHY

The use of doxorubicin (DXR) is limited by their important cardiotoxicity. In a previous study, we observed simultaneous alterations of systolic and diastolic parameters one month after the end of the chemotherapy.

The purpose of the study was to determine the evolution of these alterations. In a prospective study, twenty-three patients with a mean age of $51 \pm 13$ years, without cardiac disease were studied before chemotherapy, one and three months after the end of the chemotherapy (mean dose $233 \pm 101 \mathrm{mg} / \mathrm{m}^{2}$ ). By gated radionuclide angiography we determined : the RR interval (RR), the ejection fraction (EF), the peak ejection rate (PER), the peak filling rate (PFR) (expressed in end diastolic volume per second : EDV/sec), and the ratio PFR/PER. The statistical method uses the paired $t$-test.

$$
\begin{aligned}
& \text { RR EF PER PFR PFR/PER } \\
& \text { (msec) (\%) (-EDV/sec) (EDV/sec) }
\end{aligned}
$$

Before DXR $750 \pm 112 \quad 58 \pm 6 \quad 3.01 \pm 0.36 \quad 2.73 \pm 0.48 \quad 0.91 \pm 0.17$

1 month $\quad 798 \pm 119 \quad 55 \pm 5 \quad 2.71 \pm 0.42 \quad 2.54 \pm 0.42 \quad 0.94 \pm 0.13$

3 month $\quad 772 \pm 126 \quad 56 \pm 5 \quad 2.76 \pm 0.54 \quad 2.53 \pm 0.48 \quad 0.95 \pm 0.22$

Before vs 1 month NS $p<0.01 \quad p<0.01 \quad p<0.02 \quad$ NS

$\begin{array}{lllll}\text { Before vs 3 month NS } & \mathrm{p}<0.05 & \mathrm{p}<0.05 & \mathrm{p}<0.05 & \text { NS } \\ \text { l vs } 3 \text { month NS } & \text { NS } & \text { NS } & \text { NS } & \text { NS }\end{array}$

Our results show simultaneous alterations of the systolic and diastolic parameters one month after chemotherapy. The evolution was similar for the systolic or diastolic function and no further significant alteration was observed between one and three months after chemotherapx.

\section{8}

A. Cuocolo, M. Imbriaco, A. Nappi, S. Cardei, E. Nicolai, C. Morisco, M. Romano, B. Trimarco, M. Salvatore.University Federico II and National Cancer Institute, Napoli, Italy.

AMBULATORY MONITORING OF LEFT VENTRICULAR FUNCTION DURING CARDIOPULMONARY EXERCISE IN PATIENTS WITH DILATED CARDIOMYOPATHY.

Ambulatory left ventricular (LV) function monitor (Vest) has expanded the diagnostic potential of exercise testing in the evaluation of patients with cardiac diseases. To evaluate $L V$ response during exercise, 20 patients with dilated cardiomyopathy (DC) (5 patients with idiopathic $\mathrm{DC}$, group $\mathrm{A}$, and 15 patients with post-necrotic $\mathrm{DC}$, group B) underwent cardiopulmonary bicycle exercise testing with continuous monitoring of $\mathrm{LV}$ function. In both groups $\mathrm{LV}$ function was continuously monitored by Vest under resting conditions (RC), at anaerobic threshold (point on nonlinear increase in ventilation relative to oxygen uptake) (AT), and at peak oxygen uptake (VO2). VO2 was measured under RC and during exercise using an Aerobic Processor (MGC 2001). End diastolic volume (EDV) was expressed as percent of the value at the beginning of the study and end systolic volume (ESV) was expressed relative to EDV. In both groups heart rate (HR) and EDV significantly (all $p<0.01$ ) increased at $A T$ and at peak VO2 compared to RC. In group A, ESV did not change from RC $(65 \pm 12 \%)$ to AT $(70 \pm 14 \%)$, while it significantly (p<0.01) increased from $\mathrm{RC}$ to peak VO2 $(74 \pm 13 \%)$. In group $\mathrm{B}$, ESV significantly (0.01) increased from RC $(62 \pm 13 \%)$ to AT $(69 \pm 14 \%)$ and peak VO2 $(74 \pm 14 \%$ ). In group A, ejection fraction (EF) did not change from RC $(33 \pm 10 \%)$ to both AT $(32 \pm 9 \%)$ and peak VO2 $(32 \pm 9 \%)$. In group B, $\mathrm{EF}$ did not change from RC $(35 \pm 12 \%)$ to AT $(35 \pm 10 \%)$, while it significantly $(\mathrm{p}<0.01)$ decreased at peak VO2 $(32 \pm 10 \%)$. In conclusion, exercise-induced LV function deterioration is more evident in patients with post-necrotic DC compared to patients with idiopathic DC. Vest allows a better understanding of functional responses during cardiopulmonary exercise and may be useful to evaluate the different behavior of $\mathrm{LV}$ function in patients with idiopathic and post-necrotic DC. 


\section{9}

D. Sobic, M. Veljovic, V. Bosnjakovic, S. Pavlovic, N. Kozarevic, M. Lekic

Institute of Nuclear Medicine, CCS, Belgrade, Yugoslavia

LEFT VENTRICULAR VOLUME CALCULATIONS BY RADIONUCLIDE METHODS: ORIGINAL ("GEOMETRIC COUNT BASED") VS. CONVENTIONAL PLANAR AND SPECT ONES

The aim of the study was to evaluate left ventricular end-diastolic (EDV) and stroke volumes (SV) measured by three radionuclide (RN) methods: original geometric - count based (GCB) compared to the count-based (CB) planar and gated SPECT (GASPECT) ones. Fifty seven coronary patients - 26 with previous myocardial infarction and 31 with angina pectoris, (43 males and 14 females, 52 years old in average) underwent radionuclide ventriculography studies using our own $\mathrm{GCB}, \mathrm{CB}$ and GASPECT methods for calculating EDV and SV. In 7 patients biplane contrast ventriculography was performed and EDV and SV were calculated by "area-length" method. Correlation between $\mathrm{GCB}$ and $\mathrm{CB}$ planar methods for EDV was: $r=0.95$, and for SV: $r=0.94$. Correlation between GASPECT and GCB was, EDV: $r=0.90$ and SV: $r=0.77$, and between GASPECT and $\mathrm{CB}$ was EDV: $-r=0.81$ and $\mathrm{SV}$ : $r=0.75$. Contrast ventriculography correlated best with GCB method (EDV: $r=0.98$ ). Our results indicate that both planar methods and GASPECT are relatively accurate non-invasive $\mathrm{RN}$ techniques for measuring left ventricular volumes, with particular potentials of GCB method.

\section{0}

E. Gosiewska-Marcinkowska, S. Konieczna, E.K. Włodarska

National Institute of Cardiology, Warsaw, Poland

RADIONUCLIDE ASSESSMENT OF RIGHT AND LEFT VENTRICULAR FUNCTION IN ARRHYTHMOGENIC RIGHT VENTRICULAR DYSPLASIA

Arrhythmogenic right ventricular dysplasia (ARVD) is the disease of the right ventricular myocardium, but the left ventricle damage is also seen in these cases.

The purpose of the present analysis was to evaluate the significance of radionuclide angiography (RNA) in assessment of the right (RV) and left (LV) ventricle function in 24 pts with typical clinical, angiographic and histopathologic features of ARVD. All pts were examined in rest and during supine stress test using bicycle ergometer. To evaluation ejection fraction (EF) of both ventricles the Fourier analysis was used.

Mean RV rest EF (RVEFr) was $29,8 \% \pm-11,1$ (normal $41,3 \% \pm$ $-3,7$ ), mean RV exercise EF (RVEFex) was $27,8 \% \pm-10,7$ (normal 51,7\% $\pm-5,0$ ); mean LVEFr was $58,5 \% \pm 8,5$ (normal $59,4 \% \pm 4,3$ ), mean LVEFex was $60,0 \% \pm 8,4$ (normal $70,5 \% \pm$ $5,4)$. In the studied group following observations were made: significant decrease of RVEFr and RVEFex in comparison with normal values $(p<0,05)$, no significant decrease of RVEFex, significant difference of LVEFex in comparison with normal values $(p<0,05)$, no significant difference between LVEFr and normal values, no significant increase of LVEFex.

1. Significant damage of RV function was observed.

2. LV function impairment was observed.

\section{1}

S.Konieczna,E.Gosiewska-Marcinkowska,I.Rawczynska,J.Nowicka

National Institute of Cardiology, Warsaw, Poland

ESTIMATION OF SEVERITY OF TRICUSPID REGURGITATION (T'R) BY ANALYSING RIGHT ATRIUM (RA) TIME-ACTIVITY CURVE

The aim of the study was to estimate the value of a parameter related to the isotope outflow from the RA as an index of TR. The group of the 46 patients (pts) was studied: 34 pts with TR and 12 normal pts. The whole group was divided into subgroups (sbg) with different value of TR severity (from " + " to " ++++ ") evaluated on a basis of echocardiography. For 23 pts right ventricular pressure was measured in echocardiographic study.

First-pass radionuclid angiography were aquired in RAO projection with a bolus injection of $20 \mathrm{mCi}$ of technetium $-99 \mathrm{~m}$

The RA time-activity curve was analysed. The isotope outflow curve was approximated by the exponent curve: $p(t)=C * \exp (-a t)$ where $C$ is the maximum number of counts in the RA region. Index of tricuspid regurgitation (INT) was defined as:

$\mathrm{NT}=\mathrm{Tp} / \mathrm{Tb}$, where $\mathrm{Tp}$ is the time calculated from the equation $p(T p)=C / 2, T b$ is the time FWHM of the bolus.

\begin{tabular}{|c|c|c|c|}
\hline sbg & N pts & $\begin{array}{c}\text { Value of TR from } \\
\text { echocardiography }\end{array}$ & mean INT +/- SD \\
\hline 0 & 12 & - & $0,69+/-0,17$ \\
1 & 8 &,$+++/+$ & $1,23+/-0,68$ \\
2 & 5 &,$++++/+++$ & $3,06+/-0,60$ \\
3 & 6 &,$++++++/++++$ & $4,00+/-1,79$ \\
4 & 15 & ++++ & $7,06+/-5,20$ \\
\hline
\end{tabular}

There is no significant difference between INT values for normal pts and pts from " 1 " sbg. Mean INT value increases with the severity of TR but the ranges of INT value for $\operatorname{sbg} 2$ and 3 and also for 3 and 4 partly overlay each other. There is no significant dependence between INT and right ventricular pressure.

1. INT can be used to estimate severity of TR. 2. It is necessary to provide further research on greater number of pts, if possible, with "at operation" verification of the severity of TR.

392

D. Le Guludec, MC. Peker, G. Grimon, M. Faraggi, R. Lebtahi, M.S. Slama. Nuclear Medicine Department, Hôp. Bichat and Béclère (Paris,Clamart), France.

\section{LEFT VENTRICULAR FUNCTION IMPROVEMENT AFTER BALLOON VALVULOPLASTY IN MITRAL STENOSIS: ASSESSMENT WITH RADIONUCLIDE ANGIOGRAPHY.}

The determinants and evolution of left ventricular dysfunction occurring in 30 to $50 \%$ of patients with pure mitral stenosis are not known. The left ventricular ejection fraction (LVEF,\%), peak ejection and filling rates (PER, PFR, $\mathrm{s}^{-1}$ ) and end-diasiolic volume indexes (EDV) were prospectively studied using equilibium radionuclide angiography in 18 patients (pts), before and 3 days after successful balloon valvuloplasty (BV). The study population was $36 \pm 10$ years old, in sinus rythm before and after BV, free of coronary or other valvular disease (no mitral regurgitation > 1+ after BV). 9 pts had a baseline LVEF impairment $<55 \%(47 \pm 8 \%$, G1), and 9 pts had normal LVEF $(63 \pm 7 \%$, G2). Between the two groups, neither age, sex, baseline HR (bpm), EDV, and echographic mitral valve area $\left(\mathrm{MMA}, \mathrm{cm}^{2}\right)$ were significantly different:

\begin{tabular}{|c|c|c|c|c|c|c|}
\hline pre-BV & HR & LVEF & EDV & PER & PFR & MVA \\
\hline G 1 & $76 \pm 12$ & $47 \pm 8^{* *}$ & $150 \pm 22$ & $2.53 \pm 0.8$ & $1.87 \pm 0.5^{*}$ & $1.06 \pm 0.2$ \\
\hline G 2 & $67 \pm 11$ & $63 \pm 7^{* *}$ & $156 \pm 32$ & $3.19 \pm 0.8$ & $2.27 \pm 0.3^{*}$ & $1.01 \pm 0.2$ \\
\hline
\end{tabular}

Before and after BV, MVA was correlated with PFR $(r=0.51, p<0.05)$ but not with $L V E F$. After $B V$, no significant difference changes in $H R$ and EDV were observed in both groups. In contrast, LVEF, PER, and PFR improved significantly only in G1.

\begin{tabular}{|l|c|l|l|l|}
\hline post-BV & LVEF & PER & PFR & MVA \\
\hline G1 & $58 \pm 7^{\star \star *}$ & $3.15 \pm 0.5^{*}$ & $2.46 \pm 0.6^{* \star}$ & $2.21 \pm 0.3^{* \star *}$ \\
\hline G2 & $65 \pm 4$ & $3.41 \pm 0.6$ & $2.74 \pm 0.6$ & $1.99 \pm 0.3^{* \star *}$ \\
\hline
\end{tabular}

Conclusion: successful BV of MS increase LVEF in pts with baseline systolic dysfunction, independently of the improvement in filling parameters and preload. 
393

E. Varoğlu, A.Aktaş, T. Aras, C.F.Bekdik

Departments of Nuclear Medicine Atatürk University and Hacettepe University, Medical School Erzurum/Ankara TURKIYE

ACUTE EFFECTS OF SINGLE DOSE NIFEDIPINE ON TEE LEFT VENTRICULAR FUNCTION IN HIPERTENSIVE PATIENTS

The aim of this study was to evaluate the effect of hypertension on the left ventricular systolic and diastolic functions and the acute effect of single dose long-acting nifedipine on these functions by the use of radionuclide ventriculography. age: $48 \pm 11.2$ ) and 19 normal control subjects ( $9 \mathrm{M}_{\text {, }} 10$ $F$; mean age: $35.2 \pm 11.2$ ) were studied. Equilibrium Radionuclide ventriculography (ERNV) studies in hypertensive and normal subjects were performed by labelling the RBC in vivo..In hypertensive patients, following basal ERNV study, $20 \mathrm{mg}$ of long acting nifedipine were adninistered orally and ERNV studies were repeated at 1.5 and $4 \mathrm{~h}$. For the normal subjet only basal ERNV study was performed. Wen the left ventricular systolio and diastoli whetion indices in hypertensive and normal groups wer were copletely abnormal in the hypertensive group $(p<0.05)$. But for the systolic parameters no significant difference were found between these groups $(p>0.05)$. When the hypertensive patients were classified based on left ventricular hypertrophy no significant difference between systolic and diastolio indices were found $(\mathrm{p}>0.05)$. The acute effect of long acting nifedipine on left ventricular systolis and diatolic function in left ventricular systolic and significant improvement of these functions.

ignificant improvement of these functions. nifedipine has a positive effect oh both systolic and diastolic functions.
Twentyfive hypertensive patients $(13 \mathrm{M}, 12 \mathrm{~F}$; mean

When basal ERN study was pexformed. the normal subjets compared, diastolic parameters such as PFR and TPFR In conclusion, we found that diastolic parameters were significantly impaired in hypertensive patients and

\section{5}

WS Richter, J Hierholzer, U Keske, R Jochens, M Cordes, R Felix Strahlenklinik, Klinikum Rudolf Virchow, Freie Universität Berlin, Berlin, Germany

\section{PULMONARY ACTIVITY IN TL-201 MYOCARDIAL SCINTIGRAPHY: DISTRIBUTION AND CORRELATION WITH HEMODYNAMIC PARAMETERS}

Aim of the study was to assess the influence of parameters of left ventricular function on pulmonary thallium uptake. A further aim was to investigate inhowfar pulmonary thallium content can be accurately quantitated from the anterior SPECT projection and to determine the pattern of the intrapulmonary thallium-201 distribution.

(1) In 24 patients myocardial SPECT (30 sec/frame, $64 \times 64$ matrix) and planar anterior imaging of the thorax (acquisition time $120 \mathrm{sec}$, $256 \times 256$ matrix) were performed. The lung heart ratio (LHR) was determined from the anterior SPECT projection and from the planar anterior scintigram; the values of both methods were intercorrelated $(r=0.993 ; p<0.001$ ). (2) Regional pulmonal thallium-201 distribution in the right lung was examined in 20 patients without evidence of coronary artery disease. A lower thallium activity was recorded from the apex of the lung as compared with the basal portion. (3) In another 41 patients a radionuclide ventriculography (RNV) was performed within four weeks after myocardial SPECT. The LHR was negatively correlated $(\mathrm{p}<0.05)$ with peak ejection rate at rest $(r=-0.34)$, peak filling rate during exercise $(r=-0.41)$, left ventricular ejection fraction during exercise ( $\mathrm{LVEF}_{\mathrm{ex}}$ ) ( $\left.\mathrm{r}=-0.41\right)$, and maximal heart rate during exercise $\left(\mathrm{HR}_{\mathrm{ex}}\right)(\mathrm{r}=-0.44)$. In the multiple regression analysis $\mathrm{LVEF}_{\mathrm{ex}}$ and $H_{\mathrm{ex}}$ showed the strongest relation to LHR $\left(\mathrm{R}^{2}=0.42 ; \mathrm{p}<0.001\right)$. Using the regression equation the exspected $L_{V E F}$ was calculated using $\mathrm{HR}_{\mathrm{ex}}$ and LHR as parameters. This calculated $\mathrm{LVEF}_{\mathrm{ex}}$ correlated with the actual $\mathrm{LVEF}_{\mathrm{ex}}$ which was derived from RNV ( $r=0.53 ; \mathrm{p}<0.001)$.

In conclusion, (1) pulmonary thallium can be quantitated from the anterior SPECT projection with sufficient accuracy, (2) a pulmonary thallium gradient exists from the apex to the basis, and (3) after correction with regard to $\mathrm{HR}_{\mathrm{ex}}$ the elevated LHR points at patients with a pathological LVEFex

\section{6}

A. Loboquerreno, C. Pérault, J.C. Liełm, C. Gibold, T. Pron, J. Ouzan, A. Iepaill leur, A. Bouchard, H. Wampach Niclear Medicine Department, Institut Jean Godinot, Reims, France

Departments of Nuclear Medicine and *Hemodialysis, osijek Clinical Hospital, osijek, Croatia

CHANGE IN BLOOD VOLUME FOLLOWING CORRECTION OF ANEMIA WITH RECOMBINANT HUMAN ERYTHROPOIETIN IN PATIENTS ON MAINTENANCE HEMODIALYSIS

The aim of this study was to evaluate the effect of recombinant human erythropoietin (rHuEPO) therapy on blood volume in 19 patients (12 male, 7 female, mean age $39 \pm 11$ years, ) with severe renal anemia (hematocrit < 0.25 ) and having regular hemodyalisis (mean duration $5.0 \pm 3.4$ years). Blood volume was measured using method adjusted in our laboratory as a by-product during routine equilibrium radiornuclide ventriculography with $740 \mathrm{MBq}$ Tc-99m-human serum albumin twice: 1) before rHuEPO treatment and 2 ) at the time when target hemoglobin $(\mathrm{Hb})$ reached $100 \mathrm{~g} / 1$. Time elapsed to reach target $\mathrm{Hb}$ was $3.4 \pm 1.4$ months; $\mathrm{Hb}$ increased from $73 \pm 6$ to $111 \pm 11 \mathrm{~g} / \mathrm{l}$, $(p<0.0001)$. Red-cells volume was significantly increased from $16 \pm 3$ prior therapy to $26 \pm 6$ $\mathrm{ml} / \mathrm{kg}(\mathrm{p}<0.001)$ after therapy. Despite significant decrease in plasma volume $(53 \pm 10$ before vs. $48 \pm$ $11 \mathrm{ml} / \mathrm{kg}$ after, $\mathrm{p}<0.01$ ) total blood volume was increased from $69 \pm 12$ to $73 \pm 16 \mathrm{ml} / \mathrm{kg}(\mathrm{p}<0.01)$ after correction of anemia. Significant fall in cardiac index was found after treatment due to reduction of left ventricle end-diastolic volume ( $p<0.01$ ) and heart rate), while ejection fraction and blood pressure remained unchanged during rHuEPO treatment. We concluded that increase in red-cell volume occurs within short time period through correction of anemia, along with decrease in plasma volume, but increase in total blood volume.

\section{QUANITTATIVE COMPARISON OF PRONE AND SUPDE MYOCARDIAL SPET} MIBI TMACES

The myocardial count distribution and the stress/rest ratio in the inferior wall were compared between prone and supine SPET TC 99m MIBI acquisitions. Ninety-nine male patients with a low inferior wall count in the supine stress images underwent stress and rest acquisitions in supine and prone pasition sucoessively. For each study, values depicting the inferior, anterior, septal and lateral wall counts were extracted from a medioventricular, nomalized to ane, circumferential profile. The inferior and septal(resp. anterior and lateral) walls were significantly better visualized in the prone (resp. supine) than in the supine (resp. prone) position (Student paired t-test).

Comparison of myocardial counts between supine and prone SPET:

$\begin{array}{lllll}\mathrm{n}=198 & \text { Inferior } & \text { Anterior } & \text { Septal } & \text { Lateral } \\ \text { Supine } & 0.71 & 0.85 & 0.72 & 0.92 \\ \text { Prone } & 0.77 & 0.82 & 0.76 & 0.90 \\ \text { probability } & <0.001 & <0.001 & <0.001 & <0.01\end{array}$

probability $<0.001<0.001<0.001<0.01$

Among the 54 patients with a supine stress inferior count less than 0.8 and a supine stress/rest ratio less than 0.9 , the prone ratio, when compared to the supine one, increased by more than $5 \%$ in 34 patients and was greater than 0.95 in 20

In conclusion, with TC $99 \mathrm{~m}$ MIBI, as with Tl 201, the myocardial count distribution differs between supine and prone position, especially in the inferior wall. The inferior stress/rest differences are reduced in prone position. Prone position is preferable for the inferior and septal walls interpretation while supine position is preferable for the anterior and lateral walls. Combining both positions would probably prevent a misleading interpretation. 


\section{7}

A. Fikrle, E. P. Ritter, H. Rösler, A.R. Haldemann, M. Sulzer,

H. Ledermann, J. Kinser

Dept. of Nuclear Medicine, University of Berne, Inselspital,

CH-3010 Berne, Switzerland

MYOCARDIAL GATED 99mTc-MIBI SPET (GSPET): SUPINE VERSUS PRONE

Artificial inferior wall defects are a source of error in 201Tl supine (Sup) SPET. Prone (Pr) imaging diminishes this effect. 99mTc-MIBI has an advantage of higher energy. The aim of this study was to determine the effect of Pr position on GSPET and non-gated (NGSPET) MIBI images. Two-days stress/rest MIBI GSPET was performed with a 3-head camera in Sup and Pr in 72 pts. 49 men $(87 \pm$ $15 \mathrm{~kg}$ ) without reversibility in Sup stress/rest were selected: 34 without (Group A) and 15 with (Group B) a history and ECG evidence of inferior MI. The regional myocardial count rate distribution at rest was evaluated quantitatively with circumferential apical, middle and basal short axis profiles for diastole (D), systole (S) and NGSPET (sum of 12 frames/cardiac cycle) both for Sup and Pr. The difference between Sup and Pr was calculated as Pr minus Sup/Sup x 100 [\%]:

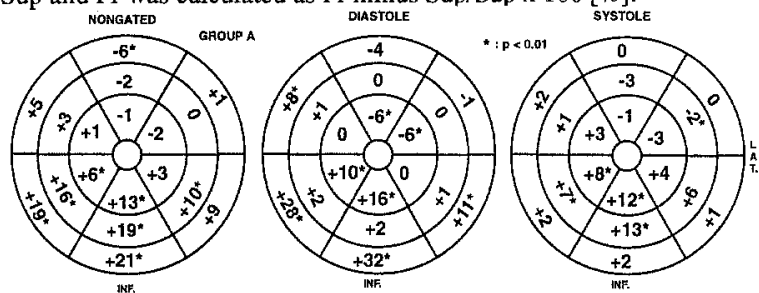

The relative regional uptake in Pr was higher $(p<0.01)$ inferior and infero-septal: in $\mathrm{D}$ apical and basal, in $\mathrm{S}$ apical and middle, in NGSPET in all slices. The highest $\mathrm{Pr} /$ Sup difference was in $D$, the smallest in S, more pronounced in (A) than in (B). Pr imaging improves the accuracy of inferior wall abnormalities in $99 \mathrm{mTc}-\mathrm{MIBI}$ SPET by reducing attenuation artefacts. The influence of $\mathrm{Pr}$ positioning is more prominent in "normal" pts than after MI and is not to the same degree in $D$ and $S(D>S)$. The quantitative parameters (i.e. "wall-thickening") may be differently influenced in Pr and Sup.

\section{8}

J.J. Bax, F.C. Visser, A. van Lingen, P.G.H.M. Raijmakers, G.J.J. Teule, C.A. Visser.

Free University Hospital Amsterdam, The Netherlands.

IMAGE QUALITY OF F18-FLUORODEOXYGLUCOSE SPECT STUDIES IN PATIENTS WITH CORONARY ARTERY DISEASE AND DIABETES MELITTUS TYPE II.

It has been described that image quality of F18-fluorodeoxyglucose (FDG) is poor in patients with diabetes mellitus. In the present study we investigated the image quality of FDG SPECT studies during hyperinsulinemic euglycemic clamping in 10 patients (pts) with chronic coronary artery disease (CAD) and in 10 pts with CAD and diabetes mellitus type II (DM II). Levels of lactate (LA in $\mathrm{mmol} / \mathrm{l}$ ), free fatty acids (FFA in $\mathrm{mmol} / \mathrm{l}$ ), glucose (GL in $\mathrm{mmol} / \mathrm{l}$ ) and insulin (INS in mU/l) were measured at the start and after 1 hour of clamping. The image quality was expressed by the myocardium-to-blood-pool (M/B) activity ratio (determined from short-axis slices).

\begin{tabular}{|c|c|c|c|c|}
\hline & start & & 1 hour & \\
\hline & $\begin{array}{l}C A D \\
1.3 \pm 0.4\end{array}$ & $1.4 \pm 0.3$ & $1.4 \pm 0.3$ & $1.6 \pm 0.5$ \\
\hline FFA & $0.07 \pm 0.06 \#$ & $0.24 \pm 0.28$ & $0.02 \pm 0.03 * *$ & $0.08 \pm 0.07 *$ \\
\hline $\begin{array}{l}\text { GL } \\
\text { INS }\end{array}$ & $\begin{array}{l}5.1 \pm 1.5 \neq \\
20.7 \pm 20.2\end{array}$ & $9.6 \pm 1.8$ & $\begin{array}{l}5.6 \pm 0.9 * \\
95.0 \pm 48.9 *\end{array}$ & $\begin{array}{l}7.3 \pm 1.6 \\
93.9 \pm 25 *\end{array}$ \\
\hline $\mathrm{M} / \mathrm{B}$ & & & $2.6 \pm 0.9$ & $2.1 \pm 0.5$ \\
\hline
\end{tabular}

Baseline levels of GL and FFA were significantly higher in the DM II group. One hour clamping increased INS levels significantly in both groups, and decreased FFA levels significantly in both groups but FFA levels remained higher in DM II pts, possible due to a relative insulin resistance. Also GL remained higher in DM II pts. Despite higher FFA levels image quality was not significantly lower in DM II pts. Thus, hyperinsulinemic glucose clamping may be advocated in DM II pts because it results in an image quality comparable to that of non-DM II pts.

\section{9}

J.J. Bax, F.C. Visser, P.G.H.M. Raijmakers, A. van Lingen, G.J.J. Teule, C.A. Visser.

Free University Hospital Amsterdam, The Netherlands.

THE RELATION BETWEEN THE EJECTION FRACTION AND THE THALLIUM CAVITY-TO-MYOCARDIUM (C/M) COUNTS RATIO; FACTORS INFLUENCING THE C/M RATIO.

Recent data suggest that the ratio of counts in the left ventricular (LV) cavity to that in normal myocardium (C/M ratio) of thallium (T1-201) scintigraphy can be used to assess LV dysfunction. This study examines the relation between the $C / M$ ratio and the $L V$ ejection fraction (EF); furthermore the influence of Tl-201 activity in blood, myocardial Tl-201 uptake and LV size on the C/M ratio was determined. Twelve patients $(9$ with previous infarction) underwent Tl-201 SPECT at rest and angiography. To calculate $\mathrm{C} / \mathrm{M}$ ratios, $2 \times 2$ pixel $\mathrm{ROIs}$ were placed in the center of the $\mathrm{LV}$ cavity and on the myocardium with the highest T1-201 activity. Venous blood samples were drawn at the start of SPECT imaging to measure T1-201 activity; LV diameter (as a measure of LV size), extent and mean severity of perfusion defects were determined from SPECT images. Multiple regression was used to delineate which of these factors contributed significantly to the $\mathrm{C} / \mathrm{M}$ ratio. $\mathrm{EF}$ varied from 13 to $80 \%$. The $\mathrm{C} / \mathrm{M}$ ratio showed a good correlation with the EF $(r=0.90, p<0.001)$.

ANOVA table of multiple regregsion p-value

LV diameter 0.002

$\begin{array}{ll}\text { Mean severity perf. defect } & 0.01 \\ \text { Extent perfubion defect } & 0.66\end{array}$

Blood TL-201 0.18

Thus, the C/M ratio shows a good relation with the LVEF and is mainly influenced by LV size and the severity of the perfusion defect, suggesting that spill-over of Tl-201 activity from myocardium to the cavity determines the $\mathrm{C} / \mathrm{M}$ ratio.

400

C.Touzery, F.X. Sotto, Y.Cottin, J. Covillard, M.Toubeau, J.M. Riedinger, A.Berriolo, F. Chossat, D. Bekhechi, D. Fagret, J.E. Wolf, F.Brunotte

Services de médecine nucléaire, CGFL-Dijon, CHU-Grenoble Service de cardiologie, CHU-Dijon, CIS biointernational (France) COMPARISON BETWEEN MYOCARDIAL UPTAKE OF 123I16-IODO-3 METHYL HEXADECANOIC ACID AT 15 AND 45 MINUTES AFTER INJECTION AT REST.

The use of 123I-16-Iodo-3 methyl hexadecanoic acid (IMHA) has been developped in order to evaluate the residual myocardial viability after a myocardial infarction. The best time for imaging is a matter of discussion. The aim of this study is to compare the relative myocardial distribution on IMHA S.P.E.C.T. 15 minutes and 45 minutes post intravenous injection.

Eleven patients were studied after a myocardial infarction. In each patient, S.P.E.C.T.was obtained 15 and 45 minutes after injection of $148 \mathrm{MBq}$ of IMHA at rest. Thallium S.P.E.C.T. was also obtained on a separate day at stress, redistribution and reinjection. Nine segments were quantitatively analysed by two observers on each tomogram set. The myocardial uptake was expressed as a percentage of the segment with the higher uptake. The uptake of IMHA was calculated in three groups of regions according to their uptake of thallium at reinjection $(<60 \%, 60 \%-80 \%,>80 \%)$.

IMHA uptake in the three groups was as follow :

Tl uptake (reinjection)

15 min Uptake (\%)

$<60 \%$ (12 segments)

$60 \%-80 \%$ (34 segments)

$72 \pm 10$

$82.1+7.7$

$73.9+7.3 \mathrm{NS}$

$>80 \%$ (53 segments)

$95.1 \pm 8.9$

$95.3+7.9$

We conclude that the relative uptake of IMHA is similar 15 and 45 minutes after intravenous injection in the three groups of segments. Further studies should focus on segments with lower thallium uptake (i.e. lower than $50 \%$ ) in which the uptake at 45 minutes could be higher than at 15 minutes. 
Sunday, 21 August - Wednesday, 24 August 1994

401

P.Bräutigam, E.Földi, T.Krause, E.Moser

Dept. of Nuclear Medicine, University of Freiburg, Germany

Földiklinik, Hospital of Lymphologic Diseases, Hinterzarten, Germany

\section{CONTRIBUTION OF SUBFASCIAL LYMPHOSCINTIGRAPHY TO THE ASSESSMENT OF THE LOWER LIMB EDEMA}

Aim: The increase of the interstitial pressure in the subfascial compartment and the fibrosis of the fascia in Iymphedema of the lower limb are well known findings and supposed to be the results of a decompensation of the subfascial lymphatics. The aim of this study was to evaluate the function of the subfascial lymphatics in edema and lymphedema by quantitative lymphoscintigraphy (QLS). Method: 20 healthy individuals and 19 patients with lower limb edema received QLS using ${ }^{99 \mathrm{~m}}$ Tc labelled nanocolloid. A wholebody scintigram was acquired $2 \mathrm{~h}$ after injection into the subfascial/epifascial compartment and after standardized stimulation of the lymphatics. The percent uptake of the colloid into the lymph nodes served as a functional parameter. Patients with edema of the lower limb were compared with normal subjects. Results: In 18 legs (12 patients) with lymphedema (stage 1 and 2) the subfascial lymphatic compartment was scintigraphically compromised in 16 legs (10 patients), the epifascial compartment only in 11 legs ( 7 patients). 2 patients with intermittent, mild lymphedema demonstrated normal findings. In 5 patients with lower limb edema ( $1 \times$ phlebedema, $4 \times$ cyclic idiopathic edema), functional disturbance of the lymphatics could be excluded by QLS, 3 of 4 patients with cyclic idiopathic edema demonstrated an increased uptake corresponding to a high volume insufficiency. Conclusion: The presented results demonstrate, that QLS allows an accurate functional assessment of the lymphatics in lower limb edema. In lymphedema, evaluation of the subfascial lymphatics by QLS is more sensitive detecting a functional disturbance than the evaluation of the epifascial system.

402

W. G. Martin, H. Bartels, Ch. Laubenbacher, K. T. E. Beckurts, J. R. Siewert, M. Schwaiger

Nuklearmedizinische Klinik und Chirurgische Klinik, Klinikum rechts der Isar, Technische Universität München, Germany

\section{C14-AMINOPYRINE BREATH TEST (ABT) AS A PREDICTOR OF POSTOPERATIVE MORTALITY}

Substantial loss of liver function is associated with increased postoperative mortality. The purpose of this study was to assess the predictive value of $A B T$ for postoperative mortality.

We studied 1491 patients between January 1, 1990, and December 31, 1993, with elective abdominal surgery. In all patients liver function was assessed by ABT prior to surgery.

All over mortality rate was $5.8 \%$ (87 pt). Preoperative ABT were significantly lower in nonsurvivors $(0.46 \pm 0.23$ [\% dose]) than in survivors $(0.53 \pm 0.21[\%$ dose]). Highest mortality rate $(33 \%)$ was seen in patients with ABT values < $0.1[\%$ dose], followed by a mortality rate of $8.2 \%$ at $A B T$ values between 0.1 to 0.19 [\% dose], and a rate of $5.5 \%$ at ABTs between 0.2 to 0.29 [\% dose]. Mortality rate was $4.4 \%$ in patients with normal liver function (ABT $\geq 0.6[\%$ dose $]$ ).

Mortality is significantly higher below, than above a separator point for the ABT at 0.4 [\% dose]. In patients dying from septical multiple organ failure ABT values were significantly lower than for other death reasons. Nonsurvivors had significantly more postoperative days at intermediate care than survivors (22.6 \pm 23.5 versus $5.2 \pm 6.9$ days).

The results of the study suggest that patients with low $A B T$ values have an increased mortality and should be followed closely for hepatic failure. Patients with normal ABTs tolerate elective surgery well. Surgery in patients with ABTs $<0.1[\%$ dose] is associated with extremely high mortality and should be avoided whenever possible.
403

M. Mysliveček, J. Králik, P. Koranda, C. Neoral V. Huß̧́k

Depts. of Nuclear Medicine and Surgery, University Hospital, Olomouc, Czech Republic

FOLLOW-UP OF LIVER PERFUSION IN PREHEPATIC PORTAL HYPERTENSION BY MEANS OF HEPATOBILIARY SCINT IGRAPHY - CLINICAL AND EXPERTMENTAL STUDY

We focused on the use of dynamic hepatobiliary scintigraphy/DHbSc/ in the follow-up of liver perfusion in patients with prehepatic portal hypertension /PPH/. Parameters of $99 \mathrm{mTc}$ HIDA transport rate were evaluated on timeactivity curves in a liver region of interest. The study was besed on the presumption that changes in extraction of radioactive pharmaceutical/RP/ by the liver and thas the shape of liver curve at DHbSc in the PPH patients with intact liver parenchyma correlate with changes of blood flow in the liver. This presumption was verified experimentally in dogs before and after artificial induction of $\mathrm{PPH}$, and in the group of $23 \mathrm{PPH}$ patients with either spontaneously developed collaterals or established portosystemic /PS/ shunt. In animal experiments, the curves of PPH-induced dogs showed a delayed RP transport. Similar liver curve pattern was observed in $\mathrm{PPH}$ patients with spontaneously developed collaterals. In patients with PS shunt the pattern of iiver curves was close to normal values. The results obtained allow to conclude that patients with PS shunt have a better liver perfusion than patients with spontaneousiy developed collaterals. Hepatobiliary scintigraphy seems to be a suitable indirect marker of the state of liver perfusion in patienst with PPH. It allows the follow-up of the disease and selection of adequate therapy.

\section{4}

A. Schomburg, C. Menzel, K. Brensing, F. Grünwald, J. Görich, T. Sauerbruch, H. J. Biersack

Depts. of Nuclear Medicine, Internal Medicine, and Radiology, University of Bonn, Bonn, Germany HEPATIC PERFUSION SCINTIGRAPHY (HPS) PRIOR TO AND AFTER TRANSJUGULAR INTRAHEPATIC PORTOSYSTEMIC SHUNTS (TIPS) IN PORTAL HYPERTENSION (PH)

TIPS-procedures are performed with an increasing frequency, and they are follwed by profound changes of liver hemodynamics; however, the role of HPS in these patients (pts.) has still to be established.

By means of time-activity curves after IV bolus injection of $555 \mathrm{Mbq}$ Tc-99m-pertechnetate we evaluated arterial and portal blood flow in the liver and calculated the relative portal perfusion index in 26 pts with portal hypertension [ $19 \mathrm{~m} ; 7 \mathrm{f}$; mean age, 52 (range, 23-81) y; diagnoses: ethylic cirrhosis (cirrh.) 17 pts, post-hepatitic cirrh. 6 pts, primary biliary cirrh. 2 pts, cryptogenic cirrh. 1 pt].

Before TIPS, the relative portal blood flow was significantly reduced compared to normal controls [mean, $30(8-56)$; ; normals $\geq 60$ \% ]. $13(5-20)$ days after TIPS, up to now 7 pts. were reevaluated and showed a further reduction of portal flow [mean 47 (31-74) $:$ of pretherapeutical levels] in 5 pts, while there was no significant change in 2 pts.

Our preliminary data show that (1) HPS can be sensitively used for the monitoring of TIPSinduced alterations in hepatic perfusion, and (2) after TIPS relative portal perfusion is further decreased in some patients when compared to pretherapeutic levels. 
405

F.D. Maul, A. Benjaminow, A. Hellstern, A. Zöller, R. Standke, D. Hellenbrecht, G. Hör

Divisions of Nuclear Medicine, Gastroenterology (Medical Clinic II), and Pharmacology, J. W. Goethe-University, Frankfurt/Main, Germany

SPLIT FUNCTIONS OF HEPATOBILIARY SCINTIGRAPHY VS. BIOCHEMICAL LIVER TESTS: COMPETING OR COMPLEMENTARY?

The objective of our investigation was to compare split functions in hepatobiliary scintigraphy by means of quantitative biochemical liver tests in patients with cirrhosis (according to Child classification $A$ and $B$ ). We investigated 37 patients: 18 suffering from biliary cirrhose, 7 from alcohol abuse and 12 from various other forms of liver cirrhosis. We applied 80 to $150 \mathrm{MBq}$ Tc-99m-bromofenine utilizing an own aquisition and processing program for quantitative hepatobiliary scintigraphy. The half live of uptake (HLU) as a clearance equivalent and the mean parenchymal transit time (MPTT) were calculated. Biochemical liver tests were: Imidocyanine green clearance (ICG), Antipyrine clearance, and Mono-ethyl-glycine-xylid concentration. A high correlation was found between all biochemical liver tests $(p<0.0001)$. The correlation between HLU and ICG was comparable high (rho $=0.68, p<0.0001$ ). Correlation between $H L U$ and the other biochemical liver tests were less but still significant. MPTT did not correlate neither with biochemical liver tests nor with HLU. We conclude that in patients with impaired liver function the clearance equivalent HLU correlates with the biochemical clearance parameters. MPTT however, which is also known to be a sensitive parameter to determine a reduction of liver function represents an additional functional parameter reflecting the reduction of transport function, and thus is complementary to the clearance function.

\section{6}

V.Artiko, M.Petrovic, M.Milicevic and K.Kostic institute of Nuclear Medicine, Medical School and

Clinical Center, Beograd - yu

\section{GALL.BL.ADDER HOTILITY IN VARIOUS FUNCTIONAL, DISORDERS} ESTIMATED BY INFUSTON CHOLESCINTYGRAPHY

The aim of the study is the assessment of the gallbladder (GB) contractile function in 8 controls (Cs), 6 patients with hypotonic/atonic GB (BD), in 6 patients with $\mathrm{GB}$ cholecystitis and calculosis $(\mathrm{CC}$ ) and especially in the postgastrectomy patients: 10 early (EPG) and 10 late (IPG) after total gastrectomy as well as 4 after Billroth I (partial) resection (Bl). The study was performed with Rota camera and Micro Delta computer during $(3 \mathrm{~h}, 10 \mathrm{ml} / \mathrm{h})$ infusion of 150 $\mathrm{MBq}, 0.25 \mathrm{mg} / \mathrm{ml}$ 99m-TC-EHIDA, preceeded by a loading dose, with two eggs given in 120. min. After generation of the liver and GB TA curves, corrections for the decay and liver radioactivity in the $G B$ region was made. In CS, emptying time (ET) was $X=37.8 \mathrm{~min}+1-$ 11.4 ) ejection fraction (EF) $X=76.7 \%+1-17.9$ ) and ejection rate (ER) $X=2.3 \% / \mathrm{min}+/-0.8$. On the contrary in $B D$ only filing phase was registered till the in BD, only filling phase was registered till the end of the study. In comparison to CS, in CC, $(X=47.2 \%+1-6.9, p<0.01)$ and $E R$ decreased $(X=1.1 \%$ mint/-0.4, $p<0.01$ ) showing impaired $G B$ motility, caused by fibrosis, thickening of the GB wall and lower number of CCK receptors. In EPG, ET (X=27.3min + 10.9) didn't differ from CS and LPG, while $E F$ $(X=28.7 \%+/-10.9)$ and $E R(X=1.1 \% / \mathrm{min}+/-0.5)$ were decreased (PS 0.01). However, in LPG, ET (X=39.6min $+/-20.0)$. EF $(X=73.3 \%+/-11.3)$ and $\mathrm{ER}(X=1.8 \% / \mathrm{min}+/-$ $0.6)$ didn't differ (p) 0.05 ) from CS, Also, in BI

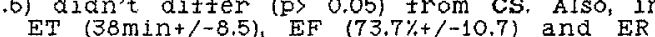

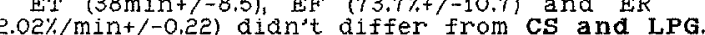
in conclusion, after total gastrectomy with vagotomy and excluded duodenal transit, impairement of vagotomy and excluded duodenal transit, impairement of the GB motility early and recovery of the physiolog contractile function late after operation might mechanisms (CCK). In the patients with partial gastrectomy, without vagal denervation and preserved duodenal tranzit, GB motilly remains undisturbed.
407

E. Øster-Jergensen, P. Thye-Ronn, N. Qvist, S.A. Pedersen, L. Rasmussen \& C.P. Hovendal.

Medical Dept., Assens Hospital, Dept. of Nuclear Medicine, Dept. of Medical Endocrinology \& Dept. of Surgical Gastroenterology, Odense University Hospital, DK-5000 Odense C, Denmark.

SIMULTANEOUS RECORDING OF GASTRIC EMPTYING AND BILE KINETIC IN DIABETICS AND NON-DIABETICS.

Gastric emptying (GE) as well as gallbladder (GB) emptying (GBE) may be delayed in diabetics. It is uncertain whether this can be ascribed to augmented blood glucose (BG), autonomous disease or other causes. We examined 4 non-diabetics $(\mathrm{N}), 4$ non-insulin dependent diabetics (NIDDM) and 8 insulin-dependent diabetics (IDDM), all healthy males. Duration of diabetes were $<6$ years, $\mathrm{HbAlc}<10 \%$, no signs of retinal or kidney complications - other late complications, e.g. neuropathy, being unlikely. GE was examined by a $100 \mathrm{~g}$ omelet $(1400 \mathrm{~kJ}, 60 \%$ fat $)$ tagged with $40 \mathrm{MBq} 99 \mathrm{mTc}$ sulphur colloid and $150 \mathrm{ml}$ water mixed with $8 \mathrm{MBq}$ 111 $\mathrm{ln}$-DTPA. GBE was examined by continuous infusion of $40 \mathrm{MBq}$ $99 \mathrm{mTc}$ Mebrofenin per $\mathrm{h}$. Time-activity curves were generated for the gastric area using geometric mean of anterior and posterior gamma camera recordings during $2 \mathrm{~h}$ and for the GB area using anterior recordings during $5 \mathrm{~h}$. BG was measured every $15 \mathrm{~min}$. Gastric retention of solids at $2 \mathrm{~h}$ ), was $21-57 \%$ in N, $42-78 \%$ in NIDDM and $19-91 \%$ in IDDM. GBE began 0-15 min after the meal in N, 5-25 min in NIDDM (absent in 1 subject) and 0-50 min in IDDM. The GBE rate, (percent of maximum per min from maximum to constant refilling) was $0.76-0.87 \% / \mathrm{min}$ in $\mathrm{N}$, $0.58-0.81 \% / \mathrm{min}$ in NIDDM and $0.23-0.78 \% / \mathrm{min}$ in IDDM (in 1 subject a net filling was observed). Following GBE a constant refilling of the GB appeared after 110-165 min in N, 150-175 min in NIDDM and 134-224 min in IDDM. Three IDDM differed from the rest in having preliminary refilling and secondary emptying periods. GE was delayed (76-91\%), GBE began late (20-50 min), net GBE was scarce $(0.23$ and $0.41 \% / \mathrm{min})$ or positive $(+0.33 \% / \mathrm{min})$. But BG in the group with abnormal emptying pattern was 4.2-16.9 mmol/l and differed not significantly from the group with normal pattern (BG 3.0-16.5 mmol/l). Augmented BG may delay .GE (and consequently GBE), but other causes must be sought; besides autonomous neuropathy, e.g. hormonal alterations.

408

D. Le Guludec, F. Delatour, R. Lebtahi, M.C. Peker, D. Daou, M. Merouche, M. Faraggi, B. Rouveix. Mèdecine Nucléaire, Hôpital Bichat, and INSERM 13, Paris, France.

SCINTIGRAPHIC EVALUATION OF EFFECTS ON GASTRIC EMPTYING OF TWO ORAL ERYTHROMYCIN ETHYLSUCCINATE FORMULATIONS.

The administration of erythromycin and other macrolides may produce gastrointestinal dose-dependent side effects due to a contractile activity of the smooth muscles. Augmented erythromycin (AE) is an amorphous form of erythromycin ethylsuccinate, with a bioavailability twice better than standard erythromycin ethylsuccinate (SE). AE 500 mg has been shown to be as effective as SE $1000 \mathrm{mg}$, but its tolerance was not evaluated. The aim of this study was to evaluate the prokinetic activity of these two formulations compared to a placebo on gastric emptying using a scintigraphic double isotope technic. This randomized double blind controlled study was performed on 12 male healthy volunteers. Each subjecl was alternatively given AE (500 mg), SE (100mg) and placebo per os during a meal labelled with Tc-99m (solid) and In-111 (liquid). Anterior and posterior $60 \mathrm{sec}-$ images were acquired in supine position over $4 \mathrm{~h}$, and solid and liquid $T 1 / 2$ values were calculated after correction for decay from exponential curve fitting. Clinical toler ance was also evaluated with a semi-quantifative score. Serum concentrations for each product were determined by microbiological method at $0 \mathrm{~h} 15,0 \mathrm{~h} 30,1 \mathrm{~h}, 2 \mathrm{~h}$ $3 h, 4 h, 6 h, 12 h$ after dosing. Statistical analysis was performed using ANOVA. Resulls:

\begin{tabular}{|c|c|c|c|}
\hline & & $T 1 / 2$ solid (min) & $T 4 / 2$ liquid (min) \\
\hline AE $500 \mathrm{mg}$ & $(n=12)$ & $88 \pm 22$ ** & $67 \pm 17$ \\
\hline SE $1000 \mathrm{mg}$ & $(n=12)$ & $94 \pm 25^{* \star}$ & $70 \pm 20^{* *}$ \\
\hline Placebo & $(n=12)$ & $130 \pm 38$ & $85 \pm 24$ \\
\hline
\end{tabular}

No difference in pharmacokinetic profile and clinical tolerance were found between $\mathrm{AE}$ and SE.

Conclusion: When compared to placebo, a single oral therapeutic dose of the two forms induced a dramatic and similar increase in gastric emptying. It confirms the interest of such a technic to "objectively" screen the gastrokinetic properties of macrolides or other drugs, reflecting their clinical tolerance. 
409

S. Adams, B. Braden, F.D. Maul, B. Lembcke, W.F. Caspary, G. Hör

Dpts. of Nuclear medicine and Gastroenterology, University Hospital Frankfurt/Main, Germany

Noninvasive ${ }^{13} \mathrm{C}$-acetate breath test for measuring gastric emptying: validation by simultaneous $99 \mathrm{~m}$ Tc-functional scintigraphy and $13 \mathrm{CO}_{2}$ breath test in dual isotope technique

Aim: ${ }^{13} \mathrm{C}$-acetate is a substrate with a low molecular weight which is rapidly absorbed and metabolized to ${ }^{13} \mathrm{CO}_{2}$ at once being emptied from the stomach. The ${ }^{13} \mathrm{C}$-acetate breath test should be validated by comparison to an established scintigraphic method $\left({ }^{99 \mathrm{~m} T c}\right.$-radiolabeled test meal as the gold standard) for determing gastric emptying time of liquids.

Method: 22 patients (14 men and 6 women) underwent gastric emptying studies for medical indications and received a standard test meal ( 60 grams of rolled oats in $75 \mathrm{ml}$ milk) containing $150 \mathrm{mg}$ of the non radioactive ${ }^{13} \mathrm{C}$ acetate and $45 \mathrm{MBq}$ of Technetium- $99 \mathrm{~m}$ labeled human albumin colloids [Solco Nanocoll, Biomedica, Düsseldorf]. Breath samples were collected before (baseline) and after receiving the test meal at 10 minutes intervals for four hours. The samples were analysed by isotope ratio mass spectrometry. The results were expressed as delta over baseline. The ${ }^{13} \mathrm{CO}_{2}$ elimination was determined as percentage of the total ${ }^{13} \mathrm{C}$ dose administered. The gastric emptying time (half time $=t 1 / 2$ ) of the radiolabeled human albumin colloids was obtained by gamma camera imaging with online computer system.

Results: The ingestion of unlabeled test meal did not cause a statistically significant elevation of the non radioactive ${ }^{13} \mathrm{C}$-isotope. 30 minutes after the preparation of the test meal $39 \%$ of the radiolabeled albumin colloids remained in the solid phase, which was shown by centrifugation and separated detection in liquid and solid phases. The scintigraphic half time of the liquid phase reached from 5.5 to $44 \mathrm{~min}$. Ten patients had normal values of the scintigraphically obtained emptying time (lees than $20 \mathrm{~min}$.) corresponding to $13 \mathrm{CO}_{2}$-peak maxima within $50 \mathrm{~min}$ (30-50 min.). The peak maxima of all other patients with delayed scintigraphic emptying rates appeared later than 50 minutes $(60-80 \mathrm{~min}$.). The percentage of $13 \mathrm{C}$ recovery after 4 hours ranged from 21 to $31 \%$ (mean $25.1 \%+/-4.7 \%$ ). There was a linear correlation of the appearance ${ }^{13} \mathrm{CO}_{2}$-peak maxima in the breath test and scintigraphic emptying time $t 1 / 2(Y=1.1 x+29.4 ; r=0.93)$. Conclusion: The ${ }^{13} \mathrm{C}$-acetate breath test shows an excellent correlation with the gastric emptying time by simultaneous $99 \mathrm{~m}$ Tc-functional scintigraphy (gold standard) in dual isotope technique

410

E. Derebek, H. Durak, M. YImaz, E. Tankut, H. Akbaylar, E. Metin

Department of Nuclear Medicine, Division of Gastroenterology, Dokuz Eylül University, Izmir - Turkey

SIGNIFICANTLY INCREASED GASTRIC UPTAKE OF TL - 201 DURING MYOCARDIAL SPECT : Its importance and possible mechanism.

It is known that slight or moderate gastric uptake of $\mathrm{TI}-201$ can be observed in rest or dipyridamole TI-201 myocardial imaging, but not during treadmill excercise studies due to diminished splanchnic blood flow. In this study we aimed to investigate the mechanism of significantly increased gastric uptake of TI-201 during excercise or dipyridamole TI201 myocardial SPECT, which is observed in some patients taking $\mathrm{H}_{2}$ -antagonists.

We have observed significantly increased gastric uptake of Tl201 in a patient who had undergone dipyridamole myocardial SPECT. This patient was on $\mathrm{H}_{2}$-antagonist. theraphy (ranitidine $300 \mathrm{mgr} /$ day) for two months. $\mathrm{H}_{2}$-antagonist treatment started on another nine valuntary patients who had been referred for myocardial SPECT. $\mathrm{H}_{-}$antagonist (ranitidine $300 \mathrm{mgr} /$ day for three patients; famotidine 40 $\mathrm{mgr} /$ day for six patients) were given for 15 days. Thereafter, dipyridamole TI-201 myocarcial SPECT was performed in two patients using ranitidine and three patients using famotidine. Treadmill excercise TI-201 SPECT was performed in the remaining four patients. Myocardial and gastric uptake of Tl-201 was visualy evaluated in the raw projection, short axis and vertical long axis images.

We observed significantly increased gastric uptake of TI-201 in all patients. But, increased gastric uptake was more marked in patients on famotidine than those on ranitidine treatment, irrespective of type of stress testing. In conslusion: 1) $\mathrm{H}_{2}$-antagonist which are $\mathrm{K}^{+}-\mathrm{H}^{+}$pomp inhibitors may increase gastric uptake of Tl-201. 2) Significantly increased gastric uptake of $T /-201$ may be due to increased blood flow to the gastric mucosa, and/or related to intracelluler homeostatic mechanisms which try to balance the intracellular $\mathrm{K}^{+}$content. 3) $\mathrm{H}_{2}$ antagonist use may be suspected, if significantly increased gastric uptake of TI-201 is seen during myocardial SPECT.
411

F.M. Akgür, O. Yenici", M. Olguner, M. Gökden ${ }^{* *}$, T. Aktuğ, M. Yilmaz *, G. Ataç**.

Depts of Pediatric Surgery, Nuclear Medicine ${ }^{*}$ and Pathology ${ }^{* *}$, Dokuz Eylül University, Medical Faculty, Izmir, Turkey. THE EFFECT OF ALIOPURINOL ON HYPOPERFUSION ENCOUNTERED AFTER CORRECTION OF INTESTINAL VOLVULUS

After reversion of blood flow following prolonged periods of ischemia, the blood flow starts for a few seconds and is reduced after then: this is called "no-reflow phenomenon". Allopurinol has been shown to prevent the occurence of this phenomenon in organs other than intestine. An experimental study was conducted to investigate the effect of allopurinol treatment before correction of intestinal volvulus in rabbits. Group 1: Intestinal blood flow (IBF) was measured using radiolabeled erthrocytes (in vivo Tc $99 \mathrm{~m}$ PYP with GE Starcam 4000 XRT) Group 2: $720^{\circ}$ intestinal volvulus was created, IBF was measured 6 hours later. Group 3: Intestinal volvulus was created, intraperitoneal $\mathrm{NaCl}(0.098)$ was injected 60 min. before correction of volvulus. IBF was measured after correction of volvulus. Group 4: The procedures performed as the group 3 , but allopurinol (200 $\mathrm{mg} / \mathrm{kg}$ ) was injected instead of $\mathrm{NaCl}$. Intestines were harvested for histopathologic examination. IBF stopped following volvulus for $6 \mathrm{hrs}$. IBF was subnormal following correction of volvulus compared to basal level $(p<0,01)$. However IBF was higher in the allopurinol treated group when compared to placebo $(p<0,01)$. Histopathologic examination showed that intestinal volvulus lead to histologic injury. Histologic injury was more pronounced in the devolvulus group. Histologic injury in allopurinol treatment group was less severe compared to placebo group. It is concluded that allopurinol pretreatment prevents intestinal hypoperfusion thus histologic injury encountered after correction of intestinal volvulus lasting 6 hours.

412

P. de Lima, J. Martin-Comin; J. Xiol; M. Roca; M. Castell; $\mathrm{J}$. Mora and $M$. Ramos Serveis de Medicina Nuclear $i$ Gastroenterologia. csuB Hospital Princeps d'Espanya. Barcelona. Spain.

THE $\mathrm{Se}^{75}$-HCAT, TEST:A DIAGNOSTIC AID IN DIARRHOEIC SYNDROMES.

The $\mathrm{se}^{75}$-HCAT test is a well established procedure to study ileal absorption of fatty acids. The aim of this work was to evaluate the usefulness of the method in selecting treatment of patients with diarrhoeic syndrome.

We retrospectively reviewed 17 test done in patients with diarrhoeic syndrome including 6 patients who have undergone diarrhoeic syndrome including 6 pati

Procedure: A capsule containing $10 \mu \mathrm{Ci}$ of $\mathrm{se}^{75}-\mathrm{HCAT}$ was administered and the percentage of abdominal retained activity (ARA) was measured at 4 and 7 days using a collimated gammacamera. Normal values of ARA were $>25$ and $>15$ at 4 th and 7 th days respectively.

Results: 1) ileal resection: All patients showed a very diminished abdominal retention at 4 and 7 days $($ values ranging from 0 to $3 z^{8}$ ). Two of them were treated with cholesteramine and reduced the number of depositions to $1-$ 2 /day. 3 out of the 4 remaining untreated patients did not show any change in their voiding frequency, 2) diarrhoeic syndrome: 6 out of the 11 patients showed a marked decrease of $\mathrm{Se}^{75}$ abdominal retention at 7 days p.a. (range: 0-8 \&). Four of them were treated with cholestiraminge: 0-8 8 ). Four of them were treated with cholestiramine and reduced patients improved with diet. In the remaining 5 patients with normal ARA, reduction of voiding frequency was obtained eithef by diet and methylcellulose treatment or was selflimited.

In summary: Though the short number of patients studied it seens that patients with diarrhoea and abnormal se ${ }^{75}$ - it test should be treated with cholesteramine while if the test is normal other treatment should be loked for. 


\section{3}

A Notghi, N Smith, $\star_{R}$ Hutchinson, $\star_{D}$ Kumar and LK Harding

Physics \& Nuclear Medicine, Dudley Road Hospital, \& *Department of Surgery, Queen Elizabeth Hospital, Birmingham, UR.

REPEATABILITY OF SEGMENTAL COLONIC TRANSIT SCINTIGRAPHY

The outcome of segmental colonic transit studies is used to decide on patient treatment and to evaluate its outcome. However there are no published data on the reproducibility of these studies. We have repeated colonic transit studies in 9 patients, 7-19 months apart. Five patients (4 IBS, 1 diabetic) with no treatment between the two studies, had no change in the patterns of colonic transit (3 normal, 1 fast, 1 right sided delay). In 3 of 4 patients, where the study was repeated after surgical treatment, the colonic transit patterns had changed:

\begin{tabular}{|l|l|l|l|}
\hline Clintcal Problem & Operation & \multicolumn{1}{|c|}{$\begin{array}{c}\text { Colonic } \\
\text { Transit 1 }\end{array}$} & $\begin{array}{c}\text { Colonic } \\
\text { Transit 2 }\end{array}$ \\
\hline Prolapse & Rectopexy & right delay & normal \\
\hline Incontinence & Rectopexy & fast transit & right delay \\
\hline Prolapse & $\begin{array}{l}\text { Left hemi- } \\
\text { colectony }\end{array}$ & right delay & right delay \\
\hline $\begin{array}{l}\text { Chronic } \\
\text { constipation }\end{array}$ & $\begin{array}{l}\text { Left hemi- } \\
\text { colectomy }\end{array}$ & right delay & $\begin{array}{l}\text { generalised } \\
\text { delay }\end{array}$ \\
\hline
\end{tabular}

Colonic transit studies using radionuclides give consistent results and clearly demonstrate changes after surgery.

\section{4}

A Notghi, *R Hutchinson, NJ Tulley, *D Kumar and LK Harding

Physics \& Nuclear Medicine, Dudley Road Hospital, \& *Department of surgery, Queen Elizabeth Hospital, Birmingham, UK.

COLONIC TRANSIT IN IRRITABLE BOWEL SYNDROME

Management of patients with irritable bowel syndrome (IBS) is difficult. Objective assessment of colonic transit may help in correct management of these patients.

segmental colonic transit was measured in 50 patients (4 male) with IBS to correlate the symptoms with colonic transit patterns. $2 \mathrm{MBg}$ lilin absorbed to resin in a $\mathrm{pH}$ sensitive capsule was given and the percentage of administered activity was calculated for 5 regions in the colon using decay corrected geometric mean counts. Presenting symptoms were: pain, constipation (Ct) and diarrhoea (Dh). Five patterns of colonic transit were identified.

Transit Pattern

\begin{tabular}{|l|c|c|c|c|c|c|}
\hline Presentation & Fast & Normal & $\begin{array}{c}\text { Right } \\
\text { Delay }\end{array}$ & $\begin{array}{c}\text { General } \\
\text { Delay }\end{array}$ & $\begin{array}{c}\text { Left } \\
\text { Delay }\end{array}$ & $\begin{array}{c}\text { No. of } \\
\text { patientB }\end{array}$ \\
\hline Pain & $14 \%$ & 728 & 148 & 08 & $0 \%$ & 7 \\
\hline Constipation & $25 \%$ & $62 \%$ & 38 & 78 & 38 & 29 \\
\hline Diarrhoea & $36 \%$ & $50 \%$ & 78 & $7 \%$ & 0 & 14 \\
\hline Total & $26 \%$ & $60 \%$ & $6 \%$ & $6 \%$ & 28 & 50 \\
\hline
\end{tabular}

Most of the patients with IBS have normal colonic transit. Fast colonic transit was most common in patients with Dh (36\%), but was also seen in $25^{\circ}$ of patients with. Ct. Interestingly delayed colonic transit occurred in 2 of the 14 patients with $D h$ and only 4 of 29 patients with Ct. Functional studies are needed for correct assessment of transit in IBS as symptoms are unreliable.
415

I. Mairal; P. de Lima; M. Roca; C. Baliellas; J. Xiol; Y. Ricart and $\mathrm{J}$. Martin-Comin.

Serveis de Medicina Nuclear y Gastroenterologia. CsUB Hospital Princeps d'Espanya. Barcelona. Spain.

SIMULTANEOUS ADMINISTRATION OF ${ }^{11}$ In-IgG AND ${ }^{99}$ TC-HMPAO LABELED LEUCOCYTES IN INFLAMMATORY BOWEI DISEASE (IBD).

The aim of the work was to study the clinical usefulness of In-IgG in diagnosis and in evaluating the extension of IBD.

15 patients ('s males), mean age 46.8 years have been studied. IBD was suspected in all cases and diagnosis was obtained by endoscopy and/or surgery.

About $1 \mathrm{mci}$ of ${ }^{11}$ In-Fuman plyclonal-IgG and $5 \mathrm{mci}$ of $99 \mathrm{~m} \mathrm{Mc}-$ HMPAO labeled autologous leucocytes was simultaneous administered to all patients. Subsequently abdominal and caudo-craneal scans were obtained at $30 \mathrm{~min}, 3-4 \mathrm{~h}$ and $18-$ $24 \mathrm{~h}$ p.i. Images were evaluated by 2 independent observers knowing only the clinical suapicion of IBD.

In 10 patients IBD was diagnosed. All showed a positive In 10 patients IBD was diagnosed. All showed a positive leucocyte scan and only 7 were positive in the IgG scan. In IgG and leucocyte scans were normal. Sensitivity and IgG and leucocyte scans were normal. Sensitivity and specificity were 100
respectively for IgG.

Concerning extension of the disease, the leucocyte scan localized 21 involved segments while only 9 were identified by the IgG (all were also seen in the leucocyte scan). The activity in the involved segments was always lower in the IgG scan.

In conclusion: ${ }^{111}$ In-IgG seems to be a valuable but less sensitive method for the diagnosis of IBD than $99_{\text {TC-HMPAO }}$ leucocytes. The method may be usefull when leucocyte labeling is not avaliable or to avoid blood handling in labeling is not avaliable or to avoid blood handling in AIDS, .... Nevertheless it does not seem
evaluate the extension of the disease.

416

I.Virgolini, M.Banyai, Ch.Konnaris, F.Rauscha, S.R.Li, A. Kurtaran, H.Graf, P.Angelberger,

Departments of Nuclear Medicine, Cardiology and Radiochemistry, Research Center Seibersdorf, Austria.

IMAGING OF HUMAN VENOUS THROMBOSIS WITH ${ }^{123}$ I-LABELED RECOMBINANI HUMAN TISSUE TYPE PLASMINOGEN ACTIVATOR

The diagnosis of human thrombi still fails to satigfy the criteria of simplicity and non-invasiveness. In $10 \mathrm{pa}$ tients with deep leg vein thrombosis, in 5 patients with Cimino shunt thrombosis, in 3 patients with vena cava ouperior thrombosis and in 5 patients with thrombophlebitis the in vivo biodiatribution and fibrin localizing capacity of ${ }^{123} \mathrm{I}$-labeled recombinant human tissue type plasminogen activator ( $r h-t P A)$ were investigated. After labeling, in vitro binding atudies with plateletg indicated the same binding behavior and biological activity of unlabeled and ${ }^{123} \mathrm{I}-$ labeled $\mathrm{rh}-\mathrm{tPA}$. ${ }^{183}$ I-rh-tPA bound to a single class of high affinity I-rh-tPA bound to a single class of high affinity binding sites indicating $>90 \%$ ligand specificity. After i.v-injection to patients $(200 \mathrm{MBg} / 2 \mathrm{mg})$ organ distriof ${ }^{123} \mathrm{I}-\mathrm{rh}-\mathrm{tPA}$ occured exclusively in the liver. In all of I-rh-tPA occured exclusively in the liver. In all patients with deep venous leg thrombosis or cimino shunt thrombosis ${ }^{123}$ I-rh-tPA scanning provided excellent imaging of venous thrombi, whereas thrombi of the vena cava accumulated in clots within 5 minutes after injection. AB jugded by ultrasound and/or phlebography no falae positive images were recorded. Blood activity declined rapidly within the first minutes after injection exhibiting a half-life of $6.3 \pm 2.1$ minutes. Thereafter slow elimination occured with a terminal half-life of $>20$ houra. In twelf patients, ${ }_{111}$ In-oxine-platelet imaging hours. In twelf patients, In-oxine-platelet imaging, ${ }^{123}$ I-plasmin or fibrinogen-acanning were compared with

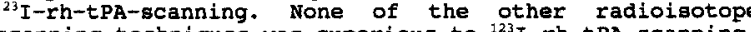
scanning techniques was superious to ${ }^{123} I-r h-t P A$ scanning We conclude that I-rh-tPA scanning could be a safe and 
417

A.Tombácz, M. Köteles, B.Spett*, Gy.A.Jánoki*

Dept. of Nuclear Medicine, Markhot F. County Hosp. Eger. *Natl."FJC" Res.Institute for Radiobiology and Radiohygiene, Budapest, Hungary

INITIAL EVALUATION OF I-123-FIBRINOGEN IN PATIENTS WITH SUSPECTED ACUTE DEEP VENOUS THROMBOSIS

l-123-hFibrinogen study for imaging acute deep vein thrombosis was carried out in comparison with Radionuclide Venography (RNV) performed with Tc-99m-MAA and Color Doppler Sonography (CDS) in 30 patients (pts) with suspected deep venous thrombosis.

Highly purified human Fibrinogen was labelled with $1-123$ using iodogene as oxidation agent. Radiochemical purity was always higher than $95 \%$ and radioisotopic clottability was more than $90 \%$.

After a single bolus injection into the superficial dorsal vein of the strangulated leg; planar scintigraphy of the leg, thigh and pelvis ( 3 regions) were performed immediately, with computer analysis of the 2,4 and 24 hours uptake. Following the $24 \mathrm{hrs}$ imaging, routine three-phase RNV and CDS were carried out. The increase of l-123-hFibrinogen uptake seems to be greater at 4 hrs image than at late image (24 hrs) in pts with anti coagulant therapy.

In 32/126 regions of 21 pts positive findings were obtained in the 1-123-Fibrinogen uptake with good correlation of CDS results. $90 \%$ of the lesions detected by l-123-Fib, did not appear during RNV when Tc-99m-MAA was used. In case of the remaining 9 pts during RNV in 23/26 region as "hot spot" was considered. The RNV indicated the filling defect both in the acute and aged diseases well, but to estimate the proper extension of the fresh and aged thrombi the CDS seems to be superior.

Based on our initial results we can concluded that I-123Fibrinogen is a promising agent for detection of fresh deep venous thrombosis.

\section{8}

D. Sandrock, A. Kühnelt, F.-E. Lüdtke, P. Schuff-Werner, A. Kögler, V. Ivancevic, D.L. Munz, D. Emrich

Departments of Nuclear Medicine, Surgery and Clinical Chemistry, Georg-August-University, Göttingen, Germany

LONG-TERM RESULTS OF LIVER-SPLEEN SCINTIGRAPHY AFTER SPLEEN SURGERY

The aim of this study was to investigate, if the results of quantitative liver-spleen scintigraphy were representative of the functional long-term outcome in patients after spleen surgery.

Overall, 59 patients ( 18 women, 41 men, age 18 - 79 years) were studied 11 years (mean) after spleen surgery. 43 patients had splenectomy (11 of them with autologous replantation), and 16 had spleen-preserving surgery after injuries. Quantitative liver-spleen scintigraphy (planar anterior and posterior views) was performed 30 min after i.v. injection of $185 \mathrm{MBq}$ Tc-99m tin-colloid and splenic uptake (in \% of the administered dose) and liver:spleen ratio were calculated. These values were compared with immunologic and hematologic parameters such as Howell-Jolly-bodies and pitted erythrocytes.

The splenic uptake had a better correlation with the immunologic and hematologic parameters than the liver:spleen ratio. Patients after spleen-preserving surgery had according to a normal functional status of the spleen (as defined by normal immunologic and hematologic parameters) a splenic uptake of $3.0+/-1.4$, which is in the normal range. All of the 10 patients with replantation revealed splenic activity (with large interindividual scatter $0.8+/-0.7$ ). After splenctomy, no activity was seen in 18 cases, minimal splenosis in $15(0.3+/$ 0.3 ) corresponding to defects of the functional status.

In conclusion, quantitative liver-spleen scintigraphy is a simple method for the assessment of the splenic functional status in the long-term follow-up of patients after spleen surgery.
419

M.Todorovic-Tirnanic, Z.Rolovic*, V.Obradovic, V.Bosnjakovic

Nuclear Medicine Institute, Hematology*

Institute, Clinical Center of Serbia, Belgrade

EFFICACY OF THE SPLENECTOMY IN CHRONIC ITP PREDICTABLE OR NOT?

The aim of this paper was to estimate the possibility of predicting the efficacy of the splenectomy in chronic ITP patients. The platelet lifespan (IS), sequestration site (SS), production (P), turnover, count, myelogram, patient's age and sex were taken into consideration as possible predictive factors.

Autologous platelet labelling with 111-Inoxinate (Mallinckrodt) was done in 48 chronic ITP patients ( 35 female and 13 male, aged from 5-76 years). Platelet count ranged from 5-140 $x$ $109 / 1$, and LS was $0,4-4,3$ days. The SS was the spleen in 32 patients, the liver in 3 , and mixte secuestration was found in 13 patients. The platelet production index was calculated in 30 patients whose lifespan was equal or longer than 24 hours, as well as the $P$ and turnover. Myelogram was examined in 30 patients. Megacaryocytes (M) were numerous in 19, only present in 8, dysplastic in 4, and in one patient small number of $M$ was found.

The solenectomy result (SR) was excellent in $50 \%$,good in $32 \%$ and bad in $18 \%$ of ITP patients. Spearman's correlation coefficient showed that highly significant correlation existed between the SS and the SR, while the correlation was not found between the SR and myelogram, P, IS, turnover, count, age or sex of patient.

The platelet SS is a good predictive factor for the efficacy of splenectomy in chronic ITP.

\section{0}

\section{I.Kostadinova}

Clinical Lentre of Nuclear Medicine ano Radiotherapy Medical Faculty, Sofia, Bulgaria

FHST METHOD FOR THE STUDY OF ERYYTHROKINETILS IN PATIENTS / PtS / WITH HAEMLLYTIC ANAEMIA

It is known that in following up the erythrokinetics, an information about the localization and the degree of erythrocytes' oestruction is acquired. One disadvantage of the method is the long duration of the investigation. The purpose of the stuoy was to wark out a fast method for the erythrokinetics. We have investigated 41 pts with haemolytic anaemia, whose erythrocytes were labelled with 51Cr-sodium chramate / 4 Meq / with determination of the difference in spleen to liver ratio/ $\mathrm{S} / \mathrm{L}$ ratio/between the third and the first day p.i. as well as $S / L$ ratio at the end of the end of the investigation/between 12-14 day/. Wa have estimated that this difference was on the average 1,12 / above 1,0 in cases with splenic destruction and below 1,0 in cases with liver destruction / and $5 / \mathrm{h}$ ratia at the end of investigation-1,44 with very good correlation betwer them both / $r=0,94 /$. In Pts with indication for splenectomy/s/h ratio above $2-2,5 /$, the differenco between the third and the first day was auove 1,3. It could be concluded that, according to the proposed method, the localization and the degree of erythrocytes' destruction can be determined with high reliability, which speeds up taking decision about eventual splenectomy. 
421

A.R. Casu, G.B. Cuccuru, M.D. Azzena, F. Dore, A. Serra, M. Longinotti, A. Spanu, G. Bertrand, S. Nuvoli, C. Todde, G. Sechi, M. Piga, P. Sulas, A. Falchi, M. Langer and G. Kadeddu.

Institutes of Nuclear Medicine, Haematology, Internal Medicine Universities and Hospitals of Sassari, Cagliari and Milan. Italy METABOLIC BONE DISORDER IN THALASSAEMIA MA.JOR (TH) AND INTERMEDIA (TI). A LOHGITUDINAL STUDY.

Bone disorders have been reported in Thalassaemia but the mechanis is complex and remains controversial. We studied 95 pts, 53 males and 47 females, affected by TM (81 cases) and TI (14 cases), 69 of these (62TM,7TI) over 7yrs. At the first observation TM pts were divided into 3 groups by age: $10\left(9-14\right.$ yrs:n.18), $2^{\circ}(15-18 y r s: n .39), 3^{\circ}$ (19-30 yrs:n.24); TI pts aged 20-42 yrs. TM pts were politransfused while II had never received blood transfusion: 42 age and sex matched controls were also studied. In all cases BMD (mo/cm2) by dual-photon absorptiometry in the distal radius was measured; serum PTH, osteocalcin (0), CT, $1 a, 25(\mathrm{OH}) 203$ and $C A$ and $P$ were also assayed. Both TH groups and $T I$ pts showed significant lower EMD values compared to controls. BMo was lower in $\mathrm{TI}$ than Groups $3 \mathrm{TM}$. Mean Ca was significantly lower andp higher than controls. PIH was within normal range but slightly elevated; hypoparathyroidisn was found in 3 Group 3 Th pts. In 2 of these 0 was also low. Individual 0 levels were variable but on everage lower than in controls, only significantly for $T$ $(4.57 \pm 1.2$ VS $7.04 \pm 1.9 \mathrm{ng} / \mathrm{ml})$. Mean $1 \mathrm{a}, 25(\mathrm{OH}) 2 \mathrm{D} 3$ levels were lower i Thalassaenic ots than controls, sianificantly only in Group 3 TM and II $(27.53 \pm 10.97$ and $18.81 \pm 16.3$ YS $45.94 \pm .7 .01 \mathrm{pg} / \mathrm{ml})$. CT was normal in all cases. After 7 yrs all TM pts, who now belonged to Group 3 , had a further significant BMD decrease in respect of controls as di $T I$. PTH levels decreased below nornal range in 13 TM and 1 II with mean values significantly lower than controls. There was a 4 th hypoparathyroidism case in TM Group. Ca remained low and $P$ high, significantly. 0 and $1 \mathrm{a}, 25$ (OH)203 were confirmed low but significantly only in the latter. Low 25 (OH)D3 levels, assayed only at 2 nd observation, were found both in TM and TI pts. Our data indicate low BMD in thalassaemia already in early age, further decreasing with diseas progression. Both well known local factors and reduced active vit. netabolites synthesis, the latter inducing intestinal Ca absorption decrease and 0 production, may contribute to bone mineralization defect. A progressive serum PIH decrement, presumably due to parathyroid hemosiderosis, nay also play a role in advanced disease stages.

Y. Duman, M. Argon. Z. B. Özan, K. Aktuğlu, S. Kara

Jepts. of Nuclear Medicine, and Urthopedics Ege University, Izmir lurkey

A NEW RADIOPHIARMACEUTICAL , Tc 99m-CITRATE, IN FOLLOW UP OF HEALING FRACTURES

Citric acid plays a spexific physiologic role in bone cell metabolism and bone formation as it is the first product of Krebs cycle. The aim of this study was to investigate the value of Tc $99 \mathrm{~m}$-Citrate in follow up of healing fractures in comparison with Tc $99 \mathrm{~m}-\mathrm{MDP}$

12 patients (pts) with healing fractures (group I), and 15 pts who failed union of the fracture (group II ) were included in the study. All pts underwent planar static imaging $2 \mathrm{hr}$. after IV injection of 640 MBq Tc $99 \mathrm{~m}$ labelled citrate. With a 3 days interval, Tc $99 \mathrm{~m}-\mathrm{MDP}$ bone scintigraphy was also performed.The findings of two scintigraphic studies were evaluated qualitatively and quantitatively by using ROI' $s$ drawn over fracture ( $\mathrm{L}$ ) and conterlateral normal bone (C)

It was noted $5 \mathrm{pts}$ in group I showed positive Tc $99 \mathrm{~m}$-MDP uptake but unexpectedly negative Te $99 \mathrm{~m}$-Citrate uptake. The mean L / C ratios of group I and group II were $1.4 \mp 0.13$ and $2.20 \mp 0.43$ respectively ( $\mathrm{p}(0.0005$ )

In conclusion. citrate compound known to have a value in bone formotion may be a promising agent in monitoring bone healing when labelled whith To $99 \mathrm{~m}$. It was observed that Tc $99 \mathrm{~m}$ citrate images corralated with clinic and radiologic findings of bone repair better than Tc $99 \mathrm{~m}-\mathrm{MDP}$.
423

M.Sahin , I. Bernay, F. Cantürk

Depts. of Nuclear Medicine and Physical Therapy and Rehabilitation, Ondokuz Mayıs University, Faculty of Medicine, SAMSUN, TURKEY

EVALUATION OF JOINTS IN RHEUMATOID ARTHRITIS AND VARIANTS WTTH Tc99m HIG , Tc99m MDP AND Tc99m HSA

Te99m polyclonal immunglobulin (HIG) has been used to detect focal infection and inflammation. We performed a comparative study between Tc $99 \mathrm{~m}$ HIG and Tc99m MDP in the visualisation of inflammed joints in 20 patients with rheumatoid arthritis (RA) and variants of RA. Additionally, in order to show that immunospecific mechanisms are effective besides increased vascular permeability in the accumulation of $\mathrm{Tc} 99 \mathrm{~m} \mathrm{HIG}$, all the joints which accumulated this radiopharmaceutical were imaged with $\mathrm{Tc} 99 \mathrm{~m}$ human serum albumin( HSA) . Tc99m HIG and Tc99m HSA scans were obtained at 2,4 and 24 hours after $5 \mathrm{mCi}$ Tc $99 \mathrm{~m}$ HSA and $9 \mathrm{mCi} \mathrm{Tc} 99 \mathrm{~m} \mathrm{HIG}$. The quantitative analysis was carried out exclusively over joint regions. We conclude that 1 The detection of joint inflammation with $\mathrm{HIG}$ was significantly superior to the conventional bone scan with MDP. 2-The increasing Tc-99m HIG uptake between 4-24 hours in contrary to Tc-99m HSA, accentuates the role of imununospecific mechanism in RA.

424

D.Rubini, G.Rubini, F.Lauriero, A. D'Addabbo.

Dept. of Nuclear Medicine, - University of Bari- Italy

THREE PHASE BONE SCINTIGRAPHY WITH 99mTc-MDP IN INSERTIONAL TENDINITIS.

The term enthesitis is used to describe nonspecfific inflammation occurring at the insertion of a tendon (ligament or capsule) during a rheumatic disorder or as the result of overexertion. Hyperactivity is observed in an enthesitic site in the angiographic, blood pool and late bone phases of the three phase bone scintigraphy. This technique was employed to examine 16 patients with a clinical suspicion of enthesitis (4) and tenosynovitis (12). Serumnegative spondyloarthritis was present in 11 and rheumatoid arthritis in 1, while 4 were free from any underlying disease. Echography was also performed in 14 patients. Three-phase bone scintigraphy with $99 \mathrm{mTC}-\mathrm{MDP}$ was positive in $4 / 4$ subjects with suspected enthesitis (2 achilles, 1 deltoid, 1 intercostals) and $8 / 12$ with suspected tenosynovitis (5 peroneals, 1 posterior tibials, 1 extensores digitorum communes, 1 extensor digiti quinti proprius). The technique proved useful in the diagnosis of enthesitis and tenosynovitis associated with enthesitis, but was insufficient for the diagnosis of tenosynovitis alone. It also disclosed enthesitis in the 2 patients not previously examined ultrasonographically. 


\section{5}

I. Makaiová, A. Sakalová, F. Makai, 5. Kováčová, L. Rajniaková, R. Synak, J. Kaušitz, I. Tomeková

Medical Faculty, National Oncological Institute, Hematologic Clinic, Orthopedic Clinic Bratislava, Slovak republ.

THE REAL ROLE OF THREE PHASE BONE SCINTIGRAPHY IN TUMOURS WITH HIGH OSTEOCLASTS ACTIVATING FACTOR / OAF /

Radionuclide bone angiography or three phase bone scintigraphy was until now used mainly in diagnostics of primary bone tumors, inflammatory bone lesions and rarely for detection of the affliction of soft parts in bone tumors.

In the present work we focused our interest of this procedure on an until now underestimated problem in the diagnosis of metastatic or systemic bone tumors with expressed s. c. asteoclasts activating factor / $\mathrm{DAF} /$. In these the sing of enlarged osteogenesis is not always clear /cold lesions / and therefore the pathologic lesion is confirmed of ten only by a pathologically higher blood flow / f. e. plasmocy toma, lymphoma, thyroid gland Ca, hypernephroma and so on /. For confirmation of this proposal we performed three phase scintigraphy in 1200 patients with above mentioned tumors. The most important group content 250 patients with plasmocytoma, in whom the investigation was performed for differential diagnosis, confirmation of diagnosis and follow up of therapy. In each patients with active plasmocytoma the blood flow was pathologically high, which decreased af ter sufficient therapy.

Conclusion: In diagnosis of bone or bone marrow involvement in tumours with increased osteoclasts activating factor / OAF / the three phase scintigraphy is an indispensable procedure, which increasses the sensitivity and specificity of bone scintigraphy.

\section{6}

\section{P.Prediě, ${ }^{*}$ D.Dodig}

Hospital Celje,Celje,Slovenia. *University Hospital Rebro Zagreb,Croatia

ARE QUANTITATIVE ANALYSIS OF RELATIVE PERFUSION IN ARTERY PHASE (3T) AND SPECT TO CONTRIBUTE TO INCREASED RELIABILITY OF DIAGNOSING ASEPTIC HIP NECROSIS

From the literature the standard 3-phase scintigraphy of aseptic hip necrosis is known for rather high sensitivity yet very low specificity and therefore also low reliability. Since we have been performing the modified examination 3T calculation and SPECT for some years, we tried to find out in a retrospective study the amount of contribution of such modifies study to the reliability of examination.

Material and methods:Our study involved 135pts with scintigraphyc diagnosis of aseptic hip necrosis and 97pts without symptoms of aseptic necrosis. All pts underwent a 3-phase scintigraphy of hips with 3 T calculation from the curve of first dynamic phase as well as SPECT of hips. In the patients with aseptic hip necrosis the process was verified.

Table 1 shows the results of examinations performed in our pts:

SC.Dg.A.H.N.

Others

$\begin{array}{rr}+ & \\ 133 & 2 \\ 1 & 96\end{array}$

The calculated sensitivity amounts to $99.3 \%$, specificity

$98 \%$ and reliability $98.9 \%$. By applying SPECT, we could exactly localize aseptic hip necrosis.

Conclusion:The obtained results of quantitative analysis of relative perfusion in the artery phase (3T) evidence somewhat increased sensitivity, high specificity of examination which allows highly reliable diagnosis of aseptic hip necrosis. SPECT examination of hips performed in addition, however, allows exact localization of the process.

\section{7}

T.Hashimoto, J.Hashimoto, K.Nakamura, A.Kubo

Department of Radiology School of Medicine Keio University

SCINTIGRAPHIC MEANINGS OF THE REFLEX SYMPATHETIC DYSTROPHY SYNDROME OF THE SHOULDER, HAND AND LOWER EXTREMITIES USING 3 PHASE BONE SCANNING. The aim of this study was to evaluate the meanings of 3 Phase Bone Scanning(3PBS)in Reflex Sympathetic Dystrophy (RSD).3PBS was performed in 61 patients suffering from RSD of the shoulder, hand and lower extremities.20m Ci (740MBq) of $\mathrm{Tc}-99 \mathrm{~m}$ MDP was administered intravenously by bolus injection. Dynamic images were acquired simultaneously, 10 minutes later early scan and 120 minutes later delayed scan were acquired.From the point of the duration of the symptoms from the onset, we have divided the patients into 3 stages. $(0-20,20-60$, over 60 weeks)In RI angiography hypervascularity was recognized in $13 / 20$ cases (65 \%) of stage 1 patients, while $1 / 10\left(10 \frac{0}{0}\right)$ and $1 / 5\left(20 \frac{\circ}{0}\right)$ in stage 2 and 3 respectively. In early scan hyperemia was recognized in $31 / 34$ cases $(91.2 \%)$ of stage 1patients, 13/18 $\left(72.2^{\circ}\right)$ in stage 2 and $5 / 8\left(62.5^{\circ}\right)$ in stage 3 .In delayed scan hyperfixation was recognized in $32 / 35$ cases ( $91.4 \frac{0}{5}$ ) in stage $1,17 / 18\left(94.4^{\circ}\right)$ and $5 / 8\left(62.5^{\circ}\right)$ in stage 2 and 3 respectively. But in 2 cases in stage 1 and 2 , although they have examined in short periods from the onset of symptoms, the ischemic changes were seen in RI angiography, early and delayed scan. Their symptoms were resistent for the persistent therapy and showed the bad prognosis. Therefore 3PBS may predict the prognosis as well as provide the useful information regarding the pathophysiological and clinical evaluation of RSD.

\section{8}

F. Montravers ${ }^{1}, N$. Younsil, C. Rousseaul, $S$. Uzan $^{2}$, V. Izrael 3 , J.N. Talbot 1. Services de Médecine Nucléaire ${ }^{1}$, Gynécologie ${ }^{2}$ Cancérologie ${ }^{3}$. Hôpital Tenon, F75020 Paris, France

EVALUATION OF THE USEFULNESS OF SYSTEMMATIC BONE SCANNING IN THE FOLLOW-UP OF BREAST CANCER

The systematic prescription of a bone scan (BS) in the follow-up of patients (pts) with breast cancer is a traditional attitude which is now questioned. The aim of our study was to estimate the yield of this systematic BS, derived from one year experience.

A BS (TC-HMDP) was performed from nov 1992 to nov 1993 in 118 women (mean age : $59 \pm 12$ yrs) with breast cancer. BS realized in the initial evaluation of the disease (reference document) and in the pts with known bone metastases were not included.

In 74 asymptomatic pts, 28 BS were norma1, 42 corresponded to a benign bone disease (BBD) and 4 were doubtful (M?).

BS were motivated by bone pain and/or signs of disease progression (local recidive, increase of CA 15-3 or visceral metastases) in 44 pts. Seven BS were normal, 20 corresponded to BBD, 9 to metastases and 8 to $\mathrm{M}$ ? ( 1 with doubtful TMR and 7 with no further examination)

These results show that no systematic BS led to the discovery of proven bone metastases. Further evaluation of the foux doubtful cases is necessary before concluding if systematic BS in breast cancer follow-up is clinically useful (and able to allow early detection of bone metastases). On the opposite, proven bone metastases were discovered in $20 \%$ of the motivated BS. 


\section{9}

A.M. A.t-Nahhas, S. Jinnouchi, C.

Anagnostopoulos, W. Hirsch, V.R. McCready, Dept. of Nuclear Medicine, The Royal Marsden Hospital, Sutton, UK.

BENIGN TECHNETIUM-99m-MDP (MDP) MYOCARDIAL UPTAKE ASSOCIATED WITH LEFT MASTECTOMY AND CA PROSTATE.

Bone seeking phosphates are known to localize in extraosseus sites such as the myocardium in myocardial infarction. A pattern of diffuse myocardial uptake of $99 \mathrm{~m}-\mathrm{TC}-\mathrm{PYP}$ and HDP (but not MDP) has also been noted in apparently healthy patients.

To assess the incidence of myocardial uptake in MDP bone scans and its relation to different etiologies and age groups, we reviewed 822 scans (656 patients) done over a period of two years for patients with ca breast and ca prostate with a matching population suffering of other malignant or non malignant conditions.All patients had no clinical or biochemical evidence of myocardial disease or other conditions known to cause such uptake. Results are shown below.

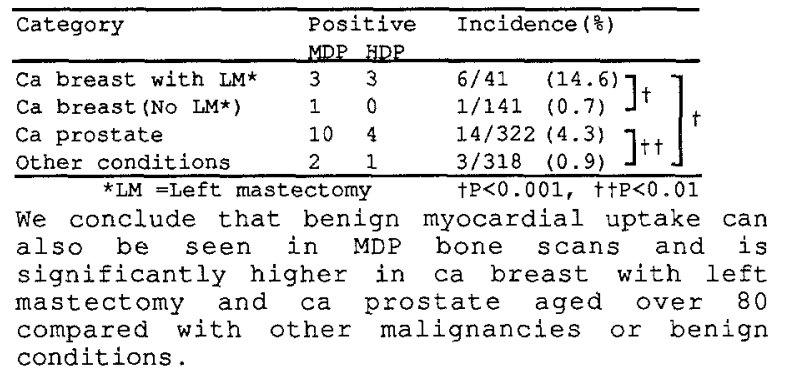

\section{0}

M. Lacić, T. Bokulić, Z. Kusić, K. Kovačić, J. Lukač, N. Đaković

Department of Nuclear Medicine and Oncology, University Hospital "Sestre Milosrdnice", Zagreb, Croatia

DISPARITY OF BONE SCAN AND BONE MARROW SCINTIGRAPHY WITH TC-LABELED ANTIGRANULOCYTE BW 250/183 ANTIBODY IN PATIENTS WITH BREAST CANCERS

Radioimmunoscintigraphy with $\mathrm{Tc}-99 \mathrm{~m}$ labeled antigranulocyte monoclonal antibody (MAb) opens the new possibility in the detection and localization of lesions which involve bone marrow (BM). In this study we describe our preliminary results comparing conventional bone scan (BS) and bone marrow scintigraphy (BMS) in detection of skeletal metastases in the follow up of breast cancer patients (pts).

We have investigated eleven pts with histologically confirmed breast cancer. Whole-body scans and single planar scans were obtained 3-6 hours post injection of a single dose of $0,25-0,50 \mathrm{mg}$ murine $\mathrm{MAb}$ BW $250 / 183$ (Behringwerke $A G$ ) labeled with $370-555 \mathrm{MBq}$ of Tc$99 \mathrm{~m}$. Conventional BS using Tc-99m MDP and BMS were performed within an interval of 4 weeks. Some of BS and BMS lesions were confirmed by standard $\mathrm{x}$-ray procedures.

BS showed bone metastases in 7 pts. BMS detected BM involvement in 8 pts, two of them with negative BS. In 6 pts with positive BS x-ray procedures confirmed osteolysis. One of these pts had negative BMS because the lesion was situated in the proximal part of the left ulna, which normally does not contain BM. Ten lesions shown on BMS were not present on BS. In 1 pt with multiple bone metastases a diffuse reduction of bone uptake on BMS associated with an intense liver and normal spleen uptake was observed.

We conclude that BMS with antigranulocye MAb plays an important role in the detection of $\mathrm{BM}$ involvement in pts with breast cancer. BMS seems to have a comparable sensitivity to the BS and these two procedures could be complementary.
431

K. Liewendahl, M. Löfberg, S. Savolainen, P. Nikkinen,

A. Lamminen, E. Tiula, H. Somer

Division of Nuclear Medicine, Depts. of Neurology, Medicine I and Radiology, Helsinki University Central Hospital, 00290 Helsinki, Finland

LESIONS ARE DETECTED BY ANTIMYOSIN SCINTIGRAPHY NOT ONLY IN ACQUIRED MUSCLE DISORDERS BUT ALSO IN CONGENITAL MUSCULAR DYSTROPHIES

In-111-labelled antimyosin has an established role in the evaluation of cardiac muscle damage. This antibody crossreacts with myosin in skeletal muscle and has been used for detection of lesions in patients with rhabdomyolysis and polymyositis. We used this method on 7 patients with Becker type muscular dystrophy with a diagnosis based on DNA deletion and/or dystrophin staining. In 6 patients weak to moderate uptake of antimyosin antibody was detected in the calves and in some patients also in the thighs and upper extremities. In 6 patients with non$\mathrm{X}$-chromosomal dystrophy uptake was recorded in the calves and in 4 patients also in the upper extremities. In dystrophy patients MRI findings were usually more prominent in the thighs, whereas in the calves the antimyosin findings were relatively stronger. This discrepancy is probably due to the better preserved muscle bulk in the calves, showing pseudohypertrophy, whereas in the thighs muscle tissue has been replaced by fat and connective tissue. In Becker dystrophy the basic defect is an abnormal structure of dystrophin, a cytoskeletal protein providing membrane integrity, whereas in non-X-chromosomal dystrophies the basic defect is unknown. Our data indicate that in muscle dystrophies there is a defect in the sarcolemmal integrity.

Of a total 24 major lesions in 9 patients with rhabdomyolysis 18 could be suspected on clinical examination leaving 6 lesions localized only radioimmunodetection. The target-to-nontarget uptake ratios varied from 1.3 to 7.6 . In 3 patients with polymyositis-dermatomyositis areas of both focal and diffuse uptake of antibody were observed.

It is concluded that antimyosin scintigraphy can be used for detecting muscle lesions not only in acquired muscular diseases but also in hereditary muscular dystrophies.

\section{2}

R.A.M.J. Claessens, E.B. Koenders, H.F. Solomon, O.C. Boerman, W.J.G. Oyen, and F.H.M. Corstens.

University Hospital, Nijmegen, The Netherlands and R.W. Johnson Pharmaceutical Research Institute, Spring House, PA, USA.

PHARMACOKINETICS OF In-111-C-14-DTPA-igG-i-123 IN RATS WITH A FOCAL INFECTION

In a previous study we demonstrated, that after administration of In111-labeled human polyclonal non-specific immunoglobulin $\mathrm{G}$ (lgG) to rats with an infection In-111 is retained in the inflammatory focus, whereas IgG is washed out at decreasing plasma concentrations. In order to further elucidate the role of the bifunctional chelating agent DTPA, we studied the behavior of In-111-C-14-DTPA-lgG-I-123 in rats with a focal infection.

C-14-DTPA-conjugated IgG was labeled with $\ln -111$ via citrate transchelation. In-111-C-14-DTPA-IgG was labeled with I-123 according to the Bolton-Hunter procedure. Labeled lgG was purified by gel filtration. HPLC was performed as quality control. Young Wistar rats with a Staph. Aur. infection of the left calf muscle were i.v. injected with $0.2 \mathrm{ml}$ of a solution containing $0.45 \mathrm{MBq} \ln -111,50$ $\mathrm{KBq} \mathrm{C}-14$, and $1.8 \mathrm{MBq} 1-123$ labeled to $2.5 \mathrm{mg} \mathrm{lgG}$. Rats were sacrificed at 2,6,24, and $48 \mathrm{hr}$. p.i.. Activity uptake was determined for plasma, urine, abscess, and various other tissues. Averages $\pm S D$ were calculated for groups of five rats. Plasma and urine samples were analyzed by HPLC.

The radiochemical purity of the IgG-preparation was $>95 \%$. The DTPA:IgG ratio was $3: 1$. In all tissues tested, except for the lung, the uptake of both In-111 and C-14-DTPA was much higher than the I-123-lgG uptake (48 hr p.i., liver: In-111: 1.1 \pm 0.1 ; DTPA: 1.0 \pm 0.1 ; IgG: $0.35 \pm 0.03$; abscess: In-111: $1.1 \pm 0.1$; DTPA: $1.7 \pm 0.2$; IgG: $0.7 \pm 0.1(\% / D / g \pm S D))$. The abscess uptake of C-14-DTPA was significantly higher than that of In-111 $(p<0.01)$. The I-123 data were similar to the C-14-lgG data from a previous experiment in the same model. In plasma release of In-111 from IgG was observed over time, whereas DTPA remained conjugated to $\mathrm{lgG}$. A small amount of $\ln -111$ in plasma was found to be transchelated to a substance with a molecular weight slightly smaller than that of IgG.

Both In-111 and DTPA appear to dissociate from $\mathrm{IgG}$. In abscesses the DTPA uptake is significantly higher than the $\ln -111$ uptake. 
433

J. Meller, D.L. Munz, V. Ivanðeví́, J. Djomotschko, D. Emrich

Division of Nuclear Medicine, University of Göttingen, Germany

SCINTIGRAPHY OF INFLAMMATION WITH MONOCLONAL ANTIGRANULOCYTE ANTIBODIES (AgAb) IN FEVER OF UNKNOWN ORIGIN (FUO)

The aim of our study was to investigate the value of 99m-Tc-anti-NCA 95 monoclonal AgAb scintigraphy in patients suffering from FUO.

Forty-five patients - 24 females and 21 males (age 1-76 years) - were examined by planar wholebody images 4 and 24 hours after i.v. injection of $185-555 \mathrm{MBq}$ of $99 \mathrm{~m}-\mathrm{Tc}-\mathrm{AgAb}$. In 24 patients SPECT was additionally performed after 24 hours. The final diagnosis was established by histology, blood cultures, routine laboratory methods, and radiology including MRI. Fifty percent of the patients had infectious, $14 \%$ autoimmune, $10 \%$ malignant, and $15 \%$ other diseases. In $11 \%$ the etiology of the fever remained unknown.

A total of 16 true positive infectious foci was found (only hot spots were considered), 5 skeletal, 5 abdominal, 2 thoracic, 2 cranial, and 2 in other locations. Three false positive findings originated from necrotising colitis uremic colitis, and a mediastinal mixed tumour. There were 9 false negative foci in patients with endocarditis, pneumonia, abdominal abscess, and asteomyelitis of the central skeleton. Sensitivity and specificity of the AgAb scintigraphy regarding infectious disease (prevalence 44 \%) amounted to $64 \%$ and $89 \%$, respectively, positive and negative predictive values were $84 \%$ and 70 \%, respectively.

In conclusion, AgAb scintigraphy is an important diagnostic tool in proving or excluding an infectious etiology of Fuo.

434

B. Grünert2, M. Cordes2, G.S. Barzen2, E. Heißler', J. Bierl, R. Felix ${ }^{2}$ 2 Strahlenklinik und Poliklinik, 1 Klinik für Mund-,Kiefer-u. Gesichtschirurgie, University Clinic Rudolf Virchow, Free University Berlin

SCINTIGRAPHY WITH ANTI-NCA-GRANULOCYTE-AB IN ORO-MAXILLO INFECTIONS

Early diagnosis of osteomyelitis and follow up in infection treatment in oro-maxillo-facial sugery is quite important. In our study we comprehended the behaviour of anti-Granulocyte $A B$ in 35 patients with osteomylitis in the oro-maxillo-region with TC $99 \mathrm{~m}$-labelled nanocolloids $(n=25) .35$ patients with a suspected infection or osteomywlitis in the head and neck region were investigated with the 123-NCA-MAB (Behring) labelled with 800$1200 \mathrm{MBq} \mathrm{TC}-99 \mathrm{~m}$. Analog and SPECT images 4 and $24 \mathrm{hrs}$. after injection were obtained. In 25 patients additional bolus injection of $740 \mathrm{Mbg}$ Tc99m-labelled nanocolloids with aquisition of analog and digital sequences were done. The results were correlated to anti-granulocytesantibody-uptake, sonography, CT and MRT. The scintigraphy with nanocolloids is a proven method in detecting chronic septic processes. In acute cases with wound trauma enlarged nanocolloid uptake was seen in the surrounding bone tissue due to the unspecific inflammation, so that infection cannot be exactly localized and differentiated to early postoperative alterations. In these cases the anti-granulocyte-scan showed in the planar and SPECT images a high concentration of antigranulocyte-AB so that localization and identification of the infection even in the SPECT-slices was better than in the nanocolloid scans.
435

F.Pons, R.Sanmarti*, M.Huguet, A.Collado*, R. Herranz, A. Martinez, J.Gratacós*, J. Muñoz-Gómez* and J. Setoain

Nuclear Medicine and Rheumatology* Departments. Hospital Clínic, University of Barcelona. Spain.

$99 \mathrm{mTc}-P O L I C L O N A L$ HUMAN IMMUNOGLOBULIN G SCINTIGRAPHY IN ACTIVE SYNOVITIS. RELATIONSHIP WITH CLINICAL AND BIOLOGICAL PARAMETERS OF INFLAMMATION.

Radiolabelled polyclonal human immunoglobulin G (HIG) has been recognized as a reliable modality for localization of infection and inflammation. The aim of this study was to evaluate if scintigraphic uptake has a relationship with clinical and biological parameters of inflammation thus allowing the measurement of the degree of synovitis.

Twenty four patients with rheumatoid arthritis and active synovitis ( 7 males 17 females, mean age: $54 \pm 11$ years) were studied. Scintigraphy with $99 \mathrm{mTc}$-HIG $(740 \mathrm{MBq})$ was performed at 4 hours $\mathrm{p} . \mathrm{i}$. The number of painful and swollen joints, the Thompson index for clinical activity and the scintigraphic uptake by a quantitative analysis were evaluated. Sedimentation rate, C-reactive protein (CRP), hemoglobin, haptoglobin and serum levels of IL-2, IL- 6 and $\alpha \mathrm{TNF}$ were taken as biological parameters of inflammation. In 8 cases serum samples radiactivity at $10 \mathrm{~min}$ and 4 hours and synovial fluid radiactivity at 4 hours were evaluated. Protein bound activity was measured by Sephadex G-25 (PD-10 columns) chromatography.

Radiochemical purity was $>99 \%$ in all labellings. Radioactivity in synovial fluid was $22.8 \pm 10.6 \%$ respect to plasmatic activity/ml and it represented only $0.05 \pm 0.04 \%$ of the total injected dose. Protein bound radiactivity in plasma and synovial fluid was higher than $95 \%$. Scans showed that $91 \%$ of swollen and $94 \%$ of painful and swollen joints had pathological uptake. Quantitative analysis of scans demostrated that the sum of scintigraphic uptake in pathologic joints correlated significantly with the number of painful joints $(r=0.46)$, the number of swollen joints $(r=0.57)$, the Thompson index $(r=0.59)$, the sedimentation rate $(r=0.55)$, CRP $(r=0.59)$ and hemoglobin $(r=0.49)$.

We can conclude that scintigraphy with $99 \mathrm{mTc}-\mathrm{HIG}$ is a reliable method to detect active synovitis and to evaluate the degree of inflammation.

\section{6}

G.E. Monsour, N.G. Hartman

Division of Nuclear Medicine, Ottawa Civic Hospital, OTTAWA (Ontario), Canada K1Y 4E9

EFFECT OF VARIOUS DRUGS ON THE LABELLING EFFICIENCY OF WHITE BLOOD CELLS WITH TC-99M HEXAMETHYLPROPYLENEAMINEOXIME (HMPAO).

Gallium- 67 has been replaced by the labelling of leukocytes with either In-111 oxine or Tc-99m HMPAO for the detection of inflammatory processes.

White blood cells (WBCs) play a major role in the body defense mechanism, and are activated at the onset of infection to immobilize, phagocytize and subsequently destroy the causative bacterial agent.

Most of the patients scanned with Tc-99m HMPAO WBCs are, by virtue of their patho-physiology, on various regimens of drugs. We have chosen a number of pharmaceuticals to evaluate their impact on the labelling efficiency (LE) of WBCs. Drugs do not only affect the morphology of the WBCs, but can have deleterious affects on the chemicals used in the labelling process (e.g. penicillin $G$ in high concentration inhibits hetastarch's effect). Edrophonium, Azathioprine, Dipyridamole, Enalapril, Isoproteronol, Penicillin G, Co-trimoxazole, Streptokinase, Diazepam, Cefazolin, Cyclosporin, Anti-human Lymphocyte $\mathrm{IgG}$, and Adriamycin were investigated. Most of these $\mathrm{did}$ not effect the LE of WBC's (even in high plasma doses), which showed a slight potentiation with Co-trimoxazole and Dipyridamole, and a $75 \%$ reduction in the presence of 0.83 $\mathrm{mg} / \mathrm{ml}$ Anti-human Lymphocyte IgG.

From a retrospective study we conducted, it is not clear whether the leukocyte count had any affect on LE. However, our results emphasize the need to screen the patients' drug regimen before embarking on a WBC-labelling test. 
437

M. Papós, I. Nehéz, I. Simonfalvi, J. Láng, L. Csernay, G. Kovács

Depts. of Nuclear Medicine, Radiology, and Cardiac Surgery, A. Szent-Györgyi Medical University, Szeged, Hungary

\section{9mTC-HM-PAO-LABELED LEUKOCYTE SCINTIGRAPHY AND COMPUTER TOMOGRAPHY FOR DETECTION OF STERNAL WOUND INFECTION}

13 studies [99mTc-HM-PAO-leukocyte scintigraphy (LS) and computer tomography (CT)] were carried out in 10 patients with a high clinical probability of infected sternal wound. Repeated investigations were made in different clinical states. The final diagnosis was verified by wound culture and surgery in all cases. Nine postoperative patients without clinical symptoms of infection were also studied.

LS was carried out with mixed, autologous leukocytes, labeled with $99 \mathrm{mTc}-\mathrm{HM}-\mathrm{PAO}$ in vitro. CT scans were obtained through the use of intravenous contrast material in the usual way.

Signs in the LS of control patients were as follows: activity irregularity, biffed sternum and diffuse, increased lung uptake of leukocytes. CT scans of the control group showed focal edema, focal hematoma and moderate sternal abnormalities. In one control patient the CT proved positive.

In 11 cases with clinically verified infection 9 proved positive on LS and 8 on CT. LS was positive in cases with either a superficial or a deep process. CT determined in all cases, whether the infection was limited to the presternal space or wether the sternum and mediastinum were also involved. In 2 cases with a negative clinical picture, both CT and LS proved negative.

Conclusion: Specific signs of infection can differentiate from the symptoms due to sternotomy when LS is used, but overlapping of the symptoms was revealed by CT. LS and CT are sensitive methods for the early detection of postoperative sternal wound infection. CT is superior for the exact localization of the process. A combination of LS and CT is suggested in the diagnosis of poststernotomy infection.

\section{8}

A.P. Iurilli, M. Iiberatore, F. Ponzo, D. Prosperi, c. Santini, P. Baiocohi, L. Rizzo, F. Speziale, A. Centi Colella

Depts.of Exper. Medicine and Vascular surgery, Inst.of 6th Medical Clinic, University of Rame "La Sapienza", Italy.

DEIBCTION OF FROSIHETIC VASOULAR GRAFT INFECITON : A COMPARISON BEIWEEN 99MIIC-MMPAO IEUKOCYTES SCAN(WBCS) AND COMFUIED TOMDCRAFHY (CIS).

The prosthetic vasoular graft infection (FVII) is considered the most seriaus and life-threatening complication in reconstnuctive vascular surgery. Although rare, FVGI seems to have a high incidence both of mortality and monbidity. Because of non specific early symptams, it is necessary an earlier and acourate diagnosis. Standard techniques (echography, angiography, CI) have not yet provided a complete and satisfactory acauracy. The aim of this study was to compare the reliability of WBCS and CTs in detecting PVGI. 22 pts. with suspected PVGI were studied. CIs and WBCs were performed in all cases. Pts. were categorized into three groazs acoording to the clinical findings. Grop A included pts who had specific signs of graft infection. In group B pts had nanspecific symptoms of graft infection. Group $C$ included pts. who had anastomotic aneurysms variably associated with nanspecific signs of PVGI. The final diagnosis was made an the basis of the intraoperative findings, culture results or 18-month follow-up in pts. who did not undergo surgical exploration. The results are shown in the following table:

\begin{tabular}{|c|c|cccc|cccc|}
\multicolumn{1}{c|}{} & $\mathrm{n}$ & $\mathrm{TP}$ & TN & FP & FN & TP & TN & FP & FN \\
Grap A & 3 & 1 & 0 & 0 & 2 & 3 & 0 & 0 & 0 \\
Grap B & 8 & 3 & 3 & 0 & 2 & 5 & 3 & 0 & 0 \\
Grap C & 11 & 2 & 7 & 2 & 0 & 2 & 8 & 1 & 0 \\
Total & 22 & 6 & 10 & 2 & 4 & 10 & 11 & 1 & 0 \\
\hline
\end{tabular}

In conclusion, diagnastic acauracy of WBCs seems to be higher than CI's's ane.
439 M.Milella R.Sara L.Ruffini M.Possa F.Banfi S.Sterzi*

Nuclear Medicine Department Division of Neurology*

Niguarda Hospital, Milan, Italy

99mTC-HMPAO LABELLED LEUKOCXTES IN THE DIAGNOSIS OF CEREBRAL ABSCESS

Differential diagnosis beetween cerebral abscess and other expansivo lesions is often difficult even if we have TC and RM results. Scintigraphy with 99mTC-HMPAO-leukocytes detection a non invasive method for infection bas shown great for ccrebral abscess diagnosis. We studied 28 patients with leukocytes (185-379 MBq di $99 \mathrm{mTC}-\mathrm{HMPAO}$ ) because of the suspicion of cerebral abscess. Scans were performed at 1,3 and 24 hours after the infection of labcledin leukocytes, of 18 patients with positive TC, 7 had positive scintigramos. Neurosurgical (3), clinical (3) and authophtic control confirmed the presence of absecs in all of them. The other patients with positive TC (11) had negative scan and in all cases it was demonstrated non infective pathology (neoplasm - lesion, MM, ematoma). Of 10 patients with negative TC, 2 had positive scintigrams confirmed by surgical control. Patient with negative TC and leuk. scan (n8) conclusive diagnosis (4 surgically and 4 clinically) of non - infective pathology. In this study sensitivity and specificity wero $100 \%$. Evcn this study rosult is ptobably releted to accurate patients selection, we think that leukocyte scan plays a centralrole in abscess diagnosis, particularly in the early stage when neuroradiologic interventhon is more efficient.

440

Z.B.Özcan, Z.Burak, C.Özcan, G.Başdenir, T.Özacar, S.Erdem,Y.Duman Ege University Medical Faculty Muclear Medicine, Pathology, $:$ : Pediatric Surgery, and Microbiology Depts. Izmir, Ti̛kKíYE

\section{CAN 201-T1 BE A RELIABLE AGENT IN LOCALIZATTON OF} INFECTIOUS PATHOLOGIES

Thallium-201. imaging has been widely used to differentiate post therapy reactions from residuel viable tumor, local recurrence or fibrosis. But the ability of $201-T 1$ to discriminate posttherapy changes superimposed with infection/inflammation is unclear. In this experimental study, it was ained to investigate the role of 201-T1 in loca lization of infected/inflanmed tissues.

24 rats infected with Staphylococcus aereus inocculation and 10 rats injected with a standart volume of saline solution (SS) into thigh muscle were studied. 24 and 48 hours after microorganisms and SS administration, $18 \mathrm{MBq} 201-\mathrm{T} 1$ was applied intravenously. $20 \mathrm{~min}$. and $3 \mathrm{hr}$. planar inages were recorded. The observed hyperactivity was evaluated qualitatively and quantitatively calculating the ratios derived from ROI's drawn over lesion and contrlateral thigh muscle $(\mathrm{L} / \mathrm{C})$. After imaging procedure, histopathological and microbiological material was obtained.

While control group showed no abnomal activity accumulation, infected rats demonstrated marked hyperactivity especially on $20 \mathrm{~min}$. images. Mean $\mathrm{L} / \mathrm{C}$ ratios of $20 \mathrm{~min}$. and $3 \mathrm{hr}$. images of infected rats were $2.18 \pm 0.20$ and $1.52 \pm 0.04$ respectively $(p<0.0005)$.

In conclusion, the unexpected accumulation of 201-T1 in infectious tissue may be a potential limitation in differentation of local recurrence in posttherapatic changes complicated with infection. Although, delayed imaging may overcame this limitation, further investigation in large series will be indicated in order to improve the reliability of $201-\mathrm{T} 1$ in oncology. 
441

Z.BURAK, Z.B.ÖZCAN, C.ÖZCAN, T.ÖZACAR, G.BASDEMIR, S.ERDEM, M.ARCON, A.ERDENER, Y.DMMAN

Depts. of Nuclear Medicine, Ped. Surgery, Microbiology and Pathology, Ege University, İzmir TRRKEY

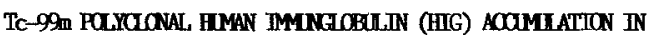
DIFFTRENT STAGES OF TNFHCTTON: AN EXPFRTMENTAL STUDY

The aim of this study was to investigate the behaviour of Tc$99 \mathrm{~m}$ labelled polyclonal human immuglobulin (HIG) in different stages of infectious pathologies in an experimental model.

Three group of rats, a total of 36, with Staph. aureus inoculation of right thigh muscle were studied. In order to investigate different stages of inflammation, $40 \mathrm{MBq} \mathrm{Tc}-99 \mathrm{~m}$ HIG was administered 24 hrs. after microorganism inoculation in Group 1 (12 rats), $48 \mathrm{hrs}$. in Group 2 (12 rats) and after $72 \mathrm{hrs}$. in Group 3 (12 rats). The scintigrams were obtained 4,24 and $48 \mathrm{hrs}$. later. Qualitative and quantitative evaluation were applied. The uptake ratios of infective focus / contrlateral site (L/C) were derived. $48 \mathrm{hrs}$. after Tc-99n HTG adninistration, the rats were sacrificed. Histological and microbiological specimen were obtained. The stage of infective process was confirmed by histological diagnosis. The mean $\mathrm{L} / \mathrm{C}$ ratios of Group 1 at 4,24 and $48 \mathrm{hrs}$. were $1.22 \neq 0.1$, $2.12 \pm 0.16$ and $4.03+0.48$, of Group 2 were $1.15 \pm 0.08,2.25 \div 0.16$ $3.87 \pm 0.02$ and of Group 3 were 1.06 $\$ 0.09,2.08 \div 0.14$ and 3.62 10.12 respectively. The mean $\mathrm{L} / \mathrm{C}$ uptake ratios of three groups were not significantly different. Histopathological examination also confirmed the findings associated with different stages of infection.

In conclusion, in this preliminary study, insignificant Tc-99n HIG accimilation was observed in acute and chronic stages of infectious pathologies. But it is believed that further investigations are indicated in order to evaluate the definite role and prognostic value of Tc-99m $\mathrm{H} \amalg \mathrm{G}$ in infections and inflammations.

442

A.Kurtaran, R.Ziesche, P.Hübsch, P.AngelI. Virgolini

Departments of Nuclear Medicine, Pulmonology, Radiology and Emergency, University of Vienna, Austria.

PULMONARY EMBOLISM (PE): IMAGING STUDY WITH

${ }^{123}$ I-VASOACTIVE INTESTINAL PEPTIDE (VIP)

Human lung tissue expresses large numbers of high affinity receptors for VIP. VIP is a neuroendocrine mediator with a broad range of biological activities. Recently, we have developed the VIP receptor scintigraphy by using ${ }^{123} I$-radiolabeled VIP as radioligand. In these studies, the lungs became visible immediately injection of ${ }^{123} I-V I P$. We have now investigated 10 patients with acute $P E$ by using ${ }^{123} \mathrm{I}$-labeled VIP. Planar imaging in a.p. p.a. and lateral views was performed. In ali patients PE was verified by a mismatch during conventional lung imaging (performed with $99_{\mathrm{Tc}} \mathrm{Tc}$ DTPA-aerosols and $99 \mathrm{mc}$-labeled albumin microspheres). Excellent imaging of these PE defects was also provided by ${ }^{123} I-V I P$. We conclude that our new radioreceptor tracer could be useful in the diagnosis of PE. Further studies in patients with suggested pulmonary embolism and negative conventional lung scans are under way.

\section{3}

A. Lepailleur, C. Pérault, A. Loboguerrero, C. Gibold, A. Bouchard

Department of Nuclear Medicine, Institut Jean Godinot, Reins, France

INITEREST OF PARAMEIRIC SUBIRACTION IMAGES IN LUNG SCAN FOR FUIMONARY EMBOLISM DIAGNOSIS

Since the diagnosis of pulmonary embolism is based on the comparison of scintigraphic images of ventilation and perfusion we studied the usefulness of a software which automatically generates the inages of the differences between ventilation and perfusion scan images.

88 patients ( 45 males, 43 females) mean age 60 years were examined. All of them had a lung scan ventilation and a lung scan perfusion with 4 views (anterior, posterior, left and right oblique posterior). The lung ventilation scan is realised with Technegas then the lung perfusion with $240 \mathrm{MBg} 99 \mathrm{~m} \mathrm{Tc}$ labelled microspheres.

According to the PIOPED classification, 26 patients were considered normal, 36 patients had a low probability of pulmonary embolism, 26 patients had a high probability. Images contained 100000 coumts for the ventilation and 400000 for the perfusion. Wre drawn regions of interest on the 4 ventilation images. After an autamatic geometric and gray level registration, images of significant differences were obtained between ventilation and perfusion images.

These images did not modifie the diagnosis obtained with original images.

However, this technique could be useful in the objective comparison of repeated perfusion scan.

\section{4}

EJR van Beek, MMC Tiel-van Buul, CA Hoefnagel, HHT Jagt, and EA van Royen.

Depts of Hemostasis/Thrombosis and Nuclear Medicine, Academic Medical Centre, Amsterdam, the Netherlands.

\section{REPORTING LUNG SCANS: PROSPECTIVE EVALUATION OF THE EFFECTS OF AN ANATOMICAL LUNG SEGMENT CHART.}

This study prospectively evaluated the effect of the consistent use of a previously described anatomical lung segment chart on the interpretation of lung scans.

Simultaneous perfusion-ventilation lung scintigraphy was performed in 221 consecutive patients with clinically suspected pulmonary embolism using $99 \mathrm{~m}-\mathrm{Tc}$ macro-aggregates of albumin and $81 \mathrm{~m}$ Krypton gas. Lung scans were immediately reported as normal, high probability or non-diagnostic, with the use of an anatomical lung segment chart. After at least six months, blinded lung scans were randomly read by a panel of nuclear medicine physicians.

Initial lung scan reports were classified as normal, high probability, and non-diagnostic in 64, 63, and 94 patients, respectively. Overall observer disagreement was $5.9 \%$ (95\% CI $3.2 \%-9.8 \%$ ), while this was $7.8 \%, 3.2 \%$ and $7.9 \%$ for lung scans that were initially reported as normal, non-diagnostic and high probability, respectively. Reclassification consisted of normal to non-diagnostic (5), non-diagnostic to normal (1), non-diagnostic to high probability (2), and high probability to non-diagnostic scan results (5). From literature, overall disagreement of $20 \%$ was expected if no chart had been used (Thromb Haemostas 1992;68:245-249).

In conclusion, this study confirms that the consistent use of a lung segment chart reduces observer disagreement in the reporting of lung scans. Routine reporting by a panel should be considered to further improve patient management. 
445

G.Rubini, D.Rubini, L.Colonna*, F.Lauriero, F.Bovenzi*, C.D'Agostino*, A.D'Addabbo.

Depts. of Nuclear Medicine, Cardiology* - University of Bari- Italy

LUNG PERFUSION SPET IN THE EVALUATION OF rt-PA THROMBOLYTIC MANAGEMENT OF PULMONARY EMBOLISM

High, average and low pulmonary embolism probabilities can be established by lung perfusion SPET. Diagnosis is confimed by arteriography. $99 \mathrm{mTC}-\mathrm{MAA}$ SPET permits assessment of the extent (voxel and $\mathrm{cm}^{\overline{3}}$ ) of perfusion defects. This technique was used to evaluate the extent of revascularised districts after rt-PA therapy in 22 patients with a clinical suspicion of embolism. Positive SPET finding obtained in all cases were followed on the same day by confimatory arteriography, $100 \mathrm{mg} / 2 \mathrm{~h}$ rt-PA i.v., and posttreatment arteriography. A second SPET on the next day showed normalisation in 9 patients (41)), improvement in 6 (27\%) and no change in 7 (32\%). Following further treatment of these 13 subjects with heparin and warfarin for 30 days, a thirth SPET showed complete normalisation in 4 and no improvement in 9. Comparison by subtraction of the pre- and post-rt-PA SPET images allowed evaluation of the extent of the revascularised districts. A good correlation was also found between the posttreatment images and the clinical pictures.

\section{6}

Y.Dunan, F. Korkut, S.Endem, Z. Burak, E. YLicesoy ,M.Ercan,N.Moğolko and Ü.Bayındr

Depts.of Nuclear Medicine, Ege University, Izmir-Turkey

A NEW VENTIIATTON AGENT TC-9ON [ABEI.TED D-GLUOOSE 1-PHOSPHALE (TC-99m GP) AND SAME HOUR PROTOCOL IN DIAGNOSIS PULONARY EMBOII

The clearance of radyoactivity from the lungs was much slower with Tc-99m GP compared to Tc-99m DTPA (T1/2=316,5-44,7 vs 80,8-13,4 min) and this was statisticaly highly significant.Various protocols were proposed for sequential pulnonary perfusion and ventilation radyonuclid studies. We ane presenting a same hour protocol. whereby Tc-99m MAA perfusion only for those patients with perfusion defect with no corresponding chest $x$-ray abnomalities. It has been taken pulmonary perfusion scintigraphy with MAA in patients with suspected pulmonary emboli. From those patients, 2l patient which had segrentally perfusion defect and normaly $x$-ray have been performed after aerosol inhalation of $30 \mathrm{mCi} \mathrm{TC}-9 \mathrm{~m} \mathrm{CP}$ following perfusion scintigraphy (Spect studies of lung were performed in four subjects, planar images of lung were obtained in 17 patients). We diagnosed pulmonary emboli in 17 patients with had perfusion-ventilation missmatch. After performing thrombolytic theraphy in these patients, it has been taken control perfusion scintigraphy. Reperfusion were observed in 15 patient had perfusion defect.

In these 15 pationts, quantification of defect and normal area on perfusion and aerosol scintigraphy were performed retrospectively.

On perfusion scintigraphy defect area/nomal area-a on aerosol scintigraphy defoct area/nomal area $=b$,filing index $=b / a$ Filing index was found $1,57^{+}-0,55$ in these 15 patient. Spect and threedimensional display showed better missmatch according to planar images. Conclusion=The clearence value of Tc-99m GP from lungs is fourth as slow as than Tc-99m DIPA aerosol. In cosecuence, it can be made together with perfusion and ventilation study respectively in an hour for diagnosing of pulmonory emboli.
447

RA Valdês Olmos, L.J. Boersma, $\mathrm{N}$ van Zandwijk, CA Hoefnagel, JV Lebesque.

The Netherlands Cancer Institute, Plesmanlaan 121, 1066 CX Amsterdam.

\section{${ }^{111}$ In-PENTETREOTIDE FOR THE DETECTION OF RADIATION PNEUMONITIS.}

Early recognition of radiation pneumonitis enables adequate treatment with a reasonable chance to prevent late sequelae. It has been suggested that peptide activation in irradiated areas may be involved in the pathogenesis of lung injury. Therefore, we have explored the feasibility of ${ }^{111}$ In-pentetreotide scintigraphy in detecting radiation pneumonitis.

Seven patients who have received radiotherapy to the mediastinum for lymphoma or to the internal mammary nodes for breast carcinoma were investigated. Planar and SPECT images were performed 24 hours after administration of $110 \mathrm{MBq}{ }^{111} \mathrm{In}$-pentetreotide. The degree of lung uptake was visually evaluated. The scintigraphic findings were correlated with the radiation field (RT-field) and compared with ventilation and perfusion lung scans (V-Q) obtained in the same week.

${ }^{111}$ In-pentetreotide was strongly positive in 4 of 6 symptomatic patients examined 2-5 months after irradiation; one of the three was receiving steroid therapy but without clinical response. In 2 other patients with good clinical response, ${ }^{111}$ In-pentetreotide, performed a few weeks after starting steroid therapy, was weakly positive and negative respectively. In one asymptomatic patient, investigated 1 month after radiotherapy, ${ }^{111}$ In-pentetreotide was negative. V-Q scans showed abnormalities in all symptomatic patients. However, the ${ }^{111}$ In-pentetreotide images we obtained so far suggest a better delineation of lung injury than standard V-Q scans. This was helpful in one case of internal mammary chain irradiation in which the $V-Q$ lung scan was not conclusive for diagnosis. ${ }^{111}$ In-pentetreotide uptake was observed only in lung areas included in the RT-field.

It is concluded that ${ }^{11.1}$ In-pentetreotide appears to be sensitive in detecting radiation pneumonitis. Uptake, which may reflect radiation-induced activation of somatostatin receptors in injuried lung areas, may be influenced by steroid therapy. ${ }^{111}$ In-pentetreotide may have a role in the differential diagnosis in patients with complaints after RT and in the assessment of response to steroid therapy.

\section{8}

S.Pavlovic, D.Jovanovic, G.Radosavljevic, D.Sobic, D.Miric, V.Bosnjakovic, A.Epenetos

Nuclear Medicine Institute and Institute for Lung Diseases, Clinical Center of Serbia,

Belgrade, Hammersmith Hospital, London

IS EARLY MONOCLONAL ANTIBODY UPTAKE IN LUNG CANCER DURING EXTERNAL BODY IRRADIATION NONSPECIEIC?

It has been reported that external body irradiation enhanced anti HMFG1-99m TC monoclonal antibody localisation in non small cell lung cancer. In order to test sensitivity and specificity of anti HMFG1-99m TC monoclonal antibodies localisation in lung carcinoma 10 patients (6 with non small cell lung cancer and 4 with small cell lung cancer) were studied while undergoing a course of external beam radiotherapy. Immunoscintigraphy was performed $10 \mathrm{~min} ., 5$ hours and 24 hours after monoclonal antibodies administration and anterior and posterior chest views were obtained. The scans were interpreted semiquantitatively using tumour to nontumour ( $\mathrm{T} / \mathrm{NT}$ ) ratio. In 4 out of 6 patients with small cell lung cancer antibody accumulation was observed in the tumour region on an early scan and $\mathrm{T} / \mathrm{NT}$ ratio ranged from 1.68 to 1.7 . After 5 and 24 hours $T / N T$ ratio increased to maximum of 2.15 . In one out of 4 patients with small cell lung cancer observable uptake of anti HMFG1-99m TC monocional antibodies was found in the tumour region. $\mathrm{T} / \mathrm{NT}$ ratio was 1.8 on early scan and on 5 hours and 24 hours 1.4 and 1.6 respectively. Our results suggest that early HMEGI-99m TC monoclonal antibody uptake in tumour region during a course of the external beam radiotherapy in patients with small cell lung cancer may be non-specific. 
Sunday, 21 August - Wednesday, 24 August 1994

449

S.Fanti, G.Compagnone, M.Marengo, G.Ferretti, A.Cavalii, A.Bini, M.Dondi, N.Monetti.

Depts. of Nuclear Medicine, Medical Physics, Pneumology and Thoracic Surgery.

S.Orsola-Malpighi Hospital,Bologna, ITALY

COMPARIBON OF QUANTITATIVE PERFUSION AND TECHNEGAB VENTILATION BCANS IN PATIENTS WITH PULMONARY CARCINOMA.

In patients with pulmonary carcinoma quantitative lung perfusion scan has been used to determine functional contribution of noncancerous lung. To assess the relative results of perfusion and Technegas ventilation scans, we studied 73 patients $(51 \mathrm{M}, 22 \mathrm{~F}$, mean age $63.7 \pm 11.6 \mathrm{yrs})$ with pulmonary carcinoma referred for surgical treatment. Ventilation scan was obtained by inhaling Technegas; perfusion scan was performed immediately after by iv. injection of $150 \mathrm{MBq}$ of TC99m-MAA. For both studies four standard views were obtained and percentage contribution of each lung calculated as mean of counts in anterior and posterior views. As regards affected lungs, mean perfusion was $42.1 \pm 10.7$ \% (range 15-56), while mean ventilation was $44.9 \pm 9.5 \%$ (range 10-57); difference was statistically significant $(p=0.034)$. In $63 / 73$ patients the contribution of the affected lung agreed within $10 \%$ for both techniques. A discrepancy was observed in the remaining 10 , and in 7 of these perfusion was less than ventilation. Our data suggest that quantitation of pulmonary function by means of Technegas may significantly differ from perfusion quantitation; this may be due to compression or invasion of arteries, or reflex vasoconstriction. These findings might be relevant for accurate estimation of pulmonary function in patients scheduled for lung surgery.

450

H Durak, Ö Ülker, B Uysal.

Dokuz Eylül University School of Medicine, Department of Nuclear Medicine, Izmir,Turkey.

DOES INCREASED LUNG UPTAKE OF TC $99 \mathrm{~m}$ HMPAO EFFECT BRAIN UPTAKE?

In order to show the effect of increased lung uptake of Tc99m HMPAO on the brain uptake in smokers, posterior thorax, head and anterior abdominal images of 16 patients( 10 women, 6 men,mean age $44 \pm 8$, with stroke, psychiatric disorders and migraine who were referred for brain SPECT) were obtained 30 minutes after injection. There were 9 smokers(S) and 7 nonsmokers(NS). S consumed $20 \pm 7$ cigarettes/day. 6 smokers smoked 3-4 hours just before injection (RS). Cigarette consumption of RS was not different than $S(22 \pm 7$ cigarettes/day). Regions of interest were placed on the lungs, liver, spleen, brain and background ((bckg), midline between the lungs). Average counts per pixel were used to calculate Lung/Liver (LL), Lung/Spleen (LS), Lung/Bckg(LB), Brain/Bck(BB) and Left/Right lung(LR) ratios.

\begin{tabular}{|c|c|c|c|c|}
\hline LL & $\begin{array}{c}\text { LS } \\
0.60+012\end{array}$ & 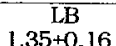 & $\overline{\mathrm{BB}}$ & \\
\hline $0.57 \pm 0.22$ & $0.87 \pm 0.42$ & $1.81 \pm 0.48$ & $1.27 \pm 0.15$ & 0 \\
\hline
\end{tabular}
$\begin{array}{llllll}S & 0.57 \pm 0.22 & 0.87 \pm 0.42 & 1.81 \pm 0.48 & 1.27 \pm 0.15 & 0.93 \pm 0.11\end{array}$ $\begin{array}{lllll}\text { RS } 0.64 \pm 0.22 & 0.95 \pm 0.48 & 1.91 \pm 0.51 & 1.26 \pm 0.19 & 0.94 \pm 0.06\end{array}$ LL, LS and LB were significantly higher in $\mathrm{S}$ than NS $(.01<\mathrm{p} \leq .025, .025<\mathrm{p} \leq .05$ and $.005<\mathrm{p} \leq .01$ respectively). There was a slight assymerical lung uptake in $S$. Though it is not statistically significant, RS seems to have slightly higher values. It was concluded that recent smoking might increase Tc 99m HMPAO retention in the lungs. Though the groups consisted of diseased brains, brain counts do not seem to be affected by increased lung uptake.
451

A. Kögler, J. Schauer, D. Sandrock, V. Ivancevic, D. L. Munz, D. Emrich

Department of Nuclear Medicine, Georg-August-University, Göttingen, and Department of Internal Medicine, University of Leipzig, Germany

VALUE OF PHASE ANALYSIS IN RADIONUCLIDE VENTRICULOGRAPHY (RNV) IN CHRONIC NONSPECIFIC PULMONARY DISEASE

The aim of this study was to assess the value of phase analysis in RNV in patients with nonspecific pulmonary disease, usually evaluated by exercise EKG and right cardiac cathetherization.

Overall, 140 patients with suspected pulmonary hypertension (PH) and without bundle brunch blocks ( 34 women, 106 men, aged $54+/-10$ years) were studied during equilibrium using Tc-99m-RNV. After Fourier analysis (ROI-technique), the phase angles of both atria, the right chamber, and regional dyskinesias of the right chamber were compared with the phase angle of the left chamber (defined as $0 \%$ ).

The number of patients with an angle $>0^{\circ}$ was significantly different among the groups "normal" $(17 / 61=28 \%)$, "latent PH" $(35 / 60=57 \%)$, and "manifest PH" $(11 / 19=58 \%)$. This resulted in a sensitivity of the angle analysis of $57 \%$ and a specificity of $72 \%$ for the detection/exclusion of $\mathrm{PH}$. Focal dyskinesias were found in $10 / 19$ patients with manifest $\mathrm{PH}$ yielding a sensitivity of $53 \%$ and specificity of $78 \%$ for the detection/exclusion of manifest $\mathrm{PH}$.

In conclusion, the phase analysis in patients with nonspecific pulmonary disease gives informations on the detection/exclusion of $\mathrm{PH}$ under rest conditions, which are available only by various exercise tests.

452

B.Fanti, M.Marengo, D.Pancaldi, R.Franchi, C.Corbelli, G.Compagnone, K.Dondi, N.Monetti. Depts. of Nuclear Medicine, Health Physics. s.orsola-Malpighi Hospital, Bologna, ITALY

EVALUATION OF PULMONARY CLEARANCE OF PERTECHNEGAS (MODIFIED TECHNEGAS).

A modified form of Technegas can be produced by combustion of $99 \mathrm{mTcO}_{4}^{-}$in a chamber filled with 96\% oxygen and $4 \%$ argon. The fine and dry aerosol obtained is called Pertechnegas and is rapidly cleared from the lungs. The present study sought to assess the reliability of ventilation scan with Pertechnegas to evaluate pulmonary epithelial permeability. Six patients without evidence of pulmonary disease were investigated. pertechnegas was inhaled by patient in sitting position; images were dynamically recorded for $30 \mathrm{~min}$ in posterior view and data elaborated to obtain timeactivity curves for each lung. Time to halfclearance $\left(T^{\frac{1}{2}}\right)$ was calculated by single exponential function fitted to the initial 7min data (Fig.1). Pertechnegas showed peripheral penetration and homogeneous distribution throughout lungs. $T \frac{1}{2}$ values are reported in Tab.1; mean $\mathrm{T} \frac{1}{2}$ was $10.7 \pm 2.1 \mathrm{~min}$.

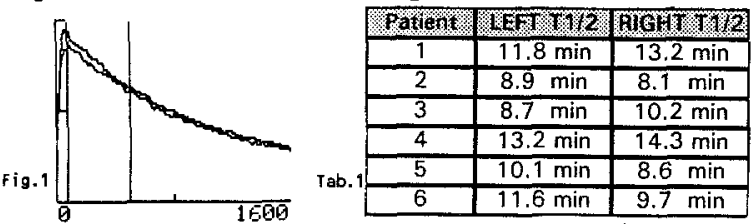

our data shows that Pertechnegas particles are rapidly cleared from the lung and suggest that this approach may have a role in studying pulmonary epithelial permeability. 


\section{3}

S.J. Wang, C.H. Kao and S.H. Yeh

Tajchung Veterans General Hospital and National Yang-Ming Medical College, Taiwan

THE RELATIONSHIP OF ALVEOLAR INTEGRIIY AND INFLAMMATION IN PATIENTS INHALATION LUNG SCINIIGRAPHY AND QUANTITATIVE GA LUNG SCAN

The alveolar integrity (AI) in 21 patients (15 males, 6 females ages: $31-74$ years, without smoking history) with interstitial lung disease (ILD) was measured by TC-99m DTPA radioaerosol jnhalation lung scintigraphy (DTPA) using a conmercial lung aerosol delivery un (AERO/VENT, MEDI-MUCLEAR) The degree of AI damage in ILD was presented as the slope (\%/min) of the time-activity curve from the dynamic lung imagings ( 1 frame/ 1 m in for 30 min over the posterior view of the chest). The AI of ILD patients were compared with the AI of 20 normal controls (ages: 29-76 years, without smoking history with normal chest $X$-ray and pulmonary function test). The patients were divided into two groups: (A) 8 ILD with normal chest X-ray $(X-$ ray) and (B) 13 ILD with abnormal $X$-ray. Meanwhile, the quantitative Ga lung scan (GA) was performed to evaluate the severity of inflammation in the lungs (IL) in ILD. The results are $Z$ tabulated as follows:

\begin{tabular}{lllllll}
\hline $\begin{array}{l}\text { Patient } \\
\text { Groups }\end{array}$ & $\begin{array}{c}\text { Slope } \\
(\% / m i n)\end{array}$ & $\begin{array}{c}\text { R value } \\
\text { with GA }\end{array}$ & $\begin{array}{c}\text { Lung } \\
(\% / m i n)\end{array}$ & $\begin{array}{c}\text { R value } \\
\text { with GA }\end{array}$ \\
\hline Normal & RUL & $1.10 \pm 1.02$ & LUL & $0.90 \pm 0.90$ & \\
controls RML & $0.97 \pm 0.74$ & LML & $0.74 \pm 0.79$ \\
\multirow{3}{*}{ ILD } & RLL & $1.66 \pm 0.95$ & & LLL & $1.27 \pm 0.95$ & \\
& RLL & $1.72 \pm 1.19$ & 0.09 & LUL & $1.45 \pm 0.86$ & 0.40 \\
& RML & $1.69 \pm 1.33$ & 0.35 & LML & $1.67 \pm 1.14$ & 0.30 \\
& RLL & $2.59 \pm 2.01$ & 0.32 & LLL & $1.92 \pm 1.73$ & 0.25
\end{tabular}

* There were significantly statistical differences between normal controls and patients with ILD ( $P<0,05$, unpaired $t$-test) as were shown in the slope. However no significant difference existed between groups $(A)$ and $(B)$. No good correlation between the degree of I damage and IL was found

In conclusion, (1) the AI of ILD is injured, as is shown by a higher slope in DTPA than that in normal controls; (2) the degree of AI damage in ILD is not consistent with the X-ray or GA results, which suggests that the mechanism involved in the evaluation of AI in ILO using DTPA could be different from those in traditional tests.

\section{4}

I.Banzo, R.Quirce, J.Jiménez-Bonilla, O.Tabuenca, R.G-Barquín, IM.Carril

Servicio de Medicina Nuclear. Hospital Universitario "M.Valdecilla" Santander. España.

CONTRIBUTION OF PERTECHNEGAS AND DTPA AEROSOL STUDIES IN PNEUMOCYSTIS CARINII PNEUMONIA

Changes of lung epithelial permeability have been documented with $99 \mathrm{mTc}$ DTPA aerosol (AERO) in several diseases. Also, lung clearances studies have been obtained with Pertechnegas (PER), a technical modification of Technegas produced by combustion in a $97 \%$ argon and $3 \%$ oxygen atmosphere. We report the results of AERO and PER studies for evaluating the alterations of lung epithelial permeability in $\mathrm{HIV}(+)$ patients with Pneumocystis Carinii Pneumonia (PCP).

This retrospective study included $8 \mathrm{HIV}(+)$ drug addicts patients with PCP confirmed by induced sputum or broncoalveolar lavage. For ventilation studies, patients were placed supine over a gammacamera with a high sensitivity collimator. The PER study was acquired after 3-5 inspirations and the pulmonary activity was recorded in $64 \times 64$ matrix during 25 minutes (1 frame $/ 30$ seconds). The AERO was performed the same day at the same conditions as PER study but the inhalation period was 2-3 minutes. Time/activity curves were obtained for each whole lung; they were corrected for a background activity area drawn over the interrenal region. T50 of lung clearance was calculated as follows: a fit of the final 7 minutes was performed to estimate the slower exponential; this curve was subtracted from the original curve and then a single exponential fit of the rapid component was performed. Final T50 was the mean of two lung regions.

All clearance studies showed biphasic curves. T50 ranged from 2.3 to 5.3 minutes (mean $3.36 \pm 0.87$ minutes) for PER; in the AERO study, the T50 range was 2.80 to 5.80 minutes (mean $4.26 \pm 1.2$ minutes)

Ventilation studies with PER and AERO showed similar results to evaluate lung epithelial permeability in PCP. The PER study is easier to perform because the inhalation period is shorter. In an attempt to provide an index for management of AIDS patients with clinical suspicion of PCP, larger patients population are needed to compare both ventilation procedures.
455

H Yağcıoğlu A Gürler, E.Özalp, N.O. Küçük, M.K.Kır,

G. Erbay.

Ankara Numune Hospital, Ankara University, Medical School,

Dept. of Dermatology and Nuclear Medicine. Ankara- TURKIYE

\section{EVALUATTON OF LUNG EPITHELLAL PERMEABILITY IN BEHCET DISEASE}

Behçet is a multisystem disorder of unknown etiology for which pulmonary manifestations are reported to be rare. Recent pathologic studies demonstrated pulmonary changes in patients with Behçet disease with obscure clinical picture.

In this study lung epithelial permeability was evaluated using Tc-99m DTPA in 13 non-smoker patients with Behçet disease( 3 female, 10 male, age between 25 -34). Patients had no pulmonary symptoms.

The inhalation studies were performed after a 3 minutes inhalation of $20 \mathrm{mCi}$ of Tc-99m DTPA in $3 \mathrm{cc}$ solution. During 20 mimutes dynamic acquisition view in a $64 \times 64$ matrix was collected from posterior thorax.

Lung epitelial permeability (LEP) was calculated from clearance curves obtained from total lung region and expressed as T1/2(min) of inhalated DTPA clearence of lungs. Results were compared to those of healthy non-smokers $(n=8)$. T1/2 value was calculated to be $55.45 \pm 29.66 \mathrm{~min}$ for patients with Behçet disease and $80.67 \pm 21.08 \mathrm{~min}$ for normal group. When compared to control group, T1/2 value in Behçet disease was significantly increased $(\mathrm{p}<0.05$ ).

Subclinic changes in lungs may occur in Behçet disease. This is a preliminary report and the value of radioaerosol studies in this respect should be investigated in larger populations.
456

A. Brut, F. Dubois, F. Blano-Jouvan, C. Pison, E Brambilla. M. Comet.

Service de Médecine Nucléaire. Service de Pneumologie, Service d'Anatomopathologie. CHRU de Grenoble. France.

DETERMINATION OF OPTIMAL VALUE OF DTPA CLEARANCE FOP THE DIAGNOSIS OF ALLOGRAFT LUNG REJECTIONBY ROC. ANALYSIS.

76 DTPA clearances were performed on 10 patients with bipulmonary lung transplantation and 2 patients with monopulmonary lung transplantation. The pulmonary Tc99m-DTPA clearance rate was calculated from negative slope of the regression line and expressed in term of percentage of the radioactivity par minute $(\% / \mathrm{min})$. Using transbronchial biopsy samples, three groups were studied: Normal lung histology (42 values), lung rejection pattern: grade I (20 values), grade 11 or III (14 values). The optimal value of DTPA clearance in terms of sensitivity and specificity for the diagnosis of lung rejection was determined by a receiver operating curve analysis (R.O.C.)

A threshold of DTPA clearance of $2,2 \% / \mathrm{min}$ was both sensitive $(76 \%)$ and specific $(61 \%)$ for the diagnosis of lung rejection (grade I to III). A threshold of DTPA clearance of $2,5 \% / \mathrm{min}$ was both sensitive $(72 \%)$ and specific $(60 \%)$ for the diagnosis of lung rejection (grade II or III).

An increase of $20 \%$ of DPTA clearance between two determinations was both sensitive $(83 \%)$ and specific $(83 \%)$ for the diagnosis of aggravation (Normal to grade 1,11 or 111; or grade I to grade 11 or 111$)$. These results suggest that DTPA clearance monitoring appear to be a good non invasive approach for the detection of lung rejection. 
461

M. Clausen, K. H. Bohuslavizki, T. Schreiber, F. Konrad, M. Schramm, H. Wolf, W. Brenner, J.-U. Eberhardt, E. Henze.

Departments of Nuclear Medicine and Anesthesia, Universities of Kiel and Ulm, Germany

\section{TRACHEOBRONCHIAL MUCUS TRANSPORT IN POST- OPERATIVE PATIENTS WITH DIFFERENT DOSAGES OF THEOPHYLLINE}

Mucociliary clearance depends in part on tracheobronchial mucus transport (TMT). A reduced TMT may result in clinical problems in postoperative patients. The purpose of this study is to define TMT in patients still during anesthesia under various dosages of theophylline.

In 30 patients informed consent was obtained, the protocol met approval by the local ethics committee. TMT was evaluated directly following surgery with the patients still under anesthesia. At the end of endocsopic cleaning $1.5 \mathrm{MBq} T \mathrm{c}-99 \mathrm{~m}$ macroaggregated human serum albumin was applied as a depot on the right and left bronchus through the scope. Dynamic data acquired for 30 min were processed by the condensed image technique and quantified: In this path-time diagramm, the slope yields TMT in $\mathrm{mm} / \mathrm{min}$.

Prior to medication by theophylline the TMT tanged from 0 to 7.8 , median $0.55 \mathrm{~mm} / \mathrm{min}$. Following $1 \mathrm{mg} / \mathrm{kg} / \mathrm{h}$ theophylline TMT increased to the median 5.4, ranging from 0.7 to $10.8 \mathrm{~mm} /$ min. However, the $0.5 \mathrm{mg} / \mathrm{kg} / \mathrm{h}$ dose of theophylline had no measureable effect on TMT. Observe: While there is a definite effect of pharmacological dosis on the TMT, the range within the groups are two- to threefold.

In conclusion, while TMT may be evaluated in the postoperative patient using the bronchoscope for application, the effects of theophylline are small when compared to the range of TMT prior to medication and have to be weighted against cardiovascular side-effects.

\section{2}

M.Toubeau, C.Touzery, A.Berriolo-Riedinger, Y.Cottin, D.Bernard, J.M.Riedinger, D.Chevet, F.Brunotte.

Service de Médecine nucléaire, Centre G.F. Leclerc, service de Nếphrologie, CHU, DIJON, FRANCE.

A NEW SIMPLE METHOD TO EXPRESS MAG-3 RENAL UPTAKE IN RENAL TRANSPLANTED PATIENTS,

The determination of plasma renal clearance using one or two blood samples has now gained wide acceptance in nuclear medicine departments. Results provided by such methods are greatly dependent on the good quality of technical parameters (activity totally injected,exact dilution). We present here a simple method based on scintigraphic data acquired before blood sampling in renal transplanted patients, that could be used to validate the quality of clearance measurements. On renal scintigraphy, an index of renal function (I.R.F.) is calculated as the ratio of renal uptake (between 1 and 2 min after injection) divided by the mean value of background counts near the renal area. No depth correction is made.

We performed MAG-3 renal scintigraphy in 67 transplanted patients (44 men 23 women aged 18-66 years). The I.R.F. was calculated and MAG-3 clearance obtained by MULLER method with a single plasma sample at $60 \mathrm{~min}$. The range of the clearances was 31-225 $\mathrm{mil} / \mathrm{min}$. A good linear regression was found between MAG-3 plasma clearance and the IR.F with a correlation coefficient of $0.76\left(\mathrm{p}<10^{-9}\right.$ SSEE $=29.8 \mathrm{ml} / \mathrm{min}$ ).

The usefulness of the IR.F. was observed in 2 transplanted patients with unexpected very high plasma clearance determinations ( 268 and $301 \mathrm{ml} / \mathrm{min}$ ) suggesting technical problems as extravasation of injected activity or wrong dilutions. Corresponding I.R.F. values gave a very good agreement between predicted and experimental clearance values, eliminating the possibility of such mistakes. This method using only scintigraphic data does not depend on plasma counting and is especially useful when an accurate clearance measurement cannot be obtained due to a technical failure.
463

M. Mitjavila, C. Bas, C. Nieto, MD. Rueda, C. Caball ero, A. Crespo.

Nuclear Medicine Department. Hospita I Ra món y Cajal Madrid. España.

RENAI FUNCTION FOLLOW-UP OF RENAL TRANSPLANTED PATIENTS ON CYCLOSPORINE.

Creatinine clearance (CCr) is not accurate for stimating of glomerular filtration rate (GFR) in renal transplants. The overstimation of GFR by CCr could be due to hypersecretion of creatinine by injured tubules. The assessment of GFR with inulin is cumbersome and time-consuming. In 19 stable renal transplanted patients on cyclosporine, with serum creatinine (SCr) between 1.0 and 4.3 $\mathrm{mg} / \mathrm{dl}$ (mean $1.9 \pm 0.8 \mathrm{mg} / \mathrm{al}$ ) GER was evaluated by clearances of imulin, CCr and by ${ }^{51} \mathrm{Cr}-\mathrm{EDTA}$. The GFR by ${ }^{51} \mathrm{Cr}$-gDTA was calculated by fitting the plasma clearance curve to a monoexponencial function using blood samples taken after 2 and 3 hours.

RESUTTS: 1 Creatinine clearance values were significativily higher than inulin clearance $(57.73 \pm 29.57$ vs $34.6 \pm 18.68) .2^{51} \mathrm{Cr}-$ EDTA clearance values $\left(3 \frac{4}{2} .29 \pm 15.87\right)$ showed no differences to those of inulin clearance. concuUsIoNs: 1 our results confirm that $\mathrm{CCr}$ overstimates GFR in renal transplanted patients. 2 Renal clearance of ${ }^{51} \mathrm{Cr}-\mathrm{EDTA}$ can be used to measure accurately GFR in renaI transplanted patients on cyclosporine.

464

${ }^{\text {DD. Huić, }}{ }^{2} \mathrm{~B}$. Brkljačić, ${ }^{3}$ V.Mrzljak, ${ }^{1}$ D.Grošev, ${ }^{1}$ M. Poropat, ${ }^{1}$ D.Dodig, 'S.Kusacić-Kuna

'University Hospital Center "Rebro", "University Hospital "Merkur", ${ }^{3}$ Institute for Diabetes "Vuk Vrhovac", Zagreb, Croatia

RADIONUCLIDE METHODS AND ULTRASOUND DOPPLER N THE ASSESSMENT OF DIABETIC NEPHROPATHY

We performed $99 \mathrm{mTc}$-DTPA renal scintigraphy and ultrasound Doppler examination in 21 patients (14 woman, 7 man, mean age 35 , 42 kidneys) with long term diabetes mellitus duration (mean 19 years). Renal blood flow expressed as a percentage of cardiac output (RBF) and glomerular filtration rate (GFR - 4 blood samples), computed for each kidney, were compared with the Doppler ratio of peak diastolic to systolic velocity (D/S), serum creatinine level (SCL) and creatinine clearance (C CR).

Obtained correlations are presented in table.

\begin{tabular}{|c|c|c|c|c|c|c|}
\hline & $\begin{array}{l}\mathrm{RBF} \\
-\mathrm{D} / \mathrm{S}\end{array}$ & $\begin{array}{l}\text { GFR } \\
-\mathrm{DS}\end{array}$ & $\begin{array}{l}\mathrm{D} / \mathrm{S} \\
\text { SCL }\end{array}$ & $\begin{array}{l}\text { GFR } \\
-\mathrm{SCL}\end{array}$ & $\begin{array}{l}\text { GFR } \\
-\mathrm{C} \mathrm{CR}\end{array}$ & $\begin{array}{l}\mathrm{RBF} \\
-\mathrm{SCL}\end{array}$ \\
\hline $\mathrm{N}$ & 34 & 34 & 18 & 18 & 18 & 17 \\
\hline $\mathrm{r}$ & 0.26 & 0.30 & -0.17 & -0.92 & 0.82 & -0.75 \\
\hline $\mathrm{p}$ & $>0.05$ & $>0.05$ & $>0.05$ & $<0.001$ & $<0.001$ & $<0.001$ \\
\hline
\end{tabular}

The results showed poor correlations among $\mathrm{D} / \mathrm{S}$ and other indicators of renal function, which could be probably improved with more precise instruments.

The radionuclide values, obtained from single injection of DTPA, correlated well with $\mathrm{SCL}$ and $\mathrm{C} C \mathrm{CR}$ values.

SCL seems to be more reliable indicator of glomerular filtration rate in diabetic nephropathy than $\mathrm{C} \mathrm{CR}$, which is greatly dependent upon accurate urine collection. 
465

Käiremo KuA, Taari K, Salo Jo, Rannikko S, Kivisaari A

Deparments of Clinical Chemistry, Urology \& Radiology, Helsinki University Central hospital, FINLAND

RBNAL EUNCTION AFTER PARTIAL NEPHRECTOMY IN PTGLETS

Twelve partiaI nephrectomies (NE) were performed in 12 piglets (11-17 kgs) using either Nd-YAG laser or steel scalpeI. Total NE was performed on the left side and partial NES on the right side (lower third of the kidney) with both techniques. Renal function was studied with Tc-99m-DTPA renography, serum urea and creatinine levels preoperatively, and 1 and 2 weeks postoperatively. The piglets were imaged in each session for $30 \mathrm{~min}$ by collecting 10-sec frames from $\mathrm{PA}$ view of an anesthesized ansmal. The injected activity was $37 \mathrm{MBq}$. Sertal blood samples were taken from the subclavian vein at $0,1,2,3,5,15,25,40,60$ and 120 min (6 animals) after TC99m-DTPA injection. The DTPA dissappearance rate (DDR) was determined from these samples and in other cases a blood sample at $20 \mathrm{~min}$ was used. The DDR was also determined from the dynamic gamma imaging data: ROI regions upper body, spleer, heart, and kidneys. The ROI analysis correlated well wth the blood sampling data ( $r=0.97, \mathrm{p}<0.00 \mathrm{i})$. The reference values for piglet DDRs were in laser group (t) $1.00 \pm 0.06 \% / \mathrm{min}$ and in scalpel growp (S) $0.97 \pm 0.09 \% / \mathrm{min}$. After 1 week postoperatively these values were: (L) $0.65 \pm 0.10$ and (S) $0.75 \pm 0.06 \% / \mathrm{min}$, and after 2 weeks: (I) $0.52 \pm 0.09$ and (S) $0.73 \pm 0.06$ $\% / \mathrm{min}$. DTPA clearance rates were preop. (L) $0.54 \pm 0.04$ and (S) $0.51 \pm 0.07 \mathrm{ml} / \mathrm{s}$; one week postop. (L) $0.35 \pm 0.05$ and (s) $0.47 \pm 0.07 \mathrm{mI} / \mathrm{s}$; and after 2 weeks (t) $0.29 \pm 0.05$ and (s) $0.40 \pm 0.07 \mathrm{ml} / \mathrm{s}$. In creatinine and urea concentrations there were no significant differences between I and $\mathrm{S}$ groups pre- and postoperatively. This data indicates that a third of kidney can be removed without a significant loss of kidney function, and there is no significant difference between surgical methods.

\section{6}

L. Wallin, M. Bajc, A. Kristjansson

Departments of Clinical Physiology and Urology University Hospital of Lund, Sweden.

COMPARISON OF GERARATE REAAL FUNCTION AGEESGED BY MAG3-RENOGRARHY AND DMSA-BPET.

The ain of the study was to find a more accurate estimation of separate renal function. Therefore comparison of separate renal function assessed by MAG3-renography and DMSA-SPET was performed.

Fourteen patients (aged 64-87 years) underwent MAG3-renography and DMSA-SPET. Separate renal function was expressed in per cent of total renal activity. At renography, total renal function was calculated as activity uptake in regions of interest over the kidneys, from 80 to 110 seconds after intravenous injection, with correction for background activity. At SPET total renal function was calculated as the activity in regions of interest over the kidneys in the sum of coronal slices.

The difference between renal function measured by renography and by SPET was $0-18$ in 4 patients, 2-4\% in 4 patients and $5-10 \%$ in 6 patients. Among patients in whon the difference of renal function measurement was 5-10\% the discrepancy could be explained by mobile kidney in one patient, and in the other five patients one kidney had slightly atypical location not visible from the dorsal view. In the group with diffrence in measurements of 2-48 there was no obvious explanation for the discrepancy.

We conclude that renography from dorsal view may not be sufficient for correct estimation of separate renal function. SPET or renography with simultaneous anterior and posterior view can provide more accurate information.

\section{7}

B. Bagni, A.R. Cavallini, M.C. Marzola

Nuclear Medicine Department. S. Anna Hospital, Ferrara, Italy.

RELATIONSHIP OF GENDER AND OF AGE TO 131I-OIH AND 99MTC-MAG3 CLEARANCE.

Technetium Mag3 Clearance is proportional to OIH Clearance in adults and can be estimated using a single sample technique similar to the method described by Tauxe and co-workers. The relationship between clearance and age and gender has not been reported. We performed a study in 97 normal adults who underwent scintigraphy to evaluate renal function. Normal renogram patterns, creatinine clearance, BUN and urea clearance as well as kidney ultrasonography were obtained in all of the patients. The simultaneous infection of $131 \mathrm{~T}-\mathrm{OIH}$ and $99 \mathrm{mTC}-\mathrm{Mag} 3$, and a single plasma sample technique was used to evaluate the ERPF in this normal population. The results show a good correlation between the values of ERPF for $O I H$ and MAG3 evaluated with the single sample technique.

A Iinear correlation between age and clearance, was found for OIF as has been previously reported and in this study for MAG3. The linear regressions for MAG3 are: MAG3 Clearance $(\mathrm{ml} / \mathrm{min})=540-5.12 \times$ age, $=-0.67, p<0.001$ in women and MAG3 Clearance $(\mathrm{ml} / \mathrm{min})=497-4.3 \mathrm{x}$ age, $r=-0.58, \mathrm{p}<$ 0.001 in men. The MAG3 clearance in women averaged $273 \mathrm{ml} / \mathrm{min} \pm 74$ and in men $278 \mathrm{ml} / \mathrm{min}$ \pm 76 .

These data reconfirm the relation between MAG3 and hippuran clearance and suggest that normal values for MAG3 should be age and gender corrected to achieve maximum accuracy.

468

K. Stipsanelli, B.Papantoniou,M.Christoforaki, E. Papadakis, Z.Anagnostopoulos, E. Neou, K. Kassiou.

Depts. of Nuclear Medicine "Alexandra" Hospital and Children's Hospital 'A.Kyriakou' 'Diabetes Center. Athens, Greece.

EARLY PREDICTORS OF DIABEITIC NEPHROPATHY IN CEILDREN AND ADOLESCENTS WITH DIABETES MELTYUS TYPF I.

The ain of the study was to estimate possible risk factors in developing diabetic nephropathy in patients with IDDM. The study consisted of 114 patients $63 \mathrm{M}, 51 \mathrm{~F} . \overline{\mathrm{X}}$ 12,5 years $S D=2,9$. Blood pressure was normal and their $u-$ sual parameters for renal function (plasma ccreatinine urea) $\mathrm{Cr}$, Ur, were normal Depending on the poor moderate or sufficient metabolic control( $\mathrm{HbAlc}$ ) the patients were divided into 2 groups.

G1. HBAlC > $72 \% \quad \mathrm{~N}=54 \quad(28 \mathrm{M} 26 \%) \quad \pi \quad=13 \quad \mathrm{SD}=2.8$ 92. HDAic $\leq 12 \% \quad \mathrm{~N}=60 \quad$ (35M 25*) $\quad \overline{\mathrm{X}}=11,8 \quad \mathrm{SD}=3$, Additionally. The patients classified in 3 groups depending on the duration of the disease.

Patients A. $23 \quad 16 \mathrm{M} \quad 7 \mathrm{~F} \quad \mathrm{X}=10,5 \quad \mathrm{SD}=2,4 \leqslant 1$ month

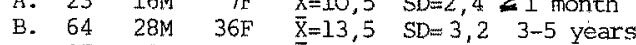
C. $27 \quad 19 \mathrm{M} \quad 8 \mathrm{~F} \quad \overline{\mathrm{X}}=13 \quad \mathrm{SD}=2,8 \quad 5$ years Nornal D. $16 \quad 10 \mathrm{M} \quad 6 \mathrm{~F} \quad \overline{\mathrm{X}}=9 \quad \mathrm{SD}=3,2$

The following parameters were measured, 1)PRA by RIA method 2) urine albumine. 3) HbAlc 4)GFR $C r-51$ EDTA. The linear regression analysis was used for A.B.C and $t$ test between G1, G2, D and all diabetics. There was very good correlation For Alb. and PRA $r=-0,368$, Alb. and GFR $r=0,6 \mathrm{GER}$ and $\mathrm{P}$ ? $\mathrm{A}$ $r=-0,58$ in group B.For GFR and PRA $r=-0,49$ in group $C$. $\mathrm{PRA}$ in group 1 was lower significantly than in group 2. GER did not differ significantly between the 2 groups. Moreover control group D was higher significantly than in alabetics. Conclusions 1) The low levels of PRA in patients with poor metabolic control is one more risk factor of developing diabetic nephropathy. 2) PRA in control group is higher than in young alabetic patients with IDDM.3)PRA $G F R$ and $A 1 B$ should be estimated as early predictors of D.N. 


\section{9}

M.Mitjavila, C. Zas, C. Nieto, C.Lancha, M E. Kioja, L. Die-..

Suclear Medicine Department. Hospital Ramón y Cajal. Madrid. España.

RENOVASCULAR DISEASE IN CHILDREN: PROGNOSTIC VALUE OF CAPTOPRIL RENAL SCINTIGRAPHY (CRS) IN TWO PATIENTS.

Disease of renal vascularity resulting from lesions that cause unilateral or bilateral impaiment of blood flow to kidneys are far more uncommon than diseases of renal parenchyma, they are about a $10 \%$ of cases of secondary hypertension in children, and trend to appear more commoly in younger children. Renal artery stenosis is the second most important forn of hypertension in children that can be surgically solved.

DTPA captopril renal scintigraphy (CRS) has proven to be very effective in the assesment of renal vascular hypertension (RVH), however cases reported in children are limited.

Two children with RVH were examined before and after surgery using DTPA scintigraphy in basal state and one hour following oral captopril administration $(0.5 \mathrm{mg} / \mathrm{Kg})$.

1.- In a seven year o1d girl with unilateral RVH shown by angiography, the test using CRS strongly predicted that percutaneos transluminal renal angioplasty (PTRA) should achieve positive results. The clinical outcome (nomalitation of blood pressure) and another DTPA CRS test confirtaed this succesfull treatment.

2.- In another two year old girl the CRS using selective RoIS was able to demonstrate a significative stenosis of both the left principal and the right polar superior renal arteries. PTRA failed to normalize blood pressure and in this case another CRS matched the presurgical test.

470

D. Sandrock, A. Kögler, V. Ivancevic, M. Steinröder, D.L. Munz, D. Emrich

Department of Nuclear Medicine, Georg August University، Göttingen, Germany

CONCORDANT DATA WITH I-131 HIPPURAN AND TC$99 \mathrm{mAG}_{3}$ ?

Aim of this intraindividual comparative parallel study was to evaluate the correlation of different parameters between 1-131 hippuran and Tc-99m MAG 3 .

52 patients lessential hypertension, 12; renal artery stenosis, 5; interstitial disease, 21; tumor, 4; obstruction, 10) aged between 22 and 65 years were studied after appropriate hydration using $18 \mathrm{MBq}$ I-131 hippuran (Oberhausen 4-probe device) and, within $4 \mathrm{hr}, 185 \mathrm{MBq}$ Tc-99m $\mathrm{MAG}_{3}$ (gamma camera, ROI technique).

Overall, there was a good correlation for the split function of the kidneys for the two tracers $(r=0.80)$ while the time-to-peak $\left(t_{\max }\right)$ and the half-life of the $t_{\max }$ gave low correlation values $(r=0.56$ and $r=0.55$, respectively). Time-to-peak was $3.54+/-1.71 \mathrm{~min}$ for hippuran (versus $3.56+/-1.48$ for $\left.\mathrm{MAG}_{3}\right)$ and half-life was $6.4+/-2.74$ (MAG $\mathbf{3}: \mathbf{8 . 9}+/-3.68)$ with more significant differences in patients with interstitial disease and tumors.

We conclude that split function has a good correlation between hippuran and $\mathrm{MAG}_{3}$ while careful comparison for other parameters from time-activity curves is recommended.
471

S.Miceva-Ristevska,N.Serafimov,S.Loparska,D.PopGorceva,V.Bogdanova,B.Andonovski,B.Badiev, L j,Lekovski Institute of Pathophysiology and Nuclear Medicine, Clinic of Urology - Medical Faculty,Skopje Republic of MACEDONIA

TG 99M DTPA AND TC 99M DMSA STUDIES BEFORE AND AFTER EXTRACORPORAL SHOCK WAVE LITHOTRIPSY

Renal function and morphology were examined in 39 patients with renal calculi before, $24 \mathrm{hr}$ and 3-4ws after extracorporal shock wave lithotripsy (ESWL), using renal radionuclide studies (RRS) with Tc-99m DTPA and $\mathrm{Tc}-99 \mathrm{~m}$ DMSA.Relative renal function, glomerular filtration rate(GFR), parenchymal transit time index (PTTI-as an index of obstructive nephropathy-ON were determined and effect of furosemide was also determined for evidence of obstructing uropathy where PTTI was not done.Static images (SI), using Tc-99m DMSA, were done looking for parenchymal defects(PD) in the kidney before and after treatment.

In 15/39 treated kidneys (k) no changes of PTTI were observed before ESWL, $24 \mathrm{hrs}$ after ESWL,PTTI was prolonged in $9 / 15 \mathrm{k}$, and $3 w s$ later it was prolonged in $3 / 15 \mathrm{k}$. In $11 / 39 \mathrm{k}$ PTTI was prolonged before ESWL and 3ws later it was prolonged in $9 / 11$, in $1 / 11 k$ it was normal and in 1 it was not done. In $7 / 39 \mathrm{k}$ with reduced GFR before ESWL no improvement was noted $24 \mathrm{hrs}$ and 3 ws after ESWL. In $6 / 39 \mathrm{k}$ GFR was reduced before ESWL and showed improvement after it.In $9 / 39 k$ with nommal GFR before ESWL was found deterioration after ESWL. In 15/39k GFR was normal before and $3 \mathrm{ws}$ after ESWL.In $28 / 39 \mathrm{~K}$ no $\mathrm{PD}$ were noted before and after ESWL and in $11 / 39$ there were PD.In $4 / 9 \mathrm{~K}$ PD seen $24 \mathrm{hrs}$ after ESWL and dissappeared 3 ws later.In $4 / 9 \mathrm{k}$ PD seen $24 \mathrm{hr}$ s after ESWL persisted still 3ws later.In 2 patient PD were noted on SI before ESWL

\section{2}

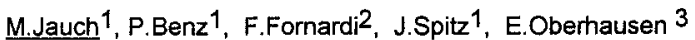

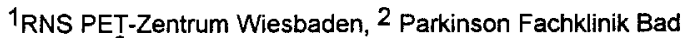
Nauheim 3Universität d.Saarlandes, Abt.f.Nuklearmedizin

\section{CAN PET HELP IN DIFFERENTIATION OF PARKINSON'S SYNDROM?}

The therapie of Parkinson's disease and diseases similar to Parkinson's has improved a lot within the last years. Using the various therapie modalities requires a differentiation between the subtypes of Parkinson syndrom. Out of 192 patients with Parkinson syndrom, provided with FDG-PET, a group of 70 patients with "Parkinson plus" disease was selected and compared with 23 patients with Parkinson's plus dementia and 17 patients with MSA respectively. All patients had a neurological examination (by the Parkinson Fachklinik).

PET: After overnight fasting and a relaxation phase (15 min.prior to injection) $200-350 \mathrm{MBq} 18$ - FDG were injected. Acqusition was performed on a GEMS 4096 system using a standard protocol:three emission scans ( $10 \mathrm{~min}$. each) $30-60 \mathrm{~min}$ p.i. were acquired. For quantification the autoradiographic method (Phelps/Huang) or semiquantitative ROI technique (ratios) was $\mathrm{u}$

Patients with "Parkinson's plus" disease showed a moderate reduction of glucose metabolism in frontal cortex and a more severe reduction in parietal and occipital cortex. Within the basal ganglia the reduction of glucose metabolism was more moderate compared to the cortex (in $60 \%$ the caudate nucleous showed a reduction, in $30 \%$ the putamen and in $10 \%$ the thalamus).

Patients with Parkinson's disease plus dementia had a reduced uptake in general, especially in the parietal and occipital cortex but nearly no disturbance of glucose metabolism in the basal ganglia. $40 \%$ of MSA patients had a moderate reduced uptake in frontal cortex, nearly no reduction in parietal and occipital cortex but a severe dropdown (in $60 \%$ ) in the putamen

There is evidence for the possibility of discrimination between the three subgroups by measuring cerebral glucose metabolis therefore it is a usefull tool for group related therapy. 
473

G. Demonceau, C. Monte, H. Colaert, J. De Vuyst, C. Vanhove.

St Elisabeth Hospital, Zottegem (Beigium) and Trionix Res. Lab., Twinsburg

PROGNOSTIC VALUE OF THE ACETAZOLAMIDE TEST IN CASE OF ACUTE CEREBRAL ISCHEMIA.

We tried to predict the capacity of recovery of patients suffering from acute ischemic attack, by testing the relation to the amplitude of the vascular reserve

Nine volunteers and 36 patients (26 strokes, 10 TIA) were included in the study. Each of them was first injected with $300 \mathrm{MBq}$ of Tc-HM-PAO and a tomography with a 3-head camera was performed 5 minutes later, 30 steps of $4^{\circ}$ per head, $75 \mathrm{sec}$ per step, in matrix $128 \times 128$. Twenty minutes before the completion, $1 \mathrm{~g}$ acetazolamide (10 cc saline in case of volunteers) was injected. At the end of the study, $520 \mathrm{MBq}$ of Tc-HM-PAO were again injected and 5 minutes later, a second tomography was obtained, using the same parameters as for the first, but 42 seconds per step. Automatic analysis included 3D geometric correction (necessary despite a dedicated brain holder), activity correction (difference in dose, acquisition time, ...), automatic detection of the pathological regions (based on flow and response to acetazolamide) and determination in the region of the area, the mean and the maximal value of the flow and the response to acetazolamide. The follow-up of the stroke patients was pursued during at leas I month and only medical treatment was applied.

Significant correlation $(\mathrm{p}=0.0 \mathrm{l})$ was found between the area of low flow normal response and the hypo density seen on the CT-scan on the 4th day after the attack. Significant correlation were also found between the clinical outcome and the defined regions, specially $(p=0.001)$ between the zone of normal flowabnormal response and the clinical outcome during the first 2 weeks.

In conclusion, the acetazolamide test combined with a full automatic software allows a good prediction of the clinical outcome of stroke patients, which looks particularly interesting for the evaluation of the therapeutic effect of drugs.

474

N. Czech ${ }^{1}$, A. Hartmann ${ }^{2}$, T. Kuwert 1, K. $-J$. Langen $^{1}$, K. Ziemons ${ }^{1}$ S. Shikare ${ }^{1}$, H. Boecker ${ }^{1}$, H. Herzogl, H.W. Mülier-Gärtner ${ }^{1}$

1) Inst. of Medicine, KFA Jülich; 2) Dept. of Neurology, University of Bonn, FRG.

ACCURACY OF GLUCOSE METABOLISM VERSUS PERFUSION SCANNING IN DIAGNOSIS OF PROBABLE ALZHEIMER'S DISEASE

The aim of this study was to compare the validity of regional glucose consumption (rCMRglc) and regional cerebral blood flow measurements ( $\mathrm{rCBF}$ ) in the diagnosis of probable Alzheimer's disease (AD).

Diagnosis of probable AD was established using NINCDS-ARDRA criteria. Ten normal volunteers and sixteen patients with probable $A D$ were studied with both modalities within one day. Regional CMRglc was measured using FDG with the PET-camera PC-4096; brain perfusion was determined using HMPAO and a triple-detector camera. The images were visually evaluated for zones of reduced rCMRglc and HMPAO uptake by three independent observers.

The results are summarised in Table 1. Table 1: Sensitivity and specificity in diagnosis of probable AD

\begin{tabular}{|c|c|c|}
\hline & $\begin{array}{c}\text { Pathological Findings in } \\
\text { Controls }(n=10)\end{array}$ & prob. AD (n=16) \\
\hline rCMRglc - PET & $0 / 10$ & $10 / 16$ \\
\hline rCBF - SPECT & $1 / 10$ & $7 / 16$ \\
\hline
\end{tabular}

The data indicate that even with a state-ofthe-art SPECT technique sensitivity in diagnosis of probable Alzheimer's disease is higher using measurements of glucose metabolism when compared to perfusion.
475

W.Burchert, T. Brunkhorst, H. Rückholdt", E. Rickels**, J. van den Hoff, G.-J. Meyer, H. Hundeshagen

Depts. of Nuclear Medicine, Anesthesia II ${ }^{*}$, Neurosurgery ${ }^{\star \star}$ Medical School Hannover, Germany

\section{CEREBRAL BLOOD FLOW AND GLUCOSE UTILIZATION MEASURED BY PET IN PATIENTS WITH CEREBRAL INJURY}

Controlled hyperventilation is supposed to be an adequate therapy in cerebral trauma patients and is often combined with intravenous administration of anaesthetics. In order to test this clinical procedure cerebral blood flow (CBF) and regional glucose utilization (MRGlc) was assessed in 27 ICU patients with dynamic positron emission tomography (PET).

The perfusion measurements were performed with [15-O]-H2O using a SIEMENS/ECAT 951 scanner. Perfusion was determined under baseline conditions (hyperventilation / analgo-sedation) and after an additional narcotic dose. In the next step perfusion was measured under normoventilation $(\mathrm{CO} 2 \mathrm{et}=5.5 \%)$. Finally, MRGlc was determined under newly adjusted baseline conditions using [18F]-Fluoro-Deoxyglucose.

Under baseline conditions the mean perfusion was $0.42 \mathrm{~m} 1 / \mathrm{min} / \mathrm{ml}$. After additonal narcotics application mean perfusion decreased to $0.33 \mathrm{ml} / \mathrm{min} / \mathrm{ml}(-21 \%)$ but maximum reduction amounted to $48 \%$. During normoventilation a mean increase in perfusion of $34 \%$ above baseline was observed. MRGlc under baseline conditions was significantly reduced as compared to normal values with a mean of $0.15 \mu \mathrm{mol} / \mathrm{min} / \mathrm{ml}$

Although the mean decrease of perfusion under hyperventilation in combination with anaesthetics is tolerable, critical values in some patients occured. Therefore, the concept of hyperventilation in the manegment of head injuries appears to be questionable. Obviously, the additional application of intraveneous narcotics can be dangerous.

\section{6}

L. Pávics ${ }^{*}$, F. Grünwald, C. Menzel, K. Reichmann, A. Hufnagel, Ch. E. Elger, $H_{2}-J$. Biersack

Depts. of Nuclear Medicine and Epileptology, University of Bonn, *Supported by the A. v. Humboldt Foundation, Germany rCBF CHANGES IN EPILEPSY USING 99mTC-ECD: A QUANTITATIVE APPROACH

Regional cerebral blood flow (rCBF) studies play an important role in the presurgical evaluation of patients with epilepsy. In the localization of epileptic foci the highest efficacy has been described for the combination of ictal and interictal studies. The application of the new rCBF tracer $99 \mathrm{mTC}-\mathrm{ECD}$ in this indication is still under evaluation. The aim of present study was the quantitative evaluation of $99 \mathrm{mTC}-E C D$ uptake changes in ictal and interictal phases of focal epilepsy. In 8 patients ictal and interictal rCBF SPECT studies using $99 \mathrm{mTC}-\mathrm{ECD}$ with a high resolution SPECT system (CERASPECT) were performed. In 5 patients the ictal SPECT study was done twice $(41 \pm 8 \mathrm{~min}$ delay) to measure the tracer washout from hyperperfused areas. The SPECT data were analysed by a standard automatic quantification method, in 10 cantomeatal (4 under, 6 over the thalamus; 24 ROIs/ 1 ring /slice) and in 4 (under the thalamus, $2 \times 24 \mathrm{ROIs} / 2 \mathrm{rings} / \mathrm{slice}$ ) horizontal orinted transversal slices. Corresponding to the visual evaluation the quantitative analysis showed a significantly increased tracer uptake at the epileptic focus in the ictal study compared to the interictal study (asymmetry index: $1.23+0.08$ and $0,88+0.03$ respectively). In patients with repeated SPECT studies the washout from the hyperperfused areas was lower than from the total brain $(8.9 \pm 2.3 \%$ and $13.5 \pm 1.6 \%$ respectively $p<0.01)$. Compared to cortical areas the difference was statistically not significant. We concluded: 1. $99 \mathrm{mTC}$-ECD SPECT is able to detect rCBF changes between ictal and interictal stages in epilepsy. 2. There is no loss of contrast (focus / other brain region) to be expected with time elapsed in the ictal study 


\section{7}

C.Hoppe $^{1}$, I. Tuxhorn ${ }^{2}$, U. Buschs ieweke 1 , A.Ebner ${ }^{2}$

1Dept.Nucl.Med., Ev.Johanneskrh.Bielefeld

$2_{\text {Epilepsy Center, Bethel, Bielefeld , Germany }}$

ICTAL TC-99M HMPAO-SPECT FINDINGS IN FIVE CHILDREN WITH EXTRATEMPORAL FOCUS EPILEPSY Successful surgery in patients (pts) with intractable focal selzures depends on precise localisation of the seizure focus. In pts with nonlesional epilepsy with focal tonic seizures this is often difficult. The purpose of the study was to investigate whether ictal Tc-99M HMPAO SPECT's are helpful in localization in this group of pts. Five children (male, range:213 years) with intractable focal tonic seizure who had intensive video-EEG-monitoring for presurgical evaluation, optimized MRI-imaging and PET-scans were studied. SPECT was performed using a rectangular single head rotating gammacamera, 128 views on a $128 \times 128$ frame matrix. In 3 pts HMPAO was injected $i . v$. within $30 \mathrm{sec}$ of seizure onset, in 1 pt periictally of seizure onset and in 1 pt interictally. 2 scans( 1 periictally, 1 interictally) demonstrated hypoperfusion in the left temporo-occipital region. congruent with a structural lesion by MRI and findings by EEG.3 ictal SPECT scans demonstrated regional increase of tracer uptake,twice in the parasagittal vertex region and once in the left temporal occipital region. In the last pt this corresponded to a structural lesion, the 2 other pts where nonlesional but showed EEG changes in the vertex region. In $1 \mathrm{pt}$ the SPECT findings were helpful in lateralization. We conclude that ictal SPECT may be a sensitive noninvasive method for localization of focus in pts.with extratemporal epilepsy.

\section{8}

H Durak, İ Durak, B Degirmenci, M Yllmaz, E Metín, M Ergin. Departments of Nuclear Medicine and Ophthalmology, Dokuz Eylül Untversity School of Medicine, İzmir, Turkey.

\section{TC 99m HMPAO BRAIN SPECT IN AMBLYOPIA: THE EFFECT OF VISUAL ACTIVATION}

Based on limited work with PET that stated visual stimulation of one eye activates contrlateral temporal and frontal cortex, 10 patients (4 women, 6 men, age range $21-37$ ) with strabismic amblyopia (4 left, 6 right eye) were imaged. If the vision of the amblyopic eye is supressed and the sound eye is used to generate image in amblyopic patients, stimulation of contrlateral cortex of the sound eye should be observed when both eyes are open. Brain SPECT with single dedector rotating gamma camera (GE XR/T) was performed twice with the administration of 555 $\mathrm{MBq}$ Tc $99 \mathrm{~m}$ HMPAO, one in basal conditions and the other following visual activation by staring at a blue spot on a pink background for 6 minutes. 2 pixel slices parallel to the orbitomeatal line were reconstructed using Butterworth filter. Activation of the brain regions were evaluated visually. In all patients, primary visual cortex (PVC) showed increased uptake after stimulation. 6 of the patients showed temporal and 5 of the patients showed frontal activation contrlateral to the sound eye. This study suggested that amblyopic model is not the same with the one eye closed model. When two eyes are open, more complex mechantsms may be involved. And there may be the contribution of the amblyopic eye in temporal and frontal cortical stimulation, though $\mathrm{its}^{\prime}$ image is not fused with the image of the sound eye in PVC.
479

M. ROUSSEAUX ; M. CABARET; M. STEINLING.

Service Central de Médecine Nucléaire and service de Rééducation et Convalescence Neurologiques. CHRU de Lille; 59037 Lille Cedex; FRANCE

NEUROPSYCHOLOGICAL CORRELATES OF REGTONAL CBF DISORDERS ASSOCIATED WITH FRONTAL MEDIO-BASAL LESIONS

Background and purpose The aim of this study was to evaluate relations between regional cerebral blood flow and neuropsychological disorders resulting from rupture of aneurysms of the anterior communicating artery.

Methods Blood flow was analysed in 22 patients at least 3 weeks after surgery using SPECT method. ICBF values were calculated in 10 regions of interest on each side of the brain. Attention, orientation, executive functions, short-term and long-term learning (verbal, visuo-spatial), semantic memory, retrograde episodic memory, general cognitive performances were investigated.

Results Flow drop was observed in the 3 frontal RoI, predominating on the right side. Stepwise variable selection $(p=0.05)$ shown that (1) right frontal and cingulate flow values entered in the best predictive model of attention disorders; but right posterior flow had inverse action on same variables, i.e. high flow was associated with longer reaction time and lower performances on go no-go test (2) right or left frontal rCBF entered in the predictive models of orientation and amnesia,

(3) frontal CBF explained performances in the Wisconsin Card sorting Test, but had adverse effects on performances in the London Tower Test evaluating planning.

conclusions Frontal rCBF drop may explain most of the cognitive consequences of frontal medio-basal lesions, with clear association between right frontal cortex rCBF and attention and non lateralized effects on memory. But posterior rCBF increase previously reported (Rousseaux et al., 1994, stroke) could also have adverse effects on attention.

\section{0}

A. Soricelli, E. Tedeschi, S. De Chiara*, L. Castelli, G. Spadaro*, M. Romano, G. Marone*, A. Postiglione* and M. Salvatore.

Nuclear Medicine, C.N.R. and National Cancer Intitute Naples and *Internal Medicine, University "Federico II", Naples.

COMPARISON BETWEEN CEREBRAL PERFUSION ALTERATIONS AND IMMUNOLOGICAL DATA IN PATIENTS WITH SYSTEMIC LUPUS ERITHEMATOSUS : PRELIMINARY RESULTS.

In patients with Systemic Lupus Erithematosus (SLE) cerebrovascular involvement can be present up to $75 \%$ of cases. To evaluate the relation between perfusion defects and the presence of anticardiolipin antibodies and lupic anticoagulant factor we have studied 14 SLE patients (13F, $1 \mathrm{M}$ mean age 32.4 years) with a disease duration ranging among 3.56.4 years. A brain perfusion SPET scan was performed after i.v. administration in resting conditions of $740 \mathrm{MBq}$ of $99 \mathrm{mTc}-\mathrm{HMPAO}$ using a dedicated device (CERASPECT, DSI, USA). SPET studies were acquired for 30 minutes and the images reconstructed using a Butterworth 2D filter and corrected for attenuation using Chang's method. The semiquantitative analysis (ratio between cerebral region/cerebellum) was performed on 10 transaxial slices $9.6 \mathrm{~mm}$ thick and the results were compared with those obtained from normal subjects sex and age matched. Cerebral perfusion abnormalities were observed in 9 patients $(64 \%)$, mainly in the frontotemporal region, in one or both hemispheres. The lupic anticoagulant factor was positive in 3 patients $(21 \%)$, all with an history of multiple trombophlebitis and peripheral arteropathy: the corresponding SPET images were normal in 2 cases while in the third a perfusion defect was evident in the right temporal region. Of the 5 patients (35\%) positive for anticardiolipin IgM antibodies, only 2 had perfusion defects, both in the fronto-temporal regions. Eight patients (57\%) had positive IgG: their SPET scans were normal in 3 cases, while perfusion defects were present in the other 5 . In 4 patients $(28 \%)$ both the antibodies were present and in one the lupic anticoagulant factor was also positive. The corresponding SPET studies were positive only in two cases.

Our data confirm the involvement of the cerebral circulation in the SLE patients and the capability of brain perfuion SPET scans to detect them, while reduced cerebral perfusion does not seem to be correlated to the presence of antibodies, well-known risk factors for thrombosis. 
Sunday, 21 August - Wednesday, 24 August 1994

481

N. Gokgora, T.Atasever, K.Gücüyener, G. Vurál, N. I Igin

Gazi University Medical School Departs. of Nuclear Hedicine and Neurology Ankara-Türiye

Te-99m HHPAO CEREBRAI. IMAGING IN YOUNG DOWN'S SYNDROKE PATTENTS

Down's syndrome(DS) is characterized by moderate mental retardation and a variety of abnormalities involving multiple organ systems. There is a high incidence of Alzheimer's disease(AD) type dementia beyond age 35. Tc-99m HAPAO SPET brain perfusion imaging of 12 DS patients (age range 3 to 24 years) vere performed and these images vere compared to a group of 10 age matched controls inorder to define brain perfusion patterns in different ages and its relation to AD type hypoperfusion pattern. SPET scans were acquired 15 minutes after $i . v$.injection of 185-555 $\mathrm{HBq}$ of Tc-HMPAO using a single head rotating gamma camera.Scans vere interpreted both visually and quantitatively by drawing multiple mirrored regions on the cerebral hemispheres. A side to side asymmetry exceeding $10 \%$ betreen the mirrored regions vere accepted to be abnormal.5 DS cases demonstrated normal brain perfusion, 3 cases shoved frontal hypoperfusion, other 4 cases revealed right or left parietotemporooccipital (PTO) hypoperfusion vith or vithout frontal hypofrontality. Briefly 7 out of 12 young DS cases vithout dementia symptoms revealed mostly frontal and/or PTO bypoperfusion. These findings suggest that DS subjeots vithout dementia symptoms may show abnormal brain perfusion. Brain perfusion imaging in younger DS cases ill be useful in identifying baseline perfusion state and may help to predict dementia earlier and in the assessment of the prognosis of the patients.

\section{2}

Krolicki L, Mikolajkow A, Trabka T, Bacia T, Graban $W$.

Dep. of Nuclear Medicine, Medical School of Warsaw.

CBF BEFORE AND AFTER PHARMACOLOGICAI PROVOCATION OF THE EPILEPTIC DISCHARGES.

The aim of this study was to evaluate $\mathrm{CBF}-$ SPECT study before, during and $3-4$ days after Ketamine or Brietal activated epileptic discharges.CT, MRI and conventional EEC examinations were non diagnostic in all cases. $99 \mathrm{mTC}-\mathrm{HM}-\mathrm{PAO}$ as a blood flow tracer and gamma camera Orbiter 75 were used.

Ketamine study: from 30 patients which were examined 10 were normal in first examination, but showed regional decrease of the blood flow during $(n=6)$ or after Ketamine stimulation $(n=8)$ : only in one case all examinations were non-diagnostic.Remaining group showed more pronounced signs after Ketamine stimulation.

Brietal study: 11 patients were examined.only during Brietal stimulation focus of decreased CBF were observed in two cases.

In 9 cases Brietal did not changed the pattern of blood flow disturbances.

In conclusion: pharmacological activation improved the sensitivity of the CBF-SPECT study in defining the focus of epileptical discharges which showed a diminished radiotracer uptake.Ketamine activation seems to be more promising method in those cases.

\section{3}

G. Blomgvist, M. Ingvar, V Grill, L. Widén, S. Stone-Elander, $J O$. Thorell

Depts.of Clinical Neuroscience, Endocrinology, and Karolinska Pharmacy, Karolinska Hospital, Stockholm, Sweden

USE OF [1-11C]- $\beta$-HYDROXYBUTYRATE AND PET IN STUDIES OF CEREBRAI UPTAKE OF KETONE BODIES IN MAN

The present study is part of a comparison between non-diabetic and IDDM subjects with respect to the brain energy metabolism. The regional uptake of ketone bodies in the human brain was investigated in four healthy, fasting volunteers. The regional time course of the tracer in the brain was measured by positron emission tomography (Scanditronix PC 2048-15B) during $10 \mathrm{~min}$ following a bolus injection of 400 MBq [1-11C]- $\beta$-hydroxybutyrate. The time course of the tracer in the arterial plasma was also measured.The plasma concentration of $\beta$-hydroxybutyrate was $20-90 \mathrm{pmol} / \mathrm{ml}$. A three compartment model with three rate constants and blood volume as parameters was applied. No correction for possible losses of tracer was made.

The average influx rate constant for ketone bodies into the brain was $\approx 0.03 \mathrm{ml} / \mathrm{g} / \mathrm{min}$, i.e. about one third of the corresponding value for glucose. This estimate is comparatively insensitive to possible tracer losses (11C-CO2) from the tissue. The uptake was largest in grey matter. The average distribution volume of ketone bodies was $\approx 0.005 \mathrm{ml} / \mathrm{g}$, indicating a very low concentration of ketone bodies in the brain, and about $1 / 20$ of the corresponding value for glucose. Any correction for tracer losses tends to lower this estimate. The results show the feasibility of determining transport kinetics of ketone bodies with PET.

\section{4}

GS Limouris ${ }^{a}$, A Kolindou ${ }^{b}$, MM Konstantoulakis ${ }^{c}$, I Fetsi ${ }^{c}$, A Labrinopoulou ${ }^{c}$, SK Shukla ${ }^{d}$ a. Nucl Med Sect, Areteion Univ Hosp, b. Nucl Med Dept, Med Coll Wisconsin, Milwaukee, c. Athens School of Medicine, Athens, d. CNR+Nucl Med Dept St Eugenio Hosp, Rome

\section{EMBRYONIC NEURAL CELL ADHESION MOLECULE (ENCAM) AND NEURON SPECIFIC ENOLASE (NSE): \\ NEW PROGNOSTIC MARKERS FOR BRAIN DAMAGES}

NSE is synthesized in high concentrations in the astrocytes and Schwann' cells. eNCAM is one of the various isoforms of the intercellular adhesion molecule, normally involved in neuron- ncuron cell recognition. As a matter of fact, every structural damage of the brain tissue should lead to a leakage of these substances both into the cerebrospinal fluid (CSF) and blood serum (BS); consequently both could be considered as suitable CSF and BS markers for cerebral tissue damage. The purpose of this study was to evaluate the clinical usefulness of eNCAM vs NSE in patients (pts) with ischemic stroke (IS).

Sequential samples of CSF and BS were taken from 56 pts $(28$ males aged 68 to $80 \mathrm{yrs}$ ) and 28 females (aged 64 to $76 \mathrm{yrs}$ ) with the diagnosis of IS, within 48 hrs and at 8,16 and 24 days after onset of the disease. NSE de-terminations were assayed with a solid phase IRMA (BYK-Sangtec-Diagnostica Germany) while eNCAM was measured with a solid phase RIA (Behringwerke AG). CT scanswere performed for correlation with the lesion size.

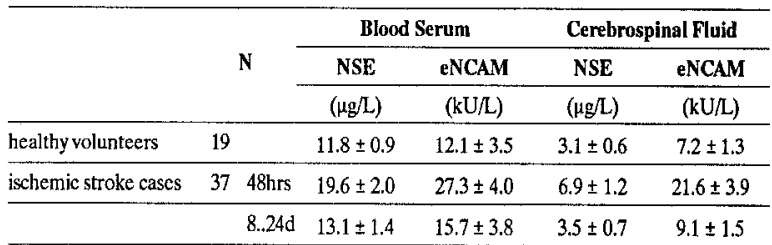

Based on our results, we can conclude: a) CFS and BS concentrations of eNCAM and NSE reflect (i) the scverity of the disease (IS) and (ii) the extent of brain damage, b) BS and CSF levels corrclate while c) repeated measurements can be used as prognostic tool of the ischemic brain damage course; d) eNCAM appears to be more sensitive compared to NSE. 


\section{5}

R. Petrović, B. Ugarković, v. Međved*, D. Dodig, D. Ivančević

Clinical Dept. of Nuclear Medicine and Radiation Protection, *Clinical Dept. of Psychiatry, University Hospital Rebro, Zagreb, Croatia

\section{DETERMINATION OF rCBF CHANGES IN PATIENTS WITH NEGATIVE SCHIZOPHRENIA AND UNIPOLAR DEPRESSION USING 99m-TC-HMPAO}

Previous studies have shown hypoperfusion of different brain regions in depressive and schizophrenic (SCH) patients using functional brain imaging technique such as the $99 \mathrm{~m}$-Tc-HMPAO-SPECT.

The aim of our study was to compare results obtained by this technique in two psychiatric disorders characterized by overlapping clinical symptomatology (negative $\mathrm{SCH}$ versus unipolar depression).

For this purpose we studied 25 patients, 15 with negative $\mathrm{SCH}$ and 10 with unipolar depression. All patients fulfilled DSM-III criteria and were on prescribed medication. SPECT images were acquired after iv. injection of $20 \mathrm{mCi} 99 \mathrm{~m}-\mathrm{Tc}-\mathrm{HMPAO}$ using a dual head rotating gamma camera. Following reconstruction and attenuation correction, coronal, sagittal and reoriented transversal slices (parallel to the orbitomeatal line) were obtained. Thirty-two Ror's were drawn in the frontal, temporal, parietal and occipital lobes, the basal ganglia and the cerebellum for semiquantitative analysis, normalized to the mean cerebral uptake. Only regional asymmetries $>15 \%$ were considered abnormal.

17 patients showed regional hypoperfusion abnormalities in frontal, parietal and temporal lobes (10 with negative $\mathrm{SCH}$ and 7 with unipolar depression), and 8 patients had no changes in rCBF ( 5 with negative SCH and 3 with unipolar depression). There were 23 regional hypoperfusion abnormalities in patients with negative $\mathrm{SCH}$ and 22 regional hypoperfusion abnormalities in patients with unipolar depression, almost all (except 3 hypoperfusions in 2 depressed patients) localized in left dominant hemisphere. Statistical analysis (2-tail localized in left dominant hemisphere. Statistical analysis (2-tail
Student's test, $\mathrm{p}>0.05$ ) showed no significant differences between SCH and depressed patients.

In conclusion, we found that 99mTc-HMPAO-SPECT could be usefull and sensible tool to diagnose $\mathrm{rCBF}$ abnormalities, but not for differentiation between patients with negative schizophrenia and unipolar depression. However, further studies are needed to evaluate the clinical significance of this findings.

\section{6}

Y. Yürekli* B. Yemez ${ }^{\star *}$, H. Durak*, E. Metin*, Ö. Kut*, M. Yılmaz*, Z. Tunca $a^{\star \star}$

Departments of *Nuclear Medicine and ** Psychiatry, School of Medicine, Dokuz Eylül University, Inciraltı , İzmir, Türkiye

\section{CORRELATION OF SYMPTOMS WITH CEREBRAL PERFUSION} ABNORMALITIES IN ATYPICAL PSYCHIATRIC PATIENTS

Although several patterns of cerebral perfusion abnormalities were described, it is not uncommon to have difficulties in interpretation of brain SPECT images of psychiatric patients. in this study, we looked for a correlation between some common symptoms and cerebral perfusion abnormalities in atypical psychiatric patients.

20 patients (mean age: $43 ; 13 \mathrm{M}, 7 \mathrm{~F}$ ) were included in the study. Brain SPECTs were performed after intravenous injection of $15-20 \mathrm{mCi}$ of Tc-99m HMPAO, using a single detector, rotating gamma camera, with eyes and ears closed, in a quiet room. Tomographic slices were evaluated visually. 32 decreased perfusion areas in 20 brains were detected. Distribution of the lesions was as follows: $50 \%$ left frontal (LF) cortex, $30 \%$ in left temporal (LT) cortex. $13 \%$ right temporal (RF) cortex, $7 \%$ right frontal (RF) cortex. Lesions were grouped as in the table:

\begin{tabular}{|l|c|c|c|c|}
\hline & Solitary $L F$ & $L F+L T$ & $L F+$ more than & \multicolumn{2}{c}{$\begin{array}{c}\text { Solitary LT or RT } \\
\text { one area and bilateral temporal }\end{array}$} \\
\hline No. of patients & 8 & 5 & 3 & 4 \\
\hline $\begin{array}{l}\text { Patients with } \\
\text { psycotic symptoms }\end{array}$ & $1(12.5 \%)$ & $2(40 \%)$ & $2(67 \%)$ & $4(100 \%)$ \\
\hline
\end{tabular}

Major symptoms were 1) depressive mood (DM), 2) social isolation (SI), 3) decreased performance (DP) ,4) psychomotor retardation (PR), 5) hallucinations $(H), 6)$ delusions (D). In patients who have LF cortex hypoperfusion the most common symptoms were DP (13.4\%), DM (11\%) and SI (11\%). We observed a tendency for $\mathrm{H}$ and $\mathrm{D}$ to be related to temporal cortex lesions.

In conclusion, it seems that solitary LF hypoperfusions tended to be with $\mathrm{DM}, \mathrm{SI}, \mathrm{DP}$ and with less probability of psycotic symptoms, while additional and temporal lesions alone were likely to be related to psycotic symptoms. Three patients with temporal lesions had a final clinical diagnosis of schizophrenia which might suggest that brain perfusion scanning can predict the possible outcome in dealing with atypical patients.
487

G. VAIVA ; P. THOMAS ; Ph. DESBONNET ; M. GOUdEMAND; STEINLING.

Department of General Psychiatry and service Central de Médecine Nucléaire; C.H.R.U. de LILLE; 59037 Lille Cedex ; FRANCE

REGIONAL CEREBRAL BLOOD FLOW BEFORE AND AFTER LOW DOSES OF AMISULPRIDE IN NEGATIVE SCHIZOPHRENIA.

Summary Twelve in-patients, fullfiling DSM III-R criteria for Schizophrenia (disorganized form) were assessed twice with SPECT, initially when drug free and secondly after 28 days treatment. All of them were assessed during a semi-structured interview with the saws at no and each week; the patients suffered from a negative form of illness defined by an initial saNs score $>65$. All of them were treated with low doses of amisulpride per os (400mg/d the first week; between 50 and $150 \mathrm{mg} / \mathrm{d}$ the two last weeks). The imaging procedure by SPECT with $99 \mathrm{~m}-T$ c HmPAo (Tomomatic 564 Medimatic) was performed after the clinical assessement, at Do and D28.

The data analysis showed significant differences in the regional cerebral Blood Flow indexes before and after treatment (Wilcaxon test). The frontal regions of interest showed the most striking variations, particulary in the right side and in the dorso-lateral prefrontal aerea $(p=0.018)$. We also found significant variation of the asymmetry in the medio-frontal aerea, before and after treatment $(p=0.05)$. We showed significant correlations between some rCBF variations and some symptomatic sans ratings improvement (spearman test).

We cannot talk about an eventual "amilsulpride effect" whithout a control goup with placebo : we supposed on an "improvement effect".

Key words : cerebral blood flow, schizophrenia, neuroleptic treatment, amisulpride

\section{8}

E. Klemm, F. Grünwald, ${ }^{*}$ G. Höflich, ${ }^{*}$ B. Martinez, ${ }^{* *}$ S. Kasper, L. Pavics, C. Menzel, H. Bender, A. L. Hotze, ${ }^{*}$ H.-J. Möller, H.-J. Biersack.

Departments of Nuclear Medicine and *Psychiatry, University of Bonn, D-53127 Bonn, Germany, and **Department of Psychiatry, University of Vienna, A-1090 Vienna, Austria

\section{${ }^{99} \mathrm{~m}$ TC-HMPAO-SPECT IN OBSESSIVE COMPULSIVE DISORDER: HIGH INCIDENCE OF PATHOLOGICAL CEREBRAL PERFUSION PATTERNS}

In patients with obsessive compulsive disorder, the mostly recent PET studies suggest an involvement of the frontal lobes and the basal ganglia. To evaluate rCBF by means of SPECT and to relate the findings to clinical variables (psychopathology, duration of disease, medication) we investigated 15 patients using high resolution SPECT (ASPECT, FWHM $5-6 \mathrm{~mm}$ ) and $99_{\mathrm{m}} \mathrm{Tc}-$ HMPAO as a tracer. In addition to visual evaluation, ROIs were drawn on the mesial and lateral, the frontal, parietal and occipital lobes, the basal ganglia, and the cerebellum. The mean age was 37 +11.3 years, the mean duration of disease ranged about $14.6+$ 10.7 years. The Yale Brown Obsessive Compulsive Scale (YBOCS) was used to quantify psychopathological symptoms. Only one patient demonstrated a normal SPECT. In 13 subjects a reduced regional perfusion was observed, while 6 patients showed an increased regional perfusion. The diminished perfusion clearly lateralized to the right side (16 right, 8 left). The predominantly affected region was the temporal lobe (6 right, 3 left). In contrast the hyperperfusion did not show major side differences ( 3 right prefrontal region, 4 left prefrontal region). It was mainly confined to the prefrontal area. Thus, our preliminary findings further suggest an involvement of frontal lobe structures in patients with obsessive compulsive disorder. Moreover, our results demonstrate hypoperfusion of the temporal lobe as it has been observed in other psychiatric diseases. Due to the number of patients evaluated up to now, valuable statistics correlating the SPECT findings to clinical variables could not be established yet. 
Sunday, 21 August - Wednesday, 24 August 1994

489

A. Dobbeleir, R. Dlerckx, P. De Deyn, M. Vandewoude. University Hospital Ghent, Belgium.

Normalisation of cerebellar uptake of TC-99m HMPAO by applying body surface and brain volume correction in 33 healthy volunteers and 13 DAT patients.

Absolute values of cerebellar uptake of To-99m HMPAO vere obtained in 33 healthy volunteers and 13 patients, euffering from probabie dementis as external standard.

If necessary, mostly for stressed volunteers, uptake values were corrected to the rest heart rate value (ER) using the previousil described equation $\Delta$ uptake $=-0.35 \Delta \mathrm{ER}$. Individual brain volume (BV) wa calculated using the sum of all vorels, above a $35 \%$ threshold of the maximal value of all slices, converted using the single voxel volume. The mean brain volume in the reference group of 18 men and 15 women With a mean age of 41 years (22-62) was $1350 \mathrm{ml}$. The DAT group consiated patients a reduced brain volume of $15 \%$ ras observed compared to the gender related bealthy younger volunteers. Body surface (BS) was

Cerebellar $x \mathrm{BO}$ (mean +-SD) is expreseed in 10-6 of the lajected lipophylic dose per $\mathrm{cm}^{3}$ brain tissue obtalned in a 11 m over the blghest activity of the cerebellum. 2 body surface correction factor BS $/ 1.73$ is epplied followed by a brain volume correction factor $\mathrm{BV} / 1350$, resulting in uniforra measurements for different subjects.

\begin{tabular}{|l|l|l|l|}
\hline & rBU at rest ER & $\begin{array}{l}\text { rBU BS } \\
\text { correated }\end{array}$ & $\begin{array}{l}\text { rBU BS+BV } \\
\text { corrected }\end{array}$ \\
\hline Volunteers (33) & $48.4+-9.7$ & $52.1+-7.7$ & $52.0+-7.3$ \\
\hline Men Vol (18) & $43.1+-7.6$ & $49.9+-8.4$ & $51.6+-8.7$ \\
\hline Women (15) & $54.8+-8.1$ & $54.7+-6.2$ & $52.5+-5.5$ \\
\hline DAT (13) & $75.2+-18.3$ & $66.2+-10.9$ & $53.9+-7.4$ \\
\hline
\end{tabular}

After applying beart rate, body surface and brain volume corrections normalized uptake values are obtained. Even in a pathologic oldez population, in which despite of the pathology, the cerebelium can be considered as normal, for the cerebellar region the same uptake is obtained as in normal healthy volunteers. The decline of the cerebella

uptake with age is negligible as shown by the equation
$\mathrm{rBO}=54.7-0.04 * \mathrm{AGE}$. Middelhelm Hospital Antwerp, Oniversity of Antwerp ora and of 10 women and 3 men wth a mean age of 76 years (67-83). For DAT obtained in the Dap group.

491

A. Soricelli, E. Tedeschi, M. Romano, P. P. Mainenti, M. Salvatore.

Nuclear Medicine, University Federico II Naples - National Research Council C.N.R. - Cancer Institute 'Fondazione Pascale' Naples, Italy.

INTRA - AND INTER- OBSERVER REPRODUCIBILITY OF VARIOUS METHODS FOR SEMTQUANTITATION OF BRAIN PERFUSION WITH SINGLE PHOTON EMISSION TOMOGRAPHY

Single Photon Emission Tomography (SPET) brain scans, performed with $99 \mathrm{mTCHMPAO}$, may be evaluated with visual and/or semiquantitative methods of regional analysis, which improve the image interpretation, but whose reproducibility must be carefully assessed. The aim of this study was to evaluate the intra- and inter- observer reproducibility of 3 semiquantitative methods in two groups of patients. In the first group - 8 patients: 2 normal (N), 4 with cerebrovascular disease (CVD) and 2 with dementia (D) - the studies were acquired with a single head rotating gamma camera (Apex SP4HR Elscint, Israel). In the second group - 10 patients: $2 \mathrm{~N}, 4 \mathrm{CVD}, 4 \mathrm{D}$ - a high resolution, brain-dedicated annular system (CERASPECT, DSI, USA) was used. On four selected brain slices, placed at standard levels above the cantomeatal $(\mathrm{CM})$ reference plane $(+2,3,5,6 \mathrm{~cm}$, respectively), one senior clinician and one first-year resident applied at both groups of patients three different sets of regions of interest (ROI), linearized to the cerebellum and consiting of: A) fixed -number and -size polygonal ROIs, B) multiple hand-drawn ROIs, and C) semiautomatic segmentation of a treshold-based, cortical ROI, plus manual definiton of deep grey matter (DGM) structures. One week later, the analysis was repeated. In both groups no significant intraobserver variation was found with any of the 3 methods. The cortical ROIs of both groups showed no difference when analyzed by the two observers with any method, while, in the first group of patients, DGM ROIs resulted significantly different $(p<0.005)$ between the 2 observers using method $B$ and $C$. In the patients evaluated with the brain-dedicated camera no difference was evident for the DGM ROIs with all methods. The data suggest that, using a high resolution system, inter-indivual variability does not affect the measurement, while, at lower spatial resolution, the choice of the semiquantitative method becomes critical for the definiton of DGM ROIs.

492

490

J. Pinkert, L. Oehme,W.-G. Franke

E. Vanninen, J.T. Kuikka, R. Vanninen,

M. Tenhunen-Eskelinen, H. Mussalo

Depts. of Clinical Physiology and Nuclear

Medicine, and Clinical Radiology,

Kuopio University Hospital, Kuopio, Finland

ACETAZOLAMIDE INDUCED INCREASE IN CARDIAC OUTPUT RATIO IS INVERSELY CORRELATED WITH CALCULATED CEREBRAL PERFUSION RESERVE

We recently observed that $1 \mathrm{~g}$ of intravenously injected acetazolamide (ACZ) increases cardiac output / blood volume ratio (COR) on the average by $10 \%$. To evaluate whether this has any effect on cerebral perfusion reserve (CPR) measurement performed with Tc-99m-HMPAO SPET, we compared ACZ induced change in COR with simultaneously measured CPR. Altogether 22 studies were analysed on 15 patients (mean age 60 yrs, range $49-72$ yrs) undergoing carotid endarterectomy, both pre- and postoperatively $(n=7)$, only preoperatively $(n=3)$ or only postoperatively $(n=5)$. COR was measured by first-pass technique by using a nuclear stethoscope simultaneously with basal and ACZenhanced Tc-99m-HMPAO injection, followed by basal and ACZ-enhanced SPET acquisition. CPR was calculated on three $10.5 \mathrm{~mm}$ thick transverse slices (OM+1 cm, $\mathrm{OM}+3 \mathrm{~cm}, \mathrm{OM}+5 \mathrm{~cm})$, and it was on the average 8,9 and $8 \%$, respectively. The corresponding correlation coefficients (Spearman rankcorrelation) with COR were -0.48 ( $p<0.05)$, $-0.71(p<0.001)$ and $-0.60(p<0.01)$. These data suggest that $A C Z$, by increasing COR, expands Tc-99m-HMPAO whole body distribution volume which may lead to underestimation of CPR.
Department of Nuclear Medicine, Technical University of Dresden

\section{ESTIMATION OF CEREBRAL BLOOD FLOW USING A BRAIN-PERFUSION-INDEX IN 99m Tc-HMPAO-STUDIES} Recently MATSUDA et al. reported a non-invasive and relatively simple procedure for estimation of total and hemispherical cerebral perfusion by calculation of a brain perfusion index (BPI) from determination of the unidirectional influx constant of HMPAO.

According to this evalutations the BPI was determinated for $57 \mathrm{pts}$. suffering from various disturbances of cerebral perfusion. The radionuclide passage in the first minute after bolus injection of $750 \mathrm{MBq}$ ${ }^{99 m} \mathrm{Tc}-\mathrm{HMPAO}$ was monitored with a dual head large field camera positioned over both, brain and thoracal region using a rapid sequence of 2 frames $/ \mathrm{sec}$. A graphical analysis of curve data obtained over aortic arch and whole brain as well as both hemispheres give the influx-constant $\left(k_{u}\right)$. To receive the BPI the $k_{u}$-values were correct for individual ROI-values. Mathematically we can show that the graphically estimated $\mathrm{k}_{\mathrm{u}}$ is a product of a influx constant (which is nearly the same in different patients) and the part of cerebral blood flow from cardiac output. Results showed a closed correlation of calculated BPI with. perfusion and the degree and number of perfusion disturbances supposed by an combination of results of physical examinations, SPECT, rentgenologic angiography, XCT and/or MRI. In patients without or with minimal disturbances of cerebral blood flow a negative correlation from BPI with age was seen. BPI-values obtained from a curve fit done between the 5.-15 $\mathrm{s}$ BPI for later time points. This finding may be attributed to fast back diffusion. If a standardisation for individual conditions of acquisition is done, this technique can complete as a helpful adjunct routine SPECT studies in a quantitative manner and alows the quantification of brain perfusion enhancement after injection of acetazolamide. compared with those between $10 .-20 \mathrm{~s}$ show a small reduction of 


\section{3}

S. Asenbaum, T. Brücke, A. Pozzera, G. Koch, P. Angelberger*, J. Kornhuber, I. Podreka

Neurological Clinic and Clinic for Nuclear Medicine, University of Vienna, For= schungszentrum Seibersdorf*, Vienna, Austria

DEGENERATION OF STRIATAL DOPAMINERGIC NEURONS IN PARKINSON'S DISEASE VISUALIZED AND QUANTIFIED BY [123I]B-CIT AND SPECT

[123I] BS-CIT, a cocaine analogue, has recently been introduced for imaging dopamine (DA) transporters primarily in the striatum. In Parkinson's disease (PD) the degeneration of nigro-striatal pathways leads to a reduction of striatal DA remuptake sites. The aim of the present study was to visualize this reduction of DA transporters and to relate it to the severity of parkinsonian symptoms.

11 volunteers (24-70a) and 30 patients with PD $(47-82 a)$ have been investigated with [123 I] isCIT and SPECT. ACquisitions started 4, 16, 20 and 24 hours after tracer application. Regions of interest (ROI) were drawn manually over the striatum and the cerebellum. Specific binding and the ratio striatum/cerebellum were calculated and compared between volunteers and patients.

Patients with PD showed a significantly reduced specific binding and reduced ratio striatum/cerebellum at every time point /f.e. $4.7+/-1.24$ vs. $8.4+/-2, \quad \mathrm{p}<0.0001$, after 20 hours). Furthermore a significant correlation was found between the severity of the disease according to Hoehn \& Yahr and the striatal ratio $(\mathrm{F}=7.02, \mathrm{p}<0.0007)$

Jsing [123I]B-CIT the degeneration of striatal dopaminergic neurons in PD can be visualized. The measured parameters show a significant relationship to the severity of symptoms.

\section{4}

M. Tondeur, R. Denays and H. Ham

Depts of Nuclear Medicine and Neurology, Hospital Saint-Pierre, Free University of Brussels, Belgium

NORMAL Tc-99m HMPAO BRAIN SPECT IN ADULTS USING A SINGLE HEADED CAMERA

This study was undertaken to determine the normal Tc-99m HMPAO brain SPECT pattern in adults.

10 volunteers, aged 20 to 30 years, were recruited: all were in good health, had no medical history and their neurological examination was normal. $555 \mathrm{MBq}$ of Tc-99m HMPAO were injected in a quiet room with open eyes, followed by 2 successive $360^{\circ}$ SPECT acquisitions. In each subject, this procedure was repeated 2 weeks later. Reconstruction, using the filtered backprojection method, allowed to obtain transaxial, coronal and sagittal slices ( 2 pixels thick $=1.3 \mathrm{cms}$ ).

The results show that the anteroposterior tracer distribution, measured on sagittal slices, was similar, in each subject, for the 4 acquisitions. Coefficients of variation of the cortico-cerebellar and cortico-cortical indexes didn't exceed $5 \%$.

Right / left tracer distribution, measured on transaxial and coronal slices with a $9 \mathrm{~cm}^{2}$ ROI showed asymmetries up to $16.9 \%$ using a Wiener filter and no orbitomeatal reorientation (OMRO), $13.3 \%$ using a Wiener filter with OMRO, and $13.1 \%$ using a Hanning filter and no OMRO. For the 40 studies, considering $12 \%$ as upper limit of normal values, we observed abnormalities in 8,1 and 1 studies using respectively a Wiener filter without and with OMRO, and a Hanning filter without OMRO.

The localisation, extent and quantitative importance of these asymmetries varied markedly in 2 successive acquisitions as well as in acquisitions obtained at different days, suggesting that these asymmetries are artifactual.

In conclusion, these results suggest that important right / left asymmetries may be observed in Tc-99m HMPAO brain SPECT from normal aduits. As these asymmetries are strongly dependent on technical aspects from the reconstruction procedure (filter, OM reorientation, ...), it is mandatory that each center determines the normal pattern according to the procedure and the equipment used.
495

I C Dormehl, W.P Pil.Loy, N Hugo, N Rossouw

AEC Institute for Life Sciences, University of Pretoria Mecical University of Southern Africa, Pretoria, and the National Accelerator Centre, Eaure

REGIONAL CEREBRAL BLOOD FLOW PATTERNS USING 123 I (IMP) AND 99mTC-HMPAO WITH SEECT IN ANAESTHETISED BABOONS

$\mathrm{N}$-isopropyl-p-(I23I)-iodoamphetamine 123I(IMP), with long-acknowledged properties of a good tracer for scintigraphic regional cerebral blood flow investigations, can be considered a second tracer in a dual-isotope SPECT study with 99m'TC-HNPPO for brain-stress testing, eg. after pharmacological intervention with acetazolamide to reflect on vasodilatory reserve. Necessary are identical $99 \mathrm{mTc}-\mathrm{HMPAO}$ and 123I(IMP) distributions in the normal brain, and again in the acetazolamide compromised brain to render diagnostically meaningful "stress" differences with pathology. Regional cerebral distributions of $99 \mathrm{mTC}-\mathrm{HMPAO}$ and $123 \mathrm{I}$ (IMP) were here investigated by two immediately consecutive SPECT studies, corrected for $99 \mathrm{mTc}$ counts in the $123 \mathrm{I}$-window, in the normal baboon $(n=6)$ and the stressed baboon $(n=6)$.

Coronal slices were generated as input for a circumferential profile programme. Nine segments of four circumferential profiles on four equidistant transaxial slices were appropriately normalised in order to compare HMPAO and IMP distributions.

Regions of interest were placed on the total brain in corresponding slices and the counts/pixel used in a comparison of the ratios of urtake from the two tracers, (IMP:HMPAO) for the normal and later the stressed baboons.

These uptake ratios, as well as the quality of the SPECT images, suggest better cerebral uptake of the IMP, and a lack of adequate agreement in the regional distributions of the two tracers for both the normal and the acetazolamide compromised animals. This finding would exclude a dual-isotope brain-stress test as proposed.

\section{6}

J Farahati, SP Müller, B Kremens, E Eising, L Geworski, Chr Reiners. Nuclear Medicine and Pediatric Oncology, University Hospital Essen, FR Germany.

\section{THE ROLE OF BONE AND MIBG SCINTIGRAPHY IN THE} EVALUATION OF CHILDREN WITH NEUROBLASTOMA.

Over the past 10 years the use of MIBG scintigraphy in the diagnostic workup and staging of children with neuroblastoma has expanded. The role of MIBG in the detection of skelettal involvement, however, is as yet controversial.

Twenty children with neuroblastoma underwent both bone scanning (99mTc-MDP) and MIBG scintigraphy (I-123-MIBG) within 4 to 11 days. In 14 children the studies were performed at staging before any treatment. $13 / 14$ had histollogicaly proven bone marrow involvement, and $10 / 14$ had bone metastases. Four children were in complete remission. In addition 2 children were studied with suspected neuroblastoma, which were later proven to be Wilms tumors.

The MIBG scan correctly identified $13 / 14$ primary tumors, $12 / 13$ patients with bone marrow involvement and $9 / 10$ children with bone metastases (34/37 true positives MIBG). All 4 children in complete remission and the 2 Wilms tumors showed no MIBG uptake (6/6 true negatives MIBG). On the bone scans we could detect $5 / 10$ patients with bone metastases and $5 / 13$ with bone marrow involvement, and $8 / 14$ primary tumors ( $18 / 37$ true positives MDP). The 2 Wilms tumors were false positive on the bone scan ( $4 / 6$ true negatives MDP). In one case the intensity of the uptake on the bone scan was superior to MIBG, and in one false-negative MIBG scan the bone metastases was diagnosed on bone scan.

In comparison to bone scanning MIBG scintigraphy has a higher sensitivity and specificity. However, because of the importance of bone and bone marrow involvement on prognosis as well as therapeutic management of children with neuroblastoma, bone scintigraphy is recommended in children with negative MIBG scan. 
497

L. BURRONI, P. BERTELLI, A. VELLA, D. VOLTERRANI and A. VATTIMO.

Nuclear Medicine Unit, University of Siena, Italy.

THERAPY-RESISTANT EPILEPSY: ITS ASSESSMENT USING SPET WITH TC-99m HMI'AO IN COMPARISON WITH MRI AND EEG.

The therapy-resistant epilepsy (TRE) represents a diagnostic challenge during childhood in view of surgical treatment: therefore an accurate localization of seizure focus is required. In this paper, brain MRI and SPET with TC99m HMPAO (PAO) and EEG were compared. The study was carried out on 37 children (20M and $17 \mathrm{~F}$ aged 18 months to 17 years) with TRE: all possessed MRIs performed elsewhere and EEGs performed in the Pediatric Department. All were submitted to the SPET study for further assessment: PAO (10 MBq/Kg) was injected in interictal period in a quiet environment and state; in 20 non cooperative children SPET was acquired on monitored sedation. Regional brain blood flow was considered pathological if the abnormality was seen in more than one slice and view. The table summarizes the cliatical data and positive results.

$\begin{array}{lrrrr} & n & \text { SPET } & \text { MRI } & \text { EEG } \\ \text { Complex Partial Seizure (a) } & 17 & 17 & 6 & 17 \\ \text { Generalized Tonic Clonic Seizure (b) } & 10 & 10 & 4 & 8 \\ \text { a+b } & 6 & 5 & 2 & 6 \\ \text { Infantile Spasm } & 2 & 2 & 1 & 2 \\ \text { Simplex Partial Seizure } & 2 & 1 & 1 & 2\end{array}$

It clearly appears that functional brain imaging usinc SPET is highly sensitive in detecting seizure foci ane promises to be superior to MRI modality in TRE children.

\section{8}

Y.Dunan, M.Kayalıoğlu, S.Erden, S.Tutitincioğlu, H.Tekgill

Depts.of Nuclear Medicine, Pediatrics,Ege Univensity, İzmir-Turkey

Tc-99m HMPAO SFECT IN ANOMALIES OF NEURONAL MIGRATION

The migrational disorders are a rare gnoup of congenital malformations of the brain. They consist of the following entities: lissencephaly. pachygria, shizencephaly, polynicrogyria, unilateral megalencephaly. These patients (pts) presented a variety of clinically serptorns. The most commons were seizures, delayed development, failure to thrive, microcephaly and occasionally hydrocephalus.

We performed brain SPECT, CT and MR on 11 childiren ( 3 years)with migrational anonalies ( 1 unilateral megalencephaly, 6 pachygria, 3 shizencephaly, 1 lissencephaly.

SPECT showed congruent or more extensive abnormalities with CT and MRI lesions in 3 pts with shizencephaly, 1 pt with lissencephaly, 3 pts with pachygyria.I pt with pachygyria demostrated no abnomality in SFECT imaging,however; calcification of bilateral capsulla interna was observed in MRT.Multiple focal perfusion defect was deteced in $2 \mathrm{pts}$ with pachygyria in SFECT, one of these patients had abnomal EEG findings. But, there was no ischeria findings in CT and MR. In one pt with unilateral hemimegolencephaly, SPECI imaging showed hypoperfusion in right hemisphere congruent with $\mathrm{CT}$ and MR findings. Noreover, additional focal hypoperfusion in left parietal lobe was also observed with HMPAO SPECT we conclude that brain SPECT is a useful adjunct to CT,MR, EFG studies in anomalies of neuronal migration.
499

A.I.van Dongen, P.M. van Roermund, Ph. Declerck, G. Fabry, J. Lammens, P.C. Anema, M.J.K. De Roo and P.P. van Rijk, University Hospital Utrecht, (The Netherlands) and University Hospital KU Leuven (Belgium)

THE VALUE OF BONE SCINTIGRAPHY IN CHILDREN WITH UNILATERAL CONGENITAL GROWTH DISORDER

25 children (age 2-16, mean 11 yrs) without abnormalities in the lower limbs and 25 children (age 4-16,mean $10 \mathrm{yrs}$ ) with a difference in leg length due to a unilateral congenital growth disorder underwent bonescintigraphy (Tc99m-HDP). Aim of the study was to determine the prognostic value in the calculation of leg length discrepancy at the end of growth.

Regions of interest were chosen over the growth plates of proximal (PF) and distal (DF) femur, proximal (PT) and distal (DT) tibia. Right to left ratios and minimum to maximum count ratios were calculated, as was the contribution in terms of percentage of each plate to the total growth capacity of the leg.

\begin{tabular}{lccc} 
NORMALS, $95 \%$ percentile: \\
\multicolumn{2}{c}{ R/L ratio } & Min/Max count ratio & Contribution in percent \\
PF & $0.92-1.06$ & $>0.92$ & $10-18$, mean $=13$ \\
DF & $0.92-1.07$ & $>0.92$ & $37-46$, mean $=41$ \\
PT & $0.94-1.06$ & $>0.93$ & $22-32$, mean $=28$ \\
DT & $0.93-1.07$ & $>0.92$ & $13-21$, mean $=18$ \\
ALL & $0.94-1.06$ & $>0.95$ &
\end{tabular}

PATIENTS, 95\% percentile:

$\begin{array}{lccc} & \text { R/L ratio } & \text { Min/Max count ratio } & \text { Contribution in percent } \\ \text { PF } & 0.86-1.22 & <0.95 & 10-17 \text {, mean }=13 \\ \text { DF } & 0.70-1.81 & <0.85 & 37-45, \text { mean }=41 \\ \text { PT } & 0.81-1.85 & <0.91 & 23-34 \text {, mean }=29 \\ \text { DT } & 0.73-1.65 & <0.90 & 14-21 \text {, mean }=17 \\ \text { ALL } & 0.82-1.63 & <0.89 & \end{array}$

The calculated values are not dependent on sex or age. The calculated $\min / \max$ count ratios show that the groups are significantly different, making it posible to differentiate between the two. The fact that contribution in percent in all patients lies within the $95 \%$ percentile of the normal group, provides a basis for prognostic calculations.

500

Y.Duman, A.Dirlik, Z. Burak, S. Tütüncii ${ }^{\mathrm{X}}$

Departments of Nuclear Medicine and Paediatrics $x$

Ege University, Faculty of Medicine, IZMİ, TURKEY

\section{MOYAMOYA DISEASE IN TWINS}

Moyamoya disease is a rare cerebrovascular pathology characterised by occlusion of cerebral arteries and development of an abnormal vascular network. Although moyamoya disease is originally thought to occur only in Japanese population, its occurance in other countries has also be reported.

In this study, we aimed to evaluate the feasibility of 99m-Tc HMPAO SPECT study in twins with moyamoya disease (age:3 years) in comparison with MRI and angiography.99mTc HMPAO SPECT study demonstrated multiple focal and/or globally decreased rCBF in both twins. MRI demonstrated significant narrowing in both supraclinoid carotid and the medial cerebral arteries, especially marked on the right side. The diagnosis in moyamoya disease was determined by angiography.

MR imaging has the greatest advantage of demonstrating blood vessels without requiring the use of the contrast medium. However; our results indicate that $99 \mathrm{~m}-\mathrm{Tc}$ HMPAO SPECT findings do correlate better with clinical symptomathology and angiographical findings.Also suggest more extensive brain involvement than did MR imaging. SPECT study is a noninvasive, inexpensive and useful modality in assessing the functional hemodynamic abnormalities in moyamoya disease. 
Sunday, 21 August - Wednesday, 24 August 1994

\section{1}

2. Maziarz, B. Malkowski, W. Tryniszewski

Dep. of Nuclear Medicine. Military Medical Academy Lodz Poland

CLINICAI USEFUINESS OF THE DYNAMIC LIVER

ANGISCYNTYGRAPHY IN PARTICULAR GASTROENTEROLOGICAL DISEASES IN CHILDREN.

Dynamic liver angioscyntygraphy (DLA) turned out to be the sensitive method estimating the perfusion of the organ with regarding on the arterial and portal blood distribution. We decided to verify the clinical usefulness of this method in particular gastroeterological diseases in children. DIA was carried out in patients with chronic persisting hepatitis (CPH), hepatomegaly in acute infectious diseases (HAID) healthy carriers of HBs antigen (CHBs), congenital hiperbilirubinemia (CH) and malapsorption syndromes (MS). Normal results of dynamic liver angioscyntygraphy are among $70-75 \%$. The greatest reduction of the portal perfusion to 58,2 and 57,38 we ascertained in the group with CPH and HAID, a little lower-reaching to $63,2 \%$ was found in CHBs slightly but statistically significant depression to $65,8 \%$ we observed in patients with $\mathrm{CH}$. In group with $\mathrm{MS}$ portal perfusion was on the normal level. In patients with $\mathrm{CPH}$ significant depression of the portal perfusion $\mathrm{CO}^{-}$ existed with the hepatomegaly, increasing activity of AIAT, increasing level of bilirubin and IgG, in children with HAID concomited with hepatomegaly and increasing activity of AlAT. In groups with CHBS, CH the depression of the perfusion coexisted only with parameters resulting from creating these groups. In the group with MS all results ware on the normal level. With the help of the method the reduction of the liver perfusion, even without the early stage symptoms of the disease, may suggest earlier reduction of the portal perfusion before laboratory and ultrasonography signs appear. Making the DLA is most helpful in clinical diagnostic in children with $\mathrm{CPH}$ $\mathrm{HC}$ and CHBs, recommended in patients with HAID, giving not much information in children with MS.

\section{2} $\frac{\text { M. Jauch }}{\text { W.Dewes }}{ }^{1}$ P.Benz ${ }^{1}$, W.Bini ${ }^{2}$, J.Spitz ${ }^{1}$, R.Schönmayr ${ }^{2}$,

${ }^{1}$ RNS-PET Center Wiesbaden, 2 Neurochirurgische Abteilung d.Städt. Kliniken Wiesbaden

COMBINATION OF CT / MRI AND SPECT /PET FOR BETTER DIAGNOSIS OF BRAIN TUMORS !!??

Imaging diagnostics in brain tumors normally means CT /MR imaging. The aim of this study was to find out if there is an improvement by adding functional imaging such as PET and SPECT.Out of a group of 65 patients with brain tumors 49 were selected (17 high grade gliomas, 12 low grade gliomas, 14 meningeomas, 6 metastases).

SPECT:130 MBq 201-Thallium and 600-750 MBq 99mTc-HMPAO respectively were injected. Data acqusition: 5 min.p.i. a $360^{\circ}$ study, $128 \times 128$ matrix, 64 frames ( 30 sec.each) was aquired. The quantification was done in ROI technique. For thallium study a ratio tumor/ normal tissue was used. PET:after overnight fasting 200 to $350 \mathrm{MBq} 18-\mathrm{FDG}$ were injected. $30 \mathrm{~min}$.p.i. three emission scans ( 10 min.each) were acquired. For quantification ROI technique was used. All patients were provided with CT or MRI scans respectively and were given biopsy or surgery of the tumor.

In high grade gliomas the thallium ratio was $1.5-2.9$, in low grade gliomas 1.2-1.5 and in meningeomas 3.1-8.5 respectively. The perfusion study showed an increased uptake for vital tumor tissue in high grade gliomas (14/17). Low grade gliomas (9/12) showed a circle-enhancement with low central uptake (necrosis, cyste). In meningeomas the perfusion uptake declined dramatically. For glucose metabolism high grade gliomas (17/17) showed an increased uptake, whereas low grade gliomas had partly increased and partly severely decreased uptake values. Meningeomas showed a low uptake and metastasis differed from high to low uptake values. The different tumor types showed different patterns in perfusion, thalium uptake and glucose metabolism. The combina-tion of morphological and functional imaging can apparently increase the specificity of diagnosis. Especially glucose metabolism gives datas about the activity of important centers in the brain, usefull for the planning of surgery.
503

Y. Duman, E. Derebek, M. Kayalıoğlu, İ. Övül, S. Erdem, Z. Burak

Depts. of Nuclear Medicine, Neurosurgery, Ege University, izmir - Turkey

THE ROLE OF EARLY AND DELAYED QUANTITATIVE TI-201 SPECT IMAGING IN THE EVALUATION OF BRAIN LESIONS

Metastatic brain tumors, menengiomas and abscesses may show Ti. 201 uptake besides low and high grade astrocytomas in the early SPECT images obtained 10-20 minutes after TI-201 injection. In this study, we aimed to investigate the role of quantitative early and delayed TI-201 SPECT imaging in various brain lesions.

Nineteen patients with brain lesions were included in the study (Age: 45-68; 11 M, 8 F). 3 mCi TI-201 was injected. 15 minutes and 3 hours after the injection, SPECT imaging was performed using a large field-of-view camera (Toshiba - 602) with a low energy general purpose collimator. Sixty-four images of $30 \mathrm{sec}$ duration in $64 \times 64$ matrix were obtained. Symmetrical ROl's were drown over the lesion and contrlateral normal tissue on the early and daleyed transaxial slices. Thereafter, early and delayed lesion (L)/ normal tissue $(\mathrm{N})$ ratios were calculated. Retantion Index (Al) was obtained for each patient using these ratios.

\begin{tabular}{|c|c|c|c|}
\hline \multirow{2}{*}{ RI } & \multicolumn{2}{|c|}{ Delayed LN } & \\
\hline & Early & $\mathrm{L} / \mathrm{N}$ & \\
\hline Diagnosis & & Early $\mathrm{L} / \mathrm{N}$ & $\mathrm{RI}$ \\
\hline \begin{tabular}{|l|} 
Infection \\
\end{tabular} & $(n=6)$ & $1.1-1.84(1.49)$ & $79-92 \%(86 \%)$ \\
\hline Primary bening tm. & $(n=9)$ & $2.05-6.43(3.56)$ & $49 \cdot 92 \%(73 \%)$ \\
\hline Metastasis & $(n=3)$ & $2.81-3.43(2.86)^{\alpha}$ & $78-86 \%(87 \%)$ \\
\hline Primary malign tm. & $(n=1)$ & 1.51 & $124 \%$ \\
\hline
\end{tabular}

For the reason that primary malignant group is limited to a patient with anaplastic astrocytoma, statistical evaluation was not performed.

In conclusion: 1) The origin of the brain lesions cainnot be predicted with early and delayed quantitative TI-201 brain SPECT 2 2) If the question is specified to the differential diagnosis between two groups-for example; infection and metastasis, or infection and primary brain bening tumor - it might provide useful information for the clinician.

504

A.T.Fonseca, L. Salgado, D. Salgado, M.R. Viera

Serviço de Medicina Nuclear

Instituto Portugues de Oncologin de Franclsco Gentil

Lisboa - Porlugal

\section{$99 \mathrm{~m}$ Te HMPAO and $201 \mathrm{TI}$ Secquential SPECT' in} Recurrent Brain Tumours

The introduction of new therapies for printary bnin tumours has lead to a better local control and, in selected groups of patients (pts), increased survival rates. Nevertheless Transuission Computerized Tomography (CT) and Magnetic Resonance Imaging art umable to reliably distingulsh persistent and recument tumor from post-thempy necrosis or edena.

Thallium 201 scintigmapy tws a high sensitivity for detecting viable tumour.Prelininary studies revealed that 99nTe HMPAO may provide prognostic information concerning tumour resporise to thempy.

The ain of the study was to determine the accuracy of sequential 20ITl and 99mTc-HMPAO SPLCT for detecting mecurrent tumour. SPECT Images were visually interpreted and sentiguantilative tumour uptake ratios were compared with the presence or absence of residual/recurnent tumor determined by stereotactic the presence or absence of residual/recurnen
biopsy or elinicul and radiological outcome.

biopsy or clinicul and radiological outcome.
We studied $18 \mathrm{pts}(13 \mathrm{M}, 5 \mathrm{~F})$, mean age 41 years (22-75); wh clinical deterioration after lerapy for astrocilona (Grade $4 / \mathrm{II}-6 \mathrm{pts}$; Grade III/IV -9 pto), ollgodendroglioma (12 pts) and linfoma (1pt). Mean interval between dlagnosis ass SPECT was 30 months. All patierts were subnitted to previcus exiemial padiation therapy (RT) (12 after surgery) and 10 also to chemotherapy.

After i.v. injection of $74-111 \mathrm{MBq}$ of $201 \mathrm{TI}$ images were nequired (64 inuges, $360^{\circ}, 30 \mathrm{seg} /$ innge, 74 to $80 \mathrm{KeV}$ photopeak). Whithou moving the $\mathrm{pt} 740$ MBy of $99 \mathrm{mT}$ c HMPAO was i.v. injecled, and another SPECT study performed (64 imnges, $360^{\circ}, 30 \mathrm{seg} /$ image, $140 \mathrm{KeV}$ photopeak).

Each SPECT study was assessed visually by two independent observers for abnomunl regional tracer uptake and judged to be hlgh, modente or low relativo to abnomunl regional tracer uptake and judged to be high, modemte or low. relative to were obtained from lrregular ROIs drawn in the 201Tl axlal slice with the highest were obtaines fro

During follow-up neurological examination and CT were performed in all pts and biopsy in 6 pts.

Recurrent tunuor was present in $12 / 18$ pts. Eleven of those 12 pts showed increased 20 I'T uplake (1 low, 8 modente, 2 high), $99 \mathrm{mT}$ T HMPAO SPECT showed decteased in 10 pts and low/nodente uptake in 2 ptE.

In $3 / 6$ pis without recurrent tumor 201 TI uptake was normal and in 3 was increased. In all the $6 \mathrm{pts} 99 \mathrm{mT}$ c HMPAO was normal.

The mean values of ${ }^{\mathrm{T} l}$ tumor/background ratios for $\mathrm{p}$ (s. with and without tumor reurrence were $3.65=1.84$ and $1.26=0.35(0<0,05)$.

In our study, 201T1 - SPECT was a neliable nethod for the ldentification of tumor recurrence. 
Sunday, 21 August - Wednesday, 24 August 1994

505

L.Collombier, L. Taillandier, M. Debouverie, M.H. Laurens, P. Thouvenot, M. Weber, A. Bertrand.

Departments of Nuclear Medicine and Neurology, NANCY, FRANC

\section{CONTRIBUTION OF THALLIUM 201 SPECT IN PRIMARY}

\section{BRAIN TUMORS GRADING}

The aim of this pilot study is to evaluate Thallium (Tl) 201 SPECT i brain tumour grading. We performed T1 201 SPECT on 30 patien with supratentorial brain tumours. Suspected diagnosis was as follows 17 grade IV, 2 grade III, 1 grade II astrocytoma, 2 metastatic brai tumours, 2 primary brain lymphoma, 2 radiation necrosis, 3 unknow processes. T1 201 is accumulated in brain tumour, not in normal brain SPECT imaging was performed $15 \mathrm{~min}$ after IV of $148 \mathrm{MBq}$ of Tl 201 with a single head tomographic camera $\left(64 \times 30 \mathrm{sec} / 360^{\circ} 64 \times 64\right.$ matrix $)$ Region of interest (ROI) were drawn on the transaxial slice showin the greatest tumour activity. For comparative measurements ROI wer determined on the homologous controlateral slide, on the controlatera scalp and over the whole cerebral contolateral hemisphere. Thre tumour Tl 201 uptake indexes were calculated using the average count: per pixel for the tumour and the control ROIs. Mann Whitney test was used for statistical analysis. Tl 201 indexes was high in 26 patients (group1) and low in 4 patients (group2). In the first group T1 201 indexes ranged to 1.35-5.97 (mean 2.64) for index 1 (Tumour/controlateral), to $0.88-3.45$ (mean 1.33) for index 2 (Tumour/scalp) and to $1.4-6.4$ (mean 2.74 ) for index 3 (tumour/hemisphere). In the second group, Tl 201 indexes ranged to 0.88-2.2 (mean 1.37) for index 1 , to $0.43-0.99$ (mean 0.6 ) for index 2 and to $0.81-1.93$ (mean 1.25) for index 3 . There were significant statistical differences between the 2 groups for the three Tl 201 uptake indexes : $p=0.0028$ for index $3, p=0.0072$ for index $2, p=0.0087$ for index 3. Predictive value of T1 201 indexes seems good : if index 1 $>1.3$ and index $2>0.9$ and index $3>1.3$ sensitivity increases to $92 \%$ and specificity to $100 \%$. Tl 201 SPECT may help to primary brain tumours grading, and to differenciate radiation necrosis versus recurent glioma. We continue this study to determine exactly threshold indexes between low and high grade gliomas. With dedicated brain gammacamera and multimodality computer it could be possible to improve anatomical data by superposing SPECT images with MRI images, and with computerized automatic calculation to assess viable tumour volume ; it would be usefulness for patients therapy and prognosis.

\section{6}

J. Tselfes, C. Giannakenas, D. Apostolopoulos, A. Mastorakou,

F. Tzortzides*, B. Michalopoulos, P.J, Vassilakos.

Dept. of Nuclear Medicine \& Neurosurgery*, Regional University Hospital, University of Patras Medical School, Patras, Greece.

IMAGING OF INTRACRANIAL TUMORS USING 111-In LABELED SOMATOSTATIN ANALOGUE - QUANTITATIVE STUDY OF THE UPTAKE IN VARIOUS TUMOR TYPES.

It is known that most neoplasms of neuroendocrine origin express somatostatin receptors. This continuing study evaluates the in vivo uptake of a somatostatin analogue (pentetreotide) by intracranial tumors.

We evaluated 49 patients, 30 males and 19 females imean age 50y range 19-811, with a variety of brain tumors. More precisely: meningiomas $(n=11)$, gliomas of various types and grades $(n=24)$, pituitary adenomas $(n=5)$ acoustic neuromas $(n=5)$ and metastatic brain tumors $(n=4)$. All patients had positive findings in previous examinations using other modalities such as CT and/or MRl scans. Planar scintigraphic imaging was performed at $6 \pm 1$ hrs $p$. following the $\mathrm{IV}$ administration of $2,2-3,0 \mathrm{mCl}$ of $111 \mathrm{ln}$-DTPA-Octreotide. The tumor/normal tissue (T/NT) uptake of the radiopharmaceutical was determined and the T/NT ratio was then calculated. All patients (with the exception of those with metastatic tumors) were surgically treated within the following 48 hours. Histologic verification of resected tissues was performed in all cases.

Positive scintigrams were obtained from all the meningiomas $(11 / 11)$, in $20 / 23$ gliomas, in all the pituitary adenomas $(5 / 5)$ and metastatic tumors $(4 / 4)$ and in $4 / 5$ of the acoustic neuromas. The quantification of the relative uptake of the radiopharmaceutical by the tumors yielded the following T/NT ratios:

\begin{tabular}{|lcc|}
\hline Tumor Type & $\mathrm{n}$ & T/NT Ratio \\
\hline Meningiomas & 11 & 7.7 \\
Astrocytomas (Grade II) & 2 & 4.5 \\
Pituitary Adenomas & 5 & 4,8 \\
Metastatic tumors & 4 & 4,2 \\
Astrocytomas (Grades III) & 14 & 3,2 \\
Acoustic Neuromas & 5 & 3,1 \\
Multiformous glloblastomas & 8 & 2,8 \\
\hline
\end{tabular}

In conclusion, 1) - the use $111 \mathrm{in}$-Octreotide may be helpful in the preoperative differentiation of meningiomas from other brain tumors in respect to the nign T/NT ratio obtained. 21 - In the remaining tumor types which were positively imaged the T/NT ratio did not show any significant differences between positively imaged the T/NT ratio
the different histologic types.
507

L.Collombier, M. Debouverie, L. Taillandier, M.H. Laurens, P. Thouvenot, M. Weber, A. Bertrand.

Depts of Nuclear Medicine and Neurology, NANCY, FRANCE VALIDATION OF BRAIN TUMORS THALLIUM 201 UPTAKE INDEXES

The aim of this study is to evaluate Thallium (T1) 201 brain tumors uptake indexes.

We performed Tl 201 SPECT on 30 patients with supratentorial brain tumours ( 22 astrocytoma, 5 others tumours, 3 unknown processes). Transaxial slices were displayed and added four by four in function of $T$ tumor uptake, resulting in 10 transaxial slices of $1,5 \mathrm{~cm}$ width. Regions of interest (ROI) were drawn on the transaxial slice showing the greatest tumour activity : a large one around all the tumour (ROI I), and a little one around the greatest $T 1$ tumor activity (ROI 1). For comparative measurements ROI were determined on the homologous controlateral slide : large (ROI II) and little (ROI 2), on the controlateral scalp(ROIscal) and over the whole cerebral contolateral hemisphere(ROIcer). Six tumour Tl 201 uptake indexes were calculated using the average counts per pixel for the tumour and the control ROIs. Mann Whitney test was used for statistical analysis.

The three indexes calculated with little tumoral ROI (ROI 1) were not pertinent for primary brain tumor grading $(\mathrm{p}=0.15)$. The 3 indexes calculated with large ROI was high in 26 patients (group1) and low in 4 patients (group2). Index 1 (ROI 1/ROI II) was at 2.64 , (mean value group $1=$ mv1) versus 1.37 (mv2), index 2 (ROI I/scal) was at 1.33 (mv1) versus 0.6 (mv2), index 3 (ROI V/ROIcer) was at 2.74 (mv1) versus 1.25 (mv2). There were significant statistical differences between the 2 groups for the 3 indexes $: p=0.0028$ (index 3), $p=0.0072$ (index 2), $p=0.0087$ (index 3). Based on the index cutoff of 1 for index 2 (ROI I/scal) sensitivity $=69 \%$ and specificity $=100 \%$, on the index cutoff of 1.5 for index 1 (ROI I/ ROI II) sensitivity $=92 \%$ and specificity $=75 \%$, on the index cutoff of 1.5 for index 3 (ROI I/cer) sensitivity $=88 \%$ and specificity $=75 \%$.

In conclusion : brain tumors Tl 201 uptake indexes are only valid if tumoral ROI is large. The choice of a $1.5 \mathrm{~cm}$ slice width is important on account of tumor heterogeneity and SPECT definition. Indexes permit to differenciate low and high grade glioma. The most specific is the index 2 (tumor/scalp), the most sensitive is the index 1 (tumor/controlateral region). The predictive value of this 3 indexes are good with index $2>1$ and index $>1.5$ and index $3>1.5$; sensitivity growth to $92 \%$ and specificity to $100 \%$. We continue this study to state exactly TI 201 uptake indexes in gliomas, and to evaluate their clinical predictive value, and their usefulness for therapy following.

508

W.-G.Franke, U.Ehrenfeld, R.Haas, H.M.Penzler, R.Koch

Clinic for Nuclear Medicine and Institute for Medical Informatics and Biometry, Technical University of Dresden, JOMOL Pharma, Inc., Regensburg, Germany

STUDIES WITH A TC-99m LABELLED IMMUNOMODULATING SUBSTANCE IN DIFFERENT TYPES OF MALIGNOMAS RESULTS AND CONCLUSION FOR DIAGNOSTIC SIGNIFICANCE AND RELLABILITY

An extract from wall of the bacterium Nocardia opaca prepared as disclosed in the German patent P 3510795 supplied by one of the authors (U.Ehrenfeld) was proved in former animal experiments as an immunomodulating substance. After administration of this preparation radiolabelled by In-111 or Tc- $99 \mathrm{~m}$ and in tracer amounts an elevated radioactivity uptake was observed at various sites of increases enzymatic activity as well as in Walker cacinsarcomas. Therefore, the reliability in diagnosing of different types of malignant tumors was studied by us. Planar imaging using a large field camera equipped with a MEHR collimator took place $3 \mathrm{hrs}$. pi. of the Tc-99m-labelled immunomodulator completed by SPECT for doubtful cases. Images were evaluated by visual interpretation as well as in a semiquantitative manner. 105 male and female pts. were studied suffering from following types of primary malignomas or metastases: breast cancer, sarcomas, malignant tumors of urogenital and gastrointestinal system, melanomas and neuroendocrine tumors. 23 lesions were proven histologically, whereas the remaining number of scintigraphic findings were confirmed by $\mathrm{CT}$ and MRI. Overall sensitivity of tumor detection was $0,97 \%$ if cut off point was set at a uptake ratio of 1.30 . The mean values of ratios differ from tumor species to species with the highest mean values for ratios of sarcomas. Advantageously, the diagnostic application of the Tc-99mlabelled IMM is not limited to a single tumor type but seems to be successfully in a broad range of different types of malignomas. Therefore, from clinical point of view the IMM seems to be suited to used for recognition of malignomas additionally to other well known tumor seeking agents. Further studies will be directed to consider the question of specificity. 
Sunday, 21 August - Wednesday, 24 August 1994

509

H-J Bair, W Becker, Th Behr, W Baum*, M Gramatzki*, F Wolf

Departments of Nuclear Medicine and *Internal Medicine III, Division of Oncology, University of Erlangen-Nuremberg, Erlangen, Germany

IMAGING CAPABILITIES AND BIOKINETICS OF A COMPLETE ANTI-CD7 ANTIBODY AND ITS FRAGMENTS $\left(\mathrm{F}\left(\mathrm{ab}^{\prime}\right) 2\right.$, Fab')

The aim of this study was to compare the biokinetic properties and imaging capabilities of an anti-CD7 antibody, using the ${ }^{131_{I}}$ labeled complete $\mathrm{IgG}$ and $\mathbf{F}\left(\mathrm{ab}^{\prime}\right) 2$-fragment and the $99 \mathrm{~m} \mathrm{Tc}$ labeled $\mathrm{Fab}^{\prime}$-fragment in a nude mouse model. The CD7 molecule is a $40 \mathrm{kD}$ membrane protein, which is expressed by mature and immature T-lymphocytes. It is not expressed on a variety of non hematolymphatic human tissues.

29 nude mice with T-cell acute lymphoblastic leukemia cells (CEM cell line; $0.75-3 \mathrm{~cm}^{3}$ tumour volume) were included. Scintigraphy was performed with a pinhole collimator (Siemens Basicam gamma camera, 15min/image) and data stored in a 256×256 matrix (Max Delta computer system). Whole body kinetics were calculated by ROI technique.

The ratio tumour activity / whole body activity increased:

for the complete antibody from $16 \%$ at $24 \mathrm{~h}$ pi up to $93 \% 10$ days pi, for the $\mathrm{F}$ (ab)2-fragment from $21 \%$ at $24 \mathrm{~h}$ pi up to $88 \% 10$ days pi, for the $\mathrm{Fab}^{\prime}$ fragment from $30 \% \mathrm{lh}$ pi up to $40 \% 2 \mathrm{~h} \mathrm{pi}$, which declined to $20 \% 72 \mathrm{~h}$ pi. The complete antibody showed whole body retention with biological half life of 6.5 days, the $F\left(\mathrm{ab}^{\prime}\right) 2$-fragment of 3.7 days and the Fab'-fragment of 0.5 days.

In conclusion, the Fab'-fragments achieve very early maximal percentage of the whole body activity in the tumour region, but in contrast to the complete antibody and the $F\left(a^{\prime}\right) 2$-fragment activity in the tumour region declines. Both effects can be explained by the more rapid whole body excretion of Fab'-fragments and their faster rate of extravasation. Presumably, there is an internalization of complete IgG and its $F(a b) 2$ fragment leading to stable tumour binding, whereas Fab'-fragments have lower affinity and no internalization takes place, leading to subsequent washout.

\section{0}

A. Kroiss, F. Böck, Ch. Auinger, G. Perneczky, P. Angelberger and A. Neumayr.

City Hospital Rudolfstiftung, Forschungszentrum Seibersdorf, Ludwig Boltzmann Institute for Clinical Geriatrics, Vienna, Austria

\section{LOCALIZATION OF BRAIN TUMORS WITH INDIUM-111 LABELED SOMATOSTATIN ANALOGUE AND IODINE-123-METHYL TYROSINE.}

The aim of this study was to compare the visualization of brain tumors with lodine-123-Methyl Tyrosine (I-123-MT) and Indium-111Octreotide (ln-111-Oc).

We used I-123-MT (FZ-Seibersdorf), administering $222 \mathrm{MBq}$ and planar images were performed $10 \mathrm{~min}$ and $1 \mathrm{hr}$ after application. In 5 ptS SPECT images were performed too.

Not earlier than $48 \mathrm{hrs} 244 \mathrm{MBq} \operatorname{In}-111-0 \mathrm{C}$ (OctreoScan®, Mallincrodt) were injected and planar images 4 and 24 hrs after application performed. In 9 pts SPECT images were performed (4hrs p.appl.). A digital Anger camera was used for data acquisition and processing (APEX 409A, Elscint).

A total of 12 pts ( 8 male, 4 female; age ranging from $45-71 \mathrm{yrs}$ ) were investigated. Using a region of interest technique tumor-to-brain tissue ratios (T/BT) were calculated. Diagnosis of tumor was estabilshed by neurosurgical procedures.

$6 \mathrm{pts}$ with glioblastoma showed a high uptake with In-111-Oc (T/BT: $1.7 \pm 0.5)$ and also with $1-123-\mathrm{MT}$ (T/BT: $1.5 \pm 0.4\left(10^{\prime}\right)$ and $1.45 \pm 0.45$ (1h)). In 2 meningiomas the images with In-111-Oc were very good (T/BT: $3.1,3.6(4 \mathrm{hr} \mathrm{pl})$ ) and were negative with 1-123-MT. In 4 metastases we found a low uptake in 2 pts with l-123-MT (T/BT (10'): 1.3 and 1.25; T/BT (1h): 1.25 and 1.2 and $\ln -111-O c$ (T/BT (4h pi): 1.6 and 1.4). These were pts with brain metastases of adeno carcinoma. in two pts with brain metastases of small cell lung cancer we found good images with both substances (I-123-MT T/BT: 1.6 and $1.7(1 \mathrm{~h})$ and $\mathrm{nn}-111-\mathrm{Oc}$ T/BT: 2.5 and 2.6 (4h pl). In summary, glioblastoma showed concordant images with both substances and also metastases, meningiomas showed discordant images. SPECT acquisition is possible with both substances and sometimes advisable.
511

*D. Franceschi, D.A. Weber, M. Ivanovic, ${ }^{\circ} \mathrm{M}$. Franceschi, E. Popenoe.

Brookhaven National Laboratory, Upton, USA, ' Sestre Milosrdnice, Zagreb, Croatia, *SUNY Stony Brook, USA

\section{UPTAKE OF I-123 IMT IN PRIMARY BRAIN TUMOR}

Various in vivo and ex vivo imaging techniques were used to evaluate and quantitate I-123 iodomethyltyrosine (IMT) uptake in primary brain tumor in rats. Quantitative imaging using tumor imaging agents TI-201 and Tc-99m MIBI was performed for comparison.

A total of 30 animals with transplanted gliosarcoma was studied. The animals were imaged using pinhole planar and pinhole SPECT. At completion of imaging, animals were sacrificed, pinhole planar ex vivo images were obtained, and well counting of tumor and control brain tissues was done. Tumor to non-tumor (T/NT) ratios on images were determined using ROI techniques.

The images showed increased I-123 IMT uptake in tumor compared to the activity in the surrounding normal brain. T/NT ratios were $1.4 \pm 0.1$ and $3.0 \pm 0.3$ on planar and SPECT studies respectively, $2.9 \pm 0.5$ on ex vivo images, and $4.4 \pm 2.2$ in the well counter. Increased tumor uptake of Tl-201 and Tc-99m MIBI was observed with T/NT ratios of $1.9 \pm 0.4$ (MIBI) and $2.1 \pm 0.7$ (Tl-201) on planar images, $10.5 \pm 2.1$ (MIBI) and 5.6 2.0 (Tl-201) on SPECT images, $10.9 \pm 4.8$ (MIBI) and 7.1 \pm 2.4 (T1-201) on ex vivo images, and $9.0 \pm 2.6$ (MIBI) and $6.6 \pm 1.6(\mathrm{Tl}-201)$ in the well counter.

The selective, increased uptake of I-123 IMT in gliosarcoma observed using high resolution imaging methods, is less than observed with Tl-201 and Tc-99m MIBI. However, the findings are adequate to suggest that quantitative metabolic brain tumor imaging is feasible using high resolution SPECT with an I-123 labeled amino acid analog in order to visualized viable tumor.

(Research supported by U.S. DOE contract DE- AC02-76CH00016.)

\section{2}

H.Moustafa;W.Omar;I.Ezzat;G.Ziada;and E.El-Ghonimy

Department of Radiation Oncology and Nuclear Medicine, Faculty of

Medicine, Cairo University - Egypt

THALLIUM-201 SINGLE PHOTON EMISSION TOMOGRAPHY

IN EVALUATION OF RESIDUAL AND RECURRENT ASTROCYTOMA

ABSTRACT:

Twenty five patients with malignant astrocytoma, either as postoperative (15 cases) or with recurrent tumour versus gliosis (10 cases) were included in this study. ${ }^{201}$ TI-SPECT scintigraphy was performed with calculation of early, delayed uptake and retention index. A high mean value of early and delayed uptake with low retention index in patients with high grade astrocytoma in both postoperative residual and recurrent groups, versus lower mean value of early and delayed uptake with high retention index in the low grade tumours. All postoperative cases with high grade and high ${ }^{201} \mathrm{TI}$ uptake $>1.5$, whereas $66.6 \%$ of cases with low grade had low ${ }^{201} \mathrm{TI}$ uptake $<1.5$. A correlation between retention index of ${ }^{201} \mathrm{TI}$ and tumour grade ( $\mathrm{r}=0.47$ ). Also, recurrent cases showed remarkable difference in early and delayed 201 TI uptake $(\mathrm{P}<0.05)$ and retention index $(\mathrm{P}<0.001)$ as compared to post radiation gliosis. Also, higher sensitivity in detection of tumour viability in second group $(100 \%)$ versus $80 \%$ in CT scanning in differentiation between recurrent tumour and post radiation gliosis

Conclusion:TI-201 can be used in differentiation of low and high grade astrocytoma and in differentiation between recurrent tumour and postradiation gliosis 
Sunday, 21 August - Wednesday, 24 August 1994

513

R.M. Moresco, M. Losa, B.W. Scheithauer, G. Lucignani, R. Casati, L. Rocca, D. Lombardi, M. Giovanelli, E. Fazio. INB-CNR, University of Milan, H S. Raffaele Milan (Italy), Mayo Clinic, Rochester, Minn, (USA).

PET AND IMMUNO-HISTOCHEMICAL ASSAY OF ESTROGEN RECEPTORS IN MENINGIOMAS

To assess the suitability of $16 \alpha[F-$ 18] fluoroestradiol ([F-18]FES) for the in-vivo imaging of estrogen receptors (ER) in human meningiomas, tumours known to be influenced by sex hormones during pregnancy and the luteal phase, the cerebral uptake of [F-18]FES was examined in 6 patients (5 female) with neuroradiologic and histologic diagnoses of meningioma. Immuno-histochemical staining for $E R$ was performed ( $A B C$ method) on paraffineembedded resection specimens using a mouse monoclonal antibody (AMAC, Corp; dil 1:20). PET studies showed [E-18]EES uptake and retention in the tumor in $4 / 6$ patients; the tumor/non-tumor activity ratio, $90 \mathrm{~min}$ after tracer injection, ranged from 1.8 and 2.3. In contrast, only sparse ER immuno-staining was noted in 1 case, the other 5 tumors being nonreactive. In view of the sensitivity of the immuno-stain method, capable of detecting ER at the $>10$ picomoles/mg protein level, the inconsistency of $\mathrm{PET}$ and immuno-stain findings suggest a non-specific uptake of [F-18]FES due either to hemodinamic processes or to binding of [F-18]FES to tumor constituents other than estrogen receptors. Whereas the significance of [F-18]FES uptake is questionable for the evaluation of ER in meningiomas, the remarkable uptake of $[F-18] \mathrm{FES}$ in such tumors warrants further studies.

\section{4}

N. Avril, F. Jănicke*, J. Dose*, S. Ziegler, I. Woll, M. Hertz, S. Bense, H. Graeff*, M. Schwaiger

Nuklearmedizinische Kininik u. Poliklinik, Frauenklinik*, Klinikum rechts der Isar, Technische Universität München, Germany

EVALUATION OF FDG-PET IN PATIENTS WITH PELVIC MASSES SUSPICIOUS FOR PRIMARY OR RECURRENT OVARIAN CANCER

Conventional imaging techniques like sonography, CT or MRI are limited in diagnosing primary or recurrent ovarian cancer. The efficacy of FDG-PET in the detection of metabolically active tumor sites was assessed in patients with suspicious pelvic masses and compared with histological tissue characterisation.

Twenty-three patients underwent PET examinations (Siemens ECAT $951 R$ ) prior to laparotomy. Dynamic FDG.PET scans of the pelvis were obtained for 60 minutes followed by PET imaging of the whole abdomen. Standardized uptake values for FDG were calculated based on attenuation corrected images and related to histological findings.

Fourteen patients with primary and two patients with recurrent ovarian cancer had an increased, but heterogenous FDG uptake (SUV 5.4 \pm 2.3 ). Necrotic/ cystic areas could be clearly seperated from metabolicly active tumor sites. Five intraabdominal abscess localisations $(n=3)$ also showed an intensive FDG accumulation (SUV 9.8+3.4). One patient with ovarial cystoma (SUV 1.1) and one case with ovarian borderline carcinoma (SUV 1.3) had low FDG uptake.

In patients with benign cystic lesions ( $n=2$ ) no significant FDG-uptake was seen. In areas with questionable CT-findings, FDG uptake aided in the accurate definition of metastatic tumor spread.

In conclusion, differentiation between abscess and malignancy may be limited in patients with large pelvic masses. Correlation of FDG uptake with CT and sonography however adds diagnostic information. FDG-PET imaging is extremely useful in the localisation of viable tumor tissue in new or recurrent ovarian cancer and may therefore guide surgical interventions.

\section{5}

Krolicki L,Andrysiak R, Mikolajkow A,Graban W.Dziuk E, Mielcarek S, Kozlowicz I, Tomaszewska A.

Dep. of Nuclear Medicine Medical School of Warsaw

IMMUNOSCINTIGRAPHY IN DIAGNOSIS OF COLORECTAE CANCER (POLISH MULTICENTER STUDY)

The aim of this study was to evaluate the role of the immunoscintigraphy in diagnosis of the colorectal carcino ma. This method was not develop till now in Poland and this multicenter study is the first project concerning usefulnes of RIS in our country.

Monoclonal antibody B 72.3 (CYT-103) labeled with 111-indium chloride were used. 42 patients with high suspiction of cancer were studied.Two group of case were selected:a.with primary tumour and b. with local recurren ce. A single dose of 1 . Omg labeled antibody (4.5-5.5 mCi) was administered.vital signs were monitored during and $60 \mathrm{~min}$. after injection. Gamma cameras ZLC Siemens were used.Examinations were performed in all cases twice or third between 24-72 hs, after injection of the tracer.

No adverse reactions after injection of CYT-103 were noted. In the group a 21 out 25 examinations showed true positive results. The sensitivity was $84 \%$. In the group b true negative results were observed in 6 cases, true positive - in 8 cases and false negative - in 2 cases. The sensitivity was $80 \%$.

In conclusion: CYT-103 scintigraphy has proven to be a safe and promising method in diagnosis of colorectal primary as well as recurrence neoplastic process.

516

G.Vural* ${ }^{*}$ T.Atasever*,1.Oznur**, A. Ozdemir**,

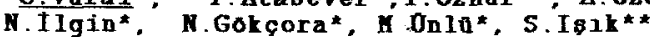

Gazi University, School of Medicine, Depart. of Nuclear Med*. and Radiology**, Ankara-Türkiye

CORRELATION OF DIFFEREYT DIAGNOSTIC HODALITIES IN BREAST LESIONS AND LYYMPHADENOPATHIES

The aim of the study was to compare different

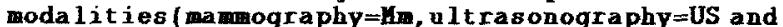
T1-201 scintigraphy=Tlsc) in deteoting breast lesions and lymphadenopathies(LAPs) 48 patients vho have mammographically or sonographically detected breast lesions vere evaluated by Tlsc for suspected axillary (Ax) and ${ }^{\circ}$ r internal mammary (IH) LAPs. Early (15min) and delayed (3hr) Tlsc images vere evaluated both by visually (Tivis) and quantitatively(Tlquan) using retention index ( $R I=$ delay-early/early). There vere 35 breast lesions, 5 microcalsifications, 24 Ax and 4 II LAPs. Sensitivity, specificity and accuracy of Tlso in detecting breast lesions vere $86 \%$, 758 and $80 \%$ respectively. Sensitivity, specificity and acouracy of LAP detection vere as follows: $\underline{\operatorname{LAP}(\mathrm{Ax}+\mathrm{II})} \quad \underline{\operatorname{LAP}(\mathrm{Ax})}$

\begin{tabular}{|l|l|l|l|l|l|l|l|}
\multicolumn{1}{c}{ sens } & spec & acc & sens & spec & acc \\
\hline U.S & $87 \%$ & $76 \%$ & $82 \%$ & $100 \%$ & $75 \%$ & $88 \%$ \\
Tlvis & $87 \%$ & $76 \%$ & $82 \%$ & $83 \%$ & $83 \%$ & $83 \%$ \\
Tlquan & $80 \%$ & $85 \%$ & $82 \%$ & $75 \%$ & $83 \%$ & $80 \%$ \\
\hline
\end{tabular}

Ouantitative assessment was more specific in differentiating malign and benign l.APs compared to US and visual assessment. In cases of breast lesions $\mathrm{Hm}$ and US vere more sensitive and specific than Tisc. Correlative evaluation of the techniques may improve management of breast lesions and metastatic I.APs 


\section{7}

P.Bourgeois $\%^{* *}$. W. Matthéiem* and J.Fruhling ${ }^{\star *}$ Services of Nuclear Medicine*** and of Surgery*, Universitary Hospital St Pierre and institut J.Bordet*/**, Universite Libre de Bruxelles, Brusseis, Belgium.

INTERNAL MAMMARY LYMPHOSCINTIGRAPHY (IMLSC) IS ONE UNDEPENDENT PROGNOSTIC VARIABLE IN BREAST CA. $(B C)$.

Several authors had reported on the value of IMLSC as a prognostic variable (PV) in BC. However, their conclusions might be criticized because they only take into account - as other potential PV - the axillary (ax) nodes status. Therefor, the follow-up (FUp) data of 860 patients ( mean FUp $=5 \mathrm{y}$.) operated for histologically proven $8 C$ from $12 / 1978$ to 12/1989 have been reviewed and analyzed (Free Disease Interval curve, Delay Before Generalisation curve, Surviva curve, Kaplan-Meier actuarial method, Logrank Test ) with regard to the various progorstic variables :clinical staging (CSt), clinical size (CSz), anatomopathological status of the ax nodes (AxAP) results of the MLSc (MLSc), hormonal ( estrogen ) receptivity (ER), hormonal treatment (HTt), menopausal status, age. differenciation of the tumor,...

In our population and whatever was the considered end-point ( $F D I, D B G, S$ ), the main PV (univariated analysis ) were the CSt or the CSz, the AXAP, the IMLSC $(2 \mathrm{p}<0.0001$ when classified as pathologic), the HTt and the ER

When MLSc was introduced in a Cox Model (Multivariated analysis ) with the other potential explicative PV, the scintigraphic result remains as one undependent explicative variable for the prognosis of $B C$.

it is concluded that MLSc represents one undependent PV in $\mathrm{BC}$ and that its result has to be taken into account in the management and treatment of $B C$.
519

R. Campini, F. Fringuelli, R. Giubbini, F.perotti, A.Colombo, E.Castellano, F.Cozzaglio.

Nuclear Medicine Dpt, Clinica Del Lavoro Foundation veruno (No), Surgery Dpts Eorgomanero and Arona Hospitals. Italy.

Could Tc99m Sestamibi scintigraphy be complementary to mammography in the diagnosis of breast cancer? preliminary results

Breast cancer frequently occurs in women and patients (pts) prognosis has been shown to improve with early detection and aggressive treatment. clinical examination, maramography (mmg) and fine needle biopsy are frequently inconclusive in order to characterise breast nodules. Aim of the present study has been the evaluation of the accuracy of rc99m MIBI imaging to define malignant lesions and its possible role in the diagnostic strategy. our study group consisted of 17 women affected by breast lesions detected by ming who should be considered a rancom sample from the clinic.. All the pts were treated surgically and had a histological evaluation.

$370-500 \mathrm{MBq}$ of MIBI were injected in the antecubital vein opposite to the involved breast and the scan was performed immediately and after 2 hours. Pts were imaged by planar scintigraphies obtained in anterior and anterior oblique views. Two independent experienced observers blinded to clinical and histological data interpreted the MIBI studies. Any disagreement was resolved by consensus. In 15 pts solitary nodules were found (tumor size $=2.4+/-.9 \mathrm{~cm}) ; 2$ pts suffered from diffuse pathology (carcinomathosis mastitis). In 13 pts a malignant disease (54\% with positive mug, $46 \%$ inconclusive) and in 4 benignant lesions (75\% negative, $25 \%$ doubtful at ma) were found. MIBI imaging showed $86 \%$ sensitivity and $75 \%$ specificity with an overall accuracy of $82 \%$. particularly MIBI imaging gave correct interpretation in 72 : of pts with inconclusive mmg. Thus MIBI scan could be considered a useful tool. complementary to mmg, in the screening of breast nodules.

520

D.W. Seldin, M. Camus, K.S. Hughes, M.A. Cox.

Departments of of Diagnostic Radiology and Surgery, Lahey Clinic, Burlington, Massachusetts, USA

PREOPERATIVE EVALUATTON OF PRTMARY BREAST CARCINOMA (CA) AND AXILLARY METASTASES USTNG A RADIOLABELED ANT IBODY FRAGMENT

We are evaluating the safety and efficacy of a TC-99m labeled Fab' anti-CEA antibody fragment (IMMU-4, Immunomedics, Inc.) for preoperative evaluation of the breasts and axillae in patients (pts) presenting with breast ca. Nine pts with 10 primary breast ca (one bilateral) received IV injections of $1 \mathrm{mg}$ of IMMU-4 labeled with $740^{\circ} \mathrm{MBq}$ of TC-99m. Planar whole body and thoracic SPECT images were obtained 6 hours post injection using a dual-head camera. In 7 pts SPECT imaging for 30 minutes was performed at 24 hours. The image results were compared with pathologic examination of surgical specimens in all pts. The primary breast ca was confirmed with RAID in 10 of 10 cases (sensitivity $100 \%$ ). The smallest tumor found was $1.1 \mathrm{~cm}$. Two pts with unexpected contralateral breast uptake are being followed. In the 9 pts who had ipsilateral axillary exploration, scans were true-negative in 4 , truepositive in 3 , and false-positive in 2 (both of which showed only slightly elevated activity). belayed SPECT imaging improved tumor detection in $4 / 7$ pts. The only adverse reactions were mild itching at the time of the injection in two pts and a metallic taste in one pt. HAMA titers remained nomal in all pts (in other studies $<1 \%$ of pts show elevated HAMA titers). TC-99m TMMU-4 Fab' appears promising for detecting primary breast ca and axillary metastases. SPECY at 24 hours is feasible and appears to aid diagnosis. 


\section{1}

GS Limouris $^{2}$, V Giannakopoulos ${ }^{\mathrm{a}}$, S Athanassiades ${ }^{\mathrm{b}}$, A Stavraka $^{\mathrm{a}}$ a. Nuclear Medicine Section, Areteion Univ Hosp, Athens b. Dept of Surgery, Areteion Univ Hosp, Athens

\section{THE EFFICACY OF In-111-PENTETREOTIDE IN BREAST CANCER STAGING}

So far, the role of the bone scan in the routine screening of breast cancer patients for metastases at presentation and during follow-up is widely agreed. However, this early enthusiasm has been recently decreased somewhat due to the relatively low true positive yield and the measurable rate of false positive studies. Furthermore the main indication for bone scanning is exclusively dealing with the osseous metastases status of the malignancy while the possible soft tissue secondary lesions cannot be explored but surgically only detected.

Since in any histopathologic type of breast cancer, somatostatin receptors has been found to be more or less, we attempt by the present to evaluate the use of radiolabelled Pentetreotide as a possible screening method in breast cancer suspected women; thereby delineating any soft tissue participation (lymph nodes tc) as well as detecting occult secondary osseous lesion

In 23 pre-operative breast cancer females, aged 32 to 51 years, $185 \mathrm{MBq}$ (5mCi) In-111-Pentetreotide [OctreoScan 111, Mallinckrodt Medical B.V., Pettenl and $740 \mathrm{MBq}(20 \mathrm{mCi}) \mathrm{Tc} 99 \mathrm{~m}-\mathrm{MDP}$ [Nucl Research Center "Demokritos», Athens] were both injected (3-4 days time interval between the two examinations). All 23 women were after scintigraphy surgically investigated.

\begin{tabular}{lccc}
\hline Radiopharmaceuticals & $\begin{array}{c}\text { Breast } \\
\text { Primary Lesion }\end{array}$ & $\begin{array}{c}\text { Lymph Node } \\
\text { Secondary Lesions }\end{array}$ & $\begin{array}{c}\text { Osseous } \\
\text { Secondary Lesions }\end{array}$ \\
\hline In-111-Pentetreotide & $23 / 23$ & $23 / 23$ & $20 / 23$ \\
\hline Tc-99m-MDP & & & $23 / 23$ \\
\hline Surgical Assessment & $23 / 23$ & $23 / 23$ & \\
\hline
\end{tabular}

Based on the results we can conclude: a) In-111-Pentetreotide i.v. application very accurately detects the extent of the maliqnancy (primary and secondary (soft tissue, bone) lesions]; b) a differential diagnosis can be done in cases where Tc-99m-MDP increased accumulation have an other than metastasis, aetiology, c) In-111-Pentetreotide scan could be used as an excellent routine pre-operative procedure for breast cancer staging as well as an accurate examination method in the follow-up of any therapeutic scheme.

\section{2}

KJA Kairemo, H Ramsay, T Raavonen, S Savolainen, JV Hiltunen, EVM Hopsu, H Jäảskelả-Saarí, S Bordestam, $\mathrm{R}$ Ljunggren, S-E Strand, $\mathrm{k}$ Sjögreen, $\mathrm{K}$ Liewendahl.

Clin Chem, Otol, Pathol, Radiol \& oncol, Helsinki Univ. Hosp MAP Medical Tech., JYväskylä, FINLAND; Radiat Phý, Lund, SWEDEN

DOSIMETRIC AND KINETIC CHARACTERISTICS OF In-111-BLMC; A POSSIBLE AGENT FOR RADIOCHEMOTHERAPY IN HEAD AND NECR CANCER Bleomycin (BLM), a natuxal antibiotic toxic to dividing cells, has been used successfully for treatment of $H \& N$ cancer, as a single agent or combined with external radiation therapy. Therefore, the idea of combining radio- and chemotherapy using BLM complex (BMLC) is a fascinating possibility. In-112-BLMC was prepared from BLM sulfate, consisting of 13 alkalire glycopeptides, with $\mathrm{In}_{\mathrm{n}} 111-\mathrm{Cl}_{3}$, at low $\mathrm{pH}$. Radiolabeled In1I1-BLMC (subfractions $\left.\mathrm{A}_{2 a-C}\right)$ (100 MBq/mg) were administered to $H \& N$ cancer patients in escalating doses of $75(4 \mathrm{pts}), 175$ (3 pts) and $375 \mathrm{MBg}(3 \mathrm{pts})$. The patients were imaged quantitively with gamna camera, at least three times prior to surgery. scintigraphic data were compared with tissue samples from surgery $48 \mathrm{~h}$ after injection. SPECT studies were also performed in 3-D format for comparison with high field-MRI (fusion imaging\}. Serial blood and urine samples were collected for radiopharmacokinetics. $T_{1 / 2}$ for radioactivity was $1.5-3.1 \mathrm{~h}$ in serum and 1.4-3.7 b in urine. About $50 \%$ of the activity was excreted in urine within $3 \mathrm{~h}$. In all patients $>95 \%$ of the activity was excreted in $22 \mathrm{~h}$. In surgical samples following uptakes were observed: fat $0.02-0.09$, bone 0.05 , salivary gland $0.04-0.17$, muscle $0.03-0.08$, blood $0.04-0.08$ and turnor 0.39 $0.95 \times 10^{-3} \frac{1}{6} / \mathrm{ID} / \mathrm{g}$ (at $48 \mathrm{~h}$ ). From the imaging data, kidneys were the critical organ; the dosimetric estimate for a $3700 \mathrm{MBq}$ dose, assuming a mean residence time of $5 \mathrm{~h}$, was $1.4 \mathrm{~Gy}$. The tumox dose was $0.66 \mu \mathrm{GY} / \mathrm{MBq}$ (a mean residence time of $16 \mathrm{~h}$ ), in a 14-gram tumor (MIRD formalism). Tumor $T_{1 / 2}$ was $16-49 \mathrm{~h}$. The activity distribution and penetration into tumor tissue was not affected by the increasing injected activity. BLMC activity correlated positively with immunohistochemietry of the proliferation maxkers $\mathrm{Ki}-67$ and Mib. Cell nuclear localization (autoradiography) and heterogeneous activity distribution was bserved (beta camera). These data are compatible with In-111BLMC being suitable for adjuvant Auger-electron therapy of $\mathrm{H}$ \& $N$ cancer. We suggest that In $114 \mathrm{~m}$ would probably be the most effective Auger-mittex.

\section{3}

Th. Behr, W. Becker, M. Klein*, H.-J. Bair, Ch. Stühler*, J. Scheele*, F. Wolf

Departments of Nuclear Medicine and Surgery* of the Friedrich-Alexander-University of Erlangen-Nuremberg, Erlangen, FRG

IMMUNOSCINTIGRAPHY OF COLORECTAL CANCER WITH $99 \mathrm{~m}$ TCLABELED ANTI-CEA MAb F(ab')2 FRAGMENTS F023C5

The aim of this study was to evaluate the sensitivity and diagnostic accuracy of the directly $99 \mathrm{~m} T \mathrm{~T}$-labeled $F\left(\mathrm{ab}^{\prime}\right) 2$ fragments of the monoclonal anti-CEA antibody (MAb) F023C5 in patients with colorectal and other CEA-expressing tumors. The MAb (M-CEATEC Fab, Sorin Biomedica, Saluggia, Italy) was labeled with $1480-1850 \mathrm{MBq}(1 \mathrm{ml})$ of a fresh technetium generator eluate. After removing $12-20 \%$ remaining frec pertechnetate by ion exchange chromatography, 740-1295 $\mathrm{MBq}(0.25-0.5 \mathrm{mg})$ were injected iv. Totally 50 patients were investigated. Planar scans were performed 1,4 and $24 \mathrm{~h}$ pi, SPECT after 4 and $24 \mathrm{~h}$. All scintigraphic results were correlated to morphologic imaging procedures (CT, MRI) and finally to histology after surgery or biopsy.

The following lesions could be detected (scintigraphically pos. / all lesions): 4/4 colonic primaries, 15/15 locoregional recurrences, 24/31 liver metastases (scintigraphically hot or with warm rim), 11/11 lymph node metastases, $1 / 4$ lung metastases, $2 / 3$ brain metastases, $2 / 3$ bone metastases and $4 / 5$ peritoneal carcinoses. Positive tumor detection was possible in $16 \%$ as early as $1 \mathrm{~h} \mathrm{pi}$, in $82 \% 4 \mathrm{~h}$ pi. SPECT was superior to planar scans in $43 \%$, especially with respect to locoregional recurrence and liver metastases. Previously unsuspected lesions were found in $42 \%$ of patients.

Summarizing, the use of $99 \mathrm{~m}$ Tc-labeled $F\left(a b^{\prime}\right) 2$ fragments could yield additional information to conventional imaging procedures (CT, MRI) in the staging of colorectal cancer patients. The sensitivity for locoreg. recurrence and lymph node metastasis detection was especially high, despite the renal elimination of MAb fragments (SPECT indispensable).

\section{4}

D.M. Goldenberg and C.M. Pinsky (for the Multicenter Immunomedics Clinical Trial Group) Garden State Cancer Center at the Center for Molecular Medicine and Immunology, Newark, NJ; and Immunomedics,

PIVOTAL STUDIES WITH A NEW MONOCLONAL ANTIBODY IMAGING AGENT FOR IMAGING COLORECTAL CANCER

Various antibody-based cancer imaging agents have been developed and evaluated over the past decade including different antibodies and antibody forms radionuclides, and scanning procedures. Use of the Fab' form of anticancer MAbs combined with direct labeling of SH-groups with $\mathrm{Tc}-99 \mathrm{~m}$ has allowed the development of rapid and simple kits of low immunogenicity when $1 \mathrm{mg} \mathrm{Fab}^{\prime}$ is used (less than $1 \frac{0}{6}$ HAMA incidence). A multicenter study of 382 patients with colorectal cancer given a CEA-MAb agent. ImmuRAID-CEA, revealed both safety and evidence of efficacy. In 122 patients with known disease by conventional imaging methods (mostly CT scans) and surgical followup, sensitivity in the Iiver, surgical followup, sensitivity in the Iiver,
extrahepatic abdomen and pelvis was $83 \%$, $78 \%$, and $73 \%$, respectively. The Positive Predictive value for respectively. The Positive Predictive value for
lesions was significantly higher when conventional lesions was significantly higher when conventional
imaging and antibody imaging were both positive (99\%), than when conventional imaging was positive and antibody imaging was negative $(68 \%)$. Antibody imaging also significantly increased imaging accuracy when combined with conventional imaging. In another series of 88 patients with suspected recurrence, but occult disease, combining antibody imaging with conventional imaging significantly enhanced diagnostic accuracy, potentially improving clinical decision-making in $42{ }^{\circ}$ of the surgical patients. In summary, this appears to be a rapid and simple test for disclosing colorectal cancer sites, including lesions in the liver, and appears to contribute significantly to presurgical appears to contribute significantly to presurgical (Supported in part by USPHS grant CA39841 from the NIH.) 
525

A. Dimitrakopoulou-Strauss, L.G. Strauss, P. Schlag, F. Helus, W.J. Lorenz, W. Maier-Borst, G, van Kaick

Department of Oncologic Diagnosis and Therapy, German Cancer Research Center, Heidelberg, FRG

PREDICTION OF THERAPY OUTCOME IN PATIENTS WITH LIVER METASTASES FROM COLORECTAL CARCINOMA*

*This study was supported by the Dr. Mildred Scheel Stiftung

Fluorouracil (FU) has been used in clinical practice to treat colorectal carcinomas for more than two decades. The average response rate is lower than $20 \%$. Since tumor response to FU chemotherapy necessitates the accumulation of the cytotumor response to FU chemotherapy necessitates the accumulation of the cytostatic agent in the metastases, PET with F-18 labeled FU can be used to assess the tracer concentration in the target area. We used a PET system to evaluate the FU uptake and trapping in patients with liver metastases prior to the FU chemotherapy. The target area corresponded to the largest tumor diameter and was determined by CT shortly before the PET study. F-18 labeled FU was administered together with $500 \mathrm{mg}$ nonlabeled FU in order to obtain therapeutic concentrations. Sequential PET images were acquired for two hours beginning with the FU infusion. Standardized uptake values (SUV) were calculated from the PET cross sections two hours after tracer application. All patients received several cycles of FU chemotherapy after the PET study. The tumor volume and growth rate were calculated from follow up CT studies during treatment and compared to the FU ac-

cumulation in the metastases prior to chemotherapy. The evaluation includes 23 examinations. A correl tion coefficient of 0.88 was obtained for the standardized FU concentrations and the growth rate of the metastases (see diagram). Response to therapy necessitates FU tracer concentrations exceeding 3.5 SUV Ou exceeding 3.5 SUV. Ou data show, that PET can be used to estimate the tumor growth rate prior to chemotherapy and might help to predict come.

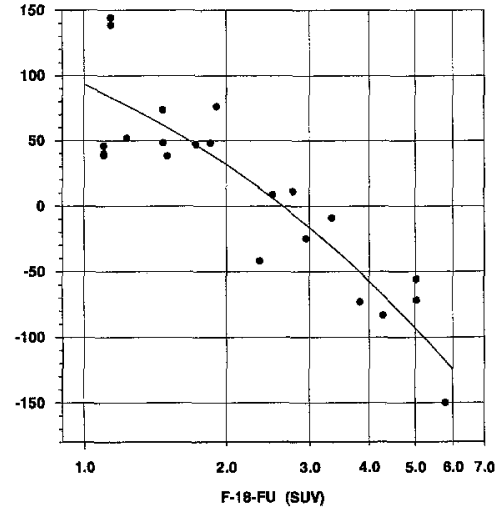

526

D.Platz, K. F. Gratz, M. Lübeck, A. Frilling*, C.E. Broelsch*

Abt. Nuklearmedizin, * Allgemeinchirurgie - UK Eppendorf, Universität Hamburg, FRG

\section{PREOPERATIVE IN-111-PENTETREOTIDE SPECT IN THE AS-} SESMENT OF GASTRO-ENTERO-PANCREATIC (GEP) TUMORS

The sensitivity of In-111-Pentetreotide (SMS) whole body scintigraphy and SPECT in patients with tumor not detected by abdominal or thoracic CT, MRI or ultrasound (US) is not well examined.Using $110 \mathrm{MBq}$ In-111-Pentetreotide, a HEAP-collimator, SPECT performed 4 and 24 hrs p.i. $\left(360^{\circ}, 64\right.$ steps, each $\left.40 \mathrm{~s}\right)$, we evaluated 2 groups of patients :

1) 6 pats. (5 carcinoids, 1 insulinoma) in whom CT, MRI and US showed isolated liver metastases, refered for livertransplantation (LTX), SMS scintigraphy revealed additional tumor sites in 5/6 (1 pleural, 1 abdominal, 2 skeletal, 1. lymphogen). The patient without extrahepatic tumor was transplanted. He is free of recurrence $3 / 4$ year later. In 3 pats. the scintigraphy failed to detect some of the known liver metastases shown by CT.

2) 6 pats. with suspected GEP tumor ( 3 primaries, 3 recurrent $t u$ ) because of elevated hormon levels in $5 \mathrm{ca}$ ses and a positive biopsy in 1 case. In all of them Cr, MRI and US imaging was negative. SMS scintigraphy detected tumor in 2 cases 11 local recurrence, 1 disseminated tumor) - true positive. In the pat. with positive biopsy the SMS scan was negative and the resection showed no tumor as well - true negative. In one pat. with a negative SMS scan and a suspectable angiographic finding, a curative resection was done - false negative. In the last 2 SMS scan negative pats. no explorative laparatomy was performed, so the result is nonconclusive. Conalusions:- In pats. referred for LTX due to metastases of GEP tumors, the SMS whole body scintigraphy detected in $80 \%$ foci not found by abdominal and thoracic CT, MR or US. Early use is recommended. In case of isolated liver metastases in SMS scan, completing CT, MR and US are required.

-In pats. with clinical high suspicion of tumor not detected by CT, MRI or US, SMS scintigraphy showed true positive or negative results in approximately $50 \%$.

527

T. Bokulić, M. Lacić, Z. Kusić, J. Lukač, S. Rončević, N. Daković

Department of Nuclear Medicine and Oncology, University Hospital "Sestre Milosrdnice", Zagreb, Croatia

RADIOIMMUNOSCINTIGRAPHY WITH Tc-LABELED ANTI-CEA. BW 431/26 ANTIBODY IN FOLLOW UP OF COLORECTAL CARCINOMA

Some recent clinical studies report excellent results, using a Tc-99m. labeled anti-CEA monoclonal antibody (MAb), in postsurgical evaluation of lesions of colorectal carcinoma (CC). In the present study our first experiences with murine MAb BW 431/2 (Behringwerke AG) have been described. We investigated postsurgically thirteen patients (pts) with high clinical suspicion of recurrent or metastatic $\mathrm{CC}$. Each patient received a single intravenous infusion of $0,65-1,0 \mathrm{mg}$ anti-CEA BW 431/26 MAb labeled with $814-1110 \mathrm{MBq}$ of Tc-99m. Whole-body and SPECT scans were acquired at 4-6 hours and 20-24 hours post infusion. Radioimmunoscintigraphic (RIS) findings were verified surgically, and/or clinically. On a per patient basis, RIS demonstrated an overall sensitivity of $83 \%$, specificity of $100 \%$ and accuracy of $85 \%$. RIS findings were positive (scintigraphically "hot" appearance) in 23 (56\%) lesions, and remaining $18(44 \%)$ lesions were "cold" or scintigraphically indeterminate.

\begin{tabular}{|l|c|c|c|}
\hline & Sensitivity & Specificity & Accuracy \\
\hline Liver & $78 \%$ & $100 \%$ & $85 \%$ \\
\hline Abdomen (extrahepatic) & $67 \%$ & $87 \%$ & $85 \%$ \\
\hline Pelvis & $83 \%$ & $100 \%$ & $92 \%$ \\
\hline
\end{tabular}

In $2 \mathrm{pts}$ with pulmonary metastases, $1 \mathrm{pt}$ with kidney involvement and 1 pt with recurrence of CC RIS findings were negative. Whole-body scan imaged $63 \%$ of described lesions, and $20-24$ hours SPECT showed more lesions than 4-6 hours one (ratio $1,2: 1,0$ ). No correlation was found between CEA serum levels and RIS results. In conclusion our results suggest that RIS is a useful procedure in the follow up of pts with CC, especially in situation when the results of other diagnostic modalities are equivocal. SPECT imaging at 20-24 hours seems to be mandatory.

528

S. Dresel ${ }^{1}$, R. Zachoval2 ${ }^{2}$ C.-M. Kirsch ${ }^{1}$, K.Hahn ${ }^{1}$

${ }^{1}$ Department of Nuclear Medicine, ${ }^{2}$ Department of Internal Medicine Klinikum Grosshadern, University of Munich

\section{IMMUNOSCINTIGRAPHY WITH AFP-MONOCLONAL- ANTIBODY - CLINICAL PRELIMINARY RESULTS}

In more than $80 \%$ of the cases, primary hepatocellular carcinoma (HCC) is associated with elevated levels of serum alpha-fetoprotein (AFP). Using specific radiolabeled antibodies as diagnostic imaging agents, AFP-antibodies may be useful diagnosing HCC.

Eighteen patients suffering from ensured or highly suspected HCC were examined. In 12 of these markedly elevated serum AFP was evident. The radiolabeled ( $900 \mathrm{MBq}$ Tc-99m) Fab-fragment (1mg), supplied as an instant labeling kit, was applied by infusion over 30min. The planar and SPECT imaging was performed $6 \mathrm{~h}$ and $24 \mathrm{~h}$ p.i.

In 12 patients there was a specific binding of the antibody to the tumor, seen at the rim of the tumor due to the physiological internal pattern of $\mathrm{HCC}$ compared to normal liver tissue. In all these cases the result could be positively correlated with the findings of computed tomography (CT) and biopsy. In four cases presenting elevated serum AFP, no specific antibody binding was found and no malignant lesion was evident in CT or biopsy. One of these showed an atypical increased uptake in the 6h-imaging. Biopsy documented an area of highly vascularized tissue related to inflammatory changes due to hepatitis-C. One lesion in another patient with a normal level of AFP did not show specific binding of the antibody. In this case, the suspected lesion could not be ensured as HCC by biopsy. In one case metastatic disease in the lower abdomen was evident. One case showing two small lesions was diagnosed false negative.

The preliminary experience in imaging of HCC using radiolabeled anti-AFP-antibody fragments clearly demonstrates the high potential of this method to diagnose HCC. With respect to the clinical impact, possible applications are recurrent tumors, non resectable tumors and the detection of metastatic disease. 
Sunday, 21 August - Wednesday, 24 August 1994

\section{9}

1. Balogh, L. Duffeko, Z. Nagy*, A. Mayer, J. Csengódy, A. Bgjtay, K. Bor*, J. Csikely

Uzsoki Hospital, Semmelweis Medical University", Posteraduate Medical School*, Budapest

WHAT IS THE DIAGNOSTIC VALUE OF MMUNOSCINTIGRAPHY WTH WN-111 ONCOSCINT CRI03 IN PRIMARY COLORECTAL CANCER AND RECURRENI DISEASE ?

Immunoscintigraphy (IMS) was perforned in patients (pts) with colorectal cancer in cases of known primary or suspected recurrence of disease.

To study the diagnostic usefulness of OncoScint CR103 (OS) we examined three groups of pts $(\mathrm{G}-1,2,3)$. In $\mathrm{G}-1$ nine pts were examined with known prinary colorectal cancer, imnediately preoperatively detecting the extension of disease. G-2 contained seven pts with suspected recurrence of disease, one to four years after operation. In G-3 five pts were examined with inoperable rectum carcinoma after irradiation. In all cases (21 pts) the histological result was known. We measured the serum level of CA 19-9, CEA and TAG-72. Planar images were obtained at 24, 72., 120. hours post OS injection from the whole body. We analysed the pictures visually and quantitatively the ratio of target-background (T/BO) r. In G-1 we measured after operation OS uptake of removed tumors, normal tissues and removed lymph nodes (in $\mathrm{kBq} / \mathrm{q}$ ), and determined the ratio of $\mathrm{OS}$ uptake of carcinoma and normal tissue (CN). The content of CA 19-9, CEA and TAG-72 of these different tissues were measured as well.

Our results compared with the surgical, histological, CT and UH findings were as follows: 1.) OS uptake of colorectal cancer was increased in 18/21 pts. False negative cases (3 pts) were in $\mathrm{G}-1$ two, because of low level of OS uptake of tumor (C/N of these pts were $1.5,1.8$ and in $\mathrm{MS}$ positive cases $\mathrm{C} N \mathrm{~N}$ were 3.4-18) and in $\mathrm{G}-2$ one, because of small size of tumor. (Sensitivity.85\%). 2.) Metastases were observed with IMS in four cases in spite of negative results of $\mathrm{CT}$ and/or $\mathrm{UH}$. We could not detect lymph node metastases in the pelvis proved by $\mathrm{CT}$ and $\mathrm{UH}$ in $3 / 6$ cases. (Sensitivity: $77 \%$ ). 3.) Inflanumation (was after irradiation and in colostoma) caused increased uptake of $O S$ in six cases, but we could distinguish tumor from inflammation analysing the value of T/BG during the series of images during the whole examination /comparing the 24.72.,120. hours pictures/. (Specificity: 100\%). 4.) Serum level of CA 19-9, CEA and TAG-72 did not correlate with the positivity of IMS, but mild to severe elevation of one or more nimor markers were found in 15 pts of 18 IMS positive cases.

In conclusion OS-IMS seems to be a sersitive and specific method for detecting the primary colorectal cancer and recurrence of disease.

\section{0}

CE Neal, DL Johnson, LC Meis, and VL Cornwell.

Department of Radiology, Southern Illinois University School of Medicine, Springfield, IL, USA.

\section{QUANTITATIVE ASSESSMENT OF In-111 SATUMOMAB PENDETIDE IMMUNOSCINTIGRAPHY IN THE DLAGNOSIS OF INTRAABDOMINAL CARCINOMATOSIS.}

The diagnosis of diffuse intraperitoneal spread of ovarian or colorectal carcinoma (carcinomatosis) is often difficult on the basis of visual analysis of immunoscintigraphic images. The purpose of this study is to assess the utility of quantitative analysis of planar In-111 satumomab pendetide images in the diagnosis of carcinomatosis.

Quantitative assessment of monoclonal antibody scans obtained two to four days post injection was performed on 29 studies of patients with ovarian or colorectal carcinoma. Regions of interest $5 \times 5 \mathrm{~cm}$ were made over the right and left sides of the pelvis between the iliac vascular bundle and the iliac crest. The counts from these two regions were averaged and an uptake ratio was created by dividing these averaged counts by the counts obtained from a similar sized background region of interest over the femoral vascular bundle. These ratios were then correlated with surgical-histologic findings.

In 22 scans on patients who did not have carcinomatosis nor focal tumor sites in either lower quadrant, there was a mean uptake ratio of 1.23 (range $0.78-1.76, S . D .=0.308$ ). This compares with a mean ratio of 2.36 (range $1.70-3.07$, S.D.= 0.585 ) in seven patients with confirmed carcinomatosis. The difference between these two means was statistically significant at $\mathrm{p}<0.001$. Using the cut-off ratio of 1.80 , all 22 scans without carcinomatosis were correctly identified. Only one of the seven cases of carcinomatosis fell below an uptake ratio of 1.80 .

Based upon this sample of patients, quantitative analysis appears to hold promise as a useful adjunct to visual interpretation of immunoscintigraphic images in the diagnosis of carcinomatosis.
531

T. Togawa, N, Yui, F, Kinoshita,

M. Yanagisawa, F. Shimada, K. Omura

Divisions of Nuclear Medicine and Head \&

Neck Surgery, Chiba Cancer Center Hospital,

Chiba 260 , Japan

VISUALIZATION OF NASOPHARYNGEAL CARCINOMA BY T1-201 SPECT.

T1-201 SPECT was performed on 19 patients, 13 males and 6 females aged 28 to 73 yrs (mean 51), with nasopharyngeal carcinoma (NPC) histologically proven to evaluate whether or not T1-201 SPECT was useful and reliable for assessing the tumor viability of NPC. Thirty seven T1-201 SPECT studies were performed for the follow-up study after radiation therapy in 9 of 19 patients and for the as sessment of tumor extent before treatment in the other 10 patients. Data acquisition was started $5 \mathrm{~min}$ after the intravenous injec tion of $111 \mathrm{MBq}$ of T1-201 chloride using three-head rotating gama camera(Toshiba GCA 9300A) equipped with fan-beam collimators. T1-201 clearly accumulated to the tumor in 10 patients before radiation treatment and increased Tl-201 uptake by the lesion disappeared after the treatment. Three of $9 \mathrm{pa}^{-}$ tients who were followed-up after radiother apy developed apparent local recurrence and T1-201 SPECT could definitely visualize these recurrent lesions. T1-201 SPECT was very useful for detecting local recurrent tumor. High resolution SPECT system with the use of Tl-201 chloride is a new reliable and accurate diagnostic tool for assessing the tumor viability of NPC.

\section{2}

M. Pérez páramo, M.J. Tabuenca, J. Ramos, J. Mulero*, J.L. Andreu*, C. Sanjuan and J. Ortiz Berrocal

Services of Nuclear Medicine and *Rheumatology. Clinica puerta de Hierro. 28035 Madrid, Spain.

ROLE OF METHOXYISOBUTYLISONITRILE-99TC BONE SCAN IN ESTABLISHING MALIGNANT NATURE OF TUMOR-LIKE LESIONS

Background: Establishing the malignant nature of a bone lesion by imaging procedures can be difficult. The aim of our study was to investigate the role of methoxyisobutylisonitrile$99 \mathrm{TC}$ (MIBI) bone scan in diagnosing the malignant nature of tumor-like bone lesions.

Methods: Patients with plain radiographs or computed tomography suggesting bone tumor were enrolled. Every patient was studied by conventional bone scan with MDP-99TC and MIBI-99TC. only patients with firm pathologic diagnosis were evaluated. Statistical significance was established by chi-square test with the Yate's correction.

Results: Sixty-six patients were enrolled. In 55 patients, it was possible to establish a pathologic diagnosis of the bone lesion. MDP99TC scan was nonspecific in establishing the malignant nature of the lesion since most of the patients had a positive scan. Twenty-two out of 29 patients with pathologic diagnosis of a malignant bone lesion had a positive MIBI$99 \mathrm{TC}$ scan. In sharp contrast, 23 out of $26 \mathrm{pa}-$ tients with pathologic diagnosis of benign bone lesion had a negative MIBI-99Tc scan ( $p<0.001)$. The sensitivity, specificity and positive predictive value of MIBI scan for malignant bone tumor were $76 \%, 88 \%$ and $86 \%$, respectively.

Conclusions: MIBI-99TC bone scan is a useful noninvasive test for the detection of the malignant nature of a tumor-like bone lesion. 


\section{3}

J.W. Braams, J. Pruim, P.G.J. Nikkels, N.J.M. Freling, J.L.N Roodenburg, A. Vermey, W. Vaalburg.

National Research PET-Centre, Dept. of Oro- and Maxillofacial Surgery, University Hospital, Groningen, The Netherlands.

DETECTION OF LYMPH NODE METASTASES IN SOUAMOUS CELL HEAD AND NECK CANCER WITH L-1-[1" $]$ TYROSINE PET.

An imaging modality able to detect lymph node (micro)metastases of head and neck cancer could be important for staging purposes. In this study we investigated the applicability of L-1- $\left[{ }^{11} \mathrm{C}\right]$-tyrosine (TYR) for the detection of lymph node involvement in squamous cell carcinoma of the oral cavity.

Seven patients with histologically verified $\mathrm{N}_{0}-\mathrm{N}_{2 C}$ squamous cell carcinoma were studied. Patients received $370 \mathrm{MBq}$ TYR. A total length of $21.6 \mathrm{~cm}$ was studied via 2 static emission scans (Siemens ECAT 951/31). Of 6 patients preoperative $T_{1}$ - and $T_{2}$-weighted MRIimages were available. Shortly after PET neck dissection followed. Comparisons were made between PET, MRI, and histopathology.

In the 7 resection specimens of the PET-studied patients a total of 130 lymph nodes were present. In 11 of these nodes metastatic disease was found. All histologically proven metastatic lymph nodes were visualized with PET. In addition, 10 other lymph nodes were visualized, being either normal or reactive on histopathology. The smallest lymph node detected was $0.5 \mathrm{~cm}$ in diameter. In all patients the submandibular and parotid glands showed high uptake of TYR. Preliminary sensitivity, specificity, and accuracy can be calculated at $100 \%, 93 \%$, and $94 \%$, respectively. In comparison, in the resection specimens of the 6 MRI-studied patients 108 lymph nodes were present. Six nodes showed metastatic disease. On MRI, 4 nodes gave a true-positive and 4 nodes gave a false-positive signal. Consequently, the figures for MRI are $67 \%, 96 \%$, and $94 \%$, respectively.

In conclusion: in the evaluation of lymph node involvement of squamous cell head and neck cancer TYR-PET can be a valid diagnostic tool. Our figures are favourable to those reported for FDG-PET.

534

C. Laubenbacher, C. Wagner-Manslau, N. Avril, D. Saumweber*, R. J. Kau*, I. Wolf, M. Herz, C. Kruschke, M. Schwaiger

Nuklearmedizinische und *HNO - Klinik und Poliklinik der Technischen Universität München, Munich, Germany

\section{F-18 FDG PET, MRI, AND ENDOSCOPY FOR PREOPERATIVE STAGING OF SQUAMOUS CELL CARCINOMAS (SCC) OF THE HEAD AND NECK}

Accurate preoperative staging is necessary for surgical treatment planning in patients with SCC of the head and neck. We compared metabolic imaging (F-18 FDG PET), morphological evaluation (MRI) and endoscopy with postoperative, histological tissue characterization.

Tumor extent was evaluated by all three modalities. Lymphnode involvement was only assessed by PET and MRI, After injection of $370 \mathrm{MBq} F-18$ FDG emission scans of the head and neck region were pertormed. Regional FDG uptake was evaluated visually without knowledge of other clinical results. In addition regional FDG uptake was quantified by relating F-18 tissue activity to injected dose (SUV). Metastatic lymphnode involvement was defined by FDG uptake or by MRI diameter greater than $1.5 \mathrm{~cm}$.

All primany tumors were clearly visualized by PET (SUV: $5.4 \pm 3.1$ ) and by MRI. Howewer, due to high FDG-Uptake in normal mucosa tumor extent was overestimated in 6 of 8 patients. Endoscopy and MRI yielded better T-staging in 5 , and 4 patients respectively. SUV in metastatic lymphnodes was 5.7 \pm 2.3 . F-18 FDG- PET correctly identified $\mathrm{N}$-Stage and lateralization in 7 out of 8 pts. One false negative lymphnode classification was due to a small, contralateral lymphnode (diameter: $0.9 \mathrm{~cm}$ ) with only partial metastatic infiltration. MRI staged lymphnode-involvement correctly only in 5 pts, while lateralization of In-metastases by MRI was correct in 6 pts.

These results suggest, that endoscopy and MRI provide better TStaging of SCC of head and neck as compared to PET. Howewer, PET yields more accurate determination of lymphnode involvement PET may be indicated in patients considered for surgical therapy.

\section{5}

S.J. Wang, C.Y. Hsu, W.Y. Lin, C.H. Kao, J.S. Jan and S.H. Yeh

Taichung Veterans General Hospital and National Yang-Ming Medical College, Taiwan

\section{COMPARISON OF TL AND MIBI SPECT STUDY IN NASOPHARYNGEAL} CARCINOMA

Among the Chinese of Taiwan, nasopharyngeal carcinoma (NPC) is the most common cancer in males and the third most cancer in females. The number of radiopharmaceuticals proposed for tumor imaging is in the hundreds, but only few have achieved widespread acceptance in clinical use. Both Thallium-201 (TL) and TC-99m methoxyisobutylisonitrile (MIBI) uptake in NPC have been reported, yet no comparison study of these two agents using SPECT irages has been performed for NPC. The aim of the present study was to compare the relative effectiveness of MIBI and TL SPECT for detecting $\mathrm{NPC}$.

A total of thirty two NPC patients ( 26 males, 6 females, age range 28-69 years) histologically confirmed by biopsy were studied. The dosage was $740 \mathrm{MBa}$ for MIBI, and $74 \mathrm{MBO}$ for TL. For both agents, SPECT images were obtained 10 to 20 minutes after intravenous injection. There was a period of 2 to 7 days between MIBI and TL images. All imaging procedures were performed using an Elscint APEX ECT 609R gamma camera and computer system. The image was interpreted as positive if there was significant uptake of MIBI (or $\mathrm{TL}$ ) in the nasopharynx. As a result, 26 (81\%) NPC patients showed TL tumor uptake while 24 (75\%) showed MIBI tumor uptake.

Our preliminary study suggests that 1 , both MIBI and TL SPECT imagings can be helpful in detecting NPC: 2 . the sensitivity of TL SPECT imaging in detecting NPC is slight $7 y$ higher than MIBI SPECT imaging.

536

Grünert B.2, Heissler E. 1, Barzen G. 2, Fritsche L.2 Cordes M.2 Felix R. 2 Depts. of Nuclear Medicine2, Maxillofacial Surgery 1 , University Clinic Rudolf Virchow Free University of Berlin, Germany

RADIOIMMUNOSCINTIGRAPHY OF SQUAMOUS CELL CARCINOMA IN THE MAXILLOFACIAL SURGERY FIRST RESULTS

Radioimmunoscintigraphy (RIS) is a new tool to assist diagnosis of squamous cell carcinoma in the head and neck region. Recently for this reason a new monoclonal antivody which specially directed against SCC became available.

Ten patients with histologically proven SCC were included into the study. Ages ranged from 51 to 73 years. Tatient received $1 \mathrm{mg}$ SQ 174 radiolabelled with 1000 to $1600 \mathrm{MBq}$ $99 \mathrm{mTc}$. Data acquisition was performed in planar and SPECT technique $3-4$ and $22-25$ hrs. after application.

RIS was able to detect 8 of the ten primary tumours. One patient had histopathologically proven nodal desease. This lymph node was seen in RIS. No false positive results were seen in RIS wheras Sonography, CT and MRI showed several false positive results. 
537

C. Laubenbacher, C. Wagner-Manslau, D. Saumweber", M. Herz, C. Kruschke, I. Wolf, R. J. Kau*, M. Schwaiger

Nuklearmedizinische und *HNO - Klinik und Poliklinik der Technischen Universität München, Munich, Germany

\section{TIME-DEPENDENCY OF FDG-UPTAKE IN TUMOURS AND LYMPHNODE-METASTASES OF SQUAMOUS CELL CARCINOMAS (SCC) OF THE HEAD AND NECK.}

The value of the SUV (= standardized uptake value) of F-18 FDG depends on the timepoint of the determination. We compared the time course of the FDG-Uptake in lymphnode metastases and in primaries.

8 men $(51.9 \pm 7.4$ a) with SCC of the head and neck were investigated at a SIEMENS ECAT 951 R PET scanner. In the tumour region a transmission scan was done for $20 \mathrm{~min}$. Immediately after i.v. injection of $370 \mathrm{MBq}$ F-18 FDG dynamic emission tomography up to $1 \mathrm{~h}$ p.i. was started $(6 \times 5 \mathrm{~min}, 3 \times 10 \mathrm{~min})$. PET images were generated by filtered backprojection to an image matrix of $128 \times 128$. Furthermore, a correction for attenuation and scatter was done. The spatia resolution was $6.2 \mathrm{~mm}$. ROI's were drawn over at least $2 \mathrm{~cm}$ large tumours or lymphnode-metastases and SUV was calculated for each time frame (activity conc. (nCi/g)/ inj. activity (nCi)/body weight (g) ). The time course of the FDG-

Uptake in primaries and lymphnode metastases showed no significant difference. SUV was increasing up to $60 \mathrm{~min}$ p.i. without a plateau phase.

There is no marked difference in the FDG-Uptake in primaries and lymph node metastases of squamous cell carcinomas of the head and neck. Because there was still no maximum reached up to 60 min p.i., further studies with longer examination time have to determine the optimal imaging determin.

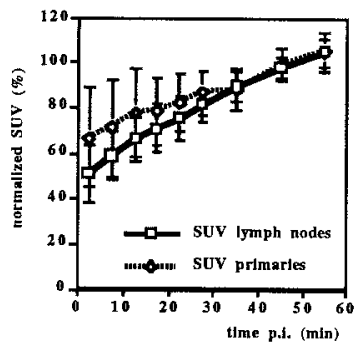

\section{9}

L.Dominguez-Gadea, LM Martin-Curto, C.Bas, MD Rueda, A.Crespo

Dept of Nuclear Medicine, Hospital Ramon Y Cajal. Madrid.

ROLE OF BONE MARROW IMMUNOSCINTIGRAPHY IN CANCER PATIENTS WITH NONDIAGNOSTIC BONE SCINTIGRAPHY

A certain number of cancer patients studied to rule out bone metastases have nondiagnostic bone scintigraphy (BS). We have assessed the clinical role of bone marrow $99 \mathrm{~m}$ Tc-Antigranulocyte monoclonal antibody scintigraphy (AGS) as complement to BS in 32 oncologic patients $(13$ breast, 5 lung, 4 prostate, 2 renal, 2 bladder, 6 other) whose BS are not definitive for bone metastases (one a two lesion). BS and AGS were obtained within the same week using the same equipement.

Bone metastases were diagnosed in 11 patients. The remaining 21 patients have benign bone lesions. In 7 patients antibody uptake was homogeneous ( 1 of them had bone metastases). In 15 patient the AGS shown focal defect coincident with the BS lesion (A cases were metastases). In two patients AGS shown a remarkably decreased uptake in the osseous structure previously irradiated. In 8 patients AGS detected additional lesions that appeared normal in the BS (six of them had bone metastases).

we conclude that, in patients with nondiagnostic BS, AGS provide valuable additional information indicative to metastases when multifocal defects or diffuse decreased uptake is shown. Irradiated territories or solitary cold lesion need additional diagnostic investigation.

540

Castellani MR Seregni E, Maffioli L, Gasparini M, Buraggi GL. Nuclear Medicine Dept. - Istituto Nazionale Tumori - Milano - Italia

RADIOIMMUNOSCINTIGRAPHY OF BONE MARROW IN LYMPHOMA Bone marrow (BM) biopsy is the main procedure to detect BM infiltration in malignant lymphomas. However, it is an invasive procedure which can be performed only at few skeletal sites, with the risk of false negative findings. Radioimmunoscintigraphy (RIS) with Tc-99m labeled antigranulocyte MAb BW250/183 seems to be efficacy in detecting $\mathrm{BM}$ or bone localization in malignant lymphomas, but its specificity is still to be determined.

The aim of the present study was to evaluate the usefulness of RIS with MAb BW250/183 labeled with Tc-99m (555-740 MBq), in malignant lymphomas, comparing the results with BM biopsy.

RIS was judged positive in case of presence of cold areas. The results in 12 patients are summarised in Table 1. In patients with documented BM invasion RIS was positive in $8 / 8$ patients. In $3 / 4$ cases with no documented BM invasion RIS was negative. In a patient heavily pretreated RIS showed several cold areas although BM negativity. In untreated patients, RIS showed a very good correlation with clinical status. In pre-treated patients the usefulness of RIS is limited because it can be positive in case of both BM infiltration and post therapy fibrosis .

Tab. 1 - Main results of RIS with BW250/183 in lymphomas.

\begin{tabular}{|l|c|c|c|c|c|c|c|c|c|}
\hline Diagnosis & Pt & \multicolumn{2}{|c|}{ Aim } & \multicolumn{3}{|c|}{ BM } & \multicolumn{4}{c|}{ RIS results } \\
\hline & nr. & stag & restag & + & - & TP & TN & FP & FN \\
\hline Hodgkin & 9 & 1 & 8 & 6 & 3 & 6 & 2 & 1 & 0 \\
\hline nonHodgkin & 3 & 1 & 2 & 2 & 1 & 2 & 1 & 0 & 0 \\
\hline TOTAL & 12 & 2 & 10 & 8 & 4 & 8 & 3 & 1 & 0 \\
\hline
\end{tabular}




\section{1}

Y.Duman, Z.Burak, K.Kumanlioğlu, Z.Balkan, U̇.Yararbas, D.Sabah, G. Yicetirk

Depts. of Nuclear medecine, Orthopaedics.Ege Univ. Izmir/TURKEY

CLTICAL VAIUE OF 99m-TC GLUTATHTONE (GSH) IN DTAGNOSIS OF PRTMARY BONE TUMORS

The purpose of this study was to evaluate the feasibility of 99m-Tc in lesion detection, differentiation of benign or nalignant nature and past therapeuthic follow-up of primary bone tumors.

A total of 27 patients were studied.Malignant bone tumors were composed of osteosancomas (5), Ewing's sarcomas (6) and Chondrosearcomas (4).6 individuals (4 osteosancoma and 2 Ewing's sarcoma) were inaged postoperativly in onder to investigate reccurrences. 6 patients with pathologically proven benign bone lesions were also studied. The patients were IV injected $555 \mathrm{MBq} 99 \mathrm{~m}-\mathrm{Tc}$ GSH.3,6 and $24 \mathrm{hrs}$.after injection planar scintigrams were obtained. Increased activity accumulation in comparison with contralateral extrenity was accepted as positive.Quantitative evaluations were also applied by ROIs drawn over lesions and contralateral site $(\mathrm{L} / \mathrm{C})$. All patients were also examined by 3 phase bone scintigraphy, $\mathrm{x}-\mathrm{ray}, \mathrm{CT}$ and MRT.Pathological examination determined final diagnosis.13 of 15 primary bone tumors demostrated positive GSH accumulation. Post therapeuthic ineging of 2 of 4 asteasarcomas and 1 of 2 patients with Ewing's sarcomas showed increased uptake pathologically consistent with local recurrence. All benign bone lesions demantrated abcent $99 \mathrm{~m}$-Tc GSH accinilation except 1 asteoblastoma. The mean L/C uptake ratios of prinary bone tumors and benign bone lesions were $2,4+0,3$ and $1,01+0,3$ respectively $(\mathrm{p} 0,05)$. The sensitivity and the specificity of $99 \mathrm{~m}-\mathrm{TC} \mathrm{GSH}$ in differantiations of malignant tumors from benign bone lesions were $87 \%$ and $92 \%$ respectively.

In conclusion, in this preliminary study $99 n-T \mathrm{C}$ GSH was found as a promising agent in the evaluation of primary bone tumors, especially in discrimination from benign lesions and in prediction of postoperative reccurrences.

\section{2}

U. Haberkorn, I. Morr, F. Oberdorfer, M.E. Bellemann, J. Blatter, J.H. Clorius, and G. van Kaick.

German Cancer Research Center (dkfz), Heidelberg, Germany.

FDG UPTAKE IN MALIGNANT CELLS: INFLUENCE OF METHODS AND EFFECTS OF CHEMOTHERAPY.

Rat prostate adenocarcinoma cells were incubated for 10 or 60 minutes in media with different glucose content. Furthermore, the cells were treated for 4 hours with different doses of gemcitabine and the FDG uptake was measured immediately and 4 hours after the end of treatment. The FDG transport was determined with a zero-trans assay and the mRNA content of the glucose transporter type 1 (GLUT1) and the hexokinase (Hk). After 60 minutes incubation a decrease in FDG uptake was found with increasing cell number in all media. A shorter incubation time of 10 minutes yielded more stable uptake data. The glucose content decreased with increasing cell number and incubation time, indicating that the glucose-to-FDG ratio is not constant in assays using glucosecontaining media. According to these data, we suggest an uptake procedure in glucose-free medium with an end concentration of 0.1 mM FDG or a zero-trans assay to determine the maximum velocity $\left(\mathrm{V}_{\max }\right)$ and the affinity $\left(\mathrm{K}_{\mathrm{m}}\right)$ of the transport system. Treatment with gemcitabine caused a time and dose dependent increase in FDG uptake. Incubation experiments with ${ }^{3} \mathrm{H}$-inulin revealed that the changes were not caused by unspecific membrane alterations. The $\mathrm{K}_{\mathrm{m}}$ of the transport system remained unchanged, whereas $V_{\text {max }}$ increased. However, the mRNA content for GLUT1 and Hk was unchanged. These data indicate that the number of glucose carriers at the plasma membrane increased after treatment with gemcitabine, probably due to redistribution phenomena from intracellular compartments to the plasma membrane. These effects may be part of cellular stress reactions after exposure to chemotherapy with gemcitabine.
543

M.N. Hosono, M.Hosono, P.O. Zamora*, S. Guhlke, H. Bender, H.J. Biersack, Y. Onoyama\#, H. Ochi\#. $\mathrm{J}$. Konishis. Nuclear Medicine, University of Bonn, Bonn, Germany, *Rhomed Inc., NM, USA, \#Osaka City University, \$kyoto University, Japan

$\begin{array}{lllll}\text { RADIOIMMUNOTARGETING } & \text { WITH } & \text { Re-188 } & \text { LABELED } \\ \text { MONOCLONAL } & \text { ANTIBODY } & \begin{array}{c}\text { IN } \\ \text { MICE }\end{array} & \text { BEARING } \\ \text { COLORECTAL } & \text { CARCINOMA } & \text { XENOGRAFTS } & \end{array}$

Re-188, which can be produced from a generator system using $\mathrm{W}-188$, has been considered suitable for the radioimmunotherapy. The purpose of this study was to determine the efficiency of Re-188 labeled monoclonal antibody (MAb) for tumortargeting. MAb B72.3, a murine IgG1 reactive with human carcinoma antigen TAG-72, was labeled with Re-188 by a direct conjugation using tin as a reduction agent. The MAb was also labeled with I125 by Chloramine-T method. Re-188 and I-125 B72.3 was injected i.v. into groups of athymic mice $(n=4)$ bearing Lovo tumors (colorectal carcinoma cell line). Biodistribution was determined at $30 \mathrm{~min}, 6 \mathrm{hr}, 1,2,3$ and 4 days. The labeling efficiency of Re-188 B72.3 was more than $80 \%$. Immunoreactive fraction of Re-188 B72.3 $(64.78)$ was similar to that of I-125 B72.3 $(72.48)$. At 2 days, the accumulations of Re-188 B72.3 in the blood, liver, kidney and tumor were $2.5 \pm 0.6,1.7 \pm 0.5,2.9 \pm 0.7$ and $3.5 \pm 0.9$ \% $\mathrm{ID} / \mathrm{g}$, respectively, while I-125 B72.3 showed uptake of $8.0 \pm 1.9,1.6 \pm 0.5,2.6 \pm 0.5$ and $7.2 \pm 2.0 \quad 8 \mathrm{ID} / \mathrm{g}$ in those organs. Tumor-to-blood ratios of Re-188 MAb were $1.47 \pm 0.54$ and $2.04 \pm 0.44$ at 2 and 4 days, in comparison with I-125 MAb showing $0.92 \pm 0.28$ and $1.05 \pm 0.28$. Re-188 B72.3 cleared from the blood faster than I-125 B72.3, resulting in higher tumor-to-blood ratios. The labeling technique was simple and efficient. Re-188 B72.3 seemed to be potent for radioimmunotargeting.

\section{4}

K.H. Bohuslavizki, D. Lennert, H. Wolf, W. Brenner, J.-U. Eberhardt, M. Schramm, M. Clausen and E. Henze

Department of Nuclear Medicine, Christian-Albrechts-University, Kiel, Germany

\section{UPTAKE OF IODO-DOXORUBICIN LABELLED WITH I-123 IN BOTH ANTHRACYCLINE RESISTANT AND SENSITIVE GASTRIC CARCINOMA CELLS.}

Drug resistance is a well-known disadvantage in chemotherapeutical cancer treatment. This has not been verified for the anthracycline derivate $4^{\prime}$-deoxy-4'-iododoxorubicin (IDOX). Therefore, this study was designed to evaluate the uptake of radiolabelled IDOX in carcinoma cells with clearly defined drug response, i.e. anthracyline resistant (ARC) and sensitive (ASC).

IDOX was radiolabelled with I-123 in conventional manner by the Iodogen method yielding radiochemical purity of $>98 \% .10 \mathrm{kBq}$ I-123labelled IDOX and $20 \mathrm{kBq}$ Tl-201 were added to each culture medium $(n=100)$, respectively. Cellular uptake was stopped by removing the medium at different incubation times and cooling. After washing, measuring cellular uptake in a well counter, and correcting for physical decay, uptake of I-123-IDOX was calculated in anthracycline resistant (D 257/85 DR) and sensitive (D 257/85 P) human gastric carcinoma cells. Results are given as percental uptake of the activity applied and as well as ratios to the uptake of T1-201

\begin{tabular}{||l|r|r|r|r|r|r|r|r|r|r||}
\hline \hline & \multicolumn{4}{|c||}{ I-123-IDOX } & \multicolumn{5}{|c|}{ Tl-201 } \\
\hline \hline [min] & 1 & 10 & 60 & 180 & 300 & 1 & 10 & 60 & 180 & 300 \\
\hline ASC & .19 & .33 & .76 & .96 & .85 & .12 & .20 & .24 & .19 & .20 \\
\hline ARC & .28 & .32 & .25 & .37 & .24 & .12 & .16 & .17 & .32 & .21 \\
\hline
\end{tabular}

All results in this table are given as medians ( $\mathrm{n}=5$ per incubation time) of percental uptake of the activity applied. Observe, in ASC I-123IDOX showed approximately 5.3 times higher uptake than T1-201. In contrast, in ARC I-123-IDOX and T1-201 showed similar values.

In conclusion, uptake of IDOX could be shown to be higher in sensitive than in resistant gastric carcinoma cells. The kinetics in ARC suggest a diminished uptake rather than an active transport out of the cells. Thus, IDOX behaves similarly to anthracylines known. 
545

K. Seybold, M. Trinkler, L.D. Frey, J.Th. Locher

Department of Nuclear Medicine, Kantonsspital Aarau CH-5001 Aarau, Switzerland

LONG-TERM HAMA-FOLLOW UP AFTER IMMUNOSCINTIGRAPHY USING ANTITUMOURS AND ANTIGRANULOCYTES MAB IN 240 PATIENTS.

Due to the increase of immunoscintigraphy (IS) in infections, tumours and their metastases, the problem of HAMA (human antimouse antibodies) has become more relevant, because HAMA may influence the diagnostic outcome of IS. So the aim of this study was to evaluate the clinical relevance of HAMA in IS using different MAbs.

In about 245 patients (mean age: 57 yrs; range: $20-89$ ) 265 HAMA serum courses were intensively documented up to eight weeks after injection of various and differently labeled monoclonal antibodies: In 197 patients (215 HAMA courses) IS of infections was performed, partially after repeated or simultaneous injections of antigranulocytes MAb [I-123-Mab47 (0.15 mg), Tc-99m-Mab47 (0.4 mg); Tc-99mBW250/183 $(0.4 \mathrm{mg})$ ]. 21 patients (23 courses) with colorectal cancer received anti-CEA MAb [Tc-99m-BW431/26 (1 mg)], and in 27 patients monoclonal anti-melanoma $F\left(a^{\prime}\right)_{2}$ fragments ('Tc-99m-TECNEMAB-K$1(0.35 \mathrm{mg})]$ were administered. HAMA serum titers were determined by an ELISA-test system.

Pathologically elevated HAMA serum levels were seen in 40/265 (15\%) courses of all patients examined [antigranulocytes MAb: 17/165 (11\%) after first, and $16 / 50(32 \%)$ after repeated injection; anti-CEA MAb: $4 / 21(19 \%)$ after first, $2 / 2$ after repeated injection; after anti-melanoma $\mathrm{F}\left(\mathrm{ab}^{\prime}\right)_{2}$ : no pathological response] mostly two and four weeks after application and a nomalization eight weeks thereafter in most of the cases. Seven patients of all (about $3 \%$ ) with strongly high HAMA titers after repeated MAb injections showed an altered biodistribution of Mabs and reduced imaging quality, but no adverse reactions.

The results show that in IS the potential human immunoreaction (about $11 \%$ after first injection) depends on the total MAb protein amount applied, especially after repeated injections. The maximum of the HAMA response, especially in IS of infections, appears two weeks after injection with a mostly complete regression after 8 weeks. So in routine diagnostic work after first injection of MAb, the problem of HAMA is negligible regarding potential disorders of the biodistribution of $\mathrm{MAb}$ or of the imaging quality of IS as well as adverse reactions.

\section{6}

S.Cammilleri*, B.Perdereau*, F.Brixy*, B. Benyahia, J.Y.Pierga*,C.Chypre**,H.Magdelenat* .

*Institut Curie - **Cis Bio Erance

BIODISTRIBUTION AND IMAGING STUDIES OF RADIOIODINE 125I TYRAMINE OLIGONUCLEOTIDE (22 MERS) IN NUDE MICE BEARING HUMAN MAMMARY TUMOR.

oligonucleotides represent a class of chemicals which pharmaceutical potential has recently been recognized. Key to developing the use of these chemicals as drugs, is the understanding of their biodistribution and metabolic properties. Material and method : Human breast cancer cells (MCF7) in co-culture with human fibroblasts were transplanted in female nude mice ( 6 weeks later, tumor weight : $0,3 \pm 0,1 \mathrm{~g})$. A 22 mer phosphodiester oligonucleotide, with a tyramine group in $5^{\prime}$ (TON) was ${ }^{125}$ I labelled (chloramine $T$ ) and intratumorally injected $(1,036 \pm 0,074 \mathrm{MBq} / \geq 16 \mu \mathrm{g}$ TON). Image recordings were performed by planar scintigraphy. Target organs were removed, weighed, and radioactivity was measured by gamma counter after imaging.

Results: Images performed lhr P.I. showed essentially tumor, liver and intestinal uptake; between $3 \mathrm{hr}$ and $6 \mathrm{hr}$ P.I., intestinal radioactivity increased whereas liver decreased and $24 \mathrm{hr}$ P.I.only the tumor was objectivated. Tumor/normal tissue ratio increased over time.

\begin{tabular}{|l|c|c|c|c|}
\hline Ratio & $3 \mathrm{hr}$ & $6 \mathrm{hr}$ & $18 \mathrm{hr}$ & $24 \mathrm{hr}$ \\
\hline Tumor/muscle & 6 & 79 & 124 & 137 \\
\hline Tumor/liver & 3 & 23 & 34 & 54 \\
\hline
\end{tabular}

The overall elimination was fast and important ( $\geq 95 \%$ at $24 \mathrm{hr}$ ) with major hepatodigestive pathway (85\% fecal, 15\% urine). Intratumoral injection of phosphodiester leads to high and increasing tumor/ratio and thus offers a potential advantage for therapeutic use.

\section{7}

U. Pietrzyk, K. Scheidhauer, E. Voth, H. Schicha Max-Planck Institute for Neurological Research and Dept. of Nuclear Medicine, University of Cologne, Germany.

EXPLORATION OF MEDICAL IMAGING TOOLS FOR APPLICATION OF PET AND SPECT IN ONCOLOGY

PET images as well as SPECT images are often difficult to evaluate due to uncertainties in the reference to the underlaying anatomical structures. Even the display of orthogonal planes cannot always eliminate the obstacles in the interpretation of images with very high contrast.

We studied several cases with high focal uptake in primaries and metastases of breast cancer with FDG-PET, (transmission and emission scans), and with MDP-SPECT (bone scan). The PET-images were acquired on a CTI ECAT Exact scanner with an axial FOV of $16.2 \mathrm{~cm}$. The bone studies were performed with a Picker Prism 3000 triple headed SPECT camera.

A multipurpose registration and imaging tool was used, to check the alignment of PET-transmission versus - emission or to match PET-versus SPECT-emission scans. The interactive, retrospective registration process is based on the visual interpretation of multiple views using internal landmarks like bony structures or characteristic areas of increased uptake. Volumetric data sets are generated by linear interpolation for both studies with subsequent application of the so-called integral shading technique. Starting at a surface pixel of the transmission data an optional number of pixels is integrated in the viewing direction. Depending on the integration length, structures within a certain depth can be visualized. This allows an integrated display of transmission and emission scans highlighting areas of focal uptake relative to the surface-rendered body outline. This technique has been extended for use with SPECT data.

This approach was appreciated in clinical cases combining multiple as pects of the tomographic studies in a single display.

\section{8}

W. Brenner, K. Erdmann, M. König, H. Wolf, J.-U. Eberhardt, K.H. Bohuslavizki, M. Schramm, M. Clausen, E. Henze Clinic of Nuclear Medicine, Christian-Albrechts-University, Kiel Germany

CARDIAC UPTAKE OF I-123 LABELLED DOXORUBICIN AND IODODOXORUBICIN IN RABBITS DUE TO VERAPAMIL

In recent years it has been shown that verapamil increases both intracellular concentration and cytotoxicity of anthracyclines like doxorubicin (DOX) or the new derivative 4-iodo-doxorubicin (IDOX) in resistant tumor cells. However, due to the well-known cardiotoxicity of anthracyclines, there are only few data concerning the impact of verapamil on myocardial accumulation of radiolabelled DOX and IDOX to estimate the potential risk of increasing cardiotoxicity.

DOX and IDOX were radioiodinated with I-123 by the lodogen method yielding radiochemical purity $>98 \% .40 \mathrm{MBq}$ of labelled DOX and IDOX were administered intravenously to four rabbits, respectively. Additionally, $1 \mathrm{mg}$ verapamil was injected i.v. prior to injection of the radiolabelled drug in two animals of each group. Subsequently, whole body sequential scintigrams in $5 \mathrm{~min}$ intervals were taken up to $100 \mathrm{~min}$ p.i.. Cardiac accumulation was determined as percental uptake of total body activity.

Cardiac accumulation of DOX, DOX + verapamil (DOX+), IDOX and IDOX + verapamil (IDOX+) were decreasing in time in a single exponential manner as shown in the following table:

\begin{tabular}{|c|c|c|c|c|}
\hline $\begin{array}{c}\text { Time } \\
{[\mathrm{min}]}\end{array}$ & $\begin{array}{c}\text { DOX } \\
{[\%]}\end{array}$ & $\begin{array}{c}\text { DOX+ } \\
{[\%]}\end{array}$ & $\begin{array}{c}\text { IDOX } \\
{[\%]}\end{array}$ & $\begin{array}{c}\text { DOX+ } \\
{[\%]}\end{array}$ \\
\hline 5 & 3,70 & 3,02 & 2,02 & 2,28 \\
\hline 30 & 1,56 & 1,80 & 0,98 & 0,97 \\
\hline 100 & 0,81 & 0,86 & 0,37 & 0,34 \\
\hline
\end{tabular}

In conclusion, the results of this preliminary study neither confirm major differences in cardiac accumulation of anthracyclines depending on verapamil nor suggest a potential risk of increasing cardiotoxity of anthracyclines in combination with verapamil. 
549

C. Stauch, H. Wolf, C. Müller, I. Stöhr, M. Clausen, E. Henze Division of Nuclear Medicine, Christian-Albrechts-University of Kiel, Germany

In vivo studies of anthracycline derivatives: comparison of organ uptake in rabbits

Antitumour efficacy of anthracyclines in malignant diseases has been proven. However, their numerous side-effects as well as tumour resistance demand research on the development of new analogues. The purpose of this study was to asses the uptake of I-123 labelled anthracycline derivatives in rabbits, an animal with kinetics similar to man for this antibiotic group (Brenner 1984). Doxorubicin (DOX), 4-Iodo-doxorubicin (IDOX) and Daunorubicin (DNR) were radioiodinated by the Iodogen method. Separation was performed by HPLC. Radiochemical purity was higher than $98 \%$ in all cases. Tracer distribution was assessed within a period of 90 minutes by whole body scintigraphy after i.v. application of $10 \mathrm{MBq} / \mathrm{kg}$ body weight.

Highest uptake was seen for the intestines when used I-123-DNR (9.5\%) followed by I-123-IDOX (4.8\%). Iodinated DNR showed a low cardiac uptake $(0.8 \%)$ and a high hepatic accumulation $(3.7 \%)$. The tested radiopharmaca showed almost constant uptake over 90 minutes.

\begin{tabular}{|lccccc|}
\hline \multicolumn{5}{c|}{ organ uptake [\%] 60 min. p.i. } \\
\hline & intestines & heart & liver & salivary gland & kidney \\
I-123-DOX & 2.0 & 2.3 & 1.6 & 1.8 & 0.8 \\
I-123-IDOX & 4.8 & 1.8 & 2.5 & 1.6 & 1.5 \\
I-123-DNR & 9.5 & 0.8 & 3.7 & 1.2 & 0.9 \\
\hline
\end{tabular}

Despite of little difference in chemical structure the organ uptake of parent compounds of the anthracycline group varies remarkably. The spectrum of I-123-DNR activity appears to have a reciprocal behaviour in intestine versus heart. This might be an advance in search of effective treatment of intestinal malignancies with contemporary reduction of cardiotoxicity.

\section{0}

K.H. Bohuslavizki, C. Richter, N. Hartkopf, D. Lennert, H. Wolf, W. Brenner, J.-U. Eberhardt, M. Schramm, M. Clausen and E. Henze Department of Nuclear Medicine, Christian-Albrechts-University, Kiel, Germany

\section{LIPOPHILICYTY IS NOT THE MAIN FACTOR WHICH AF- FECTS CELLULAR UPTAKE OF ANTHRACYCLINES IN GASTRIC TUMOUR CELLS: I-123-DOXORUBICIN VERSUS I-123-IODODOXORUBICIN.}

Physico-chemical properties, e.g. lipophilicity may effect cellular uptake This could be shown successfully for I- 123 versus Tc- $99 \mathrm{~m}$ as radiolabel. Therefore, the aim of this studie is to compare the uptake of two analogous anthracylines being different in an additional iodine only, i.e. doxorubicin (DOX) and iodo-doxorubicin (IDOX)

Both anthracyclines were radiolabelled with $\mathrm{I}-123$ in a conventional manner by the lodogen method yielding radiochemical purity of more than $98 \% .10 \mathrm{kBq}$ I-123-DOX or I-123-IDOX were added to each culture medium in addition to $20 \mathrm{kBq}$ T1-201 $(\mathrm{n}=100)$, respectively. Cellular uptake was stopped by removing the medium at different incubation times and cooling. After washing, measuring cellular uptake in a well counter, and correcting for physical decay, uptake of I-123DOX and I-123-IDOX was calculated in human gastric carcinoma cells (D 257/85 P).

\begin{tabular}{||l||r|r|r|r|r|r|r|r|r|r||}
\hline \multicolumn{1}{|c||}{} & \multicolumn{4}{c||}{ DOX } & \multicolumn{4}{c|}{ IDOX } \\
\hline \hline [min] & 1 & 10 & 60 & 120 & 240 & 1 & 10 & 60 & 120 & 240 \\
\hline I-123- & .34 & .63 & .97 & .93 & .86 & .19 & .33 & .76 & .96 & .85 \\
\hline Tl-201 & .12 & .20 & .24 & .19 & .20 & .28 & .32 & .25 & .37 & .24 \\
\hline
\end{tabular}

All results in this table are given as medians ( $\mathrm{n}=5$ per incubation time) of percental uptake of the activity applied. Observe, both I-123-DOX and I-123-IDOX showed approximately 3.4 times and 3.2 times higher uptake than Tl-201, respectively.

In conclusion, both kinetics and amount of uptake of the more lipophilic I-123-IDOX and I-123-DOX are very similar. Thus, lipohilicity is not a determining factor for cellular uptake.
551

H. Bender, A. Schomburg, C. Menzel, K. Hamad, H. Palmedo, B. Schultes, H.-J. Biersack.

Dept. of Nuclear Medicine, University of Bonn, Germany.

PREDICTIVE POTENCY OF TPS ON THE COURSE OF
BONE METASTASES AS COMPARED TO TC-99m MDP BONE SCINTIGRAPHY.

The state of progression or remission of bone metastases following therapy is currently documented mainly by standard bone scintigraphy or X-ray scans. Both methods can only be employed to a certain extent and therefore are limited as a tool for rapid decision making concerning changes in therapy concepts and/or early evaluation of therapy success or failure. The aim of this study was to estimate the use of the proliferation marker tissue polypeptide antigen specific (TPS) to predict the actual status of known bone metastases as compared to the results of standard Tc-99m-MDP bone scintigraphy. In the learning phase of this study, serum levels of TPS we correlated with the results of bone scans of patients transfered to our department for routine bone scintigraphy. At the first analysis the results of patients with no documented primary tumor $(\mathrm{N}=10)$ were correlated with those suffering from cancer $(\mathrm{N}=70)$. In a second analysis, patients with a known malignancy were subgrouped depending on the results of the bone scan as follows: 1) no bone metastases detected $(\mathrm{N}=44)$; 2) known bone metastases $(\mathrm{N}=26)$ a) in remission $(\mathrm{N}=3)$, b) stable disease $(\mathrm{N}=14)$ and c) progressive disease ( $N=9$ ) as compared to previous scans. Our preliminary data indicate significant lower TPS serum levels in patients without a known primary tumor as compared to patients suffering from a malignancy. No significant difference was found comparing TPS serum levels of the various subgroups due to high variance. Nevertheless, patients with proven bone metastases $(n=9)$ showed a dependency of TPS levels and extent of disease. On the other hand, scintigraphically documented metastases were never associated with normal TPS levels. We conclude from these preliminary data that TPS is probably not able to replace bone scintigraphy per se, but might allow to follow and supplement bone scans in the individual course of cancer.

\section{2}

K.H. Bohuslavizki, N. Hartkopf, C. Richter, H. Wolf, W. Brenner, J.U. Eberhardt, M. Schramm, M. Clausen and E. Henze

Department of Nuclear Medicine, Christian-Albrechts-University, Kiel, Germany

DIFFERENT CELLULAR UPTAKE OF DOXORUBICIN: LABELLING BY I-123 VERSUS EXCHANGE REACTION WITH C-14.

Uptake measurements of radiolabelled anthracyclines may be affected by its different physico-chemical properties due to different radiolabels. This could be shown for I- 123 versus Tc- $99 \mathrm{~m}$ as radiolabel. Therefore, the aim of this study was to compare labelling by adding (I-123) versus labelling by an exchange reaction (C-14).

Doxorubicine (DOX) was radiolabelled with I-123 in a usual manner by the lodogen method yielding radiochemical purity of $>98 \%$. C14-labelled DOX was purchased from Amersham (Braunschweig). 10 $\mathrm{kBq}$ I-123-DOX or $0.3 \mathrm{kBq}$ C-14-DOX were added to each culture medium in addition to $20 \mathrm{kBq}$ T $1-201(\mathrm{n}=110)$, respectively. Cellular uptake was stopped by removing the medium at different incubation times and cooling. After washing, measuring cellular uptake in a well counter or a beta liquid scintillation counter, and correcting for physical decay, uptake of I-123-DOX and C-14-DOX was calculated in human gastric carcinoma cells (D 257/85 P).

\begin{tabular}{||l||r|r|r|r|r||r|r|r|r|r||}
\hline \multicolumn{1}{|c||}{} & \multicolumn{4}{c||}{ I-123-labelled } & \multicolumn{5}{c||}{ C-14-labelled } \\
\hline \hline [min] & 1 & 10 & 60 & 120 & 240 & 1 & 10 & 60 & 120 & 240 \\
\hline DOX & .34 & .63 & .97 & .93 & .86 & .15 & .65 & 1.74 & 3.05 & 4.65 \\
\hline Tl-201 & .12 & .20 & .24 & .19 & .20 & .12 & .16 & .17 & .32 & .21 \\
\hline
\end{tabular}

All results in this table are given as medians ( $\mathrm{n}=5$ per incubation time) of percental uptake of the activity applied. Observe, I-123-DOX showed approximately 4.5 times higher uptake than T1-201 while C-14DOX showed approximately 18.3 times higher uptake than Tl-201.

In conclusion, uptake of the more lipophilic I-123-DOX is limited by saturation while C-14-DOX showed an almost linear uptake with time. Thus, lipohilicity is not the main factor in determining uptake of radiolabelled DOX. 
553

D.M. Goldenberg, R.M. Sharkey, M. Juweid, A.I. Markowitz, L.C. Swayne, P. Conte, S.O. Leung, and H.J. Hansen

Garden State Cancer Centex at the Center at the Center for Molecular Medicine and Immunology, Newark, NJ; and Immunomedics, Inc., Morris Plains, No

INITIAL TUMOR TARGETING SYUDIES WITH ${ }^{131}$ I-LABELED CHIMERIC LI2 IgG IN PATIENTS WITH NON-HODGKIN'S LYMPHOMA

LI2 is an IgG2a murine monoclonal antibody (MAb) directed against the $\mathrm{CD} 22$ antigen found on normal and malignant $B$ cells. Clinical studies using LL2 have shown excellent tumor targeting in patients with nonHodgkin's lymphoma (NHL). Although NHL patients are less prone to develop an anti-mouse antibody response (HAMA), HAMA will develop after multiple

administrations. In order to reduce the risk of HAMA, we have developed a human/mouse chimeric IL2 (CLI2), and have shown that it has identical binding and have shown that it has identical binding properties to the murine LL2 IgG, as evaluated by
and flow cytometry. Three patients with advanced

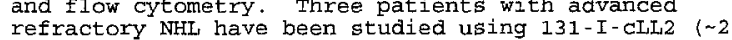
refractory NHI have been studied using $131-I-c L L 2$ ( 2 131-I-murine LL2 $\mathrm{F}\left(\mathrm{ab}^{r}\right) 2$ for comparison. In the 2 patients in whom both cLL2 IgG and murine LI2 $F$ (ab') 2 were given, tumor targeting was similar. Increasing the protein dose of the cLL2 IgG from $\sim 2 \mathrm{mg}$ to $20 \mathrm{mg}$ in 1 patient with bone marrow involvement decreased the blood clearance rate by 2 -fold, and had a variable effect on tumor targeting. These studies suggest that the CLL2 compares favorabiy to the murine LL2, and thus may be a useful agent for the radioimmunotherapy of NHL. (Supported in part by USPHS grant CA3984I from the NIH.)

\section{4}

C.Aktolun, H.Bayhan, Y.Pabuccu, H.Bilgic, H.Acar, R.Koylu

Departments of Nuclear Medicine, Diagnostic Radiology, and Chest Diseases, School of Medicine, Gulhane Military Medical Academy, Ankara, Turkey

\section{ASSESSMENT OF TUMOR NECROSIS WITH Te-99m SESTAMIBI}

It has becn shown in animal and metabolic studies that Tc-99m sestamibi does not accumulate in necrotic myocardial tissue despite nornal blood flow. If. similarly, Tc-99m sestamibi does not accumulate in nccrotic tumor tissue, then, it could be used in follow-up of patients with malignant tumors after radiotherapy, which induces tumor necrosis. The aim of this study was, therefore, to assess the effect of tumor necrosis on Tc-99m scstamibi uptake.

Thirty cight patients with 4 major types of bronchial carcinoma were included in this study. Planar $(n=38)$ and SPECT imaging $(n=29)$ was initiated 10-15 minutes after the injection of $370-740 \mathrm{MBq} \mathrm{Tc}-99 \mathrm{~m}$ sestamibi. Tumor necrosis was diagnosed in 12 patients based upon histopathology $(n=2)$, and density measurements and type of contrast enhancement on CT scan ( $\mathrm{n}=8)$. Of the patients, 34 showed Tc-99m sestamibi tumor uptake on planar and 27 on SPECT images. Four types of tumor uptake pattern was identified: focal uptake $(n=25)$, focal uptake with a central hypocative focus ( $n=7)$, ring-like uptake $(n=2)$, and no uptake (negative uptake) $(n=4)$ on planar inages. The corresponding rcsults for SPECT imaging were $17,7,3$ and 2, respectively. Of the patients with tumor necrosis, 92\% (11/12) revealed defective tumor uptake (focal uptake with a central hypoactive focus, $n=7$, ring-like uptake, $n=2$, and no tumor uptake, $n=2)$ on planar and $100 \%(12 / 12)$ on SPECT images (focal uptake with a central hypoactive focus, $n=7$, ringlike uptake, $n=3$, and no uptake, $n=2$ ).

In conclusion, this study shows that Tc-99m sestamibi does not accumulate in necrotic tumor tissue. This has significant clinical implications that Tc-99m sestamibi, particularly with SPECT imaging has a potential role in follow-up of patients with bronchial carcinoma by differentiating recurrent or residual disease from post-therapy necrosis, and in monitoring the response of tumors to radiotherapy.

\section{5}

Y.DUMAN,Z.BURAK, S.KTRAC, Z.B.ÖZCAN,A.DIRLIK,M.ERCAN, S.ÖNCHL, A. HAYDAROĞLLIART and B.BIIKAY

Depts of Nuclear Medicine, Radiation Oncology, Oncology, Fge University, IZMIR- TIRKEY

\section{THE VALUE OF" 991-TC GUITATHIONE (GSH) IN TMMOR IMAGING}

The aim of this study was to investigate the behaviour of 99r-Tc GSH in different neoplastic pathologies.

A total of 169 patients (pts) were studied.The pts were IV injected $555 \mathrm{MBq} 99 \mathrm{nr}-\mathrm{Tc}$ GSH. Increased activity accumulation in regard to nearby soft tissues were accepted as positive.Quantitative evaluation was also applied by ROIs drawn over lesions and contrlateral site ( L/C ).AIl pts were examined by other imaging modalities (US,CT, WRI etc.).Final diagnosis was determined by pathological exarination.

The pathological diagnosis and $99 \mathrm{~m}-\mathrm{Tc}$ GSI findings of 169 pts are as follows:

1)Tumors of mesenchymal origin: 33 malignant $(2.6+0.8)$ and 7 benign $(0.9+0.1)$ tumors (tms) originated from connective and endothelial tissue were included ( $p$ 0.005).12 malignant lymphones originated from lymphoid tissue did not show any (t) CSH accumulation.

2)Tumors of epithelial origin: 41 malignant melanora metastases originated from neuroectodermal origin were well visualiser $(1.84+0.2) .21 \mathrm{c}-$ pidermoid carcinonas (Ca) $(2.1+0.24)$ and 1 papilloma (negative uptake) originated from stratified squarnous tissue were also included.

3)Miscellaneous: Pleomorphic adenoma ( 4 pts), thyroid follicular adenona (3 pts), benign Iympadenitis (2 pts), breast this (12 pts), gynecologic tms (12 pts) did'nt show any GSH urtake.Moreover, neural crest tris (15 pts) with $(+)$ I-13I MTBG accumulation did'nt demonstrate any GSH uptake. 6 pts with other miscellenous tns were also (-) with 99m-Tc GSH.

As a conclusion;1) 99m-Tc GSH ray not simply a blood pool agent, 2)Active transport may be the dominant mechanism that is responsible for GSi transport into the intracellular compariment and 3) tins originated from connective and endothelial tissues of mesenchynal origin, tns originated from stratified squamous epithelium of epithelial origin and firally tms from neuroectoderm origin (nevus and malignant melanoma) showed positive 99n-Tc GSH tutake. Therefore, 99!-Tc GSI may be a promising agent in differentiation of berign and mationant lesions and in detection of metastases and recurrences of these groups of tumors.

\section{6}

L. Kostakoglu, C. Portlock, C.R. Divgi, S.J. Goldsmith, S.M. Larson, R. Castellino.

Depts. of Radiology and Medicine, Memorial Sloan Kettering Cancer Center, New York NY U.S.A

ASSESSMENT OF LOW GRADE LYMPHOMA (LGL) WITH ${ }^{201} \mathrm{TI}$ AND ${ }^{67} \mathrm{Ga}$ ON A SITE-BY-SITE CORRELATION WITH CT.

We undertook a prospective study to assess the value of scintigraphy with ${ }^{201} \mathrm{Tl}$ chloride \& ${ }^{67} \mathrm{Ga}$ citrate in determining extent of disease in previously untreated patients (pts) with LGL. 18 pts (age range: 30-80 years old, mean:50) with LGL had ${ }^{201} \mathrm{Tl}$ (15 GBq), ${ }^{67} \mathrm{Ga}$ citrate $(37 \mathrm{GBq})$ scans (planar/SPECT) and contrast-enhanced CT scans of the chest, abdomen and pelvis within a week. The radioscintigraphy studies were interpreted blinded to the results of CT scans. Tumor/Background $(\mathrm{Tm} / \mathrm{Bkg})$ ratios were obtained from planar scans of both nuclear modalities. Results for detected lesions were as follows:

\begin{tabular}{|c|c|c|c|}
\hline Modality & $\begin{array}{c}\text { Chest \& } \\
\text { Upper body }\end{array}$ & $\begin{array}{c}\text { Abdomen \& } \\
\text { Pelvis }\end{array}$ & Total \\
\hline $\mathrm{CT}$ & 22 & 34 & 56 \\
\hline${ }^{201} \mathrm{~T} 1$ & 3 & 18 & 21 \\
\hline${ }^{67} \mathrm{Ga}$ & 5 & 7 & 12 \\
\hline
\end{tabular}

There were 3 equivocal cases on ${ }^{201} \mathrm{Tl}$ and 4 on ${ }^{67} \mathrm{Ga}$ scintigraphy. Tm/Bkg ratios for positive ${ }^{201} \mathrm{Tl}$ were $\geq 2$.

In conclusion, ${ }^{201}$ Tl scintigraphy is more sensitive than ${ }^{67} \mathrm{Ga}$ in evaluation of pts with IGL, although this modality detected only $37.5 \%$ of the lesions seen on CT. 
Sunday, 21 August - Wednesday, 24 August 1994

557

R.K.L. Baidjnath Panday, I.H. Liem, E.J.Th. Rutgers, S.H. Muller, C.A. Hoefnagel, B.B.R. Kroon, A.J.M. Balm, W.J. Mooi, O.E. Nieweg

The Netherlands Cancer Institute, Plesmanlaan 121, 1066 CX Amsterdam

INTRA-OPERATIVE SENTINEL NODE (S.N.) IDENTIFICATTON USING GAMMA DETECTION PROBE IN PATIENTS WITH MELANOMA.

In melanoma patients without apparent lymph node metastases, the S.N procedure allows us to identify those who have micrometastases and need regional lymph node dissection (R.N.L.D.). We investigated the gamma detection probe and patent blue (P.B.) for this purpose.

In 9 patients with clinically localized melanoma with a mean Breslow thickness of $2.3 \mathrm{~mm}$ (range $1.1 \mathrm{~mm}-4.7 \mathrm{~mm}$ ) the SN identification and biopsy procedure was carried out. Approximately $60 \mathrm{MBq}$ of $99 \mathrm{~m} \mathrm{Tc}$ nanocolloid was injected intradermally in the margins of the tumour or at the previous tumor site and shortly thereafter the first lymphoscintigram was made. The first visualized lymph node (S.N.) was then marked on the skin. The next day P.B. was injected shortly before operation at the same location as the $99 \mathrm{mTc}$-nanocolloid. The number of identified S.N. with the gamma camera was 15 . During operation we identified 11 out of $14 \mathrm{~S}$.N. visually by its blue discoloration and 14 out of 14 with a gamma detection probe (Neoprobe 1000). The mean S.N. to background (BKG) ratio intra-operatively was 73.2 (range 3.5-359) and the mean S.N./BKG ratio ex vivo was 1125 (range 70-3957). The mean percentage of injected dose per gram tissue was 1.36 (range 0.02-5.37) Frozen section microscopy performed during surgery revealed in 2 patients micrometastases. Both patients underwent RLND.

Conclusion: Accuracy and reliability of this method appear to be very high. When carried out in a larger number of patients this procedure might be useful in the selection of patients for RLND or whether a waitand-see policy can be followed.

\section{8}

V.Rufini, M. Salvatori, I. Saletnich, G. Martino, M.L. Maussier, S.M. Corsello, L. Troncone

Depts. of Nuclear Medicine and Endocrinology, Catholic University of the Sacred Heart, Rome, Italy

RADIOLABELLED SOMATOSTATIN ANALOG (SMS) IN MEDULLARY THYROID CA. (MTC): A COMPARISON WITH TC-99m-(V)DMSA AND I-123-MIBG. PRELIMINARY RESULTS

The Authors have applied SMS scintigraphy in the imaging of MTC which shows high affinity SMS receptors. We report the results of this technique in 6 totally thyroidectomized pts. (4 F and $2 \mathrm{M}$, aged 38-69 yr) who presented recurrent and/or metastatic lesions and high levels of calcitonin (Ct) and CEA. In all pts. the $\mathrm{Ct}$ response to acute administration of octreotide (OCT) (200 $\mu \mathrm{g} \mathrm{s.c.)}$ was evaluated. Whole body SMS scans (4 to $48 \mathrm{hrs}$ ) were performed after i.v. injection of 111-158 MBq of In-111-pentetreotide. When indicated, SPET was carried out. The SMS results were compared to those of Tc-99m(V)DMSA and I-123-MIBG scans. They are listed in the table.

\begin{tabular}{|c|c|c|c|c|c|}
\hline N. & Known lesions & $O C T$ & $S M S$ scan & (VDMSA & $M I B G$ \\
\hline 1. & $\begin{array}{c}\text { widespread } \\
\text { tumoral lesions }\end{array}$ & - & Positive & Positive & Negative \\
\hline 2. & multiple LN & + & Positive & Negative & Negative \\
\hline 3. & LN & + & Positive & Positive & Negative \\
\hline 4. & multiple LN & - & Negative & Positive & Negative \\
\hline 5. & LN & - & Negative & Positive & Negative \\
\hline 6. & $\begin{array}{c}\text { widespread } \\
\text { tumoral lesions }\end{array}$ & + & Positive & Positive & Positive \\
\hline
\end{tabular}

$\mathbf{L N}=$ lymph nodes; $-=$ no response $;+=>30 \%$ decrement

Conclusions: 1) SMS scintigraphy can visualize some MIBG negative MTC lesions; 2) when compared to (V)DMSA, SMS scintigraphy gaves similar diagnostic results, however it also has a therapeutic impact, prediciting tumor response to long-term therapy with octreotide.

\section{9}

L.J.M. Rijks, K. de Bruin, E.A. van Royen, E. Busemann-Sokole, and A.G.M. Janssen.

Department of Nuclear Medicine, Academic Medical Centre, Amsterdam; Cygne B.V., Technological University, Eindhoven, The Netherlands.

THE DISTRIBUTION OF Z-113-METHOXY-17 $a$-[1-123]IODOVINYL-ESTRADIOL-173 (Z-[1-123]MIVE) AN ESTROGEN RECEPTOR SPECIFIC

The estrogen receptor specific radioligand Z-[I-123]MIVE, which in earlier studies showed high binding affinity in vitro and high target tissue uptake and selectivity in the rat in vivo, could be a suitable radioligand for estrogen receptor-positive human breast tumours. To date, its distribution was studied in three healthy female volunteers.

After I.v. injection of $185 \mathrm{MBg}$ Z-[I-123]MIVE (specific activity 200 $\mathrm{MBq} / \mathrm{nmol}$ ) a dynamic acquisition of the thorax was performed between 0 and $30 \mathrm{~min}$. Anterior and posterior whole body scans were made at 1,2,4 and $6 \mathrm{hr}$. ROI s were drawn to calculate the biodistribution. The organ activity was expressed as percentage of the total body activity. Blood samples were taken at different times after injection, and urine was collected.

No adverse effects were noticed after injection of Z-[l-123]MIVE. Lung and heart activity decreased exponentially within $10 \mathrm{~min}$ to $30 \%$ of the maximum, remaining almost constant thereafter. Liver activity increased to a steady state at $10 \mathrm{~min}$. The $\mathbf{3 0} \mathrm{min}$ blood level was about $94 \%$ of the $5 \mathrm{~min}$ activity, and decreased to $32,19,10$ and $3 \%$ at $1,2,4$ and $24 \mathrm{hr}$, resp. In agreement with the decrease in total body activity, the total urine excretion at $6 \mathrm{hr}$ was $20 \%$ of the injected dose. Urine excretion increased to $30 \%$ at $20 \mathrm{hr}$. Organ distribution was as follows

\begin{tabular}{|c|c|c|c|}
\hline $\begin{array}{l}\text { total body } \\
\text { thyroid } \\
\text { juer/gall bladder } \\
\text { intestine } \\
\text { jungs } \\
\text { bramn }\end{array}$ & $\begin{array}{r}100.8 \\
19: 7 \\
14.3 \\
3.1 \\
2.0\end{array}$ & $\begin{array}{r}100.9 \\
13.9 \\
24.2 \\
2.3 \\
1.3\end{array}$ & $\begin{array}{r}100.9 \\
3.9 \\
46.4 \\
2.4 \\
0.5\end{array}$ \\
\hline
\end{tabular}

The mean activity (counts/pixel) in the breast, expressed as percentage of the mean lung activity, increased from $97 \%$ at $1 \mathrm{hr}$ to $120 \%$ at $6 \mathrm{hr}$.

We conclude that Z-[1-123]MIVE is a promising radioligand for SPECT imaging of estrogen receptors in human breast cancer.

\section{0}

I. Saletnich, V. Rufini, M. Salvatori, MS Daidone, L. Troncone Dept. of Nuclear Medicine, Catholic University of the Sacred Heart Rome, Italy

ASSESSMENT OF T1-201 AND Tc-99m-MIBI EFFICACY IN THE FOLLOW UP OF SELECTED PATIENTS HARBOURING DIFFERENTIATED THYROID CARCINOMA (DTC).

Several papers report on the value of Tl-201 and Tc-99m-MIBI in non I-131 concentrating, or in dedifferentiated thyroid tumors. The aim of the present study is to evaluate and compare the actual role of both the tracers in selected pts. with DTC, showing high thyroglobulin (Tg) levels together with negative I-131. Among 45 pts. examined by $\mathrm{Tg}$ determination and I-131/Tl-201/Tc-99m-MIBI scans during the follow up, 12 pts. (9 $\mathrm{M}$ and $3 \mathrm{~F}$, aged 35-72 yrs; 11 papillary, 1 follicular; $\mathrm{Tg}$ levels: $67->1000 \mathrm{ng} / \mathrm{ml}$ ) were selected. All pts. had undergone total thyroidectomy followed by one or more ablative doses of I-131. I-131 $(185 \mathrm{MBq})$ and $\mathrm{Tl}-201(111 \mathrm{MBq})$ scans were obtained in all pts; Tc$99 \mathrm{~m}-\mathrm{MIBI}(555 \mathrm{MBq})$ scans were obtained in $7 \mathrm{pts}$.

T1-201 scan gave a positive result in 10 out of $12 \mathrm{pts}$. Tc-99m-MIBI was positive in 5 out of $7 \mathrm{pts}$; of the two negative studies, one was Tl201 positive. When compared with Tl-201 results, of the 5 MIBIpositive studies 3 were fully concordant with Tl-201, two had some lesions missed. Clinical follow up showed the presence of metastases in all patients.

Conclusions: 1) the need of alternative radiotracers in non I-131 concentrating tumors is confirmed. 2) T1-201 seems to have a higher sensitivity in patients with elevated $\mathrm{Tg}$ levels and negative I-131 scan than in the overall series of DTC ( $83 \%$ vs. $68 \%$ in our experience) 3) Tc-99m-MIBI which is more easily available and more suitable for SPET than T1-201, does not seem to actually improve diagnostic efficacy, at least in this limited series. 
Sunday, 21 August - Wednesday, 24 August 1994

561

A. I. Santos, P. Magro, T. Martins, G. Cantinho, M. Montciro-Grilo. Instituto de Medicina Nuclear - Serviço de Oftalmologia, Hospital de Santa Maria, Lisboa, Portugal.

IMMUNOSCINTIGRAPHY WITH F(ab') 2 OF 225.285 MONOCLONAL ANTIBODY IN UVEAL MELANOMA

In order to evaluate the use of immunoscintigraphy in uveal melanoma, both for the detection of the primary lesion and of liver metastasis, we performed this methodology in 23 patients (pts), 12 females and 11 males, with a $<$ age $>=57 \pm 17$ years (7-78 years). Three of these pts had been enucleated, 1 had had tumor resection and 2 had benign lesions. The remaining $17 \mathrm{pts}$ had uveal melanoma with an <diameter> $=8.71 \pm 4.9 \mathrm{~mm}(1.5-19.9 \mathrm{~mm})$, measured by echography. Twelve of these pts had also been submitted to CT and/or NMR.

The immunoscintigraphy was performed with fragments of the MoAb 225.28S labeled with ${ }^{99} \mathrm{~m} \mathrm{Tc}$, using $0.35 \mathrm{mg}$ and $750+186 \mathrm{MBq} / \mathrm{pt}$. All-body imaging (10 $\mathrm{min} . / \mathrm{m}$ ), planar images of the head, in anterior view, and in $20 \mathrm{pts}$ SPET study $\left(40\right.$ seconds/view $\times 32$ views, $-90^{\circ}$ to $90^{\circ}$ ) were performed at $4.75 \pm 0.75$ hours. At 24 hours post injection, planar images of the head were also done.

The first planar head images permitted a 10:17 (58.8\%) true positive (TP) results and $5: 6(83 \%)$ of true negative (TN) studies. The same results were, respectively, 15:17 (88.2\%) and 6:6(100\%) for the 24-hour image, and 15:15 $(100 \%)$ and 5:5 (100\%) for the SPET study. Evaluated as a whole group, the immunoscintigraphy permitted 16:17 (94\%) TP studies and 5:6 (83\%) TN ones. The intensity of activity was qualitatively classified as small, moderate and intense, and we tried to correlate it with the biggest diameter of the lesion. It seemed that a biggest diameter permitted a higher focal activity, although the small number of studies in each group did not permit the use of statistical tests. Five lesions were not detected by CT and 3 by NMR, and all of these were visualized with the scintigraphic study. Two pts had liver metastasis, not seen with the ${ }^{99 m} \mathrm{Tc}-\mathrm{M}_{0 A \mathrm{~A}}$

These results suggest that this method may still be used to study intra-ocular lesions suspected of being melanomic, especially if SPET acquisition is used. On the contrary, it is of no use to detect metastasis, since the main place of secondary lesions is the liver, an organ with a very high unspecific binding of the MoAb.
563

L. Troncone, V. Rufini, A. Giordano, G. Martino, I. Saletnich, M. Salvatori

Dept. of Nuclear Medicine, Catholic University of the Sacred Heart, Rome, Italy

HAS I-123-MIBG ASSOCIATED WITH SPET ANY ROLE IN THE ASSESSMENT OF NEUROBLASTOMA?

The actual value of I-123-MIBG SPET in neuroendocrine tumors, and in particular in neuroblastoma (NB), is controversial. Therefore a comparison between planar and SPET imaging in NB was undertaken We report here the results obtained in 18 patients $(9 M$ and $9 F$, aged 4 mo.-11 yr; one at stage II, six at stage III, ten at stage $\mathrm{N}$, one at stage IVS). Imaging (a total of 28 studies) was performed at diagnosis (2), after surgery (5), during or after chemotherapy (13), at follow up (5) and at relapse (3). Both planar, at 24 and occasionally 40 brs (multiple anterior and posterior images; 500,000-1,000,000 counts per scan) and SPET imaging at $24 \mathrm{hrs}$ (64 projections obtained over 360 at 30 $\mathrm{sec} / \mathrm{projection)}$ were carried out after i.v. injection of $148-296 \mathrm{MBq}$ of I-123-MIIBG.

Planar studies gave a sensitivity of $89.5 \%$ and a specificity of $88.9 \%$ In the same patients SPET gave a sensitivity of $94.7 \%$ and an equal $(88.9 \%)$ specificity. When compared with planar scanning, SPET imaging showed a total of six additional pathological areas of uptake in three patients. In another patient an equivocal abnormality on planar imaging was confirmed as pathological by SPET. Furthermore, SPEI improved the anatomical assessment of the lesions in 12/18 studies. In conclusion: the association of SPET can improve the diagnostic efficacy of MIBG scintigraphy in the evaluation of NB. In our experience SPET was conclusive in detecting lesions in one case, essential in another three cases and helpful, by giving additional information, in 12/18 true positive studies.
562

Westlin JE, Walind S, Lilja A, Ahlström $\mathrm{H}$,

Långström $\mathrm{B}$. The PET-centre, Uppsala

University, S-751 85 Uppsala, Sweden

NORMAL LUNG -AND CEREBELLAR UPTAKE

ASSESSED AS SUV-VALUES IN L-methyl-11C methionine (11C-methionine) PET

Thirtysix consecutive patients admitted for

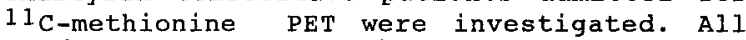
patients were admitted for primary investigation before therapy. The patients were examined with a whole body PET camera, GE 4096 (General Electric medical systems), which produces 15 simultaneous contiguous axial slices with a thickness of $6.5 \mathrm{~mm}$ and $a$ resolution of 5-6 mm.All patients fasted for at least 5 hours before scanning. An activity of $800 \mathrm{MBg}$ L-methyl-11 C-methionine was administered as an i.v. bolus injection and a scanning for 50 minutes with increasing frame times was performed.

In the SUV images regions of interest (ROI:s) covering the lung without hilar vessels were drawn.The ROI:s in the cerebellum covered the whole cerebellum.

In 36 patients 44 observations were recorded. The mean SUV uptake value was 0,59 for lung (range $0,343-0,922$ ) SD 0,14 . No correlation to plasma methionine or to diagnosis was seen. The cerebellar uptake was 1,39 (range 0,78 $1,85)$, SD 0,34 . There was a correlation between high sUV in the lungs and a high SUV in the cerebellum $(0,76)$. There are great differences between patients in the normal uptake of 11-C-methionine in both the lungs and the cerebellum. Patients with a high uptake of 11-C-methionine in the lungs also have a high uptake in the cerebellum indicating difficulties in comparing SUV values in normal organs between different patients.
564

M. Gasparini, G.L. Buraggi, C. Tondini, L. Maffioli

Istituto Nazionale dei Tumori, Milano Italy

\section{Tc-99m IMMU-LL2 MONOCLONAL ANTIBODY IN THE DIAGNOSIS OF NON HODGKIN'S LYMPHOMA'S} PATIENT. PRELIMINARY RESULTS.

Radioimmunodetection (RAID) with $99 \mathrm{~m}$ technetium labelled B cell lymphoma monoclonal antibody (MAb) was investigated in 8 patients ( 5 female and 3 male; age range $20-72$ years) with istologically proven non Hodgkin lymphoma (NHL). Of the 8 lymphomas, 5 were intermediate grade and 3 low grade. Whole body images with multiple planar views were obtained at $30 \mathrm{~min}, 4-6$ and 24 hours after the i.v. injection of $1 \mathrm{mg}$ IMMU-LL2-Fab (IMMUNOMEDICS,INC), labelled with 20-25 mCi (740-925 MBq) $99 \mathrm{~m}$ Tc. SPET of chest or abdomen was performed at 5-8 horus after injection in all patients. No adverse reactions were observed in any patient after MAb infusion and no appreciable change were seen in the blood counts, renal and liver function tests. A total of 17 from $18(94.4 \%)$ lymphoma lesions were detected by RAID All the tumor localizations were confirmed with clinical examination and with other imaging techniques, such as $\mathrm{CT}$ scan or MRI or gallium scan. In this series of patients no false positive results were noted and only 1 false negative result in a patient who had a mediastinal bulky disease. As regard the biodistribution of the immunoreagent we can do the following considerations :-) no appreciable marrow activity was seen in all patients -) spleenic targeting was demonstrated in all patients -) tumor to non tumor ratio ranged from 1.2 to 2.8 -) no difference of uptake was noted in different tumor grade. The images performed 24 hours after injection did not detect new lesions, but areas of doubful uptake were seen as positive focal areas in the delayed scan. In these preliminary results the LL2-Fab MAb seems to be useful for detection staging and follow up of NHL patients. 
565

A. Hamann, J. Kotzerke, K. F. Gratz, H. Dralle,

B. Soudah, H. Hundeshagen

Depts. of Nuclear Medicine and Biophysics, Surgery, Pathology, Medical School of Harmover, Germany

COMPARISON OF TC-99M-MIBI AND TL-201 IN THE FOLLOW-UP OF OXYPHILIC THYROID CARCINOMAS (OTC)

The application of Tc-99m-MIBI instead of T1-201 seems to be desirable because of a lower radiation exposure, a more favourable distribution and better image quality. Therefore we examined $17 / 117$ patients with OTC suspecting recurrent disease after thyroidectomy. Planar imaging of the whole body was performed 15-20 min. after administration of $74 \mathrm{MBr} T 1-201$ and $555 \mathrm{MBa} \mathrm{Tc}-99 \mathrm{~m}-\mathrm{MIBI}$. In addition all patients had a SPECT study of the neck and chest after planar imaging. 10/13 local recurrencies (LR) were detected planar with both radiopharmaceuticals (R). In one case the tumor was Tc-99m-MIBI-positive and Tl-201negativ. 2 LA could not be demonstrated neither by T1-201 nor by Tc-99m-MIBI scintigraphy. In case of distant metastases planar scintigraphy with both $R$ led to the tumor site $(n=9)$. Moreover 6 recurrent free patients as well as two patients with abnormal $\mathrm{Tg}$-serum-levels had a normal scintigraphy. That means: Tc-99n-MIBI true positive (TP) 20 , false negative (FN) 4, true negative (IN) 6 and false positive (FP) 0 ; T1-201 TP 19, FN 5, TN 6 and FP 0. SPECT was proved to be important for localisation of recurrent disease. Tumors in addition were not detectable. Quantification of the tumor uptake of T1-201 and Tc-99m-MIBI using the ROI-method led to a higher tumorbackground-ratio for Tc-99m-MIBI. The difference was proved to be highly significant ( $\leq \leq 0.0005$ ). TC-99m-MIBI planar imaging as well as SPECT showed a higher imaging quality compared to T1-201, especially in deeply situated tumor lesions.

In conclusion, Tc-99m-MIBI seems to be a promising alternative imaging agent in the follow-up of oxyphilic thyroid carcinomas.

\section{6}

H.Palmedo ${ }^{1}$,G.Meyer ${ }^{2}$, C.Dierke-Dzierzon ${ }^{3}$, P.Mallmann ${ }^{4}$, G.Schoeneich ${ }^{5}$, F.Grünwald $^{1}$,K.Reichmann ${ }^{1}$, A.Bockisch ${ }^{2}$, H.J.Biersack ${ }^{1}$

Dept. of Nucl.Med., Univ. of Bonn ${ }^{1}$,Dept. of Nucl.Med.,Univ. of Mainz ${ }^{2}$,Dept. of Anesthesiology ${ }^{3}$,Dept. of Gynecology $y^{4}$,Dept.of Urology $y^{5}$ Univ. of Bonn, Germany

Re-186 HEDP FOR PALLIATIVE THERAPY OF PAINFUL BONE METASTASES

in a prospective study, we evaluated the efficacy of Re-186 HEDP for the reduction of pain and consequently, the need for analgesics in patients with skeletal metastases. 20 single injections of $1295 \mathrm{MBq}$ were given to 18 patients (prostate cancer $n=10$, breast cancer $n=5$, lung cancer $n=1$, bladder cancer $n=1$, colorectal cancer $=1$ ) who received analgesics for the treatment of pain caused by multiple bone metastases (solitary metast. $n=2$ ). Tc-99m MDP bone scans (obtained before therapy) and Re-186 HEDP scans were used for quantitative analysis.

The mean Re-186 HEDP uptake in the skeleton was $19 \%$ of the injected dose. The average uptake in the bone metastases was found to be $3.1 \%$. There was a high correlation $(r=0.92)$ between the metastasis/normal bone-ratios of the Tc-99m MDP bone scans and the Re-186 HEDP scans. In two patients, we gave a second injection of Re-186 HEDP. There was no significant decrease in the mean Re186 HEDP uptake after the second injection.

After 1-2 weeks, 4 patients reported complete pain relief. A significant or slight reduction of pain was reported by 9 and 4 patients, respectively. In 4 cases there was no more need for analgesics after treatment with Re-186. 8 patients showed a marked decrease in the need for analgesics. The maximum interval of pain relief was 3 months. Half of the patients showed a reversible thrombopenia, in 2 cases we found a slight leukopenia. A flare reaction was reported by 7 patients $12-24$ hours after the injection.

We conclude that Re-186 HEDP uptake can be predicted by a Tc $99 \mathrm{~m}$ MDP bone scan before therapy. Therapeutical application of Re186 HEDP results in a significant relief of pain and decrease in the need for analgesics in patients with multiple bone metastases for a period of up to 3 months. No severe side effects could be observed.

\section{7}

P. Paulus, P. Rigo.

Division of Nuclear Medicine, C.H.U. LIEGE, BELGIUM

\begin{tabular}{llcll} 
DOSE & RATE & MEASUREMENT AND & \multicolumn{2}{l}{ TARGETED } \\
RADIOTHERAPY & WITH 186 RHENIUM-HEDP & FOR & THE \\
TREATMENT OF PAINFUL BONE METASTASES. & &
\end{tabular}

We have treated 21 patients with disseminated painful bone metastases with ${ }^{186} \mathrm{Re}-\mathrm{HEDP}$. Patients received $40 \mathrm{mCi}$ of $186 \mathrm{Re}-\mathrm{HEDP}$ with iterative readministration 3 to 4 months later in cases of a favourable antalgic response and after hematopoietic toxicity has subsided Rhenium-186 emits both beta and gamma rays. The beta emission provides the local irradiation of metastatic lesions while the gamma emission enables visualization of the tracer's biodistribution and dosimetric calculation. In all cases, conjugated view sequential whole body scintigraphy allows to calculate a cumulated activity curve. Knowledge of the physical characteristics of Rhenium-186 and of the accurate volume of a painful metastasic site (by tomodensitometry) associated with the activity curve allow dosimetric calculations to establish the dose rate and the total dose to the reference metastasis To appreciate the efficacy and toxicity of targeted radiotherapy, we also measure pain score, analgesic index and various biological parameters.

Analysis of these parameters suggest a correlation between the calculated dose rate and clinical and biological responses. We observe no favourable clinical response with dose rate under $0.2 \mathrm{~Gy} / \mathrm{hour}$ whereas dose rate over $0.5 \mathrm{~Gy} /$ hour are often associated with complete antalgic responses. This efficient dose rate, if not reached by the first administration, could be approached by the iterative administration of the radiopharmaceutical. An effective treatment increases the osteoblastic activity in the metastatic lesions which could increase the later uptake of the radiopharmaceutical, the dose rate and therefore progressively ameliorate the antalgic efficacy of the treatment.

\section{8}

Lluis Berná, Ignasi Carrió, Montserrat Estorch, Carmen Alonso, Belén Ojeda, Gustavo Torres.

Hospital de Sant Pau, Barcelona.

\section{BREAST CANCER PATIENTS WITH METASTATIC REFRACTORY BONE PAIN: ROLE OF STRONTIUM-89 FOR BONE PAIN PALLIATION.}

Thirteen patients with breast cancer and skeletal metastases who had bone pain refractory to opioid analgesics, and who were not eligible for or had not responded to local field radiotherapy, were treated with Sr-89. All patients had previous treatment with chemotherapy and radiotherapy for bone metastases. White cell count was $>3 \times 10^{9} / \mathrm{L}$ and platelet count was $>100 \times 10^{9} / \mathrm{L}$. Twelve patients had $>20$ bone lesions in the bone scan and 1 patient had 5 lesions. The time of evolution since the diagnosis of skeletal metastases had been made was $63 \pm 39$ months, range 22 to 135 . Severity of bone pain, mobility and dependency on analgesics were evaluated before, 4,8 and 12 weeks after $\mathrm{Sr}-89$ administration. Patients received $2 \mathrm{MBq} / \mathrm{Kg}(120-150 \mathrm{MBq})$ of $\mathrm{Sr}-89$ by i.v. injection.

Pain relief and a reduction in analgesic requirements were observed in 6 of $13(46 \%)$ patients 4 to 8 weeks after treatment: substantial improvement was observed in 2 patients and some improvement in 4 patients. Duration of the response oscillated from 3 to 7 months. Seven patients did not respond to Sr- 89 administration. A decrease in peripheral blood cell count was observed in 10 patients: a $15-66 \%$ reduction in white cell count and a $14-75 \%$ reduction in platelet count was detected at 12 weeks after treatment in these patients.

We conclude that strontium- 89 is useful for bone pain palliation in bone metastases from breast cancer. Dependency on opioid analgesics may be reduced in patients with refractory bone pain. 
Sunday, 21 August - Wednesday, 24 August 1994

569

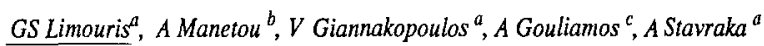
a. Nuclear Medicine Section, Areteion Univ Hosp, Athens, b. Nuclear Medicine Section, NMTS Hosp, Athens, c. Radiology Dept of Areteion Univ Hosp, Athens

\section{RENIUM-186-HEDP APPLICATION IN DISSEMINATED SKELETAL METASTASES}

The efficacy and toxicity of treatment with $1400 \pm 100 \mathrm{MBq}$ of Re186-HEDP [Mallinckrodt Medical B.V., Petten] were investigated in 27 patients who suffered from metastatic prostate, breast, bladder and colon cancer. The follow-up period was thirty weeks. The efficacy of the treatment was assessed by $(a)$ a pain and performance questionnaire that the patients were asked to complete daily and $(b)$ a CT scan comparison of a randomly preselected osseous lesion before and 30 weeks after Re-186-HEDP i.v. application. The response to treatment was also evaluated by using the Karnofsky Index.

Ten out of 12 patients with prostate cancer, 7 out of 9 with breast cancer, 2 out of 4 with bladder cancer and 1 out of 2 patients with colon cancer responded to therapy. Four patients became free of pain, 16 experienced obvious and 2 some improvement. No change was observed in 5 patients. Thirteen patients manifested a flare response to treatment, with increase in pain within the first 10 days after Re-186HEDP administration. All patients showed a definite and 6 of them a clinically important decrease of platelets and absolute number of polymorphonuclear white blood cells up to the fourth week following treatment. Three patients underwent a whole blood transfusion and in two of them neurologic side effects were observed lasting about 10 days. On CT scan comparison of the secondary osseous lesions an histopathologic improvement was noticed.

Re-186-HEDP appears to be a promissing new metal ion complex for the palliation of painful bone metastases, particularly in prostate and breast cancer.
571

D. Sandrock, C. Lexius, V. Ivancevic, A. Kögler, J. Djomotschko, A. Meller, D.L. Munz, D. Emrich

Department of Nuclear Medicine, Georg August University, Göttingen, Germany IS FLOOR CONTAMINATION A PROBLEM ON A
NUCLEAR MEDICINE THERAPY UNIT ?

Aim of this study was to yield data about floor contamination following radioiodine treatment at a nuclear medicine therapy unit.

Between 1990 and 1992, measurement of floor contamination was done in a nuclear medicine unit comprising six beds with 350 days running time per year by monitoring the floors three times a week with a xenon gas-chamber detector. During the three year observation period, 649 patients were treated with a total dose of $1147 \mathrm{GBq}$ (31 Ci) l-131. A value of $\geq 0.5 \mathrm{~Bq} / \mathrm{cm}^{2}$ was classified as "contamination".

Overall, 108 contaminations were detected, $94(87 \%)$ of them in the patients' rooms and $14(13 \%)$ in the remaining rooms, all of the latter sharing values below 5 $\mathrm{Bq} / \mathrm{cm}^{2}$. Classified by levels of contamination, $55(51 \%)$ were in the range between 0.5 and $5,37(43 \%)$ between 5 and 50 and $16(15 \%)>50 \mathrm{~Bq} / \mathrm{cm}^{2}$.

In conclusion, contaminations $>50 \mathrm{~Bq} / \mathrm{cm}^{2}$ (borderine for appropiate actions according to the German radiation safety order) occurred with a frequency of 16 events of 5250 bed-days $(0.3 \%)$ and, therefore, represent a rare event.

\section{0}

S.K. Shukla ${ }^{a}, b$, C. Cipriani ${ }^{a}$, H.L. Sharma ${ }^{c}$, G. Argiròn, G. Atzei ${ }^{a}$, S. Boemia, G.S. Limouris ${ }^{\text {, M. M. Cristallib }}$ a) Servizio di Med. Nucl., Osp. S. Eugenio, Roma; b) Ist. Cromatografia, C.N.R., Roma, Italy; c) Dep. Biophys. Univ, Manchester, U.K.; d) Nucl. Med. Athens Univ. Greece

ADVANCED LUNG CANCER THERAPY WITH Y-90 OR In-114m SPECIES

This articles reports the results of the treatment of lung cancer with cationic complexes of Y-90 or In-114m. Lung cancer patients in whom surgery, radiation therapy and chemotherapy had failed were admitted to the present radionuclide therapy. Radionuclide (Tc-99m, Ga-67, In111 and $\mathrm{In}-114 \mathrm{~m}$ ) species distribution in lung cancer patients showed that lung cancer and its bone metastases are radionuclide cationic complex affine. Y-90 and In-11.4m were found to give such complexes in citrate medium and have ideal therapeutic radionuclide properties. Three patients were chosen for Y-90 therapy and two for In-114m therapy. Initial dose of the radionuclide administered i.v. was $111 \mathrm{MBq}$ for $\mathrm{Y}-90$ and $74 \mathrm{MBq}$ for In-114m. Both radionuclides concentrated intensely and bound strongly to the primary and secondary cancer. Due to short half-life of Y-90, patients needed readministration of the radiopharmaceutical first at weekly and then at longer intervals for being free from bone pain. A single dose of In-114m on the other hand was found to keep the patient without pain for more than 3 months. Due to Ionger half-life, however, patients developed easily platelet depression. We are trying to overcome this disadvantage of $\mathrm{In}-114 \mathrm{~m}$ radiopharmaceutical. Lung cancer volume shrinked in patients of both groups. The patients are followed up with bone and Ga67 total-body scintigraphy. We have also observed complete remission of advanced lung cancer with cationic Pt(II) complex in patients kept on special diet:

\section{2}

S.K. Shuklaa,b, C. Cipriani a, G. Argiróa, G. Atzei ${ }^{a}$, F. Boccardi ${ }^{a}$ S. Boemi ${ }^{a}, K$. Schomäcker ${ }^{c}$, L. Ossicini ${ }^{b}$ a) Servizio di Med. Nucl., Osp. S. Eugenio, Roma; b) Ist. Cromatografia, C.N.R., Roma, Italy; c) Klin. u. Poliklin. Nuklearmedizin, Köln Universität, Germany

ADVANCED PROSTATE CANCER THERAPY WITH TUMOUR-AFFINE Y-90

Radionuclide cancer therapy has so far been limited to bone metastasis pain palliation with Sm-153-EDTMP, Re186-HEDP, Sr-89-Cl2, NaOrthophosphate-32 or Y-90 citrate solutions. Present paper reports the cure of 19 patients with tumour-affine $Y-90$ solution. The biochenical properties of prostate cancer and its metastases investigated with pure radionuclide (Tc-99m, Ga-67, Sm-153, and $\mathrm{Y}-90$ ) distribution in prostate cancer patients showed that prostate cancer is cation-affine while its bone metastases have high affinity for radionuclide anionic species. Studies of the complexes formed by $Y-90$ with citrate, EDTA, or DTPA as ligands showed citrate medium to be most suitable to obtain both cationic and anionic complexes of $Y-90$ for advanced prostate cancer therapy. Ninteen. patients with widely diffused bone metastasis, with PSA value above $250 \mathrm{ng} / \mathrm{ml}$ and in whom surgery, radiotherapy and hormone therapy had failed to arrest the growth of the disease were admitted to this Y-90 therapy. Depending on the seriousness of the disease, first dose given $i, v$. to the patient has been $111 \mathrm{MBq}$. Subsequent doses were $74 \mathrm{MBq}$ each week until the bone pain disappeared, PSA value returned to normal, and bone scintigrams showed no metastases. PSA value, bone scintigraphy, and blood analyses of the patients were examined first at 3 months interval and then half-yearly. No adverse effect of the therapy was so far observed. Patients resumed their normal activity. Follow-up time so far has been 2 years. 
Sunday, 21 August - Wednesday, 24 August 1994

\section{3}

Chr.Reiners, M.Lungwitz, A.te Poel, H.Schabram, K.Dutschka, H.H.Coenen

Clinic for Nuclear Medicine, University of Essen

DECREASE OF MONOMERIC SERUM CALCITONIN IN PATIENTS WITH HYPERTHYROIDISM TREATED WITH I-131

In the course of radioiodine treatment of hyperthyroidism, I131 , a beta emitter with a mean range in soft tissue of $0.5 \mathrm{~mm}$ possibly may damage $\mathrm{C}$-cells by irradiation which originates from neighboring follicular cells. Up to now, only few retrospective studies have focused on this question. We therefore started a prospective study in 15 patients with Graves' disease and 15 patients with functional autonomy. Plasma samples for the determination of calcitonin were drawn immediately before and 6 weeks after I-131 treatment. Since plasma calcitonin is undetectable in more than $50 \%$ of healthy persons, a solid phase silica gel extraction method was used to enrich monomeric calcitonin (mCT) in plasma. Afterwards mCT was measured by an immunoradiometric assay (CT-IRMA Medgenix).

The extraction procedure allowed to measure plasma $\mathrm{mCT}$ in all the patients studied. Comparing $\mathrm{mCT}$ levels before and after $\mathrm{I}-131$ treatment, a significant decrease from $6.3 \mathrm{pg} / \mathrm{ml}$ to $4.4 \mathrm{pg} / \mathrm{ml}$ could be observed in patients with Graves' disease $(P<0.05)$. However, in patients with functional autonomy, initial $\mathrm{mCT}$ levels were lower $(3.8 \mathrm{pg} / \mathrm{ml})$ as compared to Graves patients; no significant decrease could be observed after I-131 treatment $(3.4 \mathrm{pg} / \mathrm{ml})$.

The decrease of plasma mCT in Graves' patients after |-131 may be interpreted as radiation induced impairment of thyroidal C-cells. On the other hand, since this effect is not seen in patients with functional autonomy, initially higher levels of plasma mCT in Graves' disease may be disease specific in the meaning of a more relevant impairment of bone metabolism.

\section{4}

L.Mango*, M. Filesi, E. Paserio, G. Salerno, V.A.Righi, A. Signore and G. Ronga

S.S. Nuclear Medicine Clinica Medica II - University "La Sapienza" Rome - Italy

*Dept. of Nuclear Medicine - European Hospital - Rome

DIFFERENTIATED THYROID CARCINOMA (DTC): SURVIVAL FACTORS IN LUNG METHASTASES (IM) TREATED WITH 131-I

Aim of our work was to evaluate survival condictioning factors in patients with LM from DTC submitted to 131I therapy. We observed 90 patients (pts) (30 follicular, 38 papillary and 22 mixed-24 males 66 females - mean age $40.1 \pm 18.1$ years). Generally methastases were detected within one year from surgery, but in 21 cases this occured later (range 3-15 years). From the time of detection, we calculated survival curves dividing patients by age, sex, histology and 131 I uptake; then we compared obtained results at 5 , 10, 15 yrs intervals. Survival curve by age, under or over 45, shows a statistically significant longer extention of life for pts under 45 (Student two tailed t-test: $p<0.001$ at 5 and 10 yrs; $p<0.01$ at 15 yrs). We did not obtained same results by sex and histology. In the survival curve according to $131 \mathrm{I}$ uptake, we observed a good significance at 15 yrs ( $p<0.01$ ), that mostly reduces at following intervals for disappearence of pts with non uptaking lesions. The survival condictio ning factors in pts with DTC LM are primarely the age at time of methastases detection and the ability to concentrate 131I. As confirmation we observed age under 45 in all the 29 pts (32.2\% of all samples) in complete absence of disease at present time ( 5 yrs minumum from last $131 \mathrm{I}$ therapeutic administration mean dose $405.2 \pm 218.5 \mathrm{mCi}$ ).

In conclusion is confirmed the importance of more frequent controls in older pts, also using all other methods, for a precocious diagnosis of methastases and better results of radioiodine therapy.

\section{5}

E De Geeter, $H$ Andries, A Bossuyt, PR Franken.

Department of Nuclear Medicine.

Free University of Brussels (AZ VUB), Brussels, Belgium.

OPTIMAL COLLIMATOR CHOICE FOR COMBINED TC-99m/ I-123 STUDIES

Dual isotope studies with Tc-99m and $1-123$ may be useful for various organs, including brain and myocardium. For images abtained with each of the tracers to be comparable, it is important that activity ratios (activity in one part of the image / reference activity in the image) would be preserved by the imaging method. Therefore, we have used a Rollo phantom to study how collimator function affects such ratios.

All investigations were periormed with $1-123(p, 2 n)$. A Siemens Orbiter 3700 camera was used with either a HR or a ME collimator. With the ME used as a reference, the relative efficiency of the HR for $T \mathrm{c}-99 \mathrm{~m}$, as measured with a point source, was $71.9 \%$, and that for $1-123,133.4 \%$. This difference may be attributed to septal penetration. FWHM (mm) measured with a point source were :

distance source-collimator (air) HR 1-123 HR Tc-99m ME 1-123 ME Tc-99m

$\begin{array}{lllcc}0 \text {-collimator (air) } & \text { HR } 1-123 & \text { HR TC-99m } & \text { ME } 1-123 & \text { ME Tc- } \\ 0 \mathrm{~cm} & 5.1 & 5.7 & 5.2 & 4.5 \\ 8 \mathrm{~cm} & 7.6 & 7.6 & 10.4 & 10.0\end{array}$

$\begin{array}{ccccc}8 \mathrm{~cm} & 7.6 & 7.6 & 10.4 & 10.0 \\ \text { To investigate quantitative accuracy of planar images, } & 128^{* 128} & \text { images were }\end{array}$ acquired of a Rollo phantom, filled with an aqueous solution of either $234 \mathrm{MBq} \mathrm{Tc}$ $99 \mathrm{~m}$, or $292 \mathrm{MBq} \mathrm{l}-123$, and placed on the collimator surface with $8 \mathrm{~cm}$ of plex interposed. Pulse height windows were: for Tc-99m, 15\% around $140 \mathrm{keV}$, and $20 \%$ around $95 \mathrm{keV}$ (scatter); for $\mathrm{l}-123,15 \%$ around $159 \mathrm{keV}$, and $15 \%$ around $120 \mathrm{keV}$ (scatter). Activities were measured in ROIs corresponding to the cells of the phantom, and normalised to the maximum in the image.

For Tc- $99 \mathrm{~m}$, regardless of the collimator used, the ratios fairly well approximated the theoretical ones, based on the dimensions of the cells of the phantom. No further improvement was obtained by Jaszczak scatter correction with $\mathrm{k}=.35$.

For $\mathrm{l}-123$, the ratios obtained when the HR was used were consistently higher than the theoretical ones. The ME minimized the differences between the measured and the theoretical values. For $\mathrm{I}-123$ and the $\mathrm{HR}$, activity ratios were recalculated after the images were corrected for scatter using various values for $k$. On the assumption that septal penetration and scatter might induce a nonstructured homogeneaus background, we also performed homogenous background subtraction on the images using variaus threshald levels. All these methods, however, introduced further errors.

We conclude that for combined Tc- $99 \mathrm{~m} / 1-123$ studies ME is optimal, because of its higher quantitative accuracy. Spatial resolution, although less than for HR remains similar for both tracers when the ME is used.

\section{6}

B. Vollet, P. Petrusch*, J. Sciuk, O. Schober

Dept, of Nuclear Medicine, University Münster, *A\&S Agfa, Münster, Germany

\section{TRUE COLOUR DOCUMENTATION WITH DIGITAL} LASER TECHNOLOGY

Due to the development of digital gamma camera systems and the increasing importance of SPECT the percentage of digitally acquired images is growing in nuclear medicine. Digital acquisition demands large image matrixes up to $1024 \times 384$ pixels, therefore high resolution imagers are recommended, which are very expensive. Furthermore the integration of a documentation system as a network component is essential concerning the development of PACS, which offers image interpretation on digital workstations and the availability of patient and image databases.

After evaluation of the documentation systems commercially available, a colour laser copier was chosen and adapted to the required profile. The system offers:

grey scale and colour image documentation suitable for different medical imaging modalities with one system only,

image resolution comparable to analogues acquired scintigrams, grey scale linearity un to 256 shades with an optical density range $(0.1-2.0 \mathrm{OD})$ and an automatic adaptation on different colour scales, screen display appropriate to the printed image (WYSIWYG) via exact colour conversion by a 3D-colour space (CIE-XYZ) and Postscript-Standard,

network integration by Standard-Ethernet and software tools independent on hardware configurations,

reduction of operating costs and working time of staff for image generation,

use of environmentally friendly materials, abandonment of Developer/ Fixer solutions, X-ray films, colour foils and coated papers,

post processing of analogues acquired scintigrams and printing of unlimited copies.

The presented system has proven its usefulness in clinical routine. True colour printing combined with a high image resolution and the possibility of high sophisticated grey scale printing can be achieved very costeffectively. Displaying up to 256 grayshades for planar and tomographic images offers excellent conditions for image interpretation and might represent an adequate substitute or at least a complementary copy for the conventional X-ray film (NM, MRT, CT, US). 
577

M. Medvedec, S. Popović, B. Kasal, D. Huić, D. Ivančević

Department of Nuclear Medicine and Radiation Protection, University Hospital Center, Rebro, Zagreb, Croatia

\section{DESIGN AND USE OF A SLIT COLLIMATOR FOR WHOLE BODY COUNTER}

The Canberra Accuscan shadow shield whole body counter with horizontal scanning bed has $\mathrm{NaI}(\mathrm{Tl})$ detector and $\mathrm{HPGe}$ detector, both placed above a subject. A special parallel slit collimator for $10.2 \times 10.2 \times 40.6 \mathrm{~cm} \mathrm{NaI}(\mathrm{Tl})$ detector was designed to enable determination of radioactivity distribution in the subject and measurement of higher diagnostic activities.

The effects of collimator slit width on counting efficiency, spatial resolution, background and geometric uniformity were studied using gamma reference point and I-13I sources, Canberra/RMC Transfer phantom and BOMAB phantom. In vivo studies of patients after oral administration of I-131 were also performed to verify some results of phantom studies. Collimator opening was varied from 1 to $10 \mathrm{~cm}$.

Use of the collimator improves spatial resolution up to 20 times, decreases counting efficiency up to 200 times and background index up to 2 times. Total range of activities which can be measured with and without collimator is from $37 \mathrm{~Bq}$ to $37 \mathrm{MBq}$. Sum of line, Gaussian and Lorentzian distribution(s) gives the best fit of point spread function. No significant changes of geometric uniformity were indicated and different energy windows were discussed. Simultaneous measurement with both detectors is available regardless of additional shielding.

The whole body counter with new slit collimator is being used at the moment for quantitative profile scanning of patients having I-131 treatment after surgical thyroidectomy.

\section{8}

WH Thomson, AP Mills, S Williams and D Bray

Department of Physics and Nuclear Medicine, City Hospital NHS Trust, Dudley Road, Birmingham B18 7QH, UK.

\section{AUTOMATIC SCALING OF BONE IMAGES FOR DIGITAL DISPLAYS}

Modern gamma camera systems with digital displays usually scale an image to the maximum pixel value. On bone scans, this can lead to inappropriate scaling if hot areas (e.g. bladder) are present and staff must manually adjust the display maximum. We have examined the distribution of pixel count values in 30 normal bone studies with no hot areas. Histograms of the number of pixels against pixel count were created. Pixel count values of zero were omitted. The pixel count value at which $75 \%$ of the pixels had that value or below was determined (effectively, the "median" of the distribution but with $75 \%$ instead of $50 \%$ ). This was used since the median count was thought too low for accuracy.

For each view type the $75 \%$ count value was remarkably consistent (e.g. for anterior abdomen $23 / 30$ had the value of $12,5 / 30$ had the value 11 and $2 / 30$ had the value 13). Also,sampling only 1 in 4 pixels of a $256^{2}$ image gave the same $75 \%$ value, and significantly speeded up the calculation. The ratios of the maximum count to the $75 \%$ count for the 30 normals were: ant. abdomen 4.3 ; post. abdomen 3.9 ; post. pelvis 4.7 ; ant. shoulder 4.4 ; and post. skull 4.0 .

The scale maximum of an image is set by determining the $75 \%$ count value and multiplying by the appropriate ratio.

This procedure takes only a few seconds to run and allows a fully automatic protocol for the output of a standard bone study. We have found the technique reliable in clinical use; even with extensive metastatic spread, or even 'superscan', no adjustment from that set with the automatic procedure has been necessary.

\section{9}

H. Herzog, G. Schuth, H.W. Müller-Gärtner.

Institute of Medicine, Research Centre Jülich, and Department of Nuclear Medicine, Heinrich-Heine-University Düsseldorf, Jülich, Germany

THE IMPACT OF CALIBRATION ERRORS ON THE ACCURACY OF RATE CONSTANTS IN A THREE-COMPARTMENT MODEL USING EMISSION TOMOGRAPHY

The analysis of tracer kinetics including the calculation of rate constants requires the measurement of time-activity curves of the tracer concentration in tissue and plasma, which must be calibrated in respect to each other. In PET this calibration might be erroneous and in SPECT it might be doubtful due to fundamental problems of the tracer quantitation in tissue. The aim of this study was to investigate the dependency of rate constants resulting from a non-linear regression analysis on calibration errors.

Using a true arterial plasma curve of F18-fluorodeoxyglucose (FDG) an FDG-tissue curve was simulated assuming standard rate constants $\mathrm{K}_{1}, \mathrm{k}_{2}, \mathrm{k}_{3}$, and $\mathrm{k}_{4}$ and a vascular tracer signal based on a cerebral blood volume of $4 \%$. Calibration errors were simulated by multiplying the resulting tissue curve with an error factor $(E F)$ ranging from 0.5 over 1 (i.e. accurate calibration) to 1.5. In addition, these curves were distorted with different levels of gaussian noise. For each EF and noise level the curves were generated 10 times. All resulting curves were analysed with a standard non-linear regression fitting procedure based on the Marquardt-Levenberg algorithm.

When a noiseless curve was examined, $K_{1}$ and $C B V$ were regained correctly only for $\mathrm{EF}=1$ and were furthermore positively correlated with $\mathrm{EF}\left(\mathrm{r}^{2}=1\right)$. For $\mathrm{k}_{2}, \mathrm{k}_{3}$, and $\mathrm{k}_{4}$, however, the accurate values were obtained independent of EF. When noisy curves were fitted al parameters were different from their true values. $K_{1}$ and $C B V$ were still correlated to $E F\left(r^{2}=0.99\right.$ and 0.98 , respectively), but $k_{2}, k_{3}$, and $\mathrm{k}_{4}$ were not. The relative errors of the latter parameters were $10 \pm 10 \%, 13 \pm 24 \%$, and $180 \pm 95 \%$ over the range of EF from 0.5 to 1.5 when the noise level was raised to $6 \%$ of noiseless data.

Our results demonstrate that the determination of $\mathrm{K}_{1}$ in a threecompartment model depends on the accuracy of the calibration between tissue and plasma activity. The accuracy of determining $k_{2}$, $k_{3}$, and $k_{4}$ is in principle independent from calibration errors, but depends significantly on image noise characteristics.

580

D. Maintas*, R. Itti*, G. Bergeles**

*Laboratory of Biophysics. Medical School Alexis Carre 1, Lyon carrel,
of Atheratory of Aerodynamics. Tecnical University

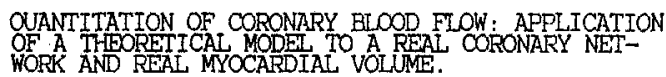

The aim of this study is to calculate the changes of coronary blood flow (CBF) using methods of coronary artery, wh a patient, with stenosis of LAD

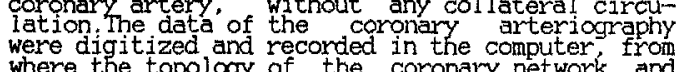
where the topology of the coronary network and branches were obtained. The diameter and density of the penetrating intThe diameter and density of the penetrating intof capillaries were obtained from existing bib of capiliaries were obtained from existing bibliographic cata. The blood viscosity is calculated difference of pressure between the aorta and left ventricle.used as pressure provoking the $\mathrm{CBF}$ during the cardiac diastole. The myocardial volume is Calculated from the tomographic slices of the TL-201 scint igraphy. The theoretical model for the various coronary branches is based on satisfying the equation of continuity at each node of the coronary network.

We assume that $\mathrm{CBF}$ is realized from the intramural arteries. which are divided according to a universal model of bifurcation in order to finiSh at the level of the capillaries. The diameter of the arteries is a function of the intramyocardial pressure and the dynamic viscosity is a Iunction the shear stress. cnder the above conditions we found that (a) The CBF is of the or der of $70 \mathrm{ml} / \mathrm{min} / 00 \mathrm{gr}$ of tissue (b) The pressure is dropped if the stenosis is greater than $80 \%$ inesunt a compensatory increase of the stenosis present $\mathrm{a}$ compensatory increase of the pressure. decreased dramatically for stenosis greater than $90 \%$ in surface. 
581

R.A.M KENGEN, D.A. PIERS

Dept. Nucl. Med., University Hospital Groningen, The Netherlands.

\section{OPTIMIZING DIAGNOSTIC PROCEDURES WITH ERROR} ANALYSIS

In most semi in vivo investigations (like cell-kinetic and clearance studies) the test result is obtained after doing several measurements (time, volume, activity) and combining them in a formula or curve (sometimes followed by curve analysis). In order to optimize these procedures, for example to determine the minimum amount of activity to be injected and the optimal counting time or to evaluate the precision of the end result, analysis of the propagation of errors is needed.

We evaluated two methods of error analysis:

1. the classical one in which propagation of errors was calculated according to standard formula's, which has disadvantages of being complex in case of complex calculations and when curve analysis has to be done. Furthermore this method requires that the interim results which are used in the calculation of the end result are independent of each other which is often not the case. 2 . Error analysis based on the simulation of spreading of the measurements according to a normal distribution (which is also an assumption with the classical method), which can be accomplished with aid of a simple spreadheat program, even in complex investigations.

We used both methods for optimizing renal clearance studies with Hippuran and thalamate to measure effective renal plasma flow (ERPF) and glomerular filtration rate (GFR). Method 2 tumed out to be very reliable, was much less subject to errors, was easier and more flexible than method 1 . For the ERPF and GFP determinations, the error analysis resulted in an improvement of the precision of the ERPF from 4 to $2,4 \%$ and a reduction in the thalamate amount which had to be administered of $50 \%$ without significant loss of precision.

We conclude that analysis of propagation of errors can be very usefult and is relatively easy with help of the error simulation method.

\section{2}

J. Hermanskáa ${ }^{a}$, J. Nèmec ${ }^{a}$, T. Blažek ${ }^{b}$, M. Kárnýc

${ }^{a}$ Faculty Hospital Motol and ${ }^{b}$ Institute of Medical Biophysics, 2nd Medical Faculty, Charles University, Prague

Institute of Information Theory and Automation, AV ČR, Prague BAYESIAN PREDICTION OF ${ }^{131}$ I KINETICS

Prediction of the amount of the accumulated activity within the patient body during diagnostics/therapy is a frequent task encountered in nuclear-medicine routine. Rather often, the prediction is based on estimates of effective half-life $T_{e f}$, i.e. the time during which activity within the region of interest falls to $1 / 2$ of its original value.

As a rule, estimates are based on least squares fit of the straightline in semi-logarithmic coordinates. Such a solution is susceptible to measurement errors, provides little information on the estimate reliability and neglects accessible prior information. These drawbacks are even enhanced when the point estimate is used for prediction of the future activity values.

Bayesian solution presented in the contribution removes the mentioned drawbacks by respecting the well defined probabilistic problem structure and by exploiting all informational sources available. Specifically, Poisson distribution of the recorded counts and well defined range of possible half-lives

$$
0<T_{e f}<T_{p}=\text { physical half-life of the radionuclide }
$$

are built-in the procedure. The proposed algorithm produces not only a half-life point estimate but complete description of its uncertainty even for a small number of measurements which are at disposal. The same outcomes can be gained for the predicted activities as they are simple deterministic functions of $T_{e f}$.

The claimed favourable properties of the procedure are demonstrated on a wide range of real-life data related to diagnostics/therapy of thyroid diseases by ${ }^{131} I$ and compared to the standard least squares fit.
583

M. Clausen, K. H. Bohuslavizki, J. Zimmermann, M. Schramm, W. Czapp, H. Wolf, W. Brenner, J.-U. Eberhardt, E. Henze. Departments of Nuclear Medicine and Radiotherapy (Radiooncology), University of Kiel, Germany

\section{SEPTAL PENETRATION IN THE SCATTER IMAGE: ARTIFACT-CORRECTION BY WEIGHTED PHOTON- PEAK IMAGE SUBTRACTION.}

Interaction of gamma radiation in tissue due to scatter may be shown by a comptonpeak image. The quality of this scatter image may be disturbed by septal penetration when using high energy photons. The purpose of this study is to minimize these artefacts by subtracting an appropriate fraction of the photonpeak image from the comptonpeak image.

A flat, rectangular acrylic glass phantom ( $490 \times 490 \times 37 \mathrm{~mm})$ was used as a water-equivalent scatter medium. A large-field of view gamma camera with a high energy all purpose collimator was completely covered by the phantom. Outside the field of view, but in contact with the lateral surface of the phantom, a $370 \mathrm{MBq}$ iodine-131 capsule was positioned. The energy windows were set at $364 \mathrm{keV} \pm 20 \%$ and $200 \mathrm{keV} \pm 60 \%$ for photonpeak and comptonpeak, respectively.

According to the hexagonal arrangement of the septa all images revealed radially arranged stripes of increased activity with an angle of 60 degrees between each of them (septal penetration). Subtration of 0.6 times photonpeak image from the scatter images resulted in an artefact-correction with well preserved scatter. The characteristics of these normalized counts as a function of distance $D$ (in $\mathrm{cm}$ ) from the source are:

$\ln ($ counts $)=0.11 * \mathrm{D}, \quad \mathrm{r}=0.998$ and SEE $=0.037$

The derived slope is close to the absorption coefficient of iodine-131 in water, thus, validating the proposed algorithm. Applications of these findings may be in dosimetry or in the developement of spatially variant absorption coefficient maps.

584

B. Bagni, I. Pareschi, R. Bentivoglio, N. Ghisi, A.R. Cavallini,

Nuclear Medicine Department. S. Anna Hospital, Ferrara, Italy.

A LOW COST WORKSTATION FOR PACS AND NUCLEAR MEDICINE.

Characteristics of workstations for use in digital images imaging have been investigates for many years. However, - much of this investigation has focused on the workstation in isolation, and has often been directed almost entirely at user interface issues. Certainly these issues are critical, but with the increasing use of commercial workstations it is important to look at the workstation in the context of the medical information environment and examine some important underlying characteristics required to meet the demands of digital radiology.

Medical View is a new software which permits to transform a personal computer (PC) in a powerful display workstation (DW) of a picture archiving and communication system (PACS). Medical view is based on a research carried out on a PACS installed at the st. Anna Hospital in Ferrara. The software kernel was enterely developed in C language on a 486 IBM compatible PC connected in LAN (local area network) to the PACS. The general structure of Medical View is imput images and data, data base mode, image processing mode, output images and data. The main menu of the image processing mode includes histogram treatment, contrast and gamma curves adjustement, pseudocolor enhancement, measures, magnify, optical lens and filters. The program is able to save images in windows 3.1 bitmap file format and to print images to a $3 M$ film laser printer. 
585

L.Tellmann, R.J. Seitz*, H. Herzog, H.W. Müller-Gärtner.

Institute of Medicine, Research Centre Jülich, Department of Nuclear Medicine and *Department of Neurology, Heinrich-Heine-University Düsseldorf, Germany

CALCULATION OF THE ARTERIAL INPUT CURVE FROM THE INTRACRANIAL CAROTID ARTERY FOR O15BUTANOL-RCBF-IMAGES

Quantitation of regional cerebral blood flow ( $\mathrm{rCBF}$ ) in studies with positron emission tomography (PET) using O15-butanol requires the precise determination of the arterial input curve. Usually, arterial data are obtained from the brachial artery using an automated blood sampling device. However, an arterial puncture bears the disadvantage of being invasive and thereby potentially dangerous to the subject. Therefore, we attempted to estimate the arterial input curve from the intracranial carotid artery that is regularly present in the lower two slices of our rCBF PET images.

In six $\mathrm{rCBF}$ studies on healthy volunteers, the arterial time activity curves recorded with a sampling rate of $1 \mathrm{~s}$ were corrected for decay, delay, and dispersion. Their maximums were reached after approximately $12 \mathrm{~s}$. The supraclinoidal part of the carotid arteries was identified on count rate images integrated over $40 \mathrm{~s}$. Mean values of 5 pixels bilaterally drawn in the centers of the carotid artery were analyzed dynamically with a sampling rate of $2 \mathrm{~s}$. The mean time activity curve calculated for the carotid arteries were corrected for decay. These curves were, however, heavily attenuated by the partial volume effect of the reconstructed PET images (full width half maximum, FWHM $=9 \mathrm{~mm}$ ). Using the diameter of the supraclinoidal carotid artery as given by Talairach and Tournoux (1988, Co-planar stereotaxic atlas of the human brain, Thieme) the recovery coefficient was calculated according to Mazziotta et al. (J Comput Assist Tomogr 5: 734-743, 1981).

Our results show that the decay and partial volume effect corrected blood curves of the intracranial carotid arteries closely overlapped with the decay, delay, and dispersion corrected blood curves of the punctured brachial artery.

In conclusion, we were able to calculate a reliable arterial input curve for quantitative $\mathrm{rCBF}$ images from PET image data. This approach i completely non-invasive, since it has the potential of allowing the calculation of functional rCBF images without need of arterial puncture.

\section{6}

J.T. Kuikka, J.B. Bassingthwaighte, R.E. Reneman, T. Arts.

Kuopio University Central Hospital, Kuopio, Finland, University of Washinton, Seattle, WA and University of Limburg, Maastricht, The Netherlands

MYOCARDIAL CAPILLARY EXTRACTION OF 2-DEOXY-DGLUCOSE IN THE ISOLATED RABBIT HEART

Positron emission tomography permits noninvasive measurement of regional fluorodeoxy glucose uptake (FDG) in the heart. The aim of the present study was to investigate the effect of myocardial blood flow on glucose extraction.

Double tracer dilution curves for an intravascular reference (I-123 RISA) and for a test tracer (H-3 labelled 2-deoxy-D-glucose) were sampled from coronary sinus of isolated Tyrode perfused rabbit hearts $(N=16)$ following bolus injection into arterial inflow. Myocardial blood flow ranged from 2.0 to $6.2 \mathrm{ml} / \mathrm{min} / \mathrm{g}$. Peak extraction of 2deoxy-D-glucose, $\mathrm{E}$ was calculated from equation: $E=1-C^{\prime}(t) / C(t)$, where $C^{\prime}(t)$ and $c(t)$ are the tracer concentrations at time $t$ for $H-3$ 2-deoxy-Dglucose and for I-131 RISA, respectively.

Peak extraction. $\mathrm{E}$ (MBF) (\%) was flow dependent in the exponential form: $E$ (MBF) $=52 \cdot \exp (-0.062 \cdot \mathrm{MBF})$ $(r=0.47 ; p<0.05)$ where MBF is myocardial blood flow $(\mathrm{ml} / \mathrm{min} / \mathrm{g})$.

The results suggest that the uptake of 2-deoxy-Dglucose into the heart is depending on myocardial blood flow (higher flow less uptake and vice versa). These changes may contribute to postischemic myocardial fluorodeoxy glucose uptake and FDG-injection should be delayed until the postischemic state.
587

A. Buck, G.Westera, C. Burger, K.Leenders++, G.K. v.Schulthess

Division of Nuclear Medicine, University Hospital, Zurich,+ PaulScherrer-Institute, Villigen,Switzerland.

MODELING ALTERNATIVES OF [11C]IOMAZENIL,

The purpose of this study was to find an appropriate mathematical model for the brain kinetics of [11ClIomazenil. Dynamic PET imaging was performed on 5 healthy young volunteers. Following injection of 150-200 MBq [11C]Iomazenil, a sequence of 16 scans were aquired until 90 minutes p.i. Arterial sampling was performed to determine the time course of the total plasma [11C] activity. The samples were corrected for metabolites to obtain the plasma input function using chloroform separation. Tissue time-activity curves were calculated over the following brain regions: occipital-(occ), frontal-(front) and cerebellar (cer) cortex, thalamus (thal), striatum (str) and pons. Tracer kinetic modeling was performed employing a 2-compartment (plasmaand 1 tissue compartment, $\mathrm{K} 1, \mathrm{k} 2$ ) and a 3-compartment model (plasma- and 2 tissue compartments, $\mathrm{K} 1^{\prime}-\mathrm{k} 4^{\prime}$ ). Parameter $\mathrm{K} 1$ $[\mathrm{ml} / \mathrm{min} / \mathrm{g}]$ reflects blood-to-brain transport, the distribution volume (DV, DV') was calculated to represent receptor density (DV=K1/k2

$\left.\mathrm{DV}^{\prime}=\mathrm{k} 33^{\prime} / \mathrm{k} 4^{\prime} * \mathrm{~K} 1^{\prime} / \mathrm{k} 2^{\prime}\right)$. Indicated are mean values and std.

$\begin{array}{llllllll} & & \text { occ } & \text { front } & \text { cer } & \text { thal } & \text { str } & \text { pons } \\ \text { K1 } & \text { 2-comp } & 0.34 & 0.30 & 0.29 & 0.29 & 0.24 & 0.23 \\ & & (0.09) & (0.08) & (0.08) & (0.10) & 0.08) & (0.09) \\ \text { K1' } & 3 \text {-comp } & 0.42 & 0.38 & 0.35 & 0.41 & 0.31 & 0.32 \\ & & (0.13) & (0.15) & (0.11) & (0.18) & 0.10) & (0.11) \\ \text { DV } & 2 \text {-comp } & 27.6 & 22.6 & 14.4 & 9.24 & 7.1 & 4.2 \\ & & (7.9) & (9.5) & (6.5) & (3.7) & (4.8) & (4.6) \\ \text { DV' } & 3 \text {-comp } & 27.6 & 20.5 & 11.7 & 7.6 & 5.4 & 3.4 \\ & & (12.6) & (9.4) & (4.5) & (3.4) & (4.5) & (3.0)\end{array}$

With both models the pattern of the regional DV values reflects the known distribution of the benzodiazepine receptors. F-test statistics revealed a significant improvement in the fit using the 3-compartment model in all regions $(\mathrm{p}<0.05)$.

Conclusion: Of the 2 models testet, the 3-compartment model is mathematically superior. However also the 2-compartment model yields quantitative information of the receptor density. This opens the possibility to calculate parametric DV maps on a pixel-by-pixel basis.
588

E. Turkheimer, V, Bettinardi, R.M. Moresco, M.C. Gilardi, G. Rizzo, A. Cardaioli, G. Lucignani, F. Fazio.

INB-CNR, H S.Raffaele Institute, University of Milan, Italy.

PARTIAL VOIUME CORRECTION IN [F18] FLUOROETHYLSPIPERONE STUDIES WITH PET. AN AUTOMATED METHOD FOR IMAGE ANALYSIS.

An automated method for the correction of partial volume effect in [F-18] fluoroethylspiperon studies with PET was imolemented. The procedure corrects the striatal uptake of the tracer by fitting the activity profile driven across the middle of putamen with a model of one-dimensional activity variation as described by Dan-Chu Yu et a1. (J.Nucl. Med 1993;34:679:687). The performance of the model was evaluated on a 3 dimensional model of the striatum created from an MRI segmented brain through mathematical simulation driving to a degradation of the EWHM of the model from the experimental $(8.5 \mathrm{~mm})$ to the value of $10.8 \mathrm{~mm}$ for an optimal recovery of counts. The three-dimensional model was then used for the simulation of errors due to noise, profiles' mispositioning variations in volume orientation.since the method revealed to be sensitive to the positioning of the profiles, an automatic 'profile positoning' procedure was developed. With a signal to background ratio of $4: 1$ the method gives an estimate of the true value of activity with a $5 \%$ error. Besides, the procedure was designed for a time-saving application in dynamic studies since the profile's center and width, are identified from the best statistics frame so that the model can be applied to the other frames through a fast linear regression. 


\section{9}

H.Herzog, A.Berner, B. Lipinski, K. Ziemons, E. Rota Kops.

Institute of Medicine, Research Centre Jülich, Jülich, and Department of Nuclear Medicine, Heinrich-Heine-University Duisseldorf, Jülich, Germany

TWO-DIMENSIONAL PREFILTERING OF SINOGRAMS IMPROVES THE SIGNAL/NOISE RATIO OF PET-IMAGES

The two-dimensional (2D) prefiltering of scintigraphic projection images yielded a considerable improvement of the signal/noise ratio (SN) of reconstructed SPECT images (M. King et al., 1984). Our work investigated whether a similar approach can be applied to PETring systems, where the projection data are not primarily two-dimensional, but recorded as one-dimensional projection profiles.

The one-dimensional projection profiles acquired from neighbouring angles inside the PET detector-ring are highly correlated in respect to their signals, but less to noise. If the neighbouring profiles are assembled in a 2D matrix, i.e. as a 2D sinogram, a 2D filter can be applied. For the 2D prefiltering a 3 by 3 kernel with symmetric weights of 1,2 and $x$ was chosen, where the central weight $x$ was varied from 4 to 16 and applied to the data of neighbouring projection angles, i.e. to the 2D sinogram data. The following 1D filter step of the filtered back projection used only a minimum low pass filtering (i.e. with cut-off frequency $=$ Nyquist frequency). For comparison, the conventional
ID low pass filtering without any $2 \mathrm{D}$ prefiltering was done with different lower cut-off frequencies. The approach was tested with simulated noise-containing projection data of a line source whose amplitude after filtering (before reconstruction) was examined. Furthermore, a phantom was scanned, in which both a line source and a homogeneous distribution of F-18 was installed. The maximum pixel value of the line source in the reconstructed image was regarded as a measure for $S$ and the variation inside the homogeneous image area as a measure for $\mathrm{N}$

The amplitude of the line source in the simulated projection data was $29 \%$ higher for $2 \mathrm{D}$ prefiltering with a central weight of 12 than for the 1D approach, although noise was decreased. The phantom study showed also an advantage of the $2 \mathrm{D}$ prefiltering. For $x$ equal 4 the $2 D$ prefiltering yielded an $\$ 10 \%$ higher than that with the conventional filtered backprojection - without any enhancement of $\mathrm{N}$.

In conclusion, also for PET ring-cameras the simple method of a 2D prefiltering of the projection data leads to an improvement of $S / N$ of reconstructed images. The 2D filter kernel must be designed in respect to the statistical characteristics of the projection data.

\section{0}

P.D. Acton, A. Jeavons*, D.L. Hastings, M.L. Waller, J.C. Hand, J.V. Moore and D. Crowther

Departments of Medical Physics and Oncology, Christie Hospital, Manchester, UK, and ${ }^{*}$ Oxford Positron Systems, Oxon, UK.

MEASUREMENT OF REGIONAL TUMOUR FUNCTION USING HIGH RESOLUTION POSITRON EMISSION TOMOGRAPHY (PET)

Advances in High Density Avalanche Chamber (HIDAC) technology, incorporating a new design of photon converter, have resulted in reliable detectors with improved sensitivity and high spatial resolution. The intrinsic spatial resolution of the detectors has been measured as $1.5 \mathrm{~mm}$ corresponding to a resolution of $2.7 \mathrm{~mm}$ in the reconstructed image in both transverse and axial planes. A prototype clinical PET scanner incorporating such detectors has been used successfully for high resolution volume imaging of head and neck lymphomas in patients using 18-FDG and 64-Cu-PTSM, and for tumours xenografted onto mice using 18-FDG. A total of 24 mice tumours were regionally damaged to varying extents with photodynamic therapy (PDT). The distribution of FDG uptake in PET images at 24 hours following PDT was identical to the distribution of viable tumour cells identified by histology, even where only a layer of 10-20 viable cells remained.

It is concluded that the high resolution capability of this type of PET system has potential for clinical and research applications where high contrast uptake in small volumes is expected, such as in oncology.

\section{1}

D. Ros, J. Pavía, A.M. Catafau, F. Lomeña, M. Huguet and $J$. Setoain.

Biophysics and Bioengineering Lab. Fac. Medicine, University of Barcelona. Nuclear Medicine Dep. Hospital clinic i Provincial of Barcelona. Spain.

REALIGNMENT OF NEUROACTIVATION BRAIN SPECT

SPECT neuroactivation studies imply the acquisition of two sets of images, basal and activation studies, which have to be registered before quantitative comparison. In this work we analyze the full 3D-realignment including translational as well as transverse, coronal and sagittal rotational corrections.

Two methods of alignment were studied. The first one is based on Principal Axes Transformation (PAT). The second one uses the results of the first method as initial values to start a leastsquares iterative process (LS) based on a downhill simplex algorithm to search for the maximum value of the correlation function in brain. The unwanted activity corresponding to salivary glands, mouth and activity below the cerebellum, was eliminated by using a mask built as an intersection of three masks drawn in the $x$, $y, z$, directions.

Both methods were tested with simulated and real studies. The results of the PAT method showed a maximum translation exror of $0.3 \pm 0.1$ pixels and a rotational error of $1.2 \pm 0.7$ degrees in a total of 100 runs. For LS method these errors were $0.2 \pm 0.1$ and $0.6 \pm 0.3$. The realignment for 11 real studies was assessed by three expert observers. The alignment was found satisfactory in all cases for LS method, and in 6 cases for PAT method. We conclude that the combination of the PAT and LS methods is a valid solution for the realignment of SPECT neuroactivation studies.

\section{2}

C. Falcón ${ }^{1}, \underline{D} \cdot \operatorname{Ros}^{1}$, I. Juvells ${ }^{2}, J \cdot$ Pavía $^{3}$

${ }^{1}$ Lab. Biophysics and Bioengineering, ${ }^{2}$ Lab. optics. University of Barcelona. ${ }^{3}$ Dept. Nuclear Medicine, Hospital Clinic P., Barcelona, Spain. RELAXATION PARAMETERS IN SPECT ITERATIVE RECONSTRUCTION ALGORITHMS

Algebraic reconstruction techniques (ART) have been intensively studied in image reconstruction. Despite ART enjoys a rapid convergence, the reconstructed images often exhibit a characteristic salt-and-pepper noise when applied to emission tomography. In this work we study the influence of a relaxation parameter $(\alpha)$ that yields smoother images while maintaining a good convergence. Each pixel value was updated according to the expression: $\quad x_{j}{ }^{k}=x_{j}{ }^{k-1}+\alpha\left[\left(p_{j}-\Sigma x_{i}^{k-1} w_{k i}\right) / \Sigma w_{k i}{ }^{2}\right] w_{k j}$.

The study included simulated and real SPECT data by using a Jaszczak phantom. In simulated studies scattering, attenuation, noise and PSF of a general purpose collimator were included. Relaxation factors ranging between 0.02 and 0.4 were considered. Total counts in projections were $4 \times 10^{5}$. The goodness of the reconstructions was assessed by using the correlation coefficient between the pattern and the actual reconstruction (CC), the background coefficient of variation (CV) and the contrast ( $\mathrm{CON}=(\mathrm{S}-\mathrm{B}) /(\mathrm{S}+\mathrm{B})$ ) and signal-to-noise ratio $\left(\mathrm{SNR}=(\mathrm{S}-\mathrm{B}) / \sigma_{\mathrm{B}}\right)$ of each circle.

The results over 10 runs for each relaxation factor showed the existence of a value near to 0.1 which yields images with good values of the parameters (CC:0.96, CV: 17\%, and CON:0.86 SNR: 4.9 for the circle of $5 \mathrm{~cm}$ of diameter). The results from the real phantom, (CC: $0.93, \mathrm{CV}: 20 \%, \mathrm{CON}: 0.87$, SNR:3.9) in agreement with those of the simulated studies, reinforce the applicability of an optimum relaxation factor in SPECT reconstruction. 
Sunday, 21 August - Wednesday, 24 August 1994

593

G. Berding, B.O. Knoop, K.F. Gratz, H. Kolbe, R. Dengler, H Hundeshagen

Abteilungen für Nuklearmedizin und Nuklearmeßtechnik und Neurologische Klinik, Medizinische Hochschule Hannover, Germany

FILTERED BACK PROJECTION AND ITERATIVE RECONSTRUCTION OF ${ }^{123}$ I-IBZM SPET STUDIES

The aim of this study was to assess the influence of reconstruction methodology in ${ }^{123}$-IBZM SPET on: (i) the statistical quality of the tomograms (total counts), (ii) the relative size of the striatal uptake (pixels in $80 \%$ isocontour ROI) and (iii) the semiquantification of uptake (striatum to frontal cortex (ST/FC) ratios). Results of iterative reconstruction (ISA) with different numbers of iteration steps and exponents and filtered back projection using low-pass or Metz prefilters with different order numbers (i.e. different suppression of higher spatial frequencies) were compared in ten ${ }^{123}$ I-IBZM SPET studies. In iterative reconstruction with the exponents one and two within the first 8 iteration steps, a variation of the reconstructed counts in tomograms on the level of the striatum between 130000 and 1900000 (mean values of ten studies) was observed. Eight iterations with the exponen 4 resulted in a loss of image information $(0$ counts in "reconstructed" tomogram) in 8 of the 10 studies. Filtered back projection led to a smaller variation of reconstructed counts ( 710000 to 840000 with the Metz and 630 000 to 780000 with the low-pass filter). Regarding the relative size of the striatum a reduction of size occurred with more iteration steps and higher exponents. Analogously a smaller representation of the striatal uptake was observed with higher filter order numbers (i.e. lesser suppression of higher spatial frequencies). Using iterative reconstruction with an exponent one, the mean ST/FC ratios were increased significantly $(p<0.0005)$ from 1.17 to 1.64 within the first 8 iteration steps. With the exponent two a ratio of 1.64 was reached already after 3 iteration steps. With an exponent of four after 3 iterations a mean $\mathrm{ST} / \mathrm{FC}$ ratio of 1.70 was obtained and in individual cases ratios higher than 2.00 were observed. Applying filtered back projection led to lower mean ratios (highest mean 1.66 with the Metz and 1.53 with the low-pass filter, in no case ratios of 2.00 or higher were observed). It is concluded that reconstruction methodology has a fundamental influence on image statistics, representation of the size of the striatum and semiquantification in ${ }^{123}$ I-IBZM SPET. Although iterative reconstruction with inadequate parameters may produces unreliable results, possibly a higher recovery can be achieved with iterative methods in comparison to filtered back projection.

\section{4}

\section{E. Voth, R. Bachmann, S. Marohl, H. Schicha}

Dept. of Nuclear Medicine, University of Cologne, Cologne, Germany

\section{END-DIASTOLIC GATED MIBI-SPECT: IMPROVED DETECTABILITY OF MYOCARDIAL INFARCTION ?}

Due to wall motion, ungated myocardial SPECT imaging may underestimate extent of myocardial infarctions. Aim of this study was to compare end-diastolic gated and ungated myocardial SPECT images concernig infarct detection, extension and severity

In 61 patients with coronary heart disease, gated SPECT (8 frames per cardiac cycle) was performed after injection of $740 \mathrm{MBq}$ $99 \mathrm{~m}$-Tc methoxy-isobutyl-isonitrile (MIBI) using a triple head SPECT camera (Picker Prism 3000). Ungated SPECT images were generated by adding the 8 acquired frames. Using low-pass prefiltering and reconstruction by filtered back-projection, gated and ungated cardiac short and long axis tomograms were produced. A standardised 14-segment scheme was used for grading of segmental myocardial MIBI-uptake (normal, mild reduction, severe reduction, absent uptake)

$739 / 854$ segments were graded identical from gated and ungated tomograms ( 615 normal, 25 mild reduction, 34 severe reduction, 65 absent uptake). Of the 115 segments graded discordantly, MIBIuptake was inferior in the gated tomograms in 102 segments, in the ungated images in 13 segments. Differences between gated and ungated images reached statistical significance (McNemar-test, $\mathrm{p}<0.05$ ) in apical, midventricular and basal segments of the inferior wall, apical and midventricular segments of the anterior wall and the midventricular segment of the inferoseptal wall. Out of the 102 segments graded inferior in the gated study, 31 corresponded to new infarct locations, 38 to extension of an infarct in a neighbouring segment and 33 to more marked infarctions in the gated study.

Compared to ungated imaging, end-diastolic gated SPECT is more sensitive in the detection of myocardial infarctions. Whether all abnormalities in gated SPECT reflect myocardial pathologies or are due to photon absorption (inferior wall) or low count statistics remains aim of further investigations.
595

V.Mongioj $j^{1}$ C.Pettinato ${ }^{1}$, L.S.Maffioli ${ }^{2}$, A.Chiti ${ }^{2}$, M.R.Castellani ${ }^{2}$, A.E.Sichirollo1, G.L.Buraggi ${ }^{2}$ 1 Health Physics Div., 2 Nuclear Medicine Div. of National Cancer Institute - Milan - Italy

Image contrast and lesion detection with $99 \mathrm{~m}_{\mathrm{Tc}}$ and $131^{I}$ in single photon emission tomography Lesion detection by a SPET system depends, principally, on image noise and contrast. Image contrast, in particular is proportional to the lesion-background uptake ratio and is degradated by finite spatial resolution and compton-scattered photons. The aim of the study was to analyze the relationship between lesion detection and image contrast for two radionuclides ( $99 \mathrm{~m}_{\mathrm{Tc}}$ and $13 \mathrm{I}_{\mathrm{I}}$ ) commonly employed in SPET studies. Eor tomographic acquisitions we used a Plexiglas phantom containing hollow spheres of different sizes.The radioactive concentrations inside the spheres were $5,3,2$ times the background concentration to simulate typical tumor/background uptake ratios. The projection data were, then, reconstructed with different filters (Shepp-Logan, Harming and 4 Butterworths) to analyze in which way a filter acts on noise level and image resolution.

Data analysis showed us that image contrast is proportional to the objects size and the lesionbackground concentration and that the contrast values are lower for images obtained with $131_{I}$. Moreover, objects smaller than $20 \mathrm{~mm}$ in diameter were not detectable in $131_{I}$ studies and were visualized in $99 \mathrm{~m}_{\mathrm{TC}}$ acquisitions only with the higher concentration ratio. As regards filters, Hamming and Butterworth with medium cut-off frequency $(0.25$ cycles/pixel) and high order values allowed to obtain high contrast images with a minimal noise increase in all studies. In conclusion, experimental evaluations and a cuantitative analysis by phantoms can give some indications about the possibilily to visualize pathological regions and to optimize the reconstructed image.

\section{6}

Th. Benoit, D. Vivegnis, S. Braat, R. Itti, A. Lahiri, P. Gomez, J. Foulon, P. Rigo.

Division of Nuclear Medicine, CHU Liege, Liege, Belgium.

\section{STRESS/REST TECHNETIUM-99m TETROFOSMIN SPECT : DEFINITION OF CRITERIA FOR ABNORMALITY USING AN AUTOMATED QUANTITATIVE ANALYSIS PROGRAM.}

Same day stress/rest $99 \mathrm{~m}_{\mathrm{Tc}-t e t r o f o s m i n}$ (Myoview ${ }^{\mathrm{TM}}$ ) myocardial perfusion tomographic images were obtained in 51 patients (pts) suspected of coronary artery disease and in 24 low likelihood $(<5 \%)$ volunteers constituting the reference normal data base. Scans were read visually by 4 investigators and by the quantitative analysis program $(Q)$. The program samples the entire left ventricle relative to the maximal uptake value. Sampling is homogeneous along the length of each radial slice ventricular contour and data are corrected for surface distortion resulting from planar projection. Threshold for abnormality is fixed pixel by pixel as normal mean (NM) - $x$ standard deviations (SD). Defect severity is "NM - pt value over NM - pt background" in abnormal regions. Ischemic size index (ISI) is defect surface $\mathrm{x}$ defect severity. ROC curve analysis using angiography as the standard was used to determine the optimal SD and regional ISI for individual vessel lesions detection. The following sensitivities (Sn), specificities $(\mathrm{Sp})$, positive predictive accuracies (PPA) and negative predictive accuracies (NPA) were obtained by $\mathrm{Q}$ and visual (V) analyses:

\begin{tabular}{|c|c|c|c|c|c|c|c|c|}
\hline$\geq 50 \%$ stenoses & \multicolumn{2}{|c|}{ Sn (\%) } & \multicolumn{2}{|c|}{ Sp (\%) } & \multicolumn{2}{|c|}{ PPA (\%) } & \multicolumn{2}{|c|}{ NPA (\%) } \\
\hline & $\mathbf{Q}$ & V & Q & V & Q & V & Q & V \\
\hline $\begin{array}{c}\text { LAD (n=35) } \\
(Q: 2.5 \text { SD; 1\% ISI) }\end{array}$ & 54 & 43 & 87 & 94 & 90 & 94 & 47 & 43 \\
\hline $\begin{array}{c}\text { LCX (n = 23) } \\
(Q: 3.7 \text { SD; } 4 \% \text { ISI) }\end{array}$ & 52 & 26 & 79 & 96 & 67 & 86 & 67 & 79 \\
\hline $\begin{array}{c}\text { RCA (n=27) } \\
(Q: 2.1 \text { SD; 6\% ISI) }\end{array}$ & 78 & 77 & 75 & 54 & 78 & 66 & 75 & 68 \\
\hline ALL (n $=85)$ & 61 & 49 & 79 & 81 & 79 & 83 & 62 & 61 \\
\hline
\end{tabular}

We conclude that quantitative analysis with optimized criteria improves the sensitivity of SPECT tetrofosmin to detect individual vessel lesions. 


\section{7}

W. Brenner, *V. Streit, *B. Lange-Aschenfeldt, K.H. Bohuslavizki, H. Wolf, J.-U. Eberhardt, M. Clausen, E. Henze

Clinic of Nuclear Medicine and *Clinic of Dermatology

Christian-Albrechts-University, Kiel, Germany

QUANTIFICATION OF ANGIOGENESIS IN A RAT MODEL BY MEASURING THE WASHOUT OF XENON-133

Quantification of neovascularization due to angiogenic factors like interleukines is difficult when only using histological techniques. However, the evaluation of tissue perfusion is a classical task of nuclear medicine. In this study the washout of Xenon-133 as an established indicator for blood flow was applied to evaluate angiogenesis quantitatively.

Two polyester foam sponges containing either interleukin 8 (IL-8) as angiogenic factor or placebo were implanted subcutaneously in the back of 5 rats. After $4,9,13,20$, and 40 days $0.1 \mathrm{ml} \mathrm{Xe-133}$ gas with a mean activity of $1 \mathrm{MBq}$ was injected first into the IL-8 sponge and 3 hours later after complete Xenon washout into the placebo sponge of the same rat. Immediately after injection the radioactivity of the sponge region was measured up to $20 \mathrm{~min}$ p.i. in 1 min intervals using a collimated gamma-scintillation detector. Washout data of $\mathrm{Xe}-133$ are given in percent of injected activity. Subsequently, the sponges were dissected and examined histologically.

In the following table only washout data of Xe-113 on day 13 are given as an example for IL- 8 induced angioproliferation between day 4 and 20 :

\begin{tabular}{|c|c|c|}
\hline time p.i. & IL-8 & placebo \\
\hline $\mathbf{5} \mathbf{~} \mathbf{~ i n}$ & $16.7 \%$ & $13.2 \%$ \\
\hline $\mathbf{1 5} \mathbf{~ m i n}$ & $39.6 \%$ & $26.1 \%$ \\
\hline
\end{tabular}

The corresponding half-life periods of Xe-133 washout on day 13 due to exponential curve fitting were $17.3 \mathrm{~min}$ and $34.7 \mathrm{~min}$ for IL8 and placebo, respectively. Histological data confirmed increased neovascularization in $\mathrm{KL}-8$ sponges compared to placebo.

Thus, measurement of Xe-133 washout seems to be a valid and easy-to-perform technique for quantitative evaluation of pharmacological effects of angiogenic factors in experimental models.

\section{8}

T. Kranert, O. Hörsch, F. D. Maul B. Wagenknecht, C. Gürtner, G. Hör

Klinikum der Johann Wolfgang Goethe-Universität Frankfurt, Abteilung Nuklearmedizin

\section{FURTHER DATA ON EXCRETION OF I-131 IN SWEAT AND SALIVA DURING RADIOTHERAPY}

The aim of the study was to examine the contamination of the environment from patients during and after radiotherapy with I-131 by measuring the excretion of activity in sweat and saliva.

For analysing the time-course of activity, sweat data from 8 different fixed parts of the body were derived and also from samples of saliva. From patients suffering from hyperthyrodism (HY) or thyroid cancer (TC) sweat activity was collected by plasters utilizing a cotton layer. Saliva was sampled after rinsening the mouth.

In saliva a $\mathrm{I}-131$ content up to $1 \mathrm{Mbq} / \mathrm{ml}\left(2.710^{-4} / \mathrm{ml}\right.$ of the applied activity) was measured during the first day after therapy in TC-Patients Differences in I-131 excretion between TC- and the HYP- patients is given in table 1 .

\begin{tabular}{|c|c|c|c|}
\hline \multicolumn{2}{|c|}{ Saliva } & \multicolumn{2}{|c|}{ Hands } \\
\hline $\begin{array}{l}\text { 1-st day } \\
/ 10^{-6} / \mathrm{ml}\end{array}$ & $\begin{array}{l}\text { last day } \\
/ 10^{-6} / \mathrm{ml}\end{array}$ & $\begin{array}{l}\text { 1-st day } \\
/ 10^{-8} / \mathrm{cm}^{2}\end{array}$ & $\begin{array}{l}\text { last day } \\
/ 10^{-8} / \mathrm{cm}^{2}\end{array}$ \\
\hline $\begin{array}{l}9927 \\
16347 \\
1.65\end{array}$ & $\begin{array}{l}1123309 \\
7322 \\
0.065\end{array}$ & $\begin{array}{l}3.42 .1 \\
1.140 .55 \\
0.38\end{array}$ & $\begin{array}{l}6327 \\
43.5 \\
0.063\end{array}$ \\
\hline
\end{tabular}

Tab.1: Comparison of mean excretion of TC and HYP patients relative to the Tab.1: Comparison of mean excretion of discharge)

The results show that hyperthyroid patients excret a higher relative amount of activity in the early phase of their treatment via saliva. Conclusion: A relative higher risk to contaminate the environment and a relative higher exposure of the salivary glands can be assumed. Before discharge, however, most of the rest activity is stored in the thyroid in this patients and thus is not available for excretion by saliva or sweat (see table 1).
599

G.Kampf , G. Knop, U. Wenzel, G.Wunderlich , R. Bergmann* W. -G. Franke

Clinic and Policlinic of Nuclear Medicine, University Clinics "Carl Gustav Carus", Technical University of Dresden

*Institute of Bioanorganic and Radiopharmaceutical Chemistry, Research Centre Rossendorf near Dresden

STUDIES OF RADIOMETAL UPTAKE BY CULTURED NORMAL AND TUMOUR CELLS

Basic studies of $169 \mathrm{yb}$ uptake in normal ( $\mathrm{V} 79 / 4$ ) and KTCTL-2 cells(Heidelberg) were started with the aim of understanding and increasing accumulation of radiometals in tumours. Cellular uptake of $169 \mathrm{Yb}$ given as citrate or NTA complex is linearly dependent on tracer concentration and incubation time, but is independent of the cell density per petri dish. $169 \mathrm{Yb}$ uptake is dependent on the metabolic activity of the cell line (checked by [ ${ }^{18}$ F]FDG) as well as on the ligand species: the stronger-metabolizing V79 cells take up more ${ }^{169} \mathrm{Yb}$ from the citrate complex than the weaker-metabolizing tumour cells, whereas from the NTA, EDTA, and DTPA complexes the uptake is higher by the tumour cells, which does not correlate with the metabolic activities.

By both cell lines by one order more $169 \mathrm{Yb}$ is taken up from the citrate complex than from the aminocarbonic acid complexes.

The results suggest the existence of different transport or uptake mechanisms i.) by the fibroblasts (V79/4) and the tumour cells, and ii.) from the citrate complex on the one hand and the NTA, EDTA, and DTPA complexes on the other. They show further that $\mathrm{Yb}$ citrate has no specific tumour affinity.

This study was enabled by the Deutsche Forschungsgemeinschaft (Fr 883/1-1).

\section{0}

McQuarrie S.A. ${ }^{1}$, McEwan A.J.B. ${ }^{1}$, Golberg L. ${ }^{2}$, Golberg K. ${ }^{1}$, Boniface G.R. ${ }^{2}$, and Noujaim A.A. ${ }^{2}$.

1. Cross Cancer Institute, 1. Biomira Inc., Edmonton, Canada.

\section{MODELLING AND DOSIMETRY OF MONOCLONAL ANTIBODY} 170H.82 (Tru-Scint $\left.{ }^{\circledR} A D^{\text {TM}}\right)$ IN BREAST CANCER.

MAb-170 (Tru-Scint ${ }^{\mathbb{Q}} A D^{\mathrm{TM}}$, Biomira Inc.) is a murine monoclonal antibody (IgG 1 Subclass) and was derived from the immunisation of mice with synthetic TF antigen. It was selected on the basis of in-vitro reactivity with human adenocarcinoma tissue. The antibody was supplied as a single vial, frozen liquid formulation. Radiolabelling was achieved with the addition of sodium pertechnetate (1600 $1800 \mathrm{MBq}$ ) to directly label the reduced thio-groups of the antibody.

Pharmacokinetic analysis was performed on a subset of breast cancer patients imaged as part of a Phase II clinical trial at the Cross Cancer Institute in order to evaluate the effect of dosage. Either 1, 2, or $4 \mathrm{mg}$ of radiolabelled antibody was administered. Serum, urine and serial whole body images were collected at defined time points and used to obtain a kinetic model to describe the in-vivo distribution of the MAb. The data were best described by a two compartment model of the form: $\mathrm{C}(\mathrm{t})=\mathrm{C}_{1} \mathrm{e}^{-\lambda 1 \mathrm{t}}+\mathrm{C}_{Z} \mathrm{e}^{-\lambda \mathrm{zt}}$. The pooled distribution half-lives were $4.3 \pm 1.8 \mathrm{~h}$ and the pooled terminal elimination half-lives were $36.9 \pm 6.2 \mathrm{~h}$. No significant difference was found between the different doses.

The radiation dose to target organs was calculated from the cumulated activity in selected source organs as defined by conjugate view gamma camera images. Standard MIRD formulae were used to calculate the absorbed dose. 
601

Yu. Lishmanov, V. Chernov, v, Skuridin, W. Ussov, N. Krivonogov, I, Dygai.

Institute of Cardiology, Tomsk, Russia.

THALL IUM-199 DIETHYLDITHIOCARBAMATE - A NEW RADIOPHARMACEUTICAL FOR BRAIN PERFUSION ESTIMATION

The alm of the study was pharmacokinetic and scintigraphy investigation of Thallium-199 diethyldithlocarbomate (199T1-DDC).

Blood and inner organs radiometry was performed in 60 rats $1-120$ minutes after intravenous 199TIDDC administration. Dynamic and static scintigraphy of 199T1-DDC studies were performed in 8 rabbits.

After intravenous injection, 199T1-DDC passed blood-brain barrier rapidly. Brain 199Tl-DDC uptake in rats became maximal imnediately after infusion and was quite high during 2 hours. Brain 19971-DDC quality scintigram in rabbits was equal that of $99 \mathrm{mTC}-\mathrm{HMPO}$. Due to the short half $11 \mathrm{fe}$ of $199 \mathrm{Tl}(7.4 \mathrm{~h}$.) the total dose to critical organs was 5-7 times lower than with conventional radionuclide - 201Tl.

Thus, 199TIDDC myocardial scintigraphy is a new perspective method for cerebral blood flow estimation.

602

J.J.J. Borm, M.J. Bruno, J.W. Ganzevoort, F. van Wagterdonk, M. Rademaker, B.J. de Bondt, R. Storm van Leeuwen, E.A. van Royen Department of Nuclear Medicine, and Department of Gastroenterology and Hepatology, Academic Medical Centre, Amsterdam. The Netherlands

\section{BIODISTRIBUTION OF ERBIUM OXIDE AFTER ORAL INTAKE}

Relevant data concerning the absorption of erbium (Er) from the gastrointestinal tract in humans is hardly available. The dosimetric tables in the ICRP-30 and ICRP-61 documents are based on the more extensively studied lanthanide cerium.

For a clinical study, we performed dual isotope gastric emptying studies, using a post-production neutron activated $170 \mathrm{Er}$ enriched erbium-oxide enteric coated pancreatic enzyme preparation. The erbium was available as Er2O3.

Four hours and eight hours after the ingestion of $4 \mathrm{MBq} 171 \mathrm{Er}$ we obtained whole body scans using a dual headed camera at a scanspeed of $10 \mathrm{~cm} / \mathrm{min}$ and a high energy collimator. Because of the $\mathrm{pH}$-sensitive enteric coat of the pancreatic enzyme preparation that regulates the release of the pancreatic enzymes and $170 \mathrm{Er}$ enriched erbium-oxide, uptake of 171 Er from the gastrointestinal tract was only possible after the gastrointestinal $\mathrm{pH}$ exceeded 5.5 which is likely to happen in the small intestine. The preparation also contained significant amounts of $24 \mathrm{Na}$, up to $400 \mathrm{kBq}$, resulting in increased background levels due to collimator septum penetration and down-scatter effects.

Preliminary data from whole body scans in five volunteers indicate that the fractional uptake from the gastrointestinal tract did not exceed 0.0003 , which matches the assumptions made in ICRP-30. Except from the gastrointestinal tract no specific uptake was seen in any organ system, especially not in the skeleton, liver or kidneys, as was previously reported by other investigators in animals. However, this may partially be due to the high level of $24 \mathrm{Na}$ downscatter

Our preliminary results confirm the assumptions used in the ICRP-30 tables. Further work on $24 \mathrm{Na}$ downscatter correction is required.
603

MRB Puncher, PJ Blower

Nuclear Medicine Dept, Kent and Canterbury Hospital, Canterbury, UK and Biological Laboratory. The University, Canterbury, UK

SUB-CELLULAR In-111 DISTRIBUTION AND MICRODOSIMETRY IN In111-OXINE LABELLED LEUCOCYTES BY AUTORADIOGRAPHY

Methods: In conjunction with frozen section microautoradiography, computer image analysis methods were applied to the analysis and quantitation of In-111-oxine labelled leukocyte sections and superimposed autoradiographs. Rapid cell fractionation was used to confirm the results. Results: The emulsion (Ilford K2) response was linear over the concentration range investigated $\left(0-33 \mathrm{MBq} \mathrm{m}^{-1}\right)$. Resolution of radionuclide distribution was better than $2 \mu \mathrm{m}$. The autoradiographs showed no dependance of radiolabel uptake on cell type. Classification of all cells into intervals according to grain density suggests an exponential rather than normal distribution, with approximately 50\% of cells having little or no radiolabel. In any one sample, cells which were heavily labeled were approximately 10 times more likely to be found in aggregates (60\% found in aggregates, mostly neutrophils) than cells which were not heavily labeled ( $6 \%$ found in aggregates); and the grain densities were at least twofold higher over nuclei than over cytoplasm. The last observation was confirmed by the rapid cell fractionation method which showed that approximately $57 \%$ of the total radioactivity was bound to nuclei. Conclusions: Frozen section microautoradiography is a practical and reliable approach to determining subcellular distribution of In-111. The radiolabeling process (not the isolation process) causes aggregation of neutrophils. Uptake is not significantly dependant on cell type, but only a fraction of cells are appreciably labeled. The radioactive concentration in cell nuclei is at least two-fold higher than in cytoplasm.

\section{4}

V. Bláha, F. Niček, J. Buk

Dept. of Nucl. Med., Faculty Hospital, Charles University

Šrobárova 50, 10034 Praha 10, Czech Republic

\section{MODIFIED METHOD OF ${ }^{99 m}$ Tc PERTECHNETATE PER RECTAL PORTAL SCINTIGRAPHY}

The method of S. Shiomi et al. is an excellent non-invasive method to prove and evaluate the grade of portosystemic shunt. PS index (PSI) may, however, be considerably discorected in three ways.

a) The heart ROI in patients with normal or close to normal portal circulation can be marked with lower degree of accuracy owing to the small number of counts in the heart area

b) The liver ROI in patients with serious portosystemic shunts can be marked with lower degree of accuracy owing to the same reason

c) The emission of ${ }^{99 . m} \mathrm{Tc}$ pertechnetate rectal activity in patients with hepatomegaly or in patients with short abdomen may influence considerably the activity of liver ROI

Our modified method solves these problems by following way: The basic five minutes dynamic portal scintigraphy after $800 \mathrm{MBq}$ ${ }^{99 m} \mathrm{Tc}$ pertechnetate per rectum continues up to ten minutes when $80 \mathrm{MBq}$ of ${ }^{99 \mathrm{~m}} \mathrm{Tc}$ sulphur colloid is administered i. v. on the start of 6th minute. The radioactive bolus in the heart and radiocolloid accumulated in the liver enable to delineate both the heart and liver ROIs by sufficient accuracy. The final static liver/kidney scintigraphy offers to obtain additional valuable informations.

The border of rectal (bowel) activity is marked as the first ROI followed by ROIs of heart and liver. The case of interference the bowel and liver ROIs is solved using our own computer programme.

The method has been tested on more than 100patients. The normal PSI was up to 7. The span of PSI in patients with liver diseases was from 9 up to 91 . 
605

W H Thomson, N R Williams and L K Harding

Department of Physics and Nuclear Medicine, City Hospital NHS Trust, Dudley Road, Birmingham, B18 7QH, UK.

A MODEL OF EXCRETED ACTIVITIES OF RADIOPHARMACEUTICALS FROM PATIENTS TO THE DRAINS.

Hospitals usually need to document the excreted activity from patients to the sewerage system. Within the United Kingdom, Her Majesty's Inspectorate of Pollution have issued guidelines which are deliberately pessimistic eg. for all long-lived radionuclides the excreted activity is assumed to be $100 \%$ of the administered activity, except $131 \mathrm{I}$ outpatient therapy and 32P therapy, where the figure is $30 \%$. All $99 \mathrm{mTc}$ radionuclides are assumed to be $30 \%$.

However, in order to assess the radiological impact of patient excretions, it is important that a more appropriate model of the situation should be used. Most excretions can be expressed as a sum of exponential components. We have derived a model for excretion for a single exponential component. In ICRP53, the whole body loss is generally expressed as a sum of exponential components with appropriate coefficients. These values have been used in conjunction with our model to derive the total excreted activity for a range of radiopharmaceuticals.

For long half-life radionuclides, we have shown that the excretion for an exponential component with activity $A$ at time $t=0$ is

$$
\text { A. } T_{1 / 2} B / T_{1 / 2} \text { E }
$$

where $T_{1 / 2} B$ is the biological loss half-life, $T_{1 / 2} E$ is the effective loss half-life.

For $99 \mathrm{mTc}$, the aspect of discontinuous bladder excretion can be taken into account and ICRP 53 assume a $3 \frac{1 / 2}{2}$ hour voiding time. Since only $6 \%$ of $99 \mathrm{mTc}$ remains after 24 hours of physical decay, excretions were derived for 7 urinary voids.

These models will be derived and explained. Using this, a comprehensive table has been collated and will be presented, but in particular the levels for some common long-lived radionuclides are 1311 (thyrotoxicosis) : $60 \%, 32 \mathrm{P}: 43 \%, 67 \mathrm{Ga}: 78 \%$, and $201 \mathrm{Tl}$ : $20 \%$.

\section{$605 a$}

S.Lejde-Svegborn, K. Stenström, B.Erlandsson, R. Hellborg, S.Mattsson, L.E.Nilsson, B. Nosslin, G. Skog, A.Wiebert

Depts. of Radiation Physics (Malmö) \& Nuclear Physics, Univ. of Lund, Lund, Sweden HIGH-SENSITIVITY MEASUREMENT OF ${ }^{14} \mathrm{CO}_{2}$ IN EXPIRED AIR WITH ACCELERATOR MASS SPECTROME TRY

organic compounds labelled with ${ }^{14} \mathrm{C}$ are used in different "breath tests". The expired metabolic end-product ${ }^{14} \mathrm{CO}_{2}$ is usually measured by liquid scintillation counting (LSC). Due to the long $\mathrm{T}_{\mathrm{w}}$ ( 5730 years) very few of the ${ }^{14} \mathrm{C}$ atoms present distintegrate during measurement. With mass spectrometry (MS) it is theoretically possible to measure all ${ }^{14} \mathrm{C}-$ atoms present.ordinary $M S$ has however a detection limit $\left(\approx 10^{-6}\right.$ of the amount of $\left.{ }^{12} \mathrm{C}\right)$ which is not sufficient for our purposes. With accelerator mass spectrometry (AMS) it is possible to measure ${ }^{14} \mathrm{C}$ down to $10^{-14}$ of the amount of ${ }^{12} \mathrm{C}$. Comparisons show that AMS is more than a hundred times more sensitive than LSC.

In ${ }^{14} \mathrm{C}$-triolein tests for fat absorption it was possible to demonstrate ${ }^{14} \mathrm{CO}_{2}$ in expired air for several months after ingestion. 30 \% of the substance was catabolised rapidly, while the remaining $70 \%$ had a very slow turnover. The measurements have given possibility to calculate a realistic radiation dose, based on data for the total amount administered. The AMS-technique makes long-term studies of biokinetics of ${ }^{14} \mathrm{C}$-labelled pharmaceuticals possible, and enables the use of much lower activity than hitherto in investigations on human.
606

S. M. Ametamey, I. Günther, A. Antonini, H. -F. Beer, K. L. Leenders, P. C. Waldmeier ${ }^{1}$ and P. A. Schubiger Division of Radiopharmacy, Paul Scherrer Institute, 5232 Villigen PSI, Switzerland, ${ }^{1}$ Ciba Geigy AG, Basle Switzerland

Radiosynthesis and evaluation of $\left.{ }^{11} \mathrm{C}\right]$-brofaromine, a potential tracer for measuring MAO-A activity with PET

Brofaromine [4-(5-methoxy-7-bromobenzofuranyl)-2-piperidine $\cdot \mathrm{HCl}]$ is a potent, selective and reversible inhibitor of monoamine oxidase $A$ (MAO-A) and its inhibition is also known to be tissue and time dependent. Two different methods for the labelling of $\left.{ }^{111} \mathrm{C}\right]$-brofaromine were applied. The one step method at low carrier concentration consisted of a direct $\left.{ }^{11} \mathrm{C}\right]$-methylation of the desmethyl precursor with [ $\left.{ }^{11} \mathrm{C}\right]-\mathrm{CH}_{3} \mathrm{I}$ in DMSO at $80^{\circ} \mathrm{C}$ for $5 \mathrm{~min}$. After separation of the $\mathrm{N}$-methylated side product (RP-18, Bondclone, MeCN/0.1\% $\mathrm{H}_{3} \mathrm{PO}_{4}=$ $31: 69 \%) 30 \%$ yield of $\left[{ }^{11} \mathrm{C}\right]$-brofaromine was obtained (EOB and decay corrected). The three step route with high carrier amounts of $\mathrm{CO}_{2}$ consisted of protection of the secondary amino functionality with the $\mathrm{t}$-BOC-group, $\mathrm{O}$-methylation with $\left[{ }^{11} \mathrm{C}\right]-\mathrm{CH}_{3} \mathrm{I}$ in $\mathrm{DMF}$ at $80^{\circ} \mathrm{C}$ for 5 min and deprotection of the t-BOC-group (TFA) resulting in a total yield of $10 \%$ (from EOB and decay corrected). Radiochemical purity of $\left[{ }^{11} \mathrm{C}\right]$-brofaromine in both cases was $>98 \%$.

PET studies using $\left[{ }^{11} \mathrm{C}\right]$-brofaromine in a thesus monkey demonstrated a slow accumulation of radioactivity in the brain. In a follow-up experiment, the monkey was given 2 doses of $2 \mathrm{mg} / \mathrm{kg}$ p.o. of the selective irreversible MAO-A inhibitor clorgyline 19.5 hours and 7 hours before injection of $\left.{ }^{11} \mathrm{C}\right]$-brofaromine. No decrease in radioactivity uptake in the monkey brain was observed. These results indicate either a high degree of non-specific binding or a slow pharmacokinetic of MAO-A inhibition by ${ }^{11} \mathrm{C}$-brofaromine as known from the literature. The available time frame of PET measurements with ${ }^{11} \mathrm{C}$ is probably too short to clarify this question. In conclusion, we therefore propose the use of $\left[{ }^{76} \mathrm{Br}\right]$ (half-life $=16 \mathrm{~h}$ ) as a suitable PET radionuclide.

\section{7}

K. Någren*, C. Halldin, L. Müller, C.-G. Swahn.

*Radiopharmaceutical Chemistry Laboratory, Turku University Cyclotron/PET Center, Turku, Finland and Department of Clinical Neuroscience, Psychiatry and Psychology section, Karolinska Institute, Stockholm, Sweden.

\section{IMPROVED SYNTHESIS OF SOME DOPAMINE REUPTAKE LIGANDS FOR PET USING [C-11]METHYL TRIFLATE.}

The dopamine reuptake system has during the last years been in the focus for studies of Parkinson's disease and other neuronal disorders with PET and SPECT. Commonly used PET radioligands are phenyltropane derivatives such as [C-11] $\beta$-CIT and [C-11] $\beta$-CFT which has been prepared by methylation of the norcompounds using [C-11]methyl iodide.

Recently a new C-11-methylation reagent, [C-11]methyl triflate has been introduced (Jewett; 1992). We have performed a comparative study using these two C-11-labelled precursors for the preparation of three radioligands, [C-11] $\beta-C I T,[C-11] \beta-C F T$ and the novel radioligand $[\mathrm{C}-11] \alpha-\mathrm{CIT}$, for examination of the dopamine reuptake system with PET.

The reactivity of [C-11]methyl triflate is very high. [C-11]ß-CIT, $[\mathrm{C}-11] \beta-\mathrm{CFT}$ and $[\mathrm{C}-11] \alpha-\mathrm{CIT}$ were all prepared in $60-90 \%$ decay corrected yield from [C-11]methyl triflate by reaction with $0.15 \mathrm{mg}$ norcompound for $1 \mathrm{~min}$ at $60^{\circ} \mathrm{C}$, while the yields using [C-11] methy] iodide and similar amounts of precursor with heating at $80^{\circ} \mathrm{C}$ for $3-5$ min were 3-4 times lower. The products were all purified using straight-phase HPLC. The total radiochemical yields of the final sterile and pyrogen-free products were $40-60 \%$ (from [C-11]CO2, decay corrected) within 30-35 min from EOB.

The low amount of precursors needed when preparing these dopamine reuptake ligands from [C-11]methyl triflate is important when considering the commercial availability and cost of the precursors and also for the final HPLC-purification. The results demonstrate that using [C-11]methyl triflate comparatively higher yields are obtained using shorter reaction times and at lower reaction temperatures, which improves the specific radioactivity of the products and are important for a reliable automated routine production for PET. 
Sunday, 21 August - Wednesday, 24 August 1994

608

K.A. Bergström ${ }^{1}$, C. Halldin, C-G Swahn, J.T. Kuikka ${ }^{1}$, J. Tiihonen 1 , L. Farde and E. Länsimies 1

Kuopio University Hospital ${ }^{1}$, Kuopio, Finland and Karolinska Institut, Stockholm, Sweden.

\section{DETERMINATION OF LABELLED METABOLITES OF} [I-123]ß-CIT IN HUMANS WTTH AN HPLC METHOD

$\beta$-CIT (2- $\beta$-carbomethoxy-3- $\beta$-(4-iodophenyl)tropane) has been developed as a radioligand for studying dopamine and serotonin reuptake in humans with both SPET and PET. [I-123]ß-CIT was labelled with a specific radioactivity of $>2900 \mathrm{Ci} / \mathrm{mmol}$ and radiochemical purity $>98 \%$. Informed consent was obtained from six subjects (three controls and three patients with Parkinson's disease) who were examined. The percentage of the radioactive metabolites and the parent compound was determined from venous plasma samples obtained up to 4 hours after injection of [I-123]ß-CIT. An HPLC method was developed that allowed a rapid analysis of many samples. Two metabolites of [I-123]ß-CIT were found, a polar metabolite $(\mathrm{P})$ and a lipophilic metabolite $(\mathrm{L})$. The relative composition of plasma at time points 1,2 and 3 hours after injection of the tracer was (mean $\pm \mathrm{SD}, \mathrm{n}=6$ ) $40 \pm 7,35 \pm 5$ and $27 \pm 7(\mathrm{P}) ; 11 \pm 2$, $29 \pm 8$ and $43 \pm 8$ (L); $49 \pm 5,36 \pm 6$ and $30 \pm 8$ (parent compound) respectively. Between 1 and 4 hours the percentage of the lipophilic metabolite increased whereas the percentage of the polar metabolite and the parent compound decreased. Compared to the results obtained from an earlier described ethyl acetate extraction method the HPLC method demonstrate a delayed appearance of the lipophilic metabolite. Due to the different labelling positions the analysis of [I-123]3-CIT metabolites provides additional information to that previously described for [C-11]ß-CIT. In conclusion two metabolites were observed with an HPLC method after injection of [I-123]ß-CIT in humans.

\section{9}

P.Benz 1 , F.Oberdorfer ${ }^{2}$, M.Orbe ${ }^{3}$

${ }^{1}$ RNS PET-Zentrum Wiesbaden, 2 DKFZ Heidelberg, , 3 GEMS Uppsala

\section{RESULTS AND QUALITY OF THE FDG-MICROLAB MODULE}

Since an increased number of clinical sites recently started with PET imaging they all are in need of getting a sufficient amount of $18-\mathrm{F}$ desoxy glucose (FDG). The way to solve the problem is to find a method for FDG production at the clinical sites themselves. We report first results of PETrace MicroLab and quality of FDG. The MicroLab module produces automaticay 2-[18F] Fluoro -2Desoxy glucose using 18-F Fluorid and Tetraacetyl-2-0-Trifly-DManose. According to the regulations of Mulholland a phase transfer catalytic method was used.The catalyst is bound covalent on a polymer which serves for trapping 18-F Fluorid. 18-F-Fluorid is given automatically or by hand to a single use cartridge where the different steps for synthesis are running. Nearly the whole amount of $18-$ F/18-O enriched $\mathrm{H}_{2} \mathrm{O}$ is saved on a reservoir and can be used again for FDG production. Quality control was done by ionexchangechromatography on a strong alkalinecolumne (CarboPac1, Dionex) with $\mathrm{NaOH}$ with gradienttechnic (start $15 \mathrm{mM}$, end $55 \mathrm{mM} \mathrm{NaOH}$, runtime $\left.50 \mathrm{~min}, \mathrm{t}_{\mathrm{ret}}(\mathrm{FDG})=35 \mathrm{~min}\right)$. A amperometric technic was used for the detection of FDG.

Within 6 month over 50 FDG production runs with MicroLab were performed. 1.5 to $12 \mathrm{GBq} 18$-F-Fluorid were used starting the production whereas production time lasted $52 \mathrm{~min}$. An overall average amount (not decay corrected) of over $32 \%$ FDG was reached. The TLC tested radiochemical purity was $>95 \%$ in $49 / 51$ production runs. In one production no injectable FDG could be produced, because the F-18 contained volatile Fluorid compounds (presumably Freones). Meanwhile more than 130 patients could be examined with FDG produced by MicroLab without any side effects. Using the GEMS MicroLab the availibility of FDG will improve with good quality of the tracer. This is a main condition for the furher developement of PET studies at clinical sites.

\section{0}

\section{Radwan ${ }^{1,2}, \underline{\text { H.L. Sharma }}^{1}$ and C.A. McAuliffe ${ }^{2}$}

Department of Medical Biophysics 1 , University of Manchester and Department of Chemistry ${ }^{2}$, UMIST, Manchester, U.K.

\section{OXINE CONJUGATES OF Co-55, Ga-66 AND Cu-64 AS POTENTIAL CELL LABELS FOR PET SCANNING}

Oxine conjugate of In-111 has been used to label a variety of cells. In recent years, other complexing agents such as tropolonate has been used for In-111. Among the PET isotopes, Ga-68 has been used to label platelets. The short half-life of $\mathrm{Ga}-68(68.1 \mathrm{~min})$ poses a severe limitation in situations where a longer waiting period is required for the tracer to accumulate in the organs of interest before imaging. We have already established procedures for a regular production of a number of medium half-life positron emitting radionuclides in our laboratory. Among these Co-55 $\left(\mathrm{T}_{1 / 2}=18 \mathrm{hrs}\right)$, $\mathrm{Cu}-64\left(\mathrm{~T}_{1 / 2}=12 \mathrm{hrs}\right)$ and $\mathrm{Ga}-66\left(\mathrm{~T}_{1 / 2}=9.4 \mathrm{hrs}\right)$ have chemical properties similar to those of In and therefore their complexes with oxine would make good potential candidates for labelling cells with these PET isotopes. With these objectives in mind, we have investigated the conjugation of $\mathrm{Co}, \mathrm{Cu}$ and $\mathrm{Ga}$ to oxine at microscale and tracer level using atomic absorption flame spectrometry and radioactive tracers. Complexes of $\mathrm{Ga}$ with oxine precipitate quantitatively at a $\mathrm{pH} 2$, complexes of $\mathrm{Co}$-oxine at a $\mathrm{pH}$ of 4.5 and $\mathrm{Cu}$-oxine at a $\mathrm{pH}$ of 2.7. In preliminary experiments, $\mathrm{Ga}-67$-oxine labelled red blood cells with a $84 \%$ labelling efficiency. Experiments are underway for the labelling of lymphocytes, platelets and neutrophils with $\mathrm{Co}-55, \mathrm{Ga}-66$ and $\mathrm{Cu}-64$.

\section{1}

R. Franceschini, M. Chinol, G. Jori, P.G. De Filippi, G. Bocchiotti, F. Lúnghi, C.J. Palestro Sorin Biomedica, University of Padua \& Turin Italy. L.I. Jewísh Med. Ctr. New Hyde Park, NY IODINE-131 LABELED $\mathrm{Zn}$ (II) -PHTHALOCYANINE: A POTENTIAL THERAPEUTIC AGENT FOR SOLID TUMORS

Phthalocyanines (PC), which are composed of four benzoindole rings bound by nitrogen bridges, are a class of photosensitizing agents which spontaneusly localize in, and are retained by, some solid tumors, causing destruction of these tumors upon illumination with red light. Feasibility of labeling Zn(II)-PC with I-13I followed by incorporation into dipalmitoylphosphatidylcholine (DPPC) liposomes has been previously shown. We now report the has been previously shown. We now report the biodistribution data of I-13I labeled $\mathrm{Zn}$ (II) -PC-
DPPC in mice bearing fibrosarcomas in the thigh $(\mathrm{N}=20)$. The uptake of this compound in tumor (T), thyroid (TR) liver (L), kidney (K) and injection was:

$\begin{array}{rrrrr} & \text { T/TR } & \text { T/L } & \text { T/K } & \text { T/M } \\ 3 \mathrm{~h} & 0.8 & 0.1 & 0.9 & 3.4 \\ & & & & \\ 15 \mathrm{~h} & 1.9 & 0.2 & 2.1 & 7.0 \\ 4 \mathrm{~h} & 3.0 & 0.2 & 2.8 & 9.1 \\ 4 \mathrm{~h} & 3.1 & 0.2 & 3.0 & 8.8 \\ 72 \mathrm{~h} & 2.4 & 0.2 & 2.8 & 10.3 \\ 9 \mathrm{~h} & 2.8 & 0.2 & 2.4 & 8.5\end{array}$

The rapid uptake in tumor, which remained constant upto $90 \mathrm{~h}$, is similar to previously published data for the unlabeled compound in the same animal model. The elevated $\mathrm{T} / \mathrm{M}$ ratios as well as the satisfactory tumor to normal (liver and kidney) and target (thyroid) organ ratios suggest the potential use of PC as carriers of long lived radionuclides for tumor therapy. 
612

B. Meller-Rehbein ${ }^{1}$, K. Schomaecker ${ }^{2}$, H. Schicha ${ }^{2}$, M. Baehre1, E. Richter ${ }^{1}$

${ }^{1}$ Clinic of Radiotherapy and Nuclear Medicine, Medical University of Luebeck, ${ }^{2}$ Clinic of Nuclear Medicine, University of Cologne, Germany

123 I-DIETHYLSTILBESTROLPHOSPHATE - A POTENTIAL TRACER FOR RECEPTORIMAGING AND THERAPY?

Diethylstilbestrolphosphate (DSEP) is known as a cytostatic active pharmaceutical in prostatic carcinoma since 1952. The therapeutical effects of DSEP are caused by the estrogenlike behaviour of diethylstilbestrol (DSE), which is formed in the tissue by dephosphorylation of the tetrapotassium salt. Different approaches have been reported intending to label DSEP with radioiodine. Unfortunately, neither the radiochemical purity nor the stability of the labelling product were sufficient. Because of the importance of radioiodinated DSEP in diagnosis and therapy (estrogenreceptor-status in breast cancer) we searched for a method of labelling and isolating ${ }^{*} I-D S E(P)$.

$50 \mu \mathrm{I}(6 \mathrm{mg} / \mathrm{ml})$ DSEP-solution and $37 \mathrm{MBq}{ }^{123} \mathrm{I}^{-}$ (NaI in $50 \mu \mathrm{l}$ ) were incubated for $1 \mathrm{~h}$ with $50 \mu \mathrm{l}$ chloramin-T-solution $(0,2 \%)$ at $\mathrm{pH} 2$ and $60^{\circ} \mathrm{C}$. The reaction mixture was purified by HPLC (conditions: Time 15 min., RP-C 18 -column, eluent $\mathrm{H}_{2} \mathrm{O}$ /ethanol/acetic acid $79 / 20 / 1$ as gradient during $5 \mathrm{~min}$. up to $19 / 70 / 1$ and then constant for 10 min.) 123 I-DSEP retention time is $10 \mathrm{~min}$. The substance was proved to be stable within $48 \mathrm{~h}$ (radiochemical purity $>97 \%$ ).

Preliminary biodistribution studies on male mice bearing mamma-carcinoma will be reported.

Our first results suggest that "I-DSEP is a useful tracer for estrogenreceptor mapping and might also be suited for radionuclide therapy.

\section{3}

\section{A. Mohammed, M. Eisenhut}

Department of Nuclear Medicine,

Radiol. Universitätsklinik, 69120 Heidelberg

Tc-99m-BAT COMPLEXES WITH QUATERNARY AMINO
GROUPS: MODEL-COMPOUNDS FOR MUSCARINIC GROUPS: MODEL-COMPOUNDS
ACETYLCHOLINE-REZEPTORS

The development of ${ }^{99} \mathrm{~m} \mathrm{Tc}$ complexes with receptor binding characteristics is one of the present challenges in synthetic radiopharmaceutical chemistry. Currently we are pursuing experimental work with Tc-BAT-complexes exhibiting a neutral complex core and substituents with quaternary ammonium functions in the side chain.
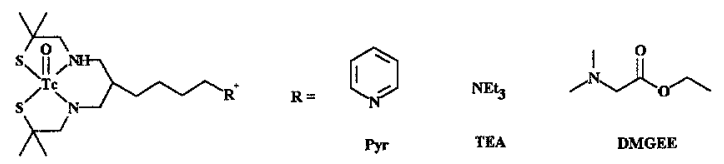

The ${ }^{99} \mathrm{~m} \mathrm{Tc}$ complexes which were obtained with high yields have been tested in animals for their affinity to organs comprising muscarinic acetylcholine (m-ACh) receptors. Since the biodistributin data proved significant myocardial uptake with delayed washout the question arose whether the uptake values of the indicated compounds are either the result of their affinity to $\mathrm{m}-\mathrm{ACh}$ receptors or to ACh esterase binding. Challenging experiments with atropin, a m-ACh antagonist, and phenyltrimethylammonium chloride, an ACh-esterase inhibitor, were performed and will be discussed. The organ distribution data, in \% inj. dose/g organ weight, 5 and 60 minutes after i.v. injection into NMRI mice are summarized below:

$$
\begin{array}{lrrrrrrrr} 
& \multicolumn{2}{c}{\text { Blood }} & \multicolumn{2}{c}{\text { Beart }} & \multicolumn{2}{c}{\text { Lung }} & \multicolumn{2}{c}{\text { Liver }} \\
& 5^{\prime} & 60^{\prime} & 5 & 50^{\prime} & 5^{\prime} & 60^{\prime} & 5 & 60^{\prime} \\
\mathbf{R}=\text { PYI } & 1.49 & 0.16 & 3.60 & 3.53 & 4.18 & 1.89 & 21.70 & 19.90 \\
\text { R = TEA } & 3.64 & 1.40 & 2.99 & 1.56 & 7.20 & 3.26 & 21.00 & 15.70 \\
\text { R = DMGII } & 1.51 & 0.15 & 1.49 & 0.89 & 2.31 & 0.77 & 20.70 & 5.61
\end{array}
$$

614

G. Demonceau, C. Van Hove, G. De Rick, K. Sunaert, H. Colaert, C. Monte.

St Elisabeth Hospital, Zottegem, Belgium.

EVALUATION OF THE EFFICACY AND SAFETY OF STABILIZED TCHM-PAO.

Weisner et al. recently (EJNM, 20, 8, 1993) succeeded in stabilising Tc- HM-PAO in vitro by the addition of cobalt chloride. Coles and Dickett were able to demonstrate in the rat its innocuousness in doses up to 1000 times the quantities applied in normal medical use (personal communication). The aim of this study was to test this observation in humans, both as to stability and harmlessness.

Thirty-four patients gave their consent to participate in the study. Ceretec $^{\circ}$ was obtained from $3.7 \mathrm{GBq}$ fresh Tc-pertechnetate from the 2nd generator elution ( 2 types of generators were used). Cobalt chloride ( $200 \mathrm{ug} / 2$ $\mathrm{ml}$ ) were added $2 \mathrm{~min}$ after reconstruction. Thirty patients were injected with 0.9 to $1.3 \mathrm{GBq}$ of Tc-HM-PAO reconstituted for less than 2 hours. The cerebral tomography was started $20 \mathrm{~min}$. after. Moreover, in 4 extra patients, leucocytes were labelled with more than one hour old tracer, the yield of the labelling being measured. Clinical parameters were followed up to 24 hours after injection. The images obtained were compared both visually and quantitatively (in the brain studies: distribution within the brain and the brain/soft tissue proportion) to those obtained in a control group, matched for age and sex, and using non-stabilised Tc-HM-PAO, injected within $30 \mathrm{~min}$.

No toxicity or other side effects were noticed. There was no significant difference between the images of both groups. The yield of leucocyte labelling was statistically unaltered.

The use of cobalt chloride as a stabilising agent for Tc-HM-PAO, proved, within the scope of this study, to be efficacious in routine application and harmless for the patient

615

J.C. Hung and L.M. Thorson

Nuclear Medicine, Department of Diagnostic Radiology, Mayo Clinic, Rochester, Minnesota, U.S.A.

\section{THE EFFECTS OF GENERATOR ELUATE AGE ON THE RADIOCHEMICAL PURITY OF FRACTIONATED Tc-99m MAG3 KITS}

The fractionation of MAG3 cold kits provides an economical way for preparing Tc-99m MAG3. However, our nuclear pharmacy recently noted that fractionated Tc-99m MAG3 kits sometimes failed radiochemical purity $(\mathrm{RCP})$ testing (i.e., $\mathrm{RCP}<90 \%$ ) when older eluates were used. The purpose of this study was to evaluate the effects of eluate age on the radiochemical purity (RCP) of fractionated Tc-99m MAG3 kits. Each of 4 MAG3 cold kits was initially diluted with $10 \mathrm{ml} \mathrm{N} \mathrm{N}_{2}$-purged $0.9 \% \mathrm{NaCl}$ solution and subdivided into 10 aliquots of $1-\mathrm{ml}$ MAG3 solution and overlayered with $\mathrm{N}_{2}$. The 40 fractionated MAG3 vials were immediately frozen at $-20^{\circ} \mathrm{C}$ for storage. Fractionated MAG3 kits were reconstituted with $1 \mathrm{ml}$ of $1,110 \mathrm{MBq} \mathrm{Tc}-99 \mathrm{~m}$ eluate from a long-ingrowth generator (i.e., $\geq 72 \mathrm{hr}$ ) at every hour during the 6hr post-elution period. The RCP of each Tc-99m MAG3 preparation was determined using the recommended Sep-Pak ${ }^{\otimes}$ C18 column chromatography at $1,2,3,4,5$, and 6 hr postreconstitution. All fractionated MAG3 kits prepared with generator eluates $4 \mathrm{hr}$ old or less maintained an average RCP value of $96.3 \pm 2.5 \%(n=144)$ throughout the 6 -hr evaluation period following preparation. However, Tc-99m eluate that was 5 $\mathrm{hr}$ and $6 \mathrm{hr}$ old after elution resulted in kit failure rates of $16.7 \%$ (1/6) with $\mathrm{RCP}=89.3 \%$ and $50 \%(3 / 6)$ with $\mathrm{RCP}=88.2 \%$, $83.9 \%$, and $87.1 \%$, respectively. These data suggest that the use of Tc-99m eluate of more than $5 \mathrm{hr}$ old for the preparation of a fractionated Tc-99m MAG3 kit is associated with a high rate of kit failure and should therefore be avoided. 


\section{6}

A. Piffanelli, M. Marengo, C. Aprile, C. Bagnara, G. Candini, F. Del Dottore, E. Di Guglielmo, P. Ferretti, S. Lazzari, D. Pancaldi.

Nucl. Med. and Med. Phys. Depts., Bologna, Cesena, Ferrara, Genova, Pavia and Reggio Emilia (Italy).

QUALITY CONTROL OF Tc $9 \mathrm{c}^{9 \mathrm{~m}}$ GENERATORS: AN ITALIAN MULTICENTER EXPERIENCE.

Evaluation of radiopharmaceutical quality is an important task for scientists in the Nuclear Medicine field, from both an economical and technical point of view. The Italian Association of Nuclear Medicine (AIMN) thus encouraged and supported a multicenter program on quality control of $\mathrm{Tc}^{99 \mathrm{~m}}$ generators supplied in our Country. Six participating Centers checked more than 100 generators over six months, measuring elution yield and profile, $\mathrm{pH}$, aluminum and other metal breakthrough, radiochemical and radionuclidic purity, by rapid semiquantitative methods and accurate reference techniques. Some of the results are reported:

\begin{tabular}{|c|c|c|c|c|c|c|c|}
\hline COMPANY & 1 & 2 & 3 & 4 & 5 & 6 & TOT \\
\hline $\begin{array}{l}\mathbf{p H} \\
\text { median } \\
\text { range }\end{array}$ & $\begin{array}{c}6 \\
5.5-6.5\end{array}$ & $\begin{array}{c}5.35 \\
4.3-6.1\end{array}$ & $\begin{array}{c}6 \\
5.0-6.0\end{array}$ & $\begin{array}{c}6 \\
4.5-7.0\end{array}$ & $\begin{array}{c}6 \\
5.0-6.0 \\
\end{array}$ & $\begin{array}{c}6 \\
5.5-7.0 \\
\end{array}$ & $\begin{array}{c}6 \\
4.3-7.0 \\
\end{array}$ \\
\hline $\begin{array}{c}\text { Al breakthr. }(\mu \mathrm{g} / \mathrm{l}) \\
\begin{array}{c}\text { median } \\
\text { range }\end{array} \\
\end{array}$ & $\begin{array}{r}563 \\
417-819 \\
\end{array}$ & \begin{tabular}{|c}
88 \\
$0.1-790$ \\
\end{tabular} & \begin{tabular}{|c|}
0.06 \\
$0.06-38$ \\
\end{tabular} & \begin{tabular}{|c|}
0.06 \\
$0.06-534$ \\
\end{tabular} & $\begin{array}{c}0.06 \\
.06-1003 \\
\end{array}$ & $\begin{array}{c}0.06 \\
0.06-514 \\
\end{array}$ & $\begin{array}{c}0.06 \\
.06-1003 \\
\end{array}$ \\
\hline $\begin{array}{l}\text { RC purity }(\%) \\
\text { mean } \\
\text { s.d. } \\
\end{array}$ & $\begin{array}{c}99.82 \\
0.34 .\end{array}$ & $\begin{array}{c}99.59 \\
0.58 \\
\end{array}$ & $\begin{array}{l}99.53 \\
0.12 \\
\end{array}$ & $\begin{array}{l}99.71 \\
0.66 \\
\end{array}$ & $\begin{array}{c}99.56 \\
0.09 \\
\end{array}$ & $\begin{array}{r}99.64 \\
0.68 \\
\end{array}$ & $\begin{array}{r}99.64 \\
0.58 \\
\end{array}$ \\
\hline \begin{tabular}{|c|}
$95 \%$ Activ. vol. (ml) \\
mean \\
s.d. \\
\end{tabular} & $\begin{array}{l}3.93 \\
1.36 \\
\end{array}$ & $\begin{array}{l}3.15 \\
1.2 \\
\end{array}$ & $\begin{array}{r}4.5 \\
0.99 \\
\end{array}$ & $\begin{array}{l}4.08 \\
1.9 \\
\end{array}$ & $\begin{array}{l}4.94 \\
0.71 \\
\end{array}$ & $\begin{array}{l}5.49 \\
1.88 \\
\end{array}$ & $\begin{array}{l}4.27 \\
1.72 \\
\end{array}$ \\
\hline $\begin{array}{c}\text { Mo-99(\%) } \\
\text { median } \\
\text { minimum value } \\
\text { maximum value }\end{array}$ & $\begin{array}{l}2.50 \mathrm{E}-05 \\
7.95 \mathrm{E}-03\end{array}$ & $\begin{array}{l}1.11 \mathrm{E}-05 \\
1.12 \mathrm{E}-03\end{array}$ & $\begin{array}{l}.24 \mathrm{E}-08 \\
2.69 \mathrm{E}-05\end{array}$ & $\begin{array}{l}4.80 \mathrm{E}-1 \\
1.40 \mathrm{E}- \\
5.89 \mathrm{E}-\end{array}$ & $\begin{array}{l}3.62 \mathrm{E}-0 \\
1.39 \mathrm{E}-0\end{array}$ & $\begin{array}{l}4.40 \mathrm{E}-05 \\
5.47 \mathrm{E}-02\end{array}$ & $5.47 \mathrm{E}-$ \\
\hline
\end{tabular}

The development of the program provided AIMN with an up to date description of the National market for one of the most important radiopharmaceuticals and helped establish a number of reference Centers for information and support on this subject for other Clinical Institutions and local Authorities.

\section{7}

Gy.A.Jánoki, L.Kórösi, L.Balogh, K.Bod6, K.Mohari and J.Láng:

F.J.C. National Research Institute for Radiobiology and Radiohygiene, Budapest, Hungary

\section{INVESTIGATIONS OF ESH GROUPS GENERATED BY ME REDUCTION FOR DIRECT LABELLING OF MOAbS WITH $99 \mathrm{mTC}$}

To define the role and quantitate endogenous sulfhydryl (eSH) groups generated by 2-mercaptoethanol (ME) reduction of antibody series of experiments were performed.

Human lgG and model anti CEA MOAb (IOR-1) were reduced with ME at molar ratio of $1000: 1 ; 3000: 1$. After 30 minutes the solution were purified on Sephadex-G-50 columns. The OD of collected fractions was determined and appropriate fractions pooled. Aliquotes were frozen and stored at $-70^{\circ} \mathrm{C}$ or lyophilized. The quantitation of $\mathrm{SH}$ concentration was accomplished by Ellman's reagent, absorbance was measured at $412 \mathrm{~nm}$. Integrity of MoAb's samples after reduction were determined by non-reduced PAGE and size exclusion HPLC. Radiolabelling with Tc-99m of thawed and freeze-dried antibody using stannous (II)-PYP was done followed by radio HPLC and biodistribution study in Balb/c/Lati/nude mice bearing CEA positive human colorectal adenocarcinoma xenografts.

The number of eSH group generated at a two ME:MoAb molar ratio were calculated to be 2.2/hlgG molecule and 3.4/anti CEA molecule. During lyophilization the number of free $\mathrm{SH}$ groups decreased by $15 \%$ (hlgG) and $30 \%$ (anti CEA). Stannous ion added prior freeze-drying prevented the re-arrangement of a eSH. At 50 ug of $\mathrm{SnCl}_{2} \times 2 \mathrm{H}_{2} \mathrm{O}$ more than $95 \%$ labelling was obtained. HPLC analysis of reduced and radiolabelled MoAbs showed one major peak $(90 \%)$ with a retention time identical to the unreduced species. Biodistribution showed high activity in blood pool, kidneys and tumour $(8.3 \% \mathrm{ID} / \mathrm{g}$ tumour at $6 \mathrm{hrs})$. eSH groups generated at controlled conditions provide a stabil binding side for $99 \mathrm{mTc}$ with retained biochemical quality and immunological function of MoAbs.

\section{8}

A. Steinsträßer, L. Kuhlmann, B. Haase, L. Warzecha

Radiochemical Laboratory, Hoechst AG; Frankfurt/Main, Germany

INFLUENCE OF LABELLING PROCEDURES ON IMMUNOREACTIVITY OF MONOCLONAL ANTIBODIES

Radiolabelling of monocional antibodies (MAb) can decrease their im munoreactivity. We studied the influence of different labelling procedures. For this a method was established to measure immunoreactivity of labelled and unlabelled MAb. After incubating the MAb with antigen positive tumorcells this procedure is able to quantify simultaneously bound activity and unbound antibodies for a direct comparison.

The labelling procedures for MAb can be divided into different categories: direct/indirect (i.e. without or with an additional linkage group) or sitespecific/allover (i.e. avoiding an impairment of the binding region of the MAb or not).

Labelling by using a chelating agent (e.g. for in-111) is an example for an indirect, non sitespecific procedure. Immunoreactivity is influenced by the amount of chelate and also by the amount of nuclide bound. In routine the decrease amounts to $15-30 \%$ for these compounds.

Most of the iodination procedures are direct but also non sitespecific. No difference is to be seen between $1-131$ and $1-125$. The decrease of immunoreactivity is $5-15 \%$. Increasing the specific activity normally has no influence on immunoreactivity but on stability of these compounds.

For the labelling of monoclonal antibodies with Technetium-99m different techniques are known, but in this context only the "Schwarz-method" is discussed. It is a direct method and is with high probability sitespecific. For this labelling procedure no influence on immunoreactivity of the monoclonal antibody was to be seen. Even increasing the activity excessively reduces not the immunoreactivity, but can decrease the radiochemical purity.

\section{9}

M. Chinol, C.V. Hamby, C.J. Palestro, S. Ferrone Long Island Jewish Medical Center, New Hyde Park, NY. New York Medical College, Valhalla, NY.

TUMOR TARGETING USING RHENIUM LABELED ANTI MELANOMA MONOCLONAL ANTIBODY

We are prospectively evaluating, in vivo, an anti-melanoma monoclonal antibody (mAb) radiolabeled with Re-188\& Re-186 for both diagnostic \& therapeutic applications. Using the direct method we labeled anti-human high molecular weight-melanoma associated antigen mab 763.74 pretreated with 100 mmol 2-mercapto ethanol, with tin reduced $\mathrm{Re}^{-188}$ or Re-186 ethanol, with tin reduced Re-188 or Re-186
overnight at $37^{\circ} \mathrm{C}$. Although labeling yields consistently exceeded 90\%, immunoreactivity decreased from $83 \%$ initially to about $50 \%$ after radiolabeling. Purifying labeled $\mathrm{mAb}$ by affinity chromatography on insolubilized anti-idiotypic mab MK2-23 yielded a preparation with nearly immunoreactivity. Radiolabeled mAb 763.74 was administered to athymic nude mice bearing human melanoma. Images \& biodistribution data were obtained $48 \mathrm{hr}$ post injection of Re-188 labeled preparation \& $96 \mathrm{hr}$ post injection of $\mathrm{Re}-186$ labeled preparation. Tumor (T) to background (BK) ratios generated from the images clearly demonstrated, as did visual inspection, the 1.4, T/BK $(96 \mathrm{~h}): 5.3$. Biodistribution data were:

\section{$\mathrm{T} / \mathrm{Blood} \mathrm{T} /$ Liver $\mathrm{T} / \mathrm{K}$ idney $\mathrm{T} / \mathrm{BK}$}

$\begin{array}{lllll}\operatorname{Re}-188(48 \mathrm{~h}) & 1.1 & 1.3 & 1.0 & 13\end{array}$

$\begin{array}{lllll}\mathrm{Re}-186(96 \mathrm{~h}) & 2.1 & 0.5 & 1.1 & 22\end{array}$

The excellent targeting of rhenium (especially Re-186) labeled anti-idiotypic purified mAb 763.74 warrants further investigation for both diagnostic \& therapeutic applications. 


\section{0}

E.Arany, P.L.Zabel, L.A.Stauder, N.J.Rideout, D.J Hill,

Lawson Research Ingtitute, St Joseph's Hospital and Department of Nuclear Medicine, University Hospital, London, ontario, CANADA

BIODISTRIBUTION OF RADIOIODINATED INSULIN-GROWTH FACTOR BINDING PROTEIN-3 (hIGFBP-3) IN RATS: A POTENTIAL ONCOLOGICAL DETECTION AGENT

Insulin-like growth factors (IGFs) are peptides that express anabolic and mitogenic activity on fetal, postnatal and transformed cells. In serum, IGF's circulate bound to IGF-binding proteins (IGFBPs...mostly IGPBP-3) which modulate their proliferative action. Several adenocarcinoma secrete IGFBP's and express binding sites for them.

Purified glycosylated human recombinant IGFBP-3 (42kDa) was labelled with I-125 and injected into non-anaesthetized rats. Concentration-time profiles were studied in blood and various organs. Autoradiography was done on major organs of localization. Uxine and blood samples were analyzed by gel permeation chromatography.

over $80 \%$ of the blood pool activity cleared by $30 \mathrm{~min}$. Gel permeation chromatography revealed radioactivity retained in the blood pool was bound in the $150 \mathrm{kDa}$ ternary complex normally found in plasma. Blood pool activity decreased to $4 \%$ by 6 hours and 0.78 at 24 hours. Liver and kidney activity peaked at 10 minutes with 248 and 5.88 respectively. In humans, the Iiver acts as the major site of IGF production. The majority of the $12.8 \%$ in the stomach at 2 hours was present in the contents. Less than is id was in the GIT and contents by 24 hours.

Autoradiography of liver samples indicated localization in Kupffer cells. In humans the MRNA for IGFBP-3 has been shown to exist in Kupffer cells. Autoradiography revealed the major kidney localization was in the glomerulus and distal tubules. Gel permeation chromatography of 10 minute urine samples indicated the protein renally excreted had been metabolized to lower molecular weight forms (<18kDa). In the stomach most of the radioactivity was localized in the epithelial mucosa. Some localization was also evident in the smooth muscle of stomach and small intestine.

somatostatin was previously found to have an improved biodistribution (Trenal, Ihepatobiliary clearance) when labelled via bifunctional chelates rather than radioiodine. Further studies are planned on tumour bearing animals and with IGFBF's radiolabelled with bifunctional chelates.

\section{1}

S. Zunic, V. Bosnjakovic,

S. Pavlovic, D.

Sobic, N. Kozarevic, M. Lekic

Institute of Nuclear Medicine, CCS, Belgrade, Yugoslavia

$\beta$-ENDORPHIN PLASMA LEVEL IN PATIENTS WITH SYSTEMIC LUPUS ERYTHEMATOSUS

Hormones, in general, especially those affecting the hypothalamo-pituitary-adrenal axis, including ACTH and POMC (proopiomelanocortine) related peptides, like beta-endorphin, may be significant in the SLF (systemic lupus erythematosus) pathogenesis. Following the previously published data about attenuated response of SLE patients to stress, the aim of our study was to compare beta-endorphin plasma levels in control volunteers and SLE patients. We investigated 9 healthy volunteers and 8 patients with clinically confirmed diagnosis of SLE. Levels of plasma beta-endorphin were estimated using Nichols Institute radioimmunoassay system. A significant decrease $(p<0.01)$ of plasma beta-endorphin in SLE patients (32.14-14.76 $\mathrm{pg} / \mathrm{ml}$ ) was found in comparison with the control group $(70.42-16.31 \mathrm{pg} / \mathrm{ml})$. Our results support the findings of suppressed response of SLE patients to stress and indicate the role of beta-endorphin in interaction between the immune and endocrine systems.
622

Giganti $\mathrm{M}^{1}$, Dittadi $\mathrm{R}^{1}$, Gion $\mathrm{M}^{1}$, Cittanti $\mathrm{C}^{\mathrm{l}}$, Malagutti $\mathrm{R}^{1}$, Brazzale $\mathrm{A}^{1}$, Capitanio $\mathrm{G}^{2}$, Gelli $\mathrm{MC}^{4}$, Menegon $\mathrm{A}^{2}$, Gardini $\mathrm{G}^{4}$, Piffanelli $\mathrm{A}^{1}$. 'Center for the Study/of Biological Markers of Malignancy and ${ }^{2}$ Service of Pathol. Anatomy, General Hospital, Venice. ${ }^{3}$ Chair of Nuclear Med., University of Ferrara. ${ }^{4}$ Inst. of Pathol. Anathomy, Hospital SM. Nuova, Reggio Emilia. Italy.

MEASUREMENT OF THE ONCOGENE cerbB-2 EXPRESSION AS PROGNOSTIC PARAMETER IN BREAST CANCER: A MULTICENTER COMPARISON STUDY OF 4 DIFFERENT DETERMINATION METHODS

The cerbB2 oncogene was proposed as a prognostic indicator in human breast cancer. In this study we evaluated 4 methods to measure the cerbB2 protein expression in order: a) to compare two IRMA kits (ELISA, Oncogene Science (ONC) and EIA, Triton Diagnostic (TRIT)), with the immunohistochemical assay (IHC) and Western blotting (WB); b) to compare the relationships between cerbB2 protein levels obtained with the different methods used and some other parameters. Tissue samples from 250 patients with primary breast cancer were partly formalin fixed and paraffin embedded for IHC (mAb clone 3B5 - ONC) and partly pulverized and homogenized in phosphate buffer. The homogenates were in part lysated and assayed for WB and ONC assays and in part ultracentrifuged. The pellets were lysated and used for TRIT assay. Positivity rates with $\mathrm{IHC}$ was $28.5 \%$. We categorized samples analyzed with WB, ONC and TRIT as overexpressing (OEx) and noOEx (-) using a cut-off obtained from non-malignant specimens. Using the second cut-off the positivity was $28 \%, 26 \%$ and $28 \%$ respectively. The differences between the 4 methods were not significative (Cochran $Q$ test: $p=0.677$ ) The concordance rates range between $76 \%$ (WB vs TRIT) and 90\% (ONC vs IHC). 78\% of the low-OEx samples identified by WB and TRIT and 93\% identified by ONC were negative with IHC. The relationships between erbB2 and both ER and PR showed an inverse association, independent of the method used. However, ONC and TRIT showed direct correlation between CerbB 2 and both ER and PR in erbB2(-) samples, and an indirect one in cerbB2 $(+) /(++)$ samples. All the methods showed a trend toward higher positivity rates of erbB2 in samples with more than 3 positive lymphnodes. Moreover, in the cerbB2 $(+) /(++)$ group assayed by ONC and WB higher cerbB2 levels in $\mathrm{N}+$ then $\mathrm{N}$ - samples were found, while an inverse trend was found in erbB2(-) samples. In conclusion, the four methods results seem to disclose confident correlation indexes.

\section{3}

\section{W.-G. Franke, E. Mueller, A. Mueller}

Clinic for Nuclear Medicine and Institute for Forensic Medicine, Technical University of Dresden, Germany

SERUM- hTG-CONCENTRATIONS IN CORPSES MEASURED BY IRMA-RESULTS OF A STUDY AND ESTIMATION OF SIGNIFICANCE FOR FORENSIC MEDICINE

Using precipitation electrophoresis Yada et al. recognised in 1971 thyroglobin in blood of corpses died by strangulation. In contrary, they could not find this hormone in other causes of death. These findings were interpreted by delivery of the intact hormone from the damaged thyrocytes and seem to present a support to prove strangulation as cause of death. However, Mueller et al found in 1990 using an IRMA-test serum Tg concentrations elevated persons died by drowning or intoxication. To clear these different results further on the study was extended at a number of further causes of unnatural death. For hormone measurements samples were measured from blood of both heart ventricles. The IRMA kit Dynotest Tg was used with a normal range of $\mathrm{Tg}$ concentration of 5-70 $\mu \mathrm{g} / \mathrm{L}$. Now the results can be presented of serum $\mathrm{Tg}$ concentration measuring accomplished in 193 male and 192 female corpses, most of them died by unnatural death. The following $\mathrm{Tg}$ mean values were found (measuring unit : $\mu \mathrm{g} / \mathrm{L}$ ): strangulation 135.5 , sudden accidental death 89.9 , delayed postaccidental death 170.6 , intoxication 194.1, hypothermia 100.5 , drownsing 155.9 , bolus- and reflex death 161.0 . Increase of mean values could be confirmed in all groups compared with normal values of living men. A very wide range of single values was seen in all groups. Furthermore the mean values found in femals were distinctly higher than in males. Until now these results are interpretable by a ante mortem disturbance of hierarchic functional structures followed by an uncontrolled delivery of thyroglobulin from thyroid in the bloodstream. The extent of hormonal increase seems to depend on duration of agonal phase. Therefore, postmortal measured serum Tg concentrations might become significance for forensic medicine because of support destination of date of death in persons died by unnatural death. 
624

K.Mohari, T.Kubasova, L.Balogh, Gy.A.Jánoki, Gy.J.Köteles, E.Duda:

F.J.C. National Research Institute for Radiobiology and Radiohygiene Budapest, Hungary

PREPARATION OF 125-I-TNF $\alpha$ AND ITS USE IN RIA, IN VITRO CELL-BINDING ASSAY AND BIOASSAY.

Because of its anti-tumour properties, we have examined the binding of tumour necrosis factor-alpha (TNF $)$ to tumour cells in vivo and in vitro. In our laboratory a new magnetic separation radioimmunoassay has been developed for measurement of TNF $\alpha$. Thereafter the radiolabelled $\mathrm{TNF}_{\alpha}$ was used in radiobioassay.

The parameters of the RIA are as follows: specific activity of tracer $7-8000 \mathrm{kBq} / \mathrm{ug}$, sensitivity: $10 \mathrm{pg} / \mathrm{ml}$, NSB: $1-2 \%$, B/B。 $40-50 \%$ (at dilution of antibody: 6000 ), recovery: $90-95 \%$, measuring range of assay: $0-10^{4} \mathrm{pg} / \mathrm{ml}$, time of assay: 24 hours. It was found that the sera of mice bearing colorectal adeno-carcinoma contained higher amounts of TNE, , (500-800 $\mathrm{pg} / \mathrm{ml}$ ) as compared to the values for normal animals: (20-100 $\mathrm{pg} / \mathrm{ml})$. For radiobioassay, labelled $\mathrm{TNF}_{\alpha \mathrm{C}}$, specific activity: 2$3000 \mathrm{kBq} / \mathrm{ug} \mathrm{TNF}_{\alpha}$, was injected intravenously into tumourbearing mice, and the distribution of tracer was followed 1,2 and 3 hours after i.v. injection. The highest level of radioactivity (9-12\% of injected activity) in tumour tissue was detected by 2 hours of i.v. injection.

In vitro studies of 125-I-TNF binding to tumour cells were performed on Calu-1 cell culture (a line established from human lung carcinoma). 125-l-TNF $(37 \mathrm{kBq} / 2000 \mathrm{U} / \mathrm{ml})$ was added to the proliferating cells at various time-points of culturing. By measurement of bound radioactivity, information was obtained on the ability of cultured tumour cells to bind TNE in the control experiments and also after the effect of ionizing radiation $(0.25$ 4 Gy). Radiation-induced cell membrane perturbation was followed by dose-dependent changes in TNF ch $_{\text {binding, too. }}$ The applied RIA method seems to be suitable for determination of the concentration of $\mathrm{TNF}_{\alpha}$ in sera. 125-I-TNF $\mathrm{T}_{\boldsymbol{C}}$ showed high localization in tumour tissue and slow blood disappearance.

\section{5}

A. Brocchi, A. Benucci, M. Tommasi, G. La Cava.

Department of Clinical Pathophysiology, Nuclear Medicine Unit, University of Firenze, Italy.

ENDOTHELIN-1-LIKE IMMUNOREACTIVITY IN HUMAN URINE Endothelin (ET) is a potent vasoconstrictor 21 aminoacid peptide originally isolated from culture supernatants of porcine endothelial cells. In search of the genomic DNA library, three distinct human ET-related genes were cloned and designated ET-1, ET-2 and ET-3; ET-1 is the classical porcine/human ET.

We report immunoreactive ET in urine. $5 \mathrm{ml}$ urine were treated with $800 \mathrm{ul}$ of diluted acetonitrile 1:1 ( $/ \mathrm{v})$ with water. ET was extracted by Sep-Pak $\mathrm{C} 18$ cartridges (Waters-Millipore) using methanol/water as the mobil phase. The peptide was eluted with methanol/water in the volume ratio of $85 / 15$, evaporated to dryness and reconstituted in 250 ul RIA buffer. We used the ETRIA system available from Biomedica, Vienna, Austria. Because of coextraction of all three isoforms of ET as well as because of crossreactivity of the anti-ET-antibody, our RIA cannot discriminate within the same. Characteristic quality control data were: the recoveries of ET added to urine and during the extraction procedure were $110 \pm 7.69 \%$ (mean $\pm S D)$; the limit of detection was $0.8 \mathrm{fmol} / \mathrm{ml}$; the precision profile shown a CV\% from 25.5 to 6.28 in the dose range from 2.53 to $131 \mathrm{fmol} / \mathrm{ml}$; within assay precision was $8.36 \%$ for a mean value of 127 $\mathrm{fmol} / \mathrm{ml}$ (SD 10.7). The hourly urinary excretion of ET (mean \pm SD) in 5 healthy females (mean age $77.2 \pm 4.02$ SD years) was $0.268 \pm 0.033 \mathrm{ng}$; the urinary excretion in 4 healthy males (mean age $79.2 \pm 8.01$ years) was $0.678 \pm 0.324 \mathrm{ng} / \mathrm{h}$; the urinary excretion in 4 healthy females (mean age $33.5 \pm 5.68$ years) was $0.418 \pm 0.221 \mathrm{ng} / \mathrm{h}$. By Wilcoxon test the only significant difference resulted between elderly males and females $(p<$ 0.02 ). Our results show that immunoreactive $E t$ is detectable in urine. The measurement of Et in urine may prove to be useful as an indicator of $24 \mathrm{~h}$ integrated ET production.

\section{6}

B. Bagni, M. Lodi, A.R. Cavallini, L.Chiccoli, S. Ferrazzini, I. Bagni, G.C. Candini Nuclear Medicine Dpt, Presidio Nultizonale USI 31, Health Physics Dpt, St Anna Hospital, 44100 Ferrara, Italy.

SENSITIVITY AND SPECIFICITY OF CYERA AND RELATED TUMOR MARKERS IN A POPULATION OF NON SMALI CELL LUNG CARCINOMA.

This study was performed on 98 patients referred to the Dpt. of Pneumology of USL 31 (Ferrara - Italy) for clinical and instrumental examination for suspicion of lung cancer (mean age 51 years, SD 3 years range 4455 years). CEA, TPA, Ca19-9, CYFRA were assayed using commercial method in a well experienced RIA laboratory.

Sensitivity and specificity were determined from a reference population and patients with liver and gastrointestinal non neoplastic involvement. The results are summarized in the following tables:

$\begin{array}{lrrcc} & \text { CYFRA } & \text { TPA } & \text { CA19-9 } & \text { CEA } \\ \text { Specificity } & 0.97 & 0.87 & 0.94 & 0.91 \\ \text { Sensitivity } & 0.58 & 0.70 & 0.53 & 0.64\end{array}$ $\begin{array}{llll}\text { Sensitivity } 0.58 & 0.70 & 0.53 & 0.64 \\ \text { A discriminant analysis among the plasma }\end{array}$ concentration data for the T.M. (Tumour Markers) were also performed:

CYFRA Threshold Error:

CYFRA $\quad 1.63$ Th

97.62

Ca19-9 15.98

The ROC curves for this T.M. evidentiate a greater surface under the curve (better response) for CYFRA $(.704)$ and TPA $(.760)$ in comparison with CEA $(.64)$ and Ca19-9 $(.52)$. our study evidentiates that CYFRA and TPA may be the best combination for the follow up of Non Small Cell Carcinoma of the lung.

\section{7}

B.Cynowska, J.M._Slominski_ J. Wyrwinski, J. Skokowsk1

Depts, Nuclear Medicine, Lung Diseases, Thoracic Surgery, Med.Academy of Gdansk, Gdansk Poland

CLINICAL EVALUATION OF CYFRA 21-1 IMMUNORADIOMETRIC ASSAY AS A MARKER FOR LUNG CANCER

Cyrokeratin 19 is a subunit of cytokeratin intermediate filament expressed particulary in epithelial tumor cells including bronchial cancers. The purpose of our study was to asses clinical value of Cyrfa 21-1 immunorad lometric assay from CIS Biointernational for lung cancer Serum levels of Cyfre 21-1 were measured in 110 patients with lung cancers $/ 96$ NSCLC and 14 SCLC/, in 20 patients with benign lung disease and in 60 heal thy individuals $/ 30$ non-smokers and 30 heavy smokers/. The mean cytokeratin 19 level in controls was $0.69 \pm 0.37 \mathrm{ng} / \mathrm{ml} / \mathrm{s} . \mathrm{D}_{\text {. }} /$. In a group of patients with non-malignant lung diseases the average value was $0.84 \pm 0.32 \mathrm{ng} / \mathrm{ml}$. Cyfra 21-1 serum level in patients with lung cancers was signiflcantly higher - all cancers $7.84 \pm 13.75 \mathrm{ng} / \mathrm{ml}$, group of NSCLC $8.69 \pm 14.52$ $\mathrm{ng} / \mathrm{ml}$ and group of SCLC $2.03 \pm 1.47$. Using a threshold of $1.33 \mathrm{ng} / \mathrm{ml}$, sensitivity and specificity were 0.68 and 0.95 , respectively. Levels of Cyfra 21-1 was strongly associated to presence of metastases and performance status in NSCLC patients $/ p<0,001$ by ANOVA for both/. Conclusions: 1/ Cyfra 21-1 assay is elinically useful in NSCLC patients, 2/ In this type of lung cancer eytokeratin is level correlates with disease stage and performance status. 


\section{8}

S.Stajić, O.Frim, R.Novaković, N.Kostić, M. Ratković, L.Rajner, V. Šobić

Institute of Nuclear Medicine, Clinical Center of Serbia, Belgrade, Yugoslavia

VASOACTIVE INTESTINAL POLYPEPTIDE (VIP) AND SOMATOSTATIN SERUM LEVELS AS VALUABLE MARKERS OF APUD ENDOCRINE CELLS DAMAGESIN ACUTE RADIATION LUNG

The purpose of the study: In past few years in 16 patients (pts.) under the radiation therapy the acute radiation pneumonitis (ARP) was discovered. In 9 patients with the small cell lung cancer (SCLC), in 5 with breast cancer and in two with Hodgkin's disease the prescribed doses were 46 and $60 \mathrm{~Gy}$ (in 11 pts. the therapy was finished). Nearly few years we tried to test the secretion of APUD endocrine cells in patients under the radiation therapy, to see if the levels of neurotransmitters may indicate the radiation damages. Methods: Somatostatin and VIP were determined in patients sera by RIA kits from Prof.Bloom Laboratory (London) and NSE (a good indicator of lung tissue and spine damages) by RIA CIS.Gastrin was determined by RIA CIS.

Results :

Patients with lung cancer, breast cancer and with Hodgkin before Rth in ARP

Somatostatin (pmol/l)

$10,6 \pm 4$,

$6,1 \pm 2,7$

$112,1 \pm 43,2$

$13,9 \pm 5,3$

$24,7: 13,1$

$34,1 \pm 9,6$ *

$9,2 \pm 3,5$

$11,5 \div 3,5$

Gastrin (pmol/1)

* $\mathrm{p}<0,01$

Conclusion: The significantly high somatostatin and VIP serum levels in ARP were caused by low gastrin levels (gastrin cells radiation damage). The spine was not seriously affected by radiation therapy (low NSE serum levels) and also that the treatment was successful in patients with SCLC.

\section{9}

J. Spitz $^{1}$, P. Benz ${ }^{1}$, M. Köllermann ${ }^{2}$

1 Private Praxis of Nuclear Medicine and 2 Clinic for Urology, Municipal Hospital (HSK), Wiesbaden, Germany

\section{Comparitive Studies with Free and Complexed Serum PSA}

This study reports the results of measurements with an established dual monoclonal reference assay in comparison with a new developed nonisotopic, chemoluminescent assay for PSA, based on both monoclonal and affinity purified polyclonal $\mathrm{Abs}$ in a single test system.

I Assays: a) Tandem PSA Hybritech b) ACS PSA Ciba-Corning II Specimen: 64 patients with 430 specimen collected between jan. ' 89 and nov. '93

Both assays showed an excellent overall correlation $(r=1.0)$ for the range from $0.1-3500 \mathrm{ng} \mathrm{PSA} / \mathrm{ml}$. This was also found for the range from $0.1-4.0 \mathrm{ng} / \mathrm{ml}(\mathrm{n}=224)$, the slope beeing almost 1.0 with a minimal offset : $\mathrm{ACS}=0.94 \times$ Hybritech $+0.11(\mathrm{r}=0.94)$. Looking for the range from $4.0-20.0 \mathrm{ng} / \mathrm{ml}(\mathrm{n}=81)$ the $\mathrm{r}$-value decreased to $\mathrm{r}=0.88$ and the slope went up: $\mathrm{ACS}=1.43 \times$ Hybritech +0.75 . Selecting the values between 20 and $3500 \mathrm{ng} / \mathrm{ml}(\mathrm{n}=123)$ the slope was lower and the offset greater: $\mathrm{ACS}=1.27 \times$ Hybritech +7.2 . After radical prostatectomy patients showed comparably low PSA values $(<0.4 \mathrm{ng} / \mathrm{ml})$ in both assays, the ACS values beeing more differentiated. In case of tumor progression ACS values rose more prounced in all cases.

Demonstrating a high correlation in the normal range, the ACS values seem to provide a more pronounced differentiation of tumor progression due to a steeper rise

On the other hand after a long period of high inter-assay comparibility for PSA values the new technique of ACS to estimate also uncomplexed PSA makes it necessary to label the results of each PSA estimation with the name of the applied kit.

\section{0}

J. Spitz ${ }^{1}$, P. Benz ${ }^{1}$, Th. Bickert ${ }^{1}$, M. Köllermann ${ }^{2}$

1 Private Praxis Nuclear Medicine and 2 Clinic for Urology, Municipal Hospital ( HSK), Wiesbaden, Germany

\section{ESTIMATION OF PSA IN URINE}

PSA-levels in blood are of great importance and interest for the diagnosis and follow up of prostate cancer in various stages. We checked the technical aspects and clinical usefulness of a PSA estimation in urine.

a) Estimation of PSA levels in urine of normal men and women, looking for circadian rythms, $24 \mathrm{~h}$ values and various influences (stability, reproducibility, $\mathrm{pH}$ values, ejaculation, location of excretion), using a commercial IRMA (Tandem-R, Hybritech). b) Specimens of 64 patients with cancer of the prostate in stage A-C were analyzed for PSA levels in blood and urine before and after radical prostatectomy.

The technical performance was as good in urine as in blood. No PSA was found in the urine collected by nephrostoma. There was a maximum of PSA at noon and at midnight. Midstream values were as representative as a $24 \mathrm{~h}$ collection. Values of normal men and patients with prostate carcinomas showed large variations. 25 patients without chemotherapy after radical prostatectomy demonstrated urine values between 0 and $8,3 \mathrm{ng} / \mathrm{ml}$ whereas $87 \%$ of 30 patients with endocrine therapy after radical prostatectomy had values below $0.3 \mathrm{ng} / \mathrm{ml}$ of PSA in urine.

PSA in urine is not excreted by the kidneys, independent from residual tumor tissue but excreted by periurethral glands, which are strongly influenced by endocrine therapy. The potential clinical use of a PSA determination in urine might be the termination of adiuvant chemotherapy and the control of patient compliance during endocrine therapy.

\section{1}

M.G. RAMOS, A. R. NOGUEIRA

Departamento de Medicina Nuclear I.P.O.F.G. Lisboa - PORTUGAL

\section{COMPARISON OF THE VALUES OF CEA AND CA19-9} DETERMINED BY IRMA AND ELISA IN THE SAME SERUM SAMPLES

The. development of fully automated analyzers that performs immunological tests using the ELISA principle is an atractive option to change the methodology of those tests from IRMA to ELISA. In the follow-up of the patients it is imperative that the values determined for the tumour markers levels by the different methods should be comparable. In order to solve this problem we have analysed the values of CEA and CA 19-9 determined in 111 and 129 serum samples respectively by both methods and distributed along all the extension of the curves and including also dilutecl samples of those serums which values were superior to the last point of the curves.

CEA: We considcred two levels of CEA values, supcrior and inferior to $7 \mathrm{ng} / \mathrm{ml}$ in IRMA. The differences of the IRMA and EI.ISA values were "marginally" different from zcro (paired t test, s.1. $=0.05$ ) for the low level $(<7 \mathrm{ng} / \mathrm{mI}$ ), and not significantly different from zero for the high level values ( $>7 \mathrm{ng} / \mathrm{ml}$ ), and for the totality of the values.

CA 19-9: For this parameter we considered three levels $0-37,37-$ 240 and $>240 \mathrm{ng} / \mathrm{ml}$. The differences of the values obtained by IRMA and ELISA were very significantly different from zero for the low level values of the parameter, "not significantly" for the medium level and " marginally" different for the high values ( paired $t$ tes, s. $t=0.05$ ). For the totality of the values the difference is also "niarginally" different from zero.

The results showing differences between the paired values that were superior to the acceptable errors of either method inclicates the necessity of study correlations between both values and to establish correlation expressions. The correlations were all statistically significant ( $t$ test of correlation coefficient, $r$ ).

The linear and logarithmic correlation expressions obtained for the different levels of tumour markers are presented and discussed. 


\section{2}

P.Goethals, W.Lodewyckx, M.van Eijkeren*, R. Dams.

Depts. of Analytical Chemistry, Radiotherapy and Nuclear Medicine*, University Gent and University Hospital, Gent, Belgium

QUANTIFICATION OF CELL PROLIFERATION BY PET AND SPECT IN TUMOURS: PRELIMTNARY RESULTS OF A COMPARISON OF 5- $\left[{ }^{123} I\right]$ IODO-2'-DEOXYURTDINE AND [METHYL $-{ }^{22} \mathrm{C}$ ] THYMIDINE RATS

PET studies using [methy $1{ }^{11} \mathrm{C}$ ] thymidine $\left({ }^{12} \mathrm{C}-\right.$ $T d R$ ) have shown to yield useful qualitative information in patients with head and neck tumours and made it possible to evaluate therapeutic techniques. The purpose of the study was to investigate whether SPECT studies using $5-\left[{ }^{123} \mathrm{I}\right]$ iodo-2'-deoxyuridine ( ${ }^{123} \mathrm{IUdR}$ ) produce the same results.

A reliable procedure, using an rodo-bead as oxidizing agent, was developed for labelling of $2^{\prime}$-deoxyuridine with ${ }^{123} \mathrm{I}$. Optimization of the reaction conditions ( $\mathrm{pH} 1 ; 15 \mathrm{~min}$; $90^{\circ} \mathrm{C}$ ) resulted in a labelling yield of at least $65 \%$. The production time was less than $1 \mathrm{~h}$.

Three adenocarcinoma-bearing rats were injected with ${ }^{11} \mathrm{C}-\mathrm{TaR}$ and ${ }^{123}$ IUdR $(370$ and 3.7 $\mathrm{MBg}$ ). The rats were recta linearly scanned with a PET-camera, a $\gamma$ - and a SPECT camera. The PET and SPECT scans were visually comparable. All animals were sacrificed and the organs measured for ${ }^{123}$ I activity.

Preliminary results suggested the behaviour of both radiopharmaceuticals being similar. Additional tests will be necessary to investigate the correlation between cell proliferation in tumours and the amount of ${ }^{123}$ IUdR uptake. ${ }^{123}$ IUdR in combination with SPECT offers the possibility of nucleoside uptake studies (DNA-synthesis) in centres without PET facilities.

\section{3}

I.Kämpfer, D.Sorger, R.Schliebs ${ }^{\star}$, W.Kärger*,

K.Günther, W.H.Knapp

Department of Nuclear Medicine and *Paul Flechsig

Institute for Brain Research, University of Leipzig, Germany

\section{SYNTHESIS AND RADIOIODINATION OF NICOTINE WITH BIGH SPECIFIC ACTIVITY AND ASSESSMENT OF ACTIVTTY DISTRIBUTION IN THE RAT BRAIN}

Muitipie neuroreceptor changes are present in Alzheimer disease (AD); in particular, a consistent loss of nicotinic receptors in cortical tissue was reported. For future clinical studies with SPET we aimed at labeling a ligand with ${ }^{123} \mathrm{I}$ and with sufficient specific activity. $1-3 \mathrm{mg}$ 5-bromonicotine were autoclaved for $60 \mathrm{~min}$ with ${ }^{123} \mathrm{I}-\mathrm{NaI}$ in the presence of $1 \mathrm{mg}$ copper chloride and glacial acetic acid (pH 4.7). The labeling efficiency was $69 \%$, the radiochemical purity exceeded $95 \%$ (HPLC and thin layer chromatography). A specific activity of 180 $\mathrm{GBq} /$ mmole was obtained by HPLC separation of the excess of 5bromo-nicotine in the reaction mixture ( $R P 18$ Eurospher $80 \mathrm{Cl}$; methanol: $10 \mathrm{mM}$ phosphate buffer $=45: 55$ ). This product $(\mathrm{H})$ and another one with lower specific activity of $0.2 \mathrm{GBq} / \mathrm{mmole}(\mathrm{L})$ were used for autoradiographic studies in a series of rat brain slices, in order to determine the binding patterns as compared with that of ${ }^{3} \mathrm{H}$-nicotine. For physical reasons, for this comparison ${ }^{125} \mathrm{I}-\mathrm{NaI}$ was used. $\mathrm{H}$ showed autoradiographic pattern similar to that of $3 \mathrm{H}$-nicotine with accumulation in cortex, hippocampus and thalamic nuclei. With $L$ these structures were not identified. Conclusion: The described procedure for 1-123-labeling of nicotine yields high specific activity. This high activity proved to be not only sufficient, but also essential to allow the compound to bind specifically to nicotinergic receptors.

\section{4}

P.0. Zamora ${ }^{\natural}$, M. Stratesteffan, K.A. Sass ${ }^{£}$, C. Menzel, H. Bender, H.J. Biersack. Institute for Clinical and Experimental Nuclear Medicine, University of Bonn, Bonn, Germany and RhoMed Incorporated $^{1}$, Albuquerque, NM, USA

\section{A THIN LAYER CHROMATOGRAPHY METHOD FOR MONITORING THE INTERACTION OF} RADIOPHARMACEUTICALS WITH BRAIN TISSUE.

A TLC method was developed to model the interaction of radiopharmaceuticals with brain tissue.

In this method, mouse brain microsomes, from cerebral cortex, were coated onto a zone of a TLC strip, the unreacted sites blocked with gelatin, and the test radiopharmaceuticals $(100-500 \mathrm{~K}$ cpm) chromatographed over the brain materials. Brain reactive materials bound to the microsomes and were separated from the unreacted materials by the mobile phase (PBS containing $60 \%$ serum and $4 \%$ ethanol). Strips coated with gelatin (no microsomes) were used to assess non-specific binding.

Using this format, Tc-99m-HMPO was found to interact with the microsomes ( $63 \%$ ) whereas the kidney imaging agent Tc-99m-MAG3 did not $(3 \%)$. Tc- $99 \mathrm{~m}$-ethyl cysteinate interacted poorly $(9.9 \%)$, a result consistent with its known inability to be metabolized by nonprimate brain tissue. In-111-DTPA-octreotide was also bound (up to $25 \%)$. Other experimental peptide radiopharmaceuticals (Tc-99moctreotide and a Tc-99m-laminin-derived peptide) were also found to bind to the microsomes. Binding to the negative control strip was consistantiy low and frequently below $5 \%$.

In this assay, there are neither wash steps nor centrifugation steps, and assays can be completed within 15 minutes. The assay may be useful in monitoring of the interaction of established and experimental brain imaging agents with brain tissue.

635

\author{
J. MALLOL \\ DEPT. TECNOLOGIA FARMACEUTICA, FACILTAD DE FARMACIA, \\ UNIVERSIDAD DE LA LAGUNA (SPAIN).
}

FALSE RADIONUCLIDIC PURITY TEST OF RADIOPHARMACEUTICALS BY GAMMA-RAY SPECTROMETRY DUE TO THE COINCIDENCE SUMMING PEAK EFFECT.

Monographs of radiopharmaceuticals (RP's) included in the European Pharmacopoeia (Eur.Ph.) indicate the energy for the most characteristic gamma photon in order to perform the radionuclidic purity test. This test can be carried out by any suitable instrument, being the NaI(T1) detector the most used at hospital level.

In the radionuclidic purity test of RP's labelled with $75 \mathrm{Se}$ and $111 \mathrm{In}$ a main peak over $400 \mathrm{kev}$, not defined as characteristic for these radionuclides, is obtained by using a 3" NaI(TI) through-hole type detector; this unexpected peak has a fractional count rate higher than $50 \frac{\circ}{5}$ of the total count rate of the spectrun of both radionuclides. However testing the same samples by using a

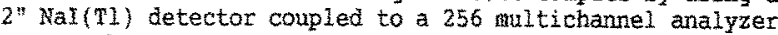
system the contribution from the abnormal peak to the total count rate registered in the whole spectrum is lower $(11.5 \%$ for $75 \mathrm{Se}$ and $3.4 \%$ for $111 \mathrm{In}$ ) and decreasing to negligible vaives when the source to detector distance is increased. These unexpected peaks are explained as an artifact due to the coincidence suming peak effect. This effect occurs with radionuclides emitting two or more cascade photons within the resolving time of the detector.

The most important consecuence of this artifact is the possibility of concluding a poor radionuclidic purity for some RP's. Therefore the possibility of the sum peak effect should be advised in the Eur. Ph. in the specific monographs for RP's for wich this effect is known. 
636

C. Piera, A. Martínez, I. Ramírez, F.J. Setoain, S. Vidal

Serv. Med. Nuclear, Hospital Clínic i Provincial and CETIR, Centre Mèdic. Barcelona. Spain.

\section{SEPARATION IN FRACTIONS OF CARDIOLITE FOR COST REDUCTION}

Separation in fractions of high cost kits is a common practice in Radiopharmacies. We have observed that good radiochemical purity (RCP) of Cardiolite cannot be obtained simply by separation in fractions and stored at $-20^{\circ} \mathrm{C}$. Since, we found that after 24 hrs of dividing the RCP of the frozen aliquots was low. Moreover, the results depended on the pertechnetate activity, RCP: $86 \pm 9 \%$ with $1073 \pm 333 \mathrm{MBq}(\mathrm{n}=6), 74 \pm 8 \%$ with $2516 \pm 444 \mathrm{MBq}(\mathrm{n}=3), 55 \pm 4 \%$ with $5661 \pm 111 \mathrm{MBq}(\mathrm{n}=3)$. These results were different from those obtained without dividing the Cardiolite and following the kit instructions, RCP:97.3 $\pm 1.5 \%$ $(\mathrm{n}=15)$.

Smaller quantities of the reducing agent in the aliquots, or damage when handling, could be the reason of the low RCP.

Accordingly, we have studied the effect of adding $\mathrm{Sn}^{+2}$. Cardiolite divided into four fractions stabilized with $25 \mathrm{mcg} \mathrm{Sn}^{+2}$ and labeled with $1850-4440 \mathrm{MBq}$ of pertechnetate resulted in the following $\mathrm{RCP}$

$$
\begin{array}{cccc}
\left(-20^{\circ} \mathrm{C}\right) \text { days } & 1-3 & 5-7 & 13-15 \\
\mathrm{RCP} & 95.5 \pm 1.3 \% & 94.2 \pm 2.9 \% & 95.9 \pm 1.3 \% \\
& (\mathrm{n}=5) & (\mathrm{n}=5) & (\mathrm{n}=5)
\end{array}
$$

High stability was found in all the experiments 7 hours after labeling, RCP: $95.0 \pm 2.1 \%(\mathrm{n}=6)$

We conclude that adding $\mathrm{Sn}^{+2}$ and separating in fractions the Cardiolite vial, a large number of low cost, high radiochemical purity doses, with an active life of 2 weeks after separation, can be obtained.

\section{7}

A.S.Hambye,L.Knaepkens,R.Vandermeiren,A.Vervaet, J.Vandevivere

Middelheim General Hospital, Antwerp, Belgium .

Failure to adequately label $\mathrm{RBC}$ in daily practice using an in vivo method : possible causes.

In 477 patients ( $>50 \%$ prior or after coronaroangiography) referred for gated blood pool study (GBP) using the in vivo method (stannous medronate), 22 studies $(4.6 \%$ ) were qualitatively considered as having poor image quality

In those patients, mean labelling efficiency of $17.8 \%$ (S.D.:14.9\%) was obtained, compared to $96.5 \%$ (S.D.:1.9\%) in a similar group of patients $(n=26)$ showing good image quality.

The possible causes for this poor binding to $\mathrm{RBC}$ was investigated. Influence of the way of administration was studied in 160 patients (15 with poor labelling), showing a highly significant relationship between direct intravenous administration ( $n=102,3.9 \%$ poor labelling), use of intravenous cannula ( $n=3,100 \%$ poor labelling) or use of butterfly needle for administration of $\mathrm{Sn}++(\mathrm{n}=13,38.5 \%$ poor labelling) and the bound fraction $(p<0.0001)$. No significant association with eventual presence of oxygen into the vial could be found

Ruling out the three patients with poor labelling and administration through an intravenous cannula, the possibie effect of the used medications on the labelling was investigated by comparing the drugs in the 19 poor labelled patients with a similar group of patients $(n=26)$ showing adequate $\mathrm{RBC}$ tagging. No significant relationship was found. Nevertheless, a $\mathrm{p}$ value of 0.1-0.2 was calculated for intravenous heparin, calcium channel blockers, chemotherapy, and digitalis derivates. Further investigation with an enlarged group of patients is required to confirm this trend

638

G. Endert, P. Kleinert, E. Klose

Klinikum Erfurt

Nuklearmedizinische Klinik

\section{"QUICK LABELLING " OF THROMBOCYTES}

- TOTAL PROCEDURE IN LESS THAN 30 MINUTES

The time expensive labelling process of radioactive labelled thrombocytes is a disadvantage in clinical usage. Also omitting certain steps the time for the whole process needs more than 45 minutes.

The reasons, why this process is time consuming, are the repeated centrifugation steps to separate the blood cells and the recommended low acceleration value (max. $1000 \mathrm{~g}$ ) to avoid the damage of thrombocytes The aim of our work was to reduce the labelling time through optimisation the steps of centrifugiation with Higher Acceleration Short Time (HAST)centrifugation.

With short-time exeeding the recommended $1000 \mathrm{~g}$ and a reduced tube filling (shorter sedimentation path) we determined the possible time reducing factor and the possible damage of platelets.

The blood cells from $25 \mathrm{ml}$ ACD-blood was selected by a first HASTcentrifugation ( $3 \mathrm{~min}, 3000 \mathrm{U} / \mathrm{min}$, $\max 1500 \mathrm{~g}, 25 \mathrm{mi}$ Monovette, 25 $\mathrm{mm}$ Diameter ). The PRP was then transferred in a $50 \mathrm{ml}$ tube followed by a second HAST-centrifugation ( $3 \mathrm{~min}, 3000 \mathrm{U} / \mathrm{min}$, $\max .1500 \mathrm{~g}$ ). The sedimented thrombocytes were redisperged and incubated ( $5 \mathrm{~min}$, $37^{\circ} \mathrm{C}$ ) with $111-\mathrm{In}-$ Oxinat. After adding of $10 \mathrm{ml}$ buffer solution the labelied thrombocytes were be selected by a third HAST-centrifugation (3 $\mathrm{min}, 3000 \mathrm{U} / \mathrm{min}$, max. $1500 \mathrm{~g}$ )

The following parameters were determined:

total labelling time:

seperation of thrombocytes:

labelling efficiency (LE):

aggregation test:

recovery (in vivo):

kinetics in patients:

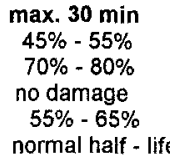

Our results show, that the labelling time can be shorten by a factor of 2 - 3 without damging the platelets.

639

S.J. Wang, C.H. Kao, W.T. Huang, Y.L. Wang and J.M. Lo

Taichung Veterans General Hospital and National Ising Hua University, Taiwan

\section{TRIAL OF TC-99m ECO AS A LEUCOCYTE LABELLING AGENT}

An Attempt was made to use Tc-99m ethyl cysteinate dimer (TC-99m ECD) to label leucocytes. The radiochemical purity of Tc-99m ECD, labelling efficiency of leucocytes, cell viability of labelled leucocytes, and stability of Tc $-99 \mathrm{~m}$ ECO labelied leucocytes were calculated.

In comparison with Tc-99m HMPAO, (1) the radiochemical purities of Tc-99m HMPAO were $84.72 \pm 0.02 \%, 61.86 \pm$ $7.77 \%, 48.92 \pm 4.65 \%, 34.65 \pm 2.60 \%, 30.69 \pm 5.21 \%, 27.03$ $\pm 2.86 \%$ and $9.23 \pm 5.18 \%$ at immediately, $1,2,4,6,8$ and $24 \mathrm{hr}$ respectively, after Tc $-99 \mathrm{~m}$ labelling $(n=8)$. The radiochemical purities of Tc-99m ECD were $97.32 \pm 0.98 \%$, $97.06 \pm 2.68 \%, 97.01 \pm 3.21 \%, 95.89 \pm 2.67 \%, 95.70 \pm$ $1.34 \%, 95.01 \pm 2.46 \%$ and $94.34 \pm 2.19 \%$ at immediately, 1 , $2,4,6,8$ and $24 \mathrm{hr}$ respectively, after Tc-99m labelling $(n=10)$; (2) The labelling efficiency of $T c-99 m$ HMPAO and Tc $-99 \mathrm{~m}$ ECD labelled leucocytes was $61.5 \pm 9.8 \%(n=21)$ and $24.5 \pm 3.7 \%(n=14)$ respectively; (3) the viability of the labelled leucocytes was both over $90 \%$ using Tc-99m HMPAO

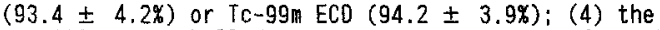
stability of labelled leucocytes were expressed as the disintegrated percentage from Tc $-99 \mathrm{~m}$ HMPAO labelled leucocytes at $1,2,4,6,8$ and 24 hours were $11.5 \pm 1.5 \%$, $16.7 \pm 1.3 \%, 20.6 \pm 2.1 \%, 25.6 \pm 2.5 \%, 29.7 \pm 2.1 \%$ and $38.7 \pm 3.1 \%$, respectively in plasma after labelling $(n=$ 21). The disintegrated percentage from Tc-99m ECD labelled leucocytes at $1,2,4,6,8$ and 24 hours were $79.6 \pm 3.9 \%$, $83.1 \pm 3.7 \%, 86.4 \pm 4.0 \%, 86.8 \pm 4.2 \%, 87.0 \pm 3.5 \%$ and $87.3 \pm 3.1 \%$, respectively, in plasma after labelling $(n=$ 14).

In conclusion, although TC-99m ECD is more stable than Tc-99m HMPAO, TC-99m ECD is not an optimal leucocyte

labelling agent due to its lower labelling efficiency and

lower stability of Tc-99m ECD labelled leucocytes. 
Sunday, 21 August - Wednesday, 24 August 1994

\section{0}

M.N. Chen, Z.T. Tsai, G. Ting, S.J. Wang, W.Y. Lin, C.H. Kao

Institute of Nuclear Energy Research and Taichung Veterans

\section{A NEW METHOD for LABELLING HUMAN SERUM ALBUMIN}

Tc-99m human serum albumin (HSA) has been used in nuclear medicine for almost 30 years. However, Tc-99m HSA is not an optimal radiopharmaceutical due to its in-vivo instability. The purpose of our study was to develop and evaluate a new method for labelling HSA.

A hexadentate nitrogen-donor chelating ligand $N, N, N$ ', $N$ '-tetrakis (2-benzymidazolylmethy1)', 2-ethanediamine (EDTB) was synthesized by condensation of 1.2-benzenediamine and ethylene-dianinete-traacetic acid (EDTA) in glycol for 20 hours followed by precipitation of the crude product with water and purification by recrystallization from hot absolute ethano7. The EDTB-HSA was prepared by mixing HSA and EOTB solutions and incubating the soluation at room temperature for 2 hours. The conjugated EDTB-HSA was separated and purified by PO-10 gel chromatography, and was added into $\mathrm{Sn}$ (II) solution, which was dispensed into vials. The labelling efficiency and in-vivo stability test of Tc-99m EDTB-HSA were performed with ITLC. The Tabelling efficiency was $92.88 \%$. The in-vivo stability was $91.19 \%$ (1 $\mathrm{hr}), 90.63 \%(2 \mathrm{hrs}), 88.93 \%$ ( $3 \mathrm{hrs}), 88.42 \%$ (4 hrs) and $85.37 \%(6 \mathrm{hrs})$, respectively.

Our preliminary study revealed the quality of EDTB-HSA was superior to the currently used commercial HSA kit, and EDTB was a potential method for labelling various protein. General Hospital, Taiwan

642

M. T. Ercan, N. C. M. Gülaldı, I. S. Ünsal, M. Kafi Ahmadi

Dept. of Nuclear Medicine, Faculty of Medicine, Hacettepe University, 06100 Sihhiye, Ankara, Turkey

Ga-67-DTPA VERSUS Ga-67-CITRATE FOR IMAGING INFLAMMATORY LESIONS

The purpose of this investigation was to evaluate $\mathrm{Ga}-67-$ DTPA for the scintigraphic visualization of experimental abscesses in comparison to Ga-67 citrate.

The biodistributions of both radiopharmaceuticals (RP's) were determined in mice with abscesses induced by turpentine 6 days previously in right thigh muscle. After i.v. injection of $3.7 \mathrm{MBq}$, the mice were sacrificed at 1, 3,6, and $24 \mathrm{~h}$ and scintigraphic images were obtained All the organs, the abscess, some muscle, blood and urine when available were obtained, weighed and counted. $\%$ uptake $/ g$ tissues and abscess/muscle (A/M), blood (A/B), intestine (A/l), liver $(A / L)$ and kidney $(A / K)$ ratios were calculated. Normal distribution of each RP was determined after i.v. administration of $37 \mathrm{MBq}$ in 3 rabbits by scintigraphic imaging up to $24 \mathrm{~h}$.

The abscesses were visualized with both RP's starting at $3 \mathrm{~h}$. The max. $A / M, A / B, A / I, A / L$, and $A / K$ ratios were $6.66 \pm 0.63$ (3 h), $8.63 \pm 0.77(3 \mathrm{~h}), 2.60 \pm 0.10(3 \mathrm{~h}), 1.57 \pm 0.12(6 \mathrm{~h})$ and $1.20 \pm 0.36(3 \mathrm{~h})$ for Ga-67-DTPA, and $4.97 \pm 2.00(6 \mathrm{~h})$, $5.24 \pm 0.37(24 \mathrm{~h}), 2.01 \pm 0.37(24 \mathrm{~h}), 1.09 \pm 0.76(6 \mathrm{~h})$ and $0.914 \pm 0.616(6 \mathrm{~h})$ for Ga-67-citrate, respectively. On normal scintigrams of rabbits the kidneys and the urinary bladder were visualized with some blood background with Ga-67DTPA.

In conclusion, Ga-67-DTPA is preferred to Ga-67-citrate, because of higher concentration ratios attained earlier and predominant excretion via kidneys.

\section{3}

F. Hosain, H.B. Pate1, R.P. Spencer

M. Giganti, L. Uccelli, A. Duatti*, C. Cittanti, P. Colamussi, A Piffanelli.

Nuclear Medicine Chair, University of Ferrara, Italy

*Chemical Physic Department, University of Bologna, Italy

EXPERIMENTAL COMPARISON STUDIES OF NEW MYOCARDIAL PERFUSION TRACERS

Experimental models of myocardial perfusion study are of paramount importance to select new radiopharmaceuticals for scintigraphic imaging evaluation of coronary artery disease. Aims of this study are addressed to evaluate: a) the biodistribution comparison results of some new tracers; b) their relative myocardium retention mechanism. a) The use of myocardial perfusion agents for the detection of areas of reduced blood flow, is dependent, at least in part, upon the single pass extraction of the respective probe compound. In Tab. 1 the behaviour of some compounds at this respect is reported in a comparative way.

\begin{tabular}{|c|c|c|c|c|}
\cline { 2 - 5 } \multicolumn{1}{c|}{} & Sestamibi & tetrofosmin & teboroxime & N-NOEt \\
\hline Charge & Cation & Cation & Neutral & Neutral \\
\hline Extraction & $1.8 \%$ & $2 \%$ & $3.5 \%$ & $4 \%$ \\
\hline
\end{tabular}

Tab.1First pass extraction of monocationic and neutral Myocardial perfusion agents.

b) The mechanism of cellular uptake and retention is the fundamental pattern for understanding the clinical meaning of scintigraphic images. In Tab. 2, are reported data obtained in our laboratory.

\begin{tabular}{|c|c|c|c|c|}
\cline { 2 - 5 } \multicolumn{1}{c|}{} & Sestamibi & tetrofosmin & teboroxime & N-NOEt \\
\hline Charge & Cation & Cation & Neutral & Neutral \\
\hline Extraction & रTl-201 & $<$ Tl-201 & $>$ T1-201 & $>$ T1-201 \\
\hline Localization & Mitochondria & Mitochondria & Lipid/hydrof. & Lipid/hydrof. \\
\hline $\begin{array}{c}\text { Metabolic } \\
\text { status }\end{array}$ & Dependence & Dependence & No Depend. & $?$ \\
\hline Heart retention & Hours & Hours & Minutes & Hours \\
\hline $\begin{array}{c}\text { Chemical } \\
\text { change }\end{array}$ & No & No & No & No \\
\hline
\end{tabular}

Tab.2: Comparative characteristics of monocationic and neutral $M$. perfusion agents.

The study of the mechanism of cellular retention of these agents, have shown that neither lipophilic properties nor the cathionic charge alone are sufficient to characterize the biologic properties of these
complexes.
Department of Nuclear Medicine, University of Connecticut Health Center, Farmington, CT-06030, USA

DIFFERENTIAL BIOLOGICAL BEHAVIOR OF RADIOTODINATED ANALOGS OF A CALICHEMICIN FRAGMENT: ESTER VERSUS ACID.

Calichemicins belong to a new class of potent antitumor antibiotics which contain a polysubstituted aromatic carboxylic acid fragment. Analogs of the fragment containing iodine have been synthesized. A methylester, Methyl 4-hydroxy-5-iodo-2,3-dimethoxy-6-methylbenzoate, was synthesized by van Laak and Scharf (Tetrahedron 45: 5511,1989 ), and was kindly supplied by Prof. H-D Scharf of the Institute fur Organische Chemie der RWTH Aachen, Germany. The acid fragment is released following hydrolysis of the ester. We investigated biological behavior of the two compounds using radionuclide tracer techniques. In the present study, iodine-131 was used to label the monoiodo methylester by exchange reactions (the yield was over $90 \%$ ). The radioiodinated ester was then hydrolyzed to obtain the acid form retaining I-131.

Biodistribution studies in mice were carried out with different times ( 3 and $10 \mathrm{~min}$, and 1,4 and $24 \mathrm{hr}$ ) following intravenous injections into tail veins. Organs were obtained, and assayed for radioactivity. Tissue distributions of these two labeled compounds were entirely different. Initial uptake in the liver, kidneys and the GI-tract was high for the ester. On the contrary, initial uptake of the labeled acid was high in the kidneys, and over $90 \%$ of radioactivity was cleared from the body in 4 hours via urinary excretion. Its biodistribution was similar to that of $\mathrm{Tc}-99 \mathrm{~m}$ labeled DTPA, as confirmed by carrying out tracer studies in mice under similar conditions. This might be useful as a radioiodinated glomerular agent for clinical applications when interfering radionuclides are present. these two labeled compounds. Animals were sacrificed at 